\title{
Novel Methods of Measuring and Visualising Youths' Physical Activity
}

Crossley, Sam G. M.

How to cite:

Crossley, Sam G. M. (2019) Novel Methods of Measuring and Visualising Youths' Physical Activity. Doctoral thesis, Swansea University.

http://cronfa.swan.ac.uk/Record/cronfa50756

Use policy:

This item is brought to you by Swansea University. Any person downloading material is agreeing to abide by the terms of the repository licence: copies of full text items may be used or reproduced in any format or medium, without prior permission for personal research or study, educational or non-commercial purposes only. The copyright for any work remains with the original author unless otherwise specified. The full-text must not be sold in any format or medium without the formal permission of the copyright holder. Permission for multiple reproductions should be obtained from the original author.

Authors are personally responsible for adhering to copyright and publisher restrictions when uploading content to the repository.

Please link to the metadata record in the Swansea University repository, Cronfa (link given in the citation reference above.)

http://www.swansea.ac.uk/library/researchsupport/ris-support/ 


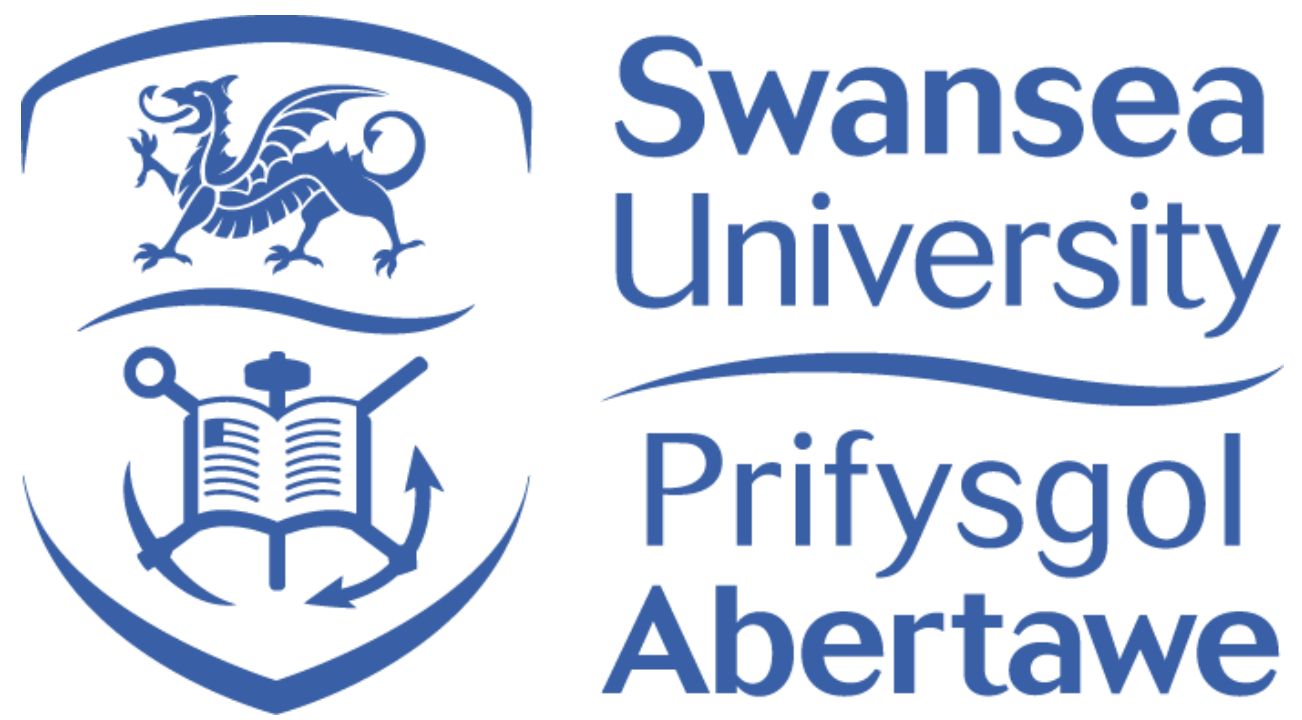

Novel Methods of Measuring and Visualising Youths' Physical Activity

\section{SAM GRAEME MORGAN CROSSLEY \\ BSc (Hons)}

Submitted to Swansea University in fulfilment of the requirements for the Degree of Doctor of Philosophy

Swansea University

School of Sport and Exercise Sciences 


\section{Dedication}

In dedication to my parents, Michael and Anne, for not only reminding me to 'stay cool' throughout but providing me with this opportunity to expand my intellectual thought.

"Knowledge speaks, but wisdom listens"

- Jimi Hendrix 


\begin{abstract}
Despite the physiological and psychosocial health benefits of youth achieving at least 60 minutes of moderate-to-vigorous physical activity (MVPA) every day, only a small proportion of youth in the UK meet this daily target. While there are several reasons for this failure to achieve the recommended amount of MVPA, recent evidence suggests that many youths lack awareness of their physical activity levels (PAL) and have difficulty interpreting and applying the guidelines to their daily activity. One solution to counteract this problem is to utilise and integrate technology, such as an objective measurement of PAL in combination with personalised feedback, to enhance youth's awareness and understanding of, and motivation for, physical activity.
\end{abstract}

Whilst accelerometers are the de facto standard in objectively measuring PAL, they have limitations when it comes to assessing non-linear movements, such as turning, that are habitual to youths' sporadic activity. Study 1, therefore, investigated the energy expenditure of turning in children, finding that the magnitude and frequency of turns completed are important considerations when measuring habitual PAL. Specifically, significant differences in energy expenditure to straight-line walking within speed were established for $2.5 \mathrm{~km} \cdot \mathrm{hr}^{-1}$ at $90^{\circ}$ turn ( $7 \%$ increase) and 3.5, 4.5 and $5.5 \mathrm{~km} \cdot \mathrm{hr}^{-1}$ for $180^{\circ}$ turns $(\sim 13 \%, \sim 14 \%$ and $\sim 30 \%$ increase, respectively). Nonetheless, one innovative method that has potential to make physical activity targets more comprehensible and actionable for youths is personalised, 3D-printed feedback that can conceptualise their PAL. Therefore, Study 2 explored youths' perceptions of, and designs for, 3D-printed visualisations of PAL. The findings revealed that youths understood the concept of visualising physical activity as a 3D object and felt that such feedback could act as a motivational tool to enhance youths PAL. Following youths' preferences for weekly models represented as abstract and bar-chart designs, two agespecific 3D models were developed to represent MVPA, across a week, with the recommended guideline depicted as a tangible goal. Study 3 sought to validate youths understanding of the age-specific 3D models and intensities of physical activity. Youth were able to correctly interpret the different components of the age-specific 3D models, although showed some misconceptions when defining moderate-intensity activities. Despite this, the age-specific 3D models showed promise to enhance youths understanding of the recommended guideline and associated MVPA intensities. Study 4 subsequently examined the efficacy of the age-specific 3D models within an intervention setting, whereby youth received personal models of their PAL. Over time, the 3D models enhanced youths' awareness of their PAL and provided a tool to compare their MVPA levels to the recommended guideline. Youths displayed their 3D models in their home environments and utilised the models as a goal-setting strategy to increase their PAL. In conclusion, the nature of the 3D models being a blend of personalised feedback, a reward and a goal-setting tool, may offer a unique strategy for the promotion of PAL and associations to the recommended guideline. 


\section{Acknowledgements}

Firstly, a very large thank you goes to my two supervisors, Dr Kelly Mackintosh and Dr Melitta McNarry for initially seeing something in me that was worth taking a chance on! Your duo insight, experience, mentorship, guidance and 24/7 academic support over the past 4 years has developed me into the researcher I am today. Of course, it wouldn't go without thanking Tizo the dog, who's forewarned barks were always a gentle reminder of how long my beard had grown!

I would like to extend my gratitude to Dr Parisa Eslambolchilar, Dr Zoe Knowles, Professor Rory Wilson, Dr Iwan Griffiths, Dr Leanne Lester, Dr Joanne Hudson and finally, Professor Michael Rosenberg, for their excellent advice and support during different stages of my $\mathrm{PhD}$.

A special mention goes to Dame Pearlette Louisy, who has not only fed me grapes from the start of my life but has continued to provide me with fruitful experiences and opportunities even within my $\mathrm{PhD}$, thank you!

To all the Swansea gang over the years: Charlie, Tom, Nick, Rich, Nils, Cain, Jess, Mayara, Zoe, Adam, Jonathan (thanks for the cake!) and Jack, thank you for all your hands and brains borrowed along the lines. Charlie, it's been a long ride together from infancy to now, we've survived shark invested scuba dives, gnarly surfs, gun point bandit attacks and now the $\mathrm{PhD}$, what's next eh?

To my parents, Anne and Michael, my brother Martin, and my señorita, Sara. I don't think the word 'acknowledgment' sums up the continuous emotional and intellectual support, wisdom, guidance and love I have received throughout this $\mathrm{PhD}$. Mum and Dad, throughout my entire life you have provided me with unwavering support through the good, bad and the ugly. I know full well that getting to this point in life is a result of your exceptional parenting, life adventures, time, understanding, friendship and more. Martin, I hope you realise the profound influence you have had on me as a big brother, how about we celebrate this with a good ol'game of $\mathrm{PhD}$ top trumps $(-)$. Sara, although you arrived at the half-way mark, I feel extremely lucky to have met and had such an understanding, supporting and loving figure beside me through this demanding time. You've played a bigger role than you think! Maybe it's time I learnt Spanish!?

Deja que las aventuras comiencen! 


\section{Declarations and Statements}

Statement 1 I, Sam Graeme Morgan Crossley, hereby declare that the work presented in this thesis has not previously been accepted in substance for any degree and is not being concurrently submitted in candidature for any degree.

Statement 2 I, Sam Graeme Morgan Crossley, hereby declare that the thesis is the result of my own investigations, except where otherwise stated and that other sources are acknowledged by footnotes giving explicit references and that a bibliography is appended.

Statement 3 I, Sam Graeme Morgan Crossley, hereby gives consent for the thesis, if accepted, to be available for photocopying and for interlibrary loan, and for the title and summary to be made available to outside organisations.

Signed: (candidate)

Date: 


\section{Contents Page}

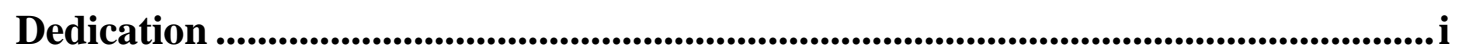

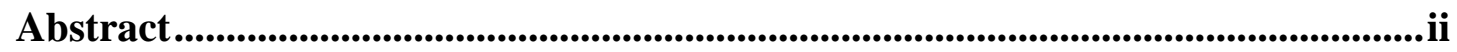

Acknowledgements...........................................................................................................................iii

Declarations and Statements ......................................................................................

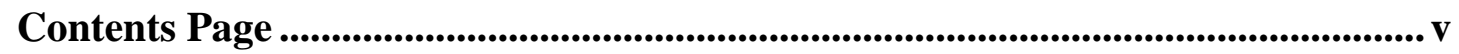

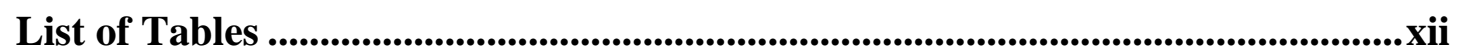

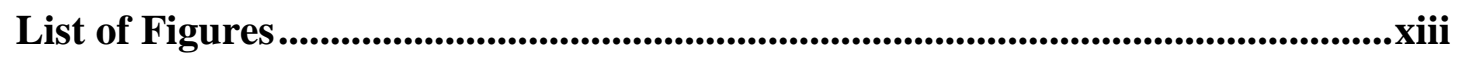

Abbreviations, Units and Symbols ............................................................................ $\mathrm{xv}$

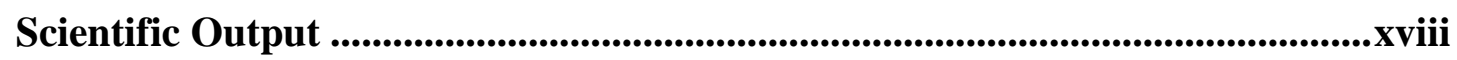

Public Engagement Output ........................................................................................... $\mathrm{xx}$

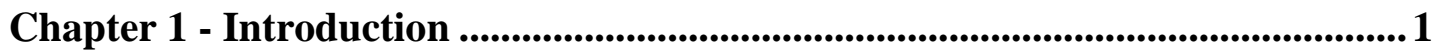

Chapter 2 - Literature Review ..........................................................................9

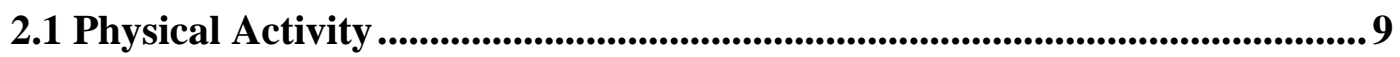

2.2 Physical Activity and Health Outcomes ........................................................... 11

2.3 Youths' Physical Activity Guidelines ................................................................... 15

2.4 Youths' Physical Activity Levels............................................................... 16

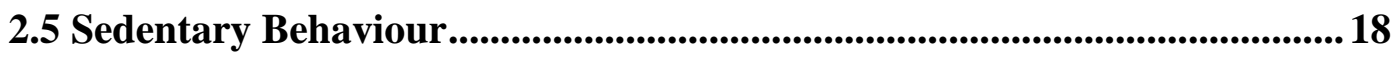

2.6 Youths' Awareness of their Physical Activity Levels....................................22

2.7 Youths' Knowledge of the Physical Activity Guidelines ............................26

2.8 Youths' Understanding of Physical Activity ....................................................227

2.9 School Context for Physical Activity Promotion...........................................332

2.10 Technology as a Barrier to Youths' Physical Activity Engagement......... 34

2.11 Technology as a Facilitator to Youths' Physical Activity Engagement ...36

2.12 Objective Measures of Physical Activity ............................................................ 40

2.13 Indirect Calorimetry for Measuring Physical Activity ............................. 41

2.14 Accelerometers for Measuring Physical Activity ....................................... 42 
2.15 The Energy Exependiture of Turning ........................................................44

2.16 The Importance of Feedback ................................................................48

2.16.1 Numerical Physical Activity Feedback ............................................................. 49

2.16.2 Numerical Visualisations of Physical Activity ................................................. 50

2.16.3 Abstract Visualisations of Physical Activity ......................................................5 53

2.16.4 Living Metaphor and Avatar Visualisations of Physical Activity...................... 55

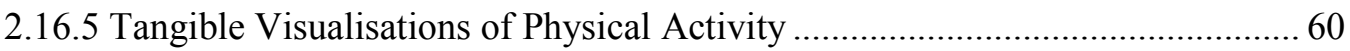

2.17 Designing Novel Technology for Youth.......................................................66

2.18 Summary and Conclusion .................................................................68

Thesis Map ............................................................................................................................ 71

Chapter 3 - Energy Expenditure Associated with Walking Speed and Angle of

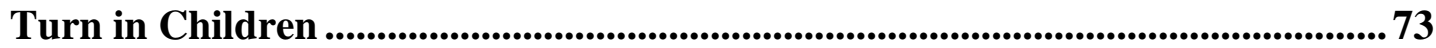

3.1 Introduction ...................................................................................................... 73

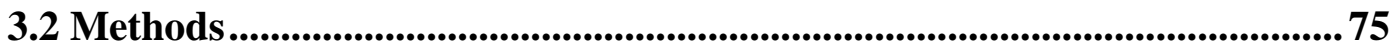

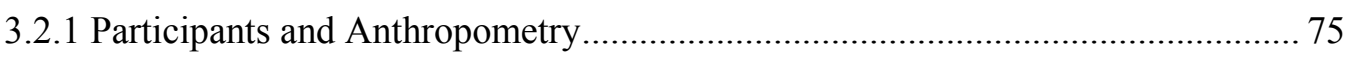

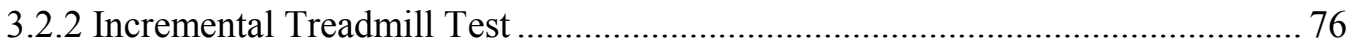

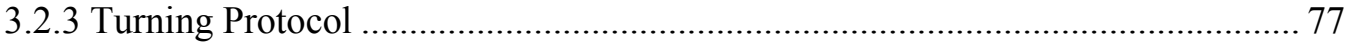

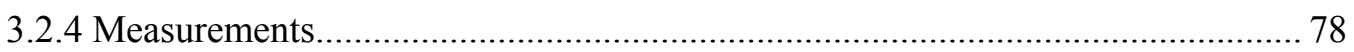

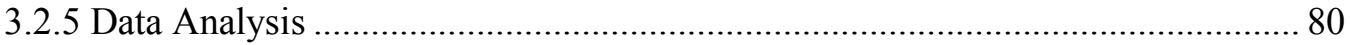

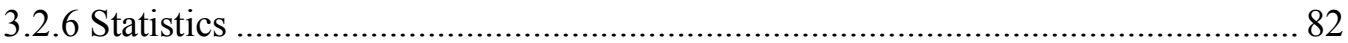

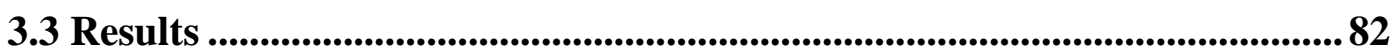

3.3.1 Pearson Product-moment Correlation Coefficient ................................................ 86

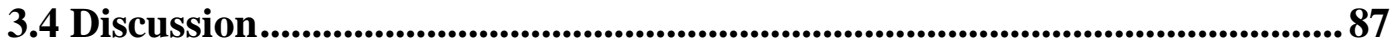

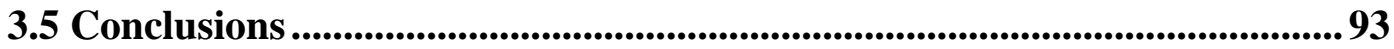

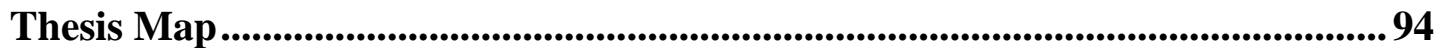

Chapter 4 - Perceptions of Visualising Physical Activity as a 3D-printed Object:

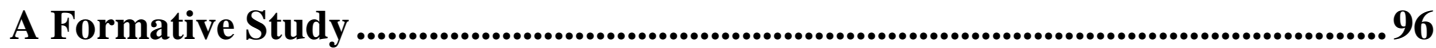




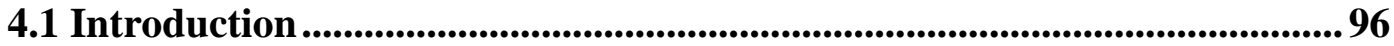

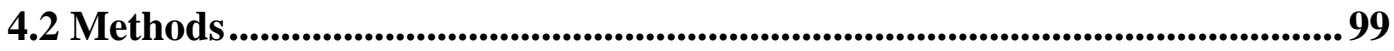

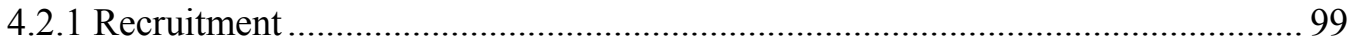

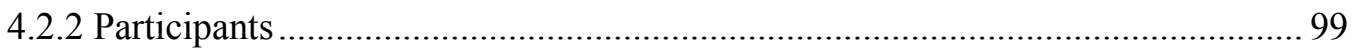

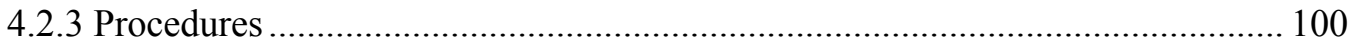

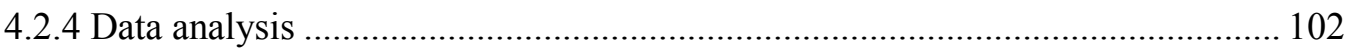

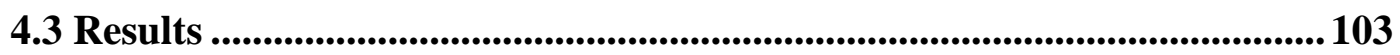

4.3.1 Children's Perceptions and Designs of 3D Physical Activity Models ............... 103

4.3.2 Adolescents' Perceptions and Designs of 3D Physical Activity Models ........... 107

4.3.3 Adults' Perceptions and Designs of 3D Physical Activity Models .................... 110

4.4 Discussion.......................................................................................................... 113

4.5 Conclusion...................................................................................................................... 120

Thesis Map ............................................................................................................................. 121

Chapter 5 - Understanding Youths' Ability to Interpret 3D-printed Physical

Activity Data and Associated Intensity Levels ....................................................123

5.1 Introduction ........................................................................................................... 123

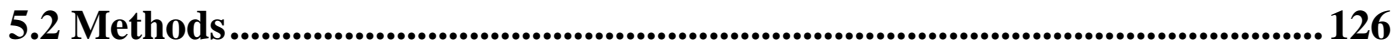

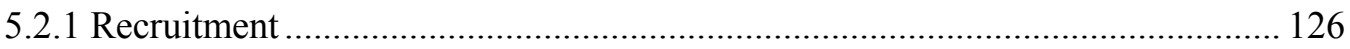

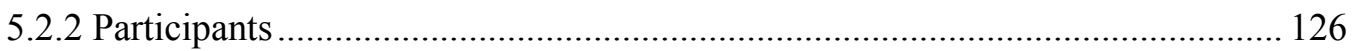

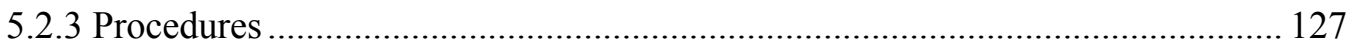

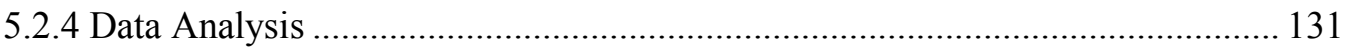

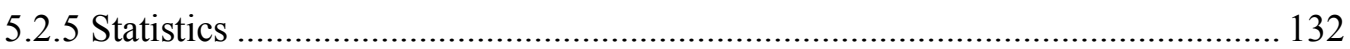

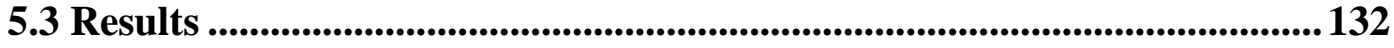

5.3.1 Children's Perceptions and Ability to Identify Physical Activity Intensities..... 133

5.3.2 Adolescents' Perceptions and Ability to Identify Physical Activity Intensities. 134

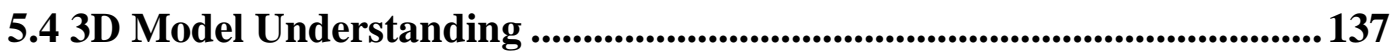

5.4.1 Children's Understanding and Ability to Interpret the 3D Model .................... 137

5.4.2 Adolescents' Understanding and Ability to Interpret the 3D Model................. 138 
Thesis Map

Chapter 6 - The Tangibility of Personalised 3D-Printed Feedback may Enhance Youths' Physical Activity Awareness ................................................................149

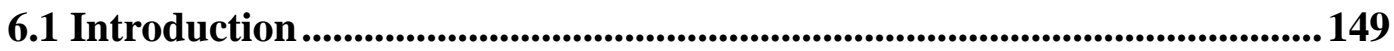

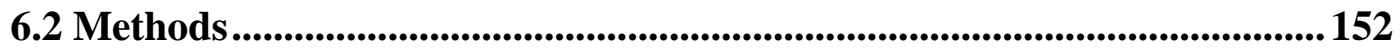

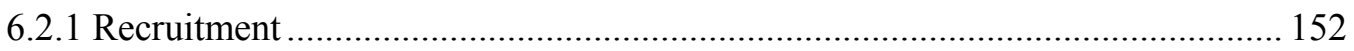

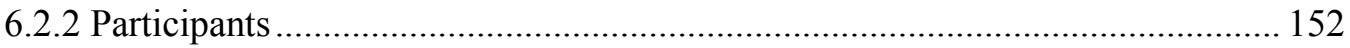

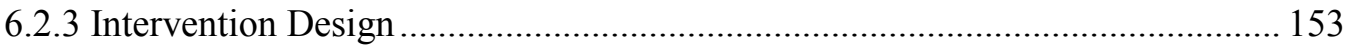

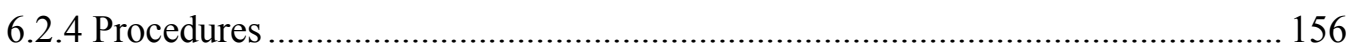

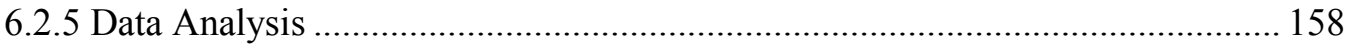

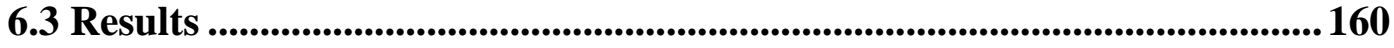

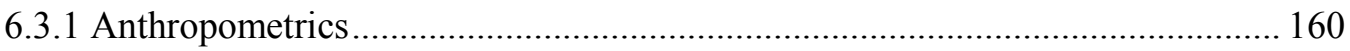

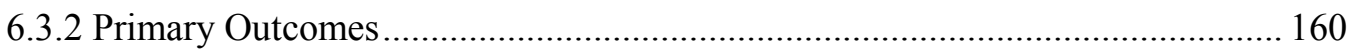

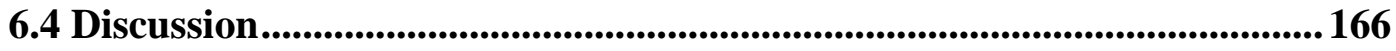

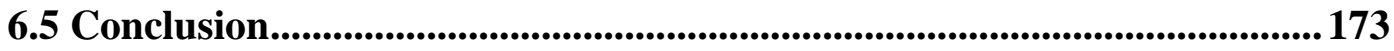

Thesis Map....................................................................................................................................... 174

Chapter 7 - Synthesis .......................................................................................................... 176

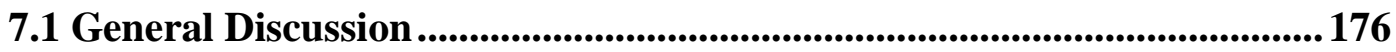

7.1.1 3D-Printed Feedback to Enhance Awareness of Physical Activity.................... 176

7.1.2 Goal-Setting and Rewards through 3D-Printed Physical Activity Feedback..... 178

7.1.3 Understanding of Physical Activity through 3D-Printed Feedback ................... 179

7.1.4 Youths' Adherence and Experience with 3D Models of Physical Activity ....... 181

7.1.5 The Use of Accelerometers to Assess Youths' Physical Activity ...................... 183

7.2 Strengths and Limitations of Thesis ....................................................... 184

7.3 Future Implications......................................................................................... 187 
7.3.1 Importance of Turning for Clinical, Health and Physical Activity Measurement

7.3.2 Self-Report Physical Activity Questionnaires: Implications for Accuracy........ 189

7.3.3 3D Printing Considerations: Practicality, Cost and Sustainability …................. 190

7.3.4 3D Printing Physical Activity as an Educational Tool ...................................... 192

7.3.5 3D Printing Personalised Multi-Dimensional Physical Activity ....................... 192

7.3.6 3D Printing Physical Activity for the Visually Impaired ................................... 193

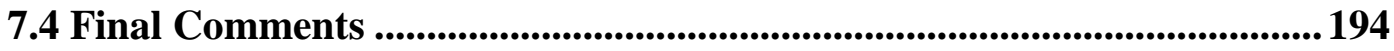

References ...................................................................................................................................... 196

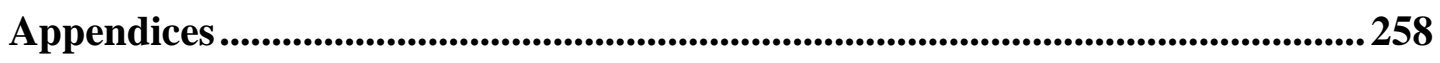

Appendix I: Study 1 - Mixed Models...........................................................258

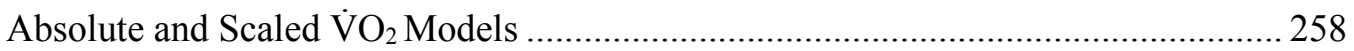

Straight and Turn Mean VeDBA Models...............................................................2259

Appendix II: Information Sheets and Consent/Assent Forms ........................260

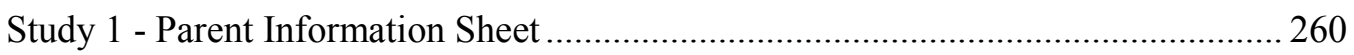

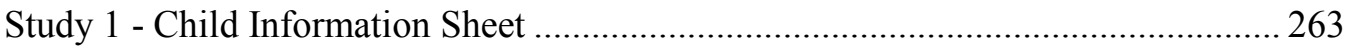

Studies 2 \& 3 - Parent Information Sheet................................................................ 266

Studies 2 \& 3 - Child Information Sheet ................................................................ 268

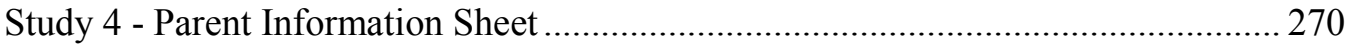

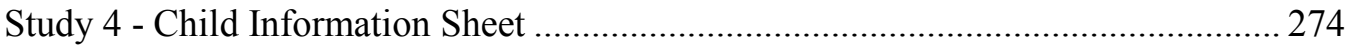

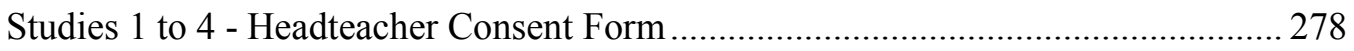

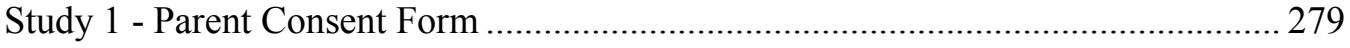

Study 1 - Child Assent Form ................................................................................ 280

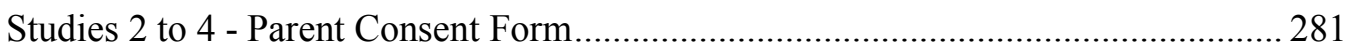

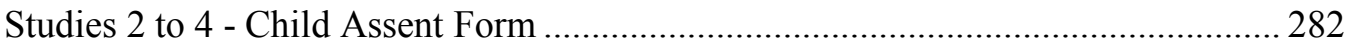

Studies 1 to 4 - Video, Audio and Photography Consent Form .................................. 283

Appendix III: Self-Report Tanner Stage Scale ................................................... 284

Appendix IV: Studies 2 to 4 Qualitative Questions and Sample Transcripts 
Study 2 - Youths' Focus Group Questions 286

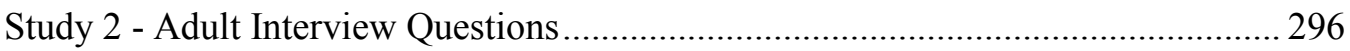

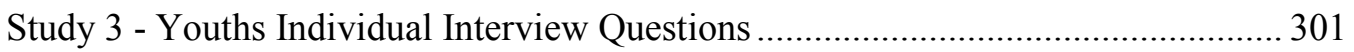

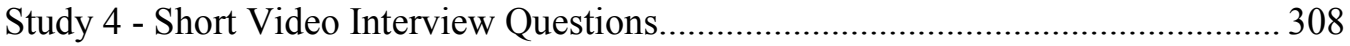

Study 2 - Sample Transcript: Secondary School Adolescents Focus Group .............. 309

Study 2 - Sample Transcript: Primary School Teacher Interview …………….......... 312

Study 3 - Sample Transcript: Primary School Child Interview …............................... 317

Study 3 - Sample Transcript: Secondary School Adolescent Interview ..................... 318

Study 4 - Sample Transcript: Primary School Child Short Interview ........................ 320

Study 4 - Sample Transcript: Secondary School Adolescent Short Interview ........... 321

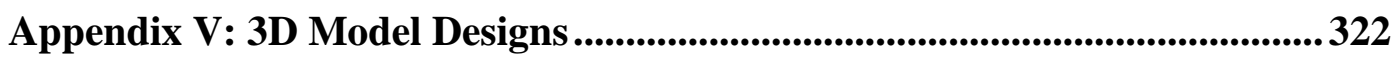

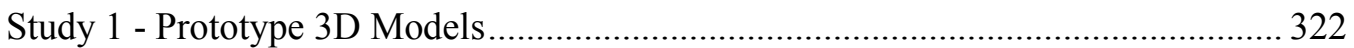

Study 1 - Age-Specific Prototype 3D Models.............................................................. 323

Study 1 - Adolescent 3D Model Prototype Designs..................................................... 325

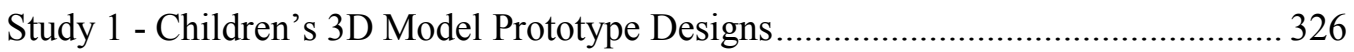

Study 1 - Children's Play-Doh Model Designs............................................................. 327

Study 1 - Adolescents' Play-Doh Model Designs....................................................... 330

Appendix VI: Scientific Outputs ...............................................................334

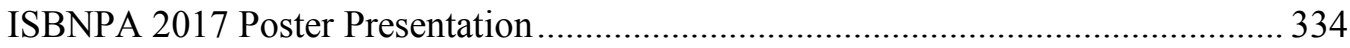

Published Paper - Energy Expenditure Associated with Walking Speed and Angle of Turn in Children. European Journal of Applied Physiology....................................... 335

Published Paper - Perceptions of Visualizing Physical Activity as a 3D-Printed Object: Formative Study. Journal of Medical Internet Research. 336

Published Paper - Understanding Youths' Ability to Interpret 3D-Printed Physical Activity Data and Identify Associated Intensity Levels: Mixed-Methods Study. Journal of Medical Internet Research.

Appendix VII: Online Articles ............................................................................... 338

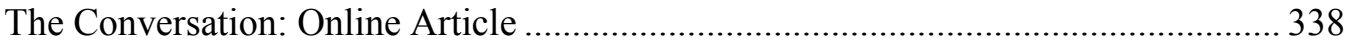

Ultimaker GB CREATE Education Blog: Online Article............................................ 341

University College London, CBC DIGI-HUB Blog: Online Article ........................... 346 
Appendix VIII: Public Engagement Outputs ...............................................350

Swansea University Research as Art Competition Submission 350

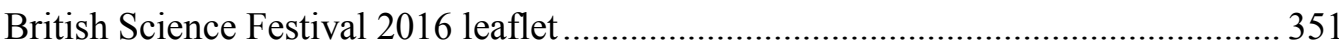

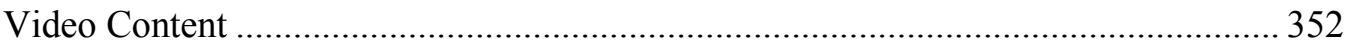

School Fortnightly Friday Flyer ........................................................................... 353

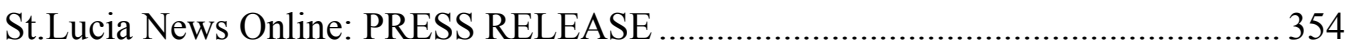

South Wales Evening Post: PRESS RELEASE ...................................................... 356 


\section{List of Tables}

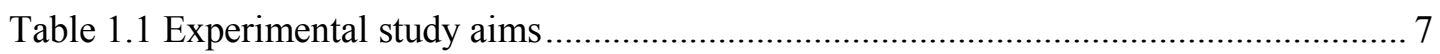

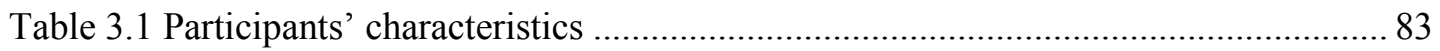

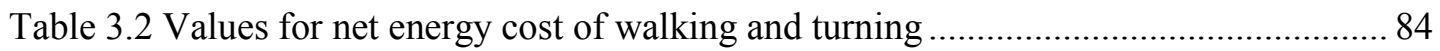

Table 3.3 Mean absolute $\dot{V} \mathrm{O}_{2}$, scaled $\dot{V} \mathrm{O}_{2}$ and caloric energy expenditure during each combination of walking velocity and angle 85

Table 3.4 Mean VeDBA, straight and turn mean VeDBA during each combination of walking velocity and angle 86

Table 4.1 Example focus group and interview questions 101

Table 5.1 Example interview questions . 128

Table 5.2 Twenty activities and respective intensity levels 129

Table 5.3 Children's perceptions and ability to identify activities to intensity. 135

Table 5.4 Adolescents' perceptions and ability to identify activities to intensity 136

Table 6.1 Example interview questions..... 158

Table 6.2 Participant anthropometric characteristics 160

Table 6.3 Youth frequency of occurrence of key themes 163 


\section{List of Figures}

Figure 2.1 Youths' time spent sedentary on weekdays.

Figure 2.2 Relative risk of obesity in youth watching TV. From Zhang et al. (2015).

Figure 2.3 Curvilinear dose-response relationship between screen-time in youth and risk of depression. From Liu et al. (2016)

Figure 2.4 The five stages of change. 23

Figure 2.5 Drawing from a boy aged 11 years old illustrating playing football with friends.

From Noonan et al. (2016). 28

Figure 2.6 The children's OMNI scale of perceived exertion. 31

Figure 2.7 Estimated weekly hours of media use in UK youth. 35

Figure 2.8 ‘Pokémon GO’ app gameplay. 39

Figure 2.9 Directions of movement. From Annegarn et al. (2012). 43

Figure 2.10 Four repeated-sprint sequences. From Buchheit et al. (2012). 45

Figure 2.11 Oxygen consumption of turning in adults.

Figure 2.12 . Numerical physical activity feedback displayed on the Omron HJ-109-E pedometer. 50

Figure 2.13 Numerical visualisations of activity feedback 51

Figure 2.14 Spark abstract visualisations of physical activity. 53

Figure 2.15 Abstract visualisations. 55

Figure 2.16 Physical activity represented as virtual growing fish. 56

Figure 2.17 Five virtual pet representations of physical activity levels. 56

Figure 2.18 Obese and healthy weight avatar. 58

Figure 2.19 Sqord avatar interface. 59

Figure 2.20 Photorealistic avatars. From Facebook.com.... 60

Figure 2.21 3D-printed representations of heart rate. 61

Figure 2.22 3D-printed chocolate representations of heart rate. 62

Figure 2.23 Human figure representing running data. 
Figure 2.24 The LOOP system. From Sauvé et al. (2017).

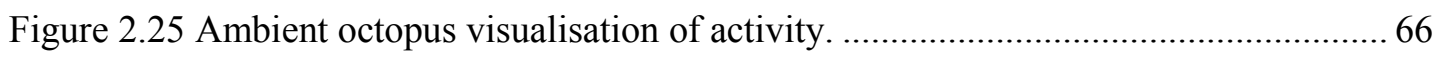

Figure 2.26 The four roles of youths to designing new technology...................................... 68

Figure 3.1 Experimental setup of the turning protocol showing $5 \mathrm{~m}$ straights interspersed by prescribed angle of turns. 78

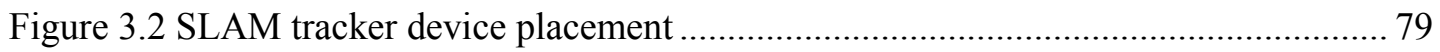

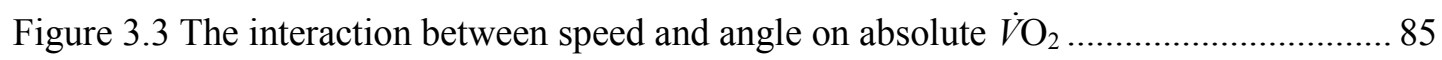

Figure 4.1 Children's Play-Doh model designs ................................................................ 104

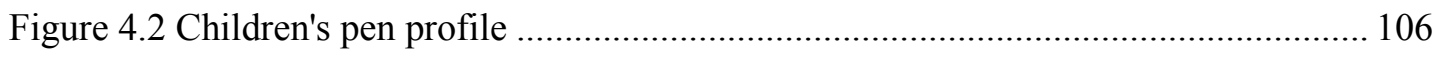

Figure 4.3 Adolescents' Play-Doh model designs ............................................................. 108

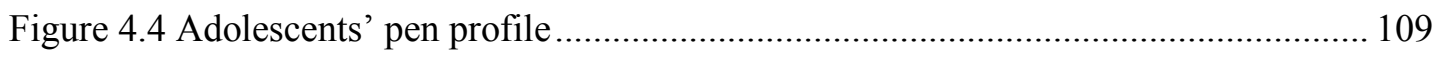

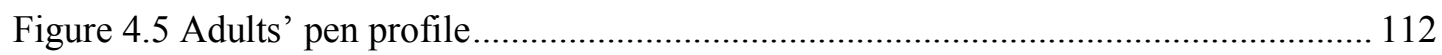

Figure 5.1 Age-specific 3D model prototypes ................................................................ 130

Figure 5.2 Children's interpretation of Sun 3D model ................................................... 138

Figure 5.3 Adolescents' interpretations of bar chart 3D model .......................................... 139

Figure 6.1 Flow chart outlining the school recruitment process, intervention allocation, intervention interview completion rate and accelerometer wear-time compliance; $\mathrm{N}=$ schools; $\mathrm{n}=$ total participants 153

Figure 6.2 The age-specific 3D models of physical activity instruction manuals ............... 155

Figure 6.3 Youths' display preferences for 3D models .................................................... 164

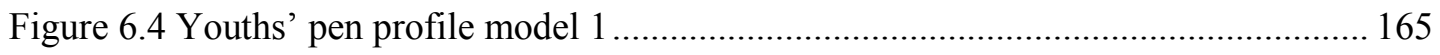




\section{Abbreviations, Units and Symbols}

\section{Abbreviations}

\begin{tabular}{|c|c|}
\hline ANOVA & Analysis of variance \\
\hline AVG & Active video games \\
\hline B & Boy \\
\hline BMI & Body mass index \\
\hline BHF & British Heart Foundation \\
\hline $\mathbf{C}_{\mathbf{r}}$ & The net energy cost of walking \\
\hline $\mathrm{CO}_{2}$ & Carbon dioxide \\
\hline DDR & Dance Dance Revolution \\
\hline DBA & Dynamic Body Acceleration \\
\hline DSH & Dynamic Similarity Hypothesis \\
\hline $\mathbf{F}$ & Female \\
\hline FITT & Frequency, intensity, time, type \\
\hline $\mathbf{G}$ & Girl \\
\hline GET & Gas exchange threshold \\
\hline HELENA & Health Lifestyle in Europe by Nutrition in Adolescence \\
\hline HSE & Health Survey England \\
\hline IMPACT & The Incorporating More Physical Activity and Calcium in Teen \\
\hline ISFE & Interactive Software Federation of Europe \\
\hline KAPM & The Knowledge, Attitude and Practice Model \\
\hline LPA & Light physical activity \\
\hline M & Male \\
\hline M1 & Model 1 \\
\hline M2 & Model 2 \\
\hline M3 & Model 3 \\
\hline M4 & Model 4 \\
\hline MET & Metabolic equivalent \\
\hline MPA & Moderate physical activity \\
\hline MVPA & Moderate-to-vigorous physical activity \\
\hline NASPE & National Association for Sport and Physical Education \\
\hline
\end{tabular}




\begin{tabular}{|c|c|}
\hline NICE & The National Institute for Health and Care Excellence \\
\hline NCDs & Non-communicable diseases \\
\hline $\mathbf{O}_{2}$ & Oxygen \\
\hline$\dot{V} \mathbf{O}_{2}$ & Oxygen uptake \\
\hline$\dot{V} \mathbf{O}_{2 \text { peak }}$ & Peak oxygen uptake \\
\hline $\mathbf{P}$ & Primary/Parent \\
\hline PA & Physical activity \\
\hline PAL & Physical activity level \\
\hline PAM & Personal activity monitor \\
\hline PAPM & Precaution Adoption Process Model \\
\hline PE & Physical Education \\
\hline PHV & Peak height velocity \\
\hline $\mathbf{S}$ & Secondary \\
\hline SED & Sedentary \\
\hline SEM & Standard error of the mean \\
\hline SLOTH & Sleep, Leisure, Occupational, Transport and Housework \\
\hline SMS & Short Messaging Services \\
\hline $\mathbf{T}$ & Teacher \\
\hline T1 & Tanner stage 1 \\
\hline $\mathbf{T} 2$ & Tanner stage 2 \\
\hline T3 & Tanner stage 3 \\
\hline TTM & The Transtheoretical Model \\
\hline TV & Television \\
\hline UK & United Kingdom \\
\hline USA & United States of America \\
\hline VPA & Vigorous physical activity \\
\hline WDST & Write, draw, show, and tell \\
\hline WHO & World Health Organisation \\
\hline VeDBA & Vectorial dynamic body acceleration \\
\hline YPAP & Youth Physical Activity Promotion Model \\
\hline 3D & Three Dimensional \\
\hline 6MWT & Six-minute walking test \\
\hline MVPA & Moderate-to-vigorous physical activity \\
\hline
\end{tabular}




\section{Units and Symbols}

$\begin{array}{ll}\mathbf{k c a l} \cdot \mathbf{k g}^{-\mathbf{1}} \cdot \mathbf{h}^{-\mathbf{1}} & \text { Kilocalorie per kilogram per hour } \\ \mathbf{k g} & \text { Kilogram } \\ \mathbf{k g} \cdot \mathbf{m}^{\mathbf{2}} & \text { Kilogram per metre squared } \\ \mathbf{k m} \cdot \mathbf{h r} \mathbf{- 1}^{-1} & \text { Kilometres per hour } \\ \mathbf{l} \cdot \mathbf{m i n} \mathbf{- 1}^{-1} & \text { Litres per minute } \\ \mathbf{m l} \cdot \mathbf{k g}^{-\mathbf{1}} \cdot \mathbf{m i n}^{-\mathbf{1}} & \text { Millilitres per kilogram per minute } \\ \mathbf{m} \cdot \mathbf{m i n} & \text { Metres per minute } \\ \mathbf{m} \cdot \mathbf{s} & \text { Metres per second } \\ \mathbf{m} & \text { Metres } \\ \mathbf{n} & \text { Sample size } \\ \boldsymbol{p} & \text { Significance value } \\ \boldsymbol{r} & \text { Pearson's correlation coefficient } \\ \mathbf{S} & \text { Seconds } \\ \circ & \text { Degree } \\ \sim & \text { Approximately }\end{array}$




\section{Scientific Output}

\section{Publications}

Crossley, S.G.M., Mackintosh, K.A., Wilson, R.P., Lester L.J., Griffiths, I.W. \& McNarry, M.A. Energy Expenditure Associated with Walking Speed and Angle of Turn in Children. 2018. European Journal of Applied Physiology.

Crossley, S.G.M., McNarry, M.A., Hudson, J., Eslambolchilar, P., Mackintosh, K.A. Perceptions of Visualising Children's Physical Activity as a 3D Object. 2019. Journal of Medical Internet Research.

Crossley, S.G.M., McNarry, M.A., Rosenberg, M., Knowles, Z., Eslambolchilar, P., Mackintosh, K.A. Understanding Youths' Ability to Interpret 3D-printed Physical Activity Data and Identify Associated Intensity Levels. 2019. Journal of Medical Internet Research.

Crossley, S.G.M., McNarry, M.A., Eslambolchlar, P., Knowles, Z.R., Mackintosh, K.A. The Tangibility of Personalised 3D-Printed Feedback May Enhance Youth's Physical Activity Awareness, Goal-Setting and Motivation. 2019. Journal of Medical Internet Research.

\section{Conferences}

Mackintosh, K.A., Crossley, S.G.M., Eslambolchilar, P., Knowles, Z. \& McNarry, M.A. Using Tangible, 3D-Printed, Objects to Enhance Children's Understanding of Physical Activity, International Society of Behavioural Nutrition and Physical Activity (ISBNPA), Hong Kong, China. June 2018. Oral Presentation.

Crossley, S.G.M., McNarry, M.A., Knowles, Z., Eslambolchilar, P., Mackintosh, K.A. Children and Adolescent's Interpretations and Understanding of 3D-printed Models of Physical Activity, 22 ${ }^{\text {nd }}$ Annual Congress of the European College of Sport Science (ECSS), MetropolisRuhr, Essen, Germany. July 2017. Oral Presentation.

Mackintosh, K.A., Crossley, S.G.M., Eslambolchilar, P., Knowles, Z. \& McNarry, M.A. 3D Printing as a Tool to Enhance Children's Understanding of Physical Activity Levels, $22^{\text {nd }}$ Annual Congress of the European College of Sport Science (ECSS), MetropolisRuhr, Essen, Germany. July 2017. Oral Presentation. 
Mackintosh, K.A., Crossley, S.G.M., Eslambolchilar, P., Knowles, Z., Hudson, J. \& McNarry, M.A. Perceptions of Visualising Children's Physical Activity as a 3D Object, International Society of Behavioural Nutrition and Physical Activity (ISBNPA), Victoria, Canada. June 2017. Poster Presentation.

Mackintosh, K.A., McNarry M.A., Eslambolchilar, P. \& Crossley, S.G.M. Grasping Physical Activity: Using 3D Printers to visualise physical activity, International Society of Behavioural Nutrition and Physical Activity (ISBNPA), Victoria, Canada. June 2017. Conference Workshop.

Eslambolchilar, P., Crossley, S.G.M., Hudson, J., Knowles, Z., Mackintosh, K.A., \& McNarry, M.A. Modelling Physical Activity: From Play-Doh to $3 D$ digital artefacts. $1^{\text {st }}$ GetAMoveOn Symposium, London, UK. May 2017. Oral Presentation.

Crossley, S.G.M., McNarry, M.A., Knowles, Z., Eslambolchilar, P., Mackintosh, K.A. Perceptions of Visualising Children's Physical Activity as a 3D Object, ${ }^{\text {st }}$ Annual Pan-Wales Postgraduate Conference in Sport and Exercise Sciences, Swansea, UK. April 2017. Oral Presentation.

Crossley, S.G.M., Mackintosh, K.A., Wilson, R.P., Lester L.J., Griffiths, I.W. \& McNarry, M.A. Investigating the Energetic Cost Associated with Turning in Pre-pubertal Children, Applied Sport Technology Exercise and Medicine Research Centre (A-STEM) Postgraduate Conference, Swansea, Wales, UK. March 2016. Oral Presentation.

Crossley, S.G.M., Mackintosh, K.A., Wilson, R.P., Lester L.J., Griffiths, I.W. \& McNarry, M.A. Investigating the Energetic Cost Associated with Turning in Pre-pubertal Children, The British Association of Sport and Exercise Sciences (BASES) Student Conference, Bangor, Wales, UK. March 2016. Oral Presentation.

Crossley, S.G.M., Mackintosh, K.A., Wilson, R.P., Lester L.J., Griffiths, I.W. \& McNarry, M.A. The Energetic Costs of Turning in Children, Applied Sport Technology Exercise and Medicine Research Centre (A-STEM) Postgraduate Conference, Swansea, Wales, UK. March 2014. Oral Presentation. 


\section{Public Engagement Output}

\section{International Presentations}

Oral Presentation by Sam Crossley, Visualising physical activity through 3D printing to get children moving more and sitting less. Sir Arthur Lewis Community College (SALCC) in association with the Office of the Governor General, St.Lucia, Caribbean. June 2017.

\section{Public Workshops}

Sports and Exercise Science: 3D Printing Physical Activity, Crucial Crew, Swansea, UK, December 2016. School Workshop.

3D Printing Physical Activity, British Science Festival, Swansea, UK. September 2016. Public Workshop.

\section{Online Articles}

Crossley, S.G.M., Mackintosh, K.A., McNarry, M.A., Eslambolchilar, P. Using 3D Printing to Enhance Children's Understanding of Physical Activity, UCL, CBBC Digi-Hub Blog, London, UK. July 2018. Online Article.

Crossley, S.G.M., Mackintosh, K.A. \& McNarry, M.A. Visualising Physical Activity through $3 D$ Printing to Get Children Moving More and Sitting Less, CREATE Education, Blog, Chorley, UK. November 2016. Online Article.

Mackintosh, K.A. \& McNarry, M.A. How 3D Printing May Help Cut Childhood Obesity by Getting Children Active, The Conversation, Health + Medicine, UK. September 2016. Online Article.

\section{Press Releases}

Physical Activity Seminar Hailed a Success, St.Lucia News Online, St.Lucia, Caribbean. June 2017. Press Release.

SALCC Host Important Physical Activity Seminar, St.Lucia News Online, St.Lucia, Caribbean. June 2017. Press Release

$3 D$ Printing Can Help Curb Childhood Obesity According to Swansea Experts, Swansea Wales Evening Post, Swansea, UK. September 2016. Press Release. 


\section{CHAPTER 1 Introduction}




\section{1}

\section{Introduction}

Physical activity is a fundamental part of life, serving our earliest and most primitive ancestors by enabling them to hunt, gather, adapt and survive different environments (Eaton et al., 1988). Indeed, human evolution has been sculpted by necessity-driven functional activities, such as foraging, farming, nurturing, building shelters and fighting (Cordain et al., 1998). As a result of persistently practising these tasks, it is likely that they contributed to the development of modern Homo sapiens. Since the industrial revolution of the 1800 's, technological innovations have created an environment where physical activity is restricted or not as valuable as a result of transitioning from hand production to machine tools, with only a select few individuals motivated to lead an active and healthy lifestyle (Hallal et al., 2012). This recent divergence between human behaviour and genetic makeup is problematic for the biochemistry and physiology of the human body that is designed to function optimally when undergoing regular bouts of daily physical activity (Eaton and Eaton, 2003).

Physical inactivity is presently considered to be one of the greatest public health problems of our time, and promotion to increase engagement in physical activity remains a high priority (Blair, 2009, Mountjoy et al., 2011, Trost et al., 2014a). The time spent and intensity of physical activity have consistently been associated with physiological and psychological health benefits in youths (Janssen \& Leblanc, 2010a). However, much concern has been expressed regarding the lack of youths engaging in sufficient sustained physical activity to accrue such health rewards (Biddle et al., 2004, Riddoch et al., 2007). For basic health benefits, the World Health Organisation (WHO) and UK Government both recommend that youths aged 5 to 18 years engage in a minimum of 60 minutes moderate-to-vigorous physical activity (MVPA) every day (Department of Health, 2011, 2011b, WHO, 2011). Recent UK figures report that only $23 \%$ of boys and $20 \%$ of girls aged $4-15$ years meet these minimum levels of physical activity (Health Survey for England, 2017b), while it is estimated that almost half the number of boys and two thirds of girls fail to achieve even half the recommended amount (Graig et al., 2009). 
Low levels of physical activity in youths are of considerable concern given the concomitant increased risk for obesity, hypertension, cardiovascular risk factors, type 2 diabetes mellitus and coronary heart disease (Mountjoy et al., 2011, Lee et al., 2012). Recent estimates suggest that one in five children in the UK are obese when they start school, rising to one in three children when leaving primary school (Department of Health, 2011, 2011b). Worryingly, evidence suggests that approximately $40 \%$ of obese children will continue to have increased weight during adolescence, with $75-80 \%$ of these obese adolescents becoming obese adults (Rowicka et al., 2017). Indeed, due to the direct results of obesity and associated deleterious non-communicable diseases (NCDs; Banjare and Bhalerao, 2016), it is currently predicted that today's youth will be the first generation with a lower life expectancy than their parents (Designed to Move, 2012).

Health-related behaviour change is a complex process determined by biological, psychological, social and environmental factors (Uphill, 2014). Awareness of physical activity levels (PAL) has been identified as an independent correlate for behaviour change and important for motivating individual's movement from pre-contemplation to contemplation of behaviour change, as described in the Transtheoretical model (TTM; Ronda et al., 2001, Kremers et al., 2008). To date, there is consensus within research supporting a lack of awareness of PAL within youths (Kremers et al., 2008, Corder et al., 2010, Xu et al., 2017). Research exploring youths' awareness showed that the majority of the study population (64\%) were unaware of their PAL (Kremers et al., 2008). Furthermore, youths that were aware of their PAL were reported to be, on average, 20 minutes per day more physically active than their unaware counterparts (Kremers et al., 2008). According to Kremers et al. (2008), this lack of awareness of PAL among youths is likely to make them less susceptible to educational programmes that aim to influence attitudes, norms, self-efficacy or other cognitive factors, because youth will not perceive a need to change. One possible reason for the limited success of physical activity interventions in youths (Mears \& Jago, 2016, Owen et al., 2017) is suggested to be the paucity of research focussing on the component of raising awareness within interventions (Corder et al., 2010). Therefore, researchers argue that more attention should be focused upon raising youths' awareness of PAL (Kremers et al., 2008, Corder et al., 2010). Equally important, understanding youths' perceptions 
about a particular behaviour, such as physical activity, should not be neglected in designing a programme aimed at improving physical activity engagement (Cottrell et al., 2012). Indeed, enhancing an individual's knowledge of the nature of physical activity and its relationship to health is likely to act as a fundamental drive towards appropriate levels of health behaviour (Bandura, 1998, Nutbeam, 2000).

Physical activity is a phenomenon that is difficult to assess and measure as activities tend change with days, weeks, and seasons (Pearce et al., 2008). Factors such as environmental context (i.e., inside or outside) and conditions (i.e., weather) can affect the energy expenditure of activities (Shephard \& Vuillemin, 2003). It could be conceivable that these interchangeable variables would make it hard for youths to interpret the concepts behind physical activity. Noonan et al. (2016) highlighted that children's interpretations of 'physical activity' revolved around traditional sports (i.e., football, basketball, gymnastics). Consequently, other important sources of physical activity, such as playing active games, walking to school and household chores, are not considered to contribute towards physical activity by children (Trost et al., 2000a), with similar findings reported in adolescents (Harris et al., 2016). Therefore, many children who do not adequately understand the concept of physical activity may erroneously consider sedentary behaviours, such as watching television (TV) or playing traditional video games, as a physical activity (Trost et al., 2000a). Perhaps more complex for youths is understanding the duration, frequency and intensities of physical activity (Pearce et al., 2008). Indeed, more recent findings show that youths have a limited ability to classify the intensity of physical activity (Harris et al., 2016). It is especially important that youths understand the type and intensity of physical activities that form the recommended guideline of 60 minutes of MVPA to gain associated metabolic health benefits (Andersen et al., 2006a, Ekelund et al., 2007). While there are several reasons that youth fail to achieve the recommended amount of MVPA, including socioeconomic status, urbanisation, social and environmental differences, it is sedentary screen-based technology that garnered most public criticism (Carrington, 2016).

As one cultural historian Steven Mintz notes (2004), young people's lifestyles have gradually shifted over the past century from outdoors to bedrooms and from improvised to fantasy toys. Consequently, these shifts in behaviour have increased 
youths' sedentary time to more electronically mediated play (i.e., watching television, video gaming and internet usage; Foley \& Maddison, 2010, Ofcom, 2014), with declining participation in unstructured play (Mintz, 2004, Gray, 2011). Indeed, these technology-based activities have been independently associated with adverse health outcomes, such as obesity (Proctor et al., 2003) and hypertension (Pardee et al., 2007). Some studies have shown promise in increasing youths' physical activity by limiting screen-time (Carlson et al., 2010, Hoyos Cillero \& Jago, 2011, Brindova et al., 2014), however, many interventions have been unsuccessful, as youths' are reluctant to relinquish such highly-valued behaviours (Timperio et al., 2004).This reluctance to relinquish technology-based behaviours is most likely attributed to technology being is an important part of youths' daily habits and lifestyle (Smahel et al., 2015). Most notably, young people are currently labelled as 'digital natives' and the 'iGeneration' (Prensky, 2012, Rosen, 2012) because they have grown up and developed with technological innovations, such as the internet, smartphones and sophisticated video games (Hillier, 2008). As technology continues to permeate, and become increasingly adopted, within young people's everyday lives (Cullen et al., 2013), it is important that we educate youth to be more responsible and aware to how they use such technology to benefit a healthy lifestyle (UNICEF, 2017). In this respect, technological innovations that can create opportunities for youth to engage in physical activity, empower them and actively protect them from damaging health behaviours, such as inactivity, are evidently required (Chaddha et al., 2017). Indeed, evidence suggests that youth prefer health support from information technology rather than traditional sources that may cause embarrassment with peers or conflicts with parents or teachers (Skinner et al., 2003). Hence the movement of technology as a 'new channel' to develop behaviour change strategies in youths (Cullen et al., 2013). Subsequent research has therefore focussed on the utility of technology as part of the solution to elicit positive health behaviour change, rather than part of the problem to youths' present physical inactivity levels (McDougall \& Duncan, 2008, Duncan \& Staples, 2010, Poole et al., 2011, Lubans et al., 2016, Mackintosh et al., 2016). In this light, the use of technology, such as objective measure of physical activity are suggested to be great tools to facilitate youths' awareness and understanding about physical activity through the monitoring of behaviours (Brusseau et al., 2011). 
Providing youth with constant feedback of their behaviours, by employing objective measures (e.g., accelerometers), may positively influence behaviour by enhanced awareness (van Sluijs et al., 2007). Indeed, the most accurate and detailed objective measure of energy expenditure to which physical activity contributes is by assessing the body's oxygen utilisation and carbon dioxide production by indirect calorimetry (Levine, 2005). This direct physiological measure is known as the 'gold standard' for assessing physical activity, although it comes at a price and is impractical in free-living settings (Strath et al., 2013, Westerterp, 2009). Accelerometers, on the other hand, are small and unobtrusive (Freedson et al., 2005a, Rowlands, 2007) and can be used to quantify physical activity into categories of intensity, frequency, duration and total volume of activity (Rowlands, 2007, Rowlands et al., 2004b). However, accelerometer measures are based upon linear regression models that emphasise that energy expenditure increases linearly with vertical acceleration during locomotive activities (Freedson et al., 2012). Therefore, this method is thought to be less accurate for nonlinear activities that are representative of daily living (Bassett \& John, 2010, Chen et al., 2007, Van Remoortel et al., 2012).

One such movement that is fundamental to performing physical activity is turning. Historically, turning was thought not to have a significant additional energetic cost to that of straight-line locomotion, however, recent studies suggest otherwise (Buchheit et al., 2010b, Buchheit et al., 2012, Buchheit et al., 2011, Dellal et al., 2010, Hatamoto et al., 2014, McNarry et al., 2017, Minetti et al., 2011, Wilson et al., 2013). Buchheit et al. (2010b) reported increased physiological changes in adult's heart rate, blood lactate and perceived exertion whilst running and turning at $180^{\circ}$, with more recent research highlighting a synergistic interaction between increasing walking speed and angle of turn in determining energy expenditure (McNarry et al., 2017). Such findings are particularly pertinent to identifying the true energetic costs associated with youths' PAL, especially given the highly sporadic and ballistic movement patterns of children that involve considerable amounts of non-linear locomotion (Adamo et al., 2009, Baquet et al., 2007, Sleap \& Warburton, 1996, Welk et al., 2000). In this respect, a greater understanding of how to measure and account for the diverse nature of physical activity more accurately is required, especially if we expect youths to understand their behaviours through monitoring devices. However, wearing an accelerometer does not develop youths' awareness or increase youths' engagement in physical activity alone 
(Vanhelst et al., 2017) as it must run parallel to personalised feedback (Bentley et al., 2012).

Over the past decade there has been an explosion in the availability of technologies facilitating self-monitoring (e.g., Fitbit) and thereby providing real-time feedback on PALs through phone applications. In the past few years, these self-monitoring technologies, coupled with online gaming with reward platforms (e.g., Sqord, iBitz, Striiv), have become more readily available to the youth consumer market (e.g., Fitbit Ace, Garmin Vivofit jr 2; Ananthanarayan, 2015). These commercially-designed health technologies are showing some degree of success within youth, improving their daily step count (Miller \& Mynatt, 2014, Hayes \& Van Camp, 2015, Hooke et al., 2016) and increasing the time spent in MVPA during school playtime (Hayes \& Van Camp, 2015). Although, contrary to adult literature, youths' are not naturally taking up these types of fitness instruments in the way that adults most commonly use them, with only $2 \%$ of youth being current users of such devices, taken from a sample of 1,156 participants (Wartella et al., 2016). With recent advances in digital fabrication, such as $3 \mathrm{D}$ printing, a new innovative approach to visualising data has been developed. Specifically, Khot et al. (2013) used 3D printing to create personalised physical activity feedback for adults, reporting that the 3D artefacts of activity data acted as a reward and created time for personal reflection and awareness of physical activity behaviours. Indeed, tangible interfaces have been shown to promote youths' engagement and reflection in learning (Rogers et al., 2002a, Price et al., 2003, Marshall, 2007). However, this type of feedback is yet to be explored in the context of physical activity within youths, which is especially pertinent given that developmental psychology promotes the use of tangible objects to stimulate intellectual development and understanding of concepts (Rita \& Dunn, 1979, Cole \& Wertsch, 1996, Piaget \& Cook, 1952, Fleming \& Mills, 1992, Montessori, 1912). Therefore, more empirical research is required to establish how $3 \mathrm{D}$ printing could offer a novel platform to conceptualise physical activity data for youths to enhance awareness and understanding of and motivation to engage in physical activity to promote positive behaviour change. 


\section{General Aim}

To this end, the overarching aim of the present thesis was to investigate the measurement and tangible visualisation of youths' physical activity. The aims of the studies within the experimental chapters (Chapter 3 to 6) are outlined in Table 1.1. In summary, Study 1 will investigate the energy expenditure associated with turning in children to facilitate more accurate measures of their habitual PAL which is essential for investigations requiring detailed physical activity feedback and knowledge of results to enforce behaviour change and health policies, respectively. Study 2, will therefore, explore youth's and adults' perceptions of and designs for detailed physical activity feedback displayed as tangible objects to promote physical activity and reinforce health messages and polices. Following this, Study 3 will seek to validate any specifically designed 3D-printed models of physical activity as a tool to enhance youths understanding of intensities of physical activity within a school-based intervention. Finally, Study 4 will investigate the efficacy of personalised 3D-printed models of youths' physical activity in a school-based intervention to elicit enhanced levels of awareness, understanding and motivation for physical activity.

\section{Table 1.1 Experimental study aims}

\section{Aims}

Study 1 To investigate the influence of walking speed and angle, and their interaction, on the energy expenditure of healthy children.

Study 2 To elicit children's, adolescents', parents' and teachers' perceptions and understanding of $3 \mathrm{D}$ physical activity objects to inform the design of future 3D models of physical activity.

Study 3 To elicit children's and adolescents' (i) interpretations of two age-specific 3D models displaying physical activity and (ii) ability to appropriately align activities to the respective intensity of physical activity.

Study 4 To investigate the efficacy of two age-specific 3D models to enhance youths' awareness and understanding of, and motivation for, physical activity during a 7-week faded intervention, whereby youth receive personal 3D-printed models displaying their PAL. 


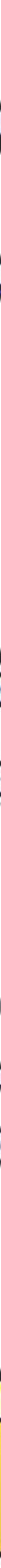




\section{2}

\section{Literature Review}

\subsection{Physical Activity}

Physical activity is defined as any bodily movement caused by the musculoskeletal system that exerts energy expenditure above that expended at rest (Caspersen et al., 1985). Energy expenditure is the sum of basal metabolic rate (i.e., the amount of energy expended by bodily systems at complete rest), the thermic effect of food (i.e., the amount of energy used to digest and absorb food), and the energy expended during physical activity (Westerterp, 2004). The basal metabolic rate and thermic effect of food account for $\sim 60 \%$ and $\sim 10 \%$ of total energy expenditure, respectively (Abadi et al., 2010). Although, basal metabolic rate and the thermic effect of food can both be subtly modified by factors such as body composition (Cunningham, 1980) and the volume and ingredients of food (Westerterp et al., 1999), physical activity is the most adjustable component of energy expenditure (Rising et al., 1994). On average, physical activity accounts for about $30 \%$ of total energy expenditure, although can vary depending on the activity level of an individual (Abadi et al., 2010). Physical activity has multiple sub-dimensions that include the frequency, intensity, time and type, collectively referred to as FITT. Frequency can be defined as the rate at which physical activity occurs over a period of time, such as a day, week or month (Gabriel et al., 2012), with intensity referring to the level of effort or physiological demand required to perform a physical activity (Gabriel et al., 2012). Time, or duration, is the amount of time (i.e., minutes or hours) spent in a physical activity (Gabriel et al., 2012), with type alluding to the physical activity being engaged in, such as walking, running or swimming (Gabriel et al., 2012).

It is important to understand that 'exercise' is a sub-domain of physical activity, which describes the premeditated and structured set of actions undertaken to achieve a goal of improved fitness or skill (Caspersen et al., 1985). With the exception of sleep, there are a number of domains in which people can engage in physical activity over the course of the day, as suggested by the socioecological 'SLOTH' model that categorizes 
an individual's day into five domains: sleep, leisure, occupation, transportation and home (Pratt et al., 2004). Leisure-based physical activity can include exercise participation and play, although this only constitutes a small percentage of an individual's total physical activity ( $\mathrm{Ng} \&$ Popkin, 2012). Activities undertaken at home (e.g., cleaning and cooking), at work or school, active travel (e.g., walking or cycling) and passive movements brought on by reactive interactions with the environment, all contribute to an individual's physical activity (Gabriel et al., 2012). Thus, the nature of physical activity is not only in the form of highly strenuous exertion, but a reflection of behavioural actions that an individual can undertake, whether it be light-, moderateor vigorous-intensity (Powell et al., 2011).

The intensity at which a physical activity is engaged in are usually estimated with metabolic equivalents (METs), with one MET referring to the resting metabolic rate, equivalent to $3.5 \mathrm{ml} \cdot \mathrm{kg}^{-1} \cdot \mathrm{min}^{-1}$ of oxygen consumption or $1 \mathrm{kcal} \cdot \mathrm{kg}^{-1} \cdot \mathrm{h}^{-1}$ of energy (Hills et al., 2014b). As such, two METs requires twice the resting metabolism (i.e., 7 $\mathrm{ml} \cdot \mathrm{kg}^{-1} \cdot \mathrm{min}^{-1}$ ) and three METs requires three times the resting metabolism (i.e., 10.5 $\mathrm{ml} \cdot \mathrm{kg}-1 \cdot \min -1$; Jette et al., 1990). However, it is important to note that adult MET values are not applicable to youths (Aull et al., 2008, Bailey \& McInnis, 2011, Banerjee \& Saha, 1972), as a result of youths' higher basal metabolic rates per unit body mass which declines as they grow and mature (Butte et al., 2017). Indeed, as in many other species, the metabolic costs within humans are shown to be correlated with age, sex, health status, fitness level, body mass and height (Ocobock, 2014). It is also important to note that energy expended is sensitive to environmental factors, such as altitude and temperature, which can alter basal metabolic rate and thermoregulation (Ocobock, 2014). Therefore, the human body relies on the interaction between morphology, physiology, behaviour and the environment to perform activities, all of which determines the energy expenditure (Ocobock, 2014). In this case, the youth compendium of physical activities defines light-intensity physical activity as $<3$ METs, moderate-intensity as between 3-6 METs and vigorous-intensity physical activities as $>6$ METs (Butte et al., 2017). However, it is important to recognise that MET levels for moderate-intensity have varied, with previous research defining it to be between 4-7 METs (Corbin \& Le Masurier, 2014). Despite this, activity intensities range from 0.9 METs for sleeping to 11.5 METs for running at a speed of $8 \mathrm{mph}$ (Butte et al., 2017, Ridley et al., 2008). These MET estimations of daily energy expenditure 
are particularly useful for epidemiological studies, as MET scores can be derived from individuals' self-reported physical activity levels (PAL; Manson et al., 1999). Furthermore, understanding the dose (i.e., frequency and duration) of physical activity, and its associated intensity, is important for identifying and applying physical activity guidelines (i.e., 60 minutes of moderate-to-vigorous physical activity every day; WHO, 2011). Indeed, the dose of physical activity is especially important when informing intervention studies to account for how much physical activity youths' need to do, but also to classify them as inactive or active for the purpose of monitoring. In this respect, physical activity is most commonly measured for interventions that are designed to effectively promote the adoption and maintenance of active lifestyles in a large number of individuals (Marcus et al., 2006). Interventions can be either acute or chronic in their nature, whereby a chronic based physical activity intervention refers to an intervention that involves participation in multiple sessions of physical activity and an acute physical activity intervention implements a single session of activity (Bouchard et al., 2012). Most commonly, studies investigating physical activity and health deploy chronic physical activity strategies (Bouchard et al., 2012). Indeed, research using chronic strategies for physical activity have led to a greater understanding of the benefits of engaging in physical activity to improve an individual's health status (Warburton \& Bredin, 2017).

\subsection{Physical Activity and Health Outcomes}

Physical inactivity is identified by the World Health Organisation (WHO) as the fourth leading risk factor for global mortality. Indeed, engaging in regular physical activity has been considered to be an integral preventative strategy to eliminate or prevent the burden of a variety of health-related risk factors associated with physical inactivity (Sallis et al., 2008, Janssen \& Leblanc, 2010a, Tremblay et al., 2011b, Trost et al., 2014a). Historically, this understanding of its importance was pioneered by the work of Jeremy Morris in the 1950's, who discovered that physically active workers had significantly fewer instances of chronic heart disease when compared to their less active counterparts (Morris \& Crawford, 1958, Morris et al., 1953). A synthesis of existing evidence comparing individuals of high and low PAL indicates individuals with high PAL have a 30\% risk reduction for all-cause mortality, 20-35\% for cardiovascular diseases, $30-40 \%$ for diabetes mellitus type II and $20-30 \%$ for colon 
and breast cancer (Department of Health, 2011a). A more recent systematic review showed that inactive individuals are $26 \%$ and $21 \%$ more likely to suffer from a stroke and coronary heart disease, respectively (Kyu et al., 2016). In this respect, there is a linear relationship between PAL and adverse health conditions, meaning that those individuals with the highest PAL have the lowest risk of contracting such adverse health conditions (Lee \& Skerrett, 2001b, Powell et al., 2011).

The dose-response relationship between physical activity and health implies that increases in physical activity results in additional improvements in health status, even when an individual is not frequently engaging in activity (Galán et al., 2013). Past research on the dose-response relationship of physical activity and health indicators has mainly focused on adult populations (Abu-Omar et al., 2004, Haennel \& Lemire, 2002, Kim et al., 2008, Lee \& Skerrett, 2001a, Martin et al., 2009, Mayer-Davis et al., 1998, Ohkawara et al., 2007), however, this focus is now changing with increasing numbers of studies examining this relationship within youth populations (Biddle et al., 2004, Biddle \& Asare, 2011, Hallal et al., 2006b, Janssen \& Leblanc, 2010a, Kesäniemi et al., 2010, Mountjoy et al., 2011, Strong et al., 2005). Nonetheless, there is still controversy on whether this dose-response relationship is linear (i.e., large improvements in health occurring with limited increases in physical activity) or curvilinear (i.e., small improvements in health occurring with increases in physical activity; Janssen \& Leblanc, 2010a, LeBlanc \& Janssen, 2010b, Mark \& Janssen, 2008), which is due part to the varying health outcomes being assessed within studies (Galán et al., 2013). Within child and adolescent populations, there is much evidence to support a positive dose-response relationship between physical activity and health benefits (Biddle et al., 2004, Biddle \& Asare, 2011, Hallal et al., 2006b, Janssen \& Leblanc, 2010a, Kesäniemi et al., 2010, Mountjoy et al., 2011, Strong et al., 2005). Indeed, engagement in physical activity can significantly reduce the occurrence of non-communicable diseases (NCD's), such as diabetes mellitus type II, cardiovascular disease and obesity (Dias et al., 2018, Shiroma et al., 2017, Weston et al., 2016). A number of physiological mechanisms may be involved in the relationship between increased PAL and the prevention of NCD's, including the improvement in body composition by reduced visceral adiposity and overall body mass, reductions in triglycerides, total cholesterol levels, blood pressure and improved blood glucose regulation and insulin sensitivity (Booth et al., 2008, Warburton et al., 2001). 
It appears that MVPA is associated with the greatest number of health benefits in youth, such as obesity prevention and improved cardiometabolic health (Janssen \& LeBlanc, 2010b, Andersen et al., 2006b, Ekelund et al., 2012, Ness et al., 2007, Steele et al., 2008, Steele et al., 2009b, Hallal et al., 2006a). In overweight youth, evidence shows that programs of moderately intense physical activity between 30 and 60 minutes in duration, 3 to 7 days a week, reduced total body fat, visceral adiposity and blood pressure in youth with mild hypertension, as well as enhancing their aerobic fitness (Gutin et al., 2002, LeMura \& Maziekas, 2002, Owens et al., 1999, Strong et al., 2005). When comparing youths who are habitually more active to those who are less-active, the former show better levels of aerobic fitness (Armstrong et al., 1990, Craft et al., 2003, Kemper et al., 2001, Rowland et al., 2000, Rowlands et al., 1999). Interestingly, a regression analysis on 285 middle school students found that moderateintensity physical activity did not contribute meaningfully to aerobic fitness after vigorous-intensity physical activity was included in the model (Moore et al., 2013). From these findings, Moore et al. (2013) suggested that public health messages should look to promote vigorous-intensity physical activity to reap cardiorespiratory health benefits among youth. In this regard, research looked to quantify whether vigorousintensity physical activity had additional health-enhancing benefits over moderateintensity physical activity on a per-minute basis (Owens et al., 2017). Wittmeier et al (2008) analysed physical activity data from 251 children, reporting that 45 minutes of moderate-intensity physical activity per day were needed to elicit the same benefits as 15 minutes per day of vigorous-intensity physical activity for reduced body fat and BMI. Similarly, Steele et al (2009a) reported that 13.6 minutes per day of moderateintensity physical activity compared with 6.5 minutes per day of vigorous-intensity physical activity were associated with a reduction in waist circumferences of 0.49 and $1.32 \mathrm{~cm}$, respectively. However, more recent controlled interventions have shown that vigorous-intensity physical activity to be less consistently superior to moderateintensity physical activity for improving aerobic fitness (Racil et al., 2013, Steele et al., 2009a). Nonetheless, bone health is more favourably affected by modest levels of high-impact weight-bearing activities, with modest effects on bone mineral density typically found anywhere from 3 to 60 minutes on at least 2 or 3 days per week (Kontulainen et al., 2002, Linden et al., 2006, MacKelvie et al., 2001, MacKelvie et al., 2004, McKay et al., 2005). In this case, vigorous-intensity physical activity is as 
effective as, but not necessarily superior to, moderate-intensity physical activity for improving youth's health. That said, vigorous-intensity physical activity does have the advantages of time efficiency for achieving health benefits, which may be an important consideration for time-restricted physical activity interventions (Owens et al., 2017).

Physical activity has also been shown to be beneficial for youths' psychological wellbeing, by enhancing physical self-perceptions, self-esteem and social interaction skills (Bunker, 1998, Lubans et al., 2016). Furthermore, previous studies have also shown how physical activity positively improves youths' measures of anxiety (Hilyer et al., 1982, Norris et al., 1992) and depression symptoms (Brown et al., 1992, Hilyer et al., 1982, Koniak-Griffin, 1994, Norris et al., 1992). Rothon and colleagues (2010) found that for every additional hour youth spent being physically active resulted in an $8 \%$ decrease in depressive symptoms. There is, however, much debate on which type of activity (i.e., aerobic vs. anaerobic) elicits the greatest improvements in symptoms associated with depression and anxiety (Azar et al., 2008, Richardson et al., 2005). However, the data suggests that aerobic activity is more effective as it can activate a chemical effect similar to that found within an 'antidepressant' (Oddie et al., 2014). One physiological-based theory for the benefits of aerobic activity is the release of dopamine and beta endorphins, which leads to a relaxation or peacefulness effect (Stella et al., 2005). The psychosocial theories on the impacts of aerobic activity are explained through distraction, task mastery and social interaction as well as improving mood and self-esteem (Annesi, 2004, Rothon et al., 2010). Numerous studies implementing physical activity interventions demonstrate reduced symptoms of depression and anxiety when performing MVPA for 30 to 45 minutes, 3 to 5 days a week (Calfas \& Taylor, 1994, Richardson et al., 2005, Strong et al., 2005). Others show similar effects following an intervention combining both an educational and physical activity component lasting 50 minutes in duration (20 minutes of physical activity) for 2 to 3 days per week (Melnyk et al., 2009). Physical activity is also associated with an improvement in youth's coping mechanisms (Gerber et al., 2012) and lowered perceived stress (Brown et al., 1992, Kantomaa et al., 2008). Therefore, physical activity can improve mental resilience and could be one potential preventative measure for those youth who may be at a higher risk of developing mental health disorders (Oddie et al., 2014). 
As the numbers of studies investigating the relationship between physical activity and health increase, so too does the application of the principles of FITT to reduce the burden of diseases (Powell et al., 2011). Given the pandemic rise of childhood physical inactivity (Kohl et al., 2012, Moore et al., 2012), public health sectors have produced and communicated physical activity recommendations that aim to guide individuals towards achieving the minimum levels of physical activity for health benefits (Blair et al., 2004).

\subsection{Youths' Physical Activity Guidelines}

The current recommendations of 60 minutes moderate-to-vigorous physical activity (MVPA) were first proposed by a consensus statement released in 1998 (Biddle et al., 1998). Whilst the government guidelines are currently being updated, the latest guidelines were released in 2011 and the WHO and UK Chief Medical Officers both recommend that youths (5-18 years old) should engage in MVPA for at least 60 minutes and up to several hours every day (WHO, Department of Health, 2011b). The concept of accumulating 60 minutes per day refers to performing shorter bouts of physical activity throughout the day (e.g., 2 bouts of 30 minutes) that can then summed to attain the 60 minute target. The term MVPA refers to activities that increase heart rate, sweating, heavier breathing or being out of breath (NICE, 2009). Indeed, there is much evidence to support the health benefits that can be accrued from children and adolescents accumulating 60 or more minutes of MVPA daily (Janssen \& Leblanc, 2010a, Janssen, 2007). However, there are a number of specific types of physical activity that youths must include into their overall physical activity to gain comprehensive health benefits (Janssen, 2007, Janssen \& LeBlanc, 2010b). These include, resistance exercise to enhance muscular strength in large muscle groups (e.g., trunk and limbs), vigorous aerobic exercise to improve cardiorespiratory fitness and weight-loading activities to promote bone health . According to Strong et al. (2005), activities such as brisk walking, riding a bicycle and active outdoor play are usually indicative of the moderate-intensity threshold. The guidelines also recommend that vigorous-intensity activities should be incorporated at least three days a week, including those that strengthen muscle and bone (i.e., gymnastics or tennis; Department of Health, 2011b). Indeed, evidence supports the use of these recommendations as an appropriate measure for detecting a target populations PAL 
and for examining the efficacy of physical activity promotion and treatment strategies (Martinez-Gomez et al., 2010c).

\subsection{Youths' Physical Activity Levels}

Studies and health surveys indicate that many youths do not meet the recommended PAL (Economos, 2001, Westerstahl et al., 2005). Studies assessing PAL are often difficult to compare because of the diversity of methodological approaches, data analysis, reporting of results and the varying definitions of what constitutes an appropriate level of activity (Livingstone et al., 2003, Reilly et al., 2006, Steinbeck, 2001). The Health Survey for England (2014) demonstrates that self-reported levels of youths meeting the current physical activity recommendations has declined from $28 \%$ to $21 \%$ for boys and $19 \%$ to $16 \%$ for girls across the years 2008 to 2014 (Health Survey for England, 2014). Similarly, the percentage of youths meeting the guidelines peaks between the ages of 8-10 years for boys (26\%) and ages 5-7 years in girls (23\%), with the decline progressing with age, decreasing to $14 \%$ in boys and $8 \%$ in girls by $13-15$ years old (Health Survey for England, 2014). However, these measures were based upon self-reported methods that are subject to limitations, such as youths overestimating their PAL (Adamo et al., 2009), which may be influenced by youths' limited ability to recall their activity as well as conforming to social desirability (Biddle et al., 2009, Corder et al., 2008, Gorely et al., 2009).

An alternative method to self-reported physical activity and a more widely adopted measure among researchers is an objective measure, thereby removing the potential issues of recall and response bias of self-reporting (Adamo et al., 2009). The doubly labelled water method (see section 2.13, this chapter) remains the 'gold standard' for assessing energy expenditure under free-living conditions; however, it is not often utilised in studies as it is expensive and has high participant burden and is timeintensive (Sylvia et al., 2014, Westerterp, 2009). Despite this, studies concerning physical activity measurement use the doubly labelled water technique to validate instruments, such as accelerometers, for assessing activity in free-living individuals (Hills et al., 2014b, Plasqui \& Westerterp, 2007). Indeed, accelerometers have gained popularity because of their ease of administration and ability to provide a reliable assessment of physical activity in terms of time spent active and the distribution of 
exercise intensities in a large population sample (Adamo et al., 2009, Westerterp, 2009). A UK study using accelerometers in 11-year old youths reported a median time spent in MVPA of 20 minutes per day, with boys accumulating 25 minutes and girls 16 minutes per day (Riddoch et al., 2007). In contrast, another UK study reported a mean time spent in MVPA of 74 minutes per day, with boys achieving 84 minutes and girls 66 minutes per day in 10-11 year old youths (van Sluijs et al., 2008).These contrasting results may be explained by the specific cut-points of accelerometer counts used by the researchers, which determines the amount of time spent in a specific physical activity intensity level (Riddoch et al., 2007). In this respect, the choice of cut-points used can produce very different estimates of MVPA ranging from 15 to 107 minutes per day of MVPA for Mattocks and Romanzini cut-points, respectively (Banda et al., 2016). Nonetheless, irrespective of the cut points used, it is consistently found that boys are more active than girls (Rowlands et al., 2008, Trost et al., 2002b, Ekelund et al., 2012, Hallal et al., 2012, Pearce et al., 2012, Telford et al., 2016). In detail, Rowlands et al. (2008) investigated the PAL of youths aged 9-11 years based in the South West of England, reporting that boys performed a greater frequency, duration and intensity of physical activity bouts compared to girls, who were more sporadic in their physical activity. Furthermore, the duration of bouts was greater on weekdays than on weekends, revealing that youths were more active on school days, as supported by Nader et al. (2008). More recent evidence suggests this trend is still apparent, with girls aged 4-18 years suggested to be performing 17\% (Ekelund et al., 2012) to $19 \%$ (Telford et al., 2016) less total daily physical activity when compared to boys. The lower physical activity among girls has been associated with weaker influences at the school environment and family levels, with a lower proportion of girls participating in extracurricular sport (Telford et al., 2016).

Of particular concern is the evidence supporting the decline in PAL as children transition into adolescence (Kimm et al., 2002, Riddoch et al., 2004, Thompson et al., 2009, Nader et al., 2008), with findings revealing that 16-17 year old adolescents are significantly less active (225 minutes per week) than children aged 8-9 years old (1038 minutes per week; Thompson et al., 2009). However, a more recent and novel 8-year longitudinal study using accelerometers concluded that PAL in both sexes declines a lot earlier than previously reported, with children as young as 6-7 years old showing a decline in their PAL, therefore reinforcing that methods should target childhood 
(Farooq et al., 2017). Indeed, childhood is an important period for developing a physically active lifestyle that encourages reduced sedentary behaviours (i.e., long periods of sitting or lying) that can continue into adulthood (Telama, 2009, Telama et al., 2005).

\subsection{Sedentary Behaviour}

It is important to understand that sedentary behaviour is a separate behaviour in its own right and not just simply a lack of physical activity or 'inactivity'. Sedentary behaviours are typically defined as pursuits that involve sitting or lying for extended periods that require low levels of energy expenditure (i.e. $<1.5$ METs; Barnes et al., 2012). Physical activity and sedentary behaviour can co-exist, as an individual can engage in the daily recommended requirement of 60 minutes of MVPA and also spend prolonged periods engaged in sedentary behaviours (Tremblay et al., 2011a). Indeed, reports suggest that youths are spending more time engaged in sedentary behaviours (Figure 2.1; Breslin et al., 2012, Colley et al., 2011, Griffiths et al., 2013, Sisson et al., 2009) and in particular, screen-based sedentary pursuits (Rideout et al., 2010). In the UK, youths aged 7 years old are reported to spend 6.4 hours or more each day in sedentary pursuits (Griffiths et al., 2013), with 8-9 year old youths from Northern Ireland reporting on average 11-12 hours of sedentary behaviour per day (Breslin et al., 2012). Worryingly, the problem is widespread, with Canadian youths (aged 6-19) spending on average 8.6 hours sedentary (Colley et al., 2011) and similar findings in American youths (aged 2-15 years) averaging 6-8 hours per day in sedentary pursuits (Sisson et al., 2009). 


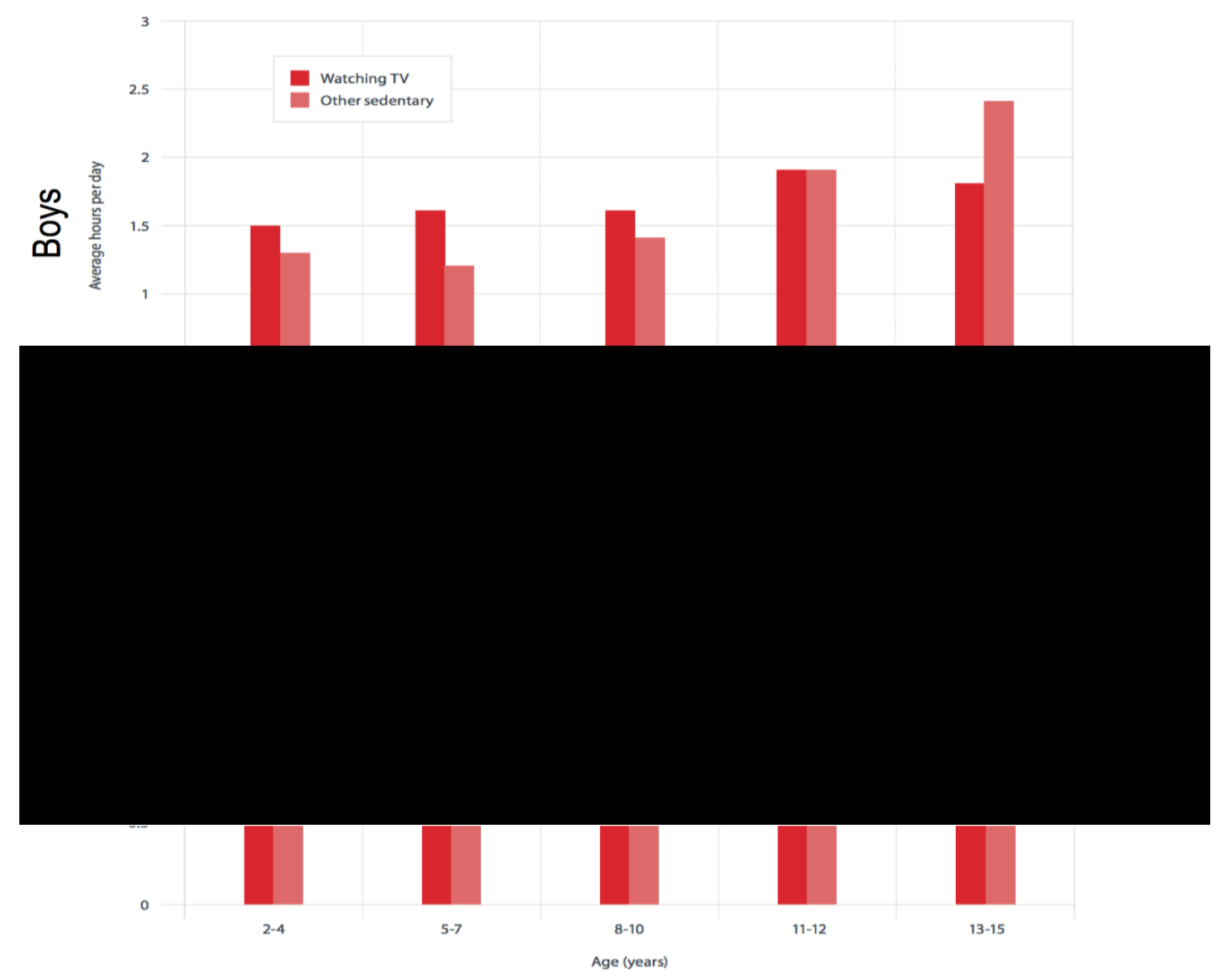

Figure 2.1 Youths' time spent sedentary on weekdays. From British Heart Foundation (2015).

Similar to physical activity, a dose-response relationship is observed with increased sedentary behaviour and a greater risk of adverse health-outcomes (Tremblay et al., 2010). Evidence suggests that prolonged sedentary behaviour is independently and positively associated with all-cause mortality and cardiometabolic risk (Katzmarzyk et al., 2009, Tremblay et al., 2011a), including increased risk of being overweight or obese (Berkey et al., 2003, Jago et al., 2005, Proctor et al., 2003, Wethington et al., 2013), hypertension (Pardee et al., 2007, Wyszynska et al., 2017), increased risk of insulin resistance (Hardy et al., 2010, Sardinha et al., 2008), alterations in lipid profiles (Aadahl et al., 2007, Martinez-Gomez et al., 2010b), back pain and headaches (Torsheim et al., 2010), respiratory symptoms (Tsai et al., 2007) and reduced fitness (Sandercock \& Ogunleye, 2013). Dietz and Gortmaker (1985) were the first to systematically explore the dose-response relationship between television (TV) viewing and health in youth, reporting that more than 5 hours TV per day resulted in a higher risk of obesity. A more recent systematic review showed that 1 hour per day 
increments of TV viewing corresponded to a $13 \%$ increased risk of obesity in youth (Figure 2.2; Zhang et al., 2015). On the contrary, data from van Ekris et al (2016) suggests close to zero effect of each additional hour of TV viewing on adiposity. Nonetheless, a number of studies have shown that higher TV viewing is significantly associated with increased cardiovascular disease risk factors when a 2 hour (Giussani et al., 2013, You \& Son, 2012), 3 hour (Martinez-Gomez et al., 2010a) and 4 hour cutpoint (Carson \& Janssen, 2011) was used. A large international cross-sectional study on 77,000 and 207,000, children and adolescents, respectively, reported a doseresponse effect for obesity in both age groups. In detail, there was a 10 to $27 \%$ increased risk of obesity in youth watching TV 1 to 3 hours per day, with adolescent girls having a $45 \%$ increase in risk when watching TV for more than 5 hours per day (Braithwaite et al., 2013). However, one major limitation to TV viewing studies and greater sedentary behaviour is the consumption of food in front of the TV. Specifically, triggers for greater snacking or unhealthy food consumption can be prompted by advertisements (Pearson \& Biddle, 2011). Indeed, within youth, TV viewing has been shown to be positively associated with the consumption of energy-dense snacks and drinks, total energy intake and fast foods, which will likely influence findings regarding the impact of sedentary behaviour on health parameters (Pearson \& Biddle, 2011).

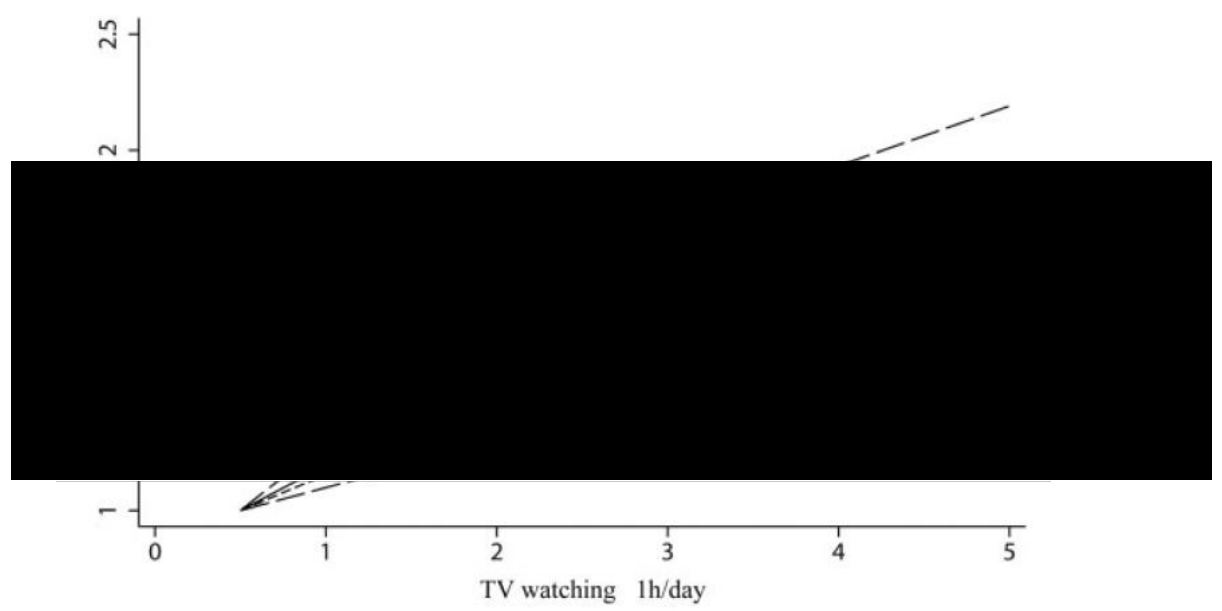

Figure 2.2 Relative risk of obesity in youth watching TV. From Zhang et al. (2015).

Sedentary behaviours can negatively impact an individuals psychosocial health (e.g., decreased self-esteem) with associations to poorer school performance (Tremblay et 
al., 2010). More worryingly, screen-time based sedentary behaviour has been associated with an increased risk of depression (Feng et al., 2014, Hamer \& Stamatakis, 2014). Depression in youth can have significant consequences on their psychological health (Thapar et al., 2012, Calles, 2007), such as substance abuse (Hersh et al., 2014), increased suicide risk (Hawton \& van Heeringen, 2009), impaired psychosocial functioning (Gotlib et al., 1995) and even cause psychological health disorders in later adulthood (Fergusson et al., 2005). Interestingly, one meta-analysis discovered a significant curvilinear dose-response associated with sedentary screentime and risk of depression in youth (Liu et al., 2016). The findings suggested that when limiting screen-time to 0 to 2 hours per day, screen-time actually decreased the risk of depression with lowest risk being detected at 1 hour per day of sedentary screentime (Figure 2.3; Liu et al., 2016). Liu et al (2016) postulated that the psychological benefits of screen-time could be related to youth processing humorous content on TV, the internet and video games (Primack et al., 2009), as well as enhancing ability to read and visualise images (Bar-On et al., 2001). Many of the aforementioned reports and studies measuring sedentary behaviour in youths have focused predominantly on television (TV) viewing which may confound the attribution of other sedentary screenbased behaviours (e.g., smartphone usage, video game playing, computer use; Biddle et al., 2016, Bouchard et al., 2015). Given the trend of sedentary technology use may continue to increase, it is critical that youths develop an awareness of their behavioral patterns to increase the likelihood of their intentions to change health-risk behaviours (Kremers et al., 2008).

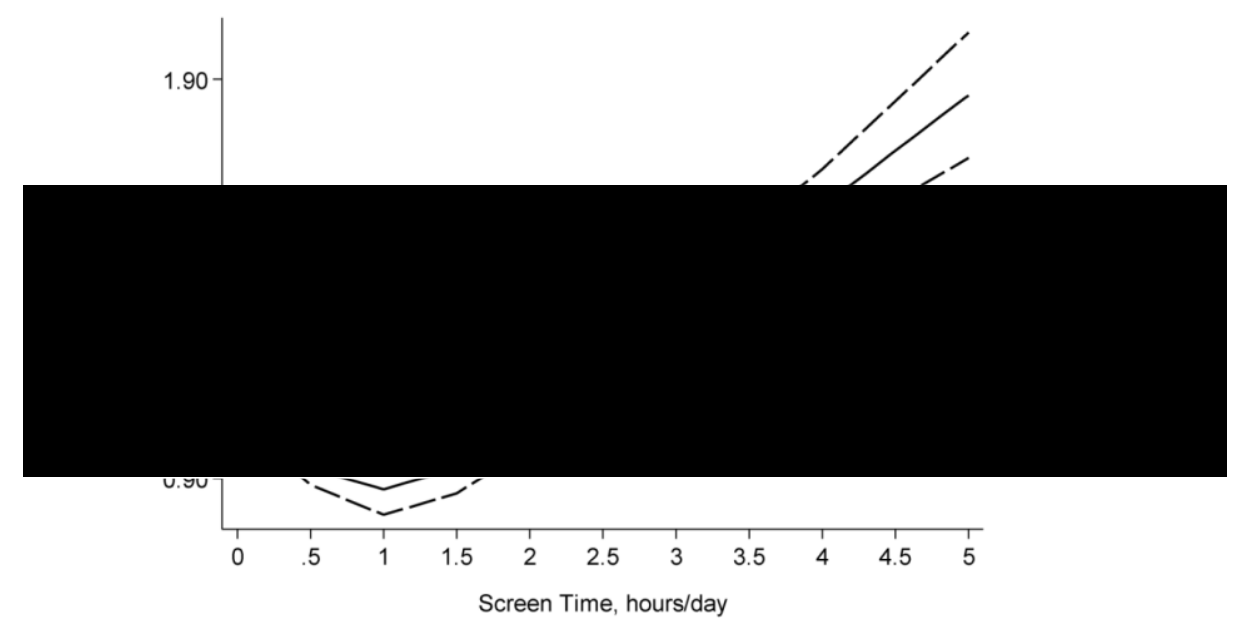

Figure 2.3 Curvilinear dose-response relationship between screen-time in youth and risk of depression. From Liu et al. (2016). 


\subsection{Youths' Awareness of their Physical Activity Levels}

Physical activity is a multi-dimensional health-related behaviour that consists of a large number of different activities (e.g., cycling, walking, running, swimming and jumping; Ronda et al., 2001) and measures of time and intensity (e.g., time engaged in moderate-intensity physical actvity; Thompson \& Batterham, 2013). More specifically, youths tend to display sporadic and irregular movement patterns, moving from one extreme intensity to another (e.g., from light- to vigorous-intensity physical activity; Adamo et al., 2009, Janssen \& LeBlanc, 2010b, Sleap \& Warburton, 1996, Baquet et al., 2007). These aforementioned complexities of physical activity make it especially hard for young people to accurately evaluate their PAL. One behavioural model that has be used by researchers to identify and work with young people to raise awareness of PAL and modify inactive behaviours is the Transtheoretical model (TTM; Prochaska \& DiClemente, 1992). The TTM, which incorporates stages of change, has been used extensively for different health-related behaviours and exercise (Ronda et al., 2001). Specifically, there are five stages of change that are distinguished (Figure 2.4): pre-contemplation (i.e., individual is not considering increasing their PAL), contemplation (i.e., individual is considering increasing their PAL), preparation (i.e., individual intends to increase PAL), action (i.e., individual has started to increase PAL more) and maintenance (i.e., individual is sustaining the desired PAL). Given that the TTM is a multistage theory, it has the potential to provide a complete picture of physical activity stage changes, which could enable conclusions to be drawn on what constructs or variables lead to behaviour change (Nigg \& Courneya, 1998). It is suggested through the TTM that there are certain cognitive processes that influence the early stages of physical activity behaviour, including consciousness raising, environmental-evaluation and self-evaluation (Maddux \& Rogers, 1983, Marcus et al., 1992, Prochaska et al., 1992, Prochaska \& Marcus, 1994). These cognitive processes encourage youth to be more aware of their activity levels, which often requires repeated awareness-raising to nudge an individual through the stages of behavioural change (Walton et al., 1999). Indeed, research has demonstrated that youth in the later stages of change (i.e., action and maintenance) reported significantly greater levels of both moderate- and vigorous-intensity physical activity to those in the earlier stages (i.e., precontemplation and contemplation; Lee et al., 2001, Wyse et al., 1995). Sanaeinasab and colleagues (2013) found a linear pattern of improvement in youth's 
time spent in MVPA per week across the stages of change (i.e., from precontemplation to the maintenance stage). However, there is some uncertainty as to how effective the stages of change are in influencing the level of light physical activity and sedentary behaviour (Haas \& Nigg, 2009, Schumann et al., 2002). Nonetheless, a more recent study found that $58 \%(\mathrm{n}=212)$ of youth resided in the precontemplation stage at the start of the study (Pope et al., 2015), which is similar to previous findings (Annesi et al., 2010, Prapavessis et al., 2004, Sanaeinasab et al., 2013, Walton et al., 1999, Wyse et al., 1995). These similar findings may suggest that future studies should investigate tailoring interventions to the first precautionary stages of change (Pope et al., 2015). In line with this notion, awareness of personal risk behaviours has been identified as an independent correlate for behaviour change, by motivating an individual to move from pre-contemplation to contemplation of behaviour change (Ronda et al., 2001, Kremers et al., 2008). Indeed, based on Weinstein's (1988) Precaution Adoption Process Model (PAPM), an individual can only be expected to proceed to contemplation when they are aware of their inadequate PAL. Despite this evidence to promote behavioural change, there is limited research investigating the awareness of PAL among children and adolescents.

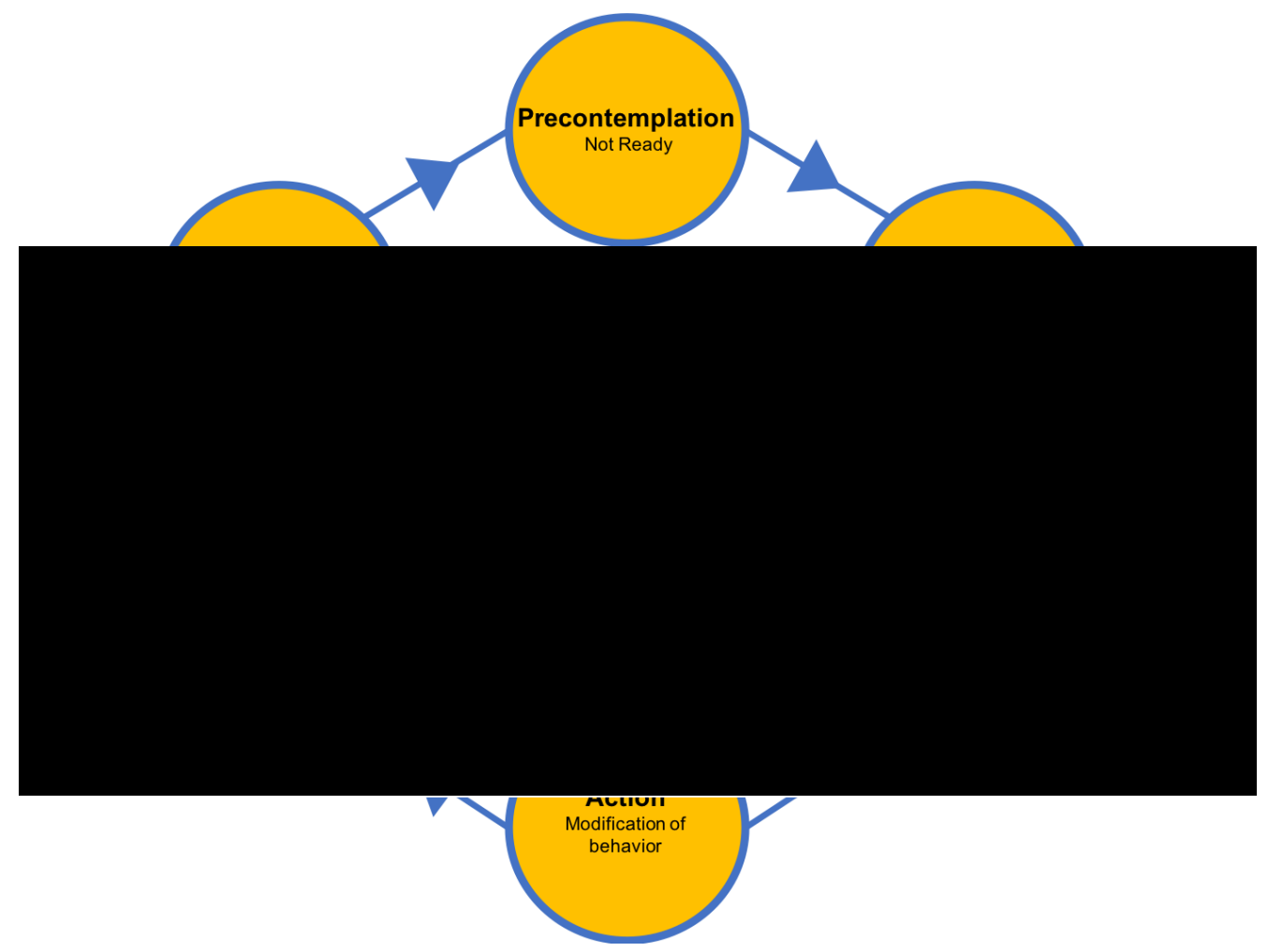

Figure 2.4 The five stages of change.

Adapted from Prochaska and DiClemente (1986). 
Physical activity awareness is defined as the agreement between self-rated and actual PAL (Van Hoye et al., 2012). Kremers et al. (2008) investigated the extent to which children, aged 8-13 years, were aware of their own PAL using a self-reported measurement. The findings revealed that $64 \%$ of the children were unaware of their PAL, with those that were aware of their physical activity behaviour reporting to be, on average, 20 minutes more active per day compared to their unaware counterparts. Of more concern perhaps was there was little consistency in children who couldn't accurately self-report, with $41 \%$ over-estimating and $23 \%$ under-estimating their PAL. However, caution must be taken as findings were based upon self-reported methods of physical activity that are known to elicit inaccurate measures of a child's activity behaviour when compared to objective methods (Adamo et al., 2009). Consequently, Corder et al. (2010) assessed physical activity awareness in youths aged 9-10 years using self-reported physical activity perceptions in contrast to an objective accelerometer measurement. In total, $31 \%$ of youths were objectively recorded as inactive, with $40 \%$ of this number overestimating their PAL and therefore lacking awareness. A more recent one-year observational study examined 1899 youths (9-12 years) response to a single question regarding their knowledge of healthy behaviour and their PAL. The findings revealed that after nine months $71 \%$ of youths demonstrated an increased awareness of the positive association between increased physical activity and reduced obesity. Furthermore, youths who demonstrated increased awareness also significantly increased their duration and frequency of physical activity by 12.7 minutes per day and 2.5 sessions of exercise per week, respectively, from baseline (Xu et al., 2017). Similarly, Huhman et al (2007a) reported a positive relationship between the frequency of youth viewing an activity health message and their behavioural outcomes related to physical activity. Specifically, youth who reported being more aware of the health message engaged in 4 weekly sessions of free-time physical activity, whereas youth who were not aware reported 3 sessions per week, which accounts for a $22 \%$ difference. According to Brusseau et al. (2011), youth construct knowledge and behaviour cumulatively, therefore, enhancing youths knowledge of the concepts and principles related to physical activity and how to apply them to daily tasks will likely increase independent cumulative learning and participation in physical activity (NASPE, 2004). Based on the 'Knowledge, Attitude and Practice Model' (KAPM), if an individual has specific knowledge about how their negative behaviours can increases their disease risk, they are more likely to modify 
their behaviour to counteract such adverse health problems (Ward et al., 2002).These findings suggest that knowledge dissemination and health education may be important strategies to promote positive behaviour modification among youths (Xu et al., 2017, Fairclough \& Stratton, 2004). Although previous research in adults and the use of health messages to increase physical activity has had little impact (Knox et al., 2013a), research among youths has shown this to have a positive influence on their physical activity attitudes and behaviours (Huhman et al., 2007b, DiLorenzo et al., 1998).

The evidence suggests that youths who are unaware of their PAL are less likely to perceive the need to change unhealthy risk behaviours (Kremers et al., 2008). According to Kremers et al. (2008), a lack of awareness among youths is likely to make them less susceptible to educational programmes that are aimed to influence attitudes, norms, self-efficacy or other cognitive means, as they will not perceive the need to change. Furthermore, there is contradictory research that the lack of effectiveness of physical activity interventions could be, in part, explained by individuals lacking awareness of their physical activity behaviours, such as believing themselves to be more active than they really are (Oenema \& Brug, 2003). Therefore, to effectively promote physical activity through health education programmes, researchers should primarily focus upon raising awareness levels among youths (Kremers et al., 2008). Indeed, the PAPM suggests that individuals are unlikely to perceive the need to change unhealthy behaviours unless they become aware that their behaviour is not optimal, such as, 'I do this much MVPA but this much MVPA is recommended' (Weinstein, 1988). In this case, the UK guidelines of 60 minutes of MVPA are used as a minimum recommended level for youths to achieve and gain associated health benefits. In line with this, evidence suggests that interventions that implement knowledge of health messages among youths, such as the guidelines, have been shown to increase physical activity and energy expenditure (Kahn et al., 2002, Belton et al., 2014, Borges et al., 2015). Therefore, it could be postulated that the lack of awareness of the guidelines may be a contributing factor to the declining PAL in youths (Roth \& Stamatakis, 2010). 


\subsection{Youths' Knowledge of the Physical Activity Guidelines}

Institutes, such as The National Institute for Health and Care Excellence (NICE) and the British Heart Foundation (BHF), support the use of the 60 minutes MVPA guidelines to promote awareness of the importance of physical activity and its health benefits among youths (NICE, 2015, Townsend et al., 2015). The Health Survey England published in 2007 investigated youths', aged 11-15 years, knowledge of the physical activity guidelines. The report revealed that out of 2,510 participants, only $10 \%$ of boys and girls were able to state the current minimum physical activity guidelines (HSE, 2007). Furthermore, a smaller number of youths cited more than the current minimum ( $8 \%$ boys, $3 \%$ girls), with $81 \%$ and $87 \%$ of boy and girls, respectively, reporting less than the current minimum or having no knowledge (HSE, 2007). In accord with Kremers et al. (2008), the HSE found that those youths who correctly reported the guidelines were in the high category of physical activity and did attain the target of 60 minutes MVPA, although their ability to articulate the guidelines was limited to time spent being active (e.g., 60 minutes).

Snethen et al. (2001) found a limited comprehension about the physical activity guidelines, including the recommended frequency, intensity and duration of physical activity in a population of overweight youths aged 8-12 years. Similar findings were reported in adolescent girls, showing a lack of knowledge of the guidelines with little awareness of the required intensity (Sleap \& Wormald, 2001). A more recent study highlighted that this lack of knowledge regarding the guidelines is still present among youths, expressing varying durations of time from 20 minutes to 5 hours, with youths most commonly suggesting 2 hours per week of physical activity (Harris et al., 2016). The lack of knowledge over the guidelines is disconcerting, especially given the number of years that public health physical activity recommendations have been available and the evidence supporting that enhanced knowledge facilitates PAL for some youths. For these reasons, better ways of marketing, disseminating and implementing physical activity recommendations are required. However, this lack of knowledge regarding the recommended targets may not be the only concern (HSE, 2007, Roth \& Stamatakis, 2010). Understanding youths' perceptions about a particular behaviour is equally important and should not be neglected when designing programs to initiate behaviour change, such as physical activity (Cottrell et al., 2012). According 
to Noonan et al. (2016), there is a lack of understanding within youths on the various forms of physical activity, specifically those of active travel and unstructured play, with a need to educate how these types of activities contribute to achieving physical activity targets. Similarly, Placek et al. (2001) highlighted that youths have certain limitations and inaccuracies in defining and relating their physical activity to the principles represented by FITT. Indeed, enhancing an individual's knowledge and understanding of the complexities of physical activity, such as the type, intensity, frequency, duration and their associations to health are likely to drive an individual towards achieving appropriate levels of health behaviour (Bandura, 1998, Nutbeam, 2000).

\subsection{Youths' Understanding of Physical Activity}

Despite the promotion of active lifestyles being a central aim of the UK government for many years, there is little evidence that it has significantly impacted youths' knowledge and understanding of physical activity and behavior (Harris et al., 2016). Since the early 1990s, findings have reported that youths have misconceptions and misunderstandings about health, physical activity and fitness (Brusseau et al., 2011, Burrows \& Wright, 2004, Burrows et al., 2002, Burrows et al., 2009, Desmond et al., 1990, Dixey et al., 2001, Harris, 1993, Harris, 1994, Keating et al., 2009, Kulinna, 2004, Lee \& Macdonald, 2009a, Lee \& Macdonald, 2010b, Merkle \& Treagust, 1993, Placek et al., 2001, Powell \& Fitzpatrick, 2015, Shea \& Beausoleil, 2012, Stewart \& Mitchell, 2003, Trost et al., 2000a). Trost et al. (2000) addressed the issues of children's understanding of the term physical activity using a 17-item checklist displaying different activities (e.g., from playing video games to climbing trees) and asked the children to write down what they thought physical activity meant. In total, 127 youths (aged 8-9 years) participated in the study and were grouped into three experimental groups, a video or verbal instruction group and a no instruction group. The video group developed the best understanding of physical activity, followed by the instruction group. Worryingly, 38\% of the control group indicated that working on the computer was a physical activity, with approximately $30 \%$ believing that sweeping the floor was not a physical activity. Nonetheless, it is important to consider the applicability of activities, such as sweeping the floor, as a lack of familiarity may lead youths erroneous conclusions regarding the intensity (Li, 2016). That said, findings 
from Trost et al. (2000) highlight that youths aged between 8-9 years, without prior intervention, demonstrate a limited understanding of physical activity, as defined by researchers and health practitioners. The following year, research from the USA revealed that youths aged between 11-12 years also had difficulty in defining physical activity, especially in terms of fitness components, activities that improve fitness and the principles of training (Placek et al., 2001). For instance, youth had trouble relating physical activities to the four main components of fitness, such as bicycling, pushups, stretching and weight lifting to enhance cardiovascular endurance, muscular endurance, flexibility and muscular strength, respectively (Placek et al., 2001). It can be seen that the types of activities associated with physical activity are largely based upon sport specific team games (Everley \& Macfadyen, 2015, Harris et al., 2016, Macdonald et al., 2005, Noonan et al., 2016, Pearce \& Bailey, 2011). Specifically, Everley and Macfayden (2015) used a drawing technique to elicit children's perceptions of physical activity, finding that boys most commonly drew themselves taking part in team games (56\%), with football the dominating activity (46\%). Noonan et al. (2016) reported similar findings using the write, draw, show, and tell (WDST) method, with physical activity most frequently associated with organized sports (e.g., football, basketball, gymnastics; Figure 2.5). Consequently, research has shown that children do not account for unstructured forms of physical activity, such as active travel, dog walking, active play, video gaming and household chores (Noonan et al., 2016, Trost et al., 2000a), even though data from Noonan et al. (2016) reported that $60 \%$ of participants walked to school regularly.

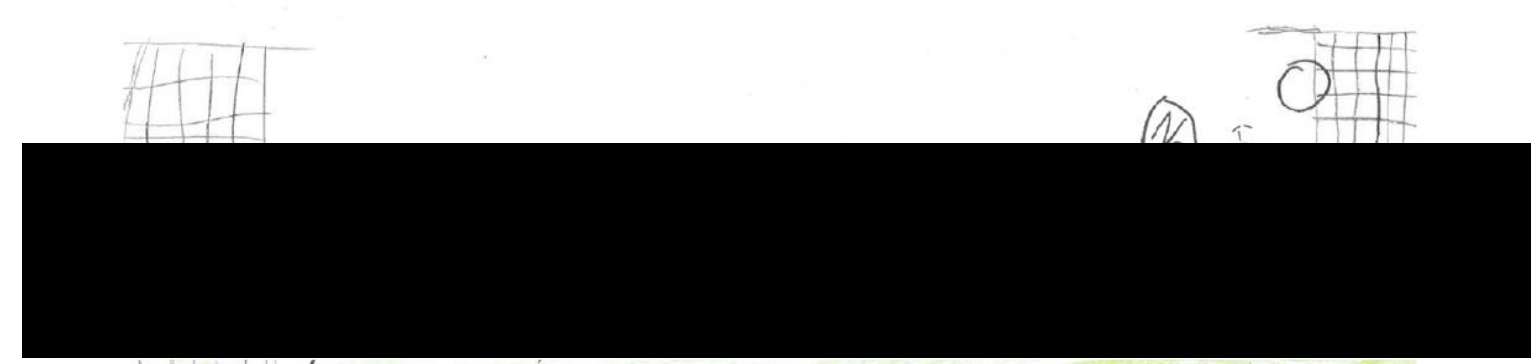

Figure 2.5 Drawing from a boy aged 11 years old illustrating playing football with friends. From Noonan et al. (2016). 
It is likely that cognitive developmental differences between children and adolescents have an impact on their understanding of the concepts related to physical activity (Piaget \& Cook, 1952). Following Piaget's developmental theory, early cognitive development in youth involves processes based upon actions, whereas later it changes to more mental operations (Piaget \& Cook, 1952). In more detail, Piaget proposed four stages of cognitive development to reflect increasing sophistication of a child's thought. These include: 1) sensorimotor stage, birth to age 2;2) pre-operational stage, from age 2 to 7 ; 3) concrete operational stage, from age 7 to 11 ; and 4) the formal operational stage, age 11+ (Piaget \& Cook, 1952). Each child goes through these stages in the same order up until adolescence, however, the rate at which a child progresses through the stages varies due to biological maturation and interaction with the environment, with some youth never attaining the later stages. Given that adolescents may differ to children as a result of their greater ability to think about abstract concepts and logically test hypotheses, it could be conceivable that their understanding of physical activity is greater. Indeed, research has demonstrated that youths' understanding of concepts related to physical activity, such as their ability to identify physical activity for health enhancement and vigorous-intensity activities, improves with age (Brusseau et al., 2011). On the contrary, more recent evidence suggests that this may not be the case (Harris et al., 2016). For example, Harris et al. (2016) reported that adolescents aged 11-15 years have a limited awareness of incidental or routine moderate-intensity activities (e.g., walking) and informal recreational activities, including scootering or roller skating. Similar findings were found within Australian adolescent girls, who did not consider walking, cycling and physical labour to be legitimate health-enhancing activities (Lee \& Macdonald, 2009b, Lee \& Macdonald, 2010a). One of the problems is that youths tend to associate physical activity with being thin and/or being good at sport, as documented in the early 90s (Harris, 1994, Harris, 1993). Youths limited conceptualisation of what types of activities form physical activity is of concern and may have potential impacts on youths' physical activity participation due to the perceived notion that they are not good at sport or believing that the activities they choose are not health enhancing.

One possible reason for youths' lack of understanding physical activity is suggested to be their limited ability to understand and identify different intensities of physical 
activity (Cowden \& Plowman, 1999, Prochaska et al., 2001, Snethen \& Broome, 2007, Placek et al., 2001, Pearce et al., 2008). Placek et al. (2001) discovered that youths had no concept of the intensity required to improve fitness levels, with no youths mentioning checking heart rate as a way of monitoring effort during running or number of repetitions and/or sets during weight training. Moreover, Pearce et al. (2008) found that youths had difficulty in categorising their perceptions of intensity as a result of skill for an activity or physical competence influencing their ability to identify the physical activity intensity. Perhaps this level of competence or skill for an activity is explained, in part, by Erikson's (1998) theory of psychosocial development, whereby youths are developing a strong sense of competence for specific activities, which in turn, could influence their ability to accurately define the physical intensity. Nevertheless, Pearce et al. (2008) did report how some youths demonstrated an ability to assess the intensity of activities by body cues, such as heartbeat and sweat. These reported types of body cues for activity intensity were similar to those found in previous research, with "tired" acting as an indicator for higher intensity activity (Lévesque et al., 2004). Indeed, the children's OMNI exertional scales was developed using the body cue "tired" (Figure 2.6; Robertson et al., 2005, Robertson et al., 2000). In this respect, youths demonstrate a greater ability to identify the intensity of their activities based in laboratory settings (Pfeiffer et al., 2002, Robertson et al., 2005, Robertson et al., 2000). A recent longitudinal analysis revealed that the validity of the OMNI scale was strong for children aged 9-10, 11-12, and $\geq 13$ years, although concluded that caution should be used when interpreting OMNI reports from children younger than 8 years (Gammon et al., 2016). The lack of ability to define intensity at younger ages aligns with previous research reporting that children do not have the cognitive ability to respond to biofeedback from their body (Brief, 1983). Therefore, it is reasonable to accept that younger children have difficulty in rating their physical activity intensity according to the OMNI scale. Although children have a limited ability to consider the intensity of physical activity, some promise has been shown in non-laboratory settings with adolescents considering that increased levels of physical activity depends on the type of activity performed (e.g., running, star jumps and Wii Fit Plus) and on its intensity and duration (Harris et al., 2016). However, these adolescents still had little understanding of routine moderate-intensity activities, such as walking to school, which highlights gaps within their understanding of intensity. 


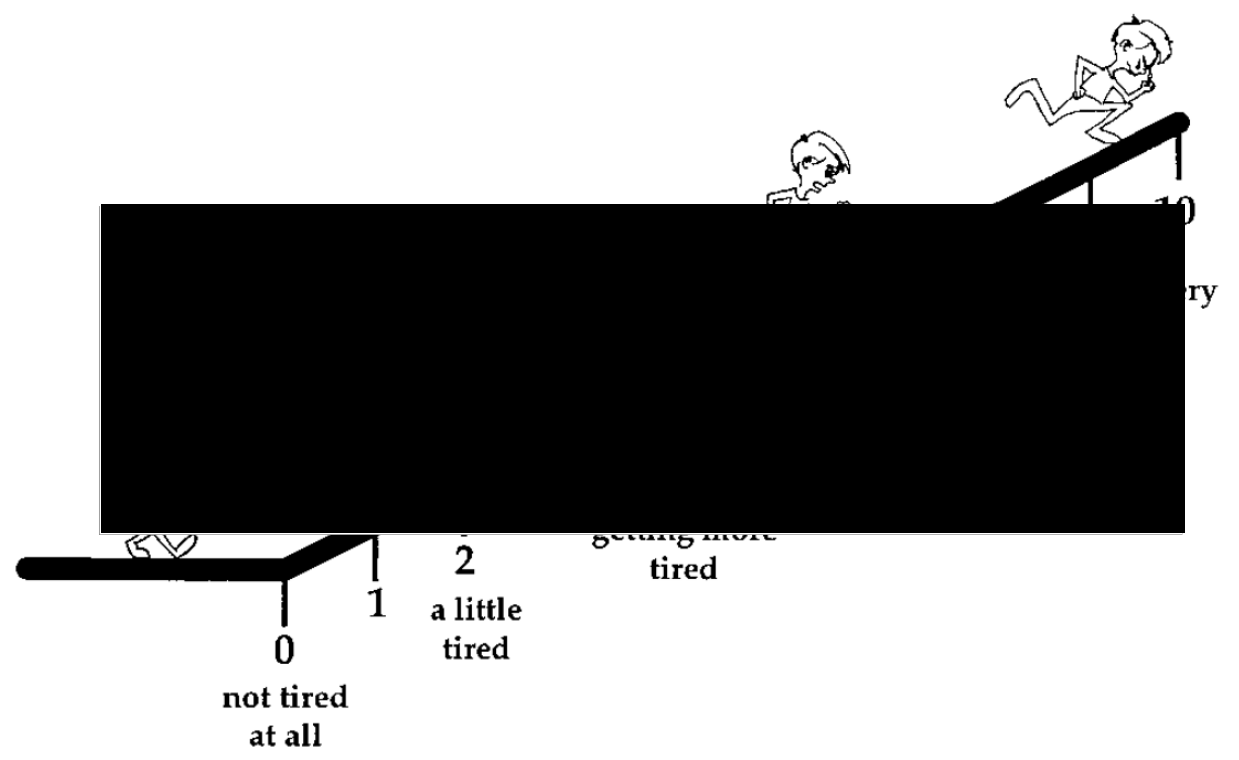

Figure 2.6 The children's OMNI scale of perceived exertion.

From Robertson et al. (2000).

Although evidence on the impact of knowledge and understanding of physical activity to significantly increase an individual's PAL is questionable (Abula et al., 2016), findings are equivocal (DiLorenzo et al., 1998). Interestingly, Nemet et al. (2012) compared knowledge of physical activity in different socio-economic groups of young children, revealing that children from lower socio-economic backgrounds reported poorer knowledge of physical activity when compared to their middle socio-economic counterparts. Although the findings from Nemet et al. (2012) were limited in detailing children's knowledge of physical activity, the findings may, in part, explain the higher prevalence of overweight British children in lower socio-economic backgrounds reported today (Bann et al., 2018). It is therefore important to recognise this lack of knowledge regarding the complexities of physical activity within youths as development of content knowledge is a critical step for youth achieving healthy and active lifestyles that can be continued into adulthood (Brusseau et al., 2011). Having this knowledge of the concepts and principles related to physical activity and how to apply them is likely to enhance the likelihood of independent cumulative learning and increased participation in physical activity (NASPE, 2004). It has been suggested that youths inadequate and/or inaccurate understandings of physical activity are the consequence of a lack of teaching within the school environment (Burrows \& Wright, 2004, Burrows et al., 2002, Lee \& Macdonald, 2009b, Lee \& Macdonald, 2010b). 
Given the role of schools in influencing healthy lifestyle choices, it is essential that they provide both opportunities for activity and a learning space for building knowledge related to physical activity and health behaviors (Brusseau et al., 2011).

\subsection{School Context for Physical Activity Promotion}

The importance of schools in promoting health behaviours and physical activity is widely acknowledged among researchers (Cale \& Harris, 2004, Harrell et al., 1999, Penney \& Jess, 2004, Salmon et al., 2007, Warren et al., 2003). Youth attend school for at least a decade of their lives (Story et al., 2006), spending approximately $40 \%$ of their waking time in a school setting (Fox et al., 2004). Some researchers have found that a majority of youths' physical activity takes place during the school day, in the form of active travel to school, physical education, playtime and extra-curricular activities (Fairclough et al., 2007, Mota et al., 2003). Schools can make a difference in providing clear and consistent messages to a large population of youths from a broad range of socio-economic backgrounds on the benefits of attaining physical activity recommendations and developing healthy lifestyle behaviours (Naylor et al., 2008). Given that schools have a pre-established organisational, social and communication structure, they are a key setting for providing youth with opportunities to engage in regular physical activity and health education that can continue into adulthood (Fairclough et al., 2013). As identified by Armour and Harris (2013), government initiatives are increasingly looking to schools as an appropriate setting for public health investment. For example, the 'Moving More, Living More' Government plan inspired by the London 2012 Olympic and Paralympic Games with the aim of having a more physically active nation (Cabinet Office, 2014). The document identifies school time and Physical Education (PE) curriculum as potential strands to increase PAL among youths. Similarly, the All-Party Commission on Physical Activity report ‘Tackling Physical Inactivity - Coordinated Approach' (All-Party Commission on Physical ActivityActivity, 2014) advocates a whole school approach, including high quality PE, the development of physical literacy, active lessons, activity breaks and active travel. Indeed, many school-based interventions have sought to change on the school environment, curriculum and structure of the school day in hope to increase youths' PAL (Timperio et al., 2004). A number of these interventions have been successful in promoting youths' physical activity through making changes to the 
playground environment (Stratton \& Mullan, 2005), delivering classroom health education (Caballero et al., 2003) and curriculum-based game sessions during playtime (Connolly \& McKenzie, 1995), as well as physical activity breaks during lessons (Donnelly et al., 2009, Kriemler et al., 2010) and providing youth with games equipment (Jago \& Baranowski, 2004). Most importantly, promotion of physical activity within the school setting has shown to not only increase in school PAL but also out of school PAL (Kriemler et al., 2011), which is crucial given that evidence suggests that youth are less physically active at weekends compared to weekdays when attending school (Fairclough et al., 2012). Additionally, school settings have the potential to create sustainable physical activity strategies that can be delivered by school personnel in 'real life' conditions (i.e., without the aid and resources of researchers; De Bourdeaudhuij et al., 2011), which lends to reduced costs to run (Warren et al., 2003) and an increased likelihood of the strategy being adopted into the existing curricula and maintained over time (Stone et al., 1998).

Despite the current drive in promoting youths' physical activity, the effectiveness of strategies within schools, or a lack thereof, appears to be a global issue (Cardon et al., 2012). Much of this concern has been expressed over the marginal status and limited attention given to health and physical activity within school contexts (Alfrey et al., 2012, Bailey, 2010, Cale, 2000, Cale \& Alfrey, 2013, Cardon et al., 2012, Marks, 2008), including teachers and PE teachers' limited knowledge and understanding of physical activity for healthy lifestyles (Trost, 2006, Tang et al., 2008, St Leger, 2004, Speller et al., 2010, McKenzie, 2007, Larsen et al., 2012, Kulinna et al., 2008, Jourdan et al., 2010, Davidson, 2007, Castelli \& Williams, 2007). Equally, numerous factors beyond the school environment influence youths' health and PAL, such as individual (e.g., age, sex, education, socio-economic status, beliefs, self-efficacy), sociocultural (e.g., social support, parental/siblings physical activity, ethnicity, social capital and norms), and the environment (e.g., seasonality, urban and transport policy, access to facilities, traffic, crime and safety rates; Davison \& Birch, 2001, Stokols et al., 2002). However, it is screen-based technology that has garnered the most public criticism for the disparity of youths engagement in physical activity (Carrington, 2016). 


\subsection{Technology as a Barrier to Youths' Physical Activity Engagement}

In today's society, technology has become an important part of youths' everyday lives (Smahel et al., 2015), with youths being labelled as 'digital natives' (Prensky, 2012) and the 'iGeneration' (Rosen, 2012). Technological innovations, including the internet, smartphones, sophisticated video games and the abundance of TV options have changed how youths engage with their environments, with much less engagement in the world outside their homes (Hillier, 2008). The adoption of these technologies is creating environments that enable youths to communicate with friends around the world faster through video gaming or social-media platforms, such as Facebook that encourage 'likes' that can "bring dings of pseudo-pleasure" (Lewis, 2017). However, this use of technology to attain functionality and pleasure can quickly become a form of addiction that encompasses sedentary behaviour (Kim et al., 2015). This advancement in technology could, at least in part, be attributable to the increasing weight status currently reported (Lakdawalla \& Philipson, 2009). Moreover, is the worrying amount of time youths spend engaging in sedentary screen-based activities, such as internet usage, watching TV and playing video games (Coleman, 2014, Foley \& Maddison, 2010). The 2017 UK Ofcom report revealed that youths aged 5-15 years typically spend 15 hours on their mobile and online, 14 hours watching TV and 10 hours playing video games per week (Figure 2.7; Ofcom, 2017). Moreover, as youths age, their time spent online per week and the percentage of youths online increases (Coleman, 2014, Greca et al., 2016), from 9 (79\%) to $13 \frac{112}{2}(94 \%)$ to 21 (99\%) hours for 5-7 years, 8-11 years and 12-15 year old youths, respectively (Ofcom, 2017). It is, however, worth bearing in mind that these Ofcom figures on technology use are derived from self-reported levels of technology use from parents' and youths', which relies heavily on an individual's ability to recall their behaviours and is open to a degree of under- and over-reporting. Given these inherent difficulties in quantifying technology use, the Ofcom figures work best as an indication of the relative balance of these types of activities and how this balance changes with time within youths' lives. 


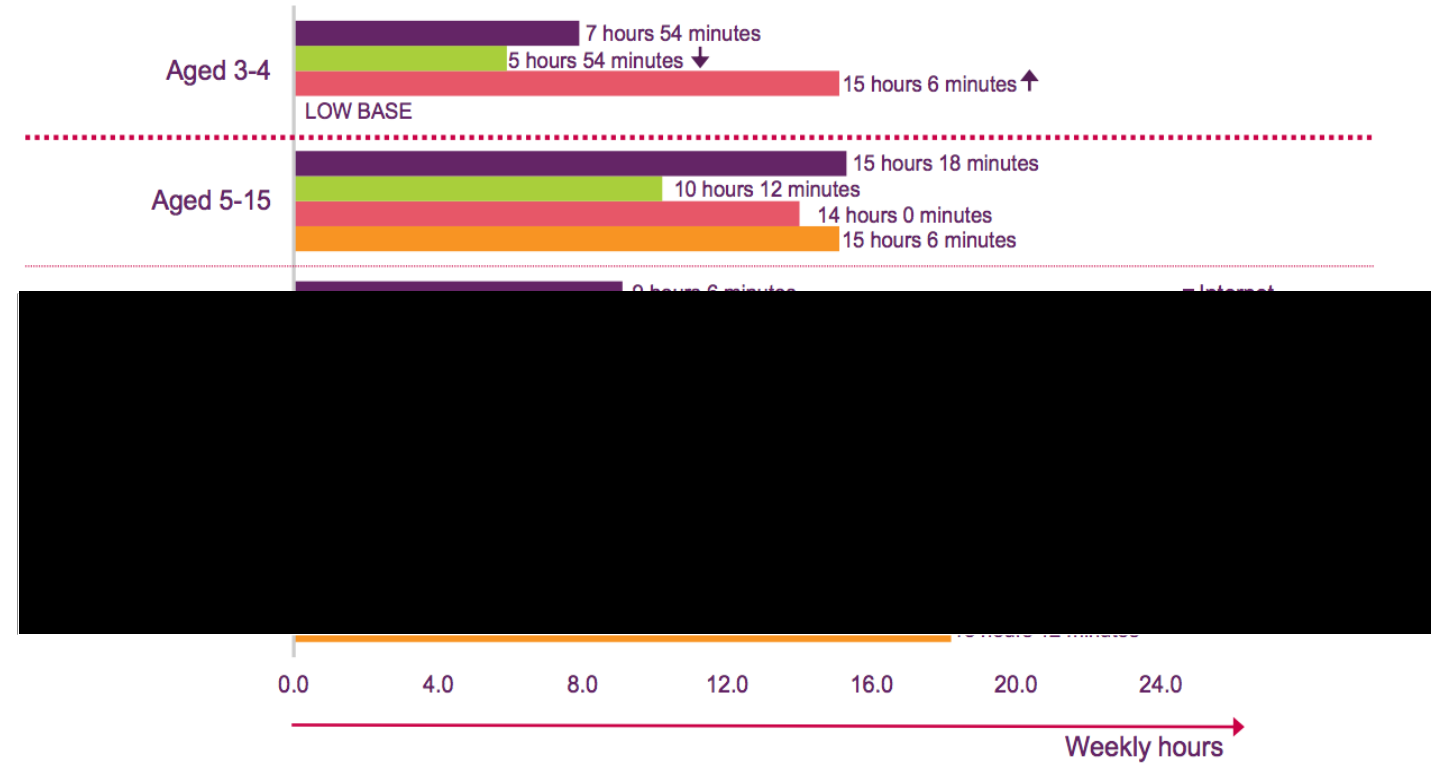

Figure 2.7 Estimated weekly hours of media use in UK youth. From Ofcom (2017).

As a result of the deleterious health consequences associated with sedentary screenbased behaviours (Wethington et al., 2013, Wyszynska et al., 2017, Martinez-Gomez et al., 2010b, Torsheim et al., 2010, Tsai et al., 2007, Sandercock \& Ogunleye, 2013, Banyai et al., 2017), countries such as the USA, Australia and Canada have adopted sedentary guideline recommendations towards limiting youths recreational screen time to less than 2 hours per day (American Academy of Pediatrics, 2001, Australian Government, 2017, Canadian Society for Exercise Physiology, 2017). However, it remains unclear as to whether screen-time is negatively associated to health simply because it displaces physical activity behaviours (Cummings \& Vandewater, 2007). Indeed, previous research supports that there is no association between youths' physical activity and the amount of time spent in sedentary screen-based activities (Serrano-Sanchez et al., 2011, Feldman et al., 2003, Silva et al., 2016), with some studies finding that screen-time did not distinguish the active and inactive youths (Santos et al., 2005, Karaca et al., 2011). On the contrary, Tammelln et al. (2007) found that youths who engaged in more than 4 hours per day watching TV had a 1.42.5-fold increased risk of not achieving the recommended MVPA guideline compared to those youths who watched less than 1 hour of TV per day. Moreover, The Health Behaviour in School-aged Children study (Melkevik et al., 2010) reported that, for both boys and girls, exceeding 2 hours of daily total screen time was negatively 
associated with MVPA. Similarly, Ferrari et al. (2015) reported that youths who viewed less than 2 hours a day of screen-time accumulated significantly more MVPA and steps on weekdays than their counterparts who reported over 2 hours of screen time.

A recent systematic review suggests that interventions aimed at reducing recreational screen-time are effective in reducing the time youths spend in sedentary screen-based behaviours and increasing PAL (Buchanan et al., 2016). The Incorporating More Physical Activity and Calcium in Teens (IMPACT; Jones et al., 2008) study sought to investigate the effect of promoting physical activity in conjunction with implementing physical activity classes (e.g., rope jumping) three times a week in adolescent girls. The findings revealed a significant increase of $45.4 \%$ in vigorous physical activity from baseline, with a significant decrease in the intervention group screen-time of $16.7 \%$ in contrast to a $17.9 \%$ increase in the control participant's screen-time (Jones et al., 2008). However, previous interventions are largely based on reducing youths' TV viewing time and neglect the potential impact of more recent technologies such as smartphones and tablets that are rarely reported (Buchanan et al., 2016). Indeed, much concern has been expressed on the impact of social media apps accessed through smartphone or tablet devices on youths' physical activity engagement, psychological health and sedentary behaviour (Eckersley, 2011, Banyai et al., 2017). Although there is a paucity of evidence on the impact of smartphone use on PAL, research has shown that smartphone use and physical activity are negatively associated in college age students (Kim et al., 2015, Lepp et al., 2013). It is unknown to what extent these devices may impact youths' physical activity behaviours, practically given that screentime is highly valued and enjoyable behaviour by youths, with attempts to restrict it usually met with resistance as they are reluctant to relinquish such technology (Faith et al., 2001). Therefore, using technological innovations and applications to promote and reverse un-healthy patterns of behaviour in youth are likely to hold more promise (Hillier, 2008).

\subsection{Technology as a Facilitator to Youths' Physical Activity Engagement}

Within the context of this thesis, technology for health purposes is defined as an application of organised health data, feedback and knowledge in the form of devices 
and ubiquitous systems developed to solve health problems, provide reflection and promote quality of life. With technology becoming ubiquitous in youths' everyday lives (Kretschmann, 2015), researchers are recognising technology as a 'new channel' in which to increase youths' PAL (Cullen et al., 2013). Technology-based methods are seen to be particularly advantageous compared to previous strategies as they can reach and enable youths who might want to seek help anonymously and autonomously, with more sensitive health topics or stigmatised behaviours (Khadjesari et al., 2011), or those individuals, particularly boys, who prefer self-help (Ellis et al., 2013). The earliest systematic review on the use of technology-based interventions to promote youths' physical activity was conducted by Norman et al. (2007), reporting that technology-based interventions were effective at changing physical activity behaviour in youths. However, the review was limited as conclusions were drawn on a larger sample of adult outcomes, lacking generalisability to youth populations. Since then, numerous additional systematic reviews have been conducted, supporting the effectiveness of utilising technology to promote behaviour change in youths (Barnett et al., 2011, Biddiss \& Irwin, 2010, Buhi et al., 2012, Campos \& del Castillo Fernández, 2016, Lappan et al., 2015, Lau et al., 2011, LeBlanc et al., 2013, Liang \& Lau, 2014, Park \& Calamaro, 2013, Peng et al., 2013, Rose et al., 2017, Shaw et al., 2015). Specifically, Lau et al. (2011) evaluated the efficacy of youth technology-based interventions, reporting that more than $65 \%$ of the interventions demonstrated a positive effect on psychosocial or behavioural physical activity outcomes. Technology-based interventions are diverse in nature, as they can be delivered through websites, text messages, games or apps, email, social media platforms or be a combination of methods. Understanding how youths interact and experience different platforms of technology is imperative for identifying and adapting future strategies for physical activity promotion.

Website-based interventions have resulted in marked improvements in PALs among youths (Chen et al., 2011, Cook et al., 2014, Cullen et al., 2013, De Bourdeaudhuij et al., 2010, Guthrie et al., 2015, Jago et al., 2006, Slootmaker et al., 2010, Whittemore et al., 2012). For example, Guthrie et al. (2015) found that participants taking part in an accelerometer-linked online website intervention with financial rewards, significantly increased their MVPA by $49 \%$ and $67 \%$ more than the passive (accelerometer no website) and active (accelerometer and dance game) control groups, 
respectively. However, incentivised physical activity through monetary rewards, such as gift cards, questions whether similar effects in MVPA could be obtained without the inclusion of financial incentives, given that individuals may only engage in physical activity to achieve the monetary reward (Lepper et al., 1973). Nonetheless, a recent systematic review provides strong evidence for the use of behavioural incentives to promote youths physical activity engagement (Corepal et al., 2018). Oliver and Brown (2012) argue that maintained behaviour change in youths could be achieved if the incentives are in place for a sufficient amount of time, however, the appropriate length of time remains unclear.

Short messaging services (SMS) or text messaging offers many benefits in comparison to other modes of communications, including speed, cost and convenience (Davie et al., 2004). Given that youths are heavy users of texting (Lenhard, 2009), a SMS intervention that encourages youths to be take more steps may be an effective strategy to increase physical activity that can be sustained throughout life (Thompson et al., 2014). Indeed, intervention delivering daily texts of encouragement to youths have had some level of success to increasing PAL (Lau et al., 2012, Sirriyeh et al., 2010). Of more recent interest are the use of social media platforms (e.g., Facebook and Instagram) to encourage and promote positive behaviour change (Bennett \& Glasgow, 2009, Pedrana et al., 2013, Vyas et al., 2012, Webb et al., 2010), although there is a paucity of literature regarding social media to elicit increased physical activity (Park \& Calamaro, 2013, Shaw et al., 2015, Cavallo et al., 2012, Kelty et al., 2012, Wojcicki et al., 2014, Mendoza et al., 2017, Pumpera et al., 2016, Ridgers et al., 2017). Specifically, a recent 10-week intervention utilising the Fitbit Flex activity tracker in partnership with a Facebook group for encouragement found no significant difference in MVPA between the intervention and control participants, however, modest differences were found for select subscales of quality of life and motivation for physical activity in the intervention group (Mendoza et al., 2017).

Over the past decade a rapidly emerging body of evidence supports the use of 'active video games' (e.g., Nintendo Wii Fit, Dance Dance Revolution, Microsoft Kinect) to promote youths' physical activity (Bethea et al., 2012, Errickson et al., 2012, Gao et al., 2011, Maloney et al., 2008, Murphy et al., 2009, Graves et al., 2010, Owens et al., 
2011, Campos \& del Castillo Fernández, 2016, Carmo \& Palmeira, 2013, Daley, 2009, Guy et al., 2011, Johnson et al., 2016, Pakarinen et al., 2017, Parisod et al., 2014, Sween et al., 2014). However, more recent statistics suggest that youths are increasingly preferring smartphone devices to computers and consoles for gaming entertainment (The NDP Group, 2015). Of note are the use of smartphone apps, such as the running app 'Zombies, Run!' and the more gaming app 'Pokémon GO', which requires a user to walk around their environment (i.e., city or park) catching virtual 'Pokémon' characters (Figure 2.8). For example, Althoff et al. (2016) found that the app 'Pokémon GO' significantly increased youths physical activity by $25 \%$ when compared to their prior activity levels. However, Rasche et al. (2017) reported that high numbers of former 'Pokémon GO' users quit after a short period of time as a result of boredom, with this duration of playing the gaming app shorter than the average time individuals abandoned activity trackers (Ledger \& McCaffrey, 2014). Indeed, previous studies utilising technology to promote physical activity highlight that maintaining user engagement can be a significant challenge (Owens et al., 2011, Staiano et al., 2012), and it is expected that more change will come in terms of how technology is utilised, especially with the on-going development of smartphones and tablets (Shaw et al., 2015).

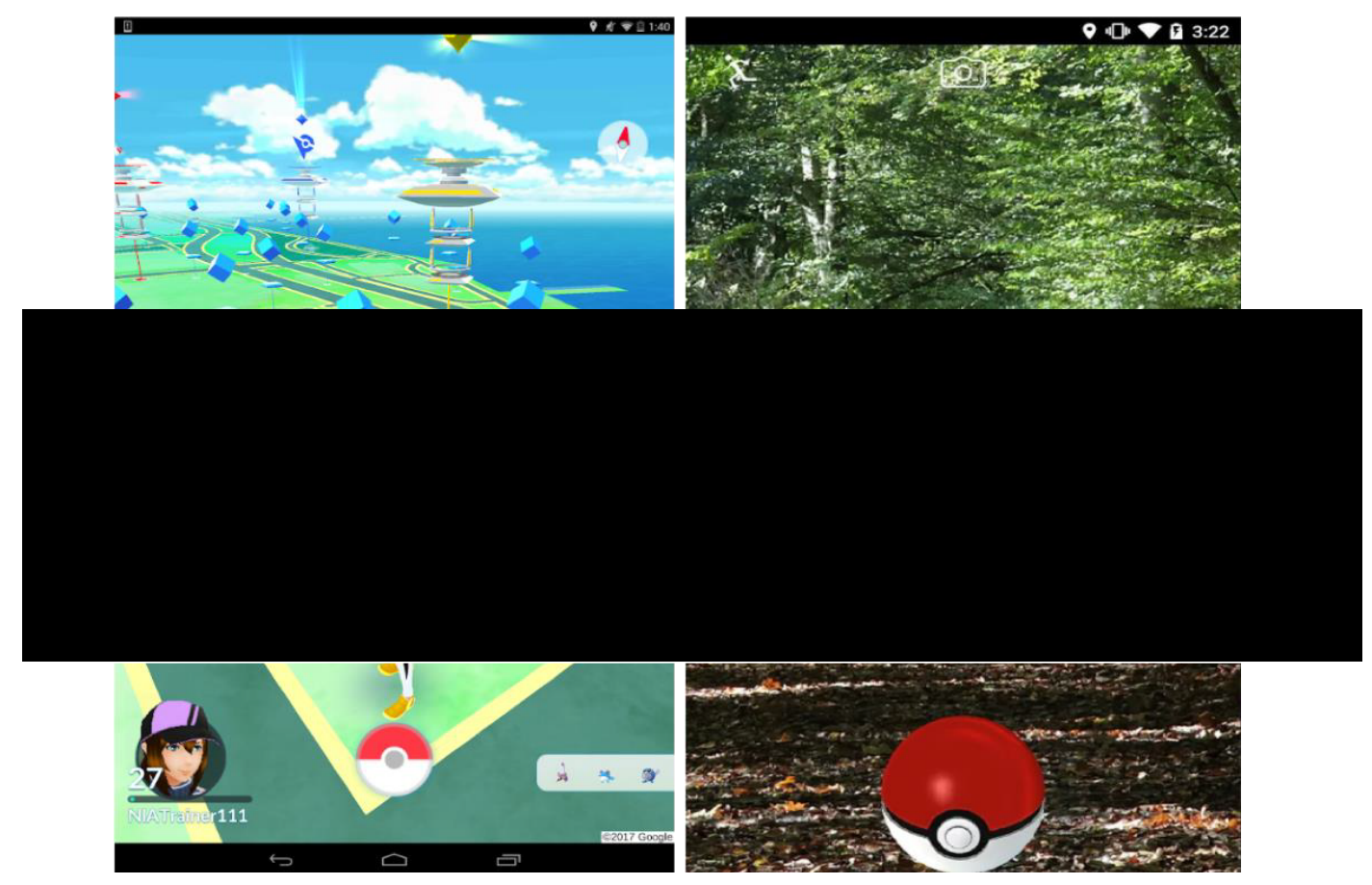

Figure 2.8 'Pokémon GO’ app gameplay.

From Pokémon GO (Pokemongo.com). 
A recent systematic review, exploring the effectiveness of the aforementioned strategies, including websites, SMS, active video games, smartphone gaming apps and social media platforms, concluded that particular interventions utilising technology should incorporate self-monitoring, goal setting, education and parental involvement (Rose et al., 2017). Taken from this, it is well documented that self-monitoring devices, such as objective measurement tools are important in raising an individual's awareness of their PAL (Van Hoye et al., 2012, Bentley et al., 2012). Furthermore, objective measures of physical activity are not only useful for monitoring youths' behaviours in and out of school (Brusseau et al., 2011), but also have potential to enhance youths' understanding of physical activity and encourage optimal levels of activity for health benefits (Harris, 2014).

\subsection{Objective Measures of Physical Activity}

Measuring physical activity is pivotal to our understanding of the protective health benefits, frequency, intensity, type and duration of activity that individuals engage in (Strath et al., 2013). Perhaps the most important feature of any monitoring method is the accuracy and precision of which it measures the desired variable (Butte et al., 2012, Kelly et al., 2016). Specifically, the accuracy (or validity) refers to how true the measurement is compared to what is really happening, whereas precision (or reliability) refers to the consistency of the measure to accurately quantify the component of interest (Bassett Jr, 2000). In the context of physical activity assessment, accuracy would relate to the appropriate estimation of energy expenditure at a given moment in time, whilst precision would note whether the same estimate of energy expenditure on a specific day and activity would be scored the same on another day, week or month. It is important to understand that the constructs of accuracy and precision are not dependent upon each other, in that a measure can be reliable but not valid and vice versa (Bassett Jr et al., 2012). For example, consistently measuring an inaccurate magnitude of energy expenditure may have benefits for assessing relative changes in a population's energy demands but consequently have implications on an individual's personal assessment of energy expended. In this respect, an inaccurate measurement of an individual's physical activity may influence their awareness of PAL, and consequently lead to a misguided appraisal of their attainment of a physical activity recommendation or goal (Ainsworth et al., 2015, Strath et al., 2013). From 
this, it could be postulated that the most appropriate method for capturing and assessing energy expenditure and thus physical activity, could be an objective tool that is highly accurate and precise with a good qualitative resolution.

\subsection{Indirect Calorimetry for Measuring Physical Activity}

The most accurate method for assessing an individual's energy expenditure is by measuring the body's oxygen $\left(\mathrm{O}_{2}\right)$ utilization and carbon dioxide production, otherwise referred to as indirect calorimetry (Levine, 2005). This measurement can be achieved in free-living settings using doubly labelled water (Ainslie et al., 2003) or in controlled setting in the form of a metabolic cart or chamber (Haugen et al., 2007). In brief, doubly labelled water involves an individual consuming water with a traceable isotopic form such as deuterium oxide which replaces the hydrogen and oxygen elements. The rate of deuterium and oxygen $\left({ }^{18} \mathrm{O}\right)$ elimination over a period of exercise is then measured in the blood, which is directly related to the carbon dioxide $\left(\mathrm{CO}_{2}\right)$ production and gives an accurate metabolic rate measurement (Coward et al., 1994, Schoeller, 1999). Indirect calorimeters are classified as the 'gold standard' as they are able to measure the fraction of inspired and expired oxygen and carbon dioxide over a given time period (da Rocha et al., 2006). According to Rosado et al. (2013), indirect calorimetry is based on the principles that there are no considerable reserves of oxygen in the body, with oxygen uptake $\left(\dot{V} \mathrm{O}_{2}\right)$ reflecting the oxidation of nutrients, including carbohydrates, fats and proteins, with the ratio of $\mathrm{O}_{2}$ and $\mathrm{CO}_{2}$ produced from the oxidation of these macronutrients being fixed. In more detail, nutrient oxidation estimates are dependent upon the respiratory quotient (RQ), which is the ratio of $\mathrm{CO}_{2}$ produced and $\mathrm{O}_{2}$ consumed. A RQ of $0.7,0.85$ and 1.0 can indicate that fat, protein and carbohydrate, respectively, are predominately being catabolized. However, studies show that it is not always that easy to determine the nutrient oxidisation, as an RQ of 0.85 could also indicate a mix of metabolic fuels for catabolism (Kaiyala \& Ramsay, 2011).

There are several physiological assumptions made when estimating an individual's energy expenditure using indirect calorimetry methods (Ocobock, 2014). Firstly, it is assumed that the individuals being tested will exhibit the same pattern of fuel catabolism as those participants that were originally tested in the experiments that 
established the relationship between metabolic rate, fuel utilisation and respirometry (Walsberg \& Hoffman, 2005, Kaiyala \& Ramsay, 2011). The second assumption is that the cost of fuel conversion through gluconeogenesis within the human body is negligible (Kaiyala \& Ramsay, 2011) and, thirdly, that the $\mathrm{CO}_{2}$ pool within the body remains constant, although, this can be violated by a number of different metabolic disorders (Kaiyala \& Ramsay, 2011). The fourth assumption is that there is no contribution of anaerobic metabolism during the measurement period (Kaiyala \& Ramsay, 2011). Lastly, the fifth assumption is that individuals are post-prandial prior to measurement (Kaiyala \& Ramsay, 2011), as indirect calorimetry can differ significantly if metabolic rates are measured post-meal consumption (Garby, 1989, Kaiyala \& Ramsay, 2011). These fundamental physiological assumptions have helped in improving the estimation of energy expenditure and population-based PAL (Ainslie et al., 2003). Although advances in technology have led to the development of indirect calorimeters that are easier to operate and more portable than previous instruments (Rosado et al., 2013, Levine, 2005), they are not without their limitations, such as being highly expensive (Westerterp, 2009) and impractical to use in free-living conditions (Strath et al., 2013). Then again, indirect calorimetry measurements do provide an essential criterion method with which to judge the accuracy and precision of smaller, cheaper and more practical instruments, such as accelerometers.

\subsection{Accelerometers for Measuring Physical Activity}

Accelerometers are a small, lightweight and un-obtrusive technology that are usually worn on an elasticated belt positioned on the right hip or lower back. Accelerometers are de facto standard for measuring physical activity and in recent decades have gained much popularity, given their ease of administration and capability to capture large amounts of varied data within large scale studies (Westerterp, 2009). Accelerometers account for physical activity in measures of acceleration (counts) in real-time, detecting movements in up to three orthogonal places (anteroposterior, mediolateral and vertical, Figure 2.9; Kong \& Bassett JR, 2005, Rachele et al., 2012). These counts of physical activity can be translated into biological metrics (e.g., energy expenditure) or physical activity patterns (e.g., moderate-intensity; Freedson et al., 2005a). Accelerometers have led to an increased precision of physical activity and are the most common method utilised to objectively assess youths' habitual physical activity 
patterns (Cain et al., 2013, Ekelund et al., 2011). Specifically, the ActiGraphs are the most widely deployed accelerometers by researchers (Cain et al., 2013, de Vries et al., 2006), as a result of their large memory capacities (Freedson \& Miller, 2000) and ability to accurately quantify the intensity, frequency, duration and total volume of physical activity (Healy et al., 2007, Matthews et al., 2008, Welk \& Corbin, 1995). Although accelerometers are praised for their robustness to measure MVPA, problems arise, however, when the translation of activity counts is made to energy expenditure as a result of linear regression models that are based upon the notion that energy expenditure increases linearly with vertical acceleration in locomotion activities (Freedson et al., 2012). Therefore, this method of assessing physical activity is thought to be less accurate at measuring non-linear activities (Bassett \& John, 2010, Van Remoortel et al., 2012, Chen et al., 2007), such as turning the body, that are highly representative of youths' sporadic and irregular physical activity patterns (Baquet et al., 2007, Sleap \& Warburton, 1996, Welk et al., 2000).

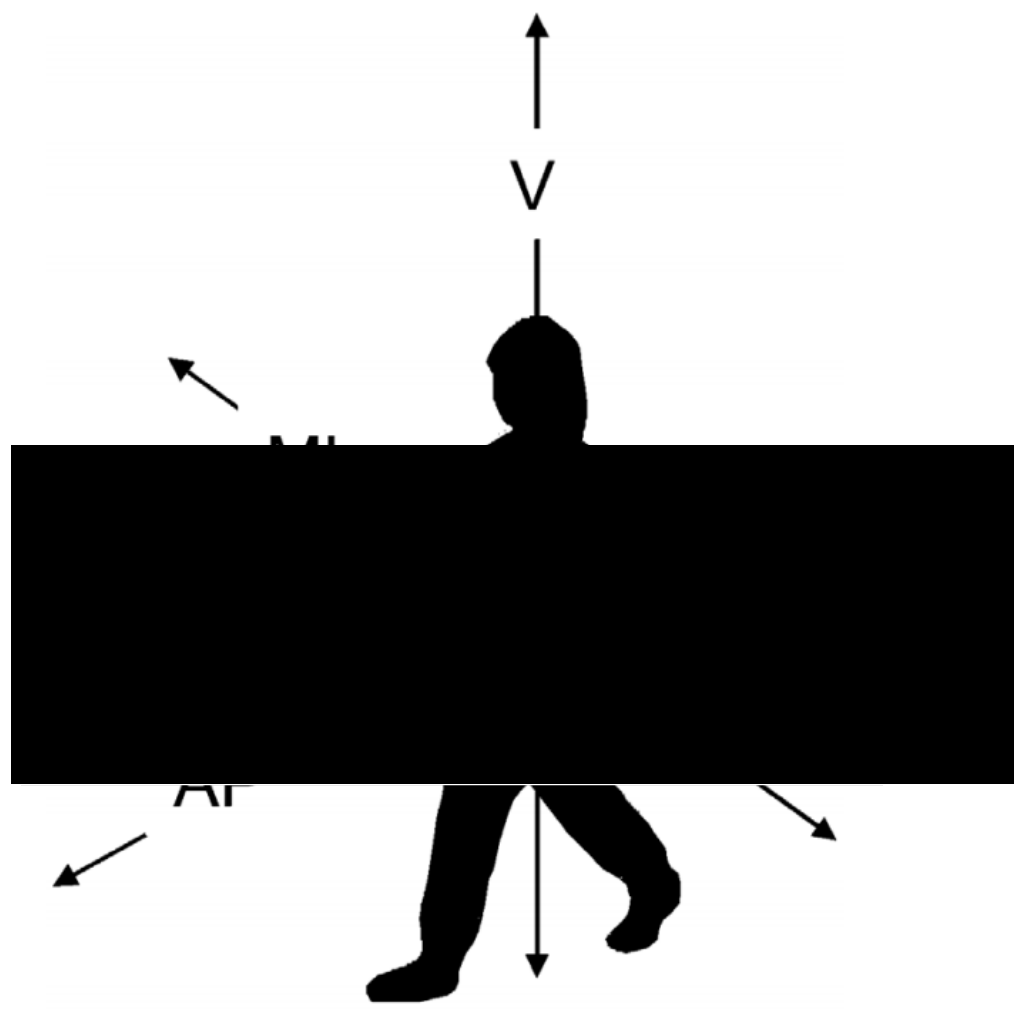

Figure 2.9 Directions of movement. From Annegarn et al. (2012).

$$
\begin{gathered}
\mathrm{AP}=\text { Anterior-posterior (forward-backward movement), } \\
\mathrm{ML}=\text { Medio-lateral (left-right movement), } \\
\mathrm{V}=\text { Vertical (up-down movement). }
\end{gathered}
$$




\subsection{The Energy Exependiture of Turning}

Few sports, fitness, or functional activities are limited to just linear locomotion, with the majority involving aspects of turning (Schot et al., 1995). For example, FA Premier League professional football players perform more than 700 turns during a match (Bloomfield et al., 2007), with turning accounting for $35-45 \%$ of all steps taken by adults in a typical day (Glaister et al., 2007). Turning is a manoeuvre that involves braking in the original direction of forward progression, translation and a reorientation in the new direction, all of which is completed without stopping the on-going locomotion (Hase \& Stein, 1999a, Schot et al., 1995, Rand \& Ohtsuki, 2000). As a result of these forces (deceleration and acceleration) acting upon the body's centre of mass during a change in direction, turns may impose a greater physiological demand to that of straight-line locomotion. Indeed, Hamill et al. (1983) were one of the first to suggest that curved path locomotion or turning may subject individuals to unique stresses, noting that research examining this non-locomotor behaviour had been largely neglected. Although there are several studies reporting the biomechanical changes during turning (Rand \& Ohtsuki, 2000, Schot et al., 1995, Besier et al., 2003, Besier et al., 2001) and the specific turning strategies utilised by individuals (Hase \& Stein, 1999a, Patla et al., 1991, Taylor et al., 2005, Dixon et al., 2013, Glaister et al., 2007, Akram et al., 2010), studies assessing turning and its physiological demand are limited. A few previous studies have compared the differences in physiological response of straight-line running and shuttle runs involving a $180^{\circ}$ turn (Buchheit et al., 2010b, Buchheit et al., 2011, Dellal et al., 2010). These studies showed that the incorporation of a $180^{\circ}$ turn during submaximal (Buchheit et al., 2011) and highintensity (Dellal et al., 2010) running elicited greater physiological demands, with an increased $\dot{V} \mathrm{O}_{2}$, heart rate and blood lactate when compared to straight-line running. Buchheit et al. (2012) extended this understanding of the physiological effects using three repeated sprint turning protocols at $45^{\circ}, 90^{\circ}$ and $135^{\circ}$ turns compared to straightline running, all completed at a self-selected pace (Figure 2.10). In contrast to Dellal et al. (2010), Buchheit et al. (2012) reported that heart rate, blood lactate and rate of perceived exertion were greater for straight-line running when compared to the three turning protocols. It was concluded that the greater physiological and perceptual strain of the straight-line protocol could be related to the faster absolute running speeds attained, which may directly influence energetic demands and trigger a greater 
contribution of anaerobic metabolism (Buchheit et al., 2011).

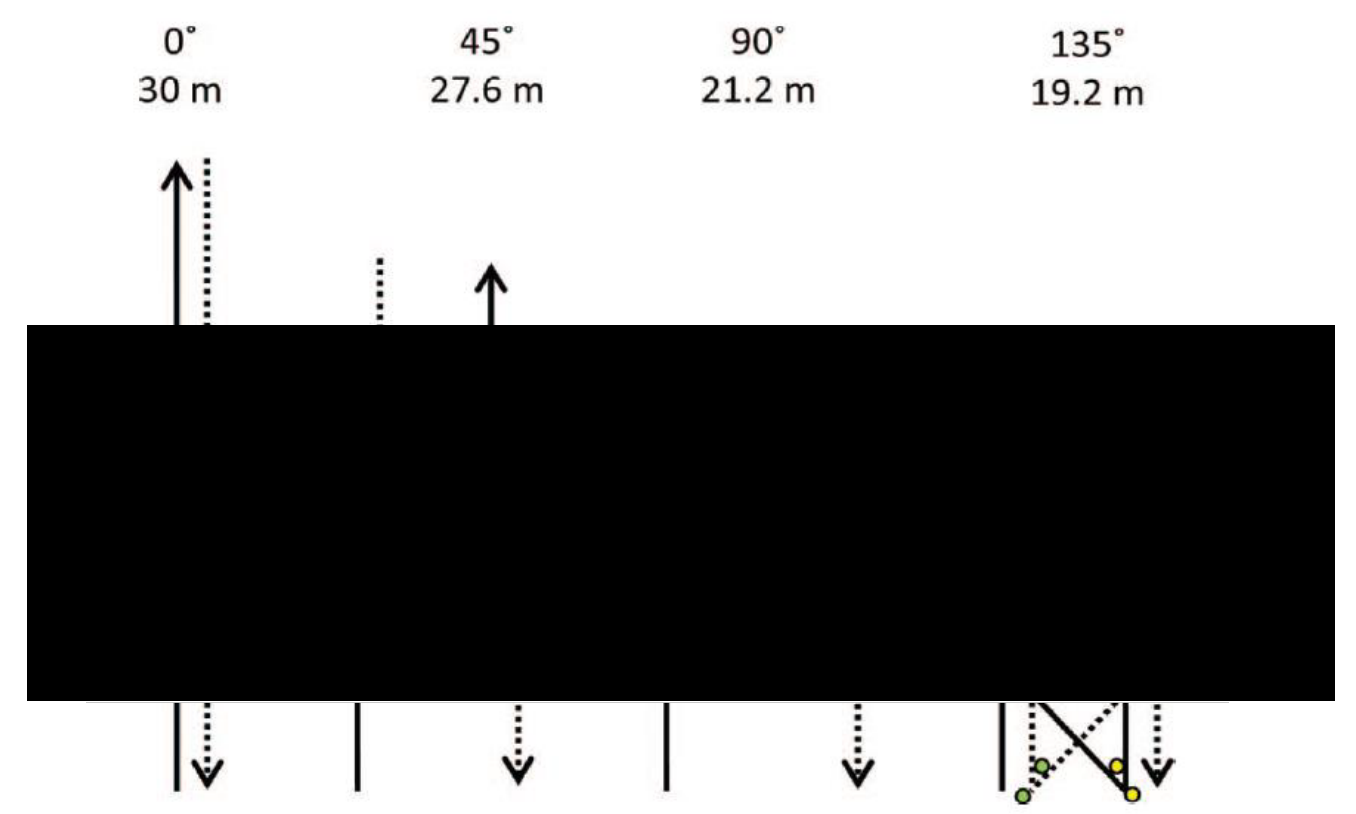

Figure 2.10 Four repeated-sprint sequences.

From Buchheit et al. (2012).

To take into account the influence of specific speeds of locomotion on turning energy expenditure, Hatamoto et al. (2013) investigated the effect of walking at 4.3 and 5.4 $\mathrm{km} \cdot \mathrm{hr}^{-1}$ and turning at $180^{\circ}$ over varying distances in young adults. Findings revealed that, irrespective of speed, as the frequency of turns increased so too did the gross $\dot{V} \mathrm{O}_{2}$ in a linear fashion, with $180^{\circ}$ turns at $5.4 \mathrm{~km} \cdot \mathrm{hr}^{-1}\left(0.55 \pm 0.09 \mathrm{ml} / \mathrm{kg} / \mathrm{min}^{-1}, p<0.001\right)$ requiring significantly greater energy demands compared to $4.5 \mathrm{~km} \cdot \mathrm{hr}^{-1}$ turns $\left(0.34 \pm 0.13 \mathrm{ml} / \mathrm{kg} / \mathrm{min}^{-1}\right)$. In this case, Hatamoto et al. (2014) extended their findings regarding the energy demands of $180^{\circ}$ turns in adults at a range of speeds $(3,4,5,6,7$ and $8 \mathrm{~km} \cdot \mathrm{hr}^{-1}$ ) and turn frequencies (13, 18, 24 and 30 per minute). In accord with Dellal et al. (2010) and Buchheit et al. (2011), Hatamoto et al (2014) showed that as speed increased during a turn, so too did the physiological responses, including heart rate, rate of perceived exertion, blood lactate and $\dot{V} \mathrm{O}_{2}$ cost. Moreover, even low walking speeds, such a $3.5 \mathrm{~km} \cdot \mathrm{hr}^{-1}$ with 30 turns per minute attained a similar metabolic demand to straight-line running at $6 \mathrm{~km} \cdot \mathrm{hr}^{-1}$. However, these aforementioned studies were limited to measuring $180^{\circ}$ turns, which ignore the energy 
demands of other important angles of turn, such as $90^{\circ}$, that are likely to be more habitual to youths' physical activities (Bloomfield et al., 2007).

To account for a larger range of angles, including $45^{\circ}, 90^{\circ}, 135^{\circ}$ and $180^{\circ}$, Wilson et al. (2013), investigated the effect of speed $6 \mathrm{~km} \cdot \mathrm{hr}^{-1}$ on the $\dot{V} \mathrm{O}_{2}$ demand in healthy adults, finding that as angle increased within speed, the energy expenditure also increased, with a single $180^{\circ}$ turn eliciting the same energy expenditure as walking 5.88 metres at the speed at $6 \mathrm{~km} \cdot \mathrm{hr}^{-1}$ in a straight line (Figure 2.11). To consider the energy expenditure of turning in more detail, McNarry et al. (2017) investigated the energy expenditure of angles $\left(45^{\circ}, 90^{\circ}\right.$, and $\left.180^{\circ}\right)$ on various walking speeds $(2.5,3.5$, 4.5, $5.5 \mathrm{~km} \cdot \mathrm{hr}^{-1}$ ) within an adult population. In conclusion, the study found a synergistic interaction between speed and angle in determining energy expenditure within walking, with $90^{\circ}$ and $180^{\circ}$ turns associated with a significant additional metabolic cost from speeds $4.5 \mathrm{~km} \cdot \mathrm{hr}^{-1}$ and above. Similar findings were reported in community-dwelling elderly (aged $>60$ years), with $180^{\circ}$ turns associated with a significantly greater $(p<0.05)$ energy expenditure to that of $90^{\circ}$ turns (Justine et al., 2014). However, given that children are not "mini-adults", the applicability of adult findings to turning in children are questionable, especially given their unique physiological and biomechanical structure (Andropoulos, 2012), which is likely to influence the energy expenditure of turning. For example, it is likely that a child's body mass is significantly less than an adult, therefore, it would be expected that the forces required in the acceleration phase to move the body through a turn would also be less. Indeed, Buchheit et al. (2011) found that even smaller adults demonstrated lower energy expenditure of turning when compared to taller adults. This follows from Newton's second law of motion, as a larger body mass requires a greater propulsive force to perform a given turn (Cohen et al., 2016). Furthermore, adults' larger skeletal structure and muscle size is also likely to influence energy expenditure given the larger moment arm length, defined as the perpendicular distance between the muscle-tendon action line and the axis about which the moment (i.e., force) is assumed to be generated (O'Brien et al., 2009). In this case, it could be postulated that the smaller body size and directional forces associated with turning in children when compared to adults would require less energy expenditure to complete. 


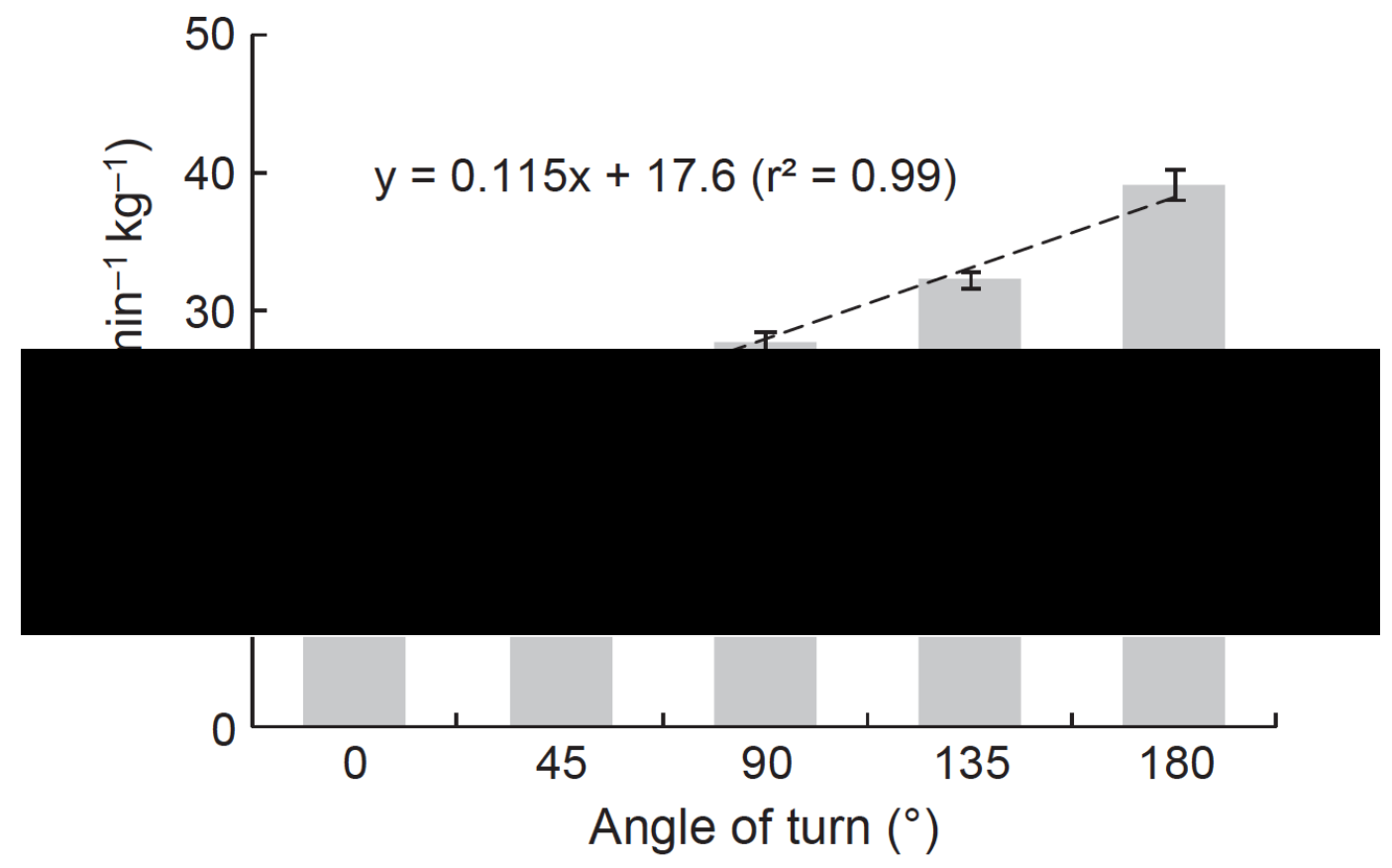

Figure 2.11 Oxygen consumption of turning in adults.

From Wilson et al. (2013).

To date, there are only two studies examining turning in children (Dixon et al., 2013, Hader et al., 2016), with only one identifying the energy costs of turning (Hader et al., 2016). Specifically, Hader et al. (2016) identified the metabolic power of turning in adolescent soccer players (aged $16 \pm 0.4$ years), finding that turning at $45^{\circ}$ and $90^{\circ}$ whilst running was less metabolically demanding than straight-line running. One possible reason for this lower metabolic demand of turning was attributed to the very low energy demand of the deceleration phase during the turn, which may not have been compensated by the re-acceleration phase. Indeed, previous research reports that a deceleration phase is characterised by a decrease in speed and an increase in eccentric muscles contractions, which is consequently estimated to be two to six time less metabolically demanding than concentric contractions at the same work output (Abbott et al., 1952, Ryschon et al., 1997). That said, the study was limited by its indirect calculation of energy expenditure estimated using the di Prampero's calculation method (2005), which is based upon adult regression models and most likely discredits other important non-locomotor muscles involved with turning (e.g., upper body and back muscles; Buchheit et al., 2010b). 
The lack of research regarding the energy expenditure of turning in youths' warrants further investigation, especially given that numerous observational studies of youths show they most commonly participate in activities, such as football, sprinting (Fjørtoft et al., 2009), brisk walking, general play and chasing games (Sleap \& Warburton, 1996), all of which involve considerable amounts of turning (Bloomfield et al., 2007). Such findings could not only have implications for the development of more sophisticated algorithms to account for turns in accelerometer-based measurements of physical activity, but also provide a more precise assessment of youths' PAL, which is essential for detailed investigations of dose-response relationships, evaluations of interventions and enhancing youths' awareness and understanding of their true PAL. However, to effectively raise an individual's awareness of their PAL, it is well documented that objective measurements, such as accelerometers, must come in partnership with personalised feedback (Bentley et al., 2012, Van Hoye et al., 2012). Indeed, previous qualitative research reports that youths would like to receive their PAL via feedback after wearing an accelerometer to encourage their engagement and understanding (Kirby et al., 2012, Zieve et al., 2017).

\subsection{The Importance of Feedback}

As defined by Abraham and Michie (2008), feedback is providing an individual with data about a recorded behaviour or performance in relation to a set standard or others' performance for evaluation. According to DiClemente et al. (2001), feedback can be generic (i.e., generalised information relevant to a whole population), targeted (i.e., adapted feedback for a specific demographic or health risk) or personalised (i.e., individual feedback based on themselves or on normative data). Personalised data can encompass information regarding risk, current state and/or change options, and is known to have benefits over other formats as it is easier for an individual to relate to and engage with (Kreuter et al., 2013, Kreuter \& Wray, 2003). Furthermore, DiClemente et al. (2001) define seven potential mechanisms of action for how feedback can promote behaviour change within an individual. These include: 1) education about the behaviour or outcome; 2) motivation or inspiration to change; 3 ) change in attitude or belief about an issue and the ability to overcome it; 4) provision of support to change; 5) providing social norms or standards to promote change; 6) increased engagement with the information supplied; and 7) the provision of critical 
risk or protective factor information. Indeed, within the context of health, it has been known for some time that providing an individual with numerical feedback of health results has a motivating effect to reduce risk behaviours (Weinberg \& Weigand, 1993).

\subsubsection{Numerical Physical Activity Feedback}

Numerous studies have utilised pedometers as a self-monitoring tool to promote physical activity in youths by providing numerical feedback (Butcher et al., 2007, Goldfield et al., 2000, Goldfield et al., 2006, Horne et al., 2009, Lieberman et al., 2006, Oliver et al., 2006, Southard \& Southard, 2006, Routen et al., 2014). Specifically, the numerical feedback provided by pedometers can inform an individual of their step count, distance travelled, and time spent partaking in physical activity. The basic premise underlying the use of pedometers is that they can provide individuals with an estimate of physical activity dose and provide immediate numerical feedback to increase awareness of how their personal behavioural patterns impact their PAL (Lubans et al., 2009). Interventions using pedometers in youths have usually employed either one or a combination of: self-monitoring (recording daily steps), pedometer feedback and step goal-setting in conjunction with additional behavioural strategies (Berry et al., 2007, Hardman et al., 2011a, Horne et al., 2009, Oliver et al., 2006). Specifically, Butcher et al. (2007) found that children who received a combination of step-count feedback and information on how to increase daily step count achieved significantly more steps when compared to a just 'feedback' group and 'no feedback and information' control group. Whilst Kang and Brinthaupt (2009) reported a 19\% increase in youths daily steps following a 6-week intervention of self-monitoring and feedback, although no control group was included. A similar study comparing a control group (pedometer use) to an intervention group (pedometer self-monitoring and feedback) found no significant difference between the groups' physical activity after a 3-week follow up (Routen et al., 2014). The major setback with the aforementioned pedometer-based measurements is their inability to account for the intensity of physical activity attained (Lubans et al., 2009) and their weak correlation to indirect measurements of physical activity. Therefore, pedometers are limited when it comes to providing detailed feedback and prescribing physical activity targets, such as 60 minutes of MVPA (Ramirez-Marrero et al., 2005). Furthermore, the nature of the feedback displayed in the aforementioned studies were predominantly through 
numerical score displays (e.g., 10,000 steps; Figure 2.12), which may limit the potential to enhance youths' awareness and understanding of physical activity than more visual and meaningful methods of representing numerical feedback through visualisations (Edwards et al., 2002, Houts et al., 2006).

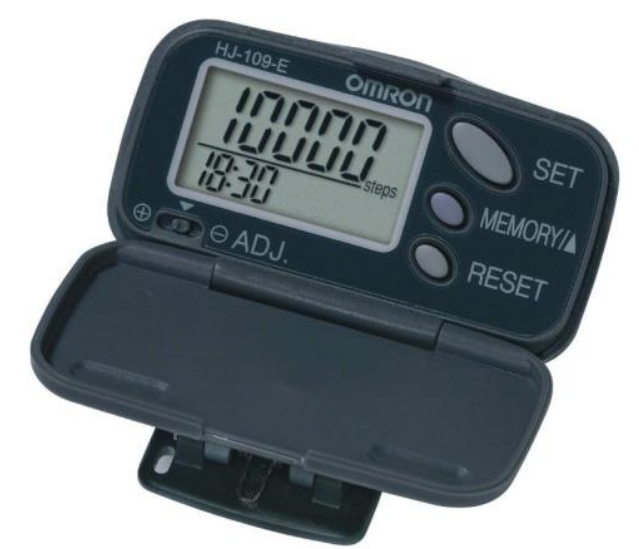

Figure 2.12 . Numerical physical activity feedback displayed on the Omron HJ-109-E pedometer

\subsubsection{Numerical Visualisations of Physical Activity}

Visualisations are particularly central to our understanding of data, as "seeing" makes knowledge credible (Bloch, 2008) and a greater visibility of information contributes to an added responsibility to act (Viseu \& Suchman, 2010). Moreover, visualisations are known to enable individuals to identify patterns and relationships within their data, leading to the discovery of new concepts and ideas that were previously unknown or only hypothesised (Card, 1999). In the context of physical activity, visualisations offer individuals awareness of their PALs, making them actionable and comprehensible in terms of health-related outcomes (Khot, 2016). Over the past 5 years, there has been an explosion of commercially-available activity trackers (e.g., Nike Fuelband, Fitbit Charge, Garmin Vivosmart and Polar loop) that incorporate a number of different sensors (e.g., pedometers, heart rate monitors, accelerometers) into one device for selfmonitoring physical activity patterns (Hooke et al., 2016). The multitude of information collected from these devices (e.g., step count, distance travelled, floors climbed, beats per minute, calories burned, sleep patterns and intensity of physical activity; Figure 2.13) can be evaluated through interfaced connections with computers, smartphones and tablets, which can provide interactive visualisations of feedback. 
Specifically, these interactive visualisations of data allow an individual to hover over a particular number or graph segment to find a specific numerical value to understand progress towards personal goals (Polzien et al., 2007).

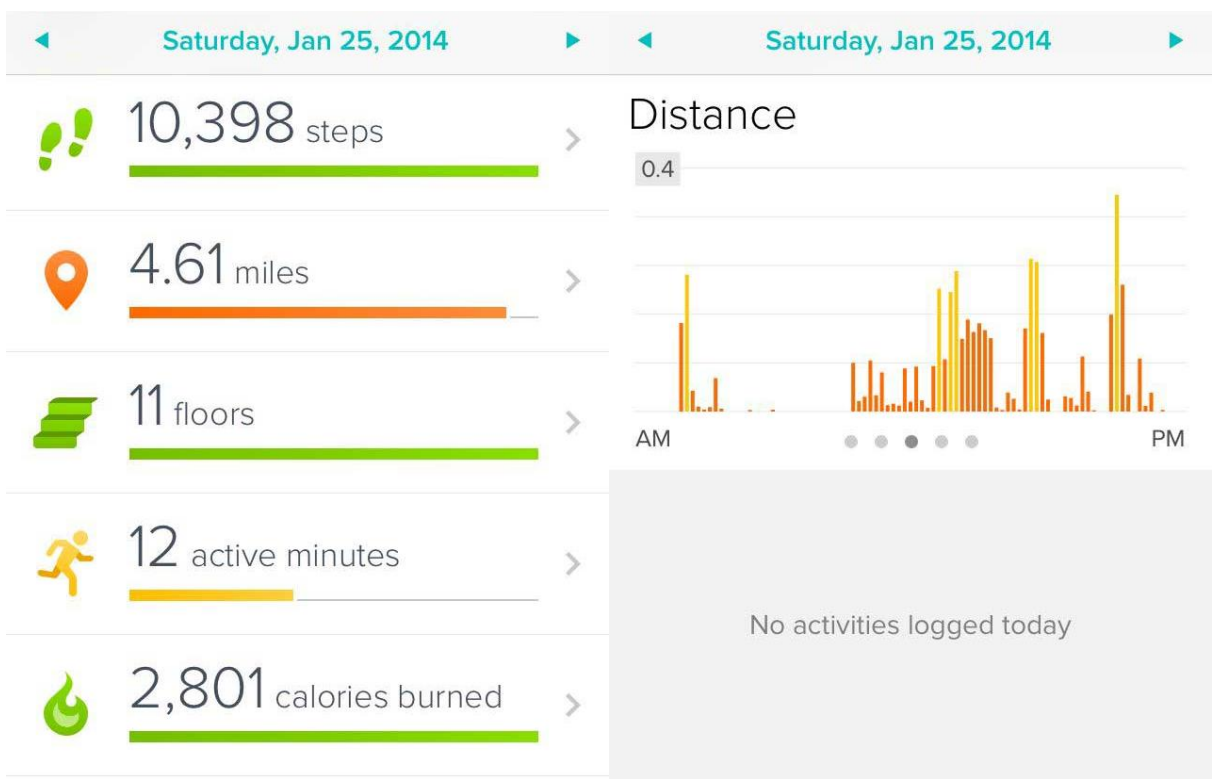

Figure 2.13 Numerical visualisations of activity feedback

The increasing field of these persuasive technologies has enormous potential for promoting physical activity (Fogg \& Eckles, 2007), with its greatest appeal for use in physical activity interventions, as they can reach large numbers of individuals and communicate large volumes of personalised visual feedback that coincides with health behaviour theory (Ramirez-Marrero et al., 2005, van Gemert-Pijnen et al., 2011). Indeed, several reviews report that using wearable activity trackers and their visual feedback can increase adults' PAL (Fanning et al., 2012, Lewis et al., 2015, Harries et al., 2016, Li et al., 2011b, Tudor-Locke \& Bassett, 2004), however, there is a paucity of evidence to support their use among youth populations (Ridgers et al., 2016, Dean et al., 2016, Hayes \& Van Camp, 2015, Jacobsen et al., 2016, Schaefer et al., 2016, Hooke et al., 2016, Gaudet et al., 2017). For example, Hooke et al (2016) examined the efficacy of the Fitbit One and it's feedback to promote youths' (6-15 years old) physical activity in a clinical setting. In detail, participants wore the Fitbit for 2-weeks, with daily screenshots of the feedback from the associated FitBit app sent via email to each individual. The findings reported no significant increase in step count; although there were marginal increases in steps per day from weeks 1-2, steps count decreased from weeks 2-3 (Hooke et al., 2016). More recently, Gaudet and colleagues (2017) 
investigated the potential of the Fitbit-Charge-HR tracker and its visual feedback to increase PAL in adolescents (aged 13-14 years) over a 7-week intervention, with levels of MVPA measured using a research-based accelerometer. It was reported that for a subset of adolescents, exposure to the Fitbit tracker and associated visual feedback was associated with a 15-minute increase in MVPA per day, with participants being in the action and maintenance stages of behaviour change in relation to physical activity. Participants in the pre-contemplation, contemplation, and preparation stages showed no change in their daily MVPA levels (Gaudet et al., 2017). One possible reason for this lack of behaviour change could be the numbers of adolescents considering the Fitbit activity tracker feedback as an “adult thing” (Wartella et al., 2016).

There are, however, many advantages to the use of activity trackers and their numerical visualisations of feedback, in that they only require a small screen (Van Wijk, 2005), with graphs or charts making data easier to understand and glance at to raise an individual's awareness of their PAL (Yi et al., 2007). On the other hand, research suggests that these numerical or graphical forms of visual feedback are too complicated for most adult users, as they are not skilled at interpreting the statistical data (Ancker \& Kaufman, 2007, Galesic \& Garcia-Retamero, 2011). As Khot (2016) noted, this could be a result of the visual data being overwhelming to generate new insights or actionable knowledge. Indeed, this numerical approach to visualising feedback could also be problematic for youths, given that numbers and graphs are associated with mathematics, which is unlikely to be meaningful and aesthetically pleasing to youth populations (Brian, 2012). As expressed by Hassenzahl et al. (2016), numbers could make physical activity feel more like work, with need to explore better and richer ways to represent data. While this is the case it can be argued that youths' lack of cohesion with on-screen feedback platforms is that current behavioural theories have not yet been adapted to leverage their advantages to promote behaviour change (Schembre et al., 2018). Nonetheless, developers and researchers are now exploring alternative ways of visualising physical activity data through abstract visualisations (Anderson et al., 2007, Fan et al., 2012). 
Abstract and metaphorical visualisations of feedback allow for data to be communicated in a symbolic way, that may have benefits to numerical feedback when data is more difficult or subjective to understand (Khot \& Mueller, 2013). Previous research has emphasised the importance of creating more abstract visualisations of feedback to support an individual's positive engagement with data (Consolvo et al., 2008b). Indeed, abstract visualisations are known to help an individual with the task of impression management, defined as the conscious or subconscious process to influence an individual's perceptions about a behaviour (Goffman, 1959), including the ambiguity to create a 'story' (Aoki \& Woodruff, 2005), which enables increased reflection of behaviours (Consolvo et al., 2009). Anderson et al. (2007) developed a mobile phone system called 'Shakra' that was designed to represent physical activity in an abstract form, finding that the visualisations encouraged individuals to reflect on their physical activity behaviours and motivated them to attain higher PAL. Congruently, Fan et al. (2012) designed a system called 'Spark' which could represent various abstract and graphical visualisations of physical activity data (Figure 2.14). The findings reported that the abstract displays increased adults' awareness of physical activity and sedentary behaviours. Interestingly, some participants preferred the graphical visualisations when looking for specific information or historical patterns of behaviour, however, all participants agreed that abstract visualisations were more appealing and aesthetically pleasing than the graph when "glancing" at their data (Fan et al., 2012).

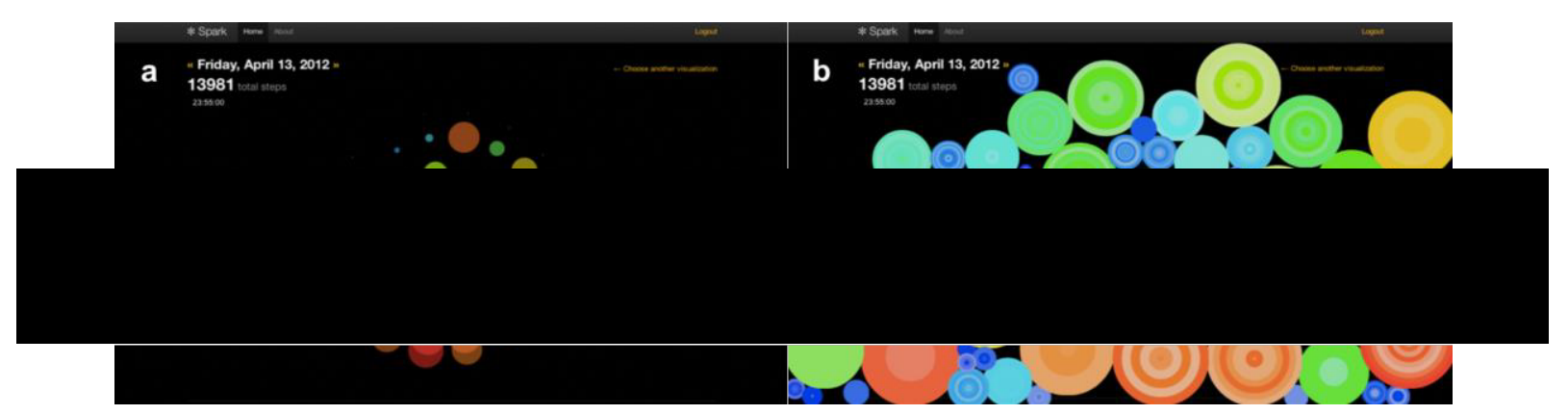

Figure 2.14 Spark abstract visualisations of physical activity.

From Fan et al. (2012).

a) Spiral, b) Bucket. 
Larsen et al. (2013) created a system called the QS (quantified self) Spiral which was designed to map 7 days of Fitbit activity data onto a spiral timeline. As shown in Figure 2.15 , the outer spirals represent an individual's most recent physical activity data with past activity data displayed towards the centre of the spiral shape. According to Larsen et al. (2013), the QS Spiral system may enable users to discover their physical activity patterns because it can present a large volume of data at a glance. However, these conclusions should be taken with caution given no evidence regarding users' perceptions of the QS Spiral were recorded, therefore, the true impact of such spiral visualisations on behaviour outcomes are unknown. Similarly, Tong et al. (2015) evaluated the readability and attractiveness of representing physical activity data on a circular Ringmap visualisation. The Ringmap was designed as a circular structure that could represent a whole month of activity data with multiple rings ( $\sim 30$ rings) used to show each day of activity. Each day ring was further split up into 5-minute segments which would shine brighter the more active an individual completed within that time segment. Although the sample size was relatively small (18 participants), which weakens the generalisability of the findings, participants stated that they found it easier to identify patterns within their activity data when structured in the Ringmap shape compared to a bar chart design (Tong et al., 2015). Despite this, Tong and colleagues (2015) concluded that the Ringmap visualisation was not helpful or intuitive enough for participants to reinterpret their activity data, which may have been influenced by the novelty of the abstract visualisation. There is, however, much praise for abstract visualisations within the commercial sector, such as the Apple watch and its ring-based visualisation of PAL with associated goals, rewards and online competitions (Figure 2.15b; Cowling, 2016). However, research is scarce on the potential of such abstract visualisations, especially among the youth populations. Tong et al. (2015) proposed that to increase readability and attractiveness of activity data, researchers should look into creating more emotionally resonant visualisations, such as virtual pet figures. 


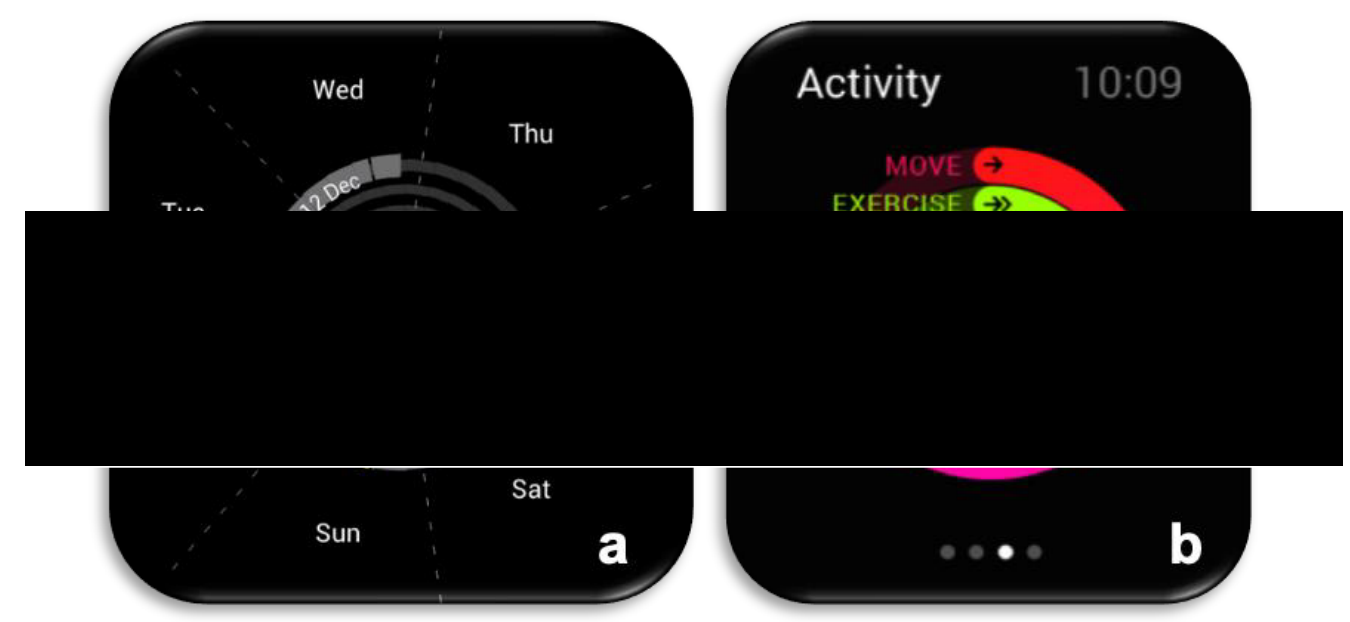

Figure 2.15 Abstract visualisations.

a) QS Spiral (from Larsen et al., 2013), b) Apple Watch rings (from Apple.com)

\subsubsection{Living Metaphor and Avatar Visualisations of Physical Activity}

Similar to abstract visualisations, living metaphors (e.g., flowers, animals) are found to be more engaging, motivating, glanceable, and ambient than graphical and numerical visualisations (Lin et al., 2006, Consolvo et al., 2008a, Consolvo et al., 2008b, Fan et al., 2012). Furthermore, living metaphors are considered to evoke more empathy and emotional experiences than abstract visualisations (Tong et al., 2015). One of the first examples of a living metaphor visualisation was the release of the Nintendo Pocket Pikachu in the late '90s, which was based upon pedometer counts and involved the user increasing or maintaining their step count to keep the virtual Pikachu alive or make them grow (Fogg, 2002). Similar to this, Lin et al. (2006) developed a living metaphor through an animated fish that's emotional state and size changed to happier and larger, respectively, in response to increased physical activity, and vice versa (Figure 2.16). The findings from Lin et al. (2006) did, however, reveal that those individuals who were inactive disengaged with the software as a result of the fish looking unhappy, highlighting that negative framing of data could result in user disengagement. Furthermore, Consolvo et al. (2008b) designed a software called 'Ubifit', which involved users growing a garden with increased levels of physical activity on their personal mobiles. The findings reported that the garden display helped raise adult's awareness and motivation to maintain their PAL. However, users PAL 
did not significantly increase, with concerns expressed over the novelty effect of the system, especially given the study was only a three-month intervention.

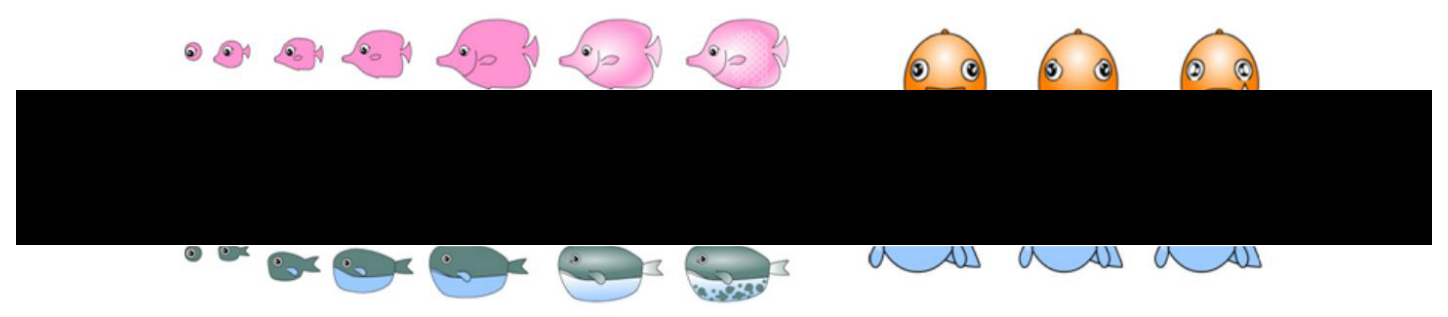

Figure 2.16 Physical activity represented as virtual growing fish.

From Lin et al. (2006).

Similar to Lin et al. (2006), Tong et al. (2015) explored how caring for virtual pets created an internal awareness of physical activity within adults. The virtual pets were categorised into five levels of physical activity: extra high, high, medium, low and extra low (Figure 2.17). The virtual pet visuals were designed to resemble an individual's approximate activity level, such as a pet being a 'couch potato' to represent a low level of activity and a 'super hero' to reflect extra high levels of activity. From the study findings, participants stated that the virtual pet visualisations created a sense of empathy and a level of emotional engagement with the feedback and associations to their physical behaviours. However, no correlation between PAL and response to receiving such virtual pet feedback were investigated. It could be theorised that this emotional response to virtual pets could be harnessed both positively and negatively, for example being perceived as a 'couch potato' could instil negative feelings and lead to disengagement from the feedback as seen in Lin et al. (2006), or conversely, elicit positive action to move more (Tong et al., 2015).

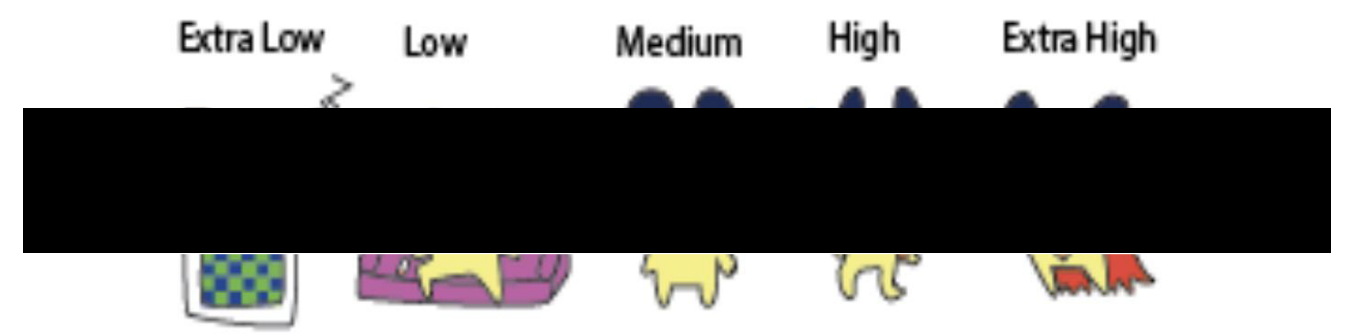

Figure 2.17 Five virtual pet representations of physical activity levels. From Tong et al. (2015). 
These living metaphor visualisations of feedback have also been represented through 'avatars' (i.e., a digital representation of a person in a virtual environment). Avatars are most commonly used to represent a person in internet chat rooms (Kang \& Yang, 2006), social networking sites (Walther et al., 2008), multiplayer online role-playing games (Yee, 2006) and social virtual worlds (Castronova, 2008). Previous research utilising avatar representations have shown them to influence an individual's health monitoring (Skalski \& Tamborini, 2007) and aid health behaviour (Eastwick \& Gardner, 2009). A meta-analysis concluded that individuals were influenced more by human-controlled avatars compared to computer-controlled representations (Fox et al., 2010). As Biocca (1997) notes, an individual can experience a feeling of avatar embodiment, whereby an individual's perception of their body image can be influenced by the geometry and topology of the virtual avatar body. To this effect, avatar embodiment may create a more meaningful experience for an individual and thus, the delivery of health messages may have greater impact than other mediated methods of feedback.

Yee and Bailenson (2007) propose the 'Proteus effect' which states that an individual may change their behaviours in accord with their avatars behaviour or characteristics. In other words, individuals are likely to adopt avatar-consistent behaviours into their real-life behaviour. This process is possible because an individual's self-concept (i.e., the collection of beliefs about oneself) is suggested to be easily changeable, therefore, having an avatar with a different identity may influence an individual's self-concept and lead to them behaving differently in a real-world setting (Yee \& Bailenson, 2007). Based on the self-concept theory, Joo et al. (2017) conducted a game-based laboratory experiment whereby participants, irrespective of their own bodyweight, were assigned to either an obese or healthy weight avatar (Figure 2.18). Participants were then given different lifestyle goals for their avatar to complete within the game. For example, participants assigned with the healthy weight avatar were told to complete at least two treadmill workouts and use the virtual home exercise equipment as much as possible whilst participants with the obese avatar were told to eat three large cheesecakes and stay on the couch or bed as much as they wanted. The findings showed that participants who controlled the healthy-weight avatar, significantly changed their real-life behaviours (e.g., increased physical activity) immediately after playing the game compared to those participants who controlled the unhealthy obese avatar. Joo et al. 
(2017) concluded that the interaction between the avatar's appearance and behaviour clearly supports the theoretical literature on how people imitate a model that is perceived to be attractive and socially rewarding (Bandura, 2009). Nickel (2013) found similar results, showing that individuals who displayed traits of body image dissatisfaction and were provided with an avatar that was an alternative view of themselves, expressed less anxiety when playing the game compared to participants with a positive body image. Nonetheless, neither of the aforementioned studies examined the role of users creating their own avatar and behaviour outcomes. Previous literature demonstrates that when an individual has an avatar similar to their ideal self, they are more motivated to play the game (Jin, 2010, Thin et al., 2013). It could be postulated that individuals who have customised their health avatars, would not only feel more emotionally connected to the game, but may also reap the benefits of enhanced lifestyle behaviours, such as increased physical activity. As argued by Druin (2002), it is important that feedback is designed to be visually stimulating and meaningful for youth, something that will excite them and aid in their development.

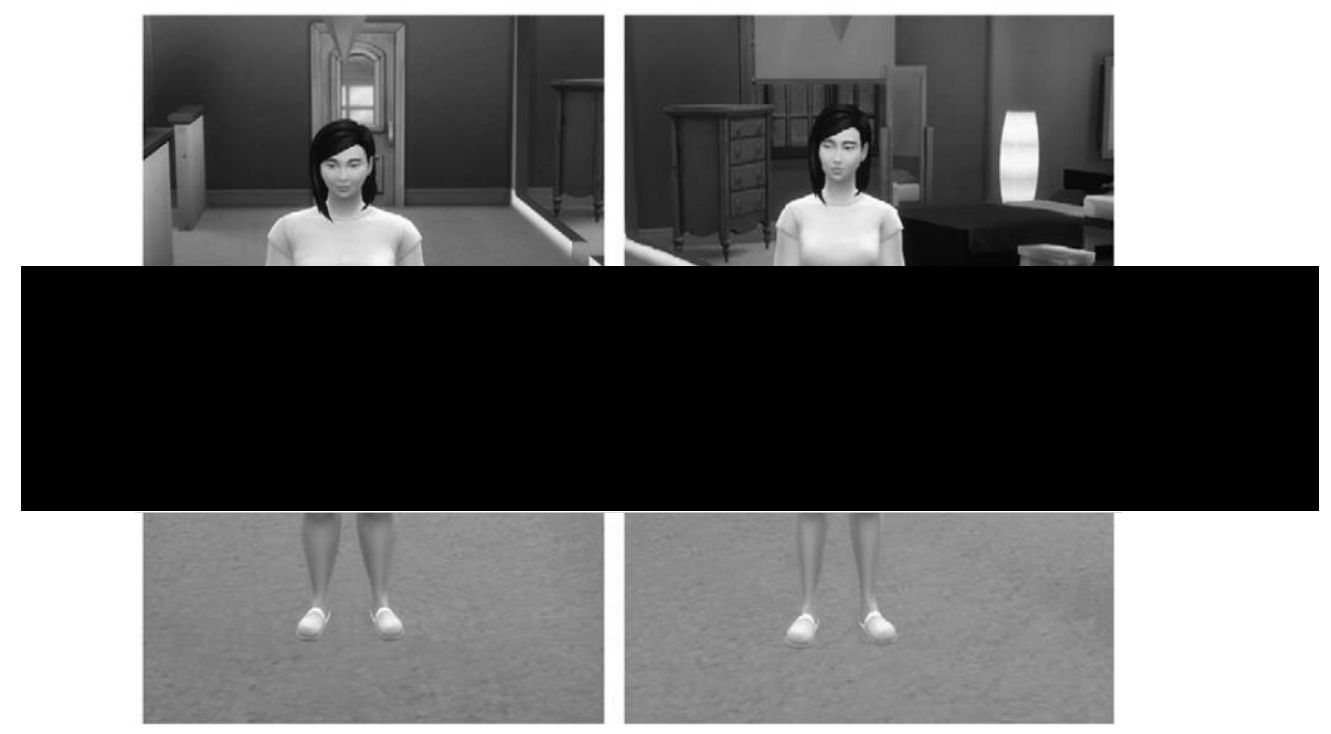

Figure 2.18 Obese and healthy weight avatar.

From Joo et al. (2017)

There are a number of activity trackers on the market, such as the Sqord activity tracker and platform where youths can create their own personal avatar to collect points and rewards for completing physical activity (Figure 2.19). Other examples include, Zamzee (zamzee.com) and Geopalz (geopalz.com), which also utilise a wearable 
tracker and online visual rewards and feedback through personalised avatars. In accord with the popularity of 'Pokémon GO' among youths (Althoff et al., 2016), Masteller et al. (2017) found that youths liked the 'avatar' feedback represented on the Sqord (75\%) and Zamzee (94\%) websites as they could change the avatar to depict themselves. Thompson et al. (2016) found that youths $(n=48)$ wanted to customise their avatars body type (96\%), clothing (94\%), hair colour/style/texture (88\%), eye colour $(79 \%)$, accessories $(79 \%)$, skin tone (77\%) and facial features $(73 \%)$. This follows the understanding of how individuals become more invested in their avatars and their virtual environment when they are able to customise and develop the avatars (Lim \& Reeves, 2010).

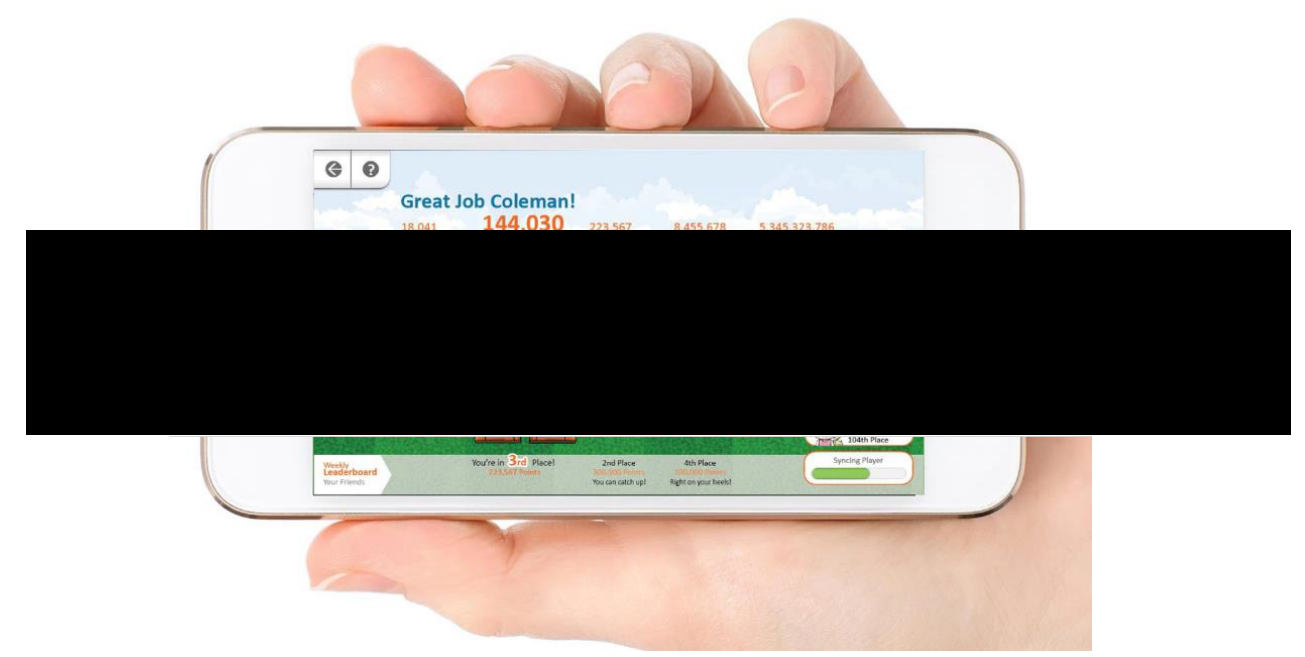

Figure 2.19 Sqord avatar interface. From Sqord (Sqord.com).

More recent technological developments have enabled researchers to use photographs or 3D scans of an individual to create a 'photorealistic' avatar that closely resembles the self (Figure 2.20; Thompson et al., 2018). Specifically, Thompson and collegues (2018) created a game called 'The Nightmare Runner' which involved youth completing physical movements in the real world to control their in-game photorealistic avatar to avoid obstacles and escape the chasing monster. The game lasted for 20 minutes with participants levels of physical activity measured using an accelerometer. The findings showed that approximately 75\% (15.9 minutes), 16\% (3.3 minutes), and 10\% (1.6 minutes) of gameplay was spent in vigorous, moderate and light physical activity, respectively. Furthermore, youth noted how their photorealistic 
avatar had a positive impact on their gameplay experience because "it's almost as if [it] was me, like if I were in the videogame I would want to get away from the monster" (P5; Thompson et al., 2018). Nonetheless, findings were based upon the short-term effects of photorealistic avatars, therefore, the influence of such methods on sustained long-term physical activity are questionable. It is also important that researchers consider the downside of encouraging youth to spend more time in front of screens when utilising avatar-based gaming, which is likely to result in less time spent outdoors (Fox, 2012). Furthermore, it can be argued that on-screen visualisations of physical activity are limited to stimulating an individual's visual and auditory senses, which ignore the abundance of other senses including touch (Ullmer \& Ishii, 2000). Indeed, a vast body of research in youths' educational science (Price et al., 2003, Marshall, 2007, Rogers et al., 2002a, Bara et al., 2004) and developmental psychology (Rita \& Dunn, 1979, Cole \& Wertsch, 1996, Piaget \& Cook, 1952, Fleming \& Mills, 1992, Montessori, 1912) suggests that manipulation of tangible objects can promote intellectual development, understanding and enable higher mental functions.

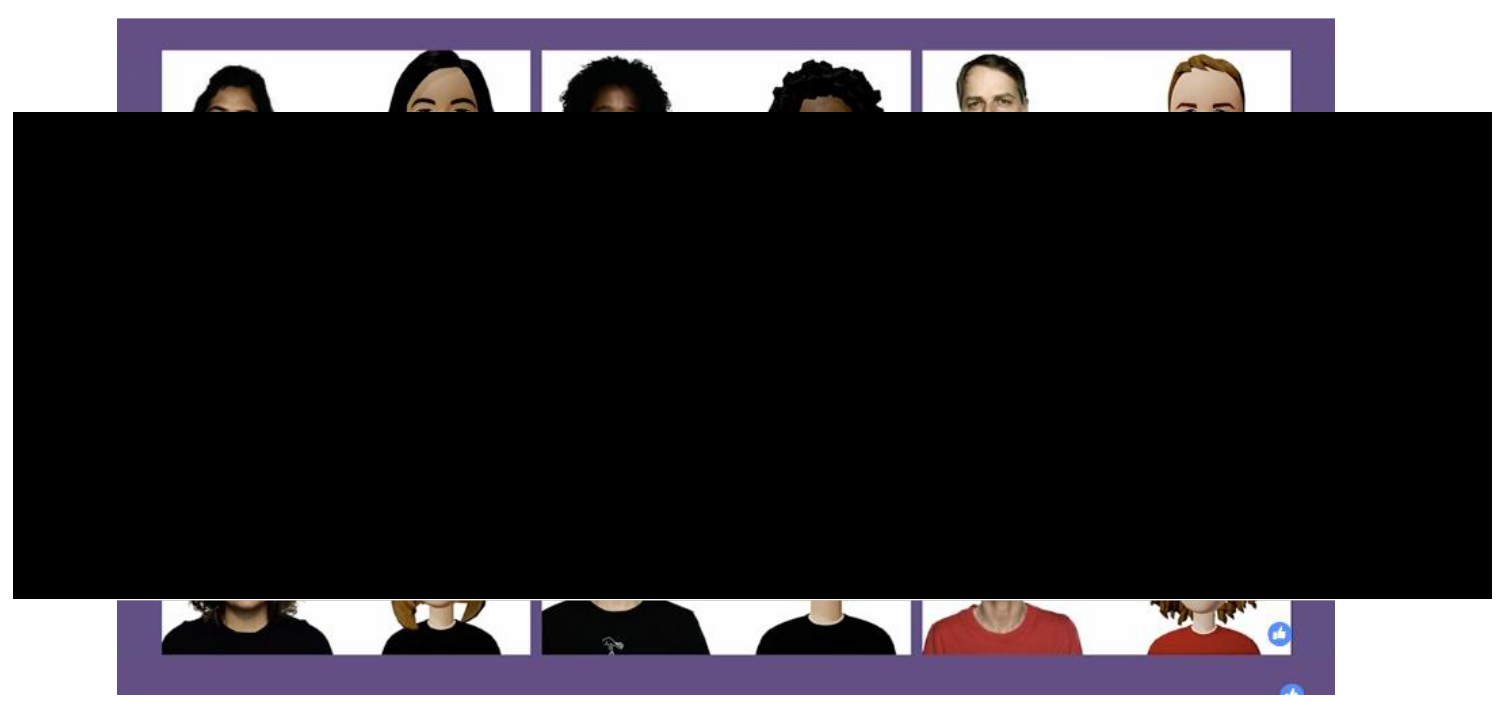

Figure 2.20 Photorealistic avatars. From Facebook.com

\subsubsection{Tangible Visualisations of Physical Activity}

As Jansen et al. (2013) advocate, there are many benefits of tangible visualisations over on-screen visualisations. These include: 1) allow for active perception; 2) can leverage non-visual senses; 3) can be integrated into the physical world; and 4) can harness the interplay of vision and touch to facilitate cognition. In addition, tangible objects can offer different opportunities for youths' interaction when compared to on- 
screen visualisations, such as being able to trade or display them on a shelf (Ananthanarayan, 2015), which historically aligns with the popularity of art installations and museums (Dragicevic, 2012). With the recent rise of the "maker movement', cost-effective 3D printers, such as the MakerBot (makerbot.com) and the Ultimaker (ultimaker.com), have given rise to health-related research utilising their capacities to create tangible visualisations of physical activity. Specifically, 3D printing is an additive manufacturing process where a tangible object is created by depositing layer by layer of a material (e.g., plastic) on a print bed. Khot et al. (2013) were the first to encapsulate adults' heart rate data into 3D-printed visualisations based upon heart rate data and took both numerical, abstract and living metaphor forms, such as a physical graph, flower, frog, dice and ring (Figure 2.21). Findings demonstrated that all the tangible representations of physical activity allowed participants to relate to their data, showing increased awareness and reflection of their physical activity patterns. As described by Khot et al (2013), this type of 3D-printed data acts as both a reward and feedback of physical activity data, which may offer more for youths given that incentive-based intervention have showed some level of promise to promoting PAL (Christian et al., 2016, Finkelstein et al., 2013, Hardman et al., 2011b).

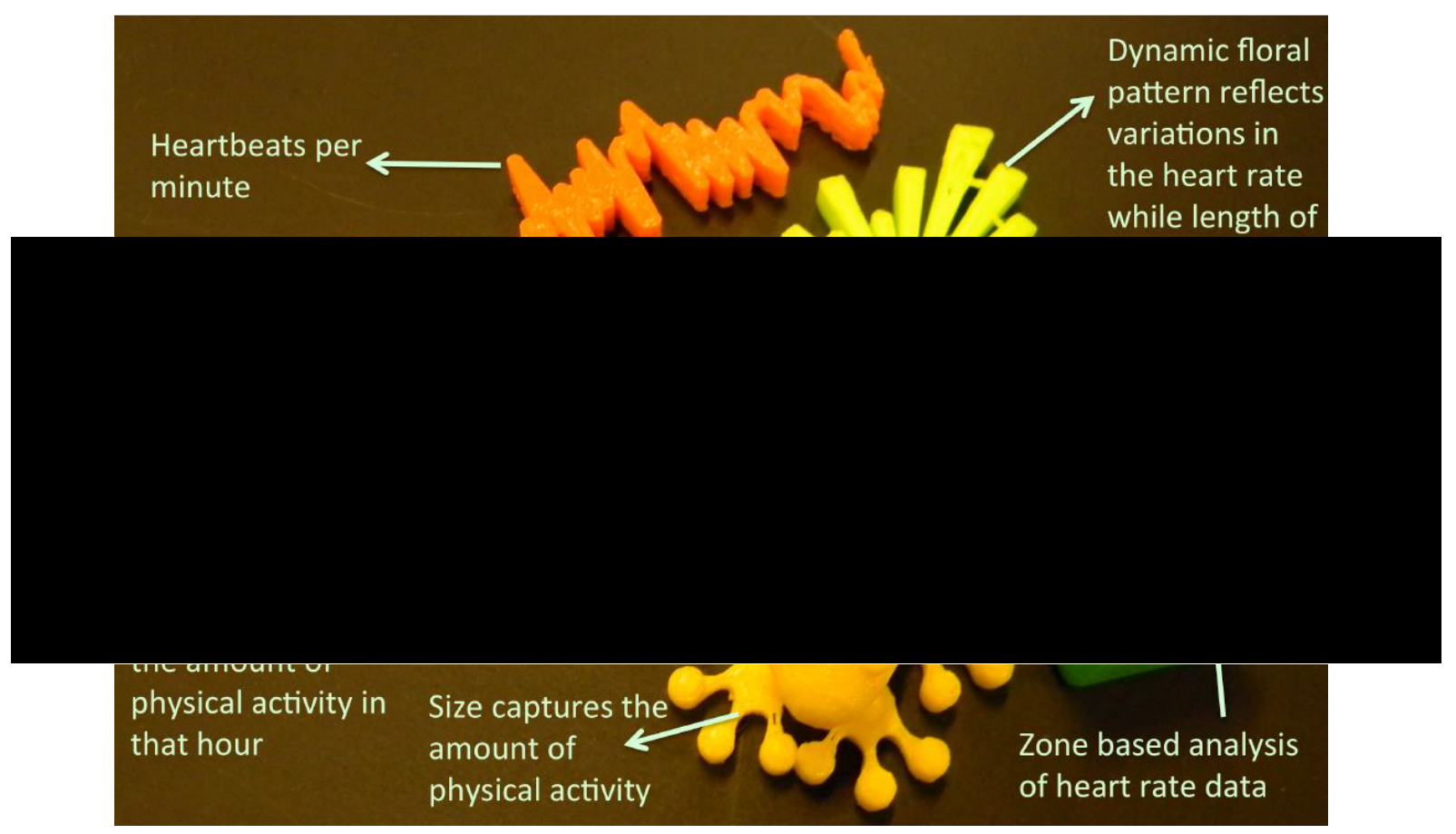

Figure 2.21 3D-printed representations of heart rate.

From Khot et al. (2013). 
Using a slightly different material output, Khot et al. (2015a) developed a system called 'EdiPulse' that translated an individual's heart rate data into 3D-printed chocolate treats. These chocolate treats were constructed with less than 20 grams of dark chocolate and embodied four different forms, including a graph, flower, emoji and slogan (Figure 2.22). The quantity of chocolate remained the same irrespective of the user's PAL achieved. The chocolate-printed emoji communicated the individuals' progress towards a self-driven goal or target, with a sad emoji appearing if the user had achieved less than $50 \%$ of their set goal and a happy emoji printed if the user attained the goal. The slogan would provide users with motivational words, whilst the flower would provide hourly physical activity with the graph displaying heart rate data across the day. In conclusion, the users reported that the 'EdiPulse' system helped manage their cravings for sweets and encouraged them to reflect upon their personal data and lifestyles in a playful way. However, one common limitation to both aforementioned studies developed by Khot et al. $(2013,2015 \mathrm{a})$ is the use of heart rate data to create tangible visualisations of feedback, as even mild mental stress in sedentary pursuits can substantially increase an individual's heart rate (Jouven et al., 2009), which could make an individual appear more physically active, and thus lead to erroneous conclusions regarding their true PAL.

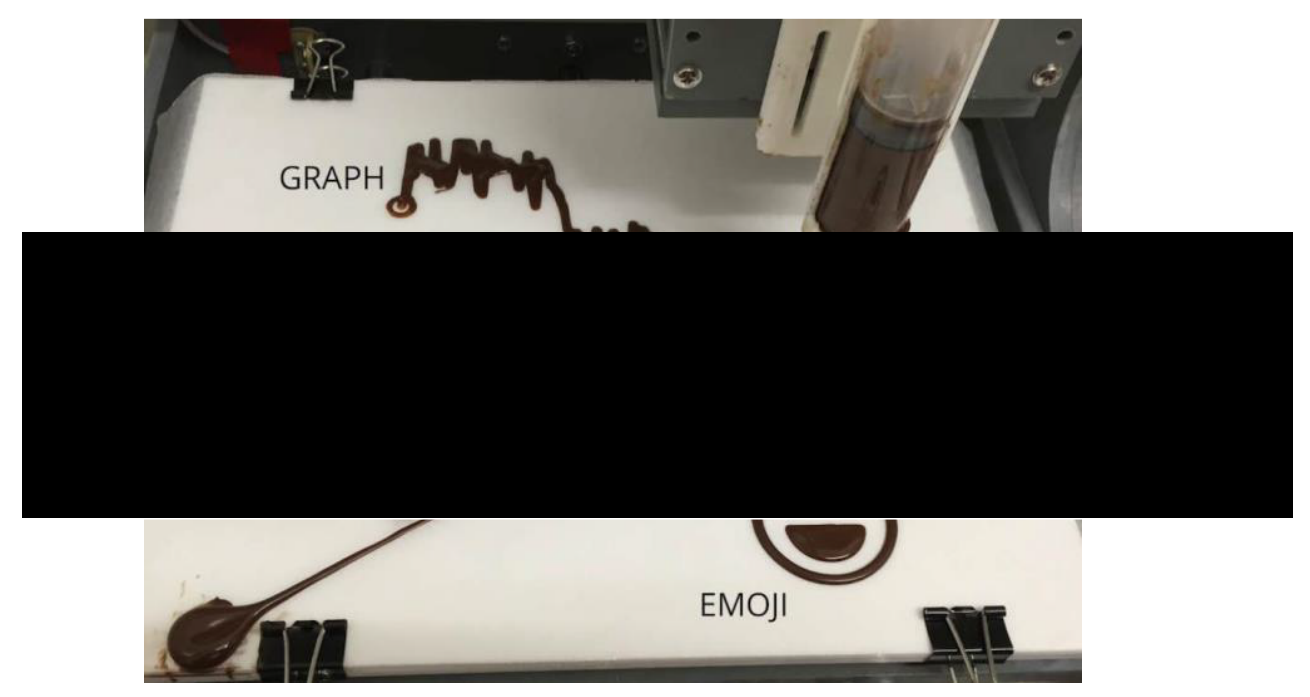

Figure 2.22 3D-printed chocolate representations of heart rate.

From Khot et al. (2015).

Similar to Khot et al. (2013), Stusak et al. (2014) designed 3D-printed sculptures, in the form of human figures, necklaces, a lamp and jar that mapped adults running data. 
However, in contrast to Khot et al (2013), Stusak and colleagues (2014) 3D-printed sculptures were created using a number of variables from running data, including duration, distance, calorie consumption and elevation gain, which allowed for alternative representations of data to be explored. As shown in Figure 2.23, the 3Dprinted human figure body would represent the run duration and the width of the leg would denote the calories burned. The 3D-printed sculptures generated curiosity, discussions and competition between participants, as well as motivating them to increase their sculptures size following receipt of previous 3D outputs. Despite this, the 3D-printed sculptures' only provided participants with a single bout of exercise data and, therefore, does not provide individuals with feedback of their overall PAL. Indeed, this is particularly important given that an individual who appears to be active in short bursts of vigorous-intensity activity can also be sedentary for prolonged periods within the same day, with very few individuals able to maintain a consistent level of activity (Thompson \& Batterham, 2013). Therefore, the 3D-printed sculptures may not provide adequate feedback to raise an individual's awareness and understanding of their true PAL.

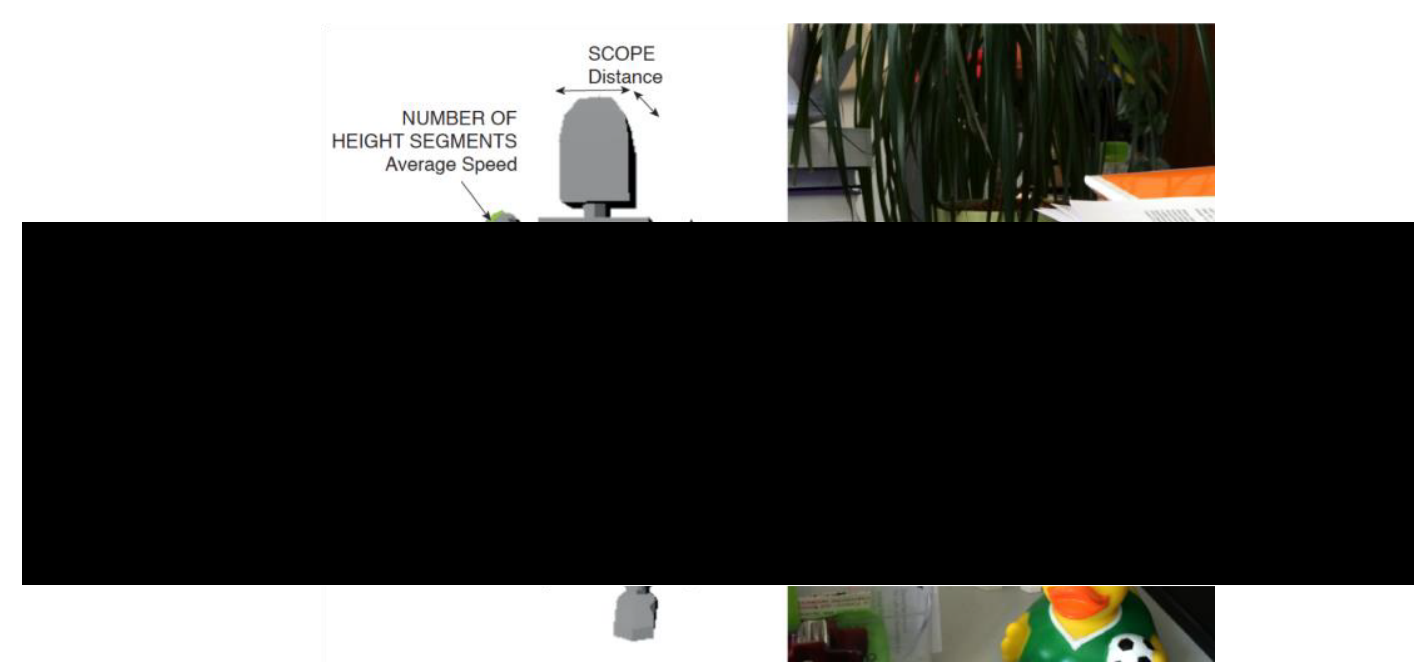

Figure 2.23 Human figure representing running data.

From Stusak et al. (2014).

Using a Fitbit activity tracker to measure physical activity, Lee et al. (2015) created a 'Patina Engraving System', which engraves a patina-like pattern onto the activity trackers wristband. Overtime the user's wristband would accumulate a visually rich activity pattern that would recognize the individual's step count, active time, calories, 
sleep and walking distance. Although the study focused more on the fashion aspect of the patina patterns for styling activity trackers, it was expressed that participants cherished the activity wristband more due to the personalisation, which led to more spontaneous interactions with other users to discuss their physical efforts. More recently, Sauvé et al. (2017) developed a system called 'LOOP' which visualises step count data recorded from a Fitbit tracker. The LOOP system is made out of eight rings, with one ring to represent the daily target (i.e., 10000 steps) and seven inner rings to represent each day of the week, with the smallest and largest rings representing Monday and Sunday, respectively (Figure 2.24). At the start of a week, each ring starts by facing downwards to represent no steps taken, with the position of the ring designed to mechanically move upwards (updated every hour) depending on the user's step count activity. Seldom to previous tangible methods is the dynamic ability of the LOOP system to change its shape or position with response to physical activity. However, the effectiveness of the LOOP system to promote physical activity is unknown due to no evaluation study completed in a real-world setting. Nonetheless, it could be postulated that the tangible and ubiquitous nature of the LOOP system could enhance an individual's awareness of their activity levels and even elicit increased physical activity through motivating an individual to raise all rings (i.e., days) to the daily target of 10,000 steps. That said, it is important to note that a majority of research surrounding tangible feedback of physical activity is in its early stages, with little supporting literature, which makes it difficult to come to definitive conclusions regarding its impact upon PAL and behaviour change (Groves, 2010).

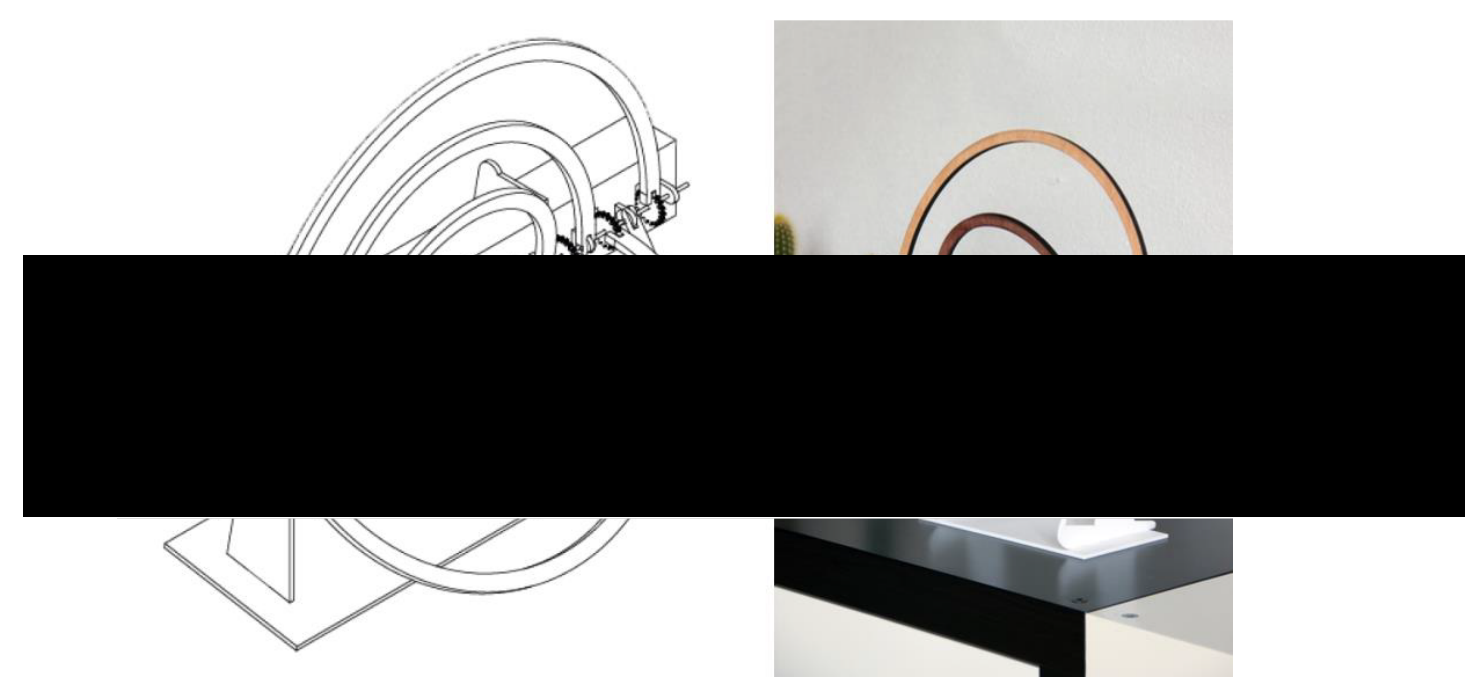

Figure 2.24 The LOOP system. From Sauvé et al. (2017). 
To date, there is only one study that has explored the utility of representing health data through personalised physical visualisations (Ananthanarayan et al., 2016). Specifically, Ananthanarayan et al. (2016) invited children to craft their own tangible visualisations using paper and a attachable pre-designed wearable UV tracker provided by the researchers to attach to their final design. The UV tracker was designed to send warning alarms, through flashing LED lights and a buzzer to inform the children they were spending too much time indoors. For example, one participant created an ambient octopus visualisation, with the eyes and tentacles of the octopus illuminating with LED lights in parallel with the sound of a buzzer from the mouth to inform the participant to play outside (see Figure 2.25). The study concluded that children's health could benefit from using the personalised health crafting approach. However, it could be argued that feedback through paper visualisations may not provide adequate haptic and proprioceptive experience for youths when compared to 3D-printed feedback of physical activity (Gillet et al., 2005). Based on Jean Ayres (2005) sensory integration theory developed in the late $60 \mathrm{~s}$, it is important to understand how an individual's brain receives and processes sensory information (i.e., sight, hearing, smell, touch and taste) to completing everyday activities. Sensory integration has become increasingly important within schools to promote youth's health and physical activity through designing activities that are rich in tactile and proprioceptive information that can stimulate youths sensory experience (Roley, 2015). Following this understanding, previous research suggests that the manipulation of tangible representations can support a more effective and natural process of learning among youth populations (Bara et al., 2004, Marshall, 2007, Price et al., 2003, Rogers et al., 2002b). In this regard, 3D-printed physical activity feedback has the potential to stimulate and develop youths sensory experience beyond the more traditional on-screen methods to promoting and eliciting greater understanding of and motivation for physical activity (Roley, 2015). At present, there has been no research to explore the utility of 3D printing as a method to visualise youths' physical activity data objectively assessed using an accelerometer, warranting further investigation. One important and common approach utlised within the majority of the aforementioned studies, is the use of a usercentred design approach, which involves stakeholders (i.e., target population) in the design process to ensure the diversity of the users' demands are met and considered within the creation of the newly designed technology (Consolvo et al., 2008a, Consolvo et al., 2006). 


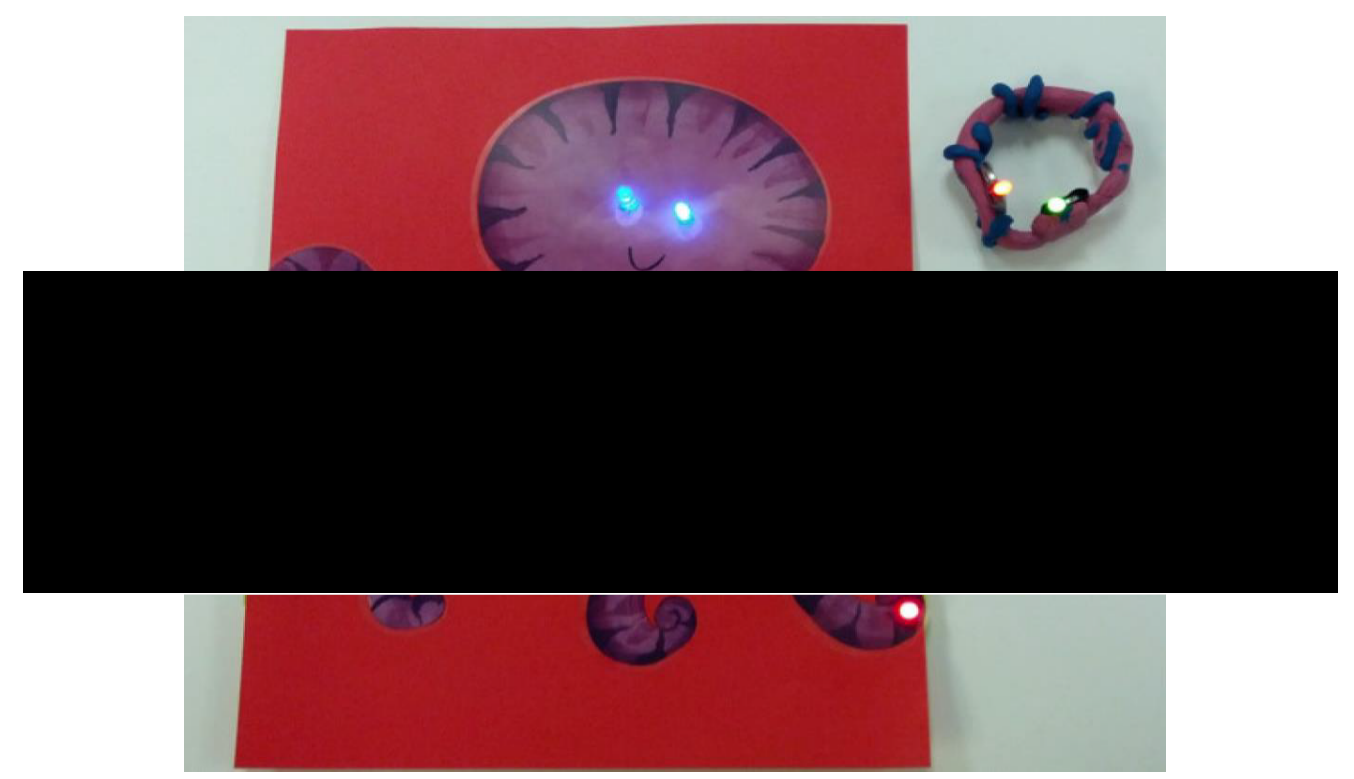

Figure 2.25 Ambient octopus visualisation of activity.

From Ananthanrayan et al. (2015).

\subsection{Designing Novel Technology for Youth}

When designing technology, a user-centred approach can have several advantages as it involves the end-users throughout the developmental process over the design and testing of the technological tool (Dabbs et al., 2009). In this way, efforts can be put into optimizing the functionality and usability of the technology and subsequent engagement to increase the likelihood of facilitating a positive behavioural change (Dabbs et al., 2009). Moreover, the involvement of the users is also likely to enhance the adherence to the implementation of an intervention and reduce the potential for abandonment of the technology (Johnson et al., 2005, Kelders et al., 2012, van GemertPijnen et al., 2011). Using qualitative methodologies to inform the design of visual representations can help refine the content and reduce individual or cultural differences (Rowsell et al., 2015). Therefore, in-depth qualitative research should be implemented to not only design and evaluate technological tools and interventions, but to develop a greater understanding of the psychosocial context of the individuals who will use them.

The used-centred design approach developed by Druin (2002) for youths is the most widely recognised and adopted framework by researchers for eliciting inventive and expressive ideas from youths to design, create and adapt new technology (Ananthanarayan, 2015, Ananthanarayan et al., 2016, Catala et al., 2018, Fitton et al., 
2018, Frauenberger et al., 2018, Lazar et al., 2018, Sharma et al., 2018, Woodward et al., 2018). According to Druin's framework (2002), there are four main roles youths can play in the technology design process, the design partner, informant, tester and the user (Figure 2.26). The methods used within the framework are influenced from the larger participatory design (Muller, 2003) and scenario-based methods (Carrol, 1999, Kankainen et al., 2012), which are known as suitable methods for motivating youths' involvement in learning activities (Sadik, 2008, van Gils, 2005). Firstly, the role of the design partner considers youths to be equal stakeholders in the design of the technology, where youths contribute to ideas and designs for the technology by creating low-tech prototypes using tools such as paper, crayons, Play-Doh, LEGO, as observed in previous health design methodologies (Ananthanarayan, 2015). The informant role, places emphasis on youths providing input on the design sketches or low-tech prototypes, offering feedback to inform the design process. Youths as the tester, involves trialing the newly developed technology before it is released into the world for evaluation, where researchers can observe youths and ask for their direct comments concerning their experiences. Finally, the role of the user, is where youths contribute to the research and development process by using the technology in a realworld setting, while researchers can observe, videotape and quantify their skill or ability with the technology. Researchers use this role to understand the impact of the technology so future technologies can be adapted or educational environments enhanced. As noted within the framework, choosing to use any of these roles is dependent upon the resources, timeframe and philosophy of the research. In this respect, the current thesis implements youths as the design partners (Study 2), tester (Study 3) and user (Study 4) to evaluate the efficacy of 3D-printed physical activity data as a tool to promote physical activity. Indeed this thesis takes note from Blandford et al. (2013) on how "the art of conducting an effective study is in pulling together appropriate ingredients to construct a recipe that is right for the occasion" (P2) - i.e. addressing the purpose of the study while working with the available resources. 


\section{The Child as...}

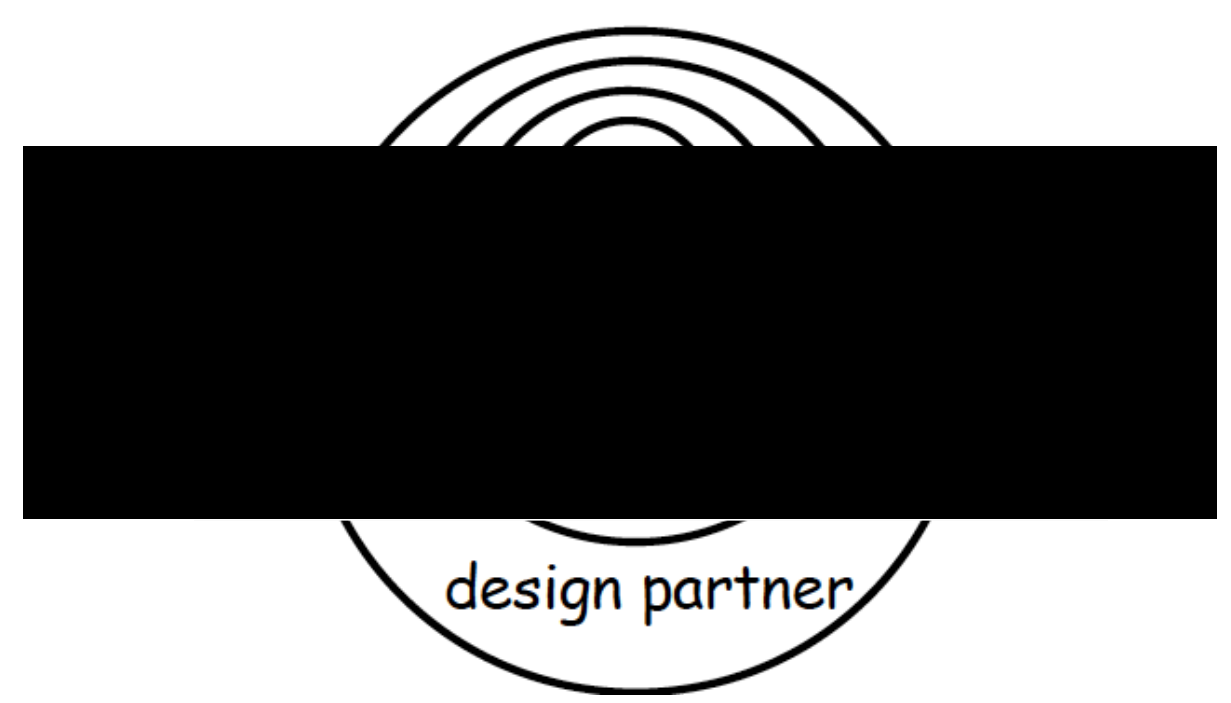

Figure 2.26 The four roles of youths to designing new technology. From Druin (1999).

\subsection{Summary and Conclusion}

The literature review in this chapter has summarised the current evidence surrounding the health benefits of increased physical activity and highlighted youths' lack of awareness and understanding of their PAL and the recommended Government guidelines, whilst considering technology as both a barrier and facilitator to youths' engagement in physical activity. Further, a comprehensive review of the literature on the accuracy of objective measurements and their ability to measure more diverse movements, and strategies to promote and design visual feedback of physical activity data is detailed.

The promotion of physical activity is identified as a public health priority (Trost et al., 2014a), with nearly a third of youths in the UK not meeting the current guideline of 60 minutes of MVPA daily (Health Survey for England, 2017b). Two frequently cited reasons for youths' underachievement of these physical activity guidelines are thought to be a lack awareness of their PAL (Corder et al., 2010, Xu et al., 2017) and limited understanding of what activities and different intensities constitute towards the daily target (Harris et al., 2016, Noonan et al., 2016). While sedentary screen-based technologies are criticised for discouraging youths from engaging in traditional 
physical activities (Brockman et al., 2011), technologies such as accelerometers in combination with personalized feedback are found to enhance an individual's awareness and understanding of physical activity (Bentley et al., 2012, Van Hoye et al., 2012). Indeed, accelerometers are praised for their ability to measure MVPA, however, they are limited when it comes to accounting for non-linear movements, such as turning, that are habitual to youths' sporadic physical activity patterns. Moreover, evidence from adult based studies suggests that turning has its own significant additional energy expenditure over straight-line walking (McNarry et al., 2017, Wilson et al., 2013). However, despite the increasing evidence on the energy costs of turning, little is known about the energy demands of turning in youths, warranting further investigation. However, research to enhance the accuracy and precision of accelerometers in estimating youths PAL will not by itself, result in positive behaviour change. Accelerometer measurements in combination with feedback that is personalised and meaningful has great potential to promote physical activity and thus positive behaviour change (Bentley et al., 2012, Van Hoye et al., 2012). More specifically, personalised feedback of physical activity data through tangible visualisations have been shown to raise adults' awareness of their PAL when compared to on-screen visualisations (Khot \& Mueller, 2013, Khot et al., 2015b, Stusak et al., 2014). Indeed, tangible visualisations have previously been shown to engage youth in playful learning, engagement and reflection (Rogers et al., 2002a, Price et al., 2003, Marshall, 2007), however, no studies to date have explored the utility of tangible physical activity feedback in youths.

Therefore, the overarching aim of this thesis it to investigate the measurement and use of tangible visualisations of youths' physical activity to positively change behaviour and improve health. The individual study objectives within the thesis are listed below (p70). 


\section{Study Objectives}

Study 1 To (i) determine the influence of walking speed and angle of turn, and their interaction on the energy expenditure of healthy children and (ii) determine the influence of sex, stature and cardiorespiratory fitness on the energy expenditure of turning in children.

Study 2 To (i) elicit children's and adolescents' perceptions of physical activity data when represented as 3D-printed objects; (ii) elicit parents' and teachers' views on the perceived benefits and barriers of 3D-printed objects of physical activity for youths; (iii) ascertain youths' designs for 3D objects of physical activity using Play-Doh and (iv) use these data to subsequently inform the design of 3D models and a school-based physical activity intervention.

Study 3 To (i) elicit children and adolescents' interpretations of the age-specific 3D model prototypes; (ii) examine children and adolescents' perceptions and ability to identify physical activity intensities (i.e., sedentary, light, moderate and vigorous) and (iii) use these data to consolidate the design of the agespecific 3D model prototypes to inform the development of a school-based physical activity intervention.

Study 4 To (i) observe youths' personal experiences with the age-specific 3D models over a 7-week faded intervention; (ii) assess youths' ability to associate their personal physical activity levels to the age-specific 3D-printed models of physical activity. 


\section{Thesis Map}

STUDY

\section{OUTCOMES}

1. Energy Expenditure Associated with

Aim Walking Speed and Angle of Turn in Children
- To investigate the influence of walking speed and angle, and their interaction, on the energy expenditure of healthy children.

Key

Findings

2. Perceptions of Visualising Physical

Aim

Activity as a 3D-printed Object: A

Formative Study

Role of DESIGN PARTNERS

Key

Findings

3. Understanding Youths' Ability to Interpret 3D-printed Physical Activity Data and Identify Associated Intensity Levels

\section{Role of TESTER} Key

Findings

4. The Tangibility of Personalised 3DAim Printed Feedback may Enhance Youths' Physical Activity Awareness 


\section{CHAPTER 3}

Energy Expenditure of Turning
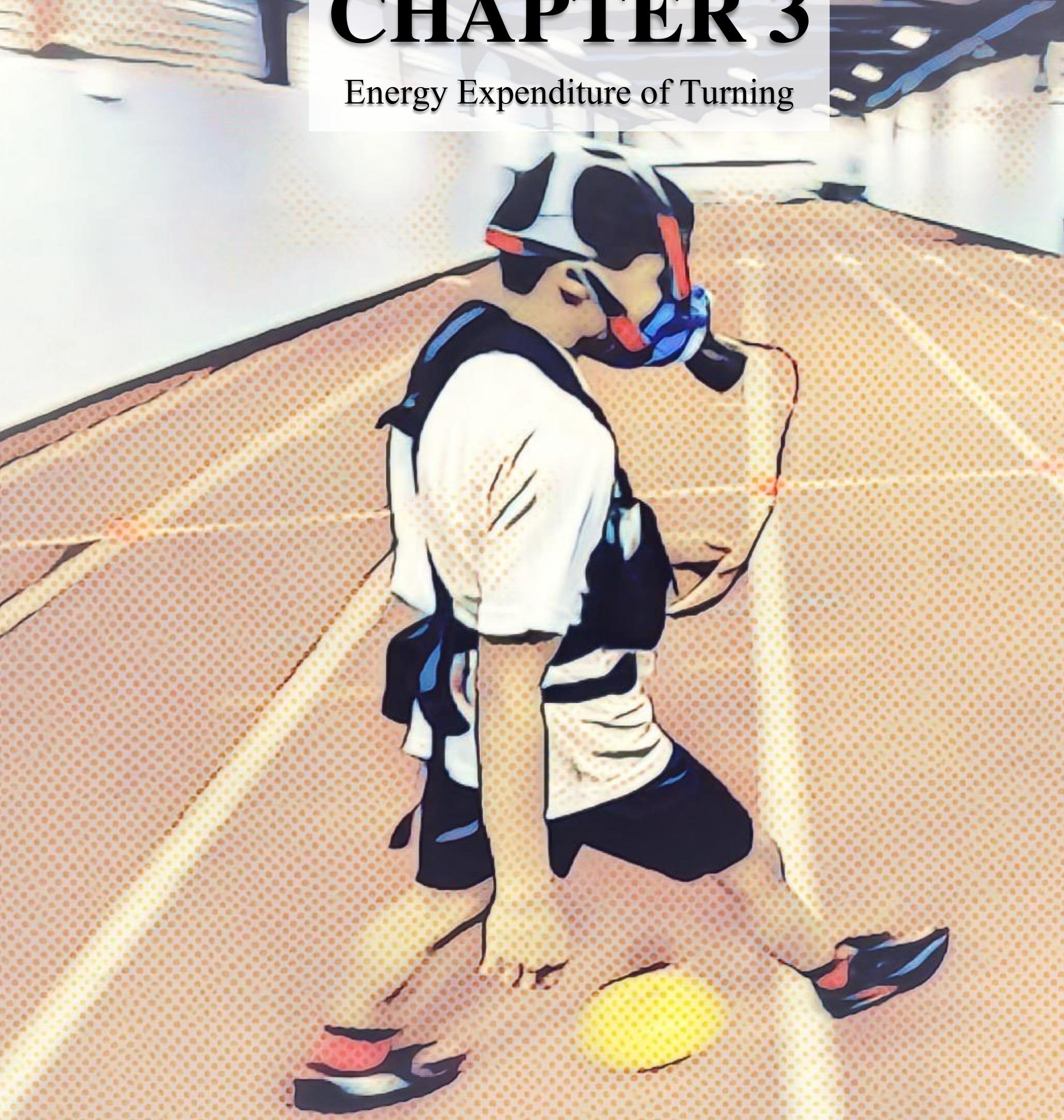


\section{Energy Expenditure Associated with Walking Speed and Angle of Turn in Children}

\subsection{Introduction}

Insufficient physical activity is one of the leading risk factors for global mortality, at least in part due to its association with obesity and non-communicable diseases (NCDs), such as cardiovascular disease, cancer and diabetes . However, despite the numerous physiological (Sothern et al., 1999, Janssen \& Leblanc, 2010a) and psychosocial benefits (Nieman, 2002, Eime et al., 2013) associated with physical activity, it is suggested that as little as $19 \%$ of boys and $16 \%$ of girls meet the current government guidelines of 60 minutes of moderate-to-vigorous physical activity every day (Townsend et al., 2015). A central tenet to these guidelines is the link between defined physical activity and energy expenditure.

The most accurate measure of an individual's physical activity and energy expenditure is by assessing the body's oxygen utilization and carbon dioxide production $\left(\dot{V} \mathrm{CO}_{2}\right)$ using indirect calorimetry methods (Levine, 2005). However, this level of measurement is expensive and impractical in free-living settings (Strath et al., 2013, Westerterp, 2009), although does provide an essential criterion method by which to judge the accuracy and precision of smaller and less obtrusive devices such as accelerometers (Freedson et al., 2005a, Rowlands, 2007). Accelerometers measure human movement through applied accelerations, usually expressed as multiples of $\mathrm{g}$ force $\left(1 \mathrm{~g}=9.8 \mathrm{~m} / \mathrm{s}^{2}\right.$, force of gravity), acting along a sensitive orthogonal axis that can be translated into the rate and intensity of body movement in up to three planes (i.e., anterior-posterior, mediolateral and vertical; Godfrey et al., 2008). The energy required to exert a force enables the use of movement acceleration to reflect the energy expenditure of an individual performing a specific physical activity (Sasaki et al., 2016). As a result, accelerometers have been widely utilized among researchers as a measurement of physical activity (Doherty et al., 2017, Lee \& Shiroma, 2014, Leung 
et al., 2017, Ward et al., 2005) due to their ability to estimate oxygen uptake ( $\left.\dot{V} \mathrm{O}_{2}\right)$ (de Almeida Mendes et al., 2018, McGregor et al., 2009). Nevertheless, there are some limitations to accelerometer measurements, including upper body movements, cycling, walking on an incline and activities that involve carrying heavy loads (Rich, 2013). In addition, accelerometer measurements are most commonly based upon linear regression models that emphasize that energy expenditure increases linearly with vertical accelerations (Freedson et al., 2012), which tend to discredit non-locomotive activities, such as turning (Bassett \& John, 2010, Chen et al., 2007, Van Remoortel et al., 2012). In this regard, children are problematic since their movement is highly sporadic (Sleap \& Warburton, 1996, Welk et al., 2000, Baquet et al., 2007), which presents challenges to power-use determination protocols that typically require steady state conditions (Reilly et al., 2004, Trost et al., 2011). Subsequently, the development of new multi-sensor devices that integrate both accelerometer and magnetometer measurements have been extensively used as a proxy of $\dot{V} \mathrm{O}_{2}$ in humans (McNarry et al., 2017, Qasem et al., 2012, Weippert et al., 2013) as a result of the combined ability to capture additional information regarding how the body rotates during pathways that require turning (Williams et al., 2017a) .

The movement of turning has three degrees of freedom because it involves the body moving through all three planes (sagittal, frontal and transverse) and around all three axes (medial-lateral, anterior-posterior and superior-inferior; Lippert, 2011). Specifically, the axial segments of the body are proactively rotated to the new direction of travel, usually following a sequential top down pattern with the eyes and head leading, followed by the trunk, pelvis and feet (Hollands et al., 2001, Hollands et al., 2004). In this respect, turning is a fundamental movement within human locomotion and is particularly prevalent in children's habitual physical activity patterns (Sleap \& Warburton, 1996). Even within adults, turning can make up $35-45 \%$ of all steps taken in a typical day (Glaister et al., 2007). Whilst turning has not generally been considered to be associated with significant additional energetic costs over straight-line walking, this attitude is now changing (Dellal et al., 2010). According to Hamill et al. (1983), curved path locomotion or turning may subject individuals to unique stresses. For example, a study in 2011 suggested that $15 \%$ of the total energy expenditure during stair climbing can be attributed to turning in adults (Minetti et al., 2011), while Buchheit et al. (2011) reported marked physiological changes associated with turning; 
increased heart rate, blood lactate and perceived exertion during intermittent shuttle run tests with a $180^{\circ}$ turn compared to straight running. Similarly, it has been shown that completing 30 turns per minute at $3 \mathrm{~km} \cdot \mathrm{hr}^{-1}$ elicits a similar energy expenditure to straight line walking at $6 \mathrm{~km} \cdot \mathrm{hr}^{-1}$ (Hatamoto et al., 2014). Furthermore, Wilson et al. (2013) extended these findings to consider a range of turning angles, demonstrating that as the angle of the turn increased, so did the associated energy expenditure. Specifically, a single $180^{\circ}$ turn elicited the same energy expenditure as walking 5.88 $\mathrm{m}$ in a straight line at a velocity of $1.67 \mathrm{~m} \cdot \mathrm{s}^{-1}\left(6 \mathrm{~km} \cdot \mathrm{hr}{ }^{-1}\right)$. To consider these findings as a function of walking speed, McNarry et al. (2017) reported a synergistic interaction between speed and angle in determining the energy expenditure associated with

walking. A similar study investigated the energy expenditure of turning and walking in community-dwelling elderly, reporting that $180^{\circ}$ turns were significantly more energy demanding than $90^{\circ}$ turns (Justine et al., 2014). However, the applicability of these findings to children is questionable, not least due to their unique physiological and biomechanical structure (Andropoulos, 2012). To date, no studies have specifically addressed the energy expenditure of turning in children. Such findings will have important implications for developing technology that enables a more precise unobtrusive assessment of children's physical activity and intensity that is essential for detailed investigations of dose-response relationships between physical activity and health, the evaluation of interventions and enhancing an individual's awareness of their physical state to enforce behaviour change.

The purpose of the present study was to investigate the influence of turn angle and walking speed on energy expenditure in children. We hypothesized that (i) as speed of walking increased, so too would the energy expenditure; (ii) as angle of turn increased, so would the energy expenditure and that (iii) walking speed and angle of turn would interact to modulate energy expenditure.

\subsection{Methods}

\subsubsection{Participants and Anthropometry}

One local primary school in Swansea was invited, via telephone, to take part in the study. Children were recruited through attending a school assembly presentation on 
the study aims and protocols in which consent forms and information sheets were distributed. From those that responded, twenty healthy children aged 9-12 years $(10.1$ $\pm 0.5 \mathrm{yrs} ; 10$ boys), split by sex and free from injury or illness, were selected by stratified randomisation to participate in the study. Child assent and parental or guardian consent were obtained prior to study participation (see Appendix II). At the start of the study and on one subsequent occasion, all participants body mass (Seca 876, Hamberg, Germany), stature (Holtain Stadiometer, Holtain Ltd) and sitting height (Holtain Sitting Height Stadiometer, Holtain Ltd) were measured to the nearest $0.1 \mathrm{~kg}$ and $0.1 \mathrm{~cm}$, respectively. For all anthropometrical measures, participants were required to be in minimal clothing (i.e., shorts and t-shirt) without shoes. Sexual maturity was assessed by self-report using the indices of pubic hair described by Tanner (1963; see Appendix C). Pre-pubertal status was defined as Tanner stage $1(\mathrm{n}=12)$, with stage 2 being early pubertal $(n=4)$, Tanner stage 3 mid-pubertal $(n=4)$, Tanner stage 4 and 5 being late pubertal and post pubertal, respectively (Chan et al., 2010). To provide an additional indicator of physical maturity, the age to peak height velocity equation devised by Mirwald et al. (2002) was used based on the measurement of standing and seated height, weight and age to calculate maturity offset. Participants were asked to arrive at the laboratory in a rested state, at least 2-hours postprandial. In total, participants were required to visit the University laboratories on three separate occasions throughout the study. All procedures employed during this study were approved by Swansea University ethics committee and were conducted in accordance with the Declaration of Helsinki (ref: PG/2014/16).

\subsubsection{Incremental Treadmill Test}

Participants were required to visit the laboratory on one occasion to perform an incremental treadmill test to volitional exhaustion for the determination of the gas exchange threshold (GET) and peak oxygen uptake $\left(\dot{V} \mathrm{O}_{2 \text { peak }}\right)$. The children were first familiarised with walking and running on the treadmill at a range of speeds (4, 6 and $8 \mathrm{~km} \cdot \mathrm{hr}^{-1}$ ) and with the testing equipment. To take into account the variation in biological ages, individual $\dot{V} \mathrm{O}_{2 \text { peak }}$ test speeds were calibrated by anchoring treadmill speeds to set Froude numbers (Houston et al., 2013). The Froude number is based on the dynamic similarity hypothesis (DSH), which implies that optimal walking speed will be at Froude number of 0.25 , and the transition from walk to run will occur close 
to a Froude number of 0.5, regardless of body size (Alexander \& Jayes, 1983). Given that gait parameters change as a direct result of both speed and size of an individual, the DSH has been shown to be invaluable for comparing both adults and children of different sizes walking at different speeds by providing a size-corrected speed (i.e., Froude number) against which gait parameters can be compared (Raichlen et al., 2013). For example, increased stride frequency is observed in shorter stature individuals compared to taller individuals during both walking and running at the same speeds (Minetti et al., 1994), which in turn leads to a higher $\dot{V} \mathrm{O}_{2}$ in shorter individuals (Rowland \& Green, 1988). The differences in $\dot{V} \mathrm{O}_{2}$ between individuals of different sizes can be accounted for by scaling the horizontal speed to leg length, as done with Froude's number, during incremental exercise (Ferretti et al., 1991, Kramer \& Sylvester, 2013, Minetti et al., 1994, Steudel \& Beattie, 1995, Steudel-Numbers et al., 2007). Therefore, to nullify size differences in the present study, treadmill speeds were calculated using the Froude number, gravity and leg length equation (Minetti, 2001). The initial stages were set at a 1\% gradient (Jones \& Doust, 1996) and increased every 2 minutes, beginning with a walking speed equivalent to Froude 0.25. Subsequent increments were determined by the calculated difference between stage 1 and 2 speeds $\left(\sim 2 \mathrm{~km} \cdot \mathrm{hr}^{-1}\right)$ until maximal running velocity was achieved. At this point, the gradient was then increased by $1 \%$ every minute until volitional exhaustion was reached.

\subsubsection{Turning Protocol}

Approximately 1-3 days after completion of the incremental treadmill test, participants completed the second part of the testing involving the turning protocol, which was repeated on two occasions, separated by a minimum of 24 hours. During this protocol, each participant was asked to complete three-minute bouts of walking interspersed by 3 minutes of seated rest. As illustrated in Figure 3.1, each participant walked at four different walking speeds $\left(2.5,3.5,4.5,5.5 \mathrm{~km} \cdot \mathrm{hr}^{-1}\right)$ in combination with four different turn angles $\left(0,45,90,180^{\circ}\right)$, in a random order generated using an excel macro randomizer. Specifically, each of the conditions involved $5 \mathrm{~m}$ straight walking stretches interspaced with prescribed turns with the speed dictated by a digital, auditory metronome. The auditory metronome sounded half-way along the $5 \mathrm{~m}$ straight and on the turns so variability in speed within conditions was minimised. 
Furthermore, all participants were accompanied by one of the research team to act as a pace-setter. Each condition incorporated an equal number of left and right turns.

A

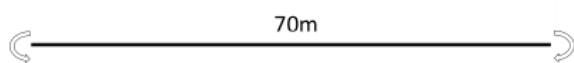

B

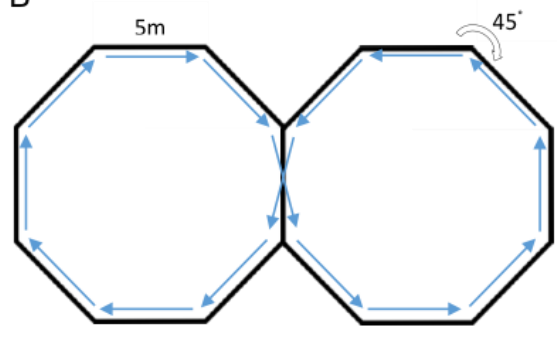

C

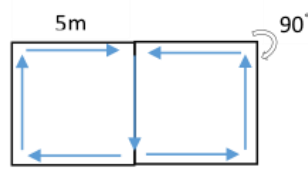

D

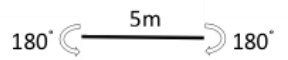

Figure 3.1 Experimental setup of the turning protocol showing $5 \mathrm{~m}$ straights interspersed by prescribed angle of turns
A) $0^{\circ}$
B) $45^{\circ}$ C) $\left.90^{\circ} \mathrm{D}\right)$
D) $180^{\circ}$ with equi

\subsubsection{Measurements}

Throughout all the tests, gas exchange variables were measured on a breath-by-breath basis using the Cortex MetaMax 3B (CORTEX Biophysik GmbH, Germany). Previous research comparing data acquired from human participants twice on different days has demonstrated that the MetaMax 3B provides a reliable measurement of $\dot{V} \mathrm{O}_{2}$ and $\dot{V} \mathrm{CO}_{2}$ (Macfarlane \& Wong, 2012, Perkins et al., 2002, Prieur et al., 2003, Vogler et al., 2010). Furthermore, the MetaMax 3B displays similar validity to other portable gas analyser systems (e.g., Jaeger Oxycon Pro; Macfarlane \& Wong, 2012, Vogler et al., 2010). Prior to each test, the MetaMax 3B was calibrated using gases of known concentration and the turbine volume transducer was calibrated using a 3-litre syringe (Hans Rudolph, Kansas City, MO). The delay in the capillary gas transit and analyser rise time were accounted for relative to the volume signal, thereby time-aligning the concentration and volume signals. Additionally, two custom-built tri-axial accelerometers and magnetometers, called SLAM Trackers (Wildbyte Technologies Ltd, Swansea, UK), measuring at $100 \mathrm{~Hz}$ on all channels, were worn by participants; one tag was worn on the right mid-axilla line at the level of the iliac crest and one tag 
at the middle of the lower back (see Figure 3.2), in accord with previous methods (McNarry et al., 2017). The SLAM tracker device has been mechanically validated as a suitable device for assessing human movement at a range of low and fast walking speeds (Clark et al., 2016). Previous research has also extensively tested the SLAM trackers reliability within various mammals (e.g., humans; Qasem et al., 2012, Wilson et al., 2013), birds and ocean dwelling creatures of differing sizes (Wilson et al., 2008). The SLAM tracker is used to derive two dynamic acceleration-based metrics, known as Overall Dynamic Body Acceleration (ODBA) and Vectorial Dynamic Body Acceleration (VeDBA). ODBA is the sum of the absolute acceleration from all three orthogonal axes (i.e., surge, heave and sway) after the static portion of the acceleration signal has been removed (Wilson et al., 2006). Research shows that ODBA correlates well with speed (Halsey et al., 2008, Wilson et al., 2008) and that it is a good proxy for movement-related metabolic rate (Wilson et al., 2006). However, Bidder et al. (2012b) found that the relationship between ODBA and speed was subject to variation within species, gait and stride frequency. Furthermore, acceleration is a vectorial quantity, which means that the summation of the three axes to attain ODBA is likely to over-estimate the physical acceleration experienced by the SLAM tracker (Bidder et al., 2012a). Therefore, researchers suggest the use of the power metric VeDBA (McNarry et al., 2017, Wilson et al., 2013, Qasem et al., 2012), as it provides values that are closer to the true physical acceleration, with the additional benefit of VeDBA being insensitive to device orientation, unlike ODBA (Gleiss et al., 2009, Qasem et al., 2012).

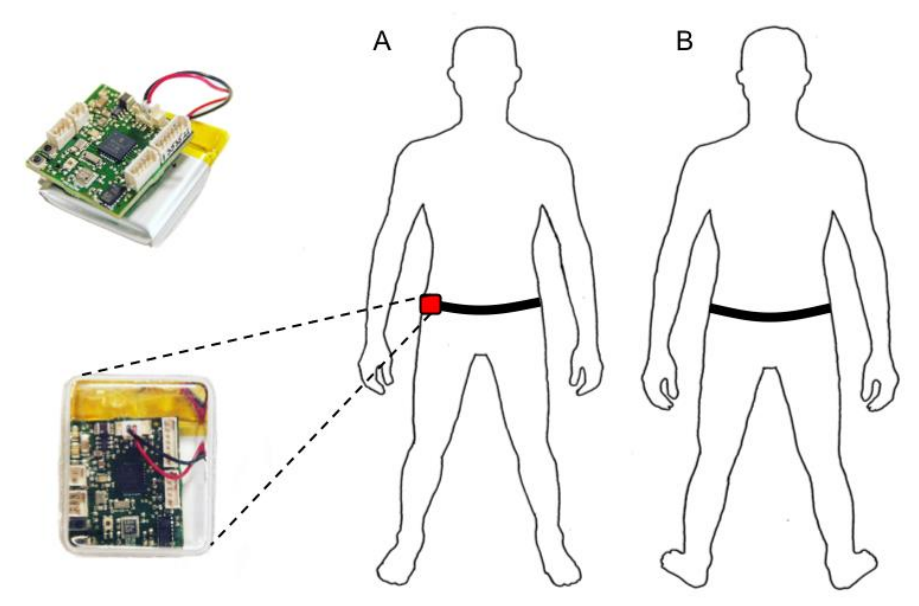

Figure 3.2 SLAM tracker device placement

A) right mid-axilla line at the level of the iliac crest; B) middle of the lower back 


\subsubsection{Data Analysis}

The $\dot{V} \mathrm{O}_{2 \text { peak }}$ was defined as the highest 10 -s stationary average during the incremental exercise test. The GET was determined by the V-slope method (Beaver et al., 1986) as the point at which carbon dioxide production began to increase disproportionately to $\dot{V} \mathrm{O}_{2}$, as identified using purpose-written software developed using LabVIEW (National Instruments, Newbury, UK). Mean absolute $\dot{V} \mathrm{O}_{2}$ values for defining steady state for each individual speed and turn were taken from the last 45 seconds of each 3 minute turning condition (Wilson et al., 2013). Analyses of turning energy expenditure were based on the premise that the additional turn cost was superimposed on the baseline of straight-line travel. Specifically, the difference in $\dot{V} \mathrm{O}_{2}$ between straight line walking $\left(0^{\circ}\right)$ at each velocity relative to the $\dot{V} \mathrm{O}_{2}$ associated with walking at 45,90 or $180^{\circ}$ turns was defined as the additional cost of turning. The net energy cost of walking $\left(\mathrm{C}_{\mathrm{r}}\right)$ was calculated from participants' absolute $\dot{V} \mathrm{O}_{2}$ values normalized per unit body mass for each experimental condition and divided by the walking speed converted to meters per minute $(\mathrm{m} / \mathrm{min})$, expressed as $\mathrm{ml} \mathrm{O}_{2} \cdot \mathrm{kg}^{-1} \cdot \mathrm{km}^{-1}$. To account for body size, the procedures proposed by Welsman and Armstrong (2000) were used to calculate the allometric scaling coefficient for absolute $\dot{V} \mathrm{O}_{2}$ for turning, straights and $\dot{V} \mathrm{O}_{2}$ peak. Firstly, the data was logarithmically transformed to determine the allometric relationship between body mass, $\dot{V} \mathrm{O}_{2}$ and $\dot{V} \mathrm{O}_{2 \text { peak. }}$ Common allometric exponents were confirmed, which were then linearly regressed to obtain a coefficient and then expressed using the formula:

Scaled $\dot{V} \mathrm{O}_{2}=\mathrm{Y} / \mathrm{X}^{\mathrm{b}}$

where $\mathrm{Y}$ is the participants $\dot{V} \mathrm{O}_{2}$ for a turn, straight or $\dot{V} \mathrm{O}_{2 \text { peak }}, \mathrm{X}$ is the body mass of the participant and $b$ is the scaling coefficient derived from the linear regression.

To estimate the caloric energy expenditure of turning, absolute $\dot{V} \mathrm{O}_{2}$ values were converted to kilocalories per min $(\mathrm{kcal} / \mathrm{min})$ based on the rounded value of $5.0 \mathrm{kcal}$ per litre of oxygen consumed (Hoeger \& Hoeger, 2015). The caloric calculation assumes that an individual's combustion of fuels is from a mixed diet (i.e., blend of carbohydrate, fat, and protein; Plowman \& Smith, 2013). However, even with large variation in metabolic mixture, the calorific value only varies by $2-4 \%$ (Plowman $\&$ 
Smith, 2013). Therefore, to obtain caloric energy expenditure of turning, absolute $\dot{V} \mathrm{O}_{2}$ was multiplied by $5.0 \mathrm{kcal}$ per litre of oxygen to provide a caloric energy expenditure per minute for reference (Hoeger \& Hoeger, 2015).

Significant absolute and scaled $\dot{V} \mathrm{O}_{2}$ outliers were established using box plots with Tukeys 1.5 multiplier of the standard deviation (Tukey, 1977). Subsequently, one participant was excluded from further analyses due to significant outliers identified in test 1 .

The raw accelerometer data was first converted to dynamic body acceleration (DBA) by smoothing each channel to derive the static acceleration using a running mean over $2 \mathrm{~s}$ (Shepard et al., 2008). The static acceleration was then subtracted from the raw acceleration data (Gleiss et al., 2011), resulting in values for dynamic acceleration that were all converted to positive values. These values for DBA were summed vectorially to provide 'vectorial dynamic body acceleration' (VeDBA):

$$
V e D B A=\sqrt{\left(A_{x}^{2}+A_{y}^{2}+A_{z}^{2}\right)}
$$

where $A_{x}, A_{y}$, and $A_{z}$ are the derived dynamic accelerations at any point in time corresponding to the three orthogonal axes of the accelerometer (Qasem et al., 2012). VeDBA has been used extensively as a proxy for $\dot{V} \mathrm{O}_{2}$ in a suite of vertebrates (cf. Halsey et al., 2011), including humans (McNarry et al., 2017, Qasem et al., 2012, Weippert et al., 2013), with appreciable success. However, many aspects of the particulars of the acceleration data recorded in such trials (e.g., lateral versus forwardbackward) as well as the effect of incline (cf. Bidder et al., 2012a) and tag mounting have not been examined critically so our use of this metric has to be seen within this context.

Using the middle minute and overall three-minute bout, both mean and summed VeDBA were derived for each individual turn and straight for each condition. The individual turns and straight sections were analysed using a custom developed $\mathrm{C}++$ software (DDMT Wildbyte Technologies Ltd, Swansea, UK) specially designed for 
visualizing the accelerometry and magnetometry traces to identify inter alia turns via systematic changes in the magnetometry data (Williams et al., 2017a).

\subsubsection{Statistics}

A Sharpiro-Wilks test was used to confirm data normality. Repeated measures linear mixed-effects models with a Tukey's test of post hoc means test were conducted using IBM SPSS Statistics 22 (Chicago, IL) to account for the repeated measures and correlated nature of the data to determine the influence of, and interaction between, independent variables walking speed and angle, with the dependent variables expressed as $\mathrm{C}_{\mathrm{r}}$, absolute $\dot{V} \mathrm{O}_{2}$, scaled $\dot{V} \mathrm{O}_{2}$ and mean VeDBA (Halsey et al., 2009, Qasem et al., 2012). All condition combinations for turning $\dot{V} \mathrm{O}_{2}$ (absolute or scaled) and Mean VeDBA (straight or turn) were placed into one mixed model analysis with covariates, sex, stature, cardiorespiratory fitness, to determine their modulatory effect. Sex and stature were chosen as covariates in the mixed model on the basis of evidence that these parameters are highly variable during childhood and between sexes, very rarely progressing at the same growth rate (Katzmarzyk et al., 1997), and thereby potentially influencing energy expenditure output. Furthermore, given that previous findings demonstrate that the training status of an individual can influence the efficiency of turning energy expenditure (Hatamoto et al., 2013, McNarry et al., 2017), participants cardiorespiratory fitness was also taken into account. A Pearson productmoment correlation coefficient was used to determine any associations between the dependent variables $\dot{V} \mathrm{O}_{2}$ and straight and turn VeDBA, including all covariates. All data are presented as mean $\pm \mathrm{SD}$, with statistical difference accepted at $p \leq 0.05$.

\subsection{Results}

The characteristics of the sample population are displayed in Table 3.1. Boys were significantly heavier, taller and demonstrated a higher peak $\dot{V} \mathrm{O}_{2}$ than girls, in both absolute and scaled terms, which is representative of European children aged approximately 10 years old (Dencker et al., 2008, Dilber et al., 2015, Graves et al., 2013). In line with the Royal College of Paediatrics and Child Health maturation reference values for Tanners stages (2013), all participants were found to be either prepubertal or early to mid-pubertal according to the self-reported Tanner stages. To 
determine the relationship between walking speed and angle on energy expenditure, a mixed model was used to examine the main effects of speed and angle, and the interaction of speed and angle while controlling for sex. Our post hoc evaluations indicated that the empirical technique utilized fully met the intended objectives.

Table 3.1 Participants' characteristics

\begin{tabular}{|c|c|c|c|}
\hline & Total & Boys & Girls \\
\hline $\mathbf{n}$ & 19 & 9 & 10 \\
\hline Age, yrs & $10.1 \pm 0.5$ & $10.2 \pm 0.6$ & $10.0 \pm 0.3$ \\
\hline Tanner Stages, \% & $60 \%^{\mathrm{T} 1}, 20 \%{ }^{\mathrm{T} 2}, 20 \%^{\mathrm{T} 3}$ & $30 \%^{\mathrm{T} 1}, 40 \%{ }^{\mathrm{T} 2}, 30 \%{ }^{\mathrm{T} 3}$ & $90 \%{ }^{\mathrm{T} 1}, 10 \%{ }^{\mathrm{T} 3}$ \\
\hline Years to PHV, yrs & $-2.4 \pm 0.8$ & $-3.03 \pm 0.3$ & $-1.9 \pm 0.6$ \\
\hline Stature, m & $1.39 \pm 0.07$ & $1.42 \pm 0.05$ & $1.38 \pm 0.09^{*}$ \\
\hline Body mass, $\mathrm{kg}$ & $33.7 \pm 5.7$ & $35.03 \pm 3.8$ & $32.6 \pm 7.0^{*}$ \\
\hline BMI, $\mathrm{kg} \cdot \mathrm{m}^{-2}$ & $17.1 \pm 2.3$ & $17.3 \pm 1.7$ & $17.2 \pm 2.8$ \\
\hline Peak $\dot{V} \mathbf{O}_{2}, \mathbf{l} \cdot \mathbf{m i n}^{-1}$ & $1.63 \pm 0.39$ & $1.79 \pm 0.44$ & $1.49 \pm 0.29^{*}$ \\
\hline Scaled peak $\dot{V} \mathrm{O}_{2}, \mathrm{l} \cdot \mathrm{kg}^{-0.79} \cdot \mathrm{min}^{-1}$ & $100.8 \pm 18.8$ & $98.8 \pm 17.4$ & $102.5 \pm 20.7^{*}$ \\
\hline GET, l- $\mathbf{m i n}^{-1}$ & $1.03 \pm 0.25$ & $1.17 \pm 0.25$ & $0.90 \pm 0.18$ \\
\hline
\end{tabular}

The values of $\mathrm{C}_{\mathrm{r}}$, for all participants under all experimental conditions are reported in Table 3.2. The $\mathrm{C}_{\mathrm{r}}$ straight $\left(0^{\circ}\right)$ decreases with speed to attain a significant $(p<0.001)$ minimum energy expenditure at $5.5 \mathrm{~km} \cdot \mathrm{hr}^{-1}$ compared to $2.5 \mathrm{~km} \cdot \mathrm{hr}^{-1}$. The effect of $45^{\circ}$ and $90^{\circ}$ turns on $\mathrm{C}_{\mathrm{r}}$ is relatively minor, with only a $90^{\circ}$ turn at $2.5 \mathrm{~km} \cdot \mathrm{hr}^{-1}$ showing a significantly $(p<0.05)$ greater energy cost $(\sim 7 \%)$ when compared to straight line walking at $2.5 \mathrm{~km} \cdot \mathrm{hr}^{-1}$. However, for $180^{\circ}$ turns at speeds $3.5,4.5$ and $5.5 \mathrm{~km} \cdot \mathrm{hr}^{-1}$, significantly greater $C_{r}(p<0.05)$ were established when compared to straight line walking within speeds. More specifically, for a $180^{\circ}$ angle, speed increased $\mathrm{C}_{\mathrm{r}}$ by $\sim 7 \%$ at $2.5 \mathrm{~km} \cdot \mathrm{hr}^{-1}$, to $\sim 13 \%$ at $3.5 \mathrm{~km} \cdot \mathrm{hr}^{-1}(p<0.05), \sim 14 \%$ at $4.5 \mathrm{~km} \cdot \mathrm{hr}^{-1}(p<0.05)$, to attain $\sim 30 \%$ increase at $5.5 \mathrm{~km} \cdot \mathrm{hr}^{-1}(p<0.001)$. 
Table 3.2 Values for net energy cost of walking and turning

\begin{tabular}{|c|c|c|c|c|}
\hline & \multicolumn{4}{|c|}{$\dot{V} \mathrm{O}_{2}\left(\mathrm{C}_{\mathrm{r}}, \mathrm{ml} \mathrm{O}_{2} \cdot \mathbf{k g}^{-1} \cdot \mathbf{k m}^{-1}\right)$} \\
\hline \multicolumn{5}{|c|}{ Speed $\left(\mathrm{km} \cdot \mathrm{hr}-{ }^{1}=\mathrm{m} / \mathrm{min}\right)$} \\
\hline & $\mathbf{0}^{\circ}$ & $45^{\circ}$ & $90^{\circ}$ & $180^{\circ}$ \\
\hline $2.5=41.67$ & $0.28 \pm 0.05$ & $0.29 \pm 0.05$ & $0.30 \pm 0.05^{\#}(1.7)$ & $0.30 \pm 0.06$ \\
\hline $3.5=58.33$ & $0.23 \pm 0.04 *(1.9)$ & $0.23 \pm 0.04 *(2.0)$ & $0.24 \pm 0.04 *(2.0)$ & $0.26 \pm 0.05^{* \#}(1.8)$ \\
\hline $4.5=75.00$ & $0.21 \pm 0.04 *(2.2)$ & $0.21 \pm 0.04 *(2.5)$ & $0.21 \pm 0.04 *(3.0)$ & $0.24 \pm 0.04^{* \#}(3.0)$ \\
\hline $5.5=91.67$ & $0.20 \pm 0.04 *$ & $0.20 \pm 0.03 *(3.5)$ & $0.20 \pm 0.04 *(3.4)$ & $0.26 \pm 0.04^{* \#}(4.2)$ \\
\hline
\end{tabular}

Participants mean absolute and scaled $\dot{V} \mathrm{O}_{2}$ are reported in Table 3.3. There was a significant main effect for speed $(F=101.13, p<0.001)$ and turn angle $(F=11.52, p$ $<0.001)$ on absolute $\dot{V} \mathrm{O}_{2}$ and a significant interaction between speed and angle $(F=$ 2.01, $p<0.05$; see Appendix I), with similar effects still observed when scaled to account for body size (speed, $F=106.30, p<0.001$; angle, $F=13.96, p<0.001$; speed and angle, $F=168.14, p<0.05)$. As shown in Figure 3.3, increasing speed was found to increase $\dot{V} \mathrm{O}_{2}$ within a turn angle, but significant increases in $\dot{V} \mathrm{O}_{2}$ due to turning relative to straight line walking were only observed at $180^{\circ}$ at the highest speed. Boys demonstrated significantly greater $\dot{V} \mathrm{O}_{2}$ than girls across all conditions $(F=6.26, p<$ $0.05)$, regardless of the method of expression for $\dot{V} \mathrm{O}_{2}$. Stature $(F=26.27, p<0.001)$ and $\dot{V} \mathrm{O}_{2}$ peak $(F=24.53, p<0.001)$ were significant predictors of $\dot{V} \mathrm{O}_{2}$ during each condition for both sexes, although when condition $\dot{V} \mathrm{O}_{2}$ was scaled, scaled $\dot{V} \mathrm{O}_{2 \text { peak }}$ was no longer a significant predictor $(F=0.98, p>0.05)$. The significant predictors for absolute and scaled $\dot{V} \mathrm{O}_{2}$ models on speed, angle and their interaction are shown in Appendix I. 
Table 3.3 Mean absolute $\dot{V} \mathrm{O}_{2}$, scaled $\dot{V} \mathrm{O}_{2}$ and caloric energy expenditure during each combination of walking velocity and angle

\begin{tabular}{|c|c|c|c|c|}
\hline & \multicolumn{4}{|c|}{ Absolute $\dot{V} \mathbf{O}_{2}\left(\mathbf{l} \cdot \mathbf{m i n}^{-1}\right)$} \\
\hline & $\mathbf{0}^{\circ}$ & $\mathbf{4 5}^{\circ}$ & $90^{\circ}$ & $180^{\circ}$ \\
\hline $2.5 \mathrm{~km} \cdot \mathrm{hr}^{-1}$ & $0.40 \pm 0.10$ & $0.40 \pm 0.10$ & $0.42 \pm 0.10$ & $0.42 \pm 0.10$ \\
\hline $3.5 \mathrm{~km} \cdot \mathrm{hr} \mathbf{r}^{-1}$ & $0.44 \pm 0.11 *$ & $0.44 \pm 0.10^{*}$ & $0.47 \pm 0.11 *$ & $0.50 \pm 0.13 * \#$ \\
\hline $4.5 \mathrm{~km} \cdot \mathrm{hr}^{-1}$ & $0.52 \pm 0.11^{*}$ & $0.51 \pm 0.12 *$ & $0.53 \pm 0.11^{*}$ & $0.60 \pm 0.15^{* \#}$ \\
\hline \multirow[t]{2}{*}{$5.5 \mathrm{~km} \cdot \mathrm{hr}^{-1}$} & $0.62 \pm 0.13^{*}$ & $0.60 \pm 0.13^{*}$ & $0.63 \pm 0.15^{*}$ & $0.75 \pm 0.17 * \#$ \\
\hline & \multicolumn{4}{|c|}{ Scaled $\dot{V} \mathrm{O}_{2}\left(\mathbf{l} \cdot \mathrm{kg}^{-0.79} \cdot \mathrm{min}^{-1}\right)$} \\
\hline $2.5 \mathrm{~km} \cdot \mathrm{hr}^{-1}$ & $24.75 \pm 4.40$ & $25.08 \pm 4.40$ & $26.25 \pm 4.34$ & $26.00 \pm 4.99$ \\
\hline $3.5 \mathrm{~km} \cdot \mathrm{hr} \mathbf{r}^{-1}$ & $27.62 \pm 5.18^{*}$ & $27.56 \pm 5.14$ & $29.27 \pm 4.58^{*}$ & $31.26 \pm 6.06^{* \#}$ \\
\hline $4.5 \mathrm{~km} \cdot \mathrm{hr}^{-1}$ & $32.84 \pm 5.55^{*}$ & $32.06 \pm 6.10^{*}$ & $33.41 \pm 5.73 *$ & $37.39 \pm 6.71^{* \#}$ \\
\hline \multirow[t]{2}{*}{$5.5 \mathrm{~km} \cdot \mathrm{hr}^{-1}$} & $38.73 \pm 7.06^{*}$ & $37.79 \pm 6.51 *$ & $39.41 \pm 7.26^{*}$ & $47.11 \pm 8.41^{* \#}$ \\
\hline & \multicolumn{4}{|c|}{ Caloric energy expenditure (kcal/min) } \\
\hline $2.5 \mathrm{~km} \cdot \mathrm{hr} \mathbf{r}^{-1}$ & $2.00 \pm 0.50$ & $2.00 \pm 0.50$ & $2.10 \pm 0.50$ & $2.10 \pm 0.50$ \\
\hline $3.5 \mathrm{~km} \cdot \mathrm{hr}^{-1}$ & $2.20 \pm 0.55^{*}$ & $2.20 \pm 0.50$ & $2.35 \pm 0.55^{*}$ & $2.50 \pm 0.65^{* \#}$ \\
\hline $4.5 \mathrm{~km} \cdot \mathrm{hr}^{-1}$ & $2.60 \pm 0.55^{*}$ & $2.55 \pm 0.60 *$ & $2.65 \pm 0.55^{*}$ & $3.00 \pm 0.75^{* \#}$ \\
\hline $5.5 \mathrm{~km} \cdot \mathrm{hr} \mathbf{r}^{-1}$ & $3.10 \pm 0.65^{*}$ & $3.00 \pm 0.65^{*}$ & $3.15 \pm 0.75^{*}$ & $3.75 \pm 0.85^{* \#}$ \\
\hline
\end{tabular}

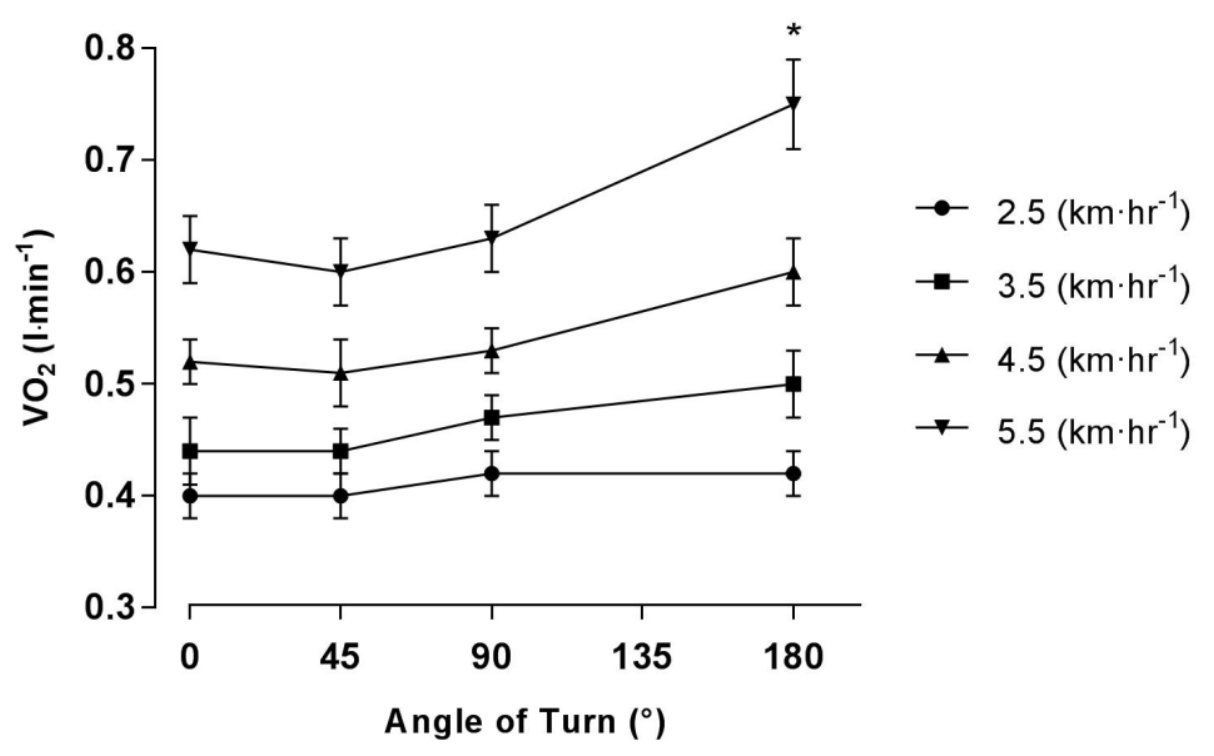

Figure 3.3 The interaction between speed and angle on absolute $\dot{\mathrm{V}} \mathrm{O}_{2}$

Displaying SEM. '*' indicates a significant difference in energy expenditure of turning relative to straight-line walking at $5.5 \mathrm{~km} \cdot \mathrm{hr}^{-1}(p<0.05)$. 
Significant effects were found for speed and angle on mean VeDBA during the straights (speed: $F=548.49, p<0.001$; angle: $F=3.66, p<0.05$ ) and turns (speed: $F$ $=724.88, p<0.001$; angle: $F=4.96, p<0.05)$. Speed and angle had no significant interaction effect on mean VeDBA during either straight $(F=0.82, p>0.05)$ or turns $(F=0.99, p>0.05)$. Specifically, both straight and turning mean VeDBA increased with speed, but the effect of angle was only manifest at $180^{\circ}$ at a speed of $5.5 \mathrm{~km} \cdot \mathrm{hr}^{-1}$ for straight and $180^{\circ}$ at a speed of $3.5 \mathrm{~km} \cdot \mathrm{hr}^{-1}$ for turns (Table 3.4). For straight walking VeDBA, there were no significant predictors (sex: $F=0.40, p>0.05 ; \dot{V} \mathrm{O}_{2 \text { peak }} F=2.62$, $p>0.05$; scaled $\left.\dot{V} \mathrm{O}_{2 \text { peak }} F=1.53, p<0.05\right)$, with turning VeDBA significantly predicted by sex $(F=14.20, p<0.001)$ with both $\dot{V} \mathrm{O}_{2 \text { peak }}(F=6.25, p<0.05)$ and scaled $\dot{V} \mathrm{O}_{2 \text { peak }}(F=2.67, p<0.05)$ not significantly predicting (see Appendix I).

Table 3.4 Mean VeDBA, straight and turn mean VeDBA during each combination of walking velocity and angle

\begin{tabular}{|c|c|c|c|c|}
\hline & \multicolumn{4}{|c|}{ Mean VeDBA $(g)$} \\
\hline & $\mathbf{0}^{\circ}$ & $45^{\circ}$ & $90^{\circ}$ & $180^{\circ}$ \\
\hline $2.5 \mathrm{~km} \cdot \mathrm{hr} \mathbf{r}^{-1}$ & $0.22 \pm 0.02$ & $0.21 \pm 0.02$ & $0.22 \pm 0.03$ & $0.22 \pm 0.03$ \\
\hline $3.5 \mathrm{~km} \cdot \mathrm{hr} \mathbf{r}^{-1}$ & $0.28 \pm 0.04 *$ & $0.29 \pm 0.05^{*}$ & $0.29 \pm 0.04 *$ & $0.31 \pm 0.04^{* \#}$ \\
\hline $4.5 \mathrm{~km} \cdot \mathrm{hr}^{-1}$ & $0.41 \pm 0.07 *$ & $0.40 \pm 0.07 *$ & $0.41 \pm 0.07 *$ & $0.44 \pm 0.06^{*}$ \\
\hline \multirow[t]{2}{*}{$5.5 \mathrm{~km} \cdot \mathrm{hr}^{-1}$} & $0.60 \pm 0.10^{*}$ & $0.59 \pm 0.11^{*}$ & $0.59 \pm 0.13^{*}$ & $0.63 \pm 0.11 *$ \\
\hline & \multicolumn{4}{|c|}{ Straight Mean VeDBA ( $g$ ) } \\
\hline $2.5 \mathrm{~km} \cdot \mathrm{hr}^{-1}$ & $0.21 \pm 0.02$ & $0.22 \pm 0.03$ & $0.22 \pm 0.03$ & $0.22 \pm 0.03$ \\
\hline $3.5 \mathrm{~km} \cdot \mathrm{hr}^{-1}$ & $0.29 \pm 0.05^{*}$ & $0.29 \pm 0.04 *$ & $0.31 \pm 0.04 *$ & $0.32 \pm 0.05^{* \#}$ \\
\hline $4.5 \mathrm{~km} \cdot \mathrm{hr} \mathbf{r}^{-1}$ & $0.40 \pm 0.07^{*}$ & $0.41 \pm 0.07 *$ & $0.44 \pm 0.06^{*}$ & $0.44 \pm 0.07 * \#$ \\
\hline \multirow[t]{2}{*}{$5.5 \mathrm{~km} \cdot \mathrm{hr}^{-1}$} & $0.59 \pm 0.11^{*}$ & $0.59 \pm 0.13^{*}$ & $0.63 \pm 0.11^{*}$ & $0.63 \pm 0.11^{\text {*\# }}$ \\
\hline & \multicolumn{4}{|c|}{ Turn Mean VeDBA $(g)$} \\
\hline $2.5 \mathrm{~km} \cdot \mathrm{hr}^{-1}$ & $0.22 \pm 0.06^{*}$ & $0.22 \pm 0.03$ & $0.22 \pm 0.03$ & $0.22 \pm 0.03$ \\
\hline $3.5 \mathrm{~km} \cdot \mathrm{hr}^{-1}$ & $0.30 \pm 0.07^{*}$ & $0.30 \pm 0.05^{*}$ & $0.29 \pm 0.05^{*}$ & $0.31 \pm 0.06^{* \#}$ \\
\hline $4.5 \mathrm{~km} \cdot \mathrm{hr}^{-1}$ & $0.39 \pm 0.06^{*}$ & $0.41 \pm 0.08^{*}$ & $0.40 \pm 0.08^{*}$ & $0.44 \pm 0.09 *$ \\
\hline $5.5 \mathrm{~km} \cdot \mathrm{hr}^{-1}$ & $0.56 \pm 0.11^{*}$ & $0.62 \pm 0.13^{*}$ & $0.59 \pm 0.13^{*}$ & $0.63 \pm 0.12 *$ \\
\hline
\end{tabular}

\subsubsection{Pearson Product-moment Correlation Coefficient}

There were significant correlations between $\dot{V} \mathrm{O}_{2}$ and straight $\left(\mathrm{r}^{2}=0.51 ; p<0.001\right)$, turning $\left(\mathrm{r}^{2}=0.54 ; p<0.001\right)$ and total mean $\operatorname{VeDBA}\left(\mathrm{r}^{2}=0.53 ; p<0.001\right)$, with a 
weaker but statistically significant relationship between $\dot{V} \mathrm{O}_{2 \text { peak }}\left(\mathrm{r}^{2}=0.30 ; p<0.001\right)$ and stature $\left(\mathrm{r}^{2}=0.32 ; p<0.001\right)$.

\subsection{Discussion}

This is the first study to consider the energy expenditure of turning in children, demonstrating that as speed increases for any given angle of turn, the associated energy expenditure also increases. However, the extent to which angle contributed to an increased energetic demand was dependent upon the degree of the angle and, indeed, its interaction with walking speed. Specifically, increasing angles of turn and increasing walking speeds are linked to an increasing energy expenditure, with $180^{\circ}$ turns requiring a significantly greater energy expenditure than $45^{\circ}$ or $90^{\circ}$ turns. The findings presented highlight the importance of accounting for the magnitude of turn angle and the frequency of turns completed when estimating the habitual physical activity and energy expenditure of children; a failure to do so is likely to lead to erroneous conclusions regarding daily energy expenditure estimated from accelerometry data.

Numerous studies have highlighted the importance of accounting for turning, as well as the physiological and the biomechanical effects of turning when compared to straight-line locomotion in adults (Patla et al., 1999, Huxham et al., 2006, Orendurff et al., 2006, Akram et al., 2010, Dellal et al., 2010, Buchheit et al., 2012, Hatamoto et al., 2013, Wilson et al., 2013, Hatamoto et al., 2014, Justine et al., 2014). A recent study found that when shuttle run distance was reduced from $7.0 \mathrm{~m}$ to $3.5 \mathrm{~m}$ and completed at the same average running speed, the $3.5 \mathrm{~m}$ shuttles induced a greater physiological response (Bekraoui et al., 2012). Hatamoto et al. (2014) found that this greater physiological demand occurred even during walking velocities as low as 3 $\mathrm{km} \cdot \mathrm{hr}^{-1}$. The present study extends these findings to children, demonstrating a synergistic interaction between increased walking speed and angle of turn. In detail, the $\mathrm{C}_{\mathrm{r}}$ in a straight line decreased with speed to a minimum energy expenditure attained at $5.5 \mathrm{~km} \cdot \mathrm{hr}^{-1}(1.5 \mathrm{~m} / \mathrm{s})$. These findings align with previous research, highlighting that human walking displays a U-shaped relationship between walking speed and energy expenditure of transport (Sparrow, 2000, Willis et al., 2005). In this case, the optimal walking speed in humans is frequently cited to be between 4.8 (1.3 
$\mathrm{m} / \mathrm{s})$ to $6 \mathrm{~km} \cdot \mathrm{hr}^{-1}(1.7 \mathrm{~m} / \mathrm{s})$, in which the energy expenditure per unit distance travelled is minimised (Bastien et al., 2005, Zarrugh et al., 1974). Furthermore, the present findings regarding the $\mathrm{C}_{\mathrm{r}}$ to straight line walking can be closely matched to Waters' and Mulroy's (Waters \& Mulroy, 1999) regression equation for children's $\dot{V} \mathrm{O}_{2}$ by dividing the walking speed by $\mathrm{m} / \mathrm{min}$ (following: $\dot{V} \mathrm{O}_{2} \operatorname{cost}=0.188+2.61 / S$ ).

More importantly, the current findings showed that $180^{\circ}$ turns had a significantly greater energy expenditure per unit body mass and distance from a speed greater than $3.5 \mathrm{~km} \cdot \mathrm{hr}^{-1}$, with a speed of $5.5 \mathrm{~km} \cdot \mathrm{hr}^{-1}$ exhibiting a $30 \%$ increase in energy expenditure compared to straight line walking at the same speed. The resultant increase in energy expenditure of a $180^{\circ}$ turn could be partly explained by the greater braking (deceleration) and propulsive (acceleration) forces encountered during turning (Schot et al., 1995). Specifically, Schot et al. (1995) found that a $90^{\circ}$ turn experiences a greater acceleration phase because an individual has to begin moving from a near stand-still position, whereas a $45^{\circ}$ turn encompasses some of the residual incoming motion prior to the turn. According to Havens and Sigward (2015), larger turn angles lead to greater alterations for both deceleration and translation subtasks. Therefore, it could be postulated that a greater angle, such as a $180^{\circ}$ turn, would experience larger deceleration and accelerations (Havens \& Sigward, 2015). As such, these findings may, in part, explain why paediatric populations with neuromuscular pathologies have trouble in turning, given their postural stability problems (Kenis-Coskun et al., 2016). In contrast, a recent study investigating the metabolic power of turning in youth soccer players concluded that turning $\left(45^{\circ}\right.$ and $\left.90^{\circ}\right)$ whilst running is less metabolically demanding than straight line running (Hader et al., 2016). It was concluded that this lower metabolic demand of turning, may have been directly related to the very low energy demands of the deceleration phase during the turn that may not be compensated by the increased requirement for the re-acceleration phase. However, the study was limited by using an indirect approach to estimate the energy demands of turning, which may ignore other non-locomotor muscles involved with turning (e.g., upper body and back muscles; Buchheit et al., 2010b). More research is warranted to investigate and strengthen our understanding of the energy expenditure associated with turning in child populations. 
It is important to acknowledge that children are not 'mini-adults' (Armstrong \& Welsman, 1997), mostly due to their anatomical and physiological differences when compared to adults (Andropoulos, 2012). Given that these biomechanical and physiological differences are likely to influence the energy expenditure of turning in children, the applicability of previous findings in adults to children must be questioned, even though the physics of force generation needed for turns makes increased energy expenditure in a turn inevitable. More specifically however, covariates such as age, stature, training status and turning technique (Buchheit et al., 2011, Zadro et al., 2011) may have a significant role in determining the energy expenditure associated with any given task and may lead to discrepancies when comparing adults to children. McNarry et al. (2017) reported that $90^{\circ}$ and $180^{\circ}$ turns were associated with a significantly greater energy cost at walking speeds of 4.5 and $5.5 \mathrm{~km} \cdot \mathrm{hr}^{-1}$. In comparison, the present study only found that walking speeds of 5.5 $\mathrm{km} \cdot \mathrm{hr}^{-1}$ at $180^{\circ}$ turn were associated with a significantly greater energy expenditure when compared to all other combinations. In more detail, the mean $\dot{V} \mathrm{O}_{2}$ for an adult turning $180^{\circ}$ at a speed of $5.5 \mathrm{~km} \cdot \mathrm{hr}^{-1}$ was $1.54 \pm 0.361 \cdot \mathrm{min}^{-1}$ (McNarry et al., 2017), which is substantially more than the value observed in the present study of $0.75 \pm 0.17$ $1 \cdot \mathrm{min}^{-1}$. This discrepancy may be explained by the much larger stature of adults when compared to young children. Adults' larger skeletal structure and muscle size is likely to change the moment arm length, both of which will result in increased energy costs (O’Brien et al., 2009). As Buchheit et al. (2011) found that shorter team sport players demonstrated less effect of a $180^{\circ}$ change of direction than their taller counterparts. Lower angles of turn are associated with greater balance and stability as a proper support base is established, therefore requiring less energy to turn (Justine et al., 2014). This is demonstrated in the present study, whereby children's stature was a significant predictor for estimating the energy expenditure of turning, as supported by a similar relationship within adults (McNarry et al., 2017). It could therefore be postulated that children's shorter moment arms and lower centre of gravity would lead to a reduced energy expenditure of turning when compared to taller adults. Furthermore, this could explain the significant differences found between boys and girls in the present study, with boys being significantly taller and demonstrating greater $\dot{V} \mathrm{O}_{2}$ demands across all combinations of turn. That said, the present study did not examine Ponderal Index, which can be used to identify children whose soft tissue mass may be below normal for the stage of skeletal development (Fayyaz, 2005), which is associated with 
decreased muscle strength and fatigue resistance (Brutsaert et al., 2011). Therefore, it is important to consider that shorter children within the current study may have been below the normal stage of skeletal development and consequently, influenced energy expenditure of turning, warranting further investigation.

The ability to maintain balance is essential for carrying out activities such as walking and a crucial component whilst turning. Dynamic postural control (i.e., balance) is defined as the ability to keep the centre of gravity within the base of support whilst performing a task in a stable condition (Winter et al., 1990). Previous studies observe deficits in postural control in children when compared to young healthy adults (Bosco \& Komi, 1980, Hytönen et al., 1993, Schärli et al., 2013), potentially due to a smaller base of support, which would be anticipated to be associated with a greater energy expenditure during turning. Furthermore, Geldhof et al. (2006) found better postural control in girls compared to boys between the ages of 9-10 years, which could explain the sex differences observed in the present study. As described by Hase and Stein (Hase \& Stein, 1999b), there are two types of turn embedded into locomotion, one being the step turn and the other the spin turn. Step turns are biomechanically more efficient (Patla et al., 1991, Taylor et al., 2005) and offer greater stability (Taylor et al., 2005) than spin turns. When observing turning strategies in adult populations, step turns are most commonly reported in both laboratory (Patla et al., 1991) and nonlaboratory environments (i.e., home or community; Glaister et al., 2007). Although findings from Dixon et al. (2013) is limited to laboratory settings, evidence suggests that children tend to adopt spin turns, with this adoption likely to be dependent on increasing gait velocity. Research suggests that spin turns limit the size of the moving base of support (Akram et al., 2010), which consequently leads to reduced stability, increasing the physiological strain on both lower limb (Hader et al., 2016) and upper body muscles (Buchheit et al., 2010a, Buchheit et al., 2010b). Therefore, it could be postulated that the increasing demands of a turn, such as a $180^{\circ}$ at a speed of $5.5 \mathrm{~km} \cdot \mathrm{hr}^{-}$ ${ }^{1}$, may expose children's gait immaturity and concurrently lead to the adoption of the more complex turning sub-strategies identified within adult populations (Dixon et al., 2013). Although turning strategies and dynamic postural control were not accounted for in the present study, it is important to consider that turning strategies may have varied between children, affecting balance and consequently the variance of the values 
observed for energy expenditure. Therefore, further work on the energy expenditure of spin and step turn strategies is warranted in non-laboratory-based environments.

Some of the present study's findings have implications for assessing children's movement patterns in both habitual and health contexts. As highlighted by McNarry et al. (2017), it is important to account for the number of turns during a clinical sixminute walking test (6MWT), designed to measure both adults' (Veloso-Guedes et al., 2011) and children's (Geiger et al., 2007) functional exercise capacity. McNarry et al. (2017) highlight that the 6MWT varies due to limited space and resources, which consequently results in distances ranging from $20 \mathrm{~m}$ to $50 \mathrm{~m}$ being used (Lipkin et al., 1986, Troosters et al., 1999), subsequently altering the frequency of turns completed from as much as 12 to 32 turns (Chetta et al., 2006). These methodological differences are likely to affect the reliability of aerobic capacity assessment using this method, especially for paediatric populations which have a restricted gait ability, such as those with cerebral palsy who show greater physiological cost of walking compared to healthy children (Liao et al., 1997). Nevertheless, a more recent study suggested that slow jogging with turns could be an effective exercise prescription to promote physical activity and fitness in inactive-healthy individuals and those who are overweight or obese. Specifically, Araki et al (2017b) demonstrated that walking at $4.2 \mathrm{~km} \cdot \mathrm{hr}^{-1}$, that is equal to 3 METs (light-intensity), to jogging at the same speed with turns, increased the intensity to 8 METs (vigorous-intensity), which resulted in a 2.7-fold increase in energy expenditure. Moreover, Araki and colleagues (2017a) showed that slow walking $\left(2.7 \mathrm{~km} \cdot \mathrm{hr}^{-1}\right)$ became moderately intense (4 METs) when turns were incorporated. Therefore, including turns may be an effective method by which to increase the amount of physical activity that inactive individuals perform, to lose weight and increase fitness. However, there is a paucity of evidence to support the use of turning as a health promotion intervention, especially in children, therefore warranting further investigation.

To fully understand the influence of turning on youth's daily energy expenditure and PALs, data from devices, such as accelerometers (i.e., counts per a given epoch or time) must be translated into a behaviorally significant variable (i.e., time spent in sedentary, light-, moderate-, and vigorous-intensity; Freedson et al., 2005b). This process is known as calibration and has been most widely utilised to establish specific 
cut-points to link between accelerometer counts and physical activity intensity (Kim et al., 2012). The majority of the protocols performed during these calibration studies have been relatively simple, with long periods of linear treadmill-based locomotion that are not true of the nature of habitual physical activity patterns. Moreover, these treadmill-based prediction equations and accelerometer-specific thresholds may be a contributing factor to the poor accuracy of energy expenditure calculations (Fortune et al., 2014, Eisenmann et al., 2004) and minutes spent in physical activity intensities (Howe et al., 2018), respectively. Unsurprisingly, protocols based on ambulatory activities, such as walking and running, have demonstrated better validity (Chu et al., 2007, Rowlands et al., 2004a, Sirard et al., 2005, van Cauwenberghe et al., 2011) than threshold derived protocols that include a combination of ambulatory and nonambulatory activities (Evenson et al., 2008a, Puyau et al., 2004, Tanaka et al., 2007). Specifically, activities, such as those that require turning, tend to exhibit lower accelerometer counts than ambulatory activities with a lower energy expenditure (Romanzini et al., 2012). Indeed, accelerometers tend to be more sensitive to activities that produce a larger vertical acceleration component, such as walking and running (Romanzini et al., 2012). This factor may explain, in part, the superior validity of accelerometer-specific thresholds derived from treadmill-based activities. In this respect, the findings in the present study question the calibration and validated accuracy of pedometers, gyroscopes and accelerometers (Crouter et al., 2003, Le Masurier \& Tudor-Locke, 2003, Mansfield \& Lyons, 2003, Salarian et al., 2004, Esliger et al., 2007, Rueterbories et al., 2010), given that children are not restricted to linear movements. Therefore, it is advisable from the present study that calibration and follow-up validation studies should include the movement of turning to develop better accelerometer-specific thresholds and predictive models of energy expenditure to correctly account for youth's habitual PAL (Welk, 2005).

One possible solution to the aforementioned inadequacies in correctly accounting for physical activity, is the use of the magnetometer utilised in the present study and how it can provide additional information on body rotation (Williams et al., 2017b), so when used in conjunction with accelerometers, it will provide more context. Indeed, using the current methods to establish VeDBA from analysing the accelerometer and magnetometer traces proved to be relatively accurate in predicting energy expenditure expressed as $\dot{V} \mathrm{O}_{2}$, aligning with previous research (McNarry et al., 2017, Qasem et al., 
2012, Weippert et al., 2013). However, from the present findings, it is apparent that there is a dissociation between VeDBA and turn angle, whereby increasing the angle of turn was not associated with significant increase in VeDBA. Similar findings were reported in adults by McNarry et al. (2017) arguing that this dissociation of VeDBA when turning could be a result of the complex and individual specific interaction between the surge, heave and sway components of DBA as well as the muscular effort involved in generating forces without the dynamism of straight locomotion. Although little is known about the benefits of including such magnetometry-derived data on the accuracy of energy expenditure prediction, the present study highlights this collective measurement as an area that warrants further investigation, especially in children who are characterised by spontaneous and transitory movement patterns (Stone et al., 2009), such as football (Fjørtoft et al., 2009) and chasing games (Sleap \& Warburton, 1996) that likely involve a considerable number of turns.

It is important to note certain limitations of the current study. As this was the first study to investigate the energy expenditure of turning in children, findings should be taken with caution, due to the limited sample size and lack of evidence on the influence of children's growth and maturation on the energy demands of turning, warranting further investigation. Additionally, the highly-controlled nature of this study may limit the generalisability of the findings and its ecological validity. Future research should seek to increase the sample size, use different age groups (i.e., adolescents) and assess participants turn strategies (i.e., pivot or step turns). Finally, to improve the accuracy of aerobic fitness data, future studies should scale $\dot{V} \mathrm{O}_{2}$ by lean mass of both legs or by DXA-estimated total lean body mass (Graves et al., 2013).

\subsection{Conclusions}

In the present study, we found that the energy expenditure of turning whilst walking was significantly greater at speeds of $3.5,4.5$ and $5.5 \mathrm{~km} \cdot \mathrm{hr}^{-1}$ at a turn angle of $180^{\circ}$ in children. The study demonstrated a synergistic interaction between turn angle and walking speeds on the energy expenditure of turning, with stature and sex adding additional determinants of the demand. More research is warranted on running speeds and the effect of turning technique on the energy expenditure of turning. These findings highlight the importance of accounting for the costs of turning in children, with implications for both sporting, habitual physical activity and health related contexts where turning is a fundamental part of movement. 


\section{Thesis Map}

\section{STUDY}

\section{OUTCOMES}

1. Energy Expenditure Associated with Walking Speed and Angle of Turn in Children

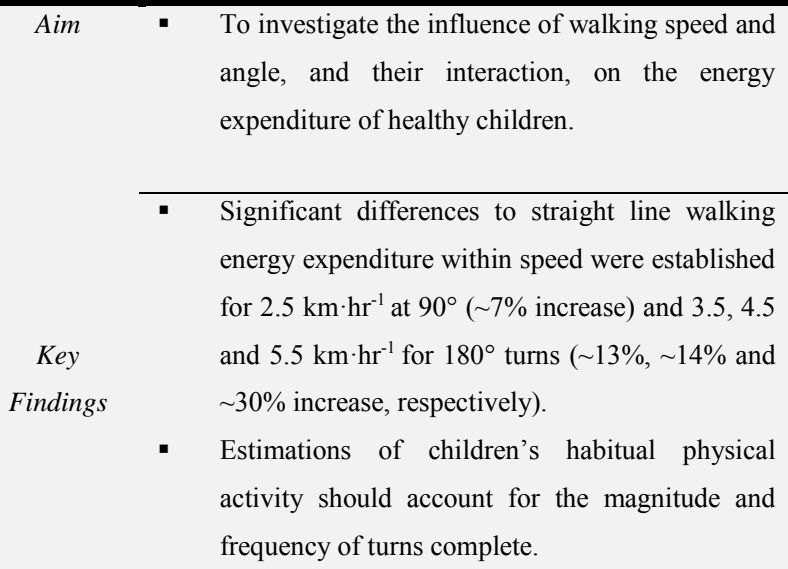

Aim

- To investigate the influence of walking speed and angle, and their interaction, on the energy expenditure of healthy children.

- Significant differences to straight line walking energy expenditure within speed were established for $2.5 \mathrm{~km} \cdot \mathrm{hr}^{-1}$ at $90^{\circ}(\sim 7 \%$ increase $)$ and $3.5,4.5$ Key and $5.5 \mathrm{~km} \cdot \mathrm{hr}^{-1}$ for $180^{\circ}$ turns $(\sim 13 \%, \sim 14 \%$ and Findings $\quad \sim 30 \%$ increase, respectively).

- Estimations of children's habitual physical activity should account for the magnitude and frequency of turns complete.

2. Perceptions of Visualising Physical Activity as a 3D-printed Object: A Formative Study Interpret 3D-printed Physical Activity Data and Identify Associated Intensity Levels

Role of DESIGN PARTNERS

\section{Aim}

- To elicit children's, adolescents', parents' and teachers' perceptions and understanding of $3 \mathrm{D}$ physical activity objects to inform the design of future 3D models of physical activity.

$$
\text { Key }
$$

\section{Findings}

\section{Aim}

Key

Findings

4. The Tangibility of Personalised 3DAim printed Feedback may Enhance Youths' Physical Activity Awareness 


\section{CHAPTER 4}

Perceptions of Visualising Physical Activity as a 3D-Printed Object 


\section{Perceptions of Visualising Physical Activity as a 3D-printed Object: A Formative Study}

\subsection{Introduction}

The UK Government recommends that children aged 5 to 18 years engage in at least 60 minutes moderate to vigorous physical activity (MVPA) every day (Department of Health, 2011, 2011b). Despite the well-established physiological and psychosocial health benefits of regular physical activity for youths (Sothern et al., 1999, Nieman, 2002, Janssen \& Leblanc, 2010a, Eime et al., 2013), many fail to meet these recommended guidelines (Hills et al., 2011). More specifically, for these populations, sedentary screen-based pursuits are thought to have displaced active behaviours and have been independently associated with adverse health outcomes, such as obesity (Proctor et al., 2003) and hypertension (Pardee et al., 2007). According to Noonan et al. (2016), there is a lack of understanding within youths on the various forms of physical activity, including those of active travel and unstructured play, with a need to educate how these types of activities contribute to achieving the physical activity recommendations. Conversely, Kremers et al. (2008) argue that a lack of awareness of physical activity among youths is likely to make them less susceptible to educational programmes that are aimed to influence attitudes, norms, self-efficacy or other cognitive means, as they will not perceive the need to change. Indeed, research supports this notion, demonstrating that youths who are aware of their physical activity levels (PAL) and the recommended guideline are, on average, 20 minutes more active than their unaware counterparts (Kremers et al., 2008), and as a result, more likely to achieve the daily 60 minutes of MVPA (Roth \& Stamatakis, 2010, Nemet et al., 2012). Therefore, developing youths' understanding and awareness of their physical activity behaviours is crucial for implementing a successful health programme designed to increase PAL (Kremers et al., 2008). 
Schools have been identified as ideal settings to integrate health-promoting interventions due to their established infrastructure and role in health education (Dobbins et al., 2013). Subsequently, researchers have developed numerous schoolbased interventions that seek to utilise technology as part of the solution, rather than part of the problem (McDougall \& Duncan, 2008, Duncan \& Staples, 2010, Poole et al., 2011, Lubans et al., 2016, Mackintosh et al., 2016). Although technology-based interventions have shown promise in improving psychosocial outcomes, efforts to elicit sustainable behaviour change have been less consistent (Lau et al., 2011). This may, at least in part, be a result of the traditional power structure of the "all-knowing" adult and the "all-learning" child, where adults' development of new technology limits the personal opinions of youths when it comes to deciding what technology should be used within a school-based environment (Druin, 2002). It is, therefore, important to acknowledge youth as the experts (Greene \& Hogan, 2005), allowing the creation of technology through the eyes of the child rather than the researcher, teacher or parent (Noonan et al., 2016). As argued by Druin (2002), children as design partners can play an impactful role in the creation of new technologies, that are not only going to be effective and meaningful, but that will excite children and aid learning.

Research shows that $80 \%$ of youths are visual and tactile learners (Rita \& Dunn, 1979); relying simply on numbers and figures as a source of knowledge is limited (Petrakaki, 2016), and richer ways of data representation are required (Hassenzahl et al., 2016). Indeed, visualisations can play a key role in motivating individuals to enhance their PALs, enabling reflection on personal performance and current level of physical activity (Li et al., 2011a). A recent school-based intervention using glanceable LED light technology to display groups' PALs reported that children wanted more personalised forms of visual feedback (Mackintosh et al., 2016), with others suggesting that material rewards are cherished more than virtual rewards (Munson \& Consolvo, 2012) due to their higher visibility and uniqueness (Kirk \& Sellen, 2010, Golsteijn et al., 2012). Indeed, previous research utilising paper and LED lights to create PA awareness promoting artefacts found that youth took incremental steps towards self-regulation through goal-setting and reflection (Ananthanarayan et al., 2016). It was concluded that although the artefacts did not elicit improved physical activity in youth, using tangible artefacts in conjunction with wearables could benefit youths' health (Ananthanarayan et al., 2016). However, it could be argued that paper 
artefacts do not provide youth with an adequate haptic and proprioceptive experience of personalised feedback to reap health benefits (Gillet et al., 2005). With the recent rise of the 'maker movement' and cost-effective 3D printers (Mueller et al., 2014), numerous opportunities in health-related research have emerged, utilising $3 \mathrm{D}$ printers to create tangible visualisations of physical activity (Khot et al., 2013, Rohit Ashok et al., 2015b, Stusak et al., 2014). As Jansen et al. (2013) advocate, there are many benefits of tangible visualisations over on-screen visualisations of data which include: (i) allowing for a more active perception; (ii) leveraging non-visual senses such as touch; (iii) integration with the physical world; and (iv) harnessing the interplay between vision and touch to facilitate cognition. For example, Khot et al. (2014) transformed adults' heart rate data into 3D-printed artefacts, with participants reporting that the artefacts acted as a reward and allowed for reflection and reminiscence on past physical activities. Indeed, tangible interfaces have been reported to involve children in playful learning (Price et al., 2003), engagement and reflection (Rogers et al., 2002a). Consistent with goal setting theory (Locke et al., 1981), incentives are important in maintaining interest in an activity, with incentive-based interventions to 'nudge' healthy behaviour change in youths demonstrating potential (Christian et al., 2016, Finkelstein et al., 2013). However, whether personalised 3Dprinted objects can be used to enhance youths' understanding, awareness and motivation relating to engagement in physical activity remains to be elucidated.

Therefore, the aims of the present study were (i) to formatively elicit children's, adolescents', teachers' and parents' perceptions of physical activity data when represented as 3D-printed objects; (ii) to ascertain how youths visualise their personal 3D objects of physical activity using Play-Doh; (iii) to obtain parents' and teachers' views on the perceived benefits and barriers of 3D-printed objects of physical activity for youths; and (iv) use these data to subsequently inform the design of 3D models to be used within a school-based physical activity intervention, whereby youth receive personal 3D-printed models displaying their PAL. 


\subsection{Methods}

\subsubsection{Recruitment}

In total, twelve primary and eight secondary schools from the Swansea region of South Wales were contacted and invited to take part via emails to the Head of School (primary schools) or Head of Physical Education (secondary schools). The schools were stratified by high and low socio economic status (SES) according to the percentage of students per school eligible to receive free school meals with the national average at 19\% (Hobbs \& Vignoles, 2010). From those schools that expressed an interest ( $35 \%, n=7 / 20$ response rate), four schools, one high and one low SES primary $(n=2)$ and secondary $(n=2)$ school, were selected based on order of availability to take part in the study. Typically developing children (aged between 7-9 years old) or adolescents (aged between 13-15 years old), which could include overweight or obese, or disadvantaged pupils, were allowed to participate in the study. According to the Estyn reports (Her Majesty's Inspectorate for Education and Training in Wales, 2017), out of the 205 and 352 pupils that attended the high and low SES primary schools, respectively, 4\% (high SES school) and 35\% (low SES school) of pupils are eligible for free school meals. Both primary and secondary schools were regular community, public schools. The high and low SES secondary schools had a total of 1,105 and 1,026 pupils, respectively, with $7 \%$ and $31 \%$ being eligible for free school meals, respectively.

\subsubsection{Participants}

In total, twenty-seven primary school children ( $8.4 \pm 0.3$ years; 15 boys) and forty-two secondary school adolescents $(14.4 \pm 0.3 ; 22$ boys $), 8$ teachers ( 2 male) and 7 parents ( 2 male) provided written informed parental or carer consent and child assent (see Appendix II), as appropriate, to participate in the study. All participants from the high SES primary and secondary school, including all parents and teachers, were White British. Within the low SES primary and secondary schools, $8 \%(n=1)$ were Black British and $6 \%(\mathrm{n}=1)$ were Asian British, respectively. All procedures were approved by the Swansea University Ethics Committee and were conducted in accordance with the Declaration of Helsinki (ref: PG/2014/40). 


\subsubsection{Procedures}

All semi-structured focus group discussions and interviews were conducted by the first author (SGMC) in a non-directive and unbiased way (Gibson, 2007), with 6 groups of children, 8 groups of adolescents and a total of 13 individual interviews with teachers and parents. Sample questions for the focus groups and one-to-one interviews are presented in Table 4.1. On two separate occasions, two parents and two teachers were interviewed together due to restricted availability (Liamputtong, 2006). Focus group discussions with youths involved 4-6 participants to allow for lively, yet manageable, interactions (Gibson, 2007, Mackintosh et al., 2011, Morgan et al., 2002), with the exception of one primary school focus group where a child with special educational needs (SEN) required a smaller group of 3 children with one support teacher. Both single and mixed sex focus groups were conducted (Hill et al., 1996). All focus group sessions were completed within the school environment, either within a familiar classroom, or in the school library, to provide comfort and reduce anxiety (Kennedy et al., 2001). Participants were seated in a circular arrangement around a table to create a relaxed and informal atmosphere (Gibson, 2007), maximizing social interaction and observer involvement (Dilorio et al., 1994). Moreover, this seating arrangement allows the facilitator to sit amongst the participants to establish a non-authoritarian approach to questioning. To ensure each of the group members was comfortable with talking aloud, and to create an environment in which sharing and listening were valued, an ice breaker question was used (Gibson, 2012). All pre-determined questions were reviewed and discussed by SGMC, MAM, PE and KAM and additional feedback was provided independently by two Health and Care Professions Council Registered Practitioner Psychologists (JH and ZRK). 
Table 4.1 Example focus group and interview questions

\begin{tabular}{|c|c|c|}
\hline Interview & Topic & Examples \\
\hline Children/Adolescents & Motivation & $\begin{array}{l}\text { What would you think if I said we could } 3 \mathrm{D} \text { print your } \\
\text { own personal model which shows how physically active } \\
\text { you are? }\end{array}$ \\
\hline Children/Adolescents & Model Design & $\begin{array}{l}\text { What sort of model would you like to develop or represent } \\
\text { your own physical activity as in the video, how would it } \\
\text { look? }\end{array}$ \\
\hline Adults & Motivation & $\begin{array}{l}\text { How do you think the } 3 \mathrm{D} \text { models of physical activity } \\
\text { could motivate children to be more physically active? }\end{array}$ \\
\hline Adults & Model Design & $\begin{array}{l}\text { Are there any models that you think would be good to help } \\
\text { children to visualize physical activity? }\end{array}$ \\
\hline
\end{tabular}

Alongside focus group discussions and one-to-one interviews, children, adolescents and adults were all shown a custom-made video on the concept of 3D printing physical activity. Following this, participants were shown three different prototype 3D-printed models displaying example accelerometry-derived physical activity data (see Appendix V), and discussions focused on how participants thought the physical activity data were represented by these models. Finally, children and adolescents were asked to independently design their own personalised model of physical activity using Play-Doh. The Play-Doh modelling process builds on the principles of the write, draw, show and tell method (Noonan et al., 2016) by replacing the write and draw components of the framework with the modelling of Play-Doh. Following the PlayDoh modelling task, the facilitator asked each child to articulate and explain the characteristics of their design in a verbal statement at their own pace. All Play-Doh models were photographed for further analyses.

Focus group discussions lasted between 60-90 minutes and 50-60 minutes for primary and secondary school groups, respectively, and adult interviews lasted approximately 25-45 minutes. All the focus groups and one-to-one interviews were digitally voice (Olympus DM-520 digital voice recorder, Shinjuku, Japan) and video (Sony Handycam HDR-PJ540, Minato, Japan) recorded. 


\subsubsection{Data analysis}

All focus group discussions and one-to-one interviews were transcribed verbatim resulting in 774 pages $(327,297$ and 150 pages for children, adolescents and adults, respectively) of raw data. Researchers SGMC, MAM and KAM read each transcript in order to familiarise and immerse themselves with the data. Out of the 774 pages of transcript, 445 pages (187, 148 and 110 pages for children, adolescents and adults, respectively) were deemed relevant to the topic of 3D-printing physical activity and consequently, further explored. Following the initial stage of data immersion, transcripts were thematically analysed by SGMC using data coding and identification of themes by a manual cut and paste technique (Clarke \& Braun, 2013). Emergent themes were explored using an inductive process to aid the exploratory nature of the research (Trochim \& Donnelly, 2001). Quantitatively, through the process of content analysis, the number of participants that raised specific statements and could correctly interpret the different components of the 3D model prototypes (i.e., data representation, length and size; Hamad et al., 2016) were noted. The process of frequency counting enables the analytic generalisation of findings (Sandelowski, 2001), which is crucial for the development of new technology as patterns and deviations within the data can be easily recognised (Kim \& Kuljis, 2010). Participants' verbatim quotations were chosen by SGMC and discussed in collaboration with MAM and KAM. The themes, frequency counts and meaningful quotations were then displayed diagrammatically using a pen profile approach. Pen profiling has been used within studies exploring perceptions and experiences of physical activity in youths (Knowles et al., 2013, Mackintosh et al., 2011) and is considered to be an accessible technique for researchers who have both quantitative and qualitative backgrounds (Krane et al., 1997). Through the process of reverse triangulation authors critically questioned and cross-examined the data in reverse from the pen profiles to the transcripts. This process was repeated, allowing authors to offer alternative interpretations of the data, until a consensus was reached to finalise the pen profile designs. In some cases, visual illustrations were presented to add more context to the data collected. Triangulation of the data tests the robustness of the findings and ensures methodological rigour using a 'trustworthiness criterion' (Ridgers et al., 2012). The criterion places trust in the researcher responsible for data collection to determine key 
findings that are worthy of attention. These were then assessed by PE, JH and ZRK who were not as directly involved in the analysis process (Hardy et al., 1996).

In addition to transcript analysis, the primary and secondary school participants' PlayDoh model photos aligned with the relevant verbal statements were analysed by SGMC, MM, JH, PE and KAM as a group to identify common trends and designs. Specifically, all Play-Doh model photos, with their respective verbal statements, were displayed on a large white board and appraised by the research team. Throughout this process, the Play-Doh models were grouped based on similar structural (e.g., sun or bar chart design) and verbal (e.g., the more physical activity you do, the larger the model) characteristics. The most common Play-Doh model designs created by children (abstract, $n=12$; graphical, $n=15$ ) and adolescents (graphical, $n=28$ ) were subsequently considered for further interpretation and 3D model design.

\subsection{Results}

In total, three separate pen profiles were constructed to represent children's (Figure 4.2), adolescents' (Figure 4.4) and adults' perceptions of 3D models. There were consistent themes identified between parents and teachers and therefore their data were combined for final analysis.

\subsubsection{Children's Perceptions and Designs of 3D Physical Activity Models}

As shown in Figure 4.2, key emergent themes were structured around 'Temporal Representation of Physical Activity', 'Motivation', 'Interpretation' and 'Physical Activity Guidelines'. The higher order theme 'Interpretation' was linked to further sub-themes 'Physical Activity Representation' and 'Design'. Primary school children demonstrated the ability to interpret and apply the different component lengths and sizes of the prototype 3D models in relation to physical activity parameters. Specifically, $92 \%$ of children were able to accurately understand how the changing length of the model represented increasing levels of physical activity. However, only $26 \%$ of children were able to understand the alternative method of increasing the size of the model to represent greater levels of physical activity. The physical activity data displayed on the models was correctly identified by $59 \%$ of the children as 
representing either hours or days of physical activity. The majority of children (81\%) preferred the 3D models to represent a week of their physical activity data, compared to a day $(n=3)$, year $(n=2)$ or month $(n=1)$.

"Because you do...you probably do more exercise in a week than a day" (G16)

From the Play-Doh modelling task, two sub-themes emerged, one being 'Abstract' and the other 'Graphical'. Children revealed no preference for abstract $(n=12)$ or graphical $(n=15)$ model representations of physical activity. Children's abstract models were characterised by the model changing shape or size, such as a volcano with more lava erupting for higher levels of physical activity (Figure 4.1A). Graphical $(n=15)$ representations, such as the flower (Figure 4.1B), distinguished between different hours, days or weeks of physical activity completed (i.e., the flower's petals resembling the different days of physical activity). Please see Appendix V: 3D Model Designs, for more Play-Doh models created by participants.

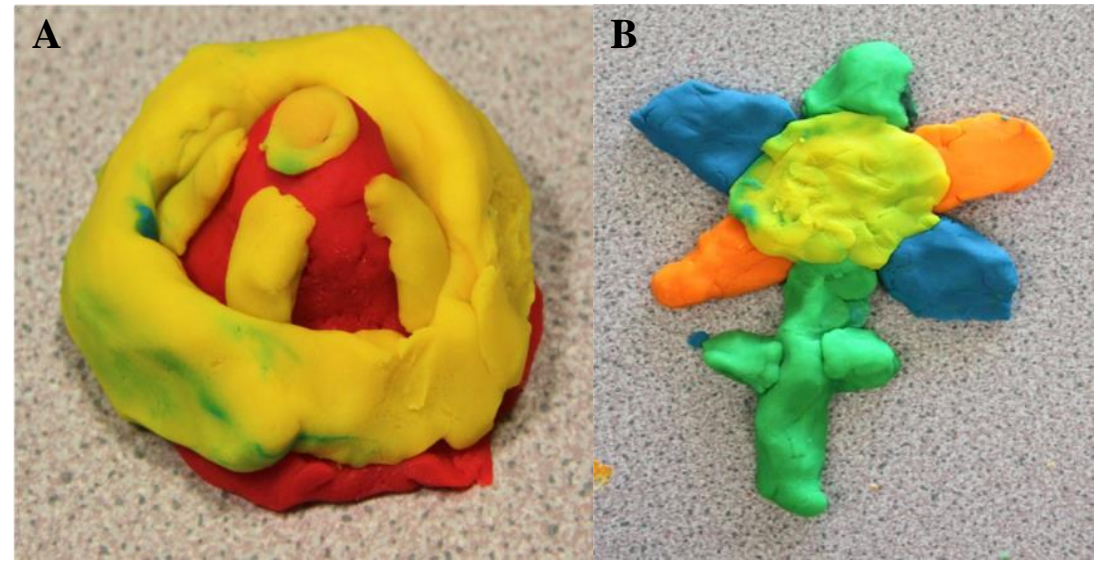

Figure 4.1 Children's Play-Doh model designs

A) 'Abstract' volcano; B) 'Graphical' flower

Twenty-one children (78\%) commented that the 3D models had potential to motivate themselves to engage in more physical activity, substantially outweighing the negatives expressed by one child. Specifically, children revealed that the 3D models would add competition between classmates and motivate them to do more. For example: 
"Because you might see how people have done much more activity than you and then you...you would think I want to be like that person and then you'd do more" (G16)

Sixteen children (59\%) displayed limited knowledge of the current UK government physical activity guidelines or how to achieve them, with only three children able to express the amount through the context of time spent being physically active, with no reference to intensity level. For example:

"...probably something like an hour, two hours a day [of physical activity]" (G9) 


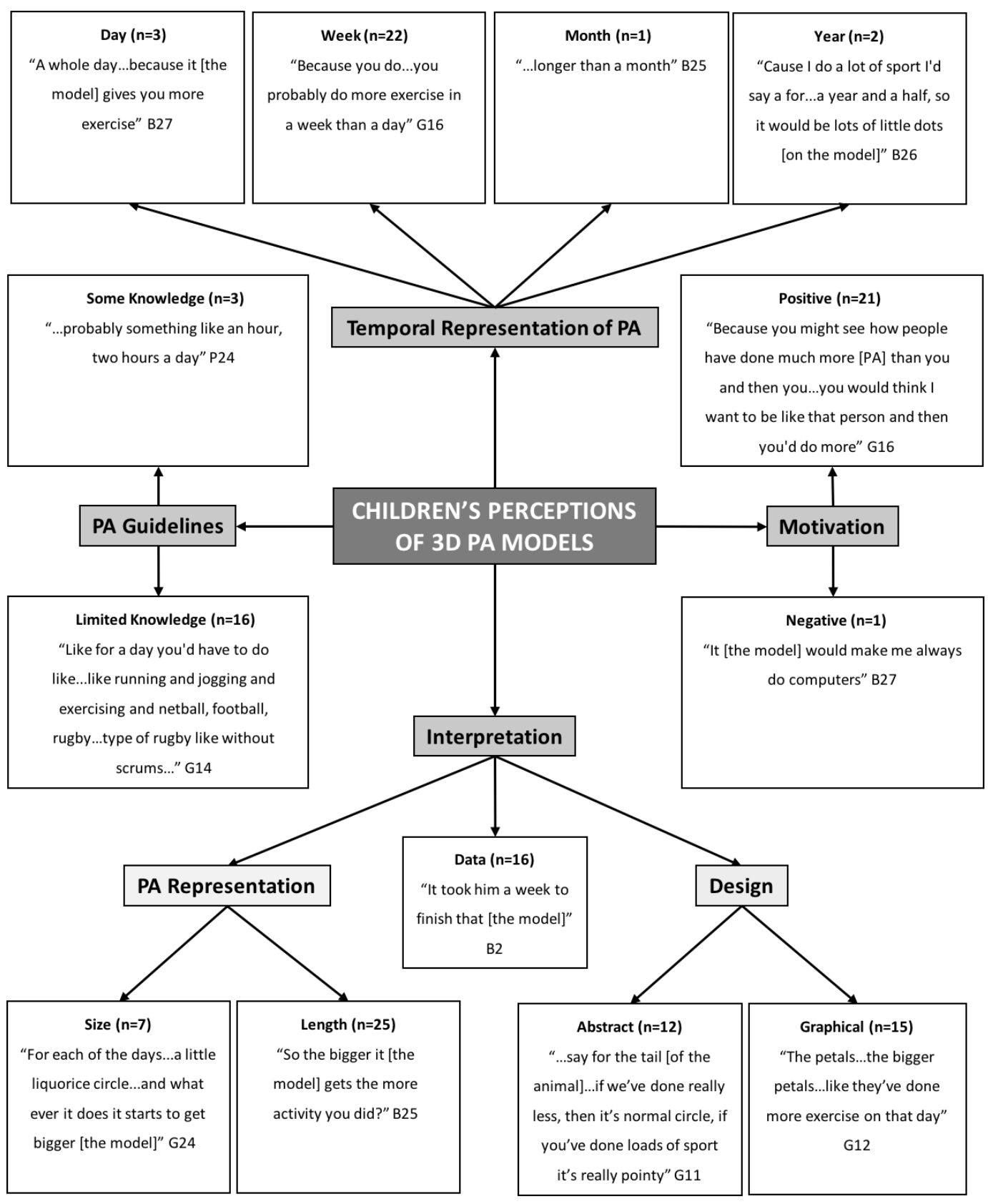

Figure 4.2 Children's pen profile

$\mathrm{B}=$ Boy, $\mathrm{G}=$ Girl, $\mathrm{PA}=$ Physical activity, $\mathrm{N}=$ frequency counts 


\subsubsection{Adolescents' Perceptions and Designs of 3D Physical Activity Models}

Four higher order themes were identified structured around 'Temporal Representation', 'Motivation', 'Interpretation' and 'Physical Activity Guidelines' (Figure 4.4). The higher order theme 'Interpretation' was further linked to sub-themes 'Physical Activity Representation' and 'Design'. Adolescents demonstrated the ability to identify and compare the different components of the prototype 3D models and their changing length and size in relation to physical activity. Specifically, the increasing size $(n=16)$ and length $(n=28)$ of the models was correctly interpreted as representing higher PALs. The majority ( $81 \%$ ) of adolescents showed a clear understanding of the represented data $(n=34)$ on the models. For example:

"The lines [on the models] are the days of the week" (B30)

“...so does that mean he's most active Tuesday, Wednesday, Thursday sort of thing" (G3)

Adolescents highlighted a preference for a week $(n=25)$ of physical activity data to be displayed on the 3D models because of the greater variety and reflection of their PALs in a week when compared to a model based on a day $(n=5)$, month $(n=4)$ or year $(n=1)$. The Play-Doh modelling task displayed similar sub-themes to those found in children, with a larger proportion of designs displaying graphical $(n=28)$ compared to abstract $(\mathrm{n}=11)$ designs. Abstract models, such as the butterfly (Figure 4.3A), were characterised by the changing size or detail of the models. Graphical representations resembled typical bar charts or line graphs (Figure 4.3B) to display different days, weeks or months of physical activity. Please see Appendix V: 3D Model Designs, for more Play-Doh models created by participants. 


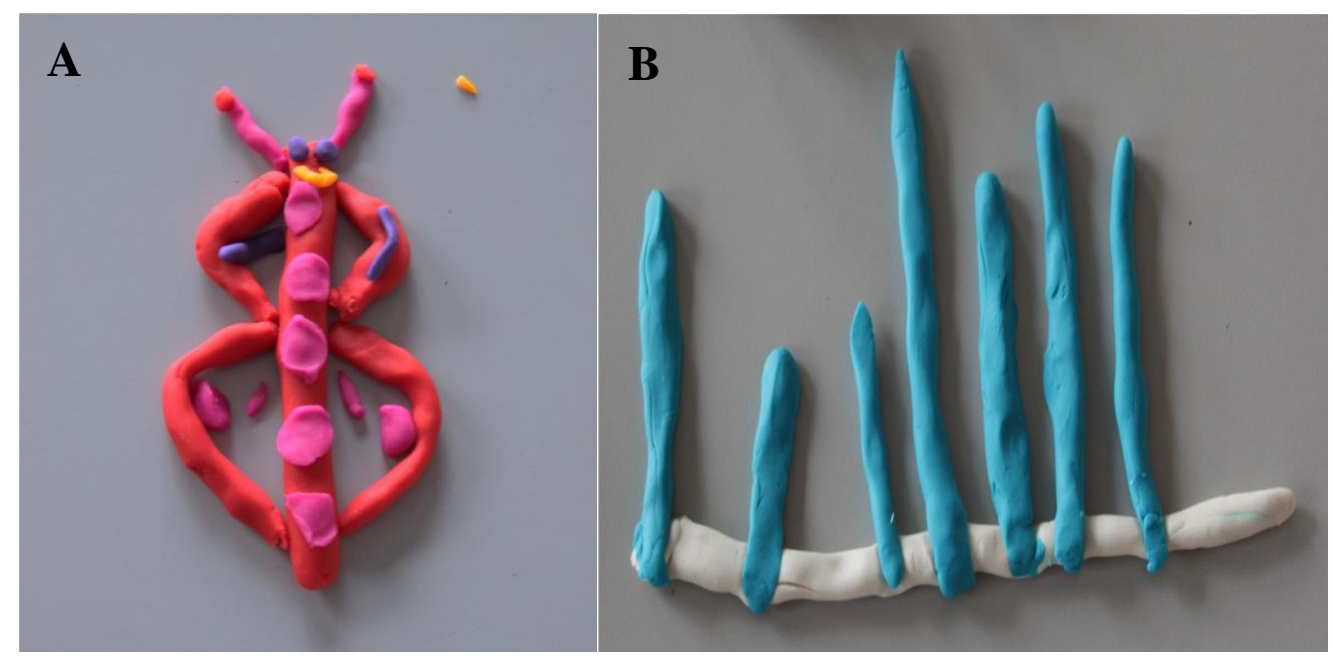

Figure 4.3 Adolescents' Play-Doh model designs

A) 'Abstract' butterfly; B), 'Graphical' bar charts

Thirty-five adolescents (83\%) expressed that the 3D models would motivate them to engage in more physical activity by beating previous models. For example:

"Oh yeah you can try and beat it [the model] the week after or the session after" (G18)

Eight adolescents thought that the 3D models may discourage engagement in physical activity because of feelings of doing worse than others and embarrassment if the model showed low PALs:

"If you don't do like a lot of exercise in compared to like people that you know... like family or friends then you might feel worse..." (G11)

"...if other people like saw the object or something it might be a bit embarrassed if you haven't done enough exercise" (G21)

Twenty-eight adolescents (67\%) showed some knowledge of the government guidelines for physical activity. A specific Sport Wales initiative called '5x60' may have influenced these findings:

"they [Sport Wales officers] try and get everyone to do five sessions of sixty minutes a week of exercise" (G3) 


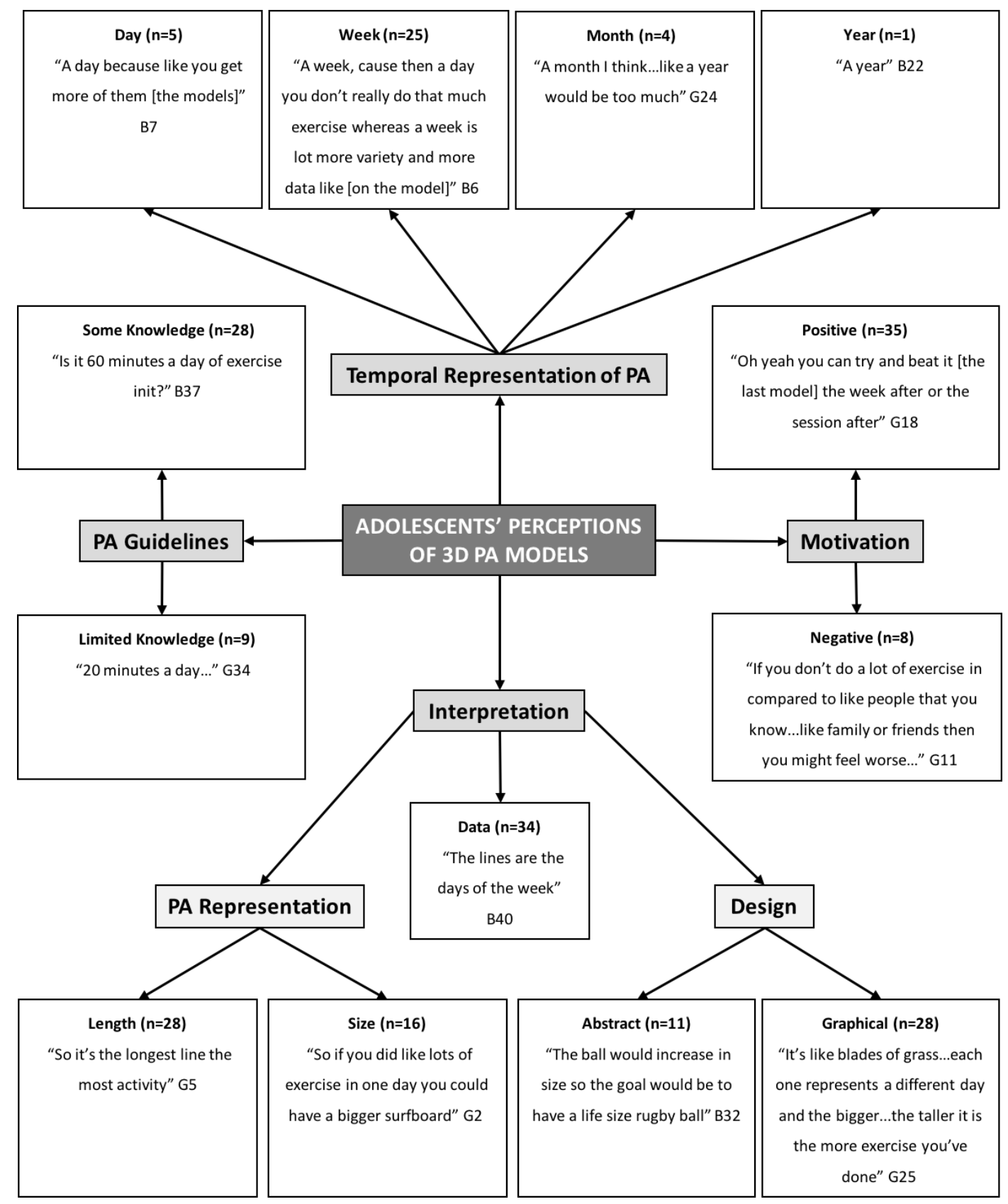

Figure 4.4 Adolescents' pen profile

$\mathrm{B}=$ Boy, $\mathrm{G}=$ Girl, $\mathrm{PA}=$ Physical activity, $\mathrm{N}=$ frequency counts 


\subsubsection{Adults' Perceptions and Designs of 3D Physical Activity Models}

The key adult emergent themes were 'Design', 'Temporal Representation of PA', 'Engagement' and 'Motivation' with a few distinct sub-themes (Figure 4.5). The higher order theme 'Engagement' was linked to additional sub-themes 'Sex Differences', 'Teacher Involvement' and 'Intervention'. Adults described characteristics similar to those used to construct both abstract $(n=7)$ and graphical $(\mathrm{n}=8)$ model representations. Adults placed emphasis on making the 3D models attractive and recognizable, but also something that challenges children's and adolescents' numeracy skills to work within the school curriculum.

"Bar charts and graphs, that's a big part of numeracy, so if you could maybe like make a physical graph... and it would go up in bars every day...” (TF8)

One parent added that a link between the 3D model and a recommended goal for physical activity could help encourage youths to achieve greater PALs.

“...you know...maybe there's strips [on the model] and each one, I don't know if you reach the sort of recommended goal you get like another strip or something [on the model]" (PF5)

Similar to youths, adults preferred a week $(n=12)$ of physical activity data represented on the model, as this was thought to have greater potential to visually guide youths, creating more awareness of their physical activity behaviours than a day $(n=3)$ or month $(n=1)$. Furthermore, some adults emphasised that changing the colour $(n=3)$ of lines on the models could visually aid participants in distinguishing the different days. The majority (87\%) of adults believed that if youths received and compared new models over time this would act as a strong motivation for increased engagement in physical activity.

"They [youths] could see at the end of five or six weeks...they could place their models and compare them then...then you're definitely motivating them [youths]"(TM4) 
Furthermore, some teachers $(n=4)$ reported that receiving their own 3D models would act as an additional competition and potential motivation for the pupils. However, some adults $(\mathrm{n}=7)$ expressed that the 3D models may ostracize youths from others if they under-achieved in physical activity.

“...they [the models] might ostracize them...you know where they might go...no that's too painful because, they're going to get a better [model]" (PF5)

Adults perceived both positives $(n=14)$ and negatives $(n=14)$ for participants' engagement with the concept of 3D printing physical activity. Positives included that the use of new technology (i.e., 3D printers) would create awareness of current technological advances, with negative responses highlighting concerns about potential disruptions to teaching during lesson time. Moreover, some adults $(n=8)$ believed that there would be no differences in how boys and girls engaged with the models, although five adults highlighted that the girls may be more reflective and the boys more competitive.

"I think the boys are more upfront and 'what did you get and what did you get, let me see yours' [the model], whereas I think a few of the girls would do that but the rest I think would do it more reflective when they're on their own" (TF5) 


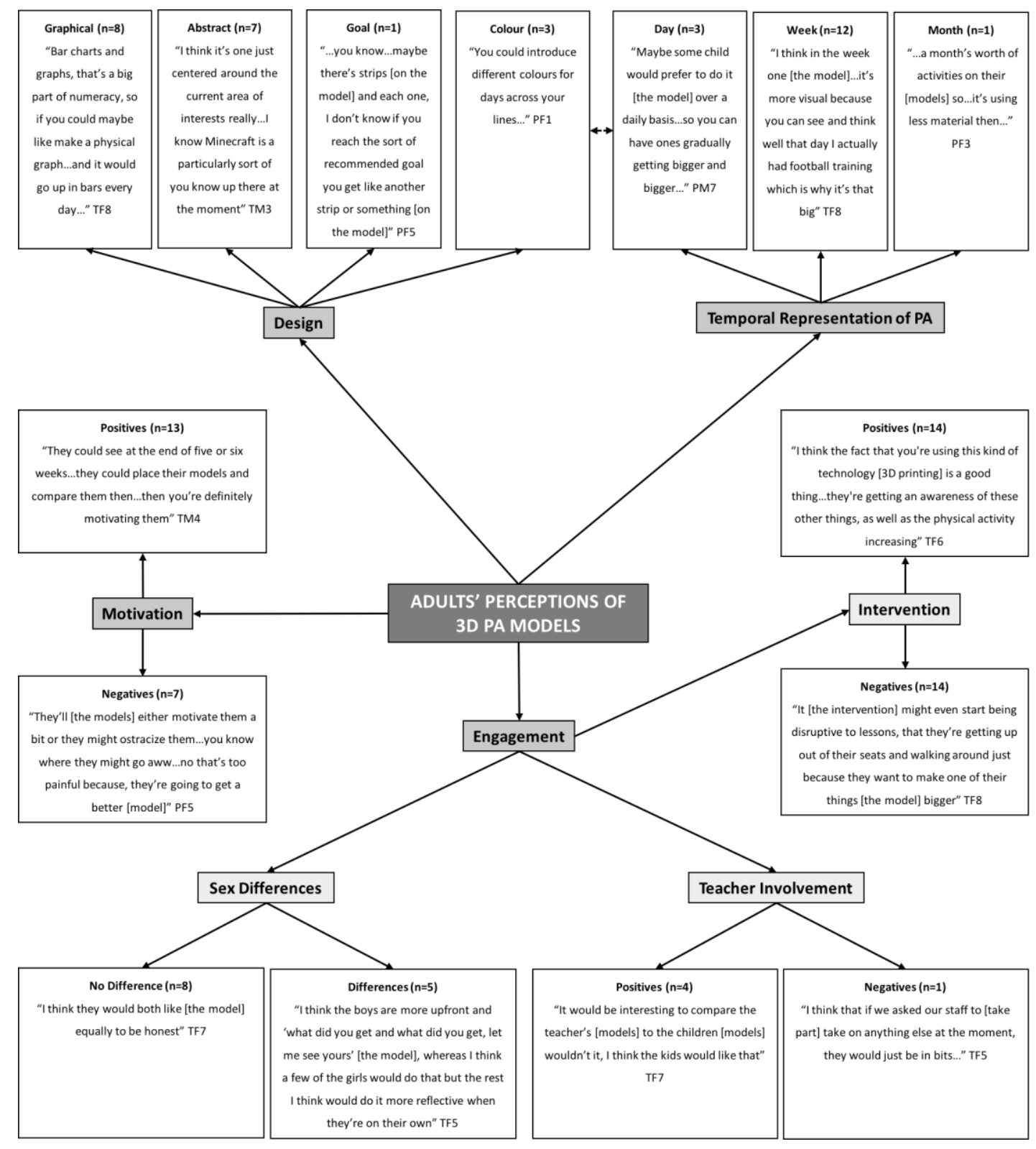

Figure 4.5 Adults' pen profile

$\mathrm{T}=$ Teacher, $\mathrm{P}=$ Parent, $\mathrm{M}=$ Male, $\mathrm{F}=$ Female,

$\mathrm{PA}=$ Physical activity, $\mathrm{n}=$ frequency counts 


\subsection{Discussion}

The aims of the research were, first, to formatively elicit children's, adolescents', teachers' and parents' perceptions of physical activity data when represented as 3Dprinted objects and their personal Play-Doh designs, and, to examine parents' and teachers' perceived benefits and barriers to 3D-printed objects of physical activity. This research extends from that of previous studies that have implemented formative research techniques to inform the development of school-based interventions (Mackintosh et al., 2011, Boddy et al., 2012). The second aim of the study was to use the formative data to inform the design of age-specific 3D models of physical activity to enhance youths' understanding, awareness and PAL.

The data indicated that youths can conceptualise physical activity data represented as a 3D object. This ability to detect and mentally represent a relationship between a symbol (i.e., 3D object) and its referent (i.e., physical activity) is known as representational insight (Uttal \& Doherty, 2008). However, the visual nature of the models does not always guarantee representational insight and its relation to the intended use (Uttal \& Doherty, 2008). For example, adolescents in this study showed greater ability to analyse and critique the physical activity behaviours represented on the prototype models. Adolescents could highlight, in some detail, differences in low and high PAL and how this related to their own and others' personal habits. These differences between adolescents and children could be explained by a greater agerelated cognitive ability in adolescents (Piaget \& Cook, 1952). However, differences in cognitive ability may be less influential as evidence suggests that visualisations help make complex information more accessible and cognitively tractable (Uttal \& Doherty, 2008). More specifically, previous research supports the use of tangible objects to stimulate youths' intellectual development as they support a more natural way of learning (Price et al., 2003, Marshall, 2007, Rogers et al., 2002a, Bara et al., 2004, Gillet et al., 2005), aligning with youths being regarded as 'visual and tactile' learners (Rita \& Dunn, 1979). For example, Gillet et al. (2005) investigated the use of 3D-printed enzyme molecules for teaching biology in youth, reporting that the tangible models provided a natural and intuitive mechanism for manipulation, exploration and a proprioceptive pathway for learning. Whilst the present findings hold promise, given that youths recognise the relationship between the tangible visualisation and its 
intended referent (i.e., physical activity), which is a necessary condition for developing a visual learning tool, others argue that this representational insight is fragile (DeLoache et al., 1998, Uttal \& Doherty, 2008, DeLoache, 2000). Specifically, even if youth initially grasp the representational relation between the $3 \mathrm{D}$ model and PAL, previous research on scaled-models shows that youth can easily lose sight of this relation (DeLoache, 2000). Based on this understanding, DeLoache and colleagues (1998) formulated the dual representation hypothesis to account for youth's success and failure in understanding visualisations and their intended use. Central to this theory is the notion that all representations (i.e., visualisations) have a dual nature, whereby they are intended to stand for something else, but at the same time, they are objects in their own right (DeLoache et al., 1998). For instance, research has demonstrated that highly attractive visualisations may actually be counterproductive, as youth (Goldstone \& Sakamoto, 2003) and even adults (Goldstone \& Son, 2005) focus more on the properties of the visualisation (e.g., shape and colours), rather than what the visualisation is intended to represent or teach. These errors illustrate that visual correspondence may not be enough to promote representational insight. In this light, future research should consider investigating 3D-printed physical activity feedback conditions to include and exclude an additional classroom educational component on PALs to fully understand the benefits of the 3D model alone.

The current study revealed that $78 \%(n=21)$ and $83 \%(n=35)$ of children and adolescents, respectively, believed the 3D models would act as a motivational tool to enhance their own and their peers PAL. These results are promising, especially for the utilisation of 3D models within a school-based intervention setting whereby youths receive their own personal $3 \mathrm{D}$ models of physical activity in the presence of peers. Indeed, previous research suggests that school-based interventions that promote youths' physical activity with peers significantly increases their motivation for physical activity (Salvy et al., 2009), as well as their enjoyment (Jago et al., 2012, Salvy et al., 2012), intensity (Barkley et al., 2014) and engagement in out of school physical activity (Pearce et al., 2014). Furthermore, the majority of primary school children expressed that the 3D models would introduce competition between classmates, motivating them to engage in more physical activity. It has been argued that competition between children can be healthy if it provides feedback about performance and improvements, where children can learn about themselves, and the 
sole or primary objective is not about winning . Conversely, adolescents placed more emphasis on how they would be motivated by beating their own personal model from the week before rather than comparing to others. These differences between youths could be, in part, explained by the adolescents' greater understanding of the concept of effort in the physical domain (Fry \& Duda, 1997) and applied ability to think independently, fostering enhanced self-evaluation skills that are important for preparation into adulthood (Jacobs \& Klaczynski, 2002).

Parents and teachers also agreed that the models would help motivate children and adolescents, allowing them to compare the models over time. Adults highlighted that boys may take a more competitive approach than girls who may engage in more reflective thoughts about the $3 \mathrm{D}$ models. Indeed, evidence suggests that young males engage in more individualistic competition than female counterparts (Benenson et al., 2008). Contrary to this, Bjorkqvist (1994) found that girls use subtler, more indirect strategies for competition than boys from childhood through to adulthood. Adolescents also displayed concerns that they might be perceived as inactive by their significant others, a consensus that was supported by the adults. Similar concerns have been raised when using digital fish avatars, the growth and emotional state of which is dependent on the participant's PALs, with participants reporting being discouraged from using the app if they saw that the avatar was unhappy (Lin et al., 2006). Of concern is the fact that material objects are more valuable than digital objects because of their higher visibility within the physical world and lower replication possibility (Golsteijn et al., 2012, Kirk \& Sellen, 2010). In this respect, material objects make physical activity data more publicly visible to peers than that of data collected and shown on a private computer or smartphone (Khot, 2016). Indeed, Khot (2016) notes that public displays of material physical activity data could lead to issues with privacy. That said, privacyrelated concerns could be tackled through the creation of abstract forms of visualisation that are meaningful to the participating-individual but not so familiar to non-participating onlookers (Khot, 2016). Another point for consideration is that material objects can gain 'autotopographical' meaning (i.e., a means of representing oneself to others through material objects; Khot \& Mueller, 2013, Petrelli et al., 2008), which links to a person's social identity (i.e., a person's sense of who they are based on their group membership), such as age, sex, sports team and ability (Reynolds et al., 2015). In this way, physical objects that display low levels physical activity may result 
in youth not wanting to display or interact with their models as it may negatively impact their social identity and result in feelings of pressure and guilt for not achieving enough physical activity (Reynolds et al., 2015). On the other hand, the visibility of physical activity data through 3D models may support two forms of social incentive: i) Competition, where youth can compare their data against others; and ii) Cooperation, where youth can motivate each other to achieve a set goal (Khot, 2016), all of which may strengthen a person's social identity (Ostrom, 2000). In this light, monitoring how youth personally evaluate models displaying low PALs, and, their support and interactions with significant others should be considered within future research.

Whilst beyond the scope of the present study, it is pertinent to note that further work is also required to adapt these models to other populations and cultures, with the current results suggesting that children with SEN may misinterpret the models with negative health consequences. For example, one child participant with SEN interpreted the $3 \mathrm{D}$ models as something that would intensify their personal need to engage in sedentary computer-based activity, stating: "It [the model] would make me always do computers." (B27). This reaction to the models may be a result of the behavioural and/or emotional problems of a child with SEN and how this interferes with their cognitive ability to interpret scientific concepts (Scruggs \& Mastropieri, 1986, Trout et al., 2003). However, evidence also suggests that there is no substantial differences in scientific understanding between SEN children and healthy children (Van Der Steen et al., 2012). Nonetheless, it is particularly important to consider those children with SEN as evidence suggests they do not engage in as much physical activity as typicallydeveloping youth (Hinckson \& Curtis, 2013). Research shows that those youth with SEN do indeed reap the physiological and psychosocial benefits of physical activity, such as improved motor skills, social interactions, cognition, language and reduced stereotypic behaviours (Lang et al., 2010). Although the present findings suggest otherwise, it could be postulated that the unique strategy of $3 \mathrm{D}$ printing physical activity for youth with SEN may facilitate increased accessibility (Zuckerman et al., 2005), interactions and offer an alternative way of promoting physical activity to that of traditional 'sport' inspired games that have been less effective for this population (Boddy et al., 2015). Therefore, more research is warranted to explore the perceptions of youth with SEN of 3D-printed feedback as a method to enhance PAL. 
For the adults, the tangibility of being able to hold something that participants have created was perceived as original and personalised. Adults expressed that the tactile forms of information would interest youths and encourage them to purposely think about the importance of physical activity, as previously identified by Mackintosh et al. (2016). Furthermore, they also believed that the 3D models could act as a material reward or medal representing achieved physical activity, something children and adolescents "could put [the models] up on their wall when they get them" (PM7). Indeed, much research suggests that material rewards are cherished more than virtual rewards (Munson \& Consolvo, 2012), as a result of their higher visibility and low replication possibility (Kirk \& Sellen, 2010, Golsteijn et al., 2012). Incentive-based interventions to encourage children to take part in more physical activity have been shown to have promising effects (Finkelstein et al., 2013, Hardman et al., 2011b), although findings have been mixed regarding sustained behaviour change following removal of incentives (Strohacker et al., 2014). Sport capitalises on this incentive form of reward system with physical medals and trophies being presented to individuals. However, while these rewards focus on the completion of certain fitness or sports goals, they do not embody any personal data or represent the active self (Khot, 2016). However, Khot (2016), notes that there is a learning value to be gained from blending rewards and representations to create more personalised and meaningful data. This concept is supported by findings from 'Pokémon GO', where children and adolescents can create and identify themselves with a visual avatar surrounded by recognizable characters (e.g., Pikachu) in a socially networked system, which was associated with significant increases in physical activity in both age groups (Althoff et al., 2016).

The current utilisation of Play-Doh enabled youths to creatively explore, adapt and develop their personal 3D model creations. This relatively inexpensive form of design prototyping has been used previously with malleable materials and is effective for brainstorming new ideas and designs from which high-tech prototypes emerge (Druin, 2002). The present findings revealed that children and adolescents preferred different types of 3D model design, leading to the development of two age-specific 3D models of physical activity. For children, a preference for a combination of both abstract (43\%, $\mathrm{n}=12)$ and graphical $(54 \%, \mathrm{n}=15)$ models was demonstrated, most commonly expressed as Play-Doh models of flower or sun like shapes. However, to avoid any 
potential sex bias resulting in boys dissociating with a flower-shaped 3D model, the more neutral sun 3D model design was chosen for further development. Interestingly, a majority of adolescents $(67 \%, n=28)$ showed a preference through Play-Doh models for a simple bar chart design. However, with regard to the two-different age-specific 3D models identified, there is limited literature as to whether the mapping of data should be abstract or graphical. Abstract data allows users to be more curious and speculative, whereas graphical representations provide more direct and comprehensive representations of data. Davis et al. (2005) suggest that more informative feedback provides greater opportunities to learn and improve performance. Indeed, it has been shown that 3D physical bar charts have benefits for information recall when compared to digital visualisations (Stusak et al., 2015). Similarly, Khot et al. (2013, 2015b) compared thoughts on both graphical and abstract 3D-printed objects of physical activity. In accord with Stusak et al. (2015), 3D-printed line graphs had more informative qualities to that of abstract objects (e.g., flowers) as participants were able to easily distinguish the low and high peaks of physical activity, which was essential for identifying and reflecting on past activities. However, participants noted that they did not find the line graphs very exciting as it was just relaying information that is commonly displayed on a screen. Participants described the abstract flower designs as more aesthetically pleasing to look at, a reflective picture of their lifestyle that highlighted their sedentary behaviours (Khot \& Mueller, 2013, Khot et al., 2015b). Stusak et al. (2014) found that participants liked abstract 3D-printed models because they cannot be identified as being related to physical activity, with Sauvé et al. (2017) noting that tangible abstract displays of physical activity can provide a level of privacy when observed by others. Nonetheless, adults believed that both methods of mapping a week of physical activity data were equally important, adding that presenting daily physical activity could potentially 'overwhelm' the children and adolescents with data. As Khot (2016) pointed out, embedding too much data can make the material model less readable, but on the other hand, with too little data the model loses its intended purpose.

Whilst physical activity recommendations for youths are set to advise them on how to achieve an active lifestyle and create awareness of the important health benefits, few children were able to identify the UK recommended amount of physical activity. Children's interpretations of how much physical activity they should achieve was 
largely based on 'how much sport' or 'how many different sports' they could complete per day (e.g., football, rugby, netball and running), aligning with previous research findings (Trost et al., 2000a). In comparison, the adolescent group showed greater knowledge of the government guidelines, but this may have been influenced by the on-going Sport Wales initiative '5x60' implemented at the time of the study and aimed at encouraging youths to engage in 60 minutes of MVPA every day within school. However, it was evident that neither children nor adolescents were able to associate their understanding of the UK government recommendations with the intensity levels of moderate-to-vigorous physical activity, which highlights the need to promote youths' knowledge of government recommendations, as reported by Mackintosh et al. (2016). As aforementioned, tangible interfaces may offer a more playful learning experience (Price et al., 2003) and natural interaction than other learning interfaces (Dourish, 2004, Jacob et al., 2002, Klahr et al., 2007), suggesting that the tangibility of data may benefit children's and adolescents' learning (Marshall, 2007). As one parent expressed, creating a recommended goal for the youths on the model could be beneficial. Therefore, by using a goal-setting strategy (Locke et al., 1981) and structurally developing the government recommendation into a tangible goal on the model, may not only help in developing children's and adolescents' understanding of the Government recommendations of 60 minutes of MVPA, but also motivate youths to increase their PAL.

One of the major strengths of this study is its originality, however, this also highlights the paucity of other supporting research for this age group and that further investigation is warranted on this tangible form of data representation. Research should focus on the relative effectiveness of different types of 3D-printed visualisations of physical activity for the promotion of active learning in youths, and a means of strengthening the articulation of such initiatives with public health guidelines (i.e., 60 minutes of MVPA) to enhance understanding and increase the motivation and engagement of youths in sustained physical activity. Given the nature of focus groups and potential for social-desirability, it is important recognise that participants may have influenced other peer-participants interpretations of the prototype 3D models and their components as there was no independence of observations. A potential limitation is that participants viewed a video of 3D model creation and were shown prototype 3D models prior to designing their own Play-Doh 
models which may have influenced their designs. This method was adopted due to the novelty of the concept of 3D printing to these children and adolescents. Furthermore, the variance in Play-Doh model designs (e.g., flowers, suns, waves, grass, animals, fruits, footballs, surfboards and bar charts) compared to the prototype models, suggests that youths were more influenced by personal possessions and hobbies. Finally, one variable that limits the generalizability of the present findings is the localised area of data collection and sample size which may underrepresent the ideologies of youth from other important social-economic groups and ethnic minorities within the UK or globally.

\subsection{Conclusion}

The present formative study provides insight into the utilisation of tangible 3D-printed objects displaying physical activity as a tool to benefit children and adolescents. The findings demonstrate how youths actively and enthusiastically engaged with the concept of 3D objects of physical activity and felt it could not only enhance their understanding of, but motivate them to increase, their PALs. From pupils' Play-Doh model outputs, two age-specific 3D models representing weekly physical activity data were developed. The motivational results will be used to inform the design of a schoolbased physical activity intervention that utilises 3D printing to create tangible, 3Dprinted models, that display youths' personal PAL data as a unique strategy to promote their engagement in physical activity. 


\section{Thesis Map}

STUDY

\section{OUTCOMES}

1. Energy Expenditure Associated with Walking Speed and Angle of Turn in Children

\author{
Aim \\ - To investigate the influence of walking speed and \\ angle, and their interaction, on the energy \\ expenditure of healthy children.
}

- Significant difference to straight-line walking energy expenditure within speed found at $2.5 \mathrm{~km} \cdot \mathrm{hr}^{-}$

Key $\quad{ }^{1}$ at $90^{\circ}$ turn, and speed $3.5,4.5$ and $5.5 \mathrm{~km} \cdot \mathrm{hr}^{-1}$ at

Findings $\quad 180^{\circ}$ turn.

- Estimations of children's habitual physical activity should account for the magnitude and frequency of turns complete.

2. Perceptions of Visualising Physical Activity as a 3D-printed Object: A Formative Study

Role of DESIGN PARTNERS
Aim

- To elicit children's, adolescent's, parents' and teachers' perceptions and understanding of 3D physical activity objects to inform the design of future 3D models of physical activity.

- Youths demonstrated a good ability to conceptualise physical activity as a 3D-printed object and

Key highlighted the potential of $3 \mathrm{D}$ models as a

Findings motivational tool.

- Two age-specific 3D models of physical activity were developed from children's preference for abstract designs and adolescents' bar chart designs.
3. Understanding Youths' Ability to Interpret 3D-printed Physical Activity Data and Identify Associated Intensity Levels

\section{Role of TESTER}

$$
\text { Key }
$$

Findings
- To elicit children's and adolescent's interpretations of two age-specific 3D models displaying physical activity.

- To assess children's and adolescent's ability to appropriately align activities to the respective intensity of physical activity.

4. The Tangibility of Personalised 3DAim printed Feedback may Enhance Youths' Physical Activity Awareness

Role of $\underline{U S E R}$ 


\section{CHAPTER 5}

Youths' Understanding of AgeSpecific 3D Models and Intensity

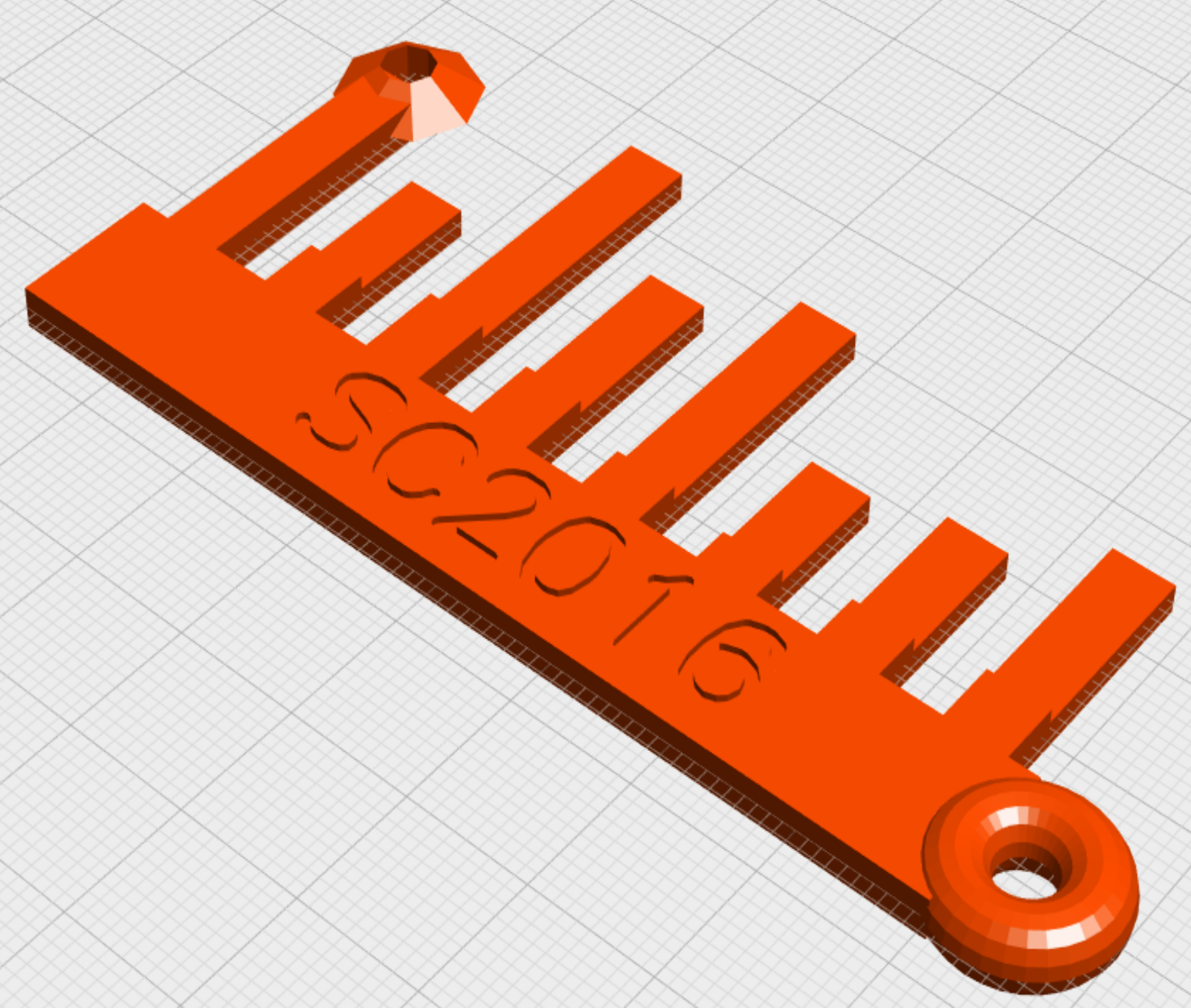




\section{5}

\section{Understanding Youths' Ability to Interpret 3D-printed Physical Activity Data and Associated Intensity Levels}

\subsection{Introduction}

Regular physical activity is considered an essential part of youths' (children and adolescents) overall physiological health and psycho-social development (Sothern et al., 1999, Nieman, 2002, Janssen \& LeBlanc, 2010b, Eime et al., 2013), providing immediate and future health benefits (Shiri et al., 2013, Tammelin et al., 2014, Wolin et al., 2009). Indeed, strong relationships exist between physical activity and health, with individuals attaining higher physical activity levels (PAL) being rewarded with a risk reduction of $30 \%$ for all-cause mortality, 20-35\% for cardiovascular diseases, 30$40 \%$ for type 2 diabetes and a $20-30 \%$ reduction in cancer when compared to an individual classified as low activity (Davies et al., 2011). Moreover, youths who frequently participate in physical activity demonstrate reduced symptoms of anxiety and depression, which subsequently leads to psycho-social benefits, such as improved self-esteem and confidence (Janssen \& LeBlanc, 2010b). Similar to physical activity, there is a dose-response relationship observed with increased sedentary behaviour (activities in a sitting or reclining position, e.g., watching TV) and greater risk of adverse health-outcomes (Tremblay et al., 2010). In the UK, youth aged 5 to 15 years have been reported to spend 7 to 8 hours per day in sedentary behaviour, which accounts for $60-65 \%$ of their day (Biddle et al., 2010). Given the pandemic rise of sedentary behaviour in youth, public health sectors have produced and communicated physical activity recommendations to guide individuals towards achieving a minimum level of physical activity to reap health benefits (Blair et al., 2004). The World Health Organisation (WHO) and UK Government both recommend that youths aged 5 to 17 years should engage in at least 60 minutes moderate-to-vigorous physical activity (MVPA) every day (Department of Health, 2011, WHO, 2011). Despite this, reports show that only $21 \%$ of boys and $16 \%$ of girls in the UK meet these current physical activity recommendations (Hills et al., 2011, Townsend et al., 2015). 
Promoting youth's physical activity relies upon understanding the underlying factors which influence the likelihood of achieving the desired behaviour. Among the most consistently reported factors are an individual's age, sex, socioeconomic status, social and environmental support, and level of education (Bauman et al., 2012, Carver et al., 2008, Trost et al., 2002a). However, little attention is given to individuals' knowledge regarding the recommended levels (Snethen \& Broome, 2007, Sleap \& Wormald, 2001, Harris et al., 2016, Roth \& Stamatakis, 2010) and intensities of physical activity (Grewal, 2013, Knox et al., 2013b) and, subsequently, manners in which to achieve the international physical activity guidelines. Of concern, youths most commonly cite 2 hours per week as the recommended PAL, as well as demonstrating a limited ability to interpret and classify the intensities associated with daily activities (Cowden \& Plowman, 1999, Prochaska et al., 2001, Snethen \& Broome, 2007, Pearce et al., 2008), therefore questioning their ability to translate their own activities to the context of the recommended levels. Furthermore, youths' inability to define and understand the intensity of physical activity may, in part, explain the inconsistent reliability and validity of children's self-reported PAL (Aggio et al., 2016, Chinapaw et al., 2010, Martinez-Gomez et al., 2009). Therefore, it is important to recognise youth's lack of knowledge regarding the complexities of physical activity; content knowledge (i.e., concepts) is a critical step towards youth achieving a healthy and sustainable active lifestyle that can be continued into adulthood (Brusseau et al., 2011). This is particularly pertinent given that adults also show lack of knowledge of their respective physical activity targets and associated activity intensities (Knox et al., 2013b). Indeed, DiClemente et al (2001) suggested that one solution to overcome youth's lack of knowledge may be the use of personalised feedback to educate an individual about a behaviour and outcome. Whilst there is currently a paucity of literature on youths' perceptions of physical activity intensity, it is evident that the development of personal feedback tools (Kremers et al., 2008), which seek to enhance their understanding of the importance of physical activity, and indeed interpret the recommended guidelines, are warranted.

Digital mediums, such as activity tracking tools and smartphone devices with assisted apps, have allowed greater accessibility for users to visualise their personal physical activity data. Visualisations are known for enabling users to understand their personal 
data and associations with PALs, making them more comprehensible and actionable in terms of health-related aims (Khot, 2016). However, on-screen visualisations are limited to visual stimulation and ignore the abundance of other senses, such as 'touch', that could potentially enrich personal engagement with data (Ullmer \& Ishii, 2000, Khot, 2016). This is especially pertinent to the current population, with $80 \%$ of youths visual and tactile learners (Rita \& Dunn, 1979). Given that physical activity happens in the physical world, tangible representations of physical activity that can be placed in the everyday environment have the potential to make data more available to an individual (Khot \& Mueller, 2013, Sauvé et al., 2017). Indeed, Khot et al. (2013) investigated the use of an innovative visualisation strategy involving $3 \mathrm{D}$ printing to create tangible physical activity data for adults, demonstrating that the visual and tactile nature of the data increased the user's awareness and reflection of their personal physical activity behaviours. Previous evidence within the educational domains suggests that tangible interfaces can play an important role in youths' active learning by increasing engagement and reflections upon a topic (Dourish, Jacob et al., 2002, Triona \& Williams, 2005, Price et al., 2003, Rogers et al., 2002a). Following these developments in understanding, recent formative research on youths has demonstrated their ability to conceptualise 3D-printed objects of physical activity, with $80 \%$ of youths expressing that the models would motivate them to engage in more physical activity (Study 2). Moreover, with the use of Play-Doh as a prototyping material to create models, youths designed 3D models that were represented through abstract and graphical representations, which led to the development of two age-specific 3Dprinted model prototypes. However, before introducing the age-specific 3D models into an intervention setting, it is important to determine their acceptability of such feedback with regards to whether youths can correctly interpret the different models in terms of the amount and intensity of daily physical activity displayed. In the absence of such formative research, researchers risk the development of 3D models and interventions that may be inappropriate or misunderstood by the target population (Bopp \& Fallon, 2008). Indeed, previous health message interventions have been limited by a lack of formative research to guide the development and delivery of messages (Martinez et al., 2012). Based on the technology design framework developed by Druin (2002), the present study implements the role of the 'tester', whereby children are the testers of the new technology and their experiences can be observed and evaluated for impact by researchers. 
The aims of this study were, therefore, to (i) examine children and adolescents' perceptions and ability to identify physical activity intensities (i.e., sedentary, light, moderate and vigorous); (ii) elicit children and adolescents' interpretations of the agespecific 3D model prototypes, and; (iii) use the data to consolidate the design of the age-specific 3D model prototypes to inform the development of a school-based physical activity intervention whereby youth receive personal age-specific 3D models displaying their PAL.

\subsection{Methods}

\subsubsection{Recruitment}

Participants were a convenience sample from one primary school and one secondary school in South Wales, UK. Schools were invited to take part in the study via email correspondence to the Head of School (primary school) or Head of Physical Education (secondary school). Participants had to be typically developing children (aged between 7-9 years old) or adolescents (aged between 13-15 years old), which could include overweight or obese, to take part in the study. The schools were regular community (primary) or comprehensive (secondary) public schools. Based on Estyn reports (Her Majesty's Inspectorate for Education and Training in Wales, 2017), out of the 205 and 1,105 pupils that attended the primary and secondary schools, respectively, $4 \%$ and $7 \%$ of pupils were eligible for free school meals.

\subsubsection{Participants}

In total, twelve primary school children ( 9 boys; $7.8 \pm 0.4$ years) and 12 secondary school adolescents ( 6 boys; $14.1 \pm 0.3$ years) participated in the study. All primary school participants were White British, with $8 \%(n=1)$ of secondary school participants being Black British. None of the children nor adolescents had participated in previous formative research regarding the concept of 3D models and their development. Parents and youths returned informed written consent and assent prior to participation (see Appendix II), respectively. All procedures were approved by the University Ethics Committee and were conducted in accordance with the Declaration of Helsinki (ref: PG/2014/40). 


\subsubsection{Procedures}

Twenty-four semi-structured individual interviews were conducted with youths by the first author, either within a familiar classroom or the school library (Kennedy et al., 2001). Individual interviews were chosen as they are a suitable method for exploratory research seeking to generate diverse and original ideas within youths (Heary \& Hennessy, 2006). Although other methods, such as questionnaires or a combination of methods (i.e., interviews and questionnaires), can provide evidence of patterns amongst large populations, they can be compromised by various methodological and cross-comparison factors, respectively (Harris \& Brown, 2010). For example, questionnaires are subject to problems such as faulty or biased questionnaire design and wording, respondent unreliability, ignorance, misunderstanding and statistical analysis errors (Oppenheim, 2000). Conversely, the questionnaire and interview approach may be seen as advantageous, as researchers gain both depth of understanding and corroboration of the same phenomenon, offsetting the weaknesses inherent to using a single approach (Symonds \& Gorard, 2008). That said, evidence suggests that a combination of structured questionnaires and semi-structured interviews provide a weak level of consistency or consensus between methods (Harris \& Brown, 2010), as interviews generally take more time to conduct, consequently exposing the variabilities and inconsistencies within human thinking (Marton \& Pong, 2005, Pajares, 1992). This variability between questionnaires and interviews makes it difficult to classify some participants' attitudes towards assessment (Harris \& Brown, 2010). Whilst individual interviews alone are also open to scrutiny, such as interviewer question manipulation and participant social desirability, they do, however, allow for participants to elaborate on ideas and explain perspectives to gather more in-depth insights (Harris \& Brown, 2010). For this reason, interview questions were adjusted for tone and structure to ensure age-appropriateness; all interview questions and tasks were reviewed, discussed and revised by authors SGMC, MAM, ZRK and KAM. The interview questions (see examples in Table 5.1) were informed by previous formative research (Study 2) and addressed concepts such as youths' knowledge of physical activity intensities and youths' interpretations of the age-specific 3D models (Figure $5.1 \mathrm{~A} \& \mathrm{~B})$. 
Complementary to the interview questions, youths were asked to complete two interactive tasks: i) a physical activity and intensity-matching task, and; ii) a 3D-model recall and interpretation task. The first task was completed at the mid-point of the interview process and invited participants to match twenty different pictures of activities (e.g., video gaming, walking, climbing stairs, football) to the correct intensity (i.e., sedentary, light, moderate and vigorous; Table 5.2). Sedentary activities were based on Trost et al. (2000a), with light, moderate and vigorous activities obtained from the youth compendium of physical activities (Ridley et al., 2008, Butte et al., 2017). After completion of the task, participants were asked to describe why they placed each activity within the specific intensity box.

Table 5.1 Example interview questions

\begin{tabular}{lll}
\hline Interview & Topic & Examples \\
\hline
\end{tabular}

Children/Adolescents PA Intensity Can you tell me what you think these different levels of intensity for physical activity might be?

Children/Adolescents PA Intensity What word would you use to describe the intensity of that activity [e.g., climbing stairs]?

Children/Adolescents PA Model What do you think the lines/bars show?

Children/Adolescents PA Model Can you tell me what you think the rest of the physical activity model shows? (Prompt: how do you think this model (sun or bar chart) shows physical activity?) 
Table 5.2 Twenty activities and respective intensity levels

\begin{tabular}{|c|c|}
\hline Intensity & Activity \\
\hline \multirow{5}{*}{ Sedentary } & Eating, sitting \\
\hline & Reading, lying down \\
\hline & Mobile phone, sitting \\
\hline & Computer, sitting \\
\hline & Video games, sitting \\
\hline \multirow{4}{*}{$\begin{array}{c}\text { Light } \\
(<3.0 \text { METs })\end{array}$} & Fishing, sitting \\
\hline & Strotching overcice \\
\hline & Darts, wall \\
\hline & Walking, slow \\
\hline \multirow{4}{*}{$\begin{array}{c}\text { Moderate } \\
(3.0-6.0 \mathrm{METs})\end{array}$} & Throwing, snowball \\
\hline & \\
\hline & Mowing lawn \\
\hline & Climbing stairs \\
\hline \multirow{7}{*}{$\begin{array}{c}\text { Vigorous } \\
\text { (>6.0 METs) }\end{array}$} & Climbing trees \\
\hline & Football/soccer \\
\hline & Tennis \\
\hline & Hockey, field \\
\hline & Running, hard effort \\
\hline & Swimming laps \\
\hline & Riding a bicycle, hard effort \\
\hline
\end{tabular}




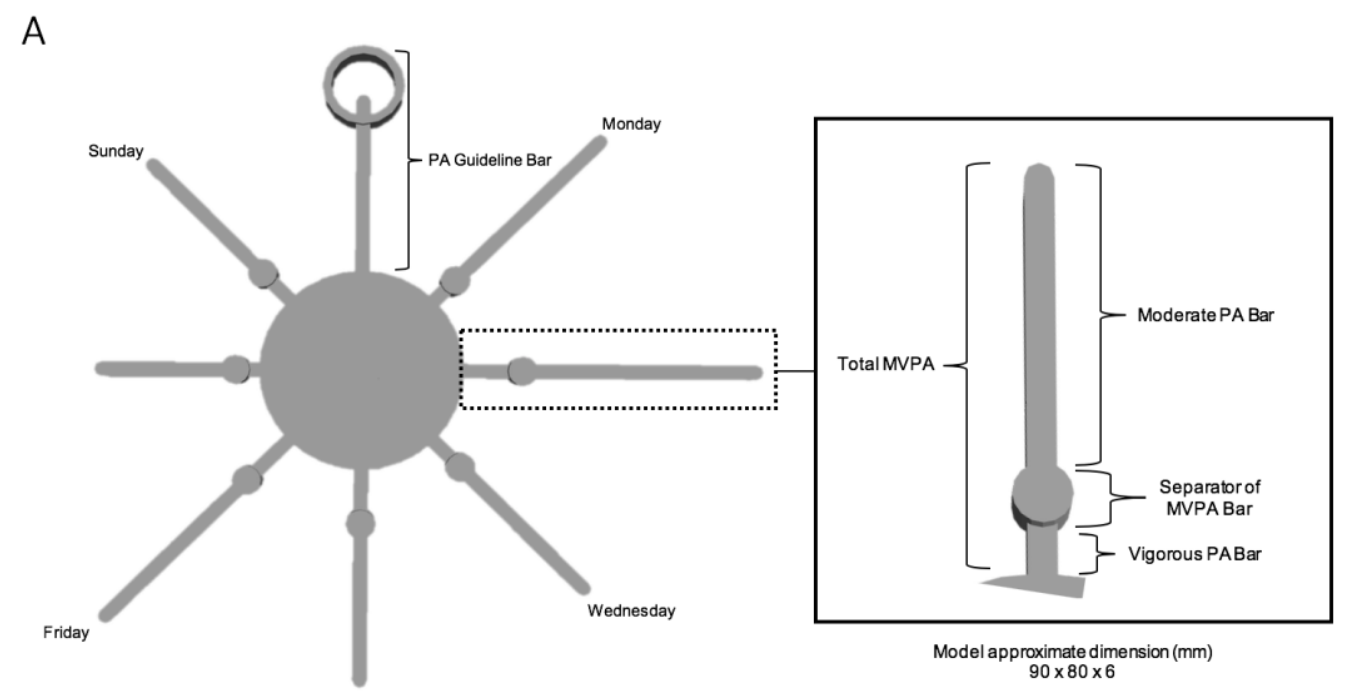

B

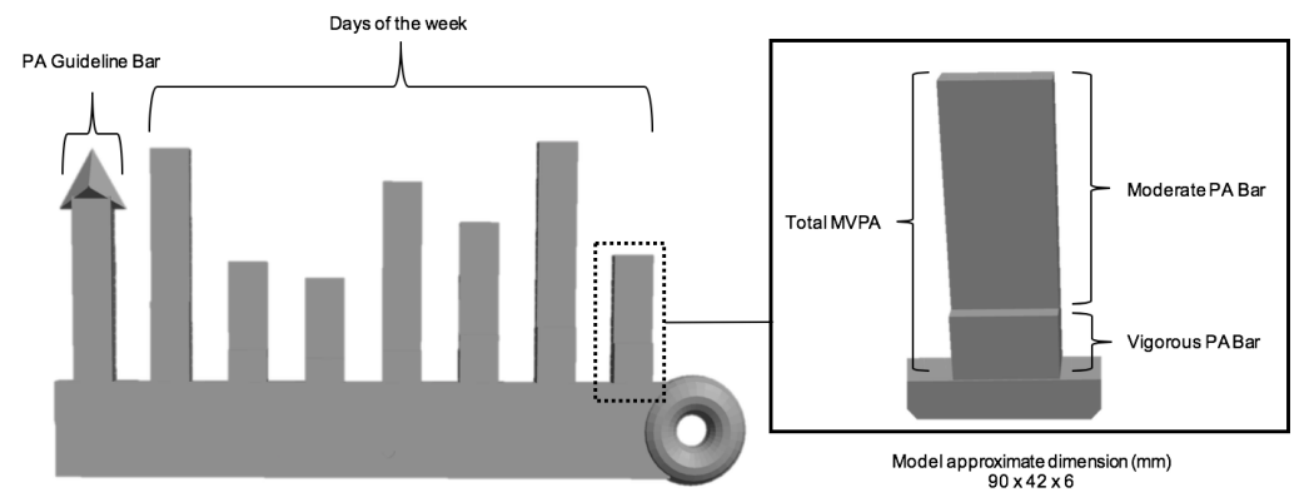

Figure 5.1 Age-specific 3D model prototypes

A) Children's Sun 3D Model, B) Adolescents' Bar Chart 3D Model; PA = Physical Activity, MVPA $=$ Moderate to Vigorous Physical Activity

The second task was completed at the end of the interview to test youths' ability to recall and interpret the different components of the age-specific 3D models. The formatively-developed 3D models were designed by children, who displayed a preference for a sun (Figure 5.1A) and adolescents bar chart (Figure 5.1B), using PlayDoh as a prototype tool for creation (Study 2). Both models depict example tri-axial accelerometry-derived (wGT3X-BT, ActiGraph LLC, Pensacola, FL) moderate and vigorous PALs achieved for each day, across a week, as well as a reference bar to the physical activity guidelines of 60 minutes MVPA. In detail, the moderate and vigorous PAL achieved for each day was calculated using Evenson's child cut-points (2008b) on ActiLife version 6.13.3 (ActiGraph LLC, Pensacola, FL). Following this, the PALs 
were inserted in to the age-specific custom developed 3D model code and loaded on OpenJSCAD version 1.8.0, and subsequently 3D-printed using polylactide (PLA) filament on the Ultimaker 2 Extended+ (Ultimaker, The Netherlands, Geldermalsen). All participants were asked to label a 2D diagram of the relevant model and to verbally describe the model's components.

Interviews lasted $35.8 \pm 5.3$ and $25.1 \pm 4.9$ minutes for children and adolescents, respectively. All the interviews were digitally voice (Olympus DM-520 digital voice recorder, Shinjuku, Japan) and video (Sony Handycam HDR-PJ540, Minato, Japan) recorded and transcribed verbatim. In total, 85 and 92 pages of raw transcription data, Arial font, size 12, double-spaced were produced for primary school children and secondary school adolescents, respectively. Unique identification codes were used to ensure anonymity of participants within all transcripts: B (boy) or G (girl), followed by participant number.

\subsubsection{Data Analysis}

Through the process of content analysis, transcripts were deductively analysed through contextual 3D model themes (separator of MVPA bar, physical activity guideline bar and the daily, moderate and vigorous physical activity bars) and activity intensities (sedentary, light-, moderate- and vigorous-intensity). Quantitatively it was noted whether the classification of data was accurate (i.e., activities to intensities and the different 3D model components; Hamad et al., 2016). This mixed-methods approach allowed for a greater insight into the meanings of the data (Holsti, 1969, Pool, 1959) and took into account the multiple aims of the research regarding youths' ability to identify physical activity intensities and accurately interpret the age-specific 3D models (Hamad et al., 2016). Firstly, transcripts were thematically analysed by the first author (SGMC) using three steps: data immersion, coding and identifying themes (Braun \& Clarke, 2006). The immersion of the data was completed in an active way of 'repeated reading' of the transcripts, searching and noting of meanings and patterns within the data set (Braun \& Clarke, 2006). The process of coding, using a manual cut and paste technique, organised the data into meaningful groups that were considered pertinent to the research questions (Braun \& Clarke, 2006). Key themes were identified by collating the relevant coded data quotes and discarding any irrelevant 
quotes from the analysis (Braun \& Clarke, 2006). A frequency count of the compiled meaningful quotes was conducted to record the number of participants that noted respective points within a theme. The meaningful quotes and frequency counts were then presented diagrammatically using a pen profile approach, which is considered an appropriate method for representing diagrams of key emergent themes (Boddy et al., 2012). The last author (KAM) independently analysed the data and discussed the outcomes with SGMC. Through the repeated process of reverse triangulation, author MAM critically cross-examined the data in reverse from the pen profiles to the transcripts until all alternative interpretations of the data were exhausted. The pen profiles were then assessed by all other authors, enabling further interpretations and adjustments prior to a final consensus was reached. For the activity intensity-matching task, the activities placed into certain key intensity boxes were counted (sedentary, light, moderate and vigorous) and aligned with direct quotations (Table 5.3 \& Table $5.4)$.

\subsubsection{Statistics}

A "N - 1" Chi-squared test was conducted using IBM SPSS Statistics 22 (Chicago, IL) to determine any significant differences between boys and girls who correctly associated activities to their respective intensity, with statistical differences accepted at $p \leq 0.05$ (Campbell, 2007, Richardson, 2011).

\subsection{Results}

Youths' understanding of physical activity intensities are presented in Table 5.3 (children) and Table 5.4 (adolescents). Specifically, the tables present the four different intensity levels (i.e., sedentary, light, moderate and vigorous) with their associated activities, as previously seen in Table 5.2. Following each activities path, within a given intensity, is a representative verbal statement that reflects the greatest intensity level frequency count. The intensity tables also show the overall percentage of youth that correctly aligned activities to their respective intensities, and sex differences therein. 


\subsubsection{Children's Perceptions and Ability to Identify Physical Activity Intensities}

From Table 5.3, children were able to correctly align sedentary-based activities with the respective intensity $62 \%$ of the time, with girls demonstrating a better understanding of sedentary behaviour than boys (girls $80 \%$ vs. boys $53 \% ; p>0.05$ ). Specifically, the sedentary activities most commonly correctly-identified were technology-based behaviours, such as playing on a mobile phone $(75 \%)$ or computer (75\%), and video gaming (75\%). A number of children (58\%) reported that eating was a light-intensity as "eating's easy cause you're just like moving your arms and putting it [food] in your mouth" (PB06). Children were only able to correctly identify lightintensity activities $31 \%$ of the time, with girls showing a better understanding of lightintensity activities than boys (girls $38 \%$ vs. boys $28 \% ; p>0.05$ ). A number of children (75\%) indicated stretching as a moderate-intensity activity because "for some people stretching is really hard..." (PB06), with one child associating stretching with "when I do rugby you have to warm up and that's not hard, easy or inactive" (PB07). Furthermore, fishing was identified by five children as a sedentary behaviour due to the nature of the sitting position, stating "he's just sitting down and waiting for a fish..." (PG11). Similarly, some children struggled to define moderate-intensity activities, with only $33 \%$ of moderate activities being correctly identified. Boys, as a group, fared somewhat better in allocating moderate-intensity activities in comparison to girls (boys 38\% vs. girls 25\%; $p>0.05$ ). Children perceived moderate activities, such as throwing $(83 \%)$, climbing stairs $(75 \%)$ and sweeping $(58 \%)$ as light-intensity activities. Specifically, climbing stairs was thought of as a light-intensity activity because "all you've got to do is lift a foot and put it on each step" (PB09), with sweeping being noted as something that "you can relax while you're doing it [sweeping]" (PB02). Vigorous activities were correctly identified 68\% of the time by children (boys $73 \%$ vs. girls $57 \% ; p>0.05$ ). Vigorous-intensity activities, such as riding a bicycle $(92 \%)$, hockey $(92 \%)$, tennis $(67 \%)$, swimming laps $(58 \%)$, football (58\%), running (50\%) and climbing trees (50\%), were all correctly classified. Children described the nature of vigorous-intensity as riding a bicycle or running making "you...really tired” (PB09) or "you get a little tired..." (PB01), respectively. When referring to swimming laps there was an emphasis on "my swimming teacher pushes me really hard" (PB07). 


\subsubsection{Adolescents' Perceptions and Ability to Identify Physical Activity Intensities}

Table 5.4 shows that adolescents correctly identified sedentary-based activities $87 \%$ of the time, with boys demonstrating a better understanding when compared to girls (boys $90 \%$ vs. girls $83 \% ; p>0.05$ ). Sedentary technology-based activities, such as playing on a mobile phone (100\%) or computer (92\%), and video gaming (75\%), were all correctly perceived as sedentary behaviours; for example: "they're just on their electronics, playing games or watching something...they don't really have to put effort into that and they're not moving around or doing anything" (SB01). Light-intensity activities were correctly placed $71 \%$ of the time, with girls displaying a better understanding than boys (girls $75 \%$ vs. boys $67 \% ; p>0.05$ ). Light-intensity activities, walking (83\%), fishing (67\%), darts (67\%) and stretching (67\%), were all consistently identified as being a light-intensity activity. Adolescents correctly identified moderateintensity activities only $10 \%$ of the time (girls $13 \%$ vs. boys $8 \% ; p>0.05$ ). All adolescents reported that the activity of throwing (100\%) was a light-intensity activity. Other moderate activities, such as mowing the lawn (75\%), climbing stairs (75\%) and sweeping (67\%), were also classified as light-intensity, expressing them as "everyday things like mowing the lawn" (SG09). Adolescents' were only able to appropriately identify vigorous-intensity activities $46 \%$ of the time, with girls demonstrating a greater ability to recognise vigorous-intensity activities than the boys (girls $62 \%$ vs. boys $24 \% ; p>0.05$ ). Adolescents correctly categorized individual fitness activities, such as cycling (75\%), running (67\%) and swimming (50\%) as vigorous-intensity activities. In contrast, organised sport activities, such as football (75\%), tennis (67\%) and hockey (58\%), were often identified as moderate-intensity, even though they regarded football and tennis as "...quite a physical sport” (SB03) or involving “...strengths" (SB04), respectively. 
Table 5.3 Children's perceptions and ability to identify activities to intensity

\begin{tabular}{|c|c|c|c|c|c|c|c|}
\hline \multirow[b]{3}{*}{ Intensity } & \multirow{3}{*}{$\begin{array}{l}\text { Age Group } \\
\text { Activity item }\end{array}$} & \multicolumn{6}{|c|}{ Children $(n=12)$} \\
\hline & & \multirow[b]{2}{*}{ Representative Verbal Statement } & \multicolumn{4}{|c|}{ Intensity Level Frequency Count (n) } & \multirow[b]{2}{*}{$\%$} \\
\hline & & & SED & LPA & MPA & VPA & \\
\hline \multirow{5}{*}{ Sedentary } & Eating, sitting & $\begin{array}{l}\text { "Eating's easy cause you're just like moving your arms and } \\
\text { putting it [food] in your mouth" PBO6 }\end{array}$ & 5 & $7^{\#}$ & 0 & 0 & \\
\hline & Reading, lying down & "That one cause you're just lying there" PBO3 & $5^{\#}$ & 3 & 3 & 1 & \\
\hline & Mobile phone, sitting & $\begin{array}{l}\text { "These [mobile phone use] are quite easy cause all you're doing } \\
\text { is basically moving your fingers" РB02 }\end{array}$ & $9^{\#}$ & 2 & 1 & 0 & $\begin{array}{c}62 \% \\
B=53 \% \\
G=80 \%\end{array}$ \\
\hline & Computer, sitting & $\begin{array}{l}\text { "Computer you just sitting down and probably typing something } \\
\text { with mouse and this you're just going [acts out typing] ..." PB07 }\end{array}$ & $9^{\#}$ & 2 & 1 & 0 & \\
\hline & Video games, sitting & $\begin{array}{l}\text { "They are like playing video games, this is inactive because } \\
\text { you're not actually like moving" PB6 }\end{array}$ & $9^{\#}$ & 2 & 1 & 0 & \\
\hline \multirow{4}{*}{ Light } & Fishing, sitting & $\begin{array}{l}\text { "He's just sitting down and waiting for a fish but when he winds } \\
\text { it in he's using kind of his muscles" PG11 }\end{array}$ & $5^{\#}$ & 1 & 4 & 2 & \\
\hline & Stretching exercises & $\begin{array}{l}\text { "Cause when I do rugby you have to warm up and that's not } \\
\text { hard, easy or inactive" PB07 }\end{array}$ & 0 & 2 & $9^{\#}$ & 1 & $\begin{array}{c}31 \% \\
B=28 \%\end{array}$ \\
\hline & Darts, wall & $\begin{array}{l}\text { "Throwing darts is pretty easy but not to hit the middle [of the } \\
\text { dart board]" PB02 }\end{array}$ & 0 & $5^{\#}$ & 4 & 3 & $G=38 \%$ \\
\hline & Walking, slow & $\begin{array}{l}\text { "Walking to school's easy, all you're doing is like moving your } \\
\text { legs" PB06 }\end{array}$ & 1 & $7^{\#}$ & 3 & 1 & \\
\hline \multirow{4}{*}{ Moderate } & Throwing, snowball & $\begin{array}{l}\text { "Throwing snowballs is quite easy because you can just throw } \\
\text { them any way you like" PBO2 }\end{array}$ & 0 & $10^{\#}$ & 2 & 0 & \multirow{4}{*}{$\begin{array}{c}33 \% \\
B=38 \% \\
G=25 \%\end{array}$} \\
\hline & Climbing stairs & $\begin{array}{l}\text { "I've put walking up steps because quite easy because all } \\
\text { you've got to do is lift a foot and put it on each step" PBO9 }\end{array}$ & 0 & $9^{\#}$ & 3 & 0 & \\
\hline & Sweeping & $\begin{array}{l}\text { "And sweeping because you can relax while you're doing it" } \\
\text { PB02 }\end{array}$ & 0 & $7^{\#}$ & 5 & 0 & \\
\hline & Mowing lawn & $\begin{array}{l}\text { "For lawn, I've done...cause it's not easy, and it's not hard and } \\
\text { it's not inactive so it's that one [moderate]" PB07 }\end{array}$ & 1 & 4 & $6^{\#}$ & 0 & \\
\hline \multirow{7}{*}{ Vigorous } & Climbing trees & $\begin{array}{l}\text { "They're using...their tummy muscles and their arms and their } \\
\text { legs" PG11 }\end{array}$ & 0 & 0 & 5 & $7^{\#}$ & \multirow{7}{*}{$\begin{array}{c}68 \% \\
B=71 \% \\
G=57 \%\end{array}$} \\
\hline & Tennis & $\begin{array}{l}\text { "Then tennis cause its quite active, you move a lot cos you hit } \\
\text { and then you have to move to hit the ball again" PBO7 }\end{array}$ & 0 & 0 & 4 & $8^{\#}$ & \\
\hline & Swimming laps & $\begin{array}{l}\text { "When I go swimming my teacher, go in the } 3 r d \text { lane and my } \\
\text { swimming teacher pushes me really hard" PB07 }\end{array}$ & 0 & 0 & 5 & $7^{\#}$ & \\
\hline & Hockey, field & $\begin{array}{l}\text { "Hockey's hard cause some people don't really know how to } \\
\text { play hockey..." PBO6 }\end{array}$ & 0 & 0 & 1 & $11^{\#}$ & \\
\hline & Football/Soccer & $\begin{array}{l}\text { "...playing football's pretty hard cause...you got to get past the } \\
\text { people who are doing skills" PB10 }\end{array}$ & 0 & 2 & 3 & $7^{\#}$ & \\
\hline & Running, hard effort & $\begin{array}{l}\text { "I think running because you run a long way, you get a little } \\
\text { tired, then you get sweaty then you can't do any more" PB01 }\end{array}$ & 0 & 1 & 5 & $6^{\#}$ & \\
\hline & $\begin{array}{l}\text { Riding a bicycle, hard } \\
\text { effort }\end{array}$ & $\begin{array}{l}\text { "I did cycling because if you go really fast you might be really } \\
\text { tired, and you might not want to do any more" PBO9 }\end{array}$ & 0 & 0 & 1 & $11^{\#}$ & \\
\hline
\end{tabular}

Figure legend: $\mathrm{P}=$ Primary, $\mathrm{B}=\mathrm{Boy}, \mathrm{G}=\mathrm{Girl}, \mathrm{n}=$ frequency count, $\mathrm{SED}=$ Sedentary, LPA = Light physical activity, MPA = Moderate physical activity, VPA = Vigorous physical activity. '\%” denotes percentage of participants correctly aligning to intensity level, “\#” denotes the representative verbal statement frequency count, ' $*$ ' denotes significant difference between gender intensity identification $(p<0.05)$ 
Table 5.4 Adolescents' perceptions and ability to identify activities to intensity

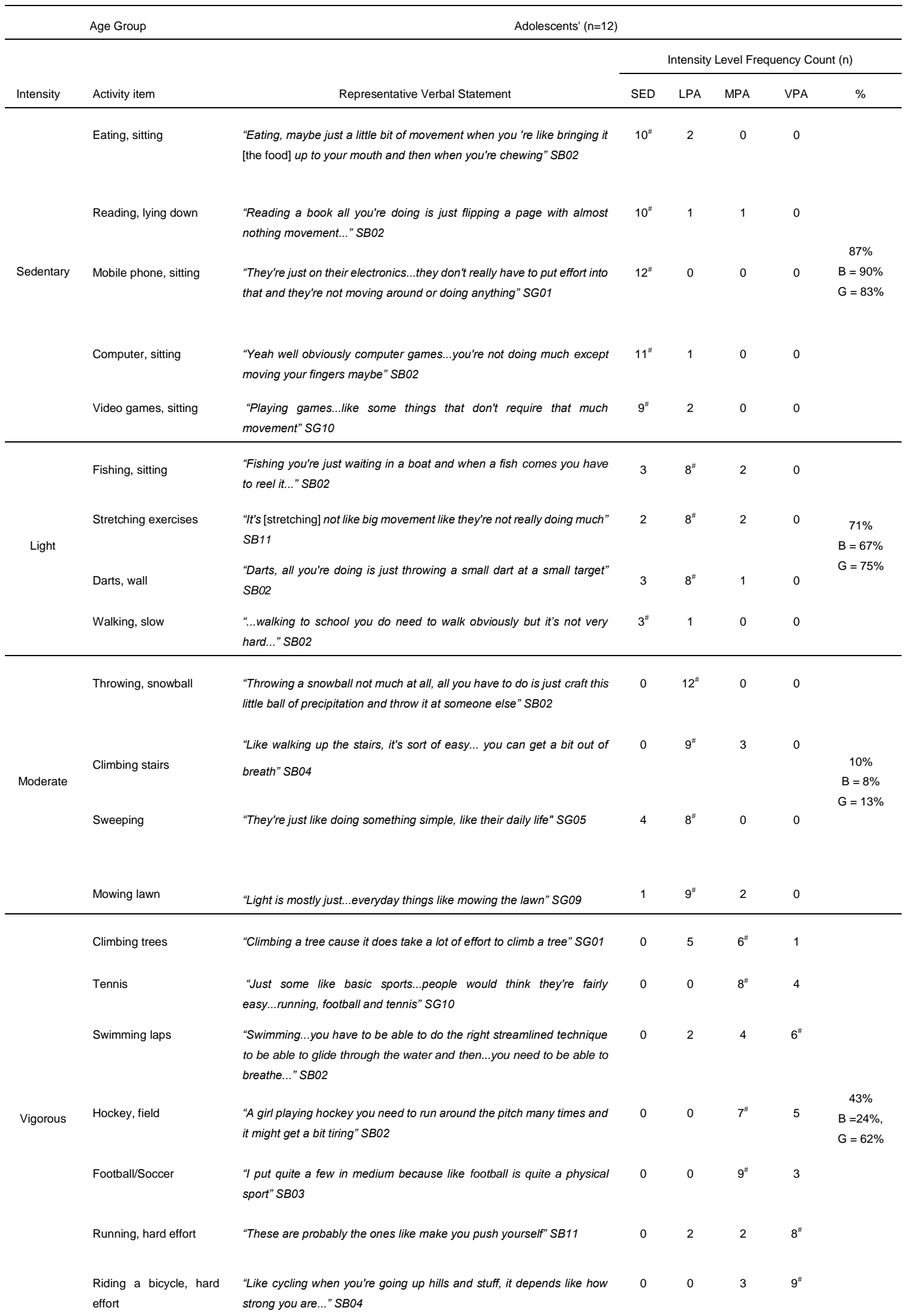

Figure legend $\mathrm{S}=$ Secondary, $\mathrm{B}=$ Boy, $\mathrm{G}=$ Girl, $\mathrm{n}=$ frequency count, $\mathrm{SED}=$ Sedentary, LPA = Light physical activity, MPA = Moderate physical activity, VPA = Vigorous physical activity. ‘\%' denotes percentage of participants correctly aligning to intensity level, “\#” denotes the representative verbal statement frequency count, ' $*$ ’ denotes significant difference between gender intensity identification $(p<0.05)$ 


\subsection{D Model Understanding}

\subsubsection{Children's Understanding and Ability to Interpret the 3D Model}

In total, six higher order themes were structured around the 3D model's components: 'Physical Activity Guideline Bar', 'Daily Physical Activity Bars', 'Moderate Physical Activity Bar', 'Vigorous Physical Activity Bar' and 'Separator of MVPA Bar' (Figure 5.2). A number of children (75\%) were able to interpret the physical activity guideline bar on the 3D model as "the 60-minute time bar" (PG10). All children correctly identified that the 3D model represented a week of physical activity "Monday they did a lot [of physical activity], on Tuesday they did a tiny bit, on Wednesday they did a tiny bit less..." (PG05). The data revealed that 58\% of children had some difficulty interpreting the moderate physical activity bar on the 3D model, with children expressing the bar as "...the easy activity to be doing because you do easy more than hard..." (PB01). Only 42\% of children were able to correctly interpret the moderate physical activity bar as the "Medium activity..." (PG05). Ten children (83\%) correctly interpreted the vigorous physical activity bar as "how much you've done of the hard level [of physical activity]" (PG11), with only two children incorrectly interpreting the bar as the time at which the physical activity was undertaken "the morning [of physical activity] and that might be the afternoon [of physical activity]". The circle separator along the Sun rays splitting the moderate and vigorous physical activity bars was correctly interpreted by $67 \%$ of children as "the blob splits the line up, so you know how many of the hard activity and how many of the medium activity" (PG11). Only two children expressed that they did not understand the meaning of the moderateto-vigorous separator along the ray. 


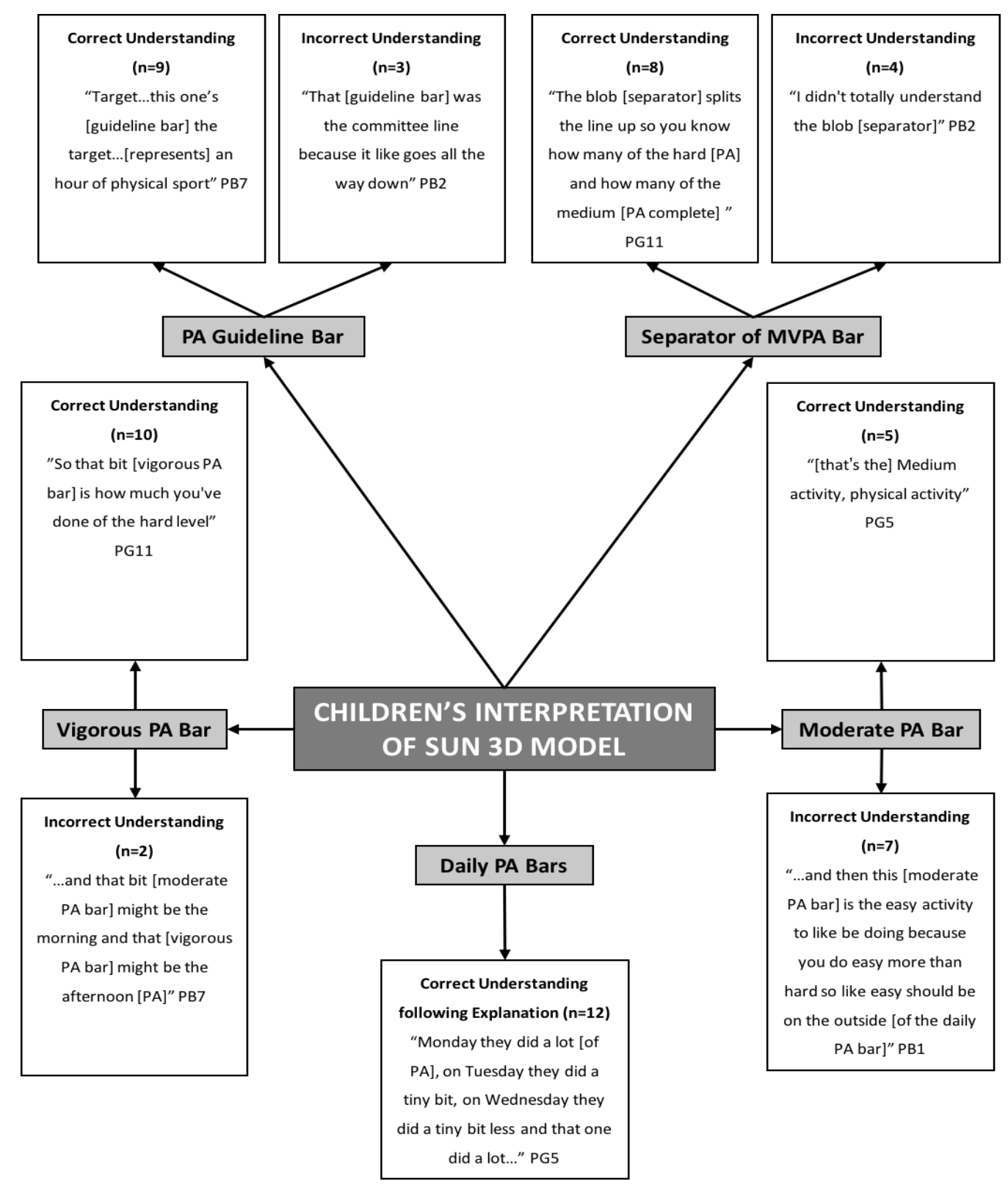

Figure 5.2 Children's interpretation of Sun 3D model

$\mathrm{P}=$ Primary, $\mathrm{B}=\mathrm{Boy}, \mathrm{G}=$ Girl, $\mathrm{PA}=$ Physical Activity, $\mathrm{N}=$ frequency counts

\subsubsection{Adolescents' Understanding and Ability to Interpret the 3D Model}

Five higher order themes were identified around the 3D model components: 'Physical Activity Guideline Bar', 'Daily Physical Activity Bars', 'Moderate Physical Activity Bar' and 'Vigorous Physical Activity Bar' (Figure 5.3). The physical activity guideline bar was correctly interpreted by $83 \%$ of adolescents as "that's the amount [of physical activity] you need to be doing or more... sixty minutes a day" (SP12), with only two participants unable to identify the meaning of the target bar. All of the adolescents had a good understanding of the physical activity data being represented as a week, with 
$42 \%$ of those adolescents able to interpret the data without any previous explanation or guidance from the facilitator. The moderate-intensity physical activity bar was correctly reported by $75 \%$ of adolescents as "...the moderate activity that you [themselves] were doing" (SG01), with only three participants incorrectly defining it as "how much sport you [themselves] have done" (SG6). All adolescents demonstrated a good understanding of the vigorous-intensity physical activity bar, stating “...this means how much hard activity you [themselves] are doing...” (SB03).

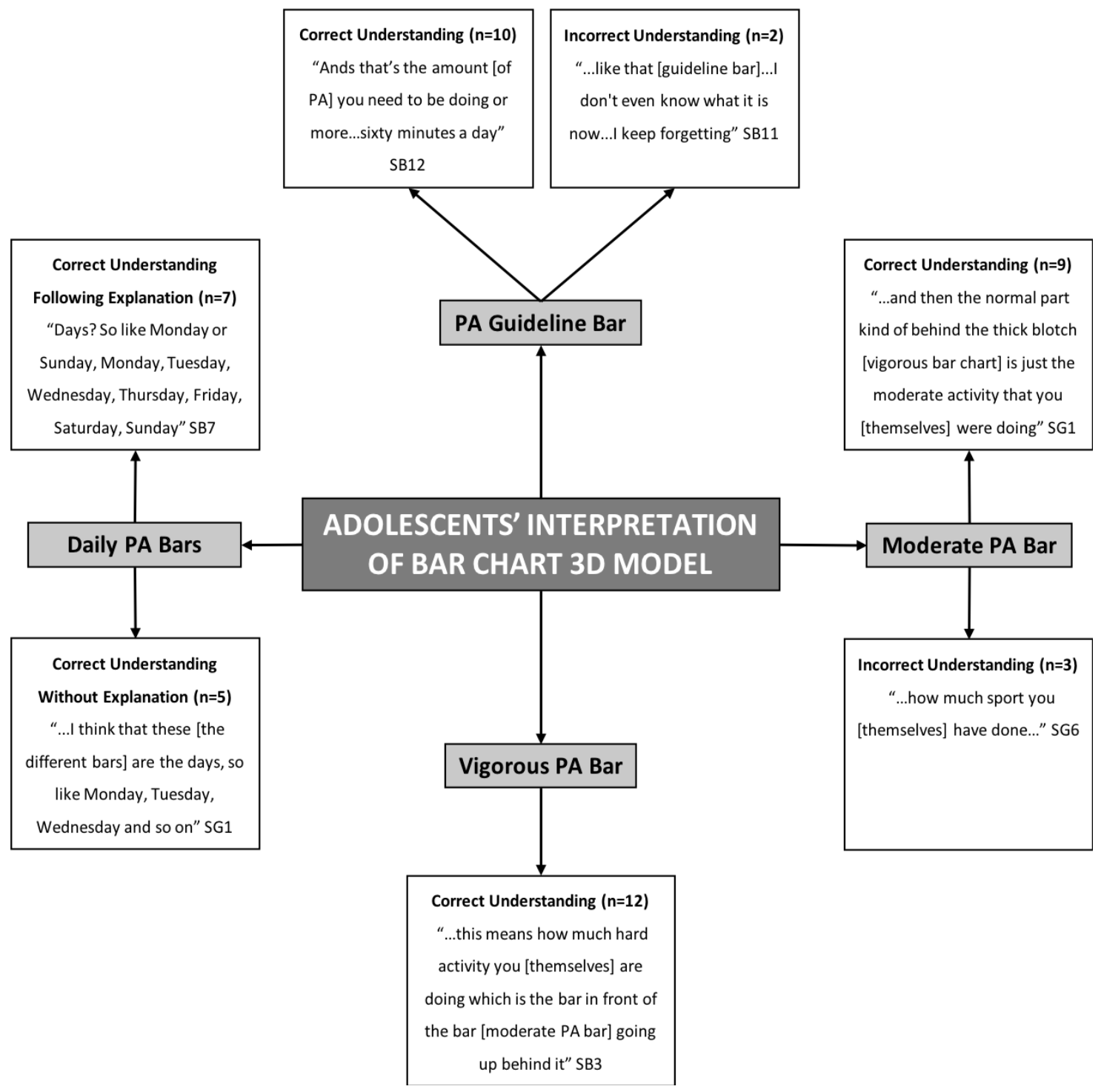

Figure 5.3 Adolescents' interpretations of bar chart 3D model

$\mathrm{S}=$ Secondary, $\mathrm{B}=\mathrm{Boy}, \mathrm{G}=$ Girl, $\mathrm{PA}=$ Physical Activity $\mathrm{N}=$ frequency counts 


\subsection{Discussion}

The aims of this study were to ascertain youths' understanding of the age-specific 3D model designs and to examine youths' perceptions and ability to identify activities according to their respective intensity. The present study findings suggest that youths demonstrate misconceptions in defining different activity intensities. However, youths' ability to interpret the age-specific 3D models supports the use of these formatively-designed tangible representations of physical activity within an intervention to aid youths understanding and awareness of the recommended 60 minutes MVPA (Study 2).

To date, little research has explored how youths understand the meaning of the term 'physical activity' (Brustad, 1991, Cardinal et al., 1998, Pearce et al., 2008, Trost et al., 2000a). It has previously been suggested that the terminology developed by adults used to describe physical activity is too complicated for youths, due to developmental and vocabulary differences (Pearce et al., 2008). As highlighted by Pearce et al. (2008), understanding how children express physical activity is a logical first step for improving overall knowledge and the development of innovative methods for enhancing physical activity. In the present study, the intensity-matching task revealed that adolescents have a greater ability to identify sedentary behaviours and lightintensity activities, whilst children showed they could more accurately identify the two extremes of intensity (i.e., sedentary behaviours and vigorous-intensity activities). It could be expected that as a result of children's sporadic and explosive patterns of activity (Sleap \& Warburton, 1996, Welk et al., 2000, Baquet et al., 2007, Adamo et al., 2009, Janssen \& LeBlanc, 2010b), moving from one extreme intensity to another, could explain, in part, the present findings demonstrating children's limited ability to identify the intermediary light and moderate-intensity activities. Furthermore, the present study showed that only $25 \%$ of children thought that working on the computer was a physical activity, with no children characterizing 'sweeping' as a sedentary behaviour when compared to Trost et al.'s (2000a) findings of $38 \%$ and $30 \%$, respectively. Whilst Trost et al. (2000a) encompassed a larger sample of 9-10 year olds, such discrepancies may be due, at least in part, to the reduced sample size and wider age range in the present study. Conversely, it could be argued that the timeframe in which the research was implemented could play an influencing role on youths' 
understandings of physical activity. For example, since the mid-2000s, the number of campaigns with mass media components have led to an increased level of exposure to the importance of physical activity behaviours within youths, with evidence supporting this exposure-response relationship (Economos et al., 2007, Huhman et al., 2007a, Sanigorski et al., 2008).

The majority of children tended to over-estimate light-intensity activities, such as stretching exercises $(75 \%)$, darts $(58 \%)$ and fishing (50\%). In some cases, children would associate stretching exercises with other more demanding activities, such as "when I do rugby you have to warm-up..." (PB07). This type of category contamination was a recurring theme within children, with other activities, such as throwing (light) and climbing trees (vigorous) being associated with “...running around..." (PB01). Other examples include fishing being of vigorous-intensity "cause you have to bring a boat down there [to the lake]" (PB02) rather than just the fishing effort alone, with the back and forth nature of darts being associated as a moderateintensity activity "cos you do move quite a bit, cause you throw [the dart], see what you got, get it [the dart] again, throw, get it again..." (PB06). In most cases, this category contamination led to an increase in intensity of the dominating activity (i.e., going from light- to moderate-intensity). Furthermore, light-intensity activities, such as darts and fishing, were often inaccurately identified because of the perceived skill or competence required to complete the activity. Specifically, playing darts was considered a vigorous-intensity activity as it required a certain skill to "...get it [the dart] in the middle [of the dart board] " (PG05), with fishing associated with moderate -intensity because it's “...quite hard to catch fish” (PB02). Skill-level was identified as a common characteristic for other activities, including football, hockey and riding a bicycle because you need to "know how to play the game" (PB07) or "it's not that easy to play" (PG05) and "you have to learn how to cycle without stabilisers" (PB01), respectively. Consistent with previous findings (Pearce et al., 2008), this study emphasises that skill in an activity, or physical competence, may have influenced children's perceptions of the intensity level. It could be speculated that children's perspectives of these skill- or physical competence-associated activities, are likely to undergo change and refinement as a consequence of time with personal experience and maturation (Brustad, 1991). However, this perspective appears unconvincing, as evidence suggests that adults also have a lack of knowledge when it comes to 
determining intensities of physical activity (Knox et al., 2013b). Given that one child even stated that reading a book was a vigorous-intensity activity because "... you have to learn how to read words" (PB06), it could be conceivable that children were simply just misinterpreting light-, moderate- and vigorous intensity as activities they personally find easy, average/normal or hard to do, respectively. This interpretation may have been reinforced by the hierarchical structure of the intensity matching task (i.e., linear path from sedentary to vigorous-intensity; Harris \& Brown, 2010), whereby children are just placing activities along a predetermined difficulty scale. In contrast, more recent evidence has demonstrated that children are able to accurately define the intensity of an activity using the OMNI exertional scale (Gammon et al., 2016). Therefore, this demonstrated inability to define intensities further highlights the importance of educating youths about different intensities of physical activity, so that as they age, their understanding of physical activity is more likely to reflect the actual intensity according to those associated with the recommended guidelines.

In the present study, youths demonstrated a limited ability to correctly identify moderate-intensity activities, although the degree of this inaccuracy was much greater in adolescents. It could be postulated that youths' inability to identify moderateintensity activities could be aligned to their limited capacity to describe how a physical activity could be performed at different intensities or effort levels (Ridley et al., 2008). In contrast, it could be argued that youth (aged 9-13 years) are reasonably good at identifying the intensity of their activities based on biofeedback using the OMNI exertional scales within a laboratory setting (Gammon et al., 2016, Pfeiffer et al., 2002, Robertson et al., 2005, Robertson et al., 2000). However, within non-laboratory settings, there is little evidence to support youths being able consider the intensity level of various physical activities (e.g., running and star jumps) with problems arising when defining the intensity of routine moderate-intensity activities, such as walking to school (Harris et al., 2016). In a similar way, the present study showed that youths underestimated the intensity levels of moderate activities related to either household chores, such as sweeping and mowing the lawn, or the daily activity of climbing stairs. Adolescents described such moderate activities as "...everyday things like mowing the lawn” (SG09) and “...like it's easy” (SB04), with children suggesting, when climbing stairs that “...all you've got to do is lift a foot and put it on each step” (PB09). These findings support those of Trost et al. (2000a), as household chores and climbing stairs 
are not considered as important contributory sources of physical activity, with the present study further highlighting that this under-estimation increased with age. Nonetheless, it is perhaps pertinent to consider the applicability of some activities, such as household chores, as a lack of familiarity may have led to exaggerated inaccuracies with respect to the intensity of these activities (Li, 2016). For adolescents, the more commonly-performed individual sports (e.g., swimming, running and cycling) were correctly identified as vigorous-intensity, with team sports such as football, hockey and tennis perceived to be of a moderate-intensity. Indeed, evidence suggests that the more the activity is considered as play or fun, the less likely youths are aware of the intensity (Pearce et al., 2008). Although there is limited evidence of this within the present findings, it could be speculated that the greater level of social interaction during team sports (Brettschneider, 2001) and the perceived conception of these team activities being for play or fun, could function as a moderator to youths' ability to correctly assess the respective intensity (Pearce et al., 2008). The present findings highlight the need to further understand how context (i.e., social settings) mediates youths' ability to interpret intensities between team sports and the more individual pursuit sports. Additionally, research is warranted to investigate the potential differences between non-athletic youths and sports orientated youths' understanding and ability to conceptualise intensities.

Inconsistencies in youths' ability to correctly identify and understand different activity intensities observed in this study are important, especially given that children's selfreport physical activity questionnaires rely on youths' ability to correctly interpret activities in accordance to the intensity level (Pearce et al., 2008). The reliability and validity of data derived from measuring youths' physical activity using self-report questionnaires is problematic (Aggio et al., 2016, Chinapaw et al., 2010, MartinezGomez et al., 2009). From the present findings, it could be postulated that the inconsistent reliability and validity of physical activity questionnaires is, at least in part, youths' misinterpretations and lack of understanding of intensity, supporting the findings of LeBlanc and Janssen (2010a). Adding to this dilemma, is that youth may find it difficult to quantify participation in physical activity of various intensities until they have gained enough experience in and reporting of these activities (Haas \& Nigg, 2009). Given that only $20 \%$ of youth in the UK are currently meeting the government guideline (National Centre for Social Research, 2017a), this could mean that a large 
percentage of youth are not gaining the invaluable experience of the health rewarding moderate- and vigorous-intensity activities, and as a consequence, be a contributing factor towards the poor accuracy of self-reported questionnaires. Additionally, although direct comparisons between sexes failed to demonstrate any significant difference in the ability to align activities with their respective activity intensities, the present findings do suggest that girls, irrespective of age, outperformed boys. For example, adolescent girls outperformed their counterparts in correctly identifying light-, moderate- and vigorous-intensity activities. Interestingly, girls in the younger age group outperformed boys in correctly identifying sedentary and light-intensity activities, though the imbalance in the number of girls $(n=3)$ to boys $(n=9)$ may limit such comparisons. Indeed, these preliminary findings could be explained by differences in cognitive development, supporting that girls have greater verbal and written language skills (Lynn, 1992, Mann et al., 1990, Martin \& Hoover, 1987, Undheim \& Nordvik, 1992) and acquire vocabulary faster (Roulstone et al., 2002) than boys until adulthood. As a consequence, it could be postulated that girls' greater level of vocabulary may put them at an advantage in aligning activities to intensities. Indeed, this is especially pertinent given that previous research has shown that girls provide more reliable and valid recollections on physical activity questionnaires than boys (Rangul et al., 2008). More research is warranted to determine whether these verbal and written advantages in girls do indeed play a significant role in the understanding of physical intensities and, if so, how this may impact upon self-report questionnaires and how best we account for these sex differences.

One method that has the potential to develop youths' comprehension of PALs and associated activity intensities is the use of personalised tangible interfaces (i.e., 3D models) to aid learning (Marshall, 2007). The present findings support this notion, with youths demonstrating a good ability to interpret and understand the age-specific $3 \mathrm{D}$ models, which is an important step towards enabling a cognitive experience whereby they can start to learn about their physical activity habits (Forlizzi \& Battarbee, 2004). Indeed, previous research has suggested that physical materials can promote playful learning in youth and might offer a more natural interaction than other types of learning interfaces (i.e., digital; Dourish, Jacob et al., 2002, Triona \& Williams, 2005, Price et al., 2003). More specifically, tangible objects can enable collaborative interactions with significant others (Fernaeus \& Tholander, 2006, Suzuki 
\& Kato, 1995), which coincides with an individual achieving social-interaction more readily to that gained from on-screen digital displays (Svendsen, 1991). Moreover, evidence suggests that physical activity with the social support of significant others can significantly increase youth's motivation for physical activity (Salvy et al., 2009), as well as their enjoyment (Jago et al., 2012, Salvy et al., 2012), intensity (Barkley et al., 2014) and overall engagement in and out of school (Pearce et al., 2014), which holds promise for the 3D models. Equally important, a large proportion of youths (79\%) could correctly identify and describe the current physical activity guidelines projected on the $3 \mathrm{D}$ models. Youths' ability to understand the physical activity guideline as a tangible representation will offer a more haptic and proprioceptive experience than visual representations alone (Gillet et al., 2005), which is especially pertinent given that youths' are regarded as visual and tactile learners (Rita \& Dunn, 1979). It is anticipated that the 3D models will act as a form of concept map, whereby youths can make connections, relationships and understand that the concepts about physical activity are not just factual, but ideas to increase comprehension and expand vocabulary (Butzow \& Butzow, 2000). Although, adolescents demonstrated a greater understanding of the age-specific 3D models and the different representations of physical intensities when compared to children, which could be explained by the Piagetian developmental theory (Piaget \& Cook, 1952). Evidence suggests that the exploratory nature of learning through tangible interfaces, such as $3 \mathrm{D}$ models of physical activity, may offer a more supportive solution to enhancing children's understanding in identifying patterns (i.e., between activities and intensities), and new concepts about physical activity than previous digital methods (Marshall, 2007). In this context, the present findings support the use of the age-specific 3D models within a school-based intervention, whereby youths receive a personalised 3D model of their objectively assessed PALs to not only enhance understanding of the recommended guidelines and associated intensities, but also as a unique motivational strategy to increase their physical activity.

Whilst data saturation was reached throughout both datasets (i.e., intensities and model understanding) and lends further credibility to the present findings, the study is limited by the relatively small sample size, age range and the geographical area of data collection, which may under-represent other social-economic groups and ethnic minorities. Furthermore, within children, sex difference comparisons regarding the 
understanding of intensities may be limited given the small number of girls who participated in the study. Therefore, the present findings on youths' understanding of the age-specific 3D models and demonstrated ability to identify activities to respective physical intensities should not be generalised but considered as a stimulus for future investigation.

\subsection{Conclusion}

This study shows that both children and adolescents have misconceptions when identifying corresponding activity intensities. Specifically, children showed recurring intensity classification errors, such as category contamination and perceived skill or competence of an activity leading to misperceptions of intensity, with both age groups severely underestimating moderate-intensity activities. However, youths demonstrated a good ability to interpret and describe the age-specific 3D model representations of physical activity, intensity and the recommended guideline. Therefore, this study highlights the potential utility of these age-specific 3D-printed models within an intervention to act as an educational tool to enhance youths understanding and awareness of the recommended physical activity guidelines and associated intensities. 


\section{Thesis Map}

STUDY

\section{OUTCOMES}

1. Energy Expenditure Associated with Walking Speed and Angle of Turn in Children

\section{Aim}

- To investigate the influence of walking speed and angle, and their interaction, on the energy expenditure of healthy children.

- Significant difference to straight-line walking energy expenditure within speed found at $2.5 \mathrm{~km} \cdot \mathrm{hr}^{-}$

Key

Findings ${ }^{1}$ at $90^{\circ}$ turn, and speed $3.5,4.5$ and $5.5 \mathrm{~km} \cdot \mathrm{hr}^{-1}$ at $180^{\circ}$ turn.

- Estimations of children's habitual physical activity should account for the magnitude and frequency of turns complete.
2. Perceptions of Visualising Physical Activity as a 3D-printed Object: A Formative Study

\section{Role of DESIGN PARTNERS}

\section{Role of TESTER}

4. The Tangibility of Personalised 3Dprinted Feedback may Enhance Youths' Physical Activity Awareness

Role of $\underline{U S E R}$

3. Understanding Youths' Ability to Interpret 3D-printed Physical Activity Data and Identify Associated Intensity Levels

\section{Aim}

-

To elicit children's, adolescent's, parents' and teachers' perceptions and understanding of 3D physical activity objects to inform the design of future 3D models of physical activity.

- Youths demonstrated a good ability to conceptualise physical activity as a 3D-printed object and highlighted the potential of $3 \mathrm{D}$ models as a motivational tool.

- Two age-specific 3D models of physical activity were developed from Children's preference for abstract designs and adolescents' bar chart designs

\section{Aim}

- To elicit children's and adolescent's interpretations of two age-specific 3D models displaying physical activity.

- To assess children's and adolescent's ability to appropriately align activities to the respective intensity of physical activity.

- Youths demonstrated a good ability to interpret their age-specific 3D model of physical activity.

Key Findings

- Youths showed misconceptions when identifying activities to respective intensities, particularly, moderate-intensity household chore activities.
Aim

- To investigate the efficacy of 3D-printed models to enhance youths' awareness and understanding of physical activity, as well as a motivational tool.
Key

Findings 


\section{CHAPTER 6}

3D-Printed Physical Activity to

\section{Enhance Youths' Awareness}

60 MVPA

\section{Tuesday} रे

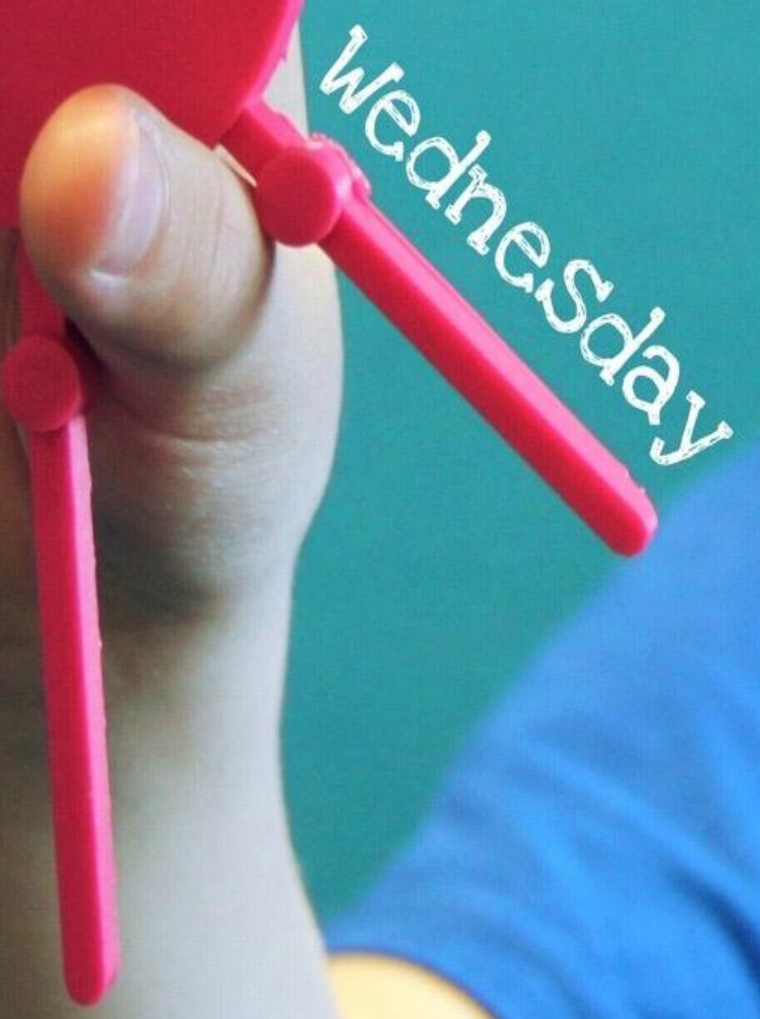




\section{The Tangibility of Personalised 3D-Printed Feedback may Enhance Youths' Physical Activity Awareness}

\subsection{Introduction}

The UK Government recommends that youths (children and adolescents) aged 5 to 18 years should engage in 60 minutes moderate-to-vigorous physical activity (MVPA) every day (Department of Health, 2011b) to accrue associated physiological (Sothern et al., 1999, Janssen \& Leblanc, 2010a) and psychosocial health benefits (Nieman, 2002, Eime et al., 2013). However, only $23 \%$ and $20 \%$ of boys and girls, respectively, aged 4-15 years in the UK meet these minimum levels of physical activity (Health Survey for England, 2017b), with almost 50\% of youths failing to achieve even half the recommended amount (Graig et al., 2009). Frequently cited reasons for youths underachievement of the physical activity guidelines are thought to be their lack of awareness of their physical activity levels (PAL; Kremers et al., 2008, Corder et al., 2010, Xu et al., 2017) and understanding of what activities and different intensities of physical activity 'count' towards the daily target (Knox et al., 2013b, Snethen \& Broome, 2007, Pearce et al., 2008, Noonan et al., 2016, Cowden \& Plowman, 1999, Prochaska et al., 2001, Placek et al., 2001, Harris et al., 2016). Given that adults also show a lack of awareness of their PAL (Godino et al., 2014), limited knowledge of their respective physical activity target and struggle to appropriately identify activity intensities (Knox et al., 2013b), addressing this during childhood is important for fostering healthy lifestyle behaviours that can continue into adulthood (Telama, 2009, Telama et al., 2005).

Based on Weinstein's (1988) Precaution Adoption Process Model (PAPM) from the Stages of Change (Prochaska \& DiClemente, 1992), an individual can only be expected to proceed to the contemplation stage of change when they become aware that their behaviours are not optimal, such as I do this much MVPA but this much MVPA is recommended'. In a similar way, the Goal Setting Theory (Locke \& Latham, 
1990) notes that setting specific and challenging, yet achievable, goals, in conjunction with feedback regarding performance towards goal attainment, is important to enhance an individual's self-efficacy (i.e., an individuals' belief to carry out a behaviour) and health behaviour change. In this respect, personalised feedback that represents an individual's PAL in contrast to the recommended level of activity (i.e., acting as a goal) is recognised as an important method for raising one's awareness of their physical activity behaviours and eliciting subsequent behaviour change (Michie et al., 2009). Therefore, for health education to be successful in youths, efforts must first raise the awareness and understanding of their PAL in the form of personalised feedback (Kremers et al., 2008) that supports goal-attainment (i.e., meeting the recommended guideline; Van Hoye et al., 2012). To make personalised feedback effective, it is important that it is visually stimulating and meaningful to the individual (Edwards et al., 2002, Houts et al., 2006), as 'seeing' makes knowledge credible (Bloch, 2008), and greater visibility of feedback contributes to an added responsibility to act (Viseu \& Suchman, 2010). The majority of personalised feedback is presented through digital, on-screen displays (e.g., smartphones or activity tracker displays; Ridgers et al., 2016, Dean et al., 2016, Hayes \& Van Camp, 2015, Jacobsen et al., 2016, Schaefer et al., 2016, Hooke et al., 2016, Gaudet et al., 2017), however, with recent advancements in 3D printing technology, Khot et al. (Khot et al., 2013) explored an innovative approach to displaying adults' heart rate data through tangible 3Dprinted artefacts to represent a day of physical activity. This novel approach demonstrated that the visual and tactile nature of the feedback increased adults' awareness of and reflection on their personal physical activity (Khot et al. 2013). Indeed, within youth populations, prior research has demonstrated that tangible interfaces can increase youths' engagement and reflection in active learning (Price et al., 2003, Rogers et al., 2002a), with several learning theories placing emphasis on tangibles as tools to stimulate intellectual development in youths (Rita \& Dunn, 1979, Piaget \& Cook, 1952, Fleming \& Mills, 1992). Building on these conclusions, more recent formative research has demonstrated that youths have the ability to conceptualise physical activity data represented as 3D-printed objects (Study 2). Moreover, two age-specific 3D model representations of youths' physical activity data were developed from the formative research (Study 2), which were further validated as a potential tool to increase youths' awareness and understanding of physical activity and the recommended guideline (Study 3). However, the efficacy of the designed age- 
specific 3D models in a real-world setting as a tool to enhance youths' awareness and understanding of physical activity is currently unknown.

In accord with Forlizzi and Battarbee (2004), understanding how a user's experiences change over time in connection to a newly designed product is an essential for developing the scalability and potential use of the technology in a realistic context. The user's experience, within the context of technology, is defined by a user's internal state (perceptions, expectations, motivation and mood), the characteristics of the product (usability, functionality and purpose) and the context (organisational or social setting) within which the interactions occur with the technology (Hassenzahl \& Tractinsky, 2006). More recently, video interview methods have become increasingly popular among researchers to assess a user's experiences, understanding and navigation of newly designed technology (Lopes, 2016, Masteller et al., 2017, Schaefer et al., 2016). However, these aforementioned video interviews have either been long in duration (e.g., 60 minutes; Lopes, 2016, Schaefer et al., 2016) and/or have been implemented with small numbers of individuals (e.g., 16-22 participants; Masteller et al., 2017, Schaefer et al., 2016), which may affect the generalisability of findings.

Therefore, the aim of the present study was to examine the efficacy of the age-specific 3D-printed models to enhance children and adolescents' levels of awareness, understanding of and motivation for physical activity during a 7-week faded intervention, whereby youth receive personal 3D-printed models displaying their PAL. It is hypothesized that receiving personalised 3D-printed physical activity feedback will enhance youths (i) awareness of their MVPA levels in comparison to the government guideline of 60 minutes of MVPA (ii) understanding of what constitutes as physical activity and of moderate- and vigorous-intensity activity and (iii) motivation to be more physically active. 


\subsection{Methods}

\subsubsection{Recruitment}

The recruitment strategy involved sending emails, detailing the research project to the Head of School (primary schools) or Head of Physical Education (PE) departments (secondary school). In total, three primary schools and one secondary school from South Wales, UK, expressed an interest in participating in the intervention study ( $n=3 / 4$ response rate), from which two primary schools and one secondary school took part in the study. A school assembly for the intervention target year groups (years 3 and 9) was organised in all of the participating schools to introduce the study with information sheets and parental/guardian consent and child assent forms distributed. Typically developing children (aged between 7-9 years old) or adolescents (aged between 13-15 years old) and adolescents, which could include overweight or obese, or disadvantaged pupils, who were free from injury and illness, were allowed to participate in the study. All schools were either community (primary) or comprehensive (secondary) schools. From Estyn reports (Her Majesty's Inspectorate for Education and Training in Wales, 2017), the primary schools sizes ranged from 205 to 352 pupils, with eligibility for free school meals ranging from $4 \%$ to $35 \%$. The secondary school constituted of 1,105 pupils with 7\% eligibility for free school meals which is below the national average of $19 \%$. A flow chart outlining the school recruitment process, intervention allocation, interview completion rate and accelerometer wear time compliance is included in Figure 6.1.

\subsubsection{Participants}

In total, 97 youths participated in the study, of which 39 were primary school children (22 boys; $7.9 \pm 0.3$ years) and 58 secondary school adolescents (37 boys; $13.8 \pm 0.3$ years). All primary school children were White British, with $96 \%$ of secondary school adolescents being White British, with the remaining 4\% being Asian (2\%; $n=1)$ and Black British $(2 \% ; n=1)$. All participants returned informed parental/guardian consent and child assent prior to participation. Ethical approval was granted by the University Ethics Committee and conducted in accordance with the Declaration of Helsinki (ref: PG/2014/40). 


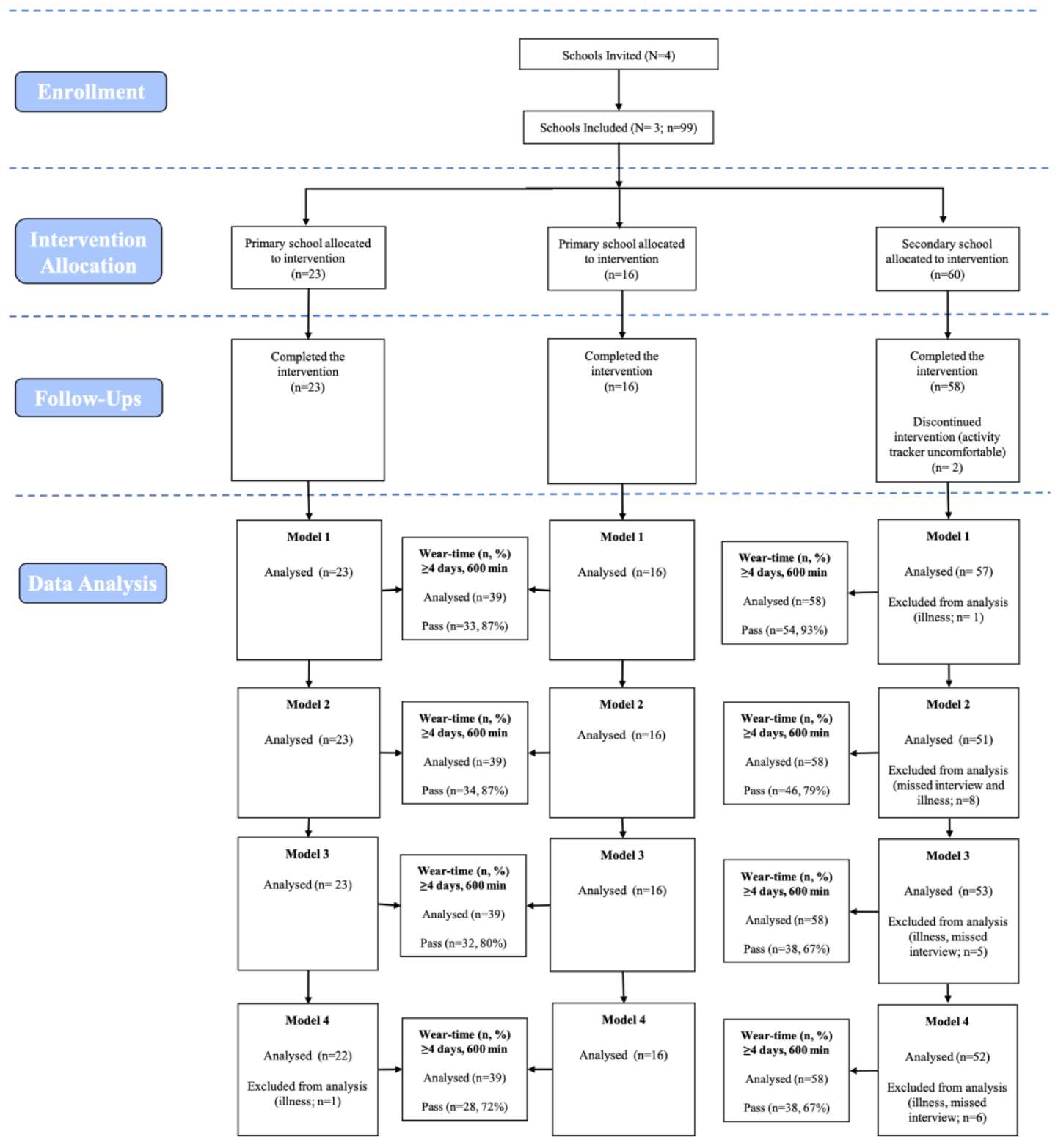

Figure 6.1 Flow chart outlining the school recruitment process, intervention allocation, intervention interview completion rate and accelerometer wear-time compliance; $\mathbf{N}=$ schools; $\mathbf{n}=$ total participants

\subsubsection{Intervention Design}

The 3D printing physical activity intervention was informed by two previous, usercentred, qualitative approaches which explored the needs, preferences for content, designs and understanding of 3D-printed models among youth (i.e., children and adolescents) as described in detail elsewhere (Study 2, Study 3). To encourage lifestyle change, the intervention was theoretically based, in part, on the notion of youths being visual and tactile learners (Marshall, 2007, Rita \& Dunn, 1979, Rogers et al., 2002b), 
with an emphasis on the PAPM (Weinstein, 1988) and Goal-setting theory (Locke \& Latham, 1990) as ideologies to enhance awareness of behaviours in relation to setgoals through personalised feedback that encompasses a physical incentive. The intervention was implemented for 7 -weeks to align with the school term time. The intervention was designed to objectively measure youths weekly PAL and use this data to generate personalised age-specific 3D-printed models to represent the moderate and vigorous PAL achieved each day across a week, as well as displaying the physical activity guideline of 60 minutes MVPA (Figure 6.2). The intervention employed a novel approach that involved participants receiving a total of four respective agespecific 3D-printed models over the course of the 7-week intervention according to a faded intervention design. For example, youths received their 3D models following baseline (model $1=\mathrm{M} 1)$ and week 1 (model $2=\mathrm{M} 2)$, week $3(\operatorname{model} 3=\mathrm{M} 3)$ and after week 6 (model $4=\mathrm{M} 4$ ). The faded approach has been proposed as a method for maximising the effectiveness of feedback contrary to frequent feedback that only provides short-term benefits (Goodman \& Wood, 2009). In this respect, the faded method is underpinned by starting with high levels of feedback and then, as the novice or participant begins to master the components of the task, gradually reduce or fade the feedback until the person is performing the task autonomously (Day et al., 2006, Edwards et al., 1995, Hesketh, 1997, Rock \& Thead, 2007, Schmidt \& Wrisberg, 2008). A key point to this faded design is to increase the sustainability and real-world 'implementability' of 3D-printing physical activity interventions by examining how the 3D models can be integrated into youth's everyday lives to determine the success of deployment and adoption of the models (Vassilev et al., 2015). Participants received their personal 3D-printed model approximately 1-3 days post physical activity measurement. Immediately following receipt of each $3 \mathrm{D}$ model, all participants completed an individual, semi-structured, short video interview conducted by the first author either during their PE class (i.e., secondary school) or in an appropriate quiet area within the school environment (i.e., primary school) to elicit information on study outcomes (Kennedy et al., 2001). Video interviews are considered a viable method for recording youths' experiences with technological designs (Masteller et al., 2017). All participants received one instruction manual (Figure 6.2) for their respective age-specific 3D model after completing their first short individual interview so to obtain baseline perceptions of primary outcome measures. To assess accelerometer wear-time compliance, data was analysed using Kinesoft 
version 3.3.67 (Kinesoft, Saskatchewan, Canada), employing 1 second epochs with sustained periods of at least 20-minutes of consecutive zeros considered to be nonwear time (Catellier et al., 2005). A minimum daily wear time of 600 minutes for any 4 days was used to calculate the compliance percentages for each time point (Trost et al., 2000b).
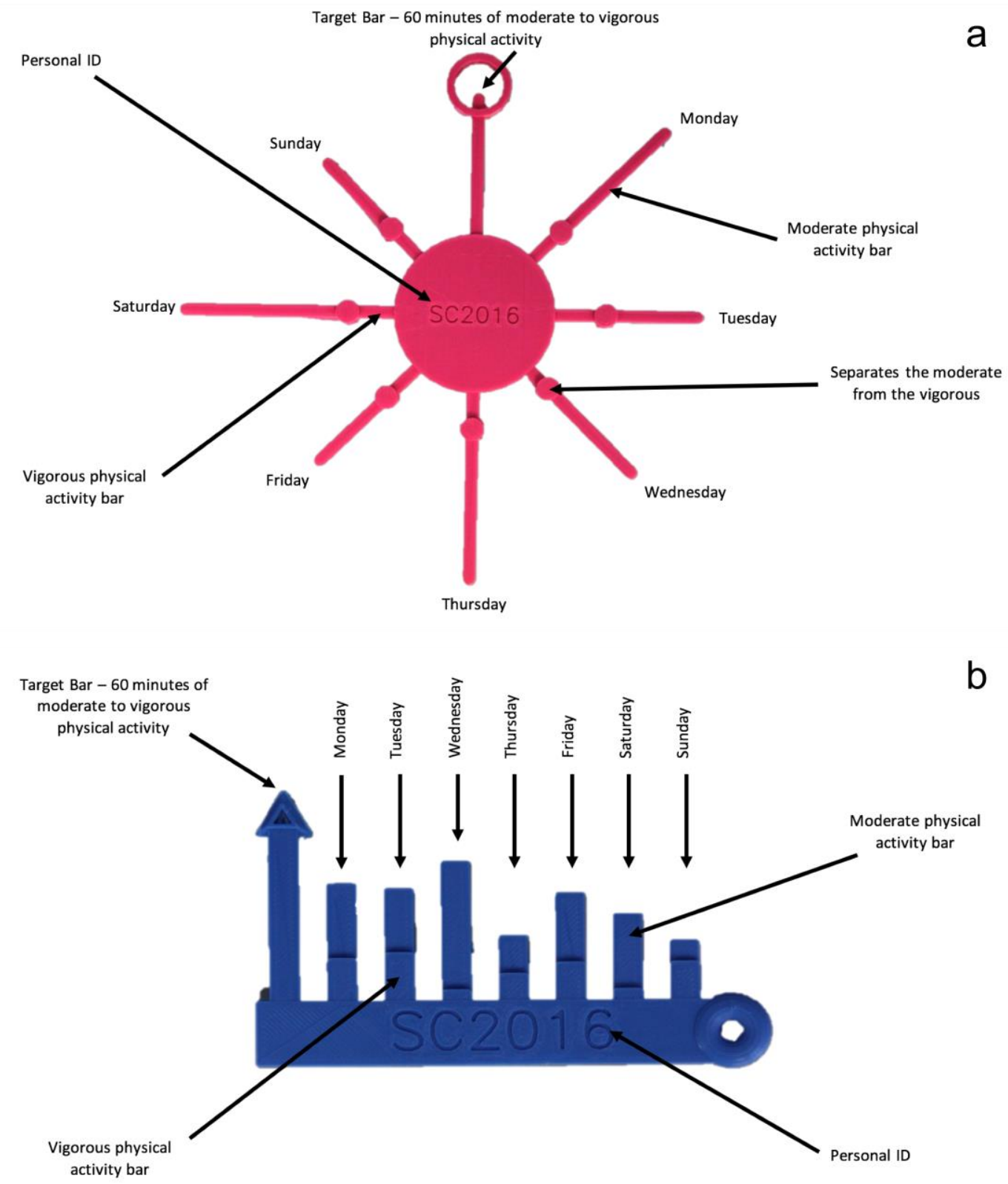

Figure 6.2 The age-specific 3D models of physical activity instruction manuals

a) Children's Sun 3D Model, b) Adolescents' Bar Chart 3D Model

$\mathrm{PA}=$ Physical Activity, MVPA = Moderate to Vigorous Physical Activity 


\subsubsection{Procedures}

\subsubsection{Anthropometrics}

All participant's standing stature, body mass and waist circumference were measured according to the techniques outlined by the International Society for the Advancement of Kinathropometry (Stewart et al.). Participants were required to be in minimal clothing (i.e., shorts and t-shirt) and without shoes. Body mass was measured to the nearest $0.1 \mathrm{~kg}$ using an electronic weighing scales (Seca 876, Hamburg, Germany), with stature assed to the nearest $0.1 \mathrm{~cm}$ using a portable stadiometer (Holtain Sitting Height Stadiometer, Holtain Ltd). Body mass index (BMI) and weight status was calculated from stature and body mass measurements as a proxy for adiposity (Cole et al., 2000). Based on BMI z-score calculations, UK age- and sex- specific BMI cut points were applied to categorise participants as underweight, normal weight, or overweight/obese (Stegenga et al., 2014). All anthropometric measurements were conducted within the school by trained male and female research assistants under supervision of SGMC. Some of the research assistants also played a role in the intervention delivery (e.g., handing out accelerometers and organisation of participants for interview).

\subsubsection{Measuring and 3D-Printing Physical Activity Data}

All participants were asked to wear the wGT3X-BT tri-axial accelerometer (ActiGraph LLC, Pensacola, FL) on an elastic belt positioned on their right mid-axilla line at the level of the iliac crest for seven consecutive days to provide an objective estimate of their PAL. Numerous studies have reported the wGT3X-BT tri-axial accelerometer to be a valid and reliable objective measurement of the quantity and frequency of physical activity (Trost, 2007, Hills et al., 2014a, Tudor-Locke et al., 2015), with previous research demonstrating that the hip placement is the most precise single location to detect everyday activities (Cleland et al., 2013, Tudor-Locke et al., 2015). All participants were shown a demonstration of the accelerometer hip-placement via SGMC and provided an information sheet regarding the use and safety of the device at baseline measurement. As far as was practically possible, participants wore the same accelerometer (serial number) at each time point, to remove 'between unit' variation (Robertson et al., 2010). Participants were instructed to wear the accelerometer all the 
time $\left(24 \mathrm{~h} \cdot \mathrm{day}^{-1}\right)$, except for when engaging in water-based activities (swimming, showering, and bathing) and contact sports. Accelerometers were initiated to run from midnight the day participants received the accelerometer until midnight seven days later and to record raw accelerations at a frequency of $100 \mathrm{~Hz}$. Data collection took place during the school term from January to April 2017, therefore, physical activity data was representative of usual winter/spring free-living activities.

Following collection of accelerometers, participants seven-day physical activity data were then downloaded and analysed using Actilife version 6.13.3 (ActiGraph LLC, Pensacola, FL). Given the intervention was designed to provide all participants with feedback on MVPA levels, even if the accelerometer was not worn at all times, no inclusion criteria were applied to the accelerometry data. Therefore, implications for youth not wearing the accelerometer on one or more days would result in them receiving a 3D model with no data displayed on that specific day. Each day's MVPA level was calculated using Evenson's child cut-points (2008b), which are known to provide the closest estimates of moderate- and vigorous-intensity PAL during freeliving measurement (Crouter et al., 2013). Participants MVPA levels and personal ID code (e.g., participant initials and model number) to distinguish participants personal age-specific 3D model, were then inserted in to the age-specific custom developed 3D model code loaded on OpenJSCAD version 1.8.0, and subsequently 3D-printed using ABSplus filament on the Objet 1000 (Statasys, United States, Eden Prairie, MN). The Sun and Bar Chart 3D models cost approximately $£ 3.20$ and $£ 6.60$ per print, respectively, based on the complexities of the model (e.g., participant ID engraving) and the infill used within the model (e.g., density).

\subsubsection{Short Individual Video Interviews}

Short, individual interviews were chosen as they lend greater control to the interviewer over the interview process relative to the unpredictable nature of focus group interactions (Morgan, 1996). Individual interviews also allow the researcher to locate specific ideologies within particular individuals (Deanscombe, 2010), which is not always possible within focus groups given that youth may tag onto the views of others without necessarily reflecting on the value or meaning (Lewis, 1992). To reinforce the interpretations of the qualitative data, each individual interview was filmed to capture 
youths non-verbal and contextual understandings of the 3D model that could be missed in a narrative statement alone (Banks, 2018). The interviews were semi-structured so that the facilitator could ask probing questions around the pre-defined topics and to keep discussions relevant to the study aims (Krueger \& Casey, 2014). The two interview types (children and adolescents) were conducted using the same research protocol and followed a pre-defined schedule of questions (see Table 6.1) sought to address concepts on youths; awareness of their PAL; understanding of intensities and interpretations of the 3D model; and motivational benefits and utility of the 3D models. A total of 369 interviews were digitally voice (Olympus DM-520 digital voice recorder, Shinjuku, Japan) and video (Sony Handycam HDR-PJ540, Minato, Japan) recorded, lasting $4.5 \pm 1.2$ and $2.2 \pm 0.6$ minutes, for children and adolescents, respectively. All interviews were transcribed verbatim, resulting in 816 pages (386 and 430 pages for children and adolescents, respectively) of raw transcription data, Arial font size 12, double spaced.

Table 6.1 Example interview questions

\begin{tabular}{ll}
\hline Topic & Examples \\
\hline
\end{tabular}

Motivation/Awareness of PAL What do you think of your first 3D model?

PA Understanding What you think physical activity means?

Awareness of PAL/Model Understanding How does your 3D-printed model show your physical activity?

Intensity Understanding What kind of activities might be vigorous and moderate physical activities?

Motivation/Model Utility

What will you do with your 3D model now?

PA = Physical Activity; PAL $=$ Physical Activity Level

\subsubsection{Data Analysis}

A Sharpio-Wilks test was used to confirm data normality within the anthropometric data sets. Once normal distributions were confirmed, independent sample t-tests were used to assess differences between sexes in children and adolescents. All statistical 
analyses were conducted using IBM SPSS Statistics 22 (Chicago, IL) and statistical differences was accepted at $p \leq 0.05$. A mixed-methods analysis was utilized to assess the qualitative outcomes to allow for greater insights into the meanings of the data (Holsti, 1969, Pool, 1959) and take into account the aims of the research regarding youths levels of awareness, understanding of and motivation for physical activity represented as 3D models (Hamad et al., 2016). Through the process of content analysis, transcripts were approached qualitatively to focus on the context of youths' awareness of their PAL and pre-understandings of intensities and the motivational aspects of the 3D models. To quantify patterns within the different time-points (i.e., receiving model 1 to 4 ), it was quantitatively noted as to the number of participants that were associated with specific statements and for the classification of categorical data being accurate (i.e., correct interpretations of the 3D model and activity intensities; Hamad et al., 2016). To aid in the identification of accurate classification of 3D model interpretations, interview videos were also assessed to examine participants non-verbal interactions with their 3D model by noting gestures (e.g., correctly points to the 60-minute MVPA guideline bar) within transcripts (Banks, 2018). All transcripts were thematically analysed by the first author, firstly by data immersion which involved 'repeated reading' of the transcripts in an active way searching and noting of meanings and patterns within the data set (Clarke \& Braun, 2013). Following the initial data immersion process, coding was undertaken, using a manual cut and paste technique, which allowed for the data to be organised into groups that were considered pertinent to the research questions. All codes were then sorted into potential themes, by collating all relevant coded data extracts to the newly identified theme. The frequency counts and themes with indicative quotes were then represented diagrammatically using a pen profile approach (Boddy et al., 2012, Mackintosh et al., 2011, Winn et al., 2017, Noonan et al., 2016), with percentages of youths expressing specific themes calculated from frequency counts. The first author discussed the identified themes with the last author to determine the existence of relationships within the data. Themes that did not have enough supportive data or were too diverse were discarded. The third author (MAM) critically cross-examined the data through reverse triangulation, from the pen profiles back to the transcripts, until all alternative interpretations of the data were exhausted. The pen profiles were then critically reviewed by all other authors, allowing further interpretations of the data until a final consensus was reached. 


\subsection{Results}

\subsubsection{Anthropometrics}

Participants anthropometric characteristics are displayed in Table 6.2. There were no significant sex differences between children, but adolescent boys were significantly taller and heavier than their counterpart girls. At baseline, $13 \%$ of children, both sexes, were overweight or obese with the remaining $87 \%$ (boys, $87 \%$; girls, $88 \%$ ) of children being classified as normal weight with no children being classified as underweight. For adolescents, 22\% (boys, 16\%; girls; 33\%) were overweight or obese and $78 \%$ (boys, 84\%; girls, 67\%) were within normal weight with no individuals categorised as underweight.

\section{Table 6.2 Participant anthropometric characteristics}

\begin{tabular}{|c|c|c|c|c|c|c|}
\hline & \multicolumn{3}{|c|}{ Primary } & \multicolumn{3}{|c|}{ Secondary } \\
\hline & Boys & Girls & Both & Boys & Girls & Both \\
\hline $\mathrm{n}$ & 22 & 17 & 39 & 37 & 21 & 58 \\
\hline Age, yrs & $7.9 \pm 0.3$ & $7.8 \pm 0.35$ & $7.9 \pm 0.3$ & $13.8 \pm 0.3$ & $13.7 \pm 0.3$ & $13.8 \pm 0.3$ \\
\hline Stature, $\mathrm{m}$ & $1.28 \pm 0.1$ & $1.25 \pm 0.1$ & $1.27 \pm 0.1$ & $1.66 \pm 0.1^{*}$ & $1.63 \pm 0.1$ & $1.65 \pm 0.1$ \\
\hline $\mathrm{WC}, \mathrm{cm}$ & $58.1 \pm 4.9$ & $59.6 \pm 5.1$ & $58.7 \pm 5.0$ & $73.3 \pm 6.0$ & $69.2 \pm 6.3$ & $72.1 \pm 6.4$ \\
\hline Body mass, $\mathrm{kg}$ & $26.1 \pm 3.5$ & $25.8 \pm 4.0$ & $26.01 \pm 3.5$ & $56.05 \pm 10.2^{*}$ & $55.8 \pm 6.8$ & $55.9 \pm 9.0$ \\
\hline BMI, kg·m-2 & $15.9 \pm 2.0$ & $16.6 \pm 2.4$ & $16.2 \pm 2.03$ & $20.2 \pm 2.4$ & $21.1 \pm 3.0$ & $20.55 \pm 2.7$ \\
\hline \multicolumn{7}{|l|}{ Weight Status, \% } \\
\hline Underweight & - & - & - & - & - & - \\
\hline Normal Weight & 87 & 88 & 87 & 84 & 67 & 78 \\
\hline Overweight/Obese & 13 & 13 & 13 & 16 & 33 & 22 \\
\hline
\end{tabular}

\subsubsection{Primary Outcomes}

The first model outcomes for children's and adolescent's data are combined and presented in one pen profile (Figure 6.4), as no different themes were found from independent analyses. To avoid duplicating the pen profiles and their identified key 
themes, Table 6.3 displays youths' frequency of occurrence of key themes for each of the four 3D models, with children, adolescents and sex independently split.

Following the first model, the majority of youths (80\%) expressed a high level of enthusiasm for their 3D model, expressing that its "really cool...because I've never seen a 3D-printed model" (PG06, M1). However, by the final model, only 4\% of children and no adolescents still expressed similar enthusiasm. Despite this, $28 \%$ of youths displayed satisfaction on how they were "very proud [of the model]" (PG07, M1) of their first 3D model, with this level of satisfaction increasing to $39 \%$ to $60 \%$ and $68 \%$, by the second, third and fourth models, respectively. Furthermore, youths demonstrated increased levels of reflection through the 3D models upon how they “...never thought Saturday was going to be that long” (PB35, M3), from 51\% to 60\% and $66 \%$, for the first, second and third models respectively, although by the fourth model, this level of reflection dropped to $58 \%$.

Overall, youths showed little difference in their interpretations of their meaning for physical activity (M1, 73\% to M4, 76\%), stating its "like doing sports and stuff that includes moving your body" (PB20, M2), with similar outcomes on their interpretations of the intensities of moderate (M1, 44\% to M4, 47\%) "like walking" (SB55, M2) and vigorous (M1, 81\% to M4, 81\%) "like sprinting so your heart rate is like beating at a fast pace” (SB45, M3). Moreover, across all time points, only 5\% of children and $17 \%$ of adolescents were able to relate the guideline bar accurately to " 60 minutes of exercise a day" (SG42, M3), with only a small proportion of adolescents $(5 \%)$ able to articulate the guideline of "... at least an hour of hard and moderate activity every day" (SB49, M3). However, youths demonstrated an accurate ability to interpret the basic components of the 3D models (e.g., days and high and low PAL) from the first (83\%) to the fourth model (88\%), such as "It [the model] means the days of the week and how much activity you've been doing" (PG31, M1), with adolescents being able to correctly distinguish "this one [vigorous bar] is the high-intensity sport activities and this one [moderate bar] is the more moderate sport activities" (SB03, M4). Moreover, youths were able to correctly interpret and identify with "the target bar...that shows how much exercise you should do in a day, which is one hour" (PB10, M3). As consequence, youths increasingly referenced their PAL to the guideline bar, adopting it as a goal-setting strategy from the first (18\%) to the second (46\%) and third models $(76 \%)$, with a small drop following the fourth model (73\%). Specifically, 
youths demonstrated this goal setting by stating how that their PAL had not "reached the target point [on] Monday" (SG09, M3) and "you have to try and be higher than that arrow [guideline bar] and that would be you reaching your target”' (SG35, M4). Conversely, some youths expressed inaccurate interpretations of their 3D models, however, this number dropped with time from the first (62\%) to the fourth model $(52 \%)$. Of note, were the small number of children $(26 \%)$ by the final model who were able to correctly interpret the moderate and vigorous bar representations, with children most commonly mistaking the bar as "the morning [vigorous bar] and that's the afternoon [moderate bar]" (PB08, M3). For adolescents, only 14\% demonstrated to incorrectly identify "the lower solid bar [vigorous bar] is walking activity, and the higher bar [moderate bar] is like sprinting activity" (SB52, M4).

In terms of the application of the 3D models, $11 \%$ of youths expressed they would "compare the next one [3D model] with it [the current model], and I'll try to do more exercise on Sundays" (SG14, M1), with this application of the models increasing following the second model (48\%), with no substantial change for time points thereafter. From the first model, $42 \%$ of youths demonstrated self-evaluation of their PAL on how "Ineed to improve certain days and do more on certain days than others" (SG32, M1), with this self-evaluation increasing to $61 \%$ to $68 \%$ for the second and third models, respectively. Interestingly, a higher percentage (81\%) of adolescent girls $(n=17)$ appeared to self-evaluate their PAL, expressing they would "see if there's anything I can change to get a higher activity than what I got”' (SG37, M2). 
Table 6.3 Youth frequency of occurrence of key themes

\begin{tabular}{|c|c|c|c|c|c|c|c|c|c|}
\hline \multirow[b]{3}{*}{ Themes } & \multicolumn{9}{|c|}{ Youths Frequency Count of Occurrence of Key Themes $(n=$} \\
\hline & \multicolumn{3}{|c|}{ Model $1\left(n(\%){ }^{G}\right)$} & \multicolumn{3}{|c|}{ Model $2(n(\%)$ G) } & \multicolumn{3}{|c|}{ Model $3\left(n(\%)^{G}\right)$} \\
\hline & Child & Adol & Total & Child & Adol & Total & Child & Adol & \\
\hline Enthusiasm & $30(37)^{15}$ & $48(84)^{19}$ & $78(80)$ & $15(39)^{4}$ & $4(7)^{2}$ & $19(20)$ & $9(23)^{3}$ & 0 & \\
\hline Level of Satisfaction & $8(21)^{3}$ & $20(35)^{6}$ & $28(29)$ & $10(26)^{2}$ & $28(49)^{14}$ & $38(39)$ & $20(51)^{10}$ & $38(67)^{16}$ & 58 \\
\hline Reflection & $13(33)^{6}$ & $37(65)^{12}$ & $50(52)$ & $24(62)^{7}$ & $38(67)^{18}$ & $62(64)$ & $20(51)^{9}$ & $44(77)^{15}$ & 64 \\
\hline Uncertainty & $2(5)$ & 0 & $2(2)$ & $2(5)$ & 0 & $2(2)$ & 0 & 0 & \\
\hline Definition & $26(67)^{7}$ & $45(79)^{18}$ & $71(73)$ & $27(69)^{9}$ & $41(72)^{14}$ & $68(70)$ & $32(82)^{11}$ & $42(74)^{17}$ & 74 \\
\hline Moderate Intensity & $5(13)^{2}$ & $38(67)^{15}$ & $43(44)$ & $15(39)^{5}$ & $34(60)^{12}$ & $49(51)$ & $9(23)^{6}$ & $36(63)^{15}$ & 45 \\
\hline Vigorous Intensity & $29(75)^{10}$ & $50(88)^{19}$ & $79(81)$ & $31(80)^{10}$ & $47(83)^{17}$ & $78(80)$ & $33(85)^{12}$ & $49(86)^{20}$ & 82 \\
\hline Accurate Interpretation & $34(88)^{14}$ & $47(83)^{18}$ & $81(84)$ & $36(92)^{14}$ & $46(81)^{17}$ & $82(85)$ & $35(90)^{14}$ & $51(90)^{21}$ & 86 \\
\hline Inaccurate Interpretation & $33(85)^{13}$ & $27(47)^{9}$ & $60(62)$ & $19(49)^{6}$ & $27(47)^{9}$ & $46(47)$ & $21(54)^{9}$ & $29(51)^{10}$ & 50 \\
\hline Comparisons & $1(3)$ & $10(17)^{3}$ & $11(11)$ & $20(51)^{8}$ & $27(47)^{10}$ & $47(49)$ & $20(51)^{6}$ & $30(53)^{13}$ & 50 \\
\hline Goal Setting & $10(26)^{2}$ & $7(12)^{3}$ & $17(18)$ & $21(54)^{9}$ & $24(42)^{8}$ & $45(46)$ & $31(80)^{13}$ & $43(75)^{16}$ & 74 \\
\hline Motivational Tool & $5(13)$ & $19(33)^{7}$ & $24(25)$ & $11(28)^{2}$ & $19(33)^{10}$ & $30(31)$ & $18(46)^{7}$ & $22(39)^{11}$ & 40 \\
\hline Recall and/or Relatedness & $18(47)^{6}$ & $42(74)^{15}$ & $60(62)$ & $19\left(49^{7}\right.$ & $38(67)^{7}$ & $57(59)$ & $28(72)^{12}$ & $40(70)^{16}$ & 68 \\
\hline Self-Evaluation & $11(28)^{4}$ & $30(53)^{11}$ & $41(42)$ & $20(51)^{6}$ & $39(68)^{15}$ & $59(61)$ & $22(56)^{9}$ & $44(77)^{18}$ & 66 \\
\hline Display & $23(59)^{10}$ & $29(51)^{10}$ & $52(54)$ & $25(64)^{10}$ & $21(37)^{5}$ & $46(47)$ & $28(72)^{14}$ & $23(40)^{7}$ & 51 \\
\hline Family & $8(20)^{4}$ & $7(12)^{4}$ & $15(16)$ & $5(13)^{2}$ & $4(7)^{4}$ & $9(9)$ & $5(13)^{2}$ & $7(12)^{6}$ & 12 \\
\hline Peers & $3(8)$ & $2(3)^{2}$ & $5(5)$ & $1(3)$ & $3(5)^{1}$ & $4(4)$ & $1(3)^{1}$ & $2(4)^{1}$ & \\
\hline
\end{tabular}

Figure legend $\mathrm{n}=$ Frequency counts, $\%=$ Frequency count percentage,${ }^{\mathrm{G}}=$ Girl frequency count, Child $=$ Children, Adol $=$ Adolesc 
Throughout all time-points (M1, 62\%; M2, 59\%; M3, 70\%; M4, 75\%), there was little change in youths' ability to recall and/or relate their 3D models to their past week of physical activity, expressing how "on Saturdays I do dance so it's bar of activity is higher than the rest of them" (SG14, M1). Some youths reported the use of the 3D models as a motivational tool because "it's [the 3D model] kind of encouraging me to do more activity, so I can get the bar higher [on the 3D model]" (SB19, M2), with this perception increasing from $25 \%$ to $31 \%$ to $41 \%$ for the first, second and third models, respectively. From all time points, only 5\% of youths expressed that they would "show it [the 3D model] to my friends" (PB01, M1), with a larger number of youths (11\%), of which were highly representative of adolescent girls and children of both sexes, expressing how they would "probably like show my parents the model" (SG43, M3). Almost half the number of the youths (48\%) mentioned that they would display their 3D models in their house (Figure 6.3), with this proportion slightly greater in children, with a preference to "hang the model up in my bedroom" (PB11, M2).

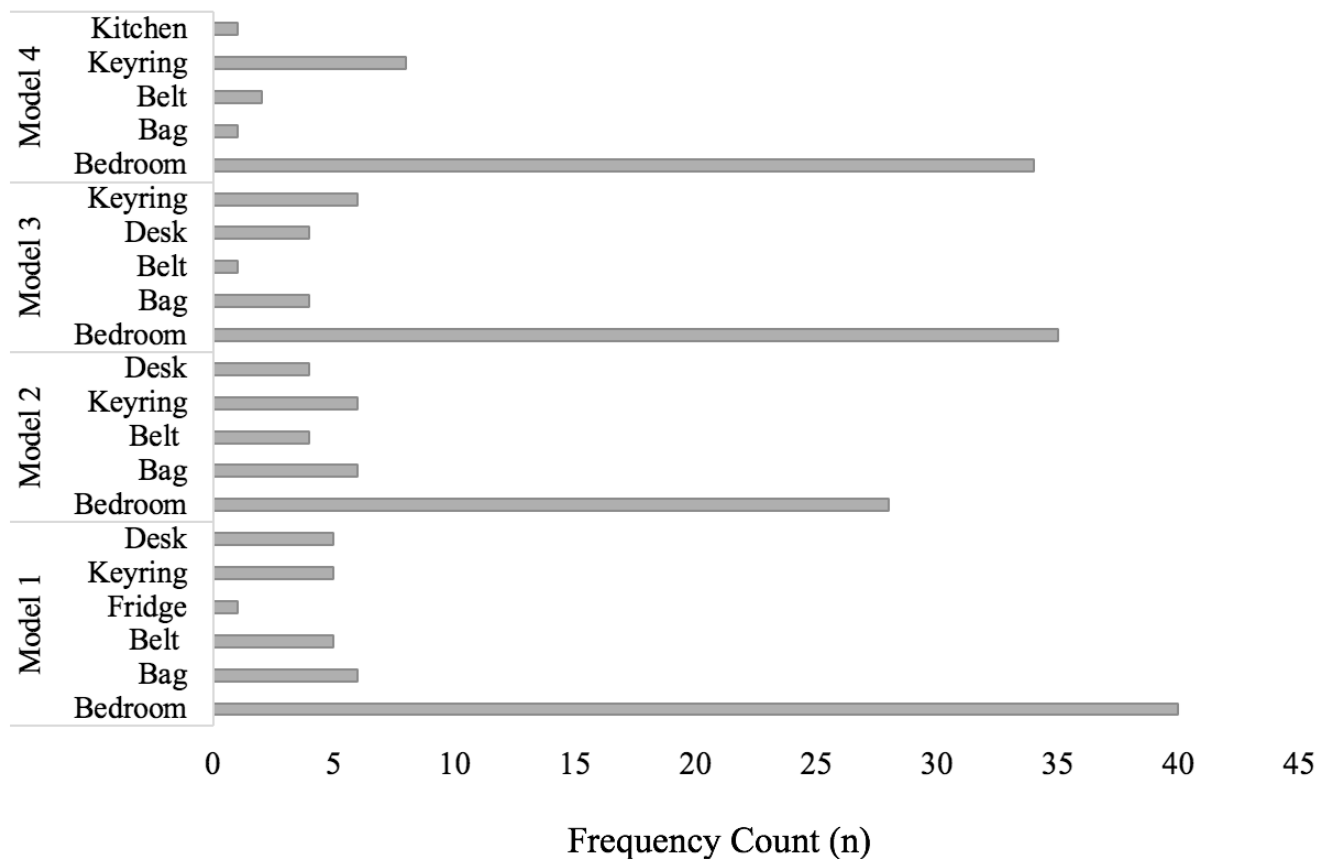

Figure 6.3 Youths' display preferences for 3D models 


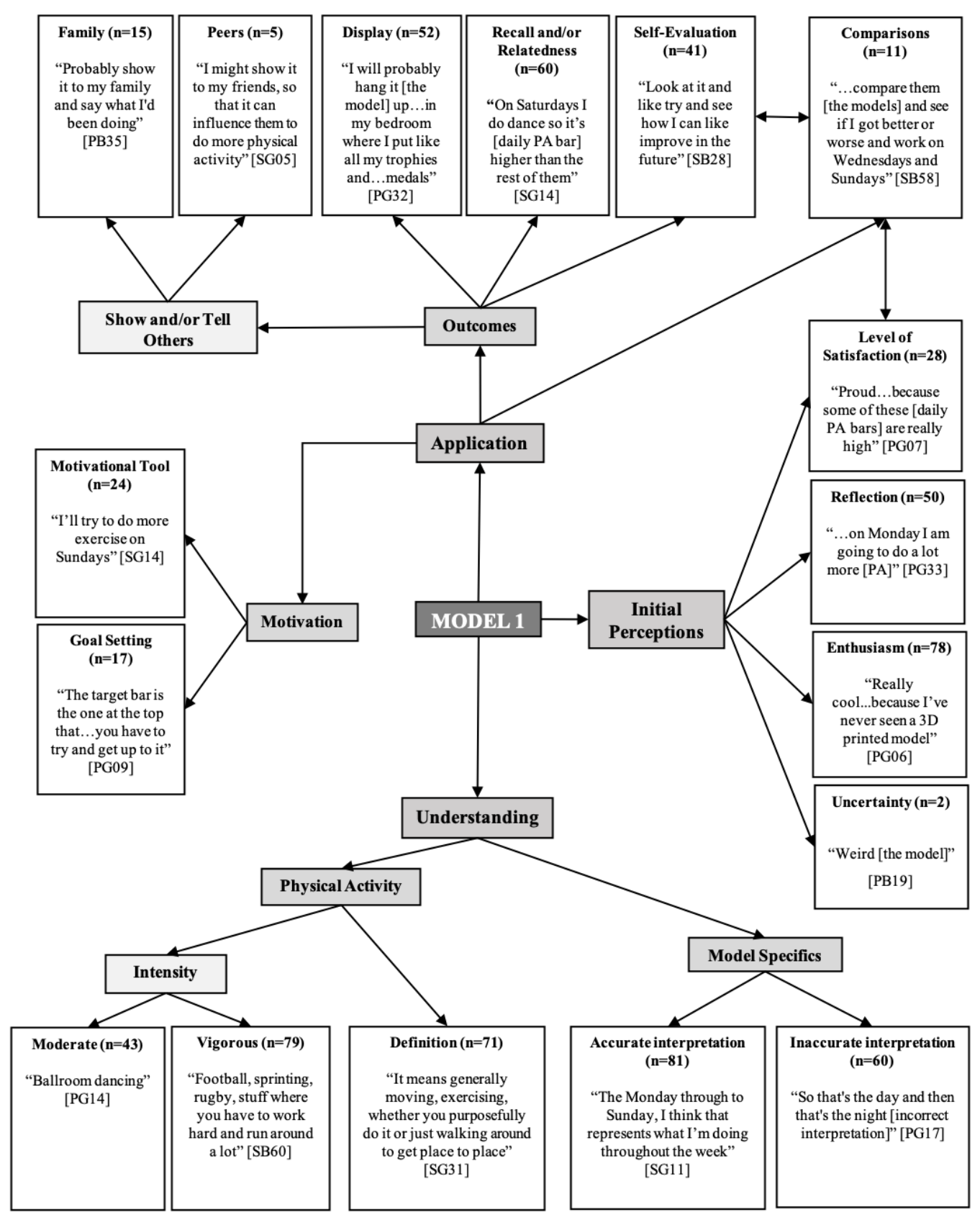

\section{Figure 6.4 Youths' pen profile model 1}

Figure legend $\mathrm{P}=$ Primary, $\mathrm{S}=$ Secondary, $\mathrm{B}=\mathrm{Boy}, \mathrm{G}=\mathrm{Girl}$, $\mathrm{PA}=$ Physical Activity, $\mathrm{N}=$ Frequency count 


\subsection{Discussion}

The primary aim of this study was to evaluate the effectiveness of age-specific 3Dprinted models of physical activity to enhance youths' awareness of their PAL, understanding of and motivation for physical activity. Taken together, the findings suggest that the 3D model feedback offered a unique strategy to enhance youths' awareness of their PAL in reference to the Government guideline and may provide a motivational tool for goal-setting.

In the present study, $63 \%$ of youths demonstrated that they were able to quickly interpret the basic components of their first 3D model (e.g., the different days of activity and their low and high PAL). Indeed, these initial interpretations of the agespecific 3D models are promising given that previous research highlights that being able to quickly interact and interpret a tool, such as a 3D model, enables an individual to learn about their behaviours from the start, all of which makes the experience with the tool rewarding and minimises the potential for abandonment (Forlizzi \& Battarbee, 2004). Following receipt of their final 3D model, 59\% of youths self-evaluated how the 3D models had made them "more aware" (SB58, M2) of their PAL. It could be argued that this raised awareness was a direct result of wearing the accelerometer rather than the $3 \mathrm{D}$ model per se, however, this is unlikely, as evidence suggests that accelerometers alone do not develop youths' awareness of physical activity (Vanhelst et al., 2017). A more likely reason for this increased awareness was the utilisation of an objective measure of physical activity in combination with personalised feedback, which has previously been suggested as an effective means to raise an individual's awareness of their physical activity (Bentley et al., 2012). Complementary to this understanding, the PAPM (Weinstein, 1988) from the Stages of Change (Prochaska \& DiClemente, 1992), suggests that an individual is unlikely to proceed to the contemplation stage unless they become aware that their behaviours are inadequate. Based on this notion, the present study demonstrated that $68 \%$ and $78 \%$ of children and adolescents, respectively, were able to identify that "some days I'm reaching the guideline bar, but some days I need to do more physical activity" (SB51, M4). This ability to apply their respective 3D model guideline bar to their personal PAL is hopeful given that previous research has shown that youths who are aware of their PAL and the recommended guideline are on average 20 minutes more active than their 
unaware counterparts, and consequently, more likely to achieve the 60 minutes of MVPA (Kremers et al., 2008, HSE, 2007, Roth \& Stamatakis, 2010, Nemet et al., 2012). Therefore, given that awareness of risk behaviours is identified as an independent correlate for behaviour change (Ronda et al., 2001), the 3D-printed feedback may not only be important to help youths categorize themselves into the correct stage of change (i.e., precontemplation, contemplation and preparation $=$ not meeting the guideline, versus action and maintenance = meeting the guideline), but also help youths perceive the need to change behaviour (De Bourdeaudhuij et al., 2004), warranting further investigation.

One important consideration with regards to 3D-printed feedback is that it possesses a higher level of visibility compared to digital feedback (i.e., on a smartphone) within the physical world (Golsteijn et al., 2012). In this way, 3D-printed physical activity data is more publicly visible to peers, teachers and family members. In contrast to previous perceptions (Study 2), only $5 \%$ and $11 \%$ of youth in the present study seemingly reported that they compared their models to their friends' models and showed their family members the models, respectively. Despite this, it could be speculated from previous research that youth may have more frequently compared their 3D models to friends within the playground and classroom environments (Ridgers et al., 2018). Moreover, it is also likely that family members did indeed come into regular contact with the 3D models given the range of ways that youth $(\sim 54 \%)$ displayed their models in the bedroom, on their school bag or attached to the house keys. In this regard, it is important to consider how the visibility of the 3D models may have stimulated more social-interactions with friends and family and thus, influenced youth's levels of self-evaluation (59\%, M4) and reflection $(58 \%, \mathrm{M} 4)$ of their PAL, rather than the 3D model itself. Indeed, the involvement of friends (Maturo \& Cunningham, 2013, Pearce et al., 2014, Salvy et al., 2009) and family (Davison et al., 2013, Haerens et al., 2007, Haerens et al., 2006, Pearce et al., 2014, Sleddens et al., 2011) can play a significant role in motivating youth to be more engaged in physical activity. On the contrary, sharing and comparing 3D models with friends or peers may increase competition, which can lead to negative feelings of the self and peer pressure to engage in an activity (Goodyear et al., 2017). Of concern are adolescent girls as they are particularly vulnerable at this age to body dissatisfaction, as this is a time when self-awareness, self-consciousness and preoccupation with self-image all dramatically 
increase (Harter, 1993). Indeed, a number of adolescent girls $(n=9,43 \%)$ in the present study reflected on how being perceived as physically active according to the $3 \mathrm{D}$ model was important because “you'll be more confident because like people won't like judge you” (SG34, M1) and worried about how "if you're not active you'll end up having a very, well kind of not nice figure [body shape]" (SG14, M2). As a consequence, youth who display such feelings of pressure and guilt for not achieving enough physical activity may remove themselves from engaging in peer-comparisons (e.g., sharing their PAL with others) altogether (Goodyear et al., 2017, Kerner \& Goodyear, 2017) and abandon the 3D model. These issues do question how public displays of physical activity data could intrude upon an individual's privacy (Khot, 2016). In this light, future research should look to monitoring more closely how youth, and in particular adolescent girls, personally reflect and evaluate their PAL with respect to body image and the influence of interactions and support from significant others on PAL.

Following receipt of the final 3D model, $72 \%$ of youths had seemingly adopted the guideline bar as a goal-setting strategy, expressing how they monitored their goalrelated progress through the guideline bar represented on the 3D models. In this way, it could be postulated that the $3 \mathrm{D}$ model guideline bar acted as an important reference for goal attainment, which subsequently led to youths' self-determined adjustment of physical activity strategies (e.g., starting to play football) and/or effort levels (e.g., try harder to do more exercise; Cullen et al., 2001, Locke \& Latham, 2002). As noted within the Goal Setting Theory (Locke \& Latham, 1990), and addressed in the Social Cognitive Theory (Bandura, 1991), setting specific and challenging (yet achievable) goals with feedback on goal attainment is an important step to enhancing an individual's self-efficacy (i.e. their belief to carry out a behaviour), and thus behaviour change. Numerous reviews support the effectiveness of goal setting to promote youths' physical activity engagement (Hynynen et al., 2016, Kyllo \& Landers, 1995, Rose et al., 2017), whilst others suggest that feedback alone has a motivating effect, regardless of whether the feedback is tied to a specific goal or not (Weinberg \& Weigand, 1993, Ivancevich \& McMahon, 1982, Hurling et al., 2007).

One particular dimension of the Goal Setting theory (Locke \& Latham, 1990) that resonates with the present findings, is the notion that goal attainment can be enhanced by incorporating feedback with rewards (e.g., monetary rewards that are linked to goal 
achievement). Indeed, throughout the intervention, $57 \%$ of youths expressed how they would display their 3D model in their bedroom, with some revealing how they placed their models next to their prized "trophies and medals" (PG32, M1). In this way, it could be argued that 3D-printed feedback is received by youths as a reward of their physical activity achievements, which is known to heighten an individual's success towards a goal as opposed to just setting a goal alone (Latham \& Locke, 1991). According to Locke and Latham (2006), rewards are important to sustain a person's interest in physical activity, which may stand true given the success of incentive-based interventions in promoting youths' physical activity (Hardman et al., 2011b, Finkelstein et al., 2013, Christian et al., 2016). On the contrary, it is important to consider the influence of a reward or incentive on youths' intrinsic interest to engage in physical activity as an explicit means to receiving the extrinsic reward (e.g., 3D model), and once removed whether their behaviour reverts back to baseline (Lepper et al., 1973, Ryan \& Deci, 2000). However, a recent systematic review provides strong evidence that behavioural incentives are an effective means of encouraging physical activity in youths, suggesting that there is a wide range of incentive designs that are yet to be explored (Corepal et al., 2018). Perhaps the novelty of 3D printing physical activity feedback may offer a greater learning value than previous incentive-based designs, as a result of the 3D models being a composite of a reward (i.e., aesthetically pleasing tangible object), feedback (i.e., personal physical activity data) and goal attainment (i.e., PAL achievement towards 60 MVPA guideline bar) that embodies personalised data and represents the active self (Khot, 2016). Therefore, the present study supports the utilisation of tangible feedback as a novel goal setting strategy for youths' physical activity through a reward, feedback and goal attainment, each of which are known to elicit greater self-efficacy (Latham \& Locke, 1991, Bandura, 1991) and youths' engagement within interventions (Shilts et al., 2004). Further research is warranted to investigate the potential utility of tangible representations of physical activity guidelines or goals to support youths' engagement and understanding of their physical activity behaviours.

Based upon previous 'learning styles' that support the use of tangibles to inform intellectual development and enable higher mental functions in youths (Cole \& Wertsch, 1996, Price et al., 2003, Rita \& Dunn, 1979, Piaget \& Cook, 1952, Fleming $\&$ Mills, 1992), it was originally postulated that the present 3D-printed feedback of 
physical activity may enhance youths' comprehension of intensities (i.e. MVPA) and associations to the Government guideline (Study 3). However, only 5\% of children and $17 \%$ of adolescents, across all timepoints, were able to interpret the guideline bar in terms of the number of minutes (i.e. 60 minutes), whilst no children and $5 \%$ of adolescents were able to cite "1 hour of physical activity whether it's moderate or vigorous" (SB60, M4). These findings align with previous research suggesting that, particularly children, have a lack of ability to define time (Baranowski, 1988, Sallis, 1991) and intensity in the context of physical activity (Cowden \& Plowman, 1999, Prochaska et al., 2001, Snethen \& Broome, 2007, Placek et al., 2001, Pearce et al., 2008). Indeed, these findings fuel the present debate to whether 'learning styles', such as youths being 'visual and tactile' learners (Rita \& Dunn, 1979), are effective strategies to enhance an individual's understanding of information (Husmann \& O'Loughlin, 2018). Previous research has demonstrated that changing the learning mode or strategy for a specific population had little improvement on learning outcomes to justify the time and financial costs involved (Coffield, 2004, Papanagnou et al., 2016, Pashler et al., 2008, Riener \& Willingham, 2010). Therefore, the present findings question the use of tangibles as an effective means to enhance youths' comprehension of the MVPA terms associated with the guidelines. Future research may wish to explore different 3D model designs using inscriptions of the intensities moderate and vigorous on the 3D models to aid youths' comprehension of terms.

There are a number of the inherent challenges associated with 3D printers and their slow development process that should be noted, otherwise the dissemination into practice is invariably unfeasible (Dishman et al., 2001, Melanson Jr et al., 1996). Specifically, the process of creating the 3D models, following the downloading, analysing and mapping of youths' physical activity data onto the $3 \mathrm{D}$ models and subsequently 3D printing, involved a considerable amount of time, which consequently delayed the delivery of the feedback to youths. It could be speculated that this delayed timing of the feedback may have impacted youths' adherence to wearing the accelerometers and consequently awareness of their PAL. Indeed, the study showed a declining trend in youths wear-time from baseline (children $n=38$, $87 \%$; adolescents $n=58,93 \%$ ) to the end of the intervention (children $=n=38,72 \%$; adolescents $n=58,68 \%$ ) for a wear-time criterion of 10 hours on any four days. In comparison, Sirard and Slater (2009) found that, using the same criteria of 10 hours 
on any four days, monetary compensation contingent was the most effective accelerometer wear-time compliance strategy $(n=22,96 \%)$. That said, the aforementioned study lacked a follow-up wear-time measurement and was based on a smaller sample size, which questions the long-term effectives of such monetary incentives (Sirard \& Slater, 2009). Furthermore, the use of monetary incentives can be costly, ranging from $£ 12$ (Sirard \& Slater, 2009) to $£ 25$ (Christian et al., 2016) per completion and return of the accelerometer, when compared to the present study costing $\sim 5$ per 3D model. It could be argued that 3D-printed physical activity feedback may be an as effective strategy to monetary incentives for wear-time compliance in youth, with the additional benefit of raising their awareness of PAL. That said, a number of youths played contact activities, which involved "taking it [the accelerometer] off because of rugby training” (SB24, M3) and consequently, "forgetting to put it [the accelerometer] back on again" (SB25, M3). In this respect, the 3D models did not account for physical activity in the form of water-based activities and contact sports, which are likely to contribute to daily MVPA, and thus will under-represent youths' achievements and awareness of their true PAL and goal attainment (i.e., meeting the MVPA guideline bar), all of which could lead to negative feelings of self (Goodyear et al., 2017). To counteract such problems, future research should look to implement 3D-printed feedback with wrist-worn, fully waterproof accelerometers as they elicit higher wear-time compliance in youth than hip-mounted devices (Trost et al., 2014b) and diary logs to account for contact sport activities (Pfitzner et al., 2013). Nevertheless, it is important to acknowledge that efforts are currently being made to make 3D printers faster, more accurate and cheaper (Mueller et al., 2014), with the potential for future research to involve youths more in the 3D printing process. Adding to this is the rise in schools owning a $3 \mathrm{D}$ printer (UK Department for Education, 2013), which makes 3D-printing interventions similar to the current study more feasible and cost-effective. In this light, it may be useful to compare 3D-printed feedback to other approaches, such as digital smartphone feedback (Larsen et al., 2013, Tong et al., 2015), LED feedback technology (Ananthanarayan et al., 2016, Mackintosh et al., 2016), 3D-printed edibles (Khot et al., 2015b) or shape changing artefacts (Sauvé et al., 2017) to determine which methods of feedback can elicit the best intervention effects, user experience and costeffectiveness. 
According to Forlizzi and Battarbee (2004), new research methods are required to better articulate the relationship between what 'we feel' and what 'we do' in connection to the utilisation of technology. The present study builds on this by illustrating a short video interview approach to eliciting how youths experienced the 3D-printed models internally, functionally and socially, all of which is essential for the development and future utilization of the designed 3D models (Hassenzahl \& Tractinsky, 2006). The short video interviews generated a large set of descriptive data that could be generalised to the study population or used to account for an individual's personal progress and experiences with the $3 \mathrm{D}$ models, which aligns with the current trend towards 'personalisation' in healthcare (Cesuroglu et al., 2016) and the 'quantified-self' movement (Almalki et al., 2015). However, one possible limitation to this aforementioned approach, could be the direct influence of the ongoing short video interviews on youths' experiences with the $3 \mathrm{D}$ models, given that previous research suggests that face-to-face support can create a more meaningful experience by reinforcing effort and goals (Fortier et al., 2012, Williams \& French, 2011). In this respect, it could argued that the on-going face-to-face short video interviews may have potentially influenced youths awareness and motivation for physical activity, rather than the 3D models per se. Indeed, there are a number of practical ways a researcher or health professional could be deployed to support such a feedback intervention, however, to make technology-based behaviour change strategies more pragmatic and cost-effective it would be useful to understand the efficacy of support through continuous interviews (Heath et al., 2012). Therefore, future research should look to break down 3D-printed feedback conditions to include and exclude support to fully understand the impact of the tangible feedback and face-to-face engagement (Moore et al., 2015). That said, the present study supports the use of short video interviews as a practical method for assessing youths' experiences, understanding of and interactions with newly designed technology.

There are, however, some additional limitations to consider to the aforementioned, such as the localised area of the data collection in South Wales, which may underrepresent the ideologies of youth from other important social-economic groups and ethnic minorities in the UK or globally. Given the paucity of research on 3Dprinted feedback, further research is required that considers the influence of age and sex specifically, as well as of baseline PALs which may be hypothesised to influence 
initial engagement with the models. Indeed, the lack of a control group within the present study questions whether the changes observed can be attributed to the impact of the 3D models per se to enhance youth's awareness, goal-setting and motivation and, therefore, findings should be considered with caution and act as a stimulus for future investigation. Finally, the study was only a 7-week intervention with no longterm follow up, therefore, it is unknown to what extent youth will sustain their engagement with the 3D models and the true benefits of such feedback on their PAL, given that most changes in behaviour take place over prolonged periods (Kwasnicka et al., 2016).

\subsection{Conclusion}

In conclusion, the present study demonstrated that the age-specific 3D models heightened youths' awareness of their PAL and enabled them to easily compare their personal PAL to the recommended guideline of 60 minutes of MVPA. Moreover, youths expressed how they displayed their 3D models in their environments, within their bedrooms or next to prized possessions, and utilised the model as a goal-setting strategy to do more physical activity. Therefore, the nature of the age-specific 3D models being a blend of feedback, reward and goal attainment that embodies personalised data may offer a unique strategy for the promotion of physical activity and associations to the recommended Government guideline. 


\section{Thesis Map}

STUDY

\section{OUTCOMES}

1. Energy Expenditure Associated with Walking Speed and Angle of Turn in Children

\begin{abstract}
Aim
- To investigate the influence of walking speed and angle, and their interaction, on the energy expenditure of healthy children.
\end{abstract}

- Significant differences to straight line walking energy expenditure within speed were established for $2.5 \mathrm{~km} \cdot \mathrm{hr}^{-1}$ at

Key $90^{\circ}\left(\sim 7 \%\right.$ increase) and $3.5,4.5$ and $5.5 \mathrm{~km} \cdot \mathrm{hr}^{-1}$ for $180^{\circ}$ turns

Findings ( $\sim 13 \%, \sim 14 \%$ and $\sim 30 \%$ increase, respectively).

- Estimations of children's habitual physical activity should account for the magnitude and frequency of turns complete.

2. Perceptions of Visualising Physical Activity as a 3D-printed Object: A Formative Study

\section{Role of DESIGN PARTNERS}

3. Understanding Youths' Ability to Interpret 3D-printed Physical Activity Data and Identify Associated Intensity Levels

Role of TESTER
Aim

To elicit children's, adolescent's, parents' and teachers' perceptions and understanding of 3D physical activity objects to inform the design of future 3D models of physical activity.

- Youths demonstrated a good ability to conceptualise physical activity as a 3D-printed object and highlighted the potential of 3D models as a motivational tool.

Findings

- Two age-specific 3D models of physical activity were developed from children's preference for abstract designs and adolescents' bar chart designs.
Aim

- To elicit children's and adolescent's interpretations of two age-specific 3D models displaying physical activity.

- To assess children's and adolescent's ability to appropriately align activities to the respective intensity of physical activity.

- Youths demonstrated a good ability to interpret their ageKey

Findings specific 3D model of physical activity.

- Youths showed misconceptions when identifying activities to respective intensities, particularly, moderate-intensity household chore activities.

4. The Tangibility of Personalised

Aim 3D-printed Feedback may Enhance Youths' Physical Activity Awareness

Role of USER
- The age-specific 3D models heightened youths' awareness of their PAL and enabled them to easily compare their Key personal PAL to the recommended guideline of 60 minutes

Findings of MVPA.

- Youths displayed their 3D models in their bedrooms or next to prized possessions and utilised the model as a goal-setting strategy to do more physical activity. 


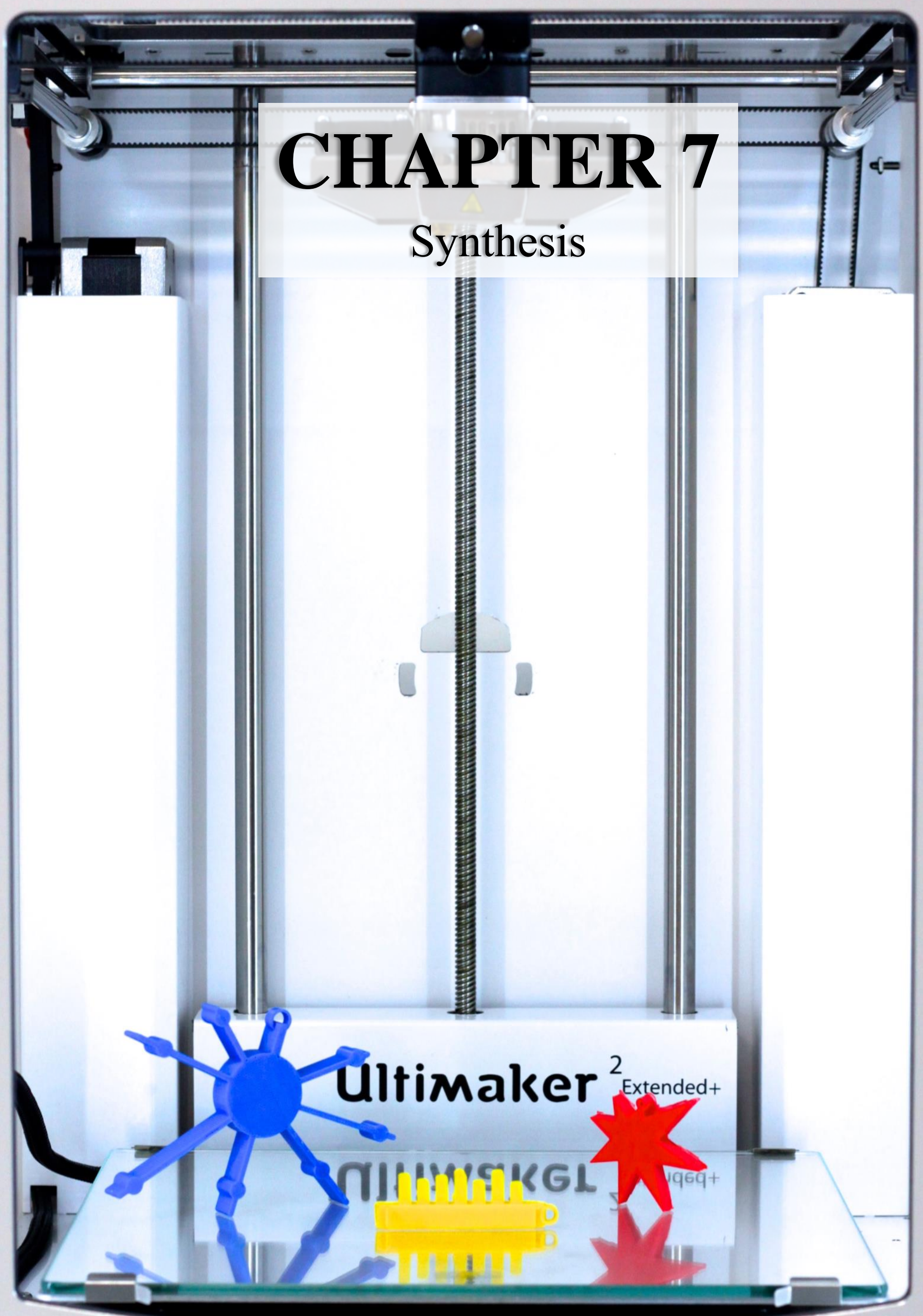




\section{7}

\section{Synthesis}

The purpose of this thesis was to contribute novel and important components that inform the measurement of physical activity and the conception of personalised visual and tangible feedback to aid youths' engagement in physical activity. Following individual study discussions in respective chapters, this chapter will seek to synthesise the overall thesis outcomes, strengths, limitations and future implications, whilst also considering the contribution to the relevant theory and literature before concluding the thesis.

\subsection{General Discussion}

\subsubsection{D-Printed Feedback to Enhance Awareness of Physical Activity}

According to Bentley and colleagues (2012), the objective measurement of physical activity in combination with personalised feedback is an effective tool to improve an individual's awareness of their physical activity levels (PAL). In line with this, the qualitative findings presented in Studies 2, 3 and 4, showed promising indications that using an objective measurement in combination with tangible, visual feedback can raise youths' awareness of their PAL. Specifically, conclusions drawn from Studies 2 and 3 highlighted the potential of the 3D-printed models to enhance youths' awareness of their PAL. Findings from Study 4 reported that $60 \%$ of youths directly expressed that the 3D models had made them "more aware" of their PAL by the end of the intervention. Furthermore, based on the Precaution Adoption Process Model (PAPM; Weinstein, 1988) within The Transtheoretical Model (TTM; Prochaska \& DiClemente, 1992), Study 4 speculated that youth are unlikely to proceed to the contemplation stage of exercise behaviour unless they become aware that their PAL are not optimal (i.e., 60 minutes of MVPA per day). In accord with PAPM, findings from Study 4 demonstrated that $68 \%$ and $75 \%$ of children and adolescents, respectively, compared their personal PAL on the $3 \mathrm{D}$ models to the guideline bar representation, similar to findings reported in Study 3 (children, 75\%; adolescents, 
$83 \%$ ). Considering that evidence reports that youths who are more aware of their PAL and the recommended guideline are to more likely to achieve the daily target (Kremers et al., 2008, HSE, 2007, Roth \& Stamatakis, 2010, Nemet et al., 2012), this holds promise for the $3 \mathrm{D}$ models to facilitate increased PAL and positive behaviour change. Nonetheless, Study 1 questioned the accuracy of data derived from accelerometers as the majority of energy expenditure prediction equations are based on linear-based locomotion protocols performed on treadmills that are not true to the habitual (Eisenmann et al., 2004, Fortune et al., 2014), sporadic (e.g., chasing games;Sleap \& Warburton, 1996, Sleap \& Wormald, 2001), physical activity patterns of youth. As a consequence, data derived from accelerometers and represented through 3D model feedback will likely be limited in accounting for youths' non-linear movements, leading to lower than true levels of physical activity being reported and thus could inhibit an individual's awareness of their PAL. Of particular concern is feedback indicating low PAL, as this could trigger denial or fatalistic attitudes, resulting in no need to change behaviours or even a decline in physical activity (Bankhead et al., 2005). Despite this, numerous studies support the use of objectively assessed physical activity feedback to promote youths PAL, regardless of whether the feedback is accurate or not (Ford et al., 2010, Hurling et al., 2007, Lim et al., 2016, Martin et al., 2015, Walsh et al., 2016).Therefore, the present body of work highlights that personalised 3D-printed feedback of PAL raises youths' awareness of their personal PAL and may serve to motivate them to transition from pre-contemplation to contemplation of the Stages of Change from the TTM (Prochaska \& DiClemente, 1992).

It is also apparent that youths' raised awareness of their PAL from the 3D model feedback may not only be important to help youths categorize themselves into the correct stage of change (i.e., precontemplation, contemplation and preparation $=$ not meeting the guideline, versus action and maintenance = meeting the guideline), but also help youths perceive the need to change (De Bourdeaudhuij et al., 2004). Indeed, research supports the use of the TTM for defining young people's readiness to participate in MVPA (Haas \& Nigg, 2009, Schumann et al., 2003, Schumann et al., 2002, Gaudet et al., 2017). However, it is also important to acknowledge that numerous reviews question the effectiveness of including the TTM into physical activity interventions (Brug et al., 2004, Adams \& White, 2004, Adams \& White, 
2003, Bridle et al., 2005, Bunton et al., 2000). For example, evidence suggests that TTM-based interventions only produce short-term change in an individual's PALs with no long-term impact on physical activity for interventions greater than 6-months (Adams \& White, 2003). In this respect, caution must be taken when interpreting the results presented in Study 4 given that the study was only a short-duration intervention.

\subsubsection{Goal-Setting and Rewards through 3D-Printed Physical Activity Feedback}

A key aim of Study 2 was to elicit youths', parents' and teachers' perceptions of and designs for 3D models. One parent from Study 2 expressed how including a "recommended goal" on the 3D models may help to encourage youths' physical activity engagement. Complementary to this, focus groups from Study 2 also found that a majority of youths had limited knowledge of the Government guideline of 60 minutes of MVPA, which consequently reinforced the importance of including the guideline in the 3D model design. Based upon the Goal Setting Theory (Locke \& Latham, 1990), Study 2, therefore, theorised that incorporating the guideline on the 3D model may help motivate youths to increase their personal PAL. Indeed, setting specific and challenging, yet achievable, goals, in conjunction with feedback regarding performance towards goal attainment is important to enhance an individual's selfefficacy (i.e., individuals' belief to carry out a behaviour) and health behaviour change (Locke et al., 1981). Additionally, 80\% of youth in Study 2 described how personalised 3D-printed feedback would motivate them to engage in more physical activity. Following the development of the two age-specific 3D models in Study 2, Study 3 demonstrated that $75 \%$ and $83 \%$ of children and adolescents, respectively, were able to correctly identify the incorporated guideline bar designed to represent the 60 minutes of MVPA on 3D model. These aforementioned findings, regarding motivation and understanding, were promising for the utility of the age-specific 3D models within an intervention setting (Study 4), as previous research demonstrates that bringing youths attention to the amount of MVPA they attained compared to the recommended amount enforces positive behaviour change (Kremers et al., 2008, HSE, 2007, Roth \& Stamatakis, 2010, Nemet et al., 2012). When the 3D models were utilised within the intervention (Study 4), 72\% of youths seemingly adopted the 3D model guideline bar as a goal-setting strategy. In this way, youths monitored their goal-related progress through the 3D models with the help of the guideline bar acting as an important 
reference for goal achievement, subsequently leading to youths' self-determined adjustment of physical activity strategies (e.g., "since I've had one of the models, I went to play girls football to build my activity") and/or effort levels (e.g., "I need to do more exercise to keep healthy and reach my target"; Cullen et al., 2001, Locke \& Latham, 2002).

According to Latham and Locke (1991), attainment of goals can be enhanced by incorporating feedback with rewards (i.e., contingent incentives that are linked to goal achievement) through the 'high-performance cycle' process (p.233). As emphasised in Study 2, and supported by Khot et al. (2016), the nature of 3D-printed feedback may offer a greater learning value through blended feedback and rewards, in comparison to the distribution of feedback and rewards in isolation (Finkelstein et al., 2013, Hardman et al., 2011b). As reported in Study 4, youths expressed how they displayed their 3D models next to their prized "trophies and medals" and "certificates", which may suggest the 3D models were received as a form of reward. Despite this, it is important to consider the over-justification effect which describes that an individual's intrinsic interest to engage in an activity may be undermined as an explicit means to receiving an extrinsic reward, such as money, and once removed the behaviour will revert back to baseline (Lepper et al., 1973). That said, this thesis draws attention to the novelty of the 3D-printed nature of the models and their capacity to not only provide youths with an extrinsic reward but to inform youths of their PAL in reference to achieving the recommended guideline, which monetary rewards cannot provide. Therefore, based on the outcomes presented in Study 4, this thesis suggests that 3D-printed models may have potential as a novel goal-setting strategy and reward system for youths, all of which may lead to greater self-efficacy (Latham \& Locke, 1991) and adherence to interventions (Shilts et al., 2004).

\subsubsection{Understanding of Physical Activity through 3D-Printed Feedback}

Previous literature has shown that youths have limited understanding of physical activity and the associated Government guidelines, particularly in terms of the recommended frequency, intensity and duration (Snethen \& Broome, 2007, Sleap \& Wormald, 2001, Harris et al., 2016, Placek et al., 2001, Pearce et al., 2008, Trost et al., 2000a). Accordingly, Studies 2, 3 and 4 found that children and adolescents had 
difficulty in identifying the different intensities of physical activity associated with the recommended guidelines. Specifically, Study 2 demonstrated that neither children nor adolescents could correctly define the Government guideline with respect to MVPA, although contrary to previous findings (Harris et al., 2016), adolescents (67\%) demonstrated the ability to define the amount of time recommended in the guidelines (e.g., "60 minutes a day"). However, Study 3 identified that both children and adolescents had misconceptions when identifying activity intensities, especially with regards to defining moderate-intensity activities that were related to household chores (e.g., sweeping the floor) or daily activities (e.g., stair climbing), as previously identified (Trost et al., 2000a). However, Study 3 showed that youths were able to correctly interpret and describe the representations of physical activity, intensity and the recommended guideline on the age-specific 3D models. Study 3, therefore, concluded that the 3D-printed feedback may have potential to enhance youths' understanding of intensities (i.e., MVPA) and associations to the recommended guideline, congruent with research supporting the use of tangible objects to promote intellectual development in youths (Price et al., 2003, Marshall, 2007, Rogers et al., 2002a, Bara et al., 2004, Rita \& Dunn, 1979, Cole \& Wertsch, 1996, Piaget \& Cook, 1952, Fleming \& Mills, 1992, Montessori, 1912).

Despite conclusions from Study 3, Study 4 showed that the 3D models did not substantially increase youths' understanding of MVPA, with no children, and only 5\% of adolescents, able to articulate the guidelines in terms of " 1 hour of physical activity whether it's moderate or vigorous" (SB60, M4) following receipt of their final 3D model. One possible explanation for youths' greater understanding to articulate the intensities and guideline reported in Study 3 when compared to Study 4, may have been the short time between youths being shown how to interpret the $3 \mathrm{D}$ model and the completion of the 3D model recall task to assess understanding. Consequently, this method for assessing youths' understanding may have been more to do with their 'working memory' (i.e., short-term memory) to recite the terms of MVPA and the recommended guideline (Novak \& Cañas, 2008), rather than their greater ability to define intensity and the guideline. Findings from Study 4, therefore, question the use of 3D-printed models to promote youths' intellectual understanding of MVPA and the recommended guideline. In this case, the present body of work adds to the current debate as to whether different 'learning styles', particularly visual and tactile learning 
styles, are effective at enhancing youths' understanding of information (Husmann \& O'Loughlin, 2018). Indeed, numerous studies have demonstrated that changing the presentation of the learning mode or strategy to align with a targeted population's learning style elicited insufficient influence on the outcome to justify the financial or temporal costs involved (Coffield, 2004, Papanagnou et al., 2016, Pashler et al., 2008, Riener \& Willingham, 2010). Whilst the present design of the 3D models did not facilitate youths understanding of intensities and associations to the recommended guideline, post-intervention focus groups did, however, reveal that youths would like future 3D models to include engravings and different colours to detail the different components (i.e. moderate- and vigorous-intensity bars, guideline bar) to heighten their understanding of terms. Therefore, future research should explore the use of multi-coloured 3D models with engravings to facilitate enhanced understanding.

\subsubsection{Youths' Adherence and Experience with 3D Models of Physical Activity}

The first step to utilising 3D-printed visualisations of physical activity was to identify whether youths could associate a 3D model (i.e., representation) with physical activity data (i.e., referent), referred to as representational insight (Uttal \& Doherty, 2008). Study 2 reported that youths, irrespective of age, were able to conceptualise physical activity represented as a 3D-printed object, which remained consistent within Studies 3 and 4 . However, Study 2 identified that children had more difficulty than adolescents in distinguishing between high and low representations of physical activity data represented on the 3D models. Similarly, Study 3 showed that children had more difficulty in defining the moderate-intensity bar on their respective 3D model. However, Study 4 demonstrated that $62 \%$ of youths, regardless of age, were able to quickly recall and/or relate their personal behaviours to their first 3D model (e.g., different days and low and high PAL), with this number increasing to $75 \%$ following receipt of their final 3D model. In accord with Forlizzi and Batterbee (2004), youths' ability to interpret and relate their behaviours to the basic functions of the 3D model will not only make outcomes rewarding from the outset, but will also minimise the potential for abandonment of the 3D model feedback, which may, in part, have explained the low intervention dropout rate $(2 \%)$. 
An equally important consideration to the adherence of the 3D models (Study 4), is whether or not the accelerometers had been worn appropriately. The time and cost burden as a result of poor accelerometer wear-time compliance, or indeed loss of monitors, remains an issue for researchers (Catellier et al., 2005, Sharpe et al., 2011, Wells et al., 2013). However, there is some evidence to suggest that children and young people would like to receive certificates, trophies or medals as a strategy to increase their wear-time compliance (McCann et al., 2016). This holds promise for the accelerometer wear-time compliance in Study 4 given that the 3D models were received as a form of reward. Despite this, Study 4 reported a decline in youths' compliance in wearing the accelerometers from baseline (children $n=39,87 \%$; adolescents $n=58,93 \%$ ) to the end of the intervention (children $=n=39,72 \%$; adolescents $n=58,67 \%$ ) for a wear-time criteria of 10 hours on any four days. In comparison, Sirard and Slater (2009) conducted three different compliance strategies in youth for wearing waist-worn accelerometers based on a wear-time criteria of 10 hours on any four days. The findings showed that monetary compensation contingent was the most effective compliance strategy $(n=22,96 \%)$, followed by daily journaling $(\mathrm{n}=20,85 \%)$ and reminder phone calls $(\mathrm{n}=21,72 \%$; Sirard \& Slater, 2009). Although direct comparisons between the 3D models and the aforementioned compliance strategies are hard to make, especially given the discrepancies in sample sizes and the lack of a follow-up measurement in the latter. However, comparing the baseline 3D model compliance rates to the monetary strategy, it could be argued that the 3D models as a wear-time strategy are just as effective. For instance, a 3D model not only provides personalised feedback that aligns with youths' needs for greater compliance (Audrey et al., 2012, Kirby et al., 2012), but is more cost-effective at $£ 5$ per model when compared to the $£ 12$ per completion and return of the accelerometer in the monetary strategy (Sirard \& Slater, 2009). Furthermore, post-intervention (Study 4) focus groups reported how youths found the accelerometers "uncomfortable", with preferences for wrist-worn devices. Indeed, evidence suggests that wrist-worn accelerometers elicit higher wear-time compliance rates than hip-mounted devices (Trost et al., 2014b). In this case, future research should investigate the benefits of using wrist-worn accelerometers in conjunction with 3D-printed feedback to enhance wear-time compliance and adherence to interventions. 
One notable experience to consider with the use of 3D models within an intervention setting is how social interactions with friends or peers influence youths' experiences with their personal model. Study 4 reported that only $5 \%$ of youths seemingly compared their $3 \mathrm{D}$ models to their friends, however, direct observations from the research team and collaborative teachers reported that these comparison interactions may have been more frequent, especially within the playground and classroom settings. Tangible objects are known to create a shared space for collaborative transactions (Fernaeus \& Tholander, 2006, Suzuki \& Kato, 1995) and to allow individuals to achieve social-interaction more easily than on-screen displays (Svendsen, 1991). Indeed, previous research suggests that peer involvement within an intervention has numerous health benefits for youths' physical activity (Barkley et al., 2014, Jago et al., 2012, Pearce et al., 2014, Salvy et al., 2012, Salvy et al., 2009). However, evidence from post-intervention (Study 4) focus groups suggested that some youths stopped comparing their 3D models to their peers' models as a result of the comparison making them "feel worse" due to their lower PAL representations, congruent with findings from Study 2. Evidence shows that social interaction with friends or peers can influence an individual's emotion with a model and can alter the meaning of 'an experience' from pleasant to unpleasant and vice-versa (Forlizzi \& Battarbee, 2004). Therefore, further research is required to assess the impact of peer involvement on youths' experiences with the 3D models as a lack of understanding of the consequences could result in individuals receiving unfavourable social feedback, and thus lead to negative associations regarding being physically active.

\subsubsection{The Use of Accelerometers to Assess Youths' Physical Activity}

Whilst Study 4 supports the use of accelerometry derived data to create tangible 3Dprinted feedback of physical activity to raise youths' awareness and goal-setting, Study 1 questions the accuracy of accelerometers to account for youths more sporadic and irregular bouts of movement (Baquet et al., 2007, Bailey et al., 1995, Welk et al., 2000, Sleap \& Warburton, 1996). Indeed, accelerometers are known to be limited when it comes to accounting for non-linear movement patterns (Bassett \& John, 2010, Van Remoortel et al., 2012, Chen et al., 2007), such as turning, that can make up 34 to $45 \%$ of all steps taken in a typical day (Glaister et al., 2007). Previous research has demonstrated that turning engenders its own independent and significant energy 
expenditure when compared to straight line locomotion in adults (Buchheit et al., 2010b, Buchheit et al., 2011, Dellal et al., 2010, McNarry et al., 2017, Wilson et al., 2013). Study 1 extends these findings to children, reinforcing the importance of accounting for the magnitude and frequency of turns completed when estimating children's habitual physical activity patterns. These findings, therefore, question the majority of energy expenditure prediction algorithms based on accelerometry data that are derived from treadmill-based locomotion protocols. Indeed, such linear modes of locomotion are not cognisant of the additional energy costs of turning and may, in part, be a contributing factor to the poor accuracy of energy expenditure calculations during free-living conditions (Fortune et al., 2014, Eisenmann et al., 2004). One possible solution, as emphasised in Study 1, is the use of a magnetometer in conjunction with accelerometery to provide more behavioural context, such as how and when the body rotates in a measurement of physical activity. The combined magnetometer and accelerometer derived data may help provide individuals with more detailed feedback, that not only accounts for the metabolic costs of turns completed in a typical day, but also enables greater awareness of their daily energy expenditure. Indeed, this is particularly important given that activity trackers are increasingly adopted within interventions as they are easy to disseminate and communicate large volumes of personalised content that coincides with health behaviour change (Ramirez-Marrero et al., 2005, van Gemert-Pijnen et al., 2011). Therefore, this thesis highlights that more research is warranted to investigate the benefits of including such magnetometryderived data to enhance the accuracy of energy expenditure predictions equations.

\subsection{Strengths and Limitations of Thesis}

The major strength of this thesis is the novelty of each study, with Study 1 and Studies 2 to 4 , being the first to investigate the energy expenditure of turning in children and the perceptions, design and utilisation of 3D-printed physical activity data feedback method for youths, respectively. Conversely, the paucity of literature regarding the topics undertaken in this thesis, as noted in Chapter 2, may also be a limiting factor towards the findings presented. For example, an a priori sample size calculation could not be conducted for Study 1 as there was no comparable data in the literature. Whilst there was a larger variability in data found in children (Study 1) when compared to the adult study (McNarry et al., 2017), which is associated with a decreased statistical 
power to detect subtle differences in energy expenditure, the sophisticated statistical analyses conducted were able to adequately account for this level of variance. Nonetheless, the highly-controlled laboratory nature of Study 1 may have limited the generalisability of the results and its ecological validity, especially given that turn strategies were not accounted for. Indeed, previous research has reported two common types of turn strategy, one being a step turn and the other a spin turn. Laboratory-based findings suggest that young people most commonly adopt a spin turn (Akram et al., 2010), which is associated with reduced stability and increased physiological strain (Hader et al., 2016, Buchheit, 2010, Buchheit et al., 2010b). Conversely, the only nonlaboratory study assessing turn strategy noted that adults did not complete a single spin turn (Glaister et al., 2007). Although it could be argued that children tend to adopt the more complex spin turns as a result of their gait immaturity (Dixon et al., 2013), more research is required to investigate children's turn strategies in a real-world setting.

One common methodological strength included within Studies 2, 3 and 4, was the use of pen profiles to illustrate the consistency of themes in the qualitative data, which prevents the over-representation of minority views (Anderson, 2010b). Further methodological rigour was demonstrated using 'trustworthiness criteria' (Ridgers et al., 2012, Boddy et al., 2012, Mackintosh et al., 2011, Mackintosh et al., 2016), whereby the triangulation of data between researchers afforded credibility, dependability and transferability of findings (Carcary, 2009). However, one variable that limited the generalisability of findings from Studies 2, 3 and 4 was the localised area of data collection in South Wales. This level of localised data collection to not only inform the perceptions, but the design and utilisation of 3D feedback, may underrepresent the ideologies of youths from other important social-economic groups and ethnic minorities in the UK or at a global level. Furthermore, the present research was also limited by the lack of health literacy assessment within youths, which is important to consider given the profound impact on health it can have (Zimmerman \& Woolf, 2014). For example, a systematic review found that individuals with lower health literacy had poorer health knowledge, comprehension and ability to understand health messages (Berkman et al., 2011). In this respect, the present findings from Studies 2, 3 and 4, demonstrating youths' interpretations of intensities and understanding, designs and utilisation of 3D-printed feedback, should be carefully generalised and considered more as a stimulus for future investigation. 
A major strength of Studies 2, 3 and 4 is the systematic framework undertaken for designing and evaluating technology developed by Druin (2002). The framework is designed to elicit inventive and expressive ideas from youths to design, create and adapt new technology (Druin, 2002). In this way, the framework can build knowledge and structure on the development of more meaningful technologies to enable better theories for future education and teaching strategies (Druin, 2002). From the framework, the studies were structured to follow youths as (a) design partners (Study 2), (b) testers (Study 3) and (c) users (Study 4) of novel 3D-printed feedback. Of note was the utilisation of Play-Doh as a prototyping material for youths to create personal 3D models in conjunction with their narrative statements (Druin, 2002). This aforementioned approach facilitated the triangulation of youths' Play-Doh tangible designs and supporting narratives, which meant that the analysis was not solely dependent upon the researcher's interpretations of the data, therefore simultaneously reducing the risk of misinterpreted views whilst enhancing the credibility and confidence in the findings (Darbyshire et al., 2005, Smith \& Noble, 2014). Arguably, one of the biggest concerns with regards to developing technology is whether individuals sustain their engagement with the technology over time (Ledger \& McCaffrey, 2014). In this respect, it is apparent that a limitation to Study 4 was the short 7-week intervention duration and with no follow-up period to determine the longterm benefits of the 3D models. As a result, it is difficult to draw any clear conclusions about youths' long-term engagement with the 3D-printed models during the user phase (Study 4). However, there is some previous evidence to suggest that youths may regularly utilise technology to self-monitor their PAL when the technology is integrated into an intervention setting (Slootmaker et al., 2010, Hooke et al., 2016). On the hand, sustained use of the technology may not be observed in the long-term when the technology is simply provided for youths to utilise (Schaefer et al., 2016). It is, therefore, important to consider the novelty effect of the $3 \mathrm{D}$ models, as their effectiveness to promote physical activity may diminish with time, as previously observed with wearable trackers (Ledger \& McCaffrey, 2014). Nonetheless, it is worthy to note that youth's adoption of the 3D models in Study 4 as a goal-setting strategy holds promise for the long-term sustainability of the 3D-printed feedback and highlights the need for a long-term investigation. 
One methodological limitation to consider within Study 4 is how the implementation of the on-going short video interviews and researchers' presence may have influenced youths' understanding and awareness of PAL (Anderson, 2010b), rather than the 3D models per se. Although the interviews conducted were not specifically aimed to encourage or support youths' engagement in physical activity, the face-to-face engagement may have helped foster a heightened level of self-efficacy and selfdetermination for physical activity by creating a more meaningful experience, reinforcing effort or goals (Fortier et al., 2012, Williams \& French, 2011). Therefore, it remains unclear how the face-to-face interviews may have influenced youth's awareness and engagement with their 3D-printed model and their physical activity behaviours. There are a number of ways that a researcher or health professional could be deployed to support such an intervention, however, to make technology-based behaviour change strategies more pragmatic and cost-effective it would be useful to understand the efficacy of such support (Heath et al., 2012). For future research, it may be important to break down 3D-printed feedback conditions to include and exclude support to fully understand the impact of the tangible feedback and face-to-face engagement (Moore et al., 2015).

\subsection{Future Implications}

Based on the considerations discussed in this chapter and the wider emerging literature, there are a number of proposed recommendations for future research to explore the accuracy of physical activity measurement, and the design and evaluation of 3D-printed feedback as a tool to promote physical activity among youths.

\subsubsection{Importance of Turning for Clinical, Health and Physical Activity Measurement}

As alluded to in Study 1, there are a number of implications for turning in clinical practice, as a health promotion strategy and for enhanced physical activity measurement. Of particular concern are the discrepancies reported within the clinical six-minute walking test (6MWT), with methodologies varying due to limited space and resources, which results in distances used ranging from $20 \mathrm{~m}$ to $50 \mathrm{~m}$ with the frequency of turns completed ranging from 12 to 32 turns (Chetta et al., 2006). Congruent with McNarry et al. (2017), Study 1 concluded that altering the frequency 
of turns completed during the 6MWT will have a significant impact on the reliability of aerobic capacity assessment in patients. Therefore, future research should seek to generate algorithms that account for the distance and turns completed during a 6MWT to facilitate standardisation between health centres. On the other hand, treatment for obesity includes weight loss by increased physical activity, which reduces the risk of many non-communicable diseases associated with obesity (Avenell et al., 2004). Walking is the most commonly prescribed exercise for obese and overweight children and adults as it is an easy aerobic activity that leads to a high-calorie energy expenditure (Avenell et al., 2004, Baker et al., 2015, Blank et al., 2012, Martí et al., 2015). The intensity of regular walking $\left(4.2 \mathrm{~km} \cdot \mathrm{hr}^{-1}\right.$ ) is reported to be 3 METs (ACSM, 2013), however, recent evidence suggests that slow jogging at the same speed with turns can increase the intensity to 8 METs, resulting in a 2.7-fold increase in energy expenditure (Araki et al., 2017b). Moreover, even slow walking at $2.7 \mathrm{~km} \cdot \mathrm{hr}^{-1}$ becomes moderately intense (4 METs) when turns are incorporated. In this case, turning may offer a unique strategy to increase daily energy expenditure, increase fitness and lose weight that could be harnessed by future health promotion interventions, therefore warranting further investigation.

It is also pertinent to note the importance of accounting for turning when estimating children's highly sporadic physical activity patterns (Baquet et al., 2007). Of note, are the number of sports (e.g., football, basketball and netball; Fjørtoft et al., 2009) and general play and chasing games activities that children typically participate in (Sleap \& Warburton, 1996), all of which are likely to involve considerable amounts of turning. For example, Bloomfield et al. (2007) reported that elite football players can perform over 700 turns during a single match. The ability to more accurately account for youths' non-linear movements would assist in understanding their typical patterns of physical activity (i.e., type and intensity) and daily energy expenditure, which could lead to better informed physical activity interventions and health promotion strategies. However, there is a paucity of research regarding the energy expenditure of turning in young people, especially across a greater range of speeds to account for the more explosive sporting contexts and different ages, warranting further investigation. 


\subsubsection{Self-Report Physical Activity Questionnaires: Implications for Accuracy}

Self-report questionnaires are frequently used to estimate youths levels of physical activity and sedentary behaviour because they are cost-effective, non-invasive and can be self-administered (Pols et al., 1998, Dishman et al., 2001). Nonetheless, these questionnaires only provide a subjective estimate of physical activity and sedentary behaviour over a reference period, questioning the reliability and validity of data derived from such measurements (Aggio et al., 2016, Chinapaw et al., 2010, MartinezGomez et al., 2009). The data collected from self-report questionnaires relies on the respondent's ability to recall their activities, which is associated with numerous coding errors, such as misclassification of intensity, duration and frequency of physical activity bouts (Vandoni et al., 2017). These coding errors also may vary according to the demographic characteristics, including sex and age (Ainsworth, 2009), with selfreports also being subject to social desirability bias (Adams et al., 2005). Of concern is self-report data collected from youth populations as research suggests they have a limited ability to define and classify the intensity at which an activity occurs (Pearce et al., 2008, Trost et al., 2000a). In accord with Noonan et al. (2016), Study 3 highlighted that youths have particular problems when it comes to defining the intensity of non-routine light-and moderate-intensity activities (e.g., sweeping the floor, mowing the lawn and stair climbing) as they aren't perceived as sport-based activities (e.g., football or running) that are typically of vigorous-intensity. Complementary to this, research also supports that youth have difficulties in recalling non-routine activities within self-report questionnaires (Wolin et al., 2008), with validation studies reporting high correlations between self-report questionnaires and accelerometers for vigorous-intensity activities (Hagströmer et al., 2008, Lachat et al., 2008, Ottevaere et al., 2011). One explanation for this phenomenon is that vigorousintensity activities are easier to recall because they are more 'retrievable' from memory than the non-routine based activities (Biddle et al., 2011, Shephard, 2003). Similarly, results obtained from Study 3 (Chapter 5) showed how both children and adolescents were able to better define vigorous-intensity activities over the non-routine light- and moderate-intensity activities. In this light, future research should seek to explore youths' interpretations and understanding of non-routine-based activities to aid in the development and design of self-report questionnaires that better enable youth 
to correctly classify and recall non-routine activities and to enhance the accuracy of physical activity estimates.

As discussed in Study 3 (Chapter 5) there appeared to be a pattern whereby girls, irrespective of age, outperformed the boys at correctly aligning activities to their respective light-, moderate- and vigorous-intensity level. Indeed, previous research shows that girls do provide more reliable and valid self-reports of their true PAL than boys (Rangul et al., 2008). Based on cognitive developmental research, these sex difference could be, in part, explained by girls greater verbal and written language skills (Lynn, 1992, Mann et al., 1990, Martin \& Hoover, 1987, Undheim \& Nordvik, 1992) and ability to acquire vocabulary faster than boys up until adulthood (Roulstone et al., 2002). In this respect, girls may be at an advantage to boys in aligning activities to intensities. Therefore, future research should look to explore whether girls greater verbal and written language skills do indeed play a significant role in the understanding of intensities, and if so, how does this impact these measures and are sex-specific selfreport questionnaires required.

\subsubsection{D Printing Considerations: Practicality, Cost and Sustainability}

The practicality and cost of a tool within an intervention is important to consider, otherwise the dissemination into practice is invariably unfeasible (Dishman et al., 2001, Melanson Jr et al., 1996). From Studies 2, 3 and 4 it is evident that the sample size of future research will determine the size of the 3D printer required. However, there are some important practical implications to consider when choosing the optimal 3D printer for an intervention. Smaller 3D printers (e.g., Ultimaker, MakerBot) are usually simple to navigate and can be easily transferred to a new location, although are limited by the number of models printed per batch (2-4 models per print). In comparison, a larger 3D printer (e.g., HP Design jet, Stratasys Objet 1000 Plus) can print between 15-100 models per print. Additionally, the process of manually downloading, analysing, creating and printing youths' physical activity models involved a considerable amount of time and leaves much room for improvement. One solution would be to automate the creation process of the 3D models to reduce manual labour time. On the other hand, youths could be involved in the creation process of their $3 \mathrm{D}$ models as part of the intervention experience to engage them in healthful 
thinking (Khot, 2016, Ananthanarayan, 2015). In light of this, future research should seek to explore how youths can be involved within the 3D model creation process for the development of the importance of physical activity, as well as the sustained engagement with and understanding of the technology.

One notable consideration is the cost of 3D printing. Specifically, smaller 3D printers are more reasonably priced ( $£ 500$ to $£ 2,500)$ compared to large $3 \mathrm{D}$ printers $(\sim £ 13,000$ to $£ 500,000$ ), making them more feasible within a school-based setting. Furthermore, the specific filament (material) utilised to create the 3D models will also determine the cost, with filaments ranging from $\sim £ 20 / \mathrm{kg}$ to $\sim £ 70 / \mathrm{kg}$. A more expensive filament may involve a mixture of materials to reinforce the strength and flexibility of the 3D models, which is important to consider given that some youths in Study 4 alluded to the fact that their models had broken. Additionally, a better-quality filament also gives a more attractive and aesthetically pleasing finish to touch, which may positively influence youths' interactions with the 3D-printed feedback (Gillet et al., 2005). It is also important to consider the complexity of the designed 3D model, as the number of details on the model (e.g., engraving, patterns), the infill used within the model (e.g., hollow, light, dense or solid) and support structure (i.e., supporting structure used to prevent the $3 \mathrm{D}$ print from sagging or printing in mid-air), will each contribute to an increased cost of printing. Less obvious is the preparation time after a completed print, as supporting structures must be cut off or power-washed away from the 3D model itself. It is anticipated that $3 \mathrm{D}$ printing will become more accurate and cheaper (Mueller et al., 2014), with frequent use of 3D printers in everyday life expected in the near future (Anderson, 2010a). That said, the time and costs involved with 3D printing raises questions surrounding the sustainability of the technology to create 3D-printed physical activity feedback, especially given the limited number of 3D printing filaments that are recyclable at present. Congruent with Sauvé et al. (2017), creating more dynamic tangible feedback of physical activity that can change in shape over time via different moving parts to represent youths' PAL, may be one solution. In this case, future research should look to explore how shape-changing $3 \mathrm{D}$ models of physical activity feedback can be appropriately designed to represent different PAL to help reduce the number of models created per individual and associated resources utilised (Rasmussen et al., 2012). 


\subsubsection{D Printing Physical Activity as an Educational Tool}

Although youths showed no substantial increase in their understanding of intensities of MVPA associated with the Government guideline, the exploratory nature of the 3D models did aid youths in identifying their personal PAL to the recommended amount of physical activity (Marshall, 2007). In this way, using the 3D models in conjunction with school personal, social and health education (PSHE) lessons may offer a more meaningful, playful and interactive experience for learning about the importance of the Government guidelines and staying physically active and healthy, in comparison to traditional methods (Price et al., 2003). Indeed, there is an increasing recognition of 3D printing being a relevant and engaging educational tool within schools, with advances in user-friendly software making 3D printing more available and accessible for teachers (Biggs, 2017). In this light, this thesis puts forward the idea of integrating $3 \mathrm{D}$ printing physical activity as an educational tool to be utilised by teachers within the school PSHE curriculum, to enhance youths' awareness of the importance of physical activity for health. It is therefore important that teachers are included in the decision-making process on how best to integrate this novel technology into a schoolbased setting, through qualitative interview methods as utilised within Study 2 (Bitner \& Bitner, 2002).

\subsubsection{D Printing Personalised Multi-Dimensional Physical Activity}

The central focus of this thesis has been upon raising youths' MVPA as targeted by the UK Government guidelines for physical activity (Department of Health, 2011b). Indeed, it is important to acknowledge that physical activity is a much more heterogeneous behaviour than this thesis implies, with various other dimensions of physical activity having clear biological health benefits (Thompson et al., 2015). For example, an individual may score high in one physical activity domain (e.g., time spent in moderate-intensity physical activity) and low in another (e.g., total physical activity), with very few individuals able to consistently meet all physical activity dimensions (Thompson \& Batterham, 2013). In this way, feedback provided on one physical activity measure could impact the message youths receive, and as a consequence, youths could form incomplete or inaccurate perceptions of what types of behaviours are health rewarding. Therefore, it is especially important that we provide youths with knowledge regarding all types of behaviour, such as time spent 
being sedentary and their PAL for light-, moderate- and vigorous-intensity. Future research is required to explore the use of 3D printing personalised, multi-dimensional physical activity feedback, as a tool to promote youths' greater awareness and understanding of physical activity.

\subsubsection{D Printing Physical Activity for the Visually Impaired}

One noteworthy population that may benefit from 3D-printed physical activity data is those who are visually impaired. Indeed, visually impaired children (Aslan et al., 2012) and adults (Marmeleira et al., 2014) are reported to be less physically active when compared to their sighted peers. Visually impaired people rely primarily on their sense of touch to learn geometric shapes and the orientation of an object, as well as the spatial relationships among them (Jafri et al., 2015). In this way, 3D-printed tangible data of physical activity can provide visually impaired individuals with a tactile experience and understanding of their behaviours, once taught, on how to interpret the different components on the model (e.g., different days, intensities and recommended amount). It could be postulated that this increased level of interaction with their personal physical activity behaviours through the 3D models may provide visually impaired individuals with greater autonomy, personal interpretation and awareness of recommended guidelines and their relative PAL, or indeed risk behaviours. This thesis, therefore, suggests the need for future research to explore visually impaired youths' and adults' perceptions of 3D-printed feedback to promote physical activity engagement. 


\subsection{Final Comments}

This thesis presents an initial enquiry into the efficacy of measuring physical activity and the development of 3D-printed physical activity feedback. Whilst the results in Study 1 are preliminary and require further investigation, they indicate that measuring youths' physical activity is clearly still a big challenge with cause for concern around the capacity of accelerometers to account for children's sporadic movement patterns. However, there are early indications that tangible, 3D-printed physical activity feedback is a promising strategy to promote youths' awareness of their PAL, with potential to evoke the formation of goal-setting and improved self-determined motivation towards physical activity engagement. The positive findings in the series of studies presented in this thesis provide a strong foundation from which to develop future investigations and interventions, drawing on the strengths and future directions for research, and addressing the limitations acknowledged. As technology advances and becomes increasingly affordable in the near future, there will be exciting opportunities to further develop the capacities of tools to measure and tangibly conceptualise physical activity feedback on a wider scale, thereby reaching individuals who could, arguably, benefit the most from increased PAL. 


\section{REFERENCES}

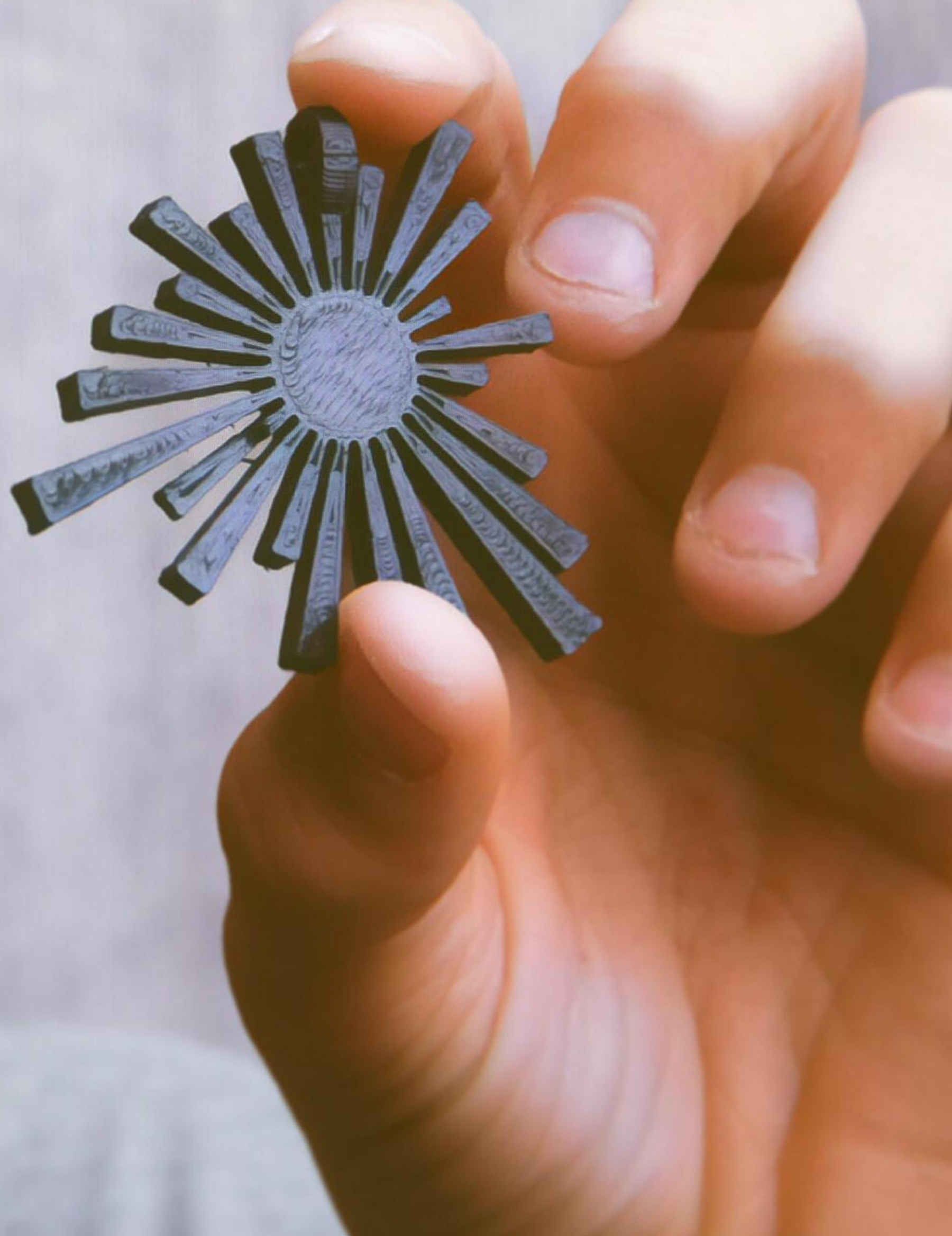




\section{References}

AADAHL, M., KJAER, M. \& JORGENSEN, T. 2007. Influence of time spent on TV viewing and vigorous intensity physical activity on cardiovascular biomarkers. The Inter 99 study. Eur J Cardiovasc Prev Rehabil, 14, 660-5.

ABADI, F. H., MUHAMAD, T. A. \& SALAMUDDIN, N. 2010. Energy expenditure through walking: Meta analysis on gender and age. Procedia-Social and Behavioral Sciences, 7, 512-521.

ABBOTT, B., BIGLAND, B. \& RITCHIE, J. 1952. The physiological cost of negative work. The Journal of physiology, 117, 380-390.

ABRAHAM, C. \& MICHIE, S. 2008. A taxonomy of behavior change techniques used in interventions. Health psychology, 27, 379.

ABU-OMAR, K., RÜTTEN, A. \& ROBINE, J.-M. 2004. Self-rated health and physical activity in the European Union. Sozial-und Präventivmedizin/Social and Preventive Medicine, 49, 235-242.

ABULA, K., GRÖPEL, P., CHEN, K. \& BECKMANN, J. 2016. Does knowledge of physical activity recommendations increase physical activity among Chinese college students? Empirical investigations based on the transtheoretical model. Journal of Sport and Health Science.

ACTIVITY, A.-P. C. O. P. 2014. Tackling Physical Inactivity - A Coordinated Approach.

ADAMO, K. B., PRINCE, S. A., TRICCO, A. C., CONNOR-GORBER, S. \& TREMBLAY, M. 2009. A comparison of indirect versus direct measures for assessing physical activity in the pediatric population: a systematic review. Int $J$ Pediatr Obes, 4, $2-27$.

ADAMS, J. \& WHITE, M. 2003. Are activity promotion interventions based on the transtheoretical model effective? A critical review. British Journal of Sports Medicine, 37, 106-114.

ADAMS, J. \& WHITE, M. 2004. Why don't stage-based activity promotion interventions work? Health education research, 20, 237-243.

ADAMS, S. A., MATTHEWS, C. E., EBBELING, C. B., MOORE, C. G., CUNNINGHAM, J. E., FULTON, J. \& HEBERT, J. R. 2005. The effect of social desirability and social approval on self-reports of physical activity. American journal of epidemiology, 161, 389-398.

AGGIO, D., FAIRCLOUGH, S., KNOWLES, Z. \& GRAVES, L. 2016. Validity and reliability of a modified english version of the physical activity questionnaire for adolescents. Arch Public Health, 74, 3.

AINSLIE, P. N., REILLY, T. \& WESTERTERP, K. R. 2003. Estimating human energy expenditure. Sports medicine, 33, 683-698.

AINSWORTH, B., CAHALIN, L., BUMAN, M. \& ROSS, R. 2015. The current state of physical activity assessment tools. Progress in cardiovascular diseases, 57, 387-395.

AINSWORTH, B. E. 2009. How do I measure physical activity in my patients? Questionnaires and objective methods. British Journal of Sports Medicine, 43, 6-9.

AKRAM, S. B., FRANK, J. S. \& CHENOURI, S. 2010. Turning behavior in healthy older adults: Is there a preference for step versus spin turns? Gait Posture, 31, 23-6. 
ALEXANDER, R. M. \& JAYES, A. 1983. A dynamic similarity hypothesis for the gaits of quadrupedal mammals. Journal of zoology, 201, 135-152.

ALFREY, L., CALE, L. \& A. WEBB, L. 2012. Physical education teachers' continuing professional development in health-related exercise. Physical education and sport pedagogy, 17, 477-491.

ALMALKI, M., GRAY, K. \& SANCHEZ, F. M. 2015. The use of self-quantification systems for personal health information: big data management activities and prospects. Health Information Science and Systems, 3, S1-S1.

ALTHOFF, T., WHITE, R. W. \& HORVITZ, E. 2016. Influence of Pokemon Go on Physical Activity: Study and Implications. J Med Internet Res, 18, e315.

ANANTHANARAYAN, S. 2015. Health Craft: A Computational Toolkit for Motivating Health Awareness in Children. Computer Science Graduate Theses \& Dissertations, University of Colorado at Boulder.

ANANTHANARAYAN, S., SIEK, K. \& EISENBERG, M. 2016. A Craft Approach to Health Awareness in Children. Proceedings of the 2016 ACM Conference on Designing Interactive Systems. Brisbane, QLD, Australia: ACM.

ANCKER, J. S. \& KAUFMAN, D. 2007. Rethinking health numeracy: A multidisciplinary literature review. Journal of the American Medical Informatics Association, 14, 713-721.

ANDERSEN, L. B., HARRO, M., SARDINHA, L. B., FROBERG, K., EKELUND, U., BRAGE, S. \& ANDERSSEN, S. A. 2006a. Physical activity and clustered cardiovascular risk in children: a cross-sectional study (The European Youth Heart Study). Lancet, 368, 299-304.

ANDERSEN, L. B., HARRO, M., SARDINHA, L. B., FROBERG, K., EKELUND, U., BRAGE, S. \& ANDERSSEN, S. A. 2006b. Physical activity and clustered cardiovascular risk in children: a cross-sectional study (The European Youth Heart Study). The Lancet, 368, 299-304.

ANDERSON, C. 2010a. In the next industrial revolution, atoms are the new bits. Wired magazine, 1, 2010.

ANDERSON, C. 2010b. Presenting and evaluating qualitative research. American journal of pharmaceutical education, 74, 141.

ANDERSON, I., MAITLAND, J., SHERWOOD, S., BARKHUUS, L., CHALMERS, M., HALL, M., BROWN, B. \& MULLER, H. 2007. Shakra: Tracking and Sharing Daily Activity Levels with Unaugmented Mobile Phones. Mobile Networks and Applications, 12, 185-199.

ANDROPOULOS, D. B. 2012. Pediatric Physiology: How Does it Differ from Adults? In: MASON, K. P. (ed.) Pediatric Sedation Outside of the Operating Room: A Multispecialty International Collaboration. New York, NY: Springer New York.

ANNEGARN, J., SPRUIT, M. A., SAVELBERG, H. H., WILLEMS, P. J., VAN DE BOOL, C., SCHOLS, A. M., WOUTERS, E. F. \& MEIJER, K. 2012. Differences in walking pattern during 6-min walk test between patients with COPD and healthy subjects. PloS one, 7, e37329.

ANNESI, J. J. 2004. Relationship between self-efficacy and changes in rated tension and depression for 9-to 12-yr.-old children enrolled in a 12-wk. after-school physical activity program. Perceptual and motor skills, 99, 191-194.

ANNESI, J. J., FAIGENBAUM, A. D. \& WESTCOTT, W. L. 2010. Relations of transtheoretical model stage, self-efficacy, and voluntary physical activity in African American preadolescents. Research quarterly for exercise and sport, 81, 239-244. 
AOKI, P. M. \& WOODRUFF, A. Making space for stories: ambiguity in the design of personal communication systems. Proceedings of the SIGCHI conference on Human factors in computing systems, 2005. ACM, 181-190.

ARAKI, M., HATAMOTO, Y., HIGAKI, Y. \& TANAKA, H. 2017a. "Slow walking with turns" increases quadriceps and erector spinae muscle activity. $J$ Phys Ther Sci, 29, 419-424.

ARAKI, M., HATAMOTO, Y., TAKAE, R., JACKOWSKA, M. \& TANAKA, H. 2017b. Turns in Jogging Increase Energy Expenditure: Proposed Home Exercise for Sedentary People. J Nov Physiother, 7.

ARMOUR, K. \& HARRIS, J. 2013. Making the case for developing new PE-for-health pedagogies. Quest, 65, 201-219.

ARMSTRONG, N., BALDING, J., GENTLE, P., WILLIAMS, J. \& KIRBY, B. 1990. Peak oxygen uptake and physical activity in 11-to 16-year-olds. Pediatric exercise science, 2, 349-358.

ARMSTRONG, N. \& WELSMAN, J. 1997. Young People and Physical Activity, Oxford University Press.

ASLAN, U. B., CALIK, B. B. \& KITIS, A. 2012. The effect of gender and level of vision on the physical activity level of children and adolescents with visual impairment. Res Dev Disabil, 33, 1799-804.

AUDREY, S., BELL, S., HUGHES, R. \& CAMPBELL, R. 2012. Adolescent perspectives on wearing accelerometers to measure physical activity in population-based trials. The European Journal of Public Health, 23, 475-480.

AULL, J. L., ROWE, D. A., HICKNER, R. C., MALINAUSKAS, B. M. \& MAHAR, M. T. 2008. Energy expenditure of obese, overweight, and normal weight females during lifestyle physical activities. Pediatric Obesity, 3, 177-185.

AVENEll, A., BROOM, J., BROWN, T. J., POOBALAN, A., AUCOTT, L. S., STEARNS, S., SMITH, W. C., JUNG, R., CAMPBELL, M. K. \& GRANT, A. M. 2004. Systematic review of the long-term effects and economic consequences of treatments for obesity and implications for health improvement. Health technology assessment.

AYRES, A. J. \& ROBBINS, J. 2005. Sensory integration and the child: Understanding hidden sensory challenges, Western Psychological Services.

AZAR, D., BALL, K., SALMON, J. \& CLELAND, V. 2008. The association between physical activity and depressive symptoms in young women: a review. Mental Health and Physical Activity, 1, 82-88.

BAILEY, B. W. \& MCINNIS, K. 2011. Energy cost of exergaming: a comparison of the energy cost of 6 forms of exergaming. Archives of pediatrics \& adolescent medicine, 165, 597-602.

BAILEY, R. 2010. Physical education for learning: a guide for secondary schools, A\&C Black.

BAILEY, R. C., OLSON, J., PEPPER, S. L., PORSZASZ, J., BARSTOW, T. J. \& COOPER, D. M. 1995. The level and tempo of children's physical activities: an observational study. Medicine and science in sports and exercise, 27, 10331041.

BAKER, E. H., MILNER, A. N. \& CAMPBELL, A. D. 2015. Walking Programs to Promote Weight Loss among Obese and Overweight Individuals: Walking Buses for Adults. Public health, 129, 822.

BANDA, J. A., HAYDEL, K. F., DAVILA, T., DESAI, M., BRYSON, S., HASKELL, W. L., MATHESON, D. \& ROBINSON, T. N. 2016. Effects of varying epoch lengths, wear time algorithms, and activity cut-points on 
estimates of child sedentary behavior and physical activity from accelerometer data. PloS one, 11, e0150534.

BANDURA, A. 1991. Social cognitive theory of self-regulation. Organizational Behavior and Human Decision Processes, 50, 248-287.

BANDURA, A. 1998. Health promotion from the perspective of social cognitive theory. Psychology \& Health, 13, 623-649.

BANDURA, A. 2009. Social cognitive theory of mass communication. Media effects. Routledge.

BANERJEE, B. \& SAHA, N. 1972. Energy intake and expenditure of Indian schoolboys. British Journal of Nutrition, 27, 483-490.

BANJARE, J. \& BHALERAO, S. 2016. Obesity associated noncommunicable disease burden. International Journal of Health \& Allied Sciences, 5, 81-87.

BANKHEAD, C., BRETT, J., BUKACH, C., WEBSTER, P., STEWART-BROWN, S., MUNAFO, M. \& AUSTOKER, J. 2005. Impact of screening on future health-promoting behaviors and health beliefs: A systematic review. International Journal of Technology Assessment in Health Care, 21, 147-147.

BANKS, M. 2018. Using visual data in qualitative research, Sage.

BANN, D., JOHNSON, W., LI, L., KUH, D. \& HARDY, R. 2018. Socioeconomic inequalities in childhood and adolescent body-mass index, weight, and height from 1953 to 2015: an analysis of four longitudinal, observational, British birth cohort studies. Lancet Public Health, 3, e194-e203.

BANYAI, F., ZSILA, A., KIRALY, O., MARAZ, A., ELEKES, Z., GRIFFITHS, M. D., ANDREASSEN, C. S. \& DEMETROVICS, Z. 2017. Problematic Social Media Use: Results from a Large-Scale Nationally Representative Adolescent Sample. PLoS One, 12, e0169839.

BAQUET, G., STRATTON, G., VAN PRAAGH, E. \& BERTHOIN, S. 2007. Improving physical activity assessment in prepubertal children with highfrequency accelerometry monitoring: a methodological issue. Prev Med, 44, 143-7.

BAR-ON, M. E., BROUGHTON, D. D., BUTTROSS, S., CORRIGAN, S., GEDISSMAN, A., DE RIVAS, M. R., RICH, M., SHIFRIN, D. L., BRODY, M. \& WILCOX, B. 2001. Children, adolescents, and television. Pediatrics, 107, 423-426.

BARA, F., GENTAZ, E., COLÉ, P. \& SPRENGER-CHAROLLES, L. 2004. The visuo-haptic and haptic exploration of letters increases the kindergartenchildren's understanding of the alphabetic principle. Cognitive development, 19, 433-449.

BARANOWSKI, T. 1988. Validity and Reliability of Self Report Measures of Physical Activity: An Information-Processing Perspective. Research Quarterly for Exercise and Sport, 59, 314-327.

BARKLEY, J. E., SALVY, S. J., SANDERS, G. J., DEY, S., VON CARLOWITZ, K. P. \& WILLIAMSON, M. L. 2014. Peer influence and physical activity behavior in young children: an experimental study. J Phys Act Health, 11, 4049.

BARNES, J., BEHRENS, T. K., BENDEN, M. E., BIDDLE, S., BOND, D., BRASSARD, P., BROWN, H., CARR, L., CHAPUT, J.-P. \& CHRISTIAN, H. 2012. Letter to the Editor: Standardized use of the terms" sedentary" and" sedentary behaviours". Applied Physiology Nutrition and MetabolismPhysiologie Appliquee Nutrition Et Metabolisme, 37, 540-542. 
BARNETT, A., CERIN, E. \& BARANOWSKI, T. 2011. Active video games for youth: a systematic review. J Phys Act Health, 8, 724-37.

BASSETT, D. R. \& JOHN, D. 2010. Use of pedometers and accelerometers in clinical populations: validity and reliability issues. Physical Therapy Reviews, 15, 135142.

BASSETT JR, D. R. 2000. Validity and reliability issues in objective monitoring of physical activity. Research quarterly for exercise and sport, 71, 30-36.

BASSETT JR, D. R., ROWLANDS, A. V. \& TROST, S. G. 2012. Calibration and validation of wearable monitors. Medicine and science in sports and exercise, 44, S32.

BASTIEN, G. J., WILLEMS, P. A., SCHEPENS, B. \& HEGLUND, N. C. 2005. Effect of load and speed on the energetic cost of human walking. Eur J Appl Physiol, 94, 76-83.

BAUMAN, A. E., REIS, R. S., SALLIS, J. F., WELLS, J. C., LOOS, R. J., MARTIN, B. W. \& GROUP, L. P. A. S. W. 2012. Correlates of physical activity: why are some people physically active and others not? The lancet, 380, 258-271.

BEAVER, W. L., WASSERMAN, K. \& WHIPP, B. J. 1986. A new method for detecting anaerobic threshold by gas exchange. J Appl Physiol (1985), 60, 2020-7.

BEKRAOUI, N., FARGEAS-GLUCK, M.-A. \& LÉGER, L. 2012. Oxygen uptake and heart rate response of 6 standardized tennis drills. Applied Physiology, Nutrition, and Metabolism, 37, 982-989.

BELTON, S., O'BRIEN, W., MEEGAN, S., WOODS, C. \& ISSARTEL, J. 2014. Youth-Physical Activity Towards Health: evidence and background to the development of the Y-PATH physical activity intervention for adolescents. BMC Public Health, 14, 122.

BENENSON, J. F., ANTONELlis, T. J., COTTON, B. J., NODDIN, K. E. \& CAMPBELL, K. A. 2008. Sex differences in children's formation of exclusionary alliances under scarce resource conditions. Animal Behaviour, 76, 497-505.

BENNETT, G. G. \& GLASGOW, R. E. 2009. The delivery of public health interventions via the Internet: actualizing their potential. Annu Rev Public Health, 30, 273-92.

BENTLEY, G. F., GOODRED, J. K., JAGO, R., SEBIRE, S. J., LUCAS, P. J., FOX, K. R., STEWART-BROWN, S. \& TURNER, K. M. 2012. Parents' views on child physical activity and their implications for physical activity parenting interventions: a qualitative study. BMC Pediatrics, 12, 180.

BERKEY, C. S., ROCKETT, H. R., GILlMAN, M. W. \& COLDITZ, G. A. 2003. One-year changes in activity and in inactivity among 10- to 15-year-old boys and girls: relationship to change in body mass index. Pediatrics, 111, 836-43.

BERKMAN, N. D., SHERIDAN, S. L., DONAHUE, K. E., HALPERN, D. J. \& CROTTY, K. 2011. Low health literacy and health outcomes: an updated systematic review. Annals of internal medicine, 155, 97-107.

BERRY, D., SAVOYE, M., MELKUS, G. \& GREY, M. 2007. An intervention for multiethnic obese parents and overweight children. Applied Nursing Research, 20, 63-71.

BESIER, T. F., LLOYD, D. G. \& ACKLAND, T. R. 2003. Muscle activation strategies at the knee during running and cutting maneuvers. Medicine \& Science in Sports \& Exercise, 35, 119-127. 
BESIER, T. F., LLOYD, D. G., COCHRANE, J. L. \& ACKLAND, T. R. 2001. External loading of the knee joint during running and cutting maneuvers. Medicine \& Science in Sports \& Exercise, 33, 1168-1175.

BETHEA, T. C., BERRY, D., MALONEY, A. E. \& SIKICH, L. 2012. Pilot Study of an Active Screen Time Game Correlates with Improved Physical Fitness in Minority Elementary School Youth. Games Health J, 1, 29-36.

BIDDER, O. R., QASEM, L. A. \& WILSON, R. P. 2012a. On higher ground: how well can dynamic body acceleration determine speed in variable terrain? PLoS One, 7, e50556.

BIDDER, O. R., SORESINA, M., SHEPARD, E. L., HALSEY, L. G., QUINTANA, F., GÓMEZ-LAICH, A. \& WILSON, R. P. 2012b. The need for speed: testing acceleration for estimating animal travel rates in terrestrial dead-reckoning systems. Zoology, 115, 58-64.

BIDDISS, E. \& IRWIN, J. 2010. Active video games to promote physical activity in children and youth: a systematic review. Arch Pediatr Adolesc Med, 164, 66472.

BIDDLE, S., CAVILL, N., EKELUND, U., GORELY, T., GRIFFITHS, M., JAGO, R., OPPERT, J., RAATS, M., SALMON, J. \& STRATTON, G. 2010. Sedentary behaviour and obesity: review of the current scientific evidence.

BIDDLE, S., SALLIS, J. F. \& CAVILL, N. 1998. Young and active? Young people and health enhancing physical activity - Evidence and implications: A report of the health education authority symposium, young and active?

BIDDLE, S. J., BENNIE, J. A., BAUMAN, A. E., CHAU, J. Y., DUNSTAN, D., OWEN, N., STAMATAKIS, E. \& VAN UFFELEN, J. G. 2016. Too much sitting and all-cause mortality: is there a causal link? BMC Public Health, 16, 635.

BIDDLE, S. J., GORELY, T., MARSHALL, S. J. \& CAMERON, N. 2009. The prevalence of sedentary behavior and physical activity in leisure time: A study of Scottish adolescents using ecological momentary assessment. Prev Med, 48, 151-5.

BIDDLE, S. J., GORELY, T., PEARSON, N. \& BULL, F. C. 2011. An assessment of self-reported physical activity instruments in young people for population surveillance: Project ALPHA. International Journal of Behavioral Nutrition and Physical Activity, 8, 1.

BIDDLE, S. J., GORELY, T. \& STENSEL, D. J. 2004. Health-enhancing physical activity and sedentary behaviour in children and adolescents. J Sports Sci, 22, 679-701.

BIDDLE, S. J. H. \& ASARE, M. 2011. Physical activity and mental health in children and adolescents: a review of reviews. British Journal of Sports Medicine, 45, 886.

BIGGS, S. 2017. The future of 3D printing in education. Eureka! London.

BIOCCA, F. 1997. The cyborg's dilemma: Progressive embodiment in virtual environments. Journal of computer-mediated communication, 3, JCMC324.

BITNER, N. \& BITNER, J. 2002. Integrating technology into the classroom: Eight keys to success. Journal of technology and teacher education, 10, 95-100.

BJÖRKQVIST, K. 1994. Sex differences in physical, verbal, and indirect aggression: A review of recent research. Sex Roles, 30, 177-188.

BLAIR, S. N. 2009. Physical inactivity: the biggest public health problem of the $21 \mathrm{st}$ century. Br J Sports Med, 43, 1-2. 
BLAIR, S. N., LAMONTE, M. J. \& NICHAMAN, M. Z. 2004. The evolution of physical activity recommendations: how much is enough? Am J Clin Nutr, 79, 913s-920s.

BLANDFORD, A. 2013. Semi-structured qualitative studies. Interaction Design Foundation.

BLANK, L., JONES, R., WOODS, H. B. \& PAYNE, N. 2012. Systematic review and narrative synthesis of the effectiveness of local interventions to promote cycling and walking for recreational and travel purposes. School of Health and Related Research. Sheffield.

BLOCH, M. 2008. Truth and sight: generalizing without universalizing. Journal of the Royal Anthropological Institute, 14.

BLOOMFIELD, J., POLMAN, R. \& O'DONOGHUE, P. 2007. Physical Demands of Different Positions in FA Premier League Soccer. J Sports Sci Med, 6, 63-70.

BODDY, L. M., DOWNS, S. J., KNOWLES, Z. R. \& FAIRCLOUGH, S. J. 2015. Physical activity and play behaviours in children and young people with intellectual disabilities: A cross-sectional observational study. School Psychology International, 36, 154-171.

BODDY, L. M., KNOWLES, Z. R., DAVIES, I. G., WARBURTON, G. L., MACKINTOSH, K. A., HOUGHTON, L. \& FAIRCLOUGH, S. J. 2012. Using formative research to develop the healthy eating component of the CHANGE! school-based curriculum intervention. BMC Public Health, 12, 710.

BOOTH, F. W., LAYE, M. J., LEES, S. J., RECTOR, R. S. \& THYFAULT, J. P. 2008. Reduced physical activity and risk of chronic disease: the biology behind the consequences. Eur J Appl Physiol, 102, 381-90.

BOPP, M. \& FALLON, E. 2008. Community-based interventions to promote increased physical activity. Applied health economics and health policy, 6, 173-187.

BORGES, T. T., HALLAL, P. C., DA SILVA, I. C. M., MIELKE, G. I., ROMBALDI, A. J. \& BARROS, F. C. 2015. Association Between Knowledge and Practice in the Field of Physical Activity and Health: A Population-Based Study. Journal of Physical Activity and Health, 12, 1005-1009.

BOSCO, C. \& KOMI, P. V. 1980. Influence of aging on the mechanical behavior of leg extensor muscles. Eur J Appl Physiol Occup Physiol, 45, 209-19.

BOUCHARD, C., BLAIR, S. N. \& HASKELL, W. L. 2012. Physical activity and health, Leeds.

BOUCHARD, C., BLAIR, S. N. \& KATZMARZYK, P. T. Less sitting, more physical activity, or higher fitness? Mayo Clinic Proceedings, 2015. Elsevier, 15331540.

BRAITHWAITE, I., STEWART, A. W., HANCOX, R. J., BEASLEY, R., MURPHY, R., MITCHELL, E. A. \& GROUP, I. P. T. S. 2013. The worldwide association between television viewing and obesity in children and adolescents: cross sectional study. PloS one, 8, e74263.

BRAUN, V. \& CLARKE, V. 2006. Using thematic analysis in psychology. Qualitative Research in Psychology, 3, 77-101.

BRESLIN, G., BRENNAN, D., RAFFERTY, R., GALLAGHER, A. M. \& HANNA, D. 2012. The effect of a healthy lifestyle programme on 8-9 year olds from social disadvantage. Archives of disease in childhood, archdischild-2011301108. 
BRETTSCHNEIDER, W.-D. 2001. Effects of sport club activities on adolescent development in Germany. European Journal of Sport Science, 1, 1-11.

BRIAN, K. 2012. Maths anxiety: the numbers are mounting. The Guardian.

BRIDLE, C., RIEMSMA, R. P., PATTENDEN, J., SOWDEN, A. J., MATHER, L., WATT, I. S. \& WALKER, A. 2005. Systematic review of the effectiveness of health behavior interventions based on the transtheoretical model. Psychology \& Health, 20, 283-301.

BRIEF, J.-C. 1983. Beyond Piaget : a philosophical psychology, New York.

BRINDOVA, D., PAVELKA, J., ŠEVČIKOVA, A., ŽEŽULA, I., DIJK, J. P., REIJNEVELD, S. A. \& GECKOVA, A. M. 2014. How parents can affect excessive spending of time on screen-based activities. BMC Public Health., 14.

BROCKMAN, R., JAGO, R. \& FOX, K. R. 2011. Children's active play: self-reported motivators, barriers and facilitators. BMC public health, 11, 461.

BROWN, S. W., WELSH, M. C., LABBÉ, E. E., VITULLI, W. F. \& KULKARNI, P. 1992. Aerobic exercise in the psychological treatment of adolescents. Perceptual and motor skills, 74, 555-560.

BRUG, J., CONNER, M., HARRE, N., KREMERS, S., MCKELlAR, S. \& WHITELAW, S. 2004. The Transtheoretical Model and stages of change: a critique: observations by five commentators on the paper by Adams, J. and White, M.(2004) why don't stage-based activity promotion interventions work? Health education research, 20, 244-258.

BRUSSEAU, T., KULINNA, P. H. \& COTHRAN, D. J. 2011. Health and physical activity content knowledge of Pima children. Physical Educator, 68, 66.

BRUSTAD, R. J. 1991. Children's perspectives on exercise and physical activity: measurement issues and concerns. Journal of School Health, 61, 228-231.

BRUTSAERT, T. D., TAMVADA, K. H., KIYAMU, M., WHITE, D. D. \& GAGE, T. B. 2011. Low ponderal index is associated with decreased muscle strength and fatigue resistance in college-aged women. Early human development, 87, 663-669.

BUCHHEIT, M. 2010. Performance and physiological responses to repeated-sprint and jump sequences. Eur J Appl Physiol, 110, 1007-18.

BUCHHEIT, M., BISHOP, D., HAYDAR, B., NAKAMURA, F. Y. \& AHMAIDI, S. 2010a. Physiological responses to shuttle repeated-sprint running. Int J Sports Med, 31, 402-9.

BUCHHEIT, M., BISHOP, D., HAYDAR, B., NAKAMURA, F. Y. \& AHMAIDI, S. 2010b. Physiological responses to shuttle repeated-sprint running. International journal of sports medicine, 31, 402-409.

BUCHHEIT, M., HAYDAR, B. \& AHMAIDI, S. 2012. Repeated sprints with directional changes: do angles matter? J Sports Sci, 30, 555-62.

BUCHHEIT, M., HAYDAR, B., HADER, K., UFLAND, P. \& AHMAIDI, S. 2011. Assessing running economy during field running with changes of direction: application to $20 \mathrm{~m}$ shuttle runs. Int J Sports Physiol Perform, 6, 380-95.

BUHI, E. R., TRUDNAK, T. E., MARTINASEK, M. P., OBERNE, A. B., FUHRMANN, H. J. \& MCDERMOTT, R. J. 2012. Mobile phone-based behavioural interventions for health: A systematic review. Health Education Journal, 72, 564-583.

BUNKER, L. K. 1998. Psycho-Physiological Contributions of Physical Activity and Sports for Girls. President's Council on Physical Fitness and Sports Research Digest. 
BUNTON, R., BALDWIN, S., FLYNN, D. \& WHITELAW, S. 2000. The 'stages of change'model in health promotion: science and ideology. Critical Public Health, 10, 55-70.

BURROWS, L. \& WRIGHT, J. 2004. The good life: New Zealand children's perspectives on health and self. Sport, Education and Society, 9, 193-205.

BURROWS, L., WRIGHT, J. \& JUNGERSEN-SMITH, J. 2002. "Measure Your Belly." New Zealand Children's Constructions of Health and Fitness. Journal of Teaching in Physical Education, 22, 39-48.

BURROWS, L., WRIGHT, J. \& MCCORMACK, J. 2009. Dosing up on food and physical activity: New Zealand children's ideas about 'health'. Health Education Journal, 68, 157-169.

BUTCHER, Z., FAIRCLOUGH, S., STRATTON, G. \& RICHARDSON, D. 2007. The effect of feedback and information on children's pedometer step counts at school. Pediatric exercise science, 19, 29-38.

BUTTE, N. F., EKELUND, U. \& WESTERTERP, K. R. 2012. Assessing physical activity using wearable monitors: measures of physical activity. Medicine \& Science in Sports \& Exercise, 44, S5-S12.

BUTTE, N. F., WATSON, K. B., RIDLEY, K., ZAKERI, I. F., MCMURRAY, R. G., PFEIFFER, K. A., CROUTER, S. E., HERRMANN, S. D., BASSETT, D. R., LONG, A., BERHANE, Z., TROST, S. G., AINSWORTH, B. E., BERRIGAN, D. \& FULTON, J. E. 2017. A Youth Compendium of Physical Activities: Activity Codes and Metabolic Intensities. Med Sci Sports Exerc.

BUTZOW, C. M. \& BUTZOW, J. W. 2000. Science through children's literature: An integrated approach, Libraries Unlimited.

CABAllERO, B., ClAY, T., DAVIS, S. M., ETHELBAH, B., ROCK, B. H., LOHMAN, T., NORMAN, J., STORY, M., STONE, E. J. \& STEPHENSON, L. 2003. Pathways: a school-based, randomized controlled trial for the prevention of obesity in American Indian schoolchildren. The American journal of clinical nutrition, 78, 1030-1038.

CAIN, K. L., CONWAY, T. L., ADAMS, M. A., HUSAK, L. E. \& SALLIS, J. F. 2013. Comparison of older and newer generations of ActiGraph accelerometers with the normal filter and the low frequency extension. International Journal of Behavioral Nutrition and Physical Activity, 10, 51.

CALE, L. 2000. Physical activity promotion in secondary schools. European Physical Education Review, 6, 71-90.

CALE, L. \& ALFREY, L. 2013. Physical education and health: moving forwards or 'going round in circles'?

CALE, L. \& HARRIS, J. 2004. Exercise and young people: Issues, implications and initiatives, Palgrave Macmillan.

CALFAS, K. J. \& TAYLOR, W. C. 1994. Effects of physical activity on psychological variables in adolescents. Pediatric exercise science, 6, 406-423.

CALLES, J. L. 2007. Depression in Children and Adolescents. Primary Care: Clinics in Office Practice, 34, 243-258.

CAMPBELL, I. 2007. Chi-squared and Fisher-Irwin tests of two-by-two tables with small sample recommendations. Stat Med, 26, 3661-75.

CAMPOS, C. M. \& DEL CASTILLO FERNÁNDEZ, H. 2016. The benefits of active video games for educational and physical activity approaches: A systematic review. Journal of New Approaches in Educational Research, 5, 115.

CARCARY, M. 2009. The Research Audit Trial--Enhancing Trustworthiness in Qualitative Inquiry. Electronic Journal of Business Research Methods, 7. 
CARD, S., MACKINLAY, J AND SCHNEIDERMAN, B 1999. Readings in information visualisation, San Francisco: Kaufmann.

CARDINAL, B. J., ENGELS, H.-J. \& ZHU, W. 1998. Application of the Transtheoretical Model of Behavior Change to Preadolescents' Physical Activity and Exercise Behavior. Pediatric Exercise Science, 10, 69-80.

CARDON, G. M., VAN ACKER, R., SEGHERS, J., DE MARTELAER, K., HAERENS, L. L. \& DE BOURDEAUDHUIJ, I. M. 2012. Physical activity promotion in schools: which strategies do schools (not) implement and which socioecological factors are associated with implementation? Health education research, 27, 470-483.

CARlson, S. A., FUltON, J. E., LEE, S. M., FOLEY, J. T., HEITZLER, C. \& HUHMAN, M. 2010. Influence of Limit-Setting and Participation in Physical Activity on Youth Screen Time. Pediatrics., 126.

CARMO, J. L. F. D. \& PALMEIRA, A. L. 2013. Can active video games be part of the solution to promote physical activity in youth? A systematic review. Archives of Exercise in Health and Disease, 4, 216-226.

CARRINGTON, D. 2016. Three-quarters of UK children spend less time outdoors than prison inmates - survey. The Guardian.

CARROL, J. M. Five reasons for scenario-based design. Systems Sciences, 1999. HICSS-32. Proceedings of the 32nd Annual Hawaii International Conference on, 1999. IEEE, $11 \mathrm{pp}$.

CARSON, V. \& JANSSEN, I. 2011. Volume, patterns, and types of sedentary behavior and cardio-metabolic health in children and adolescents: a crosssectional study. BMC public health, 11, 274.

CARVER, A., TIMPERIO, A. \& CRAWFORD, D. 2008. Playing it safe: The influence of neighbourhood safety on children's physical activity-A review. Health \& place, 14, 217-227.

CASPERSEN, C. J., POWELL, K. E. \& CHRISTENSON, G. M. 1985. Physical activity, exercise, and physical fitness: definitions and distinctions for healthrelated research. Public Health Rep, 100, 126-31.

CASTELLI, D. \& WILLIAMS, L. 2007. Health-related fitness and physical education teachers' content knowledge. Journal of Teaching in Physical Education, 26, 3-19.

CASTRONOVA, E. 2008. Synthetic worlds: The business and culture of online games, University of Chicago press.

CATALA, A., SYLLA, C., THEUNE, M., BROOKS, E. \& READ, J. C. Rethinking children's co-creation processes beyond the design of TUIs. Proceedings of the 17th ACM Conference on Interaction Design and Children, 2018. ACM, 733-740.

CATELLIER, D. J., HANNAN, P. J., MURRAY, D. M., ADDY, C. L., CONWAY, T. L., YANG, S. \& RICE, J. C. 2005. Imputation of missing data when measuring physical activity by accelerometry. Medicine and science in sports and exercise, 37, S555.

CAVALLO, D. N., TATE, D. F., RIES, A. V., BROWN, J. D., DEVELLIS, R. F. \& AMMERMAN, A. S. 2012. A social media-based physical activity intervention: a randomized controlled trial. Am J Prev Med, 43, 527-32.

CESUROGLU, T., SYURINA, E., FERON, F. \& KRUMEICH, A. 2016. Other side of the coin for personalised medicine and healthcare: content analysis of 'personalised' practices in the literature. BMJ Open, 6. 
CHADDHA, A., JACKSON, E. A., RICHARDSON, C. R. \& FRANKLIN, B. A. 2017. Technology to help promote physical activity. American Journal of Cardiology, 119, 149-152.

CHAN, N. P., SUNG, R. Y., NELSON, E. A., SO, H. K., TSE, Y. K. \& KONG, A. P. 2010. Measurement of pubertal status with a Chinese self-report Pubertal Development Scale. Matern Child Health J, 14, 466-73.

CHEN, J. L., WEISS, S., HEYMAN, M. B., COOPER, B. \& LUSTIG, R. H. 2011. The efficacy of the web-based childhood obesity prevention program in Chinese American adolescents (Web ABC study). J Adolesc Health, 49, 14854.

CHEN, K. Y., ROTHNEY, M. P. \& BRYCHTA, R. J. 2007. Physical Activity Monitors: Do More Sensors Mean Better Precision? Journal of diabetes science and technology (Online), 1, 768-770.

CHETTA, A., ZANINI, A., PISI, G., AIELlO, M., TZANI, P., NERI, M. \& OLIVIERI, D. 2006. Reference values for the 6-min walk test in healthy subjects 20-50 years old. Respir Med, 100, 1573-8.

CHINAPAW, M. J., MOKKINK, L. B., VAN POPPEL, M. N., VAN MECHELEN, W. \& TERWEE, C. B. 2010. Physical activity questionnaires for youth: a systematic review of measurement properties. Sports Med, 40, 539-63.

CHRISTIAN, D., TODD, C., HILL, R., RANCE, J., MACKINTOSH, K., STRATTON, G. \& BROPHY, S. 2016. Active children through incentive vouchers - evaluation (ACTIVE): a mixed-method feasibility study. BMC Public Health, 16, 890.

CHU, E., MCMANUS, A. M. \& YU, C. 2007. Calibration of the RT3 accelerometer for ambulation and nonambulation in children. Medicine and science in sports and exercise, 39, 2085-2091.

CLARK, C. C., BARNES, C. M., HOLTON, M., SUMMERS, H. D. \& STRATTON, G. 2016. SlamTracker Accuracy under Static and Controlled Movement Conditions. Sport Science Review, 25, 374-383.

CLARKE, V. \& BRAUN, V. 2013. Teaching thematic analysis: Overcoming challenges and developing strategies for effective learning. The psychologist, 26, 120-123.

CLELAND, I., KIKHIA, B., NUGENT, C., BOYTSOV, A., HALLBERG, J., SYNNES, K., MCCLEAN, S. \& FINLAY, D. 2013. Optimal placement of accelerometers for the detection of everyday activities. Sensors, 13, 91839200.

COFFIELD, F. 2004. Should we be using learning styles? What research has to say to practice, Learning and Skills Research Centre.

COHEN, I. B., WHITMAN, A. \& BUDENZ, J. 2016. The Principia: The Authoritative Translation: Mathematical Principles of Natural Philosophy.

COLE, M. \& WERTSCH, J. V. 1996. Beyond the Individual-Social Antinomy in Discussions of Piaget and Vygotsky. Human Development, 39, 250-256.

COLE, T. J., BELLIZZI, M. C., FLEGAL, K. M. \& DIETZ, W. H. 2000. Establishing a standard definition for child overweight and obesity worldwide: international survey. Bmj, 320, 1240.

COLEMAN, T. 2014. Amy B. Jordan and Daniel Romer (eds.): Media and the Wellbeing of Children and Adolescents. A Multidisciplinary Research Publication. Boston.

COlley, R. C., GARRIGUET, D., JANSSEN, I., CRAIG, C. L., ClARKE, J. \& TREMBLAY, M. S. 2011. Physical activity of Canadian children and youth: 
accelerometer results from the 2007 to 2009 Canadian Health Measures Survey. Health reports, 22, 15.

CONNOLLY, P. \& MCKENZIE, T. 1995. Effects of a games intervention on the physical activity levels of children at recess. Res $Q$ Exerc Sport, 66, A60.

CONSOLVO, S., EVERITT, K., SMITH, I. \& LANDAY, J. A. Design requirements for technologies that encourage physical activity. Proceedings of the SIGCHI conference on Human Factors in computing systems, 2006. ACM, 457-466.

CONSOLVO, S., KLASNJA, P., MCDONALD, D. W., AVRAHAMI, D., FROEHLICH, J., LEGRAND, L., LIBBY, R., MOSHER, K. \& LANDAY, J. A. 2008a. Flowers or a robot army?: encouraging awareness \& activity with personal, mobile displays. Proceedings of the 10th international conference on Ubiquitous computing. Seoul, Korea: ACM.

CONSOLVO, S., MCDONALD, D. W., TOSCOS, T., CHEN, M. Y., FROEHLICH, J., HARRISON, B., KLASNJA, P., LAMARCA, A., LEGRAND, L., LIBBY, R., SMITH, I. \& LANDAY, J. A. 2008b. Activity sensing in the wild: a field trial of ubifit garden. Proceedings of the SIGCHI Conference on Human Factors in Computing Systems. Florence, Italy: ACM.

COOK, T. L., DE BOURDEAUDHUIJ, I., MAES, L., HAERENS, L., GRAMMATIKAKI, E., WIDHALM, K., KWAK, L., PLADA, M., MORENO, L. A., ZAMPELAS, A., TOUNTAS, Y. \& MANIOS, Y. 2014. Moderators of the effectiveness of a web-based tailored intervention promoting physical activity in adolescents: the HELENA Activ-O-Meter. J Sch Health, 84, 256-66.

CORBIN, C. B. \& LE MASURIER, G. 2014. Fitness for Life, 6E, Human Kinetics.

CORDAIN, L., GOTSHALL, R. W., EATON, S. B. \& EATON, S. B., 3RD 1998. Physical activity, energy expenditure and fitness: an evolutionary perspective. Int J Sports Med, 19, 328-35.

CORDER, K., EKELUND, U., STEELE, R. M., WAREHAM, N. J. \& BRAGE, S. 2008. Assessment of physical activity in youth. J Appl Physiol (1985), 105, 977-87.

CORDER, K., VAN SLUIJS, E. M., MCMINN, A. M., EKELUND, U., CASSIDY, A. \& GRIFFIN, S. J. 2010. Perception versus reality awareness of physical activity levels of British children. Am J Prev Med, 38, 1-8.

COREPAL, R., TULLY, M. A., KEE, F., MILLER, S. J. \& HUNTER, R. F. 2018. Behavioural incentive interventions for health behaviour change in young people (5-18 years old): A systematic review and meta-analysis. Preventive Medicine, 110, 55-66.

COTTRELl, L., HARRIS, C. V., BRADLYN, A., GUNEL, E., NEAL, W. A., ABILDSO, L. \& COFFMAN, J. W. 2012. Identifying the People and Factors That Influence Children's Intentions to Make Lifestyle Changes. Health Promotion Practice, 13, 183-189.

COWARD, W., RITZ, P. \& COLE, T. 1994. Revision of calculations in the doubly labeled water method for measurement of energy expenditure in humans. American Journal of Physiology-Endocrinology and Metabolism, 267, E805E807.

COWDEN, R. D. \& PLOWMAN, S. A. 1999. The Self-Regulation and Perception of Exercise Intensity in Children in a Field Setting. Pediatric Exercise Science, $11,32-43$.

COWLING, M. 2016. How Apple Watch and pervasive computing can lure you into leveling up your fitness. The Conversation. [Online]. [Accessed]. 
CRAFT, L. L., PFEIFFER, K. A. \& PIVARNIK, J. M. 2003. Predictors of physical competence in adolescent girls. Journal of Youth and Adolescence, 32, 431438.

CROUTER, S. E., HORTON, M. \& BASSETT JR, D. R. 2013. Validity of ActiGraph child-specific equations during various physical activities. Medicine and science in sports and exercise, 45, 1403.

CROUTER, S. E., SCHNEIDER, P. L., KARABULUT, M. \& BASSETT, D. R., JR. 2003. Validity of 10 electronic pedometers for measuring steps, distance, and energy cost. Med Sci Sports Exerc, 35, 1455-60.

CULLEN, K. W., BARANOWSKI, T. \& SMITH, S. P. 2001. Using goal setting as a strategy for dietary behavior change. Journal of the American Dietetic Association, 101, 562-566.

CULLEN, K. W., THOMPSON, D., BOUSHEY, C., KONZELMANN, K. \& CHEN, T. A. 2013. Evaluation of a web-based program promoting healthy eating and physical activity for adolescents: teen choice: food and fitness. Health Educ Res, 28, 704-14.

CUMMINGS, H. M. \& VANDEWATER, E. A. 2007. Relation of Adolescent Video Game Play to Time Spent in Other Activities. Archives of pediatrics \& adolescent medicine, 161, 684-689.

CUNNINGHAM, J. J. 1980. A reanalysis of the factors influencing basal metabolic rate in normal adults. Am J Clin Nutr, 33, 2372-4.

DA ROCHA, E. E. M., ALVES, V. G. F. \& DA FONSECA, R. B. V. 2006. Indirect calorimetry: methodology, instruments and clinical application. Current Opinion in Clinical Nutrition \& Metabolic Care, 9, 247-256.

DABBS, A. D. V., MYERS, B. A., MC CURRY, K. R., DUNBAR-JACOB, J., HAWKINS, R. P., BEGEY, A. \& DEW, M. A. 2009. User-centered design and interactive health technologies for patients. Computers, informatics, nursing: CIN, 27, 175.

DALEY, A. J. 2009. Can exergaming contribute to improving physical activity levels and health outcomes in children? Pediatrics, 124, 763-71.

DARBYSHIRE, P., MACDOUGALL, C. \& SCHILLER, W. 2005. Multiple methods in qualitative research with children: more insight or just more? Qualitative research, 5, 417-436.

DAVIDSON, F. 2007. Childhood obesity prevention and physical activity in schools. Health Education, 107, 377-395.

DAVIE, R., PANTING, C. \& CHARLTON, T. 2004. Mobile phone ownership and usage among pre-adolescents. Telematics and Informatics, 21, 359-373.

DAVIES, S., BURNS, H., JEWELL, T. \& MCBRIDE, M. 2011. Start active, stay active: a report on physical activity from the four home countries. Chief Medical Officers, 16306, 1-62.

DAVIS, W. D. 2005. The Interactive Effects of Goal Orientation and Feedback Specificity on Task Performance. Human Performance, 18, 409-426.

DAVISON, K. K. \& BIRCH, L. L. 2001. Childhood overweight: a contextual model and recommendations for future research. Obesity reviews, 2, 159-171.

DAVISON, K. K., MÂSSE, L. C., TIMPERIO, A., FRENN, M. D., SAUNDERS, J., MENDOZA, J. A., GOBBI, E., HANSON, P. \& TROST, S. G. 2013. Physical activity parenting measurement and research: challenges, explanations, and solutions. Childhood Obesity, 9, S-103-S-109.

DAY, E. A., BLAIR, C., DANIELS, S., KLIGYTE, V. \& MUMFORD, M. D. 2006. Linking instructional objectives to the design of instructional environments: 
The Integrative Training Design Matrix. Human Resource Management Review, 16, 376-395.

DE ALMEIDA MENDES, M., DA SILVA, I. C. M., RAMIRES, V. V., REICHERT, F. F., MARTINS, R. C. \& TOMASI, E. 2018. Calibration of raw accelerometer data to measure physical activity: A systematic review. Gait Posture, 61, 98110.

DE BOURDEAUDHUiJ, I., MAES, L., DE HENAUW, S., DE VRIENDT, T., MORENO, L. A., KERSTING, M., SARRI, K., MANIOS, Y., WIDHALM, K., SJOSTROM, M., RUIZ, J. R. \& HAERENS, L. 2010. Evaluation of a computer-tailored physical activity intervention in adolescents in six European countries: the Activ-O-Meter in the HELENA intervention study. $J$ Adolesc Health, 46, 458-66.

DE BOURDEAUDHUIJ, I., PHILIPPAERTS, R., CROMBEZ, G., MATTON, L., WIJNDAELE, K., BALDUCK, A.-L. \& LEFEVRE, J. 2004. Stages of change for physical activity in a community sample of adolescents. Health education research, 20, 357-366.

DE BOURDEAUDHUIJ, I., VAN CAUWENBERGHE, E., SPITTAELS, H., OPPERT, J. M., ROSTAMI, C., BRUG, J., VAN LENTHE, F., LOBSTEIN, T. \& MAES, L. 2011. School-based interventions promoting both physical activity and healthy eating in Europe: a systematic review within the HOPE project. Obesity Reviews, 12, 205-216.

DE VRIES, S. I., BAKKER, I., HOPMAN-ROCK, M., HIRASING, R. A. \& VAN MECHELEN, W. 2006. Clinimetric review of motion sensors in children and adolescents. J Clin Epidemiol, 59, 670-80.

DEAN, P., VOSS, C., DE SOUZA, A. \& HARRIS, K. 2016. Assessment, Assurance, and Actuation: the use of activity trackers to monitor physical activity in a pediatric population with congenital heart disease. Canadian Journal of Cardiology, 32, S130-S131.

DEANSCOMBE, M. 2010. The good research guide for small scale research projects. Berkshire: Open University Press.

DELlAL, A., KELLER, D., CARLING, C., CHAOUACHI, A., WONG DEL, P. \& CHAMARI, K. 2010. Physiologic effects of directional changes in intermittent exercise in soccer players. $J$ Strength Cond Res, 24, 3219-26.

DELOACHE, J. S. 2000. Dual representation and young children's use of scale models. Child development, 71, 329-338.

DELOACHE, J. S., PIERROUTSAKOS, S. L., UTTAL, D. H., ROSENGREN, K. S. $\&$ GOTTLIEB, A. 1998. Grasping the nature of pictures. Psychological Science, 9, 205-210.

DENCKER, M., THORSSON, O., KARLSSON, M. K., LINDÉN, C., WOLLMER, P. \& ANDERSEN, L. B. 2008. Maximal oxygen uptake versus maximal power output in children. Journal of sports sciences, 26, 1397-1402.

DEPARTMENT OF HEALTH. 2011. Start Active, Stay Active. A report on physical activity for health from the four home countries' Chief Medical Officers, London.

DESMOND, S. M., PRICE, J. H., LOCK, R. S., SMITH, D. \& STEWART, P. W. 1990. Urban black and white adolescents' physical fitness status and perceptions of exercise. $J$ Sch Health, 60, 220-6.

DI PRAMPERO, P., FUSI, S., SEPULCRI, L., MORIN, J., BELLI, A. \& ANTONUTTO, G. 2005. Sprint running: a new energetic approach. Journal of experimental Biology, 208, 2809-2816. 
DIAS, K. A., INGUL, C. B., TJONNA, A. E., KEATING, S. E., GOMERSALL, S. R., FOLLESTAD, T., HOSSEINI, M. S., HOLLEKIM-STRAND, S. M., RO, T. B., HARAM, M., HUUSE, E. M., DAVIES, P. S. W., CAIN, P. A., LEONG, G. M. \& COOMBES, J. S. 2018. Effect of High-Intensity Interval Training on Fitness, Fat Mass and Cardiometabolic Biomarkers in Children with Obesity: A Randomised Controlled Trial. Sports Med, 48, 733-746.

DICLEMENTE, C. C., MARINILLI, A. S., SINGH, M. \& BELLINO, L. E. 2001. The role of feedback in the process of health behavior change. American journal of health behavior, 25, 217-227.

DIETZ, W. H. \& GORTMAKER, S. L. 1985. Do we fatten our children at the television set? Obesity and television viewing in children and adolescents. Pediatrics, 75, 807-812.

DILBER, D., MALČIĆ, I., ĆALETA, T. \& ZOVKO, A. 2015. Reference values for cardiopulmonary exercise testing in children and adolescents in northwest Croatia. Paediatria Croatica, 59, 195-201.

DILORENZO, T. M., STUCKY-ROPP, R. C., VANDER WAL, J. S. \& GOTHAM, H. J. 1998. Determinants of exercise among children. II. A longitudinal analysis. Prev Med, 27, 470-7.

DILORIO, C., HOCKENBERRY-EATON, M., MAIBACH, E. \& RIVERO, T. 1994. Focus groups: an interview method for nursing research. J Neurosci Nurs, 26, 175-80.

DISHMAN, R. K., WASHBURN, R. A. \& SCHOELLER, D. A. 2001. Measurement of Physical Activity. Quest, 53, 295-309.

DIXEY, R., SAHOTA, P., ATWAL, S. \& TURNER, A. 2001. "Ha ha, you're fat, we're strong"; a qualitative study of boys' and girls' perceptions of fatness, thinness, social pressures and health using focus groups. Health Education, 101, 206-216.

DIXON, P. C., STEBBINS, J., THEOLOGIS, T. \& ZAVATSKY, A. B. 2013. Spatiotemporal parameters and lower-limb kinematics of turning gait in typically developing children. Gait Posture, 38, 870-5.

DOBBINS, M., HUSSON, H., DECORBY, K. \& LAROCCA, R. L. 2013. Schoolbased physical activity programs for promoting physical activity and fitness in children and adolescents aged 6 to 18. Cochrane Database Syst Rev, Cd007651.

DOHERTY, A., JACKSON, D., HAMMERLA, N., PLOTZ, T., OLIVIER, P., GRANAT, M. H., WHITE, T., VAN HEES, V. T., TRENELL, M. I., OWEN, C. G., PREECE, S. J., GILLIONS, R., SHEARD, S., PEAKMAN, T., BRAGE, S. \& WAREHAM, N. J. 2017. Large Scale Population Assessment of Physical Activity Using Wrist Worn Accelerometers: The UK Biobank Study. PLoS One, 12, $\mathrm{e} 0169649$.

DONNELLY, J. E., GREENE, J. L., GIBSON, C. A., SMITH, B. K., WASHBURN, R. A., SULLIVAN, D. K., DUBOSE, K., MAYO, M. S., SCHMELZLE, K. H. \& RYAN, J. J. 2009. Physical Activity Across the Curriculum (PAAC): a randomized controlled trial to promote physical activity and diminish overweight and obesity in elementary school children. Preventive medicine, 49, 336-341.

DOURISH, P. Where the Action Is: The Foundations of Embodied Interaction. 2004.

DRAGICEVIC, P. A. J., Y 2012. List of physical visualizations. dataphys.org/list.

DRUIN, A. 2002. The role of children in the design of new technology. Behaviour and information technology, 21, 1-25. 
DUNCAN, M. \& STAPLES, V. 2010. The impact of a school-based active video game play intervention on children's physical activity during recess. Human Movement, 11, 95-99.

EASTWICK, P. W. \& GARDNER, W. L. 2009. Is it a game? Evidence for social influence in the virtual world. Social Influence, 4, 18-32.

EATON, S. B. \& EATON, S. B. 2003. An evolutionary perspective on human physical activity: implications for health. Comp Biochem Physiol A Mol Integr Physiol, 136, 153-9.

EATON, S. B., KONNER, M. \& SHOSTAK, M. 1988. Stone agers in the fast lane: chronic degenerative diseases in evolutionary perspective. Am J Med, 84, 73949.

ECKERSLEY, R. 2011. A new narrative of young people's health and well-being. Journal of Youth Studies, 14, 627-638.

ECONOMOS, C. D. 2001. Less Exercise Now, More Disease Later? The Critical Role of Childhood Exercise Interventions in Reducing Chronic Disease Burden. Nutrition in Clinical Care, 4, 306-313.

ECONOMOS, C. D., HYATT, R. R., GOLDBERG, J. P., MUST, A., NAUMOVA, E. N., COLLINS, J. J. \& NELSON, M. E. 2007. A community intervention reduces BMI z-score in children: Shape Up Somerville first year results. Obesity (Silver Spring), 15, 1325-36.

EDUCATION, U. D. F. 2013. 3D printers in the schools: uses in the curriculum [Online]. [Accessed].

EDWARDS, A., ELWYN, G. \& MULLEY, A. 2002. Explaining risks: turning numerical data into meaningful pictures. Bmj, 324, 827-830.

EDWARDS, L., SALANT, V., HOWARD, V. F., BROUGHER, J. \& MCLAUGHLIN, T. 1995. Effectiveness of self-management on attentional behavior and reading comprehension for children with attention deficit disorder. Child \& Family Behavior Therapy, 17, 1-17.

EIME, R. M., YOUNG, J. A., HARVEY, J. T., CHARITY, M. J. \& PAYNE, W. R. 2013. A systematic review of the psychological and social benefits of participation in sport for children and adolescents: informing development of a conceptual model of health through sport. Int J Behav Nutr Phys Act, 10, 98.

EISENMANN, J. C., STRATH, S. J., SHADRICK, D., RIGSBY, P., HIRSCH, N. \& JACOBSON, L. 2004. Validity of uniaxial accelerometry during activities of daily living in children. Eur J Appl Physiol, 91, 259-63.

EKELUND, U., ANDERSSEN, S. A., FROBERG, K., SARDINHA, L. B., ANDERSEN, L. B. \& BRAGE, S. 2007. Independent associations of physical activity and cardiorespiratory fitness with metabolic risk factors in children: the European youth heart study. Diabetologia, 50, 1832-1840.

EKELUND, U., LUAN, J., SHERAR, L. B., ESLIGER, D. W., GRIEW, P. \& COOPER, A. 2012. Moderate to vigorous physical activity and sedentary time and cardiometabolic risk factors in children and adolescents. Jama, 307, 70412.

EKELUND, U., TOMKINSON, G. \& ARMSTRONG, N. 2011. What proportion of youth are physically active? Measurement issues, levels and recent time trends. British Journal of Sports Medicine, 45, 859-865.

ELLIS, L. A., COLLIN, P., HURLEY, P. J., DAVENPORT, T. A., BURNS, J. M. \& HICKIE, I. B. 2013. Young men's attitudes and behaviour in relation to mental health and technology: implications for the development of online mental health services. BMC Psychiatry, 13, 119. 
ENGLAND, H. S. F. 2014. Children's body mass index, overweight and obesity. NHS Digital.

ERIKSON, E. H. \& ERIKSON, J. M. 1998. The life cycle completed (extended version), WW Norton \& Company.

ERRICKSON, S. P., MALONEY, A. E., THORPE, D., GIULIANI, C. \& ROSENBERG, A. M. 2012. "Dance Dance Revolution" Used by 7- and 8Year-Olds to Boost Physical Activity: Is Coaching Necessary for Adherence to an Exercise Prescription? Games Health J, 1, 45-50.

ESLIGER, D. W., PROBERT, A., CONNOR GORBER, S., BRYAN, S., LAVIOLETTE, M. \& TREMBLAY, M. S. 2007. Validity of the Actical accelerometer step-count function. Med Sci Sports Exerc, 39, 1200-4.

EVENSON, K. R., CATELLIER, D. J., GILL, K., ONDRAK, K. S. \& MCMURRAY, R. G. 2008a. Calibration of two objective measures of physical activity for children. Journal of sports sciences, 26, 1557-1565.

EVENSON, K. R., CATELLIER, D. J., GILL, K., ONDRAK, K. S. \& MCMURRAY, R. G. 2008b. Calibration of two objective measures of physical activity for children. J Sports Sci, 26, 1557-65.

EVERLEY, S. \& MACFADYEN, T. 2015. 'I like playing on my trampoline; it makes me feel alive.' Valuing physical activity: perceptions and meanings for children and implications for primary schools. Education 3-13, 45, 151-175.

F. SALLIS, J., OWEN, N. \& FISHER, E. 2008. Ecological Models of Health Behavior.

FAIRCLOUGH, S. \& STRATTON, G. 2004. 'Physical education makes you fit and healthy'. Physical education's contribution to young people's physical activity levels. Health education research, 20, 14-23.

FAIRCLOUGH, S. J., BUTCHER, Z. H. \& STRATTON, G. 2007. Whole-day and segmented-day physical activity variability of northwest England school children. Preventive medicine, 44, 421-425.

FAIRCLOUGH, S. J., HACKETT, A. F., DAVIES, I. G., GOBBI, R., MACKINTOSH, K. A., WARBURTON, G. L., STRATTON, G., VAN SLUIJS, E. M. \& BODDY, L. M. 2013. Promoting healthy weight in primary school children through physical activity and nutrition education: a pragmatic evaluation of the CHANGE! randomised intervention study. BMC public health, 13, 626.

FAIRCLOUGH, S. J., RIDGERS, N. D. \& WELK, G. 2012. Correlates of children's moderate and vigorous physical activity during weekdays and weekends. Journal of Physical Activity and Health, 9, 129-137.

FAITH, M. S., BERMAN, N., HEO, M., PIETROBELli, A., GALlAGHER, D., EPSTEIN, L. H., EIDEN, M. T. \& ALLISON, D. B. 2001. Effects of contingent television on physical activity and television viewing in obese children. Pediatrics, 107, 1043-8.

FAN, C., FORLIZZI, J. \& DEY, A. K. 2012. A spark of activity: exploring informative art as visualization for physical activity. Proceedings of the 2012 ACM Conference on Ubiquitous Computing. Pittsburgh, Pennsylvania: ACM.

FANNING, J., MULLEN, S. P. \& MCAULEY, E. 2012. Increasing physical activity with mobile devices: a meta-analysis. Journal of medical Internet research, 14.

FAROOQ, M. A., PARKINSON, K. N., ADAMSON, A. J., PEARCE, M. S., REILLY, J. K., HUGHES, A. R., JANSSEN, X., BASTERFIELD, L. \& REILLY, J. J. 2017. Timing of the decline in physical activity in childhood and adolescence: Gateshead Millennium Cohort Study. Br J Sports Med. 
FAYYAZ, J. 2005. Ponderal index.

FELDMAN, D. E., BARNETT, T., SHRIER, I., ROSSIGNOL, M. \& ABENHAIM, L. 2003. Is physical activity differentially associated with different types of sedentary pursuits? Arch Pediatr Adolesc Med, 157, 797-802.

FENG, Q., DU, Y., YE, Y.-L. \& HE, Q.-Q. 2014. Associations of physical activity, screen time with depression, anxiety and sleep quality among Chinese college freshmen. PloS one, 9, e100914.

FERGUSSON, D. M., HORWOOD, L. J., RIDDER, E. M. \& BEAUTRAIS, A. L. 2005. Subthreshold depression in adolescence and mental health outcomes in adulthood. Archives of general psychiatry, 62, 66-72.

FERNAEUS, Y. \& THOLANDER, J. 2006. "Looking At the Computer but Doing It On Land": Children's Interactions in a Tangible Programming Space. In: MCEWAN, T., GULLIKSEN, J. \& BENYON, D. (eds.) People and Computers XIX - The Bigger Picture: Proceedings of HCI 2005. London: Springer London.

FERRARI, G. L., ARAUJO, T. L., OLIVEIRA, L., MATSUDO, V., MIRE, E., BARREIRA, T. V., TUDOR-LOCKE, C. \& KATZMARZYK, P. T. 2015. Association Between Television Viewing and Physical Activity in 10-YearOld Brazilian Children. J Phys Act Health, 12, 1401-8.

FERRETTI, G., ATCHOU, G., GRASSI, B., MARCONI, C. \& CERRETELLI, P. 1991. Energetics of locomotion in African pygmies. European journal of applied physiology and occupational physiology, 62, 7-10.

FINKELSTEIN, E. A., TAN, Y.-T., MALHOTRA, R., LEE, C.-F., GOH, S.-S. \& SAW, S.-M. 2013. A Cluster Randomized Controlled Trial of an IncentiveBased Outdoor Physical Activity Program. The Journal of Pediatrics, 163, 167-172.e1.

FITTON, D., READ, J. C., SIM, G. \& CASSIDY, B. Co-designing voice user interfaces with teenagers in the context of smart homes. Proceedings of the 17th ACM Conference on Interaction Design and Children, 2018. ACM, 5566.

FJØRTOFT, I., KRISTOFFERSEN, B. \& SAGEIE, J. 2009. Children in schoolyards: Tracking movement patterns and physical activity in schoolyards using global positioning system and heart rate monitoring. Landscape and urban planning, 93, 210-217.

FLEMING, N. D. \& MILLS, C. 1992. Not another inventory, rather a catalyst for reflection. To improve the academy, 11, 137-155.

FOGG, B. \& ECKLES, D. The behavior chain for online participation: how successful web services structure persuasion. International Conference on Persuasive Technology, 2007. Springer, 199-209.

FOGG, B. J. 2002. Persuasive technology: using computers to change what we think and do. Ubiquity, 2002, 2.

FOLEY, L. \& MADDISON, R. 2010. Use of active video games to increase physical activity in children: a (virtual) reality? Pediatr Exerc Sci, 22, 7-20.

FORD, A. L., BERGH, C., SÖDERSTEN, P., SABIN, M. A., HOLLINGHURST, S., HUNT, L. P. \& SHIELD, J. P. 2010. Treatment of childhood obesity by retraining eating behaviour: randomised controlled trial. Bmj, 340, b5388.

FORLIZZI, J. \& BATTARBEE, K. 2004. Understanding experience in interactive systems. Proceedings of the 5th conference on Designing interactive systems: processes, practices, methods, and techniques. Cambridge, MA, USA: ACM. 
FORTIER, M. S., DUDA, J. L., GUERIN, E. \& TEIXEIRA, P. J. 2012. Promoting physical activity: development and testing of self-determination theory-based interventions. International Journal of Behavioral Nutrition and Physical Activity, 9, 20.

FORTUNE, E., LUGADE, V., MORROW, M. \& KAUFMAN, K. 2014. Validity of using tri-axial accelerometers to measure human movement - Part II: Step counts at a wide range of gait velocities. Med Eng Phys, 36, 659-69.

FOX, J. 2012. Avatars in health communication contexts. eHealth applications: Promising strategies for behavior change, 96-109.

FOX, J., YEYKELIS, L., JANSSEN, J., AHN, S., SEGOVIA, K. \& BAILENSON, J. A meta-analysis quantifying the effects of avatars and agents on social influence. conference; NCA 96th Annual Convention, 2010.

FOX, K. R., COOPER, A. \& MCKENNA, J. 2004. The School and Promotion of Children's Health-Enhancing Physical Activity: Perspectives from the United Kingdom. Journal of Teaching in Physical Education, 23, 338-358.

FRAUENBERGER, C., ANTLE, A. N., LANDONI, M., READ, J. C. \& FAILS, J. A. Ethics in interaction design and children: a panel and community dialogue. Proceedings of the 17th ACM Conference on Interaction Design and Children, 2018. ACM, 748-752.

FREEDSON, P., BOWLES, H. R., TROIANO, R. \& HASKELL, W. 2012. Assessment of physical activity using wearable monitors: recommendations for monitor calibration and use in the field. Med Sci Sports Exerc, 44, S1-4.

FREEDSON, P., POBER, D. \& JANZ, K. F. 2005a. Calibration of accelerometer output for children. Med Sci Sports Exerc, 37, S523-30.

FREEDSON, P., POBER, D. \& KATHLEEN, J. 2005b. Calibration of accelerometer output for children.

FREEDSON, P. S. \& MILLER, K. 2000. Objective monitoring of physical activity using motion sensors and heart rate. Research quarterly for exercise and sport, 71, 21-29.

FRY, M. D. \& DUDA, J. L. 1997. A Developmental Examination of Children's Understanding of Effort and Ability in the Physical and Academic Domains. Research Quarterly for Exercise and Sport, 68, 331-344.

GABRIEL, P. K. K., MORROW, J. R., JR. \& WOOLSEY, A. L. 2012. Framework for physical activity as a complex and multidimensional behavior. $J$ Phys Act Health, 9 Suppl 1, S11-8.

GALÁN, I., BOIX, R., MEDRANO, M. J., RAMOS, P., RIVERA, F., PASTORBARRIUSO, R. \& MORENO, C. 2013. Physical activity and self-reported health status among adolescents: a cross-sectional population-based study. BMJ open, 3, e002644.

GALESIC, M. \& GARCIA-RETAMERO, R. 2011. Graph literacy: A cross-cultural comparison. Medical Decision Making, 31, 444-457.

GAMMON, C., PFEIFFER, K. A., PIVARNIK, J. M., MOORE, R. W., RICE, K. R. \& TROST, S. G. 2016. Age-Related Differences in OMNI-RPE Scale Validity in Youth: A Longitudinal Analysis. Medicine and science in sports and exercise, 48, 1590-1594.

GAO, Z., HANNON, J. C., NEWTON, M. \& HUANG, C. 2011. Effects of curricular activity on students' situational motivation and physical activity levels. Res $Q$ Exerc Sport, 82, 536-44.

GARBY, L. 1989. Enthalpy changes and heat production induced by a meal. European journal of clinical nutrition, 43, 637-639. 
GAUDET, J., GALLANT, F. \& BÉLANGER, M. 2017. A bit of fit: minimalist intervention in adolescents based on a physical activity tracker. JMIR mHealth and uHealth, 5.

GEIGER, R., STRASAK, A., TREML, B., GASSER, K., KLEINSASSER, A., FISCHER, V., GEIGER, H., LOECKINGER, A. \& STEIN, J. I. 2007. Sixminute walk test in children and adolescents. J Pediatr, 150, 395-9, 399.e1-2.

GELDHOF, E., CARDON, G., DE BOURDEAUDHUIJ, I., DANNEELS, L., COOREVITS, P., VANDERSTRAETEN, G. \& DE CLERCQ, D. 2006. Static and dynamic standing balance: test-retest reliability and reference values in 9 to 10 year old children. European Journal of Pediatrics, 165, 779-786.

GERBER, M., KALAK, N., LEMOLA, S., CLOUGH, P. J., PÜHSE, U., ELLIOT, C., HOLSBOER-TRACHSLER, E. \& BRAND, S. 2012. Adolescents' exercise and physical activity are associated with mental toughness. Mental health and physical activity, 5, 35-42.

GIBSON, F. 2007. Conducting focus groups with children and young people: strategies for success. Journal of Research in Nursing, 12, 473-483.

GIBSON, J. E. 2012. Interviews and Focus Groups With Children: Methods That Match Children's Developing Competencies. J Fam Theory Rev, 4.

GILLET, A., SANNER, M., STOFFLER, D. \& OLSON, A. 2005. Tangible interfaces for structural molecular biology. Structure, 13, 483-491.

GIUSSANI, M., ANTOLINI, L., BRAMBILLA, P., PAGANI, M., ZUCCOTTI, G., VALSECCHI, M. G., LUCINI, D. \& GENOVESI, S. 2013. Cardiovascular risk assessment in children: role of physical activity, family history and parental smoking on BMI and blood pressure. Journal of hypertension, 31, 983-992.

GLAISTER, B. C., BERNATZ, G. C., KLUTE, G. K. \& ORENDURFF, M. S. 2007. Video task analysis of turning during activities of daily living. Gait Posture, 25, 289-94.

GLEISS, A. C., GRUBER, S. H. \& WILSON, R. P. 2009. Multi-channel data-logging: towards determination of behaviour and metabolic rate in free-swimming sharks. Tagging and tracking of marine animals with electronic devices. Springer.

GLEISS, A. C., WILSON, R. P. \& SHEPARD, E. L. C. 2011. Making overall dynamic body acceleration work: on the theory of acceleration as a proxy for energy expenditure. Methods in Ecology and Evolution, 2, 23-33.

GODFREY, A., CONWAY, R., MEAGHER, D. \& ÓLAIGHIN, G. 2008. Direct measurement of human movement by accelerometry. Medical engineering \& physics, 30, 1364-1386.

GODINO, J. G., WATKINSON, C., CORDER, K., SUTTON, S., GRIFFIN, S. J. \& VAN SLUIJS, E. M. F. 2014. Awareness of physical activity in healthy middle-aged adults: a cross-sectional study of associations with sociodemographic, biological, behavioural, and psychological factors. BMC Public Health, 14, 421-421.

GOFFMAN, E. 1959. Thepresentation ofselfin everyday life. GardenC'ity, NY: Doubleday/An-chor Books.

GOLDFIELD, G., KALAKANIS, L., ERNST, M. \& EPSTEIN, L. 2000. Open-loop feedback to increase physical activity in obese children. International journal of obesity, 24, 888.

GOLDFIELD, G. S., MALLORY, R., PARKER, T., CUNNINGHAM, T., LEGG, C., LUMB, A., PARKER, K., PRUD'HOMME, D., GABOURY, I. \& ADAMO, 
K. B. 2006. Effects of open-loop feedback on physical activity and television viewing in overweight and obese children: a randomized, controlled trial. Pediatrics, 118, e157-e166.

GOLDSTONE, R. L. \& SAKAMOTO, Y. 2003. The transfer of abstract principles governing complex adaptive systems. Cognitive psychology, 46, 414-466.

GOLDSTONE, R. L. \& SON, J. Y. 2005. The transfer of scientific principles using concrete and idealized simulations. The Journal of the Learning Sciences, 14, 69-110.

GOLSTEIJN, C., HOVEN, E. V. D., FROHLICH, D. \& SELLEN, A. 2012. Towards a more cherishable digital object. Proceedings of the Designing Interactive Systems Conference. Newcastle Upon Tyne, United Kingdom: ACM.

GOODMAN, J. S. \& WOOD, R. E. 2009. Faded versus increasing feedback, task variability trajectories, and transfer of training. Human Performance, 22, 6485.

GOODYEAR, V. A., KERNER, C. \& QUENNERSTEDT, M. 2017. Young people's uses of wearable healthy lifestyle technologies; surveillance, self-surveillance and resistance. Sport, education and society, 1-14.

GORELY, T., BIDDLE, S. J., MARSHALL, S. J. \& CAMERON, N. 2009. The prevalence of leisure time sedentary behaviour and physical activity in adolescent boys: an ecological momentary assessment approach. Int J Pediatr Obes, 4, 289-98.

GOTLIB, I. H., LEWINSOHN, P. M. \& SEELEY, J. R. 1995. Symptoms versus a diagnosis of depression: differences in psychosocial functioning. Journal of consulting and clinical psychology, 63, 90.

GOVERNMENT, A. 2017. Australia's Physical Activity and Sedentary Behaviour Guidelines. In: HEALTH, D. O. (ed.).

GRAIG, R., MINDELL, J. \& HIRANI, V. 2009. Health Survey for England 2008: Physical activity and fitness. Leeds: Health and Social Care Information.

GRAVES, L. E., RIDGERS, N. D., ATKINSON, G. \& STRATTON, G. 2010. The effect of active video gaming on children's physical activity, behavior preferences and body composition. Pediatr Exerc Sci, 22, 535-46.

GRAVES, L. E. F., NEVILL, A., FOWEATHER, L., MCWHANNELL, N., HOPKINS, N. D., BODDY, L. M. \& STRATTON, G. 2013. Scaling of peak oxygen uptake in children. A comparison of body size index models. Medicine \& Science in Sports \& Exercise, 45, 2341-2345.

GRAY, P. 2011. The decline of play and the rise of psychopathology in children and adolescents. American Journal of Play, 3, 443-463.

GRECA, J. P. D. A., SILVA, D. A. S. \& LOCH, M. R. 2016. Physical activity and screen time in children and adolescents in a medium size town in the South of Brazil. Revista Paulista de Pediatria (English Edition), 34, 316-322.

GREENE, S. \& HOGAN, D. 2005. Researching Children's Experience: Exploring Children's Views through Focus Groups. In: GREENE, S. \& HOGAN, D. (eds.) Researching Children's Experience. London: SAGE Publications Ltd.

GREWAL, T. S. K. 2013. Awareness of Physical Activity Levels and Sedentary Behaviour: An Assessment of Awareness of Physical Activity Levels and Sedentary Behaviour Among Parents and Children. Electronic Thesis and Dissertation Repository, The University of Western Ontario.

GRIFFITHS, L. J., CORTINA-BORJA, M., SERA, F., POULIOU, T., GERACI, M., RICH, C., COLE, T. J., LAW, C., JOSHI, H. \& NESS, A. R. 2013. How active 
are our children? Findings from the Millennium Cohort Study. BMJ open, 3, $\mathrm{e} 002893$.

GROUP, T. N. 2015. Kids Move Away From Home Computers For Gaming In Droves.

GROVES, T. 2010. What makes a high quality clinical research paper? Oral diseases, $16,313-315$.

GUTHRIE, N., BRADLYN, A., THOMPSON, S. K., YEN, S., HARITATOS, J., DILLON, F. \& COLE, S. W. 2015. Development of an accelerometer-linked online intervention system to promote physical activity in adolescents. PLoS One, 10, e0128639.

GUTIN, B., BARBEAU, P., OWENS, S., LEMMON, C. R., BAUMAN, M., ALLISON, J., KANG, H.-S. \& LITAKER, M. S. 2002. Effects of exercise intensity on cardiovascular fitness, total body composition, and visceral adiposity of obese adolescents. The American journal of clinical nutrition, 75, 818-826.

GUY, S., RATZKI-LEEWING, A. \& GWADRY-SRIDHAR, F. 2011. Moving Beyond the Stigma: Systematic Review of Video Games and Their Potential to Combat Obesity. International Journal of Hypertension, 2011, 13.

HAAS, S. \& NIGG, C. R. 2009. Construct validation of the stages of change with strenuous, moderate, and mild physical activity and sedentary behaviour among children. Journal of Science and Medicine in Sport, 12, 586-591.

HADER, K., MENDEZ-VILLANUEVA, A., PALAZZI, D., AHMAIDI, S. \& BUCHHEIT, M. 2016. Metabolic Power Requirement of Change of Direction Speed in Young Soccer Players: Not All Is What It Seems. PLOS ONE, 11, e0149839.

HAENNEL, R. G. \& LEMIRE, F. 2002. Physical activity to prevent cardiovascular disease. How much is enough? Canadian Family Physician, 48, 65-71.

HAERENS, L., DE BOURDEAUDHUIJ, I., MAES, L., CARDON, G. \& DEFORCHE, B. 2007. School-based randomized controlled trial of a physical activity intervention among adolescents. Journal of Adolescent Health, 40, 258-265.

HAERENS, L., DEFORCHE, B., MAES, L., CARDON, G., STEVENS, V. \& DE BOURDEAUDHUIJ, I. 2006. Evaluation of a 2-year physical activity and healthy eating intervention in middle school children. Health education research, 21, 911-921.

HAGSTRÖMER, M., BERGMAN, P., DE BOURDEAUDHUIJ, I., ORTEGA, F. B., RUIZ, J. R., MANIOS, Y., REY-LOPEZ, J., PHILLIPP, K., VON BERLEPSCH, J. \& SJÖSTRÖM, M. 2008. Concurrent validity of a modified version of the International Physical Activity Questionnaire (IPAQ-A) in European adolescents: The HELENA Study. International journal of obesity, 32, S42.

HALLAL, P. C., ANDERSEN, L. B., BULL, F. C., GUTHOLD, R., HASKELL, W. \& EKELUND, U. 2012. Global physical activity levels: surveillance progress, pitfalls, and prospects. Lancet, 380, 247-57.

HALLAL, P. C., VICTORA, C. G., AZEVEDO, M. R. \& WELLS, J. C. 2006a. Adolescent physical activity and health. Sports medicine, 36, 1019-1030.

HALlAL, P. C., VICTORA, C. G., AZEVEDO, M. R. \& WELlS, J. C. 2006 b. Adolescent physical activity and health: a systematic review. Sports Med, 36, 1019-30. 
HALSEY, L. G., GREEN, J. A., WILSON, R. P. \& FRAPPELL, P. B. 2009. Accelerometry to Estimate Energy Expenditure during Activity: Best Practice with Data Loggers. Physiol Biochem Zool, 82, 396-404.

HALSEY, L. G., SHEPARD, E. L., HULSTON, C. J., VENABLES, M. C., WHITE, C. R., JEUKENDRUP, A. E. \& WILSON, R. P. 2008. Acceleration versus heart rate for estimating energy expenditure and speed during locomotion in animals: tests with an easy model species, Homo sapiens. Zoology (Jena), 111, 231-41.

HALSEY, L. G., SHEPARD, E. L. \& WILSON, R. P. 2011. Assessing the development and application of the accelerometry technique for estimating energy expenditure. Comp Biochem Physiol A Mol Integr Physiol, 158, 30514.

HAMAD, E. O., SAVUNDRANAYAGAM, M. Y., HOLMES, J. D., KINSELLA, E. A. \& JOHNSON, A. M. 2016. Toward a mixed-methods research approach to content analysis in the digital age: the combined content-analysis model and its applications to health care Twitter feeds. Journal of medical Internet research, 18.

HAMER, M. \& STAMATAKIS, E. 2014. Prospective study of sedentary behavior, risk of depression, and cognitive impairment. Medicine and science in sports and exercise, 46, 718.

HAMILL, J., BATES, B. T., KNUTZEN, K. M. \& SAWHILL, J. A. 1983. Variations in ground reaction force parameters at different running speeds. Human Movement Science, 2, 47-56.

HARDMAN, C. A., HORNE, P. J. \& FERGUS LOWE, C. 2011a. Effects of rewards, peer-modelling and pedometer targets on children's physical activity: A school-based intervention study. Psychology and Health, 26, 3-21.

HARDMAN, C. A., HORNE, P. J. \& FERGUS LOWE, C. 2011b. Effects of rewards, peer-modelling and pedometer targets on children's physical activity: a schoolbased intervention study. Psychol Health, 26, 3-21.

HARDY, L., JONES, G. \& GOULD, D. 1996. Understanding Psychological Preparation for Sport: Theory and Practice of Elite Performers, Wiley.

HARDY, L. L., DENNEY-WILSON, E., THRIFT, A. P., OKELY, A. D. \& BAUR, L. A. 2010. Screen time and metabolic risk factors among adolescents. Arch Pediatr Adolesc Med, 164, 643-9.

HARRELL, J. S., MCMURRAY, R. G., GANSKY, S. A., BANGDIWALA, S. I. \& BRADLEY, C. B. 1999. A public health vs a risk-based intervention to improve cardiovascular health in elementary school children: the Cardiovascular Health in Children Study. American Journal of Public Health, 89, 1529-1535.

HARRIES, T., ESLAMBOLCHILAR, P., RETTIE, R., STRIDE, C., WALTON, S. \& VAN WOERDEN, H. C. 2016. Effectiveness of a smartphone app in increasing physical activity amongst male adults: a randomised controlled trial. BMC public health, 16, 925.

HARRIS, J. 1993. Young people's perceptions of health, fitness and exercise. British journal of physical education, 24, 5-5.

HARRIS, J. 1994. Young people's perception of health, fitness and exercise: implications for the teaching of health related exercise. Physical Education Review, 17, 143-151. 
HARRIS, J. 2014. Physical education teacher education students' knowledge, perceptions and experiences of promoting healthy, active lifestyles in secondary schools. Physical Education and Sport Pedagogy, 19, 466-480.

HARRIS, J., CALE, L., DUNCOMBE, R. \& MUSSON, H. 2016. Young people's knowledge and understanding of health, fitness and physical activity: issues, divides and dilemmas. Sport, Education and Society, 23, 407-420.

HARRIS, L. R. \& BROWN, G. T. 2010. Mixing interview and questionnaire methods: Practical problems in aligning data.

HARTER, S. 1993. Causes and consequences of low self-esteem in children and adolescents. Self-esteem. Springer.

HASE, K. \& STEIN, R. 1999a. Turning strategies during human walking. Journal of Neurophysiology, 81, 2914-2922.

HASE, K. \& STEIN, R. B. 1999b. Turning strategies during human walking. $J$ Neurophysiol, 81, 2914-22.

HASSENZAHL, M., LASCHKE, M. \& PRAEST, J. 2016. On the stories activity trackers tell. Proceedings of the 2016 ACM International Joint Conference on Pervasive and Ubiquitous Computing: Adjunct. Heidelberg, Germany: ACM.

HASSENZAHL, M. \& TRACTINSKY, N. 2006. User experience - a research agenda. Behaviour \& Information Technology, 25, 91-97.

HATAMOTO, Y., YAMADA, Y., FUJII, T., HIGAKI, Y., KIYONAGA, A. \& TANAKA, H. 2013. A novel method for calculating the energy cost of turning during running. Open Access J Sports Med, 4, 117-22.

HATAMOTO, Y., YAMADA, Y., SAGAYAMA, H., HIGAKI, Y., KIYONAGA, A. \& TANAKA, H. 2014. The relationship between running velocity and the energy cost of turning during running. PLoS One, 9, e81850.

HAUGEN, H. A., CHAN, L.-N. \& LI, F. 2007. Indirect calorimetry: a practical guide for clinicians. Nutrition in Clinical Practice, 22, 377-388.

HAVENS, K. L. \& SIGWARD, S. M. 2015. Whole body mechanics differ among running and cutting maneuvers in skilled athletes. Gait Posture, 42, 240-5.

HAWTON, K. \& VAN HEERINGEN, K. 2009. Suicide. The Lancet, 373, 1372-1381.

HAYES, L. B. \& VAN CAMP, C. M. 2015. Increasing physical activity of children during school recess. Journal of applied behavior analysis, 48, 690-695.

HEALTH, D. O. 2011a. Department of Health. 2011. NHS Choice: Live Well. London.

HEALTH, D. O. 2011b. Start Active, Stay Active: a report on physical activity for health from the four home countries' Chief Medical Officers. London. [Online]. [Accessed].

HEALTH, R. C. O. P. A. C. 2013. UK-WHO growth charts - 2-18 years [Online]. Available: https://www.rcpch.ac.uk/resources/uk-who-growth-charts-2-18years [Accessed].

HEALY, G. N., DUNSTAN, D. W., SAlMON, J., CERIN, E., SHAW, J. E., ZIMMET, P. Z. \& OWEN, N. 2007. Objectively measured light-intensity physical activity is independently associated with 2-h plasma glucose. Diabetes care, 30, 1384-1389.

HEARY, C. \& HENNESSY, E. 2006. Focus Groups Versus Individual Interviews with Children: A Comparison of Data. The Irish Journal of Psychology, 27, 58-68.

HEATH, G. W., PARRA, D. C., SARMIENTO, O. L., ANDERSEN, L. B., OWEN, N., GOENKA, S., MONTES, F., BROWNSON, R. C. \& GROUP, L. P. A. S. 
W. 2012. Evidence-based intervention in physical activity: lessons from around the world. The lancet, 380, 272-281.

HERSH, J., CURRY, J. F. \& KAMINER, Y. 2014. What is the impact of comorbid depression on adolescent substance abuse treatment? Substance Abuse, 35, 364-375.

HER MAJESTY'S INSPECTORATE FOR EDUCATION AND TRAINING IN WALES, 2017. Estyn. Annual Report.

HESKETH, B. 1997. Dilemmas in training for transfer and retention. Applied Psychology, 46, 317-339.

HILL, M., LAYBOURN, A. \& BORLAND, M. 1996. Engaging with Primary-aged Children about their Emotions and Well-being: Methodological Considerations. Children \& Society, 10, 129-144.

HILLIER, A. 2008. Childhood Overweight and the Built Environment: Making Technology Part of the Solution Rather than Part of the Problem. The Annals of the American Academy of Political and Social Science, 615, 56-82.

HILLS, A. P., ANDERSEN, L. B. \& BYRNE, N. M. 2011. Physical activity and obesity in children. British Journal of Sports Medicine, 45, 866-870.

HILLS, A. P., MOKHTAR, N. \& BYRNE, N. M. 2014a. Assessment of physical activity and energy expenditure: an overview of objective measures. Frontiers in nutrition, 1, 5.

HILLS, A. P., MOKHTAR, N. \& BYRNE, N. M. 2014b. Assessment of physical activity and energy expenditure: an overview of objective measures. Front Nutr, 1, 5.

HILYER, J. C., WILSON, D. G., DILLON, C., CARO, L., JENKINS, C., SPENCER, W. A., MEADOWS, M. E. \& BOOKER, W. 1982. Physical fitness training and counseling as treatment for youthful offenders. Journal of Counseling Psychology, 29, 292.

HINCKSON, E. A. \& CURTIS, A. 2013. Measuring physical activity in children and youth living with intellectual disabilities: a systematic review. Research in developmental disabilities, 34, 72-86.

HOBBS, G. \& VIGNOLES, A. 2010. Is children's free school meal 'eligibility' a good proxy for family income? British Educational Research Journal, 36, 673-690.

HOEGER, W. W. \& HOEGER, S. A. 2015. Principles and labs for fitness and wellness, Cengage Learning.

HOLLANDS, M., SORENSEN, K. \& PATLA, A. 2001. Effects of head immobilization on the coordination and control of head and body reorientation and translation during steering. Experimental brain research, 140, 223-233.

HOLLANDS, M. A., ZIAVRA, N. V. \& BRONSTEIN, A. M. 2004. A new paradigm to investigate the roles of head and eye movements in the coordination of whole-body movements. Experimental brain research, 154, 261-266.

HOLSTI, O. R. 1969. Content analysis for the social sciences and humanities. Reading. MA: Addison-Wesley (content analysis).

HOOKE, M. C., GILCHRIST, L., TANNER, L., HART, N. \& WITHYCOMBE, J. S. 2016. Use of a fitness tracker to promote physical activity in children with acute lymphoblastic leukemia. Pediatric blood \& cancer, 63, 684-689.

HORNE, P., HARDMAN, C., LOWE, C. \& ROWLANDS, A. V. 2009. Increasing children's physical activity: a peer modelling, rewards and pedometer-based intervention. European Journal of clinical nutrition, 63, 191.

HOUSTON, E. L., BAKER, J. S., BUCHAN, D. S., STRATTON, G., FAIRCLOUGH, S. J., FOWEATHER, L., GOBBI, R., GRAVES, L. E., 
HOPKINS, N. \& BODDY, L. M. 2013. Cardiorespiratory fitness predicts clustered cardiometabolic risk in 10-11.9-year-olds. Eur J Pediatr, 172, 9138.

HOUTS, P. S., DOAK, C. C., DOAK, L. G. \& LOSCALZO, M. J. 2006. The role of pictures in improving health communication: a review of research on attention, comprehension, recall, and adherence. Patient education and counseling, 61, 173-190.

HOWE, C., CLEVENGER, K., LESLIE, R. \& RAGAN, M. 2018. Comparison of Accelerometer-Based Cut-Points for Children's Physical Activity: Counts vs. Steps. Children, 5, 105.

HOYOS CILLERO, I. \& JAGO, R. 2011. Sociodemographic and home environment predictors of screen viewing among Spanish school children(). J Public Health (Oxf), 33, 392-402.

HSE 2007. Healthy lifestyles: knowledge, attitudes and behaviour. Health Survey for England. NHS.

HUHMAN, M. E., POTTER, L. D., DUKE, J. C., JUDKINS, D. R., HEITZLER, C. D. \& WONG, F. L. 2007a. Evaluation of a national physical activity intervention for children: VERB campaign, 2002-2004. Am J Prev Med, 32, 38-43.

HUHMAN, M. E., POTTER, L. D., DUKE, J. C., JUDKINS, D. R., HEITZLER, C. D. \& WONG, F. L. 2007b. Evaluation of a national physical activity intervention for children: VERB ${ }^{\mathrm{TM}}$ campaign, 2002-2004. American journal of preventive medicine, 32, 38-43.

HURLING, R., CATT, M., BONI, M. D., FAIRLEY, B. W., HURST, T., MURRAY, P., RICHARDSON, A. \& SODHI, J. S. 2007. Using internet and mobile phone technology to deliver an automated physical activity program: randomized controlled trial. J Med Internet Res, 9, e7.

HUSMANN, P. R. \& O'LOUGHLIN, V. D. 2018. Another nail in the coffin for learning styles? Disparities among undergraduate anatomy students' study strategies, class performance, and reported VARK learning styles. Anatomical sciences education.

HUXHAM, F., GONG, J., BAKER, R., MORRIS, M. \& IANSEK, R. 2006. Defining spatial parameters for non-linear walking. Gait Posture, 23, 159-63.

HYNYNEN, S., VAN STRALEN, M., SNIEHOTTA, F., ARAÚJO-SOARES, V., HARDEMAN, W., CHINAPAW, M. J., VASANKARI, T. \& HANKONEN, N. 2016. A systematic review of school-based interventions targeting physical activity and sedentary behaviour among older adolescents. International review of sport and exercise psychology, 9, 22-44.

HYTÖNEN, M., PYYKKÖ, I., AALTO, H. \& STARCK, J. 1993. Postural Control and Age. Acta Oto-Laryngologica, 113, 119-122.

IVANCEVICH, J. M. \& MCMAHON, J. T. 1982. The effects of goal setting, external feedback, and self-generated feedback on outcome variables: A field experiment. Academy of Management Journal, 25, 359-372.

JACOB, R. J., ISHII, H., PANGARO, G. \& PATTEN, J. A tangible interface for organizing information using a grid. Proceedings of the SIGCHI Conference on Human Factors in Computing Systems, 2002. ACM, 339-346.

JACOBS, J. E. \& KLACZYNSKI, P. A. 2002. The Development of Judgment and Decision Making During Childhood and Adolescence. Current Directions in Psychological Science, 11, 145-149. 
JACOBSEN, R. M., GINDE, S., MUSSATTO, K., NEUBAUER, J., EARING, M. \& DANDURAN, M. 2016. Can a Home-based Cardiac Physical Activity Program Improve the Physical Function Quality of Life in Children with F ontan Circulation? Congenital heart disease, 11, 175-182.

JAFRI, R., ALJUHANI, A. M. \& ALI, S. A. 2015. A tangible interface-based application for teaching tactual shape perception and spatial awareness subconcepts to visually impaired children. Procedia Manufacturing, 3, 55625569.

JAGO, R. \& BARANOWSKI, T. 2004. Non-curricular approaches for increasing physical activity in youth: a review. Preventive medicine, 39, 157-163.

JAGO, R., BARANOWSKI, T., BARANOWSKI, J. C., THOMPSON, D., CULLEN, K. W., WATSON, K. \& LIU, Y. 2006. Fit for Life Boy Scout badge: outcome evaluation of a troop and Internet intervention. Prev Med, 42, 181-7.

JAGO, R., BARANOWSKI, T., BARANOWSKI, J. C., THOMPSON, D. \& GREAVES, K. A. 2005. BMI from 3-6 y of age is predicted by TV viewing and physical activity, not diet. Int J Obes (Lond), 29, 557-64.

JAGO, R., PAGE, A. S. \& COOPER, A. R. 2012. Friends and physical activity during the transition from primary to secondary school. Med Sci Sports Exerc, 44, 111-7.

JANSEN, Y., DRAGICEVIC, P. \& FEKETE, J.-D. Evaluating the efficiency of physical visualizations. Proceedings of the SIGCHI Conference on Human Factors in Computing Systems, 2013. ACM, 2593-2602.

JANSSEN, I. 2007. Physical activity guidelines for children and youth. Can J Public Health, 98 Suppl 2, S109-21.

JANSSEN, I. \& LEBLANC, A. G. 2010a. Systematic review of the health benefits of physical activity and fitness in school-aged children and youth. Int J Behav Nutr Phys Act, 7, 40.

JANSSEN, I. \& LEBLANC, A. G. 2010b. Systematic review of the health benefits of physical activity and fitness in school-aged children and youth. International Journal of Behavioral Nutrition and Physical Activity, 7, 40.

JETTE, M., SIDNEY, K. \& BLUMCHEN, G. 1990. Metabolic equivalents (METS) in exercise testing, exercise prescription, and evaluation of functional capacity. Clin Cardiol, 13, 555-65.

JIN, S.-A. A. 2010. "I Feel More Connected to the Physically Ideal Mini Me than the Mirror-Image Mini Me": Theoretical Implications of the "Malleable Self" for Speculations on the Effects of Avatar Creation on Avatar-Self Connection in Wii. Cyberpsychology, Behavior, and Social Networking, 13, 567-570.

JOHNSON, C. M., JOHNSON, T. R. \& ZHANG, J. 2005. A user-centered framework for redesigning health care interfaces. Journal of biomedical informatics, 38, 75-87.

JOHNSON, D., DETERDING, S., KUHN, K.-A., STANEVA, A., STOYANOV, S. \& HIDES, L. 2016. Gamification for Health and Wellbeing:A Systematic Review of the Literature.

JONES, A. M. \& DOUST, J. H. 1996. A 1\% treadmill grade most accurately reflects the energetic cost of outdoor running. Journal of Sports Sciences, 14, 321-327.

JONES, D., HOELSCHER, D. M., KELDER, S. H., HERGENROEDER, A. \& SHARMA, S. V. 2008. Increasing physical activity and decreasing sedentary activity in adolescent girls - The Incorporating More Physical Activity and Calcium in Teens (IMPACT) study. The International Journal of Behavioral Nutrition and Physical Activity, 5, 42-42. 
JOO, Y. K. \& KIM, K. 2017. When You Exercise Your Avatar in a Virtual Game: The Role of Avatars' Body Shape and Behavior in Users' Health Behavior. Interacting with Computers, 29, 455-466.

JOURDAN, D., MANNIX MCNAMARA, P., SIMAR, C., GEARY, T. \& POMMIER, J. 2010. Factors influencing the contribution of staff to health education in schools. Health Education Research, 25, 519-530.

JOUVEN, X., SCHWARTZ, P. J., ESCOLANO, S., STRACZEK, C., TAFFLET, M., DESNOS, M., EMPANA, J. P. \& DUCIMETIÈRE, P. 2009. Excessive heart rate increase during mild mental stress in preparation for exercise predicts sudden death in the general population. European heart journal, 30, 17031710.

JUSTINE, M., MANAF, H., SULAIMAN, A., RAZI, S. \& ALIAS, H. A. 2014. Sharp turning and corner turning: comparison of energy expenditure, gait parameters, and level of fatigue among community-dwelling elderly. Biomed Res Int, 2014, 640321.

KAHN, E. B., RAMSEY, L. T., BROWNSON, R. C., HEATH, G. W., HOWZE, E. H., POWELL, K. E., STONE, E. J., RAJAB, M. W. \& CORSO, P. 2002. The effectiveness of interventions to increase physical activity. A systematic review. Am J Prev Med, 22, 73-107.

KAIYALA, K. J. \& RAMSAY, D. S. 2011. Direct animal calorimetry, the underused gold standard for quantifying the fire of life. Comparative Biochemistry and Physiology Part A: Molecular \& Integrative Physiology, 158, 252-264.

KANG, H.-S. \& YANG, H.-D. 2006. The visual characteristics of avatars in computermediated communication: Comparison of Internet Relay Chat and Instant Messenger as of 2003. International Journal of Human-Computer Studies, 64, 1173-1183.

KANG, M. \& BRINTHAUPT, T. M. 2009. Effects of group-and individual-based step goals on children's physical activity levels in school. Pediatric exercise science, 21, 148-158.

KANKAINEN, A., VAAJAKALLIO, K., KANTOLA, V. \& MATTELMÄKI, T. 2012. Storytelling Group-a co-design method for service design. Behaviour \& Information Technology, 31, 221-230.

KANTOMAA, M. T., TAMMELIN, T. H., EBELING, H. E. \& TAANILA, A. M. 2008. Emotional and behavioral problems in relation to physical activity in youth. Medicine and Science in Sports and Exercise, 40, 1749-1756.

KARACA, A., CAGLAR, E., BILGILI, N. \& AYAZ, S. 2011. Screen time of adolescents in an economically developing country: the case of Turkey. Ann Hum Biol, 38, 28-33.

KATZMARZYK, P., MALINA, R. \& BEUNEN, G. 1997. The contribution of biological maturation to the strength and motor fitness of children. Annals of human biology, 24, 493-505.

KATZMARZYK, P. T., CHURCH, T. S., CRAIG, C. L. \& BOUCHARD, C. 2009. Sitting time and mortality from all causes, cardiovascular disease, and cancer. Medicine \& Science in Sports \& Exercise, 41, 998-1005.

KEATING, X. D., HARRISON, L., CHEN, L., XIANG, P., LAMBDIN, D., DAUENHAUER, B., ROTICH, W. \& PIÑERO, J. C. 2009. An analysis of research on student health-related fitness knowledge in $\mathrm{K}-16$ physical education programs. Journal of Teaching in Physical Education, 28, 333-349.

KELDERS, S. M., KOK, R. N., OSSEBAARD, H. C. \& VAN GEMERT-PIJNEN, J. E. 2012. Persuasive system design does matter: a systematic review of 
adherence to web-based interventions. Journal of medical Internet research, 14.

KELLY, P., FITZSIMONS, C. \& BAKER, G. 2016. Should we reframe how we think about physical activity and sedentary behaviour measurement? Validity and reliability reconsidered. Int J Behav Nutr Phys Act, 13, 32.

KELTY, T. L., MORGAN, P. J. \& LUBANS, D. R. 2012. Efficacy and feasibility of the "Girls' Recreational Activity Support Program Using Information Technology": a pilot randomised controlled trial.

KEMPER, H. C., TWISK, J. W., KOPPES, L. L., VAN MECHELEN, W. \& POST, G. B. 2001. A 15-year physical activity pattern is positively related to aerobic fitness in young males and females (13-27 years). European Journal of Applied Physiology, 84, 395-402.

KENIS-COSKUN, O., GIRAY, E., EREN, B., OZKOK, O. \& KARADAG-SAYGI, E. 2016. Evaluation of postural stability in children with hemiplegic cerebral palsy. Journal of Physical Therapy Science, 28, 1398-1402.

KENNEDY, C., KOOLS, S. \& KRUEGER, R. 2001. Methodological considerations in children's focus groups. Nurs Res, 50, 184-7.

KERNER, C. \& GOODYEAR, V. A. 2017. The motivational impact of wearable healthy lifestyle technologies: a self-determination perspective on Fitbits with adolescents. American Journal of Health Education, 48, 287-297.

KESÄNIEMI, A., RIDDOCH, C. J., REEDER, B., BLAIR, S. N. \& SØRENSEN, T. I. 2010. Advancing the future of physical activity guidelines in Canada: an independent expert panel interpretation of the evidence. International Journal of Behavioral Nutrition and Physical Activity, 7, 41.

KHADJESARI, Z., MURRAY, E., HEWITT, C., HARTLEY, S. \& GODFREY, C. 2011. Can stand-alone computer-based interventions reduce alcohol consumption? A systematic review. Addiction, 106, 267-82.

KHOT, R. A. 2016. Understanding material representations of physical activity.

KHOT, R. A. \& MUELLER, F. F. 2013. Sweat-atoms: turning physical exercise into physical objects. CHI'13 Extended Abstracts on Human Factors in Computing Systems. Paris, France: ACM.

KHOT, R. A., MUELLER, F. F. \& HJORTH, L. 2013. SweatAtoms: materializing physical activity. Proceedings of The 9th Australasian Conference on Interactive Entertainment: Matters of Life and Death. Melbourne, Australia: ACM.

KIM, I. \& KULJIS, J. 2010. Applying content analysis to web-based content. Journal of Computing and Information Technology, 18, 369-375.

KIM, K., SHIN, Y. J., NAM, J. H., CHOI, B. Y. \& KIM, M. K. 2008. A dose-response relationship between types of physical activity and distress. Journal of Korean medical science, 23, 218-225.

KIM, S.-E., KIM, J.-W. \& JEE, Y.-S. 2015. Relationship between smartphone addiction and physical activity in Chinese international students in Korea. Journal of Behavioral Addictions, 4, 200-205.

KIM, Y., BEETS, M. W. \& WELK, G. J. 2012. Everything you wanted to know about selecting the "right" Actigraph accelerometer cut-points for youth, but...: a systematic review. Journal of Science and Medicine in Sport, 15, 311-321.

KIMM, S. Y., GLYNN, N. W., KRISKA, A. M., BARTON, B. A., KRONSBERG, S. S., DANIELS, S. R., CRAWFORD, P. B., SABRY, Z. I. \& LIU, K. 2002. Decline in physical activity in black girls and white girls during adolescence. $N$ Engl J Med, 347, 709-15. 
KIRBY, J., TIBBINS, C., CALLENS, C., LANG, B., THOROGOOD, M., TIGBE, W. \& ROBERTSON, W. 2012. Young people's views on accelerometer use in physical activity research: Findings from a user involvement investigation. ISRN obesity, 2012.

KIRK, D. S. \& SELLEN, A. 2010. On human remains: Values and practice in the home archiving of cherished objects. ACM Trans. Comput.-Hum. Interact., 17, $1-43$.

KLAHR, D., TRIONA, L. M. \& WILLIAMS, C. 2007. Hands on what? The relative effectiveness of physical versus virtual materials in an engineering design project by middle school children. Journal of Research in Science Teaching, 44, 183-203.

KNOWLES, Z. R., PARNELL, D., STRATTON, G. \& RIDGERS, N. D. 2013. Learning From the Experts: Exploring Playground Experience and Activities Using a Write and Draw Technique. Journal of Physical Activity \& Health, 10, 406-415.

KNOX, E. C., WEBB, O. J., ESLIGER, D. W., BIDDLE, S. J. \& SHERAR, L. B. 2013a. Using threshold messages to promote physical activity: Implications for public perceptions of health effects. The European Journal of Public Health, 24, 195-199.

KNOX, E. C. L., ESLIGER, D. W., BIDDLE, S. J. H. \& SHERAR, L. B. 2013b. Lack of knowledge of physical activity guidelines: can physical activity promotion campaigns do better? BMJ Open, 3, e003633.

KOHL, H. W., 3RD, CRAIG, C. L., LAMBERT, E. V., INOUE, S., ALKANDARI, J. R., LEETONGIN, G. \& KAHLMEIER, S. 2012. The pandemic of physical inactivity: global action for public health. Lancet, 380, 294-305.

KONG, C. Y. \& BASSETT JR, D. R. 2005. The technology of accelerometry-based activity monitors: current and future. Medicine \& Science in Sports \& Exercise, 37, S490-S500.

KONIAK-GRIFFIN, D. 1994. Aerobic exercise, psychological well-being, and physical discomforts during adolescent pregnancy. Research in nursing \& health, 17, 253-263.

KONTULAINEN, S., KANNUS, P., PASANEN, M., SIEVÄNEN, H., HEINONEN, A., OJA, P. \& VUORI, I. 2002. Does previous participation in high-impact training result in residual bone gain in growing girls? International journal of sports medicine, 23, 575-581.

KRAMER, P. A. \& SYLVESTER, A. D. 2013. Humans, geometric similarity and the Froude number: is "reasonably close"'really close enough? Biology open, 2, 111-120.

KRANE, V., ANDERSEN, M. B. \& STREAN, W. B. 1997. Issues of Qualitative Research Methods and Presentation. Journal of Sport and Exercise Psychology, 19, 213-218.

KREMERS, S. P. J., DIJKMAN, M. A. M., DE MEIJ, J. S. B., JURG, M. E. \& BRUG, J. 2008. Awareness and habit: important factors in physical activity in children. Health Education, 108, 475-488.

KRETSCHMANN, R. 2015. Physical education teachers' subjective theories about integrating information and communication technology (ICT) into physical education.

KREUTER, M. W., FARRELL, D. W., OLEVITCH, L. R. \& BRENNAN, L. K. 2013. Tailoring health messages: Customizing communication with computer technology, Routledge. 
KREUTER, M. W. \& WRAY, R. J. 2003. Tailored and targeted health communication: strategies for enhancing information relevance. American journal of health behavior, 27, S227-S232.

KRIEMLER, S., MEYER, U., MARTIN, E., VAN SLUIJS, E. M., ANDERSEN, L. B. \& MARTIN, B. W. 2011. Effect of school-based interventions on physical activity and fitness in children and adolescents: a review of reviews and systematic update. Br J Sports Med, 45, 923-30.

KRIEMLER, S., ZAHNER, L., SCHINDLER, C., MEYER, U., HARTMANN, T., HEBESTREIT, H., BRUNNER-LA ROCCA, H. P., VAN MECHELEN, W. \& PUDER, J. J. 2010. Effect of school based physical activity programme (KISS) on fitness and adiposity in primary schoolchildren: cluster randomised controlled trial. Bmj, 340, c785.

KRUEGER, R. A. \& CASEY, M. A. 2014. Focus groups: A practical guide for applied research, Sage publications.

KULINNA, P. 2004. Physical activity and fitness knowledge: How much 1-6 grade students know. International Journal of Physical Education, 41, 111-121.

KULINNA, P. H., MCCAUGHTRY, N., MARTIN, J. J., COTHRAN, D. \& FAUST, R. 2008. The influence of professional development on teachers' psychosocial perceptions of teaching a health-related physical education curriculum. Journal of Teaching in Physical Education, 27, 292-307.

KWASNICKA, D., DOMBROWSKI, S. U., WHITE, M. \& SNIEHOTTA, F. 2016. Theoretical explanations for maintenance of behaviour change: a systematic review of behaviour theories. Health psychology review, 10, 277-296.

KYLLO, L. B. \& LANDERS, D. M. 1995. Goal setting in sport and exercise: A research synthesis to resolve the controversy. Journal of Sport and Exercise Psychology, 17, 117-137.

KYU, H. H., BACHMAN, V. F., ALEXANDER, L. T., MUMFORD, J. E., AFSHIN, A., ESTEP, K., VEERMAN, J. L., DELWICHE, K., IANNARONE, M. L., MOYER, M. L., CERCY, K., VOS, T., MURRAY, C. J. \& FOROUZANFAR, M. H. 2016. Physical activity and risk of breast cancer, colon cancer, diabetes, ischemic heart disease, and ischemic stroke events: systematic review and dose-response meta-analysis for the Global Burden of Disease Study 2013. Bmj, 354, i3857.

LACHAT, C. K., VERSTRAETEN, R., HAGSTRÖMER, M., KHAN, N. C., VAN, N. D. A., DUNG, N. Q. \& KOLSTEREN, P. W. 2008. Validity of two physical activity questionnaires (IPAQ and PAQA) for Vietnamese adolescents in rural and urban areas. International Journal of Behavioral Nutrition and Physical Activity, 5, 37.

LAKDAWALLA, D. \& PHILIPSON, T. 2009. The growth of obesity and technological change. Econ Hum Biol, 7, 283-93.

LANG, R., O'REILLY, M., SIGAFOOS, J., MACHALICEK, W., RISPOLI, M., LANCIONI, G. E., AGUILAR, J. \& FRAGALE, C. 2010. The effects of an abolishing operation intervention component on play skills, challenging behavior, and stereotypy. Behav Modif, 34, 267-89.

LAPPAN, L., YEH, M. C. \& LEUNG, M. M. 2015. Technology as a Platform for Improving Healthy Behaviors and Weight Status in Children and Adolescents: A Review. Obes Open Access. Obes Open Access

LARSEN, J. E., CUTTONE, A. \& JØRGENSEN, S. L. QS Spiral: Visualizing Periodic Quantified Self Data. CHI 2013 Workshop on Personal Informatics in the Wild: Hacking Habits for Health \& Happiness, 2013. 
LARSEN, T., SAMDAL, O. \& TJOMSLAND, H. 2012. Physical activity in schools: a qualitative case study of eight Norwegian schools' experiences with the implementation of a national policy. Health Education, 113, 52-63.

LATHAM, G. P. \& LOCKE, E. A. 1991. Self-regulation through goal setting. Organizational behavior and human decision processes, 50, 212-247.

LAU, E. Y., LAU, P. W., CHUNG, P. K., RANSDELL, L. B. \& ARCHER, E. 2012. Evaluation of an Internet-short message service-based intervention for promoting physical activity in Hong Kong Chinese adolescent school children: a pilot study. Cyberpsychol Behav Soc Netw, 15, 425-34.

LAU, P. W. C., LAU, E. Y., WONG, D. P. \& RANSDELL, L. 2011. A Systematic Review of Information and Communication Technology-Based Interventions for Promoting Physical Activity Behavior Change in Children and Adolescents. Journal of Medical Internet Research, 13, e48.

LAZAR, J., WOGLOM, C., CHUNG, J., SCHWARTZ, A., HSIEH, Y. G., MOORE, R., CROWLEY, D. \& SKOTKO, B. 2018. Co-Design Process of a Smart Phone App to Help People With Down Syndrome Manage Their Nutritional Habits. Journal of Usability Studies, 13.

LE MASURIER, G. C. \& TUDOR-LOCKE, C. 2003. Comparison of pedometer and accelerometer accuracy under controlled conditions. Med Sci Sports Exerc, 35, 867-71.

LEBLANC, A. G., CHAPUT, J. P., MCFARLANE, A., COLLEY, R. C., THIVEL, D., BIDDLE, S. J., MADDISON, R., LEATHERDALE, S. T. \& TREMBLAY, M. S. 2013. Active video games and health indicators in children and youth: a systematic review. PLoS One, 8, e65351.

LEBLANC, A. G. \& JANSSEN, I. 2010a. Difference between self-reported and accelerometer measured moderate-to-vigorous physical activity in youth. Pediatr Exerc Sci, 22, 523-34.

LEBLANC, A. G. \& JANSSEN, I. 2010b. Dose-response relationship between physical activity and dyslipidemia in youth. Canadian Journal of Cardiology, 26, e201-e205.

LEDGER, D. \& MCCAFFREY, D. 2014. Inside wearables: How the science of human behavior change offers the secret to long-Term engagement.

LEE, I.-M. \& SKERRETT, P. J. 2001a. Physical activity and all-cause mortality: what is the dose-response relation? Medicine \& Science in Sports \& Exercise, 33, S459-S471.

LEE, I. M. \& SHIROMA, E. J. 2014. Using accelerometers to measure physical activity in large-scale epidemiological studies: issues and challenges. $\mathrm{Br} J$ Sports Med, 48, 197-201.

LEE, I. M., SHIROMA, E. J., LOBELO, F., PUSKA, P., BLAIR, S. N. \& KATZMARZYK, P. T. 2012. Impact of Physical Inactivity on the World's Major Non-Communicable Diseases. Lancet, 380, 219-229.

LEE, I. M. \& SKERRETT, P. J. 2001b. Physical activity and all-cause mortality: what is the dose-response relation? Med Sci Sports Exerc, 33, S459-71; discussion S493-4.

LEE, J. \& MACDONALD, D. 2009a. Rural young people and physical activity: understanding participation through social theory. Sociology of health \& illness, 31, 360-374.

LEE, J. \& MACDONALD, D. 2010a. 'Are they just checking our obesity or what?' The healthism discourse and rural young women. Sport, Education and Society, 15, 203-219. 
LEE, M.-H., CHA, S. \& NAM, T.-J. Patina engraver: visualizing activity logs as patina in fashionable trackers. Proceedings of the 33rd Annual ACM Conference on Human Factors in Computing Systems, 2015. ACM, 1173-1182.

LEE, R. E., NIGG, C. R., DICLEMENTE, C. C. \& COURNEYA, K. S. 2001. Validating motivational readiness for exercise behavior with adolescents. Research Quarterly for Exercise and Sport, 72, 401-410.

LEMURA, L. M. \& MAZIEKAS, M. T. 2002. Factors that alter body fat, body mass, and fat-free mass in pediatric obesity. Medicine and Science in Sports and Exercise, 34, 487-496.

LENHARD, A. 2009. Teens and mobile phones over the past five years: Pew Internet looks back. Washington, DC: Pew Internet \& American Life Project.

LEPP, A., BARKLEY, J. E., SANDERS, G. J., REBOLD, M. \& GATES, P. 2013. The relationship between cell phone use, physical and sedentary activity, and cardiorespiratory fitness in a sample of U.S. college students. The International Journal of Behavioral Nutrition and Physical Activity, 10, 79-79.

LEPPER, M. R., GREENE, D. \& NISBETT, R. E. 1973. Undermining children's intrinsic interest with extrinsic reward: A test of the" overjustification" hypothesis. Journal of Personality and social Psychology, 28, 129.

LEUNG, W., SIEBERT, E. A. \& YUN, J. 2017. Measuring physical activity with accelerometers for individuals with intellectual disability: A systematic review. Res Dev Disabil, 67, 60-70.

LÉVESQUE, L., CARGO, M. \& SALSBERG, J. 2004. Development of the Physical Activity Interactive Recall (PAIR) for Aboriginal children. The international journal of behavioral nutrition and physical activity, 1, 8 .

LEVINE, J. A. 2005. Measurement of energy expenditure. Public Health Nutr, 8, 1123-32.

LEWIS, A. 1992. Group child interviews as a research tool. British Educational Research Journal, 18, 413-421.

LEWIS, P. 2017. 'Our minds can be hijacked': the tech insiders who fear a smartphone dystopia. The Guardian.

LEWIS, Z. H., LYONS, E. J., JARVIS, J. M. \& BAILLARGEON, J. 2015. Using an electronic activity monitor system as an intervention modality: a systematic review. BMC public health, 15, 585.

LI, I., DEY, A. K. \& FORLIZZI, J. 2011a. Understanding my data, myself: supporting self-reflection with ubicomp technologies. Proceedings of the 13th international conference on Ubiquitous computing. Beijing, China: ACM.

LI, I., DEY, A. K. \& FORLIZZI, J. Understanding my data, myself: supporting selfreflection with ubicomp technologies. Proceedings of the 13th international conference on Ubiquitous computing, 2011b. ACM, 405-414.

LI, S. 2016. 'It's all about me, me, me!' Why children are spending less time doing household chores. Education [Online]. Available: https://theconversation.com/its-all-about-me-me-me-why-children-arespending-less-time-doing-household-chores-66134.

LIAMPUTTONG, P. 2006. Researching the vulnerable: A guide to sensitive research methods, Sage.

LIANG, Y. \& LAU, P. W. 2014. Effects of Active Videogames on Physical Activity and Related Outcomes Among Healthy Children: A Systematic Review. Games Health J, 3, 122-44. 
LIAO, H. F., JENG, S. F., LAI, J. S., CHENG, C. K. \& HU, M. H. 1997. The relation between standing balance and walking function in children with spastic diplegic cerebral palsy. Dev Med Child Neurol, 39, 106-12.

LIEBERMAN, L. J., STUART, M. E., HAND, K. \& ROBINSON, B. 2006. An investigation of the motivational effects of talking pedometers among children with visual impairments and deaf-blindness. Journal of Visual Impairment \& Blindness, 100, 726.

LIM, S., KANG, S. M., KIM, K. M., MOON, J. H., CHOI, S. H., HWANG, H., JUNG, H. S., PARK, K. S., RYU, J. O. \& JANG, H. C. 2016. Multifactorial intervention in diabetes care using real-time monitoring and tailored feedback in type 2 diabetes. Acta diabetologica, 53, 189-198.

LIM, S. \& REEVES, B. 2010. Computer agents versus avatars: Responses to interactive game characters controlled by a computer or other player. International Journal of Human-Computer Studies, 68, 57-68.

LIN, J., MAMYKINA, L., LINDTNER, S., DELAJOUX, G. \& STRUB, H. 2006. Fish'n'Steps: Encouraging Physical Activity with an Interactive Computer Game. In: DOURISH, P. \& FRIDAY, A. (eds.) UbiComp 2006: Ubiquitous Computing. Springer Berlin Heidelberg.

LINDEN, C., AHLBORG, H. G., BESJAKOV, J., GARDSELL, P. \& KARLSSON, M. K. 2006. A School Curriculum-Based Exercise Program Increases Bone Mineral Accrual and Bone Size in Prepubertal Girls: Two-Year Data From the Pediatric Osteoporosis Prevention (POP) Study. Journal of bone and mineral research, 21, 829-835.

LIPKIN, D. P., SCRIVEN, A. J., CRAKE, T. \& POOLE-WILSON, P. A. 1986. Six minute walking test for assessing exercise capacity in chronic heart failure. British Medical Journal (Clinical research ed.), 292, 653-655.

LIPPERT, L. 2011. Clinical Kinesiology and Anatomy: FA Davis Company.

LIU, M., WU, L. \& YAO, S. 2016. Dose-response association of screen time-based sedentary behaviour in children and adolescents and depression: a metaanalysis of observational studies. Br J Sports Med, 50, 1252-1258.

LIVINGSTONE, M. B., ROBSON, P. J., WALLACE, J. M. \& MCKINLEY, M. C. 2003. How active are we? Levels of routine physical activity in children and adults. Proc Nutr Soc, 62, 681-701.

LOCKE, E. A. \& LATHAM, G. P. 1990. A theory of goal setting \& task performance, Prentice-Hall, Inc.

LOCKE, E. A. \& LATHAM, G. P. 2002. Building a practically useful theory of goal setting and task motivation: A 35-year odyssey. American psychologist, 57, 705.

LOCKE, E. A. \& LATHAM, G. P. 2006. New Directions in Goal-Setting Theory. Current Directions in Psychological Science, 15, 265-268.

LOCKE, E. A., SHAW, K. N., SAARI, L. M. \& LATHAM, G. P. 1981. Goal setting and task performance: 1969-1980. Psychological bulletin, 90, 125.

LOPES, A. G. 2016. Using research methods in human computer interaction to design technology for resilience. JISTEM-Journal of Information Systems and Technology Management, 13, 363-388.

LUBANS, D. R., MORGAN, P. J. \& TUDOR-LOCKE, C. 2009. A systematic review of studies using pedometers to promote physical activity among youth. Preventive medicine, 48, 307-315.

LUBANS, D. R., SMITH, J. J., PERALTA, L. R., PLOTNIKOFF, R. C., OKELY, A. D., SALMON, J., EATHER, N., DEWAR, D. L., KENNEDY, S., 
LONSDAlE, C., HILlAND, T. A., ESTABROOKS, P., FINN, T. L., POLLOCK, E. \& MORGAN, P. J. 2016. A school-based intervention incorporating smartphone technology to improve health-related fitness among adolescents: rationale and study protocol for the NEAT and ATLAS 2.0 cluster randomised controlled trial and dissemination study. BMJ Open, 6.

LYNN, R. 1992. Sex differences on the differential aptitude test in British and American adolescents. Educational Psychology, 12, 101-102.

MACDONALD, D., RODGER, S., ABBOTT, R., ZIVIANI, J. \& JONES, J. 2005. 'I could do with a pair of wings': perspectives on physical activity, bodies and health from young Australian children. Sport, Education and Society, 10, 195209.

MACFARLANE, D. \& WONG, P. 2012. Validity, reliability and stability of the portable Cortex Metamax 3B gas analysis system. European journal of applied physiology, 112, 2539-2547.

MACKELVIE, K. J., MCKAY, H. A., KHAN, K. M. \& CROCKER, P. R. 2001. A school-based exercise intervention augments bone mineral accrual in early pubertal girls. The Journal of pediatrics, 139, 501-508.

MACKELVIE, K. J., PETIT, M. A., KHAN, K. M., BECK, T. J. \& MCKAY, H. A. 2004. Bone mass and structure are enhanced following a 2-year randomized controlled trial of exercise in prepubertal boys. Bone, 34, 755-764.

MACKINTOSH, K., NIEZEN, G. \& ESLAMBOLCHILAR, P. 2016. Mission possible: Using ubiquitous social goal sharing technology to promote physical activity in children. 2016, 5 .

MACKINTOSH, K. A., KNOWLES, Z. R., RIDGERS, N. D. \& FAIRCLOUGH, S. J. 2011. Using formative research to develop CHANGE!: a curriculum-based physical activity promoting intervention. BMC Public Health, 11, 831.

MADDUX, J. E. \& ROGERS, R. W. 1983. Protection motivation and self-efficacy: A revised theory of fear appeals and attitude change. Journal of experimental social psychology, 19, 469-479.

MALONEY, A. E., BETHEA, T. C., KELSEY, K. S., MARKS, J. T., PAEZ, S., ROSENBERG, A. M., CATELLIER, D. J., HAMER, R. M. \& SIKICH, L. 2008. A pilot of a video game (DDR) to promote physical activity and decrease sedentary screen time. Obesity (Silver Spring), 16, 2074-80.

MANN, V. A., SASANUMA, S., SAKUMA, N. \& MASAKI, S. 1990. Sex differences in cognitive abilities: A cross-cultural perspective. Neuropsychologia, 28, 1063-1077.

MANSFIELD, A. \& LYONS, G. M. 2003. The use of accelerometry to detect heel contact events for use as a sensor in FES assisted walking. Med Eng Phys, 25, 879-85.

MANSON, J. E., HU, F. B., RICH-EDWARDS, J. W., COLDITZ, G. A., STAMPFER, M. J., WILLETT, W. C., SPEIZER, F. E. \& HENNEKENS, C. H. 1999. A prospective study of walking as compared with vigorous exercise in the prevention of coronary heart disease in women. $N$ Engl J Med, 341, 6508.

MARCUS, B. H., ROSSI, J. S., SELBY, V. C., NIAURA, R. S. \& ABRAMS, D. B. 1992. The stages and processes of exercise adoption and maintenance in a worksite sample. Health psychology, 11, 386.

MARCUS, B. H., WILLIAMS, D. M., DUBBERT, P. M., SALLIS, J. F., KING, A. C., YANCEY, A. K., FRANKLIN, B. A., BUCHNER, D., DANIELS, S. R. \& 
CLAYTOR, R. P. 2006. Physical Activity Intervention Studies. Circulation, $114,2739$.

MARK, A. E. \& JANSSEN, I. 2008. Dose-response relation between physical activity and blood pressure in youth. Medicine and science in sports and exercise, 40, 1007-1012.

MARKS, R. 2008. Schools and health education: what works, what is needed, and why? Health Education, 109, 4-8.

MARMELEIRA, J., LARANJO, L., MARQUES, O. \& PEREIRA, C. 2014. Physical activity patterns in adults who are blind as assessed by accelerometry. Adapted Physical Activity Quarterly, 31, 283-296.

MARSHALL, P. 2007. Do tangible interfaces enhance learning? Proceedings of the 1st international conference on Tangible and embedded interaction. Baton Rouge, Louisiana: ACM.

MARTÍ, A. C., PITTI, J. C. A., PROVINCIALE, J. G., LISÓN, J. F. \& RIVERA, R. M. B. Alternative options for prescribing physical activity among obese children and adolescents: brisk walking supported by an exergaming platform. Nutricion hospitalaria, 2015. 841-848.

MARTIN, C. K., CHURCH, T. S., THOMPSON, A. M., EARNEST, C. P. \& BLAIR, S. N. 2009. Exercise dose and quality of life: a randomized controlled trial. Archives of internal medicine, 169, 269-278.

MARTIN, D. J. \& HOOVER, H. 1987. Sex differences in educational achievement: A longitudinal study. The Journal of Early Adolescence, 7, 65-83.

MARTIN, S. S., FELDMAN, D. I., BLUMENTHAL, R. S., JONES, S. R., POST, W. S., MCKIBBEN, R. A., MICHOS, E. D., NDUMELE, C. E., RATCHFORD, E. V. \& CORESH, J. 2015. mActive: a randomized clinical trial of an automated mHealth intervention for physical activity promotion. Journal of the American Heart Association, 4, e002239.

MARTINEZ, J. L., LATIMER, A. E., RIVERS, S. E. \& SALOVEY, P. 2012. Formative research for a community-based message-framing intervention. American journal of health behavior, 36, 335-347.

MARTINEZ-GOMEZ, D., MARTINEZ-DE-HARO, V., POZO, T., WELK, G. J., VILLAGRA, A., CALLE, M. E., MARCOS, A. \& VEIGA, O. L. 2009. [Reliability and validity of the PAQ-A questionnaire to assess physical activity in Spanish adolescents]. Rev Esp Salud Publica, 83, 427-39.

MARTINEZ-GOMEZ, D., REY-LÓPEZ, J. P., CHILLÓN, P., GÓMEZMARTÍNEZ, S., VICENTE-RODRÍGUEZ, G., MARTÍN-MATILLAS, M., GARCIA-FUENTES, M., DELGADO, M., MORENO, L. A. \& VEIGA, O. L. 2010a. Excessive TV viewing and cardiovascular disease risk factors in adolescents. The AVENA cross-sectional study. BMC Public Health, 10, 274.

MARTINEZ-GOMEZ, D., REY-LOPEZ, J. P., CHILLON, P., GOMEZMARTINEZ, S., VICENTE-RODRIGUEZ, G., MARTIN-MATILLAS, M., GARCIA-FUENTES, M., DELGADO, M., MORENO, L. A., VEIGA, O. L., EISENMANN, J. C. \& MARCOS, A. 2010b. Excessive TV viewing and cardiovascular disease risk factors in adolescents. The AVENA cross-sectional study. BMC Public Health, 10, 274.

MARTINEZ-GOMEZ, D., RUIZ, J. R., ORTEGA, F. B., CASAJUS, J. A., VEIGA, O. L., WIDHALM, K., MANIOS, Y., BEGHIN, L., GONZALEZ-GROSS, M., KAFATOS, A., ESPANA-ROMERO, V., MOLNAR, D., MORENO, L. A., MARCOS, A., CASTILlO, M. J. \& SJOSTROM, M. 2010c. Recommended levels and intensities of physical activity to avoid low- 
cardiorespiratory fitness in European adolescents: The HELENA study. Am $J$ Hum Biol, 22, 750-6.

MARTON, F. \& PONG, W. Y. 2005. On the unit of description in phenomenography. Higher education research \& development, 24, 335-348.

MASTELLER, B., SIRARD, J. \& FREEDSON, P. 2017. The Physical Activity Tracker Testing in Youth (PATTY) Study: Content Analysis and Children's Perceptions. JMIR mHealth and uHealth, 5.

MATTHEWS, C. E., CHEN, K. Y., FREEDSON, P. S., BUCHOWSKI, M. S., BEECH, B. M., PATE, R. R. \& TROIANO, R. P. 2008. Amount of time spent in sedentary behaviors in the United States, 2003-2004. American journal of epidemiology, 167, 875-881.

MATURO, C. C. \& CUNNINGHAM, S. A. 2013. Influence of friends on children's physical activity: a review. American Journal of Public Health, 103, e23-e38.

MAYER-DAVIS, E. J., D'AGOSTINO JR, R., KARTER, A. J., HAFFNER, S. M., REWERS, M. J., SAAD, M., BERGMAN, R. N. \& INVESTIGATORS, I. 1998. Intensity and amount of physical activity in relation to insulin sensitivity: the Insulin Resistance Atherosclerosis Study. Jama, 279, 669-674.

MCCANN, D. A., KNOWLES, Z. R., FAIRCLOUGH, S. J. \& GRAVES, L. E. 2016. A protocol to encourage accelerometer wear in children and young people. Qualitative Research in Sport, Exercise and Health, 8, 319-331.

MCDOUGALL, J. \& DUNCAN, M. J. 2008. Children, video games and physical activity: An exploratory study. International Journal on Disability and Human Development, 7, 89-94.

MCGREGOR, S. J., BUSA, M. A., YAGGIE, J. A. \& BOLLT, E. M. 2009. High Resolution MEMS Accelerometers to Estimate $\mathrm{VO}(2)$ and Compare Running Mechanics between Highly Trained Inter-Collegiate and Untrained Runners. PLOS ONE, 4, e7355.

MCKAY, H., MACLEAN, L., PETIT, M., MACKELVIE-O'BRIEN, K., JANSSEN, P., BECK, T. \& KHAN, K. 2005. "Bounce at the Bell": a novel program of short bouts of exercise improves proximal femur bone mass in early pubertal children. British journal of sports medicine, 39, 521-526.

MCKENZIE, T. L. 2007. The preparation of physical educators: A public health perspective. Quest, 59, 345-357.

MCNARRY, M. A., WILSON, R. P., HOLTON, M. D., GRIFFITHS, I. W. \& MACKINTOSH, K. A. 2017. Investigating the relationship between energy expenditure, walking speed and angle of turning in humans. PLOS ONE, 12, $\mathrm{e} 0182333$.

MEARS, R. \& JAGO, R. 2016. Effectiveness of after-school interventions at increasing moderate-to-vigorous physical activity levels in 5-to 18-year olds: a systematic review and meta-analysis. Br J Sports Med, 50, 1315-1324.

MEDICINE, A. C. O. S. 2013. ACSM's guidelines for exercise testing and prescription, Lippincott Williams \& Wilkins.

MELANSON JR, E. L., FREEDSON, P. S. \& BLAIR, S. 1996. Physical activity assessment: a review of methods. Critical Reviews in Food Science \& Nutrition, 36, 385-396.

MELKEVIK, O., TORSHEIM, T., IANNOTTI, R. J. \& WOLD, B. 2010. Is spending time in screen-based sedentary behaviors associated with less physical activity: a cross national investigation. Int J Behav Nutr Phys Act, 7, 46.

MELNYK, B. M., JACOBSON, D., KELLY, S., O'HAVER, J., SMALL, L. \& MAYS, M. Z. 2009. Improving the mental health, healthy lifestyle choices, and 
physical health of Hispanic adolescents: A randomized controlled pilot study. Journal of School Health, 79, 575-584.

MENDOZA, J. A., BAKER, K. S., MORENO, M. A., WHITLOCK, K., ABBEYLAMBERTZ, M., WAITE, A., COLBURN, T. \& CHOW, E. J. 2017. A Fitbit and Facebook mHealth intervention for promoting physical activity among adolescent and young adult childhood cancer survivors: A pilot study. Pediatric blood \& cancer, 64.

MERKLE, D. G. \& TREAGUST, D. F. 1993. Student knowledge of health and fitness concepts and its relation to locus of control. School Science and Mathematics, 93, 355-359.

MICHIE, S., ABRAHAM, C., WHITTINGTON, C., MCATEER, J. \& GUPTA, S. 2009. Effective techniques in healthy eating and physical activity interventions: a meta-regression. Health Psychol, 28, 690-701.

MILLER, A. D. \& MYNATT, E. D. 2014. StepStream: a school-based pervasive social fitness system for everyday adolescent health. Proceedings of the 32nd annual ACM conference on Human factors in computing systems. Toronto, Ontario, Canada: ACM.

MINETTI, A. E. 2001. Invariant aspects of human locomotion in different gravitational environments. Acta Astronaut, 49, 191-8.

MINETTI, A. E., CAZZOLA, D., SEMINATI, E., GIACOMETTI, M. \& ROI, G. S. 2011. Skyscraper running: physiological and biomechanical profile of a novel sport activity. Scand J Med Sci Sports, 21, 293-301.

MINETTI, A. E., SAIBENE, F., ARDiGÒ, L. P., ATCHOU, G., SCHENA, F. \& FERRETTI, G. 1994. Pygmy locomotion. European Journal of Applied Physiology and Occupational Physiology, 68, 285-290.

MINTZ, S. 2004. Huck's Raft: A History of American Childhood, Cambridge, MA, Belknap Press of Harvard

University Press.

MIRWALD, R. L., BAXTER-JONES, A. D., BAILEY, D. A. \& BEUNEN, G. P. 2002. An assessment of maturity from anthropometric measurements. Med Sci Sports Exerc, 34, 689-94.

MONTESSORI, M. 1912. The Montessori Method: Scientific Pedagogy as Applied to Child Education in" The Children's House", with Additions and Revisions by the Author; Translated from the Italian by Anne E. George; with an Introduction by Professor Henry W. Holmes, FA Stokes.

MOORE, G. F., AUDREY, S., BARKER, M., BOND, L., BONELL, C., HARDEMAN, W., MOORE, L., O'CATHAIN, A., TINATI, T. \& WIGHT, D. 2015. Process evaluation of complex interventions: Medical Research Council guidance. $b m j, 350, \mathrm{~h} 1258$.

MOORE, J. B., BEETS, M. W., BARR-ANDERSON, D. J. \& EVENSON, K. R. 2013. Sedentary time and vigorous physical activity are independently associated with cardiorespiratory fitness in middle school youth. Journal of sports sciences, 31, 1520-1525.

MOORE, L. V., HARRIS, C. D., CARLSON, S. A., KRUGER, J. \& FULTON, J. E. 2012. Trends in no leisure-time physical activity--United States, 1988-2010. Res Q Exerc Sport, 83, 587-91.

MORGAN, D. L. 1996. Focus groups as qualitative research, Sage publications.

MORGAN, M., GIBBS, S., MAXWELL, K. \& BRITTEN, N. 2002. Hearing children's voices: methodological issues in conducting focus groups with children aged 7-11 years. Qualitative Research, 2, 5-20. 
MORRIS, J. N. \& CRAWFORD, M. D. 1958. Coronary Heart Disease and Physical Activity of Work. British Medical Journal, 2, 1485-1496.

MORRIS, J. N., HEADY, J. A., RAFFLE, P. A. B., ROBERTS, C. G. \& PARKS, J. W. 1953. Coronary heart-disease and physical activity of work. The Lancet, 262, 1111-1120.

MOTA, J., SANTOS, P., GUERRA, S., RIBEIRO, J. C. \& DUARTE, J. A. 2003. Patterns of daily physical activity during school days in children and adolescents. American Journal of Human Biology, 15, 547-553.

MOUNTJOY, M., ANDERSEN, L. B., ARMSTRONG, N., BIDDLE, S., BOREHAM, C., BEDENBECK, H. P., EKELUND, U., ENGEBRETSEN, L., HARDMAN, K., HILLS, A. P., KAHLMEIER, S., KRIEMLER, S., LAMBERT, E., LJUNGQVIST, A., MATSUDO, V., MCKAY, H., MICHELI, L., PATE, R., RIDDOCH, C., SCHAMASCH, P., SUNDBERG, C. J., TOMKINSON, G., VAN SLUIJS, E. \& VAN MECHELEN, W. 2011. International Olympic Committee consensus statement on the health and fitness of young people through physical activity and sport. Br J Sports Med, $45,839-48$.

MOVE, D. T. 2012. Designed to Move: A Physical Activity Agenda. Beaverton: Nike Inc.

MUELLER, S., MOHR, T., GUENTHER, K., FROHNHOFEN, J. \& BAUDISCH, P. faBrickation: fast 3D printing of functional objects by integrating construction kit building blocks. Proceedings of the SIGCHI Conference on Human Factors in Computing Systems, 2014. ACM, 3827-3834.

MULLER, M. J. 2003. Participatory design: the third space in HCI. Human-computer interaction: Development process, 4235, 165-185.

MUNSON, S. A. \& CONSOLVO, S. Exploring goal-setting, rewards, self-monitoring, and sharing to motivate physical activity. Pervasive computing technologies for healthcare (PervasiveHealth), 2012 6th international conference on, 2012. IEEE, 25-32.

MURPHY, E. C., CARSON, L., NEAL, W., BAYLIS, C., DONLEY, D. \& YEATER, R. 2009. Effects of an exercise intervention using Dance Dance Revolution on endothelial function and other risk factors in overweight children. Int J Pediatr Obes, 4, 205-14.

NADER, P. R., BRADLEY, R. H., HOUTS, R. M., MCRITCHIE, S. L. \& O'BRIEN, M. 2008. Moderate-to-vigorous physical activity from ages 9 to 15 years. Jama, 300, 295-305.

NASPE, N. A. F. S. A. P. E. 2004. Moving into the future: National standards for physical education (2nd ed). American Alliance for Health, Physical Education, Recreation, and Dance, Reston, VA.

NAYLOR, P.-J., MACDONALD, H. M., WARBURTON, D. E., REED, K. E. \& MCKAY, H. A. 2008. An active school model to promote physical activity in elementary schools: action schools! BC. British Journal of Sports Medicine.

NEMET, D., GEVA, D., MECKEL, Y. \& ELIAKIM, A. 2012. Health-related knowledge and preferences in low socio-economic kindergarteners. Int $J$ Behav Nutr Phys Act, 9, 1.

NESS, A. R., LEARY, S. D., MATTOCKS, C., BLAIR, S. N., REILLY, J. J., WELLS, J., INGLE, S., TILling, K., SMITH, G. D. \& RIDDOCH, C. 2007. Objectively measured physical activity and fat mass in a large cohort of children. PLoS medicine, 4, e97. 
NG, S. W. \& POPKIN, B. M. 2012. Time use and physical activity: a shift away from movement across the globe. Obes Rev, 13, 659-80.

NICE 2009. Promoting physical activity for children and young people: Guidance. In: EXCELLENCE, N. I. F. H. A. C. (ed.).

NICE 2015. What Works in Schools and Collegesto Increase Physical Activity? A briefing for head teachers, college principals, staff working in education settings, directors of public health and wider partners Public Health England.

NICKEL, A. 2013. Designing better exergames: Application of flow concepts and the FITT principle to full body exertion video games and flexible challenge systems. The University of North Carolina at Charlotte.

NIEMAN, P. 2002. Psychosocial aspects of physical activity. Paediatr Child Health, 7, 309-12.

NIGG, C. R. \& COURNEYA, K. S. 1998. Transtheoretical model: Examining adolescent exercise behavior. Journal of adolescent health, 22, 214-224.

NOONAN, R. J., BODDY, L. M., FAIRCLOUGH, S. J. \& KNOWLES, Z. R. 2016. Write, draw, show, and tell: a child-centred dual methodology to explore perceptions of out-of-school physical activity. BMC Public Health, 16, 326.

NORMAN, G. J., ZABINSKI, M. F., ADAMS, M. A., ROSENBERG, D. E., YAROCH, A. L. \& ATIENZA, A. A. 2007. A review of eHealth interventions for physical activity and dietary behavior change. Am J Prev Med, 33, 336345.

NORRIS, R., CARROLL, D. \& COCHRANE, R. 1992. The effects of physical activity and exercise training on psychological stress and well-being in an adolescent population. Journal of psychosomatic research, 36, 55-65.

NOVAK, J. D. \& CAÑAS, A. J. 2008. The theory underlying concept maps and how to construct and use them.

NUTBEAM, D. 2000. Health literacy as a public health goal: a challenge for contemporary health education and communication strategies into the $21 \mathrm{st}$ century. Health Promotion International, 15, 259-267.

O'BRIEN, T. D., REEVES, N. D., BALTZOPOUlOS, V., JONES, D. A. \& MAGANARIS, C. N. 2009. Moment arms of the knee extensor mechanism in children and adults. Journal of Anatomy, 215, 198-205.

OCOBOCK, C. 2014. Measuring and predicting total energy expenditure among highly active humans in natural environments.

ODDIE, S., FREDEEN, D., WILLIAMSON, B., DECLERCK, D., DOE, S. \& MOSLENKO, K. 2014. Can Physical Activity Improve Depression, Coping \& Motivation to Exercise in Children and Youth Experiencing Challenges to Mental Wellness? Psychology, 5, 2147.

OENEMA, A. \& BRUG, J. 2003. Feedback strategies to raise awareness of personal dietary intake: results of a randomized controlled trial. Preventive medicine, 36, 429-439.

OFCOM 2014. Children and Parents: Media Use and Attitudes Report.

OFCOM 2017. Children and Parents: Media Use and Attitudes Report.

OFFICE, C. 2014. Moving More, Living More, The Physical Activity Olympic and Paralympic Legacy for the Nation. London.

OHKAWARA, K., TANAKA, S., MIYACHI, M., ISHIKAWA-TAKATA, K. \& TABATA, I. 2007. A dose-response relation between aerobic exercise and visceral fat reduction: systematic review of clinical trials. International journal of obesity, 31, 1786. 
OLIVER, A. \& BROWN, L. D. 2012. A consideration of user financial incentives to address health inequalities. Journal of health politics, policy and law, 37, 201226.

OLIVER, M., SCHOFIELD, G. \& MCEVOY, E. 2006. An integrated curriculum approach to increasing habitual physical activity in children: a feasibility study. Journal of School Health, 76, 74-79.

OPPENHEIM, A. N. 2000. Questionnaire design, interviewing and attitude measurement, Bloomsbury Publishing.

ORENDURFF, M. S., SEGAL, A. D., BERGE, J. S., FLICK, K. C., SPANIER, D. \& KLUTE, G. K. 2006. The kinematics and kinetics of turning: limb asymmetries associated with walking a circular path. Gait Posture, 23, 106-11.

OSTROM, E. 2000. Collective action and the evolution of social norms. Journal of economic perspectives, 14, 137-158.

OTD, S. S. R. \& JULIE BISSELL, O. 2015. Occupational therapy for children and youth using sensory integration theory and methods in school-based practice. The American Journal of Occupational Therapy, 69, 1.

OTTEVAERE, C., HUYBRECHTS, I., DE BOURDEAUDHUIJ, I., SJÖSTRÖM, M., RUIZ, J. R., ORTEGA, F. B., HAGSTRÖMER, M., WIDHALM, K., MOLNÁR, D. \& MORENO, L. A. 2011. Comparison of the IPAQ-A and actigraph in relation to VO2max among European adolescents: the HELENA study. Journal of Science and Medicine in Sport, 14, 317-324.

OWEN, M. B., CURRY, W. B., KERNER, C., NEWSON, L. \& FAIRCLOUGH, S. J. 2017. The effectiveness of school-based physical activity interventions for adolescent girls: A systematic review and meta-analysis. Preventive medicine.

OWENS, S., GALLOWAY, R. \& GUTIN, B. 2017. The case for vigorous physical activity in youth. American journal of lifestyle medicine, 11, 96-115.

OWENS, S., GUTIN, B., ALLISON, J., RIGGS, S., FERGUSON, M., LITAKER, M. \& THOMPSON, W. 1999. Effect of physical training on total and visceral fat in obese children. Medicine and science in sports and exercise, 31, 143-148.

OWENS, S. G., GARNER, J. C., 3RD, LOFTIN, J. M., VAN BLERK, N. \& ERMIN, K. 2011. Changes in physical activity and fitness after 3 months of home Wii Fit use. J Strength Cond Res, 25, 3191-7.

PAJARES, M. F. 1992. Teachers' beliefs and educational research: Cleaning up a messy construct. Review of educational research, 62, 307-332.

PAKARINEN, A., PARISOD, H., SMED, J. \& SALANTERA, S. 2017. Health game interventions to enhance physical activity self-efficacy of children: a quantitative systematic review. J Adv Nurs, 73, 794-811.

PAPANAGNOU, D., SERRANO, A., BARKLEY, K., CHANDRA, S., GOVERNATORI, N., PIELA, N., WANNER, G. K. \& SHIN, R. 2016. Does tailoring instructional style to a medical student's self-perceived learning style improve performance when teaching intravenous catheter placement? A randomized controlled study. BMC medical education, 16, 205.

PARDEE, P. E., NORMAN, G. J., LUSTIG, R. H., PREUD'HOMME, D. \& SCHWIMMER, J. B. 2007. Television viewing and hypertension in obese children. Am J Prev Med, 33, 439-43.

PARISOD, H., PAKARINEN, A., KAUHANEN, L., AROMAA, M., LEPPANEN, V., LIUKKONEN, T. N., SMED, J. \& SALANTERA, S. 2014. Promoting Children's Health with Digital Games: A Review of Reviews. Games Health $J, 3,145-56$. 
PARK, B. K. \& CALAMARO, C. 2013. A systematic review of social networking sites: innovative platforms for health research targeting adolescents and young adults. J Nurs Scholarsh, 45, 256-64.

PASHLER, H., MCDANIEL, M., ROHRER, D. \& BJORK, R. 2008. Learning styles: Concepts and evidence. Psychological science in the public interest, 9, 105119.

PATLA, A. E., ADKIN, A. \& BALLARD, T. 1999. Online steering: coordination and control of body center of mass, head and body reorientation. Exp Brain Res, 129, 629-34.

PATLA, A. E., PRENTICE, S. D., ROBINSON, C. \& NEUFELD, J. 1991. Visual control of locomotion: strategies for changing direction and for going over obstacles. J Exp Psychol Hum Percept Perform, 17, 603-34.

PEARCE, G. \& BAILEY, R. P. 2011. Football pitches and Barbie dolls: young children's perceptions of their school playground. Early Child Development and Care, 181, 1361-1379.

PEARCE, M., PAGE, A. S., GRIFFIN, T. P. \& COOPER, A. R. 2014. Who children spend time with after school: associations with objectively recorded indoor and outdoor physical activity. Int J Behav Nutr Phys Act, 11, 45.

PEARCE, M. S., BASTERFIELD, L., MANN, K. D., PARKINSON, K. N., ADAMSON, A. J. \& REILLY, J. J. 2012. Early predictors of objectively measured physical activity and sedentary behaviour in 8-10 year old children: the Gateshead Millennium Study. PLoS One, 7, e37975.

PEARCE, P. F., HARRELL, J. S. \& MCMURRAY, R. G. 2008. Middle-school children's understanding of physical activity: "if you're moving, you're doing physical activity". J Pediatr Nurs, 23, 169-82.

PEARSON, N. \& BIDDLE, S. J. 2011. Sedentary behavior and dietary intake in children, adolescents, and adults: a systematic review. American journal of preventive medicine, $41,178-188$.

PEDIATRICS, A. A. O. 2001. Children, adolescents, and television. Pediatrics, 107, 423-6.

PEDRANA, A., HELlARD, M., GOLD, J., ATA, N., CHANG, S., HOWARD, S., ASSELIN, J., ILIC, O., BATROUNEY, C. \& STOOVE, M. 2013. Queer as $\mathrm{F}^{* *} \mathrm{k}$ : reaching and engaging gay men in sexual health promotion through social networking sites. J Med Internet Res, 15, e25.

PENG, W., CROUSE, J. C. \& LIN, J. H. 2013. Using active video games for physical activity promotion: a systematic review of the current state of research. Health Educ Behav, 40, 171-92.

PENNEY, D. \& JESS, M. 2004. Physical education and physically active lives: A lifelong approach to curriculum development. Sport, Education and Society, 9, 269-287.

PERKINS, C. D., GREEN, M. R. \& PIVARNIK, J. M. 2002. Reliability and validity of the sensormedics VmaxST portable metabolic analyzer. Medicine \& Science in Sports \& Exercise, 34, S4.

PETRAKAKI, D. 2016. AU - Lupton, D . The Quantified Self: A Sociology of SelfTracking. Cambridge: PB - Polity . 2016. 240pp $£ 15.99$ (pbk) $£ 50$ (hbk) ISBN 978-1-5095-0059-8. Sociology of Health \& Illness, n/a-n/a.

PETRELLI, D., WHITTAKER, S. \& BROCKMEIER, J. 2008. AutoTopography: what can physical mementos tell us about digital memories? Proceedings of the SIGCHI Conference on Human Factors in Computing Systems. Florence, Italy: ACM. 
PFEIFFER, K. A., PIVARNIK, J. M., WOMACK, C. J., REEVES, M. J. \& MALINA, R. M. 2002. Reliability and validity of the Borg and OMNI rating of perceived exertion scales in adolescent girls. Med Sci Sports Exerc, 34, 2057-61.

PFITZNER, R., GORZELNIAK, L., HEINRICH, J., VON BERG, A., KLÜMPER, C., BAUER, C. P., KOLETZKO, S., BERDEL, D., HORSCH, A. \& SCHULZ, H. 2013. Physical activity in German adolescents measured by accelerometry and activity diary: introducing a comprehensive approach for data management and preliminary results. PLoS one, 8, e65192.

PHYSIOLOGY, C. S. F. E. 2017. Canadian Sedentary Behaviour Guidelines: 12-17 years.

PIAGET, J. \& COOK, M. 1952. The origins of intelligence in children, International Universities Press New York.

PLACEK, J. H., GRIFFIN, L. L., DODDS, P., RAYMOND, C., TREMINO, F. \& JAMES, A. 2001. Chapter 3: Middle School Students' Conceptions of Fitness: The Long Road to a Healthy Lifestyle. Journal of Teaching in Physical Education, 20, 314-323.

PLASQUI, G. \& WESTERTERP, K. R. 2007. Physical activity assessment with accelerometers: an evaluation against doubly labeled water. Obesity, 15, 23712379.

PLOWMAN, S. A. \& SMITH, D. L. 2013. Exercise physiology for health fitness and performance, Lippincott Williams \& Wilkins.

POLS, M. A., PEETERS, P. H., KEMPER, H. C. \& GROBBEE, D. E. 1998. Methodological aspects of physical activity assessment in epidemiological studies. European Journal of Epidemiology, 14, 63-70.

POLZIEN, K. M., JAKICIC, J. M., TATE, D. F. \& OTTO, A. D. 2007. The efficacy of a technology-based system in a short-term behavioral weight loss intervention. Obesity, 15, 825-830.

POOL, I. D. S. 1959. Trends in content analysis.

POOLE, E. S., MILLER, A. D., XU, Y., EIRIKSDOTTIR, E., CATRAMBONE, R. \& MYNATT, E. D. 2011. The place for ubiquitous computing in schools: lessons learned from a school-based intervention for youth physical activity. Proceedings of the 13th international conference on Ubiquitous computing. Beijing, China: ACM.

POPE, Z. C., LEWIS, B. A. \& GAO, Z. 2015. Using the transtheoretical model to examine the effects of exergaming on physical activity among children. Journal of Physical Activity and Health, 12, 1205-1212.

POWELL, D. \& FITZPATRICK, K. 2015. 'Getting fit basically just means, like, nonfat': children's lessons in fitness and fatness. Sport, Education and Society, 20, 463-484.

POWELL, K. E., PALUCH, A. E. \& BLAIR, S. N. 2011. Physical activity for health: What kind? How much? How intense? On top of what? Annu Rev Public Health, 32, 349-65.

PRAPAVESSIS, H., MADDISON, R. \& BRADING, F. 2004. Understanding exercise behavior among New Zealand adolescents: A test of the Transtheoretical Model. Journal of adolescent health, 35, 346. e17-346. e27.

PRATT, M., MACERA, C. A., SALLIS, J. F., O'DONNELL, M. \& FRANK, L. D. 2004. Economic interventions to promote physical activity: application of the SLOTH model. Am J Prev Med, 27, 136-45.

PRENSKY, M. 2012. From Digital Natives to Digital Wisdom: Hopeful Essays for 21 st Century Learning. Thousand Oaks, California. 
PRICE, S., ROGERS, Y., SCAIFE, M., STANTON, D. \& NEALE, H. 2003. Using 'tangibles' to promote novel forms of playful learning. Interacting with Computers, 15, 169-185.

PRIEUR, F., CASTELLS, J. \& DENIS, C. 2003. A methodology to assess the accuracy of a portable metabolic system (VmaxST). Medicine and science in sports and exercise, 35, 879-885.

PRIMACK, B. A., SWANIER, B., GEORGIOPOULOS, A. M., LAND, S. R. \& FINE, M. J. 2009. Association between media use in adolescence and depression in young adulthood: a longitudinal study. Archives of general psychiatry, 66, 181188.

PROCHASKA, J. J., SALLIS, J. F. \& LONG, B. 2001. A physical activity screening measure for use with adolescents in primary care. Archives of Pediatrics \& Adolescent Medicine, 155, 554-559.

PROCHASKA, J. O. \& DICLEMENTE, C. C. 1992. Stages of change in the modification of problem behaviors. Prog Behav Modif, 28, 183-218.

PROCHASKA, J. O., DICLEMENTE, C. C. \& NORCROSS, J. C. 1992. In search of how people change: applications to addictive behaviors. American psychologist, 47, 1102.

PROCHASKA, J. O. \& MARCUS, B. H. 1994. The transtheoretical model: Applications to exercise.

PROCTOR, M. H., MOORE, L. L., GAO, D., CUPPLES, L. A., BRADLEE, M. L., HOOD, M. Y. \& ELLISON, R. C. 2003. Television viewing and change in body fat from preschool to early adolescence: The Framingham Children's Study. Int J Obes Relat Metab Disord, 27, 827-833.

PUMPERA, M. A., MENDOZAA, J. A., ARSENIEV-KOEHLERA, A., HOLMA, M., WAITEA, A. \& MORENOA, M. A. 2016. Using A Facebook Group As An Adjunct To A Pilot ealth Physical Activity Intervention: A Mixed Methods Approach. Annual Review of Cybertherapy and Telemedicine 2015: Virtual Reality in Healthcare: Medical Simulation and Experiential Interface, 219, 97.

PUYAU, M. R., ADOLPH, A. L., VOHRA, F. A., ZAKERI, I. \& BUTTE, N. F. 2004. Prediction of activity energy expenditure using accelerometers in children. Medicine \& Science in Sports \& Exercise, 36, 1625-1631.

QASEM, L., CARDEW, A., WILSON, A., GRIFFITHS, I., HALSEY, L. G., SHEPARD, E. L., GLEISS, A. C. \& WILSON, R. 2012. Tri-axial dynamic acceleration as a proxy for animal energy expenditure; should we be summing values or calculating the vector? PLoS One, 7, e31187.

RACHELE, J. N., MCPHAIL, S. M., WASHINGTON, T. L. \& CUDDIHY, T. F. 2012. Practical physical activity measurement in youth: a review of contemporary approaches. World Journal of Pediatrics, 8, 207-216.

RACIL, G., OUNIS, O. B., HAMMOUDA, O., KALLEL, A., ZOUHAL, H., CHAMARI, K. \& AMRI, M. 2013. Effects of high vs. moderate exercise intensity during interval training on lipids and adiponectin levels in obese young females. European journal of applied physiology, 113, 2531-2540.

RAICHLEN, D. A., PONTZER, H. \& SHAPIRO, L. J. 2013. A new look at the Dynamic Similarity Hypothesis: the importance of swing phase. Biology open, BIO20135165.

RAMIREZ-MARRERO, F., SMITH, B., SHERMAN, W. \& KIRBY, T. 2005. Comparison of methods to estimate physical activity and energy expenditure in African American children. International Journal of Sports Medicine, 26, 363-371. 
RAMSEY BUCHANAN, L., ROOKS-PECK, C. R., FINNIE, R. K. C., WETHINGTON, H. R., JACOB, V., FULTON, J. E., JOHNSON, D. B., KAHWATI, L. C., PRATT, C. A., RAMIREZ, G., MERCER, S. L. \& GLANZ, K. 2016. Reducing Recreational Sedentary Screen Time: A Community Guide Systematic Review. Am J Prev Med, 50, 402-415.

RAND, M. K. \& OHTSUKI, T. 2000. EMG analysis of lower limb muscles in humans during quick change in running directions. Gait Posture, 12, 169-83.

RANGUL, V., HOLMEN, T. L., KURTZE, N., CUYPERS, K. \& MIDTHJELL, K. 2008. Reliability and validity of two frequently used self-administered physical activity questionnaires in adolescents. BMC medical research methodology, 8, 47.

RASCHE, P., SCHLOMANN, A. \& MERTENS, A. 2017. Who Is Still Playing Pokémon Go? A Web-Based Survey. JMIR Serious Games, 5, e7.

RASMUSSEN, M. K., PEDERSEN, E. W., PETERSEN, M. G. \& HORNBÆK, K. Shape-changing interfaces: a review of the design space and open research questions. Proceedings of the SIGCHI Conference on Human Factors in Computing Systems, 2012. ACM, 735-744.

REILLY, J. J., JACKSON, D. M., MONTGOMERY, C., KELLY, L. A., SLATER, C., GRANT, S. \& PATON, J. Y. 2004. Total energy expenditure and physical activity in young Scottish children: mixed longitudinal study. Lancet, 363, 211-2.

REILLY, J. J., KELLY, L., MONTGOMERY, C., WILLIAMSON, A., FISHER, A., MCCOLL, J. H., LO CONTE, R., PATON, J. Y. \& GRANT, S. 2006. Physical activity to prevent obesity in young children: cluster randomised controlled trial. Bmj, 333, 1041.

RESEARCH, N. C. F. S. 2017a. Health Survey for England 2016: Children's Health [Online]. [Accessed].

RESEARCH, N. S. 2017b. Health Survey for England 2016 Children's health. In: CENTRE, H. A. S. C. I. (ed.). UCL.

REYNOLDS, K. J., SUBAŠIĆ, E. \& TINDALL, K. 2015. The problem of behaviour change: From social norms to an ingroup focus. Social and Personality Psychology Compass, 9, 45-56.

RICH, C. 2013. Methodological considerations in large scale accelerometer-based studies of childhood physical activity. UCL (University College London).

RICHARDSON, C. R., FAULKNER, G., MCDEVITT, J., SKRINAR, G. S., HUTCHINSON, D. S. \& PIETTE, J. D. 2005. Integrating physical activity into mental health services for persons with serious mental illness. Psychiatric services, 56, 324-331.

RICHARDSON, J. T. 2011. The analysis of 2 x 2 contingency tables--yet again. Stat Med, 30, 890; author reply 891-2.

RIDDOCH, C. J., BO ANDERSEN, L., WEDDERKOPP, N., HARRO, M., KLASSON-HEGGEBO, L., SARDINHA, L. B., COOPER, A. R. \& EKELUND, U. 2004. Physical activity levels and patterns of 9- and 15-yr-old European children. Med Sci Sports Exerc, 36, 86-92.

RIDDOCH, C. J., MATTOCKS, C., DEERE, K., SAUNDERS, J., KIRKBY, J., TILLING, K., LEARY, S. D., BLAIR, S. N. \& NESS, A. R. 2007. Objective measurement of levels and patterns of physical activity. Archives of Disease in Childhood, 92, 963.

RIDEOUT, V. J., FOEHR, U. G. \& ROBERTS, D. F. 2010. Generation M 2: Media in the Lives of 8-to 18-Year-Olds. Henry J. Kaiser Family Foundation. 
RIDGERS, N. D., KNOWLES, Z. R. \& SAYERS, J. 2012. Encouraging play in the natural environment: a child-focused case study of Forest School. Children's Geographies, 10, 49-65.

RIDGERS, N. D., MCNARRY, M. A. \& MACKINTOSH, K. A. 2016. Feasibility and effectiveness of using wearable activity trackers in youth: a systematic review. JMIR mHealth and uHealth, 4.

RIDGERS, N. D., TIMPERIO, A., BROWN, H., BALL, K., MACFARLANE, S., LAI, S. K., RICHARDS, K., MACKINTOSH, K. A., MCNARRY, M. A., FOSTER, M. \& SALMON, J. 2018. Wearable Activity Tracker Use Among Australian Adolescents: Usability and Acceptability Study. JMIR mHealth and uHealth, 6, e86.

RIDGERS, N. D., TIMPERIO, A., BROWN, H., BALL, K., MACFARLANE, S., LAI, S. K., RICHARDS, K., NGAN, W. \& SALMON, J. 2017. A clusterrandomised controlled trial to promote physical activity in adolescents: the Raising Awareness of Physical Activity (RAW-PA) Study. BMC public health, 17,6 .

RIDLEY, K., AINSWORTH, B. E. \& OLDS, T. S. 2008. Development of a compendium of energy expenditures for youth. International Journal of Behavioral Nutrition and Physical Activity, 5, 45.

RIENER, C. \& WILLINGHAM, D. 2010. The myth of learning styles. Change: The magazine of higher learning, 42, 32-35.

RISING, R., HARPER, I. T., FONTVIELLE, A. M., FERRARO, R. T., SPRAUL, M. \& RAVUSSIN, E. 1994. Determinants of total daily energy expenditure: variability in physical activity. Am J Clin Nutr, 59, 800-4.

RITA, D. \& DUNN, K. 1979. Learning Styles/Teaching Styles: Should They•• Can They... Be Matched.

ROBERTSON, R. J., GOSS, F. L., ANDREACCI, J. L., DUBE, J. J., RUTKOWSKI, J. J., SNEE, B. M., KOWALLIS, R. A., CRAWFORD, K., AARON, D. J. \& METZ, K. F. 2005. Validation of the children's OMNI RPE scale for stepping exercise. Med Sci Sports Exerc, 37, 290-8.

ROBERTSON, R. J., GOSS, F. L., BOER, N. F., PEOPLES, J. A., FOREMAN, A. J., DABAYEBEH, I. M., MILLICH, N. B., BALASEKARAN, G., RIECHMAN, S. E., GALLAGHER, J. D. \& THOMPKINS, T. 2000. Children's OMNI scale of perceived exertion: mixed gender and race validation. Med Sci Sports Exerc, 32, $452-8$.

ROBERTSON, W., STEWART-BROWN, S., WILCOCK, E., OLDFIELD, M. \& THOROGOOD, M. 2010. Utility of accelerometers to measure physical activity in children attending an obesity treatment intervention. Journal of obesity, 2011.

ROCK, M. L. \& THEAD, B. K. 2007. The effects of fading a strategic self-monitoring intervention on students' academic engagement, accuracy, and productivity. Journal of Behavioral Education, 16, 389-412.

ROGERS, Y., SCAIFE, M., GABRIELLI, S., SMITH, H. \& HARRIS, E. 2002a. A Conceptual Framework for Mixed Reality Environments: Designing Novel Learning Activities for Young Children. Presence: Teleoperators and Virtual Environments, 11, 677-686.

ROGERS, Y., SCAIFE, M., GABRIELLI, S., SMITH, H. \& HARRIS, E. 2002b. A conceptual framework for mixed reality environments: Designing novel learning activities for young children. Presence Teleoperators and Virtual Environments httpsdoiorg101162105474602321050776, 11, 677-686. 
ROHIT ASHOK, K. 2014. Exploring material representations of physical activity. Proceedings of the 2014 companion publication on Designing interactive systems. Vancouver, BC, Canada: ACM.

ROHIT ASHOK, K., FLORIAN 'FLOYD, M. \& LARISSA, H. 2013. SweatAtoms: materializing physical activity. Proceedings of The 9th Australasian Conference on Interactive Entertainment: Matters of Life and Death. Melbourne, Australia: ACM.

ROHIT ASHOK, K., JEEWON, L., DEEPTI, A., LARISSA, H. \& FLORIAN 'FLOYD, M. 2015a. TastyBeats: Designing Palatable Representations of Physical Activity. Proceedings of the 33rd Annual ACM Conference on Human Factors in Computing Systems. Seoul, Republic of Korea: ACM.

ROHIT ASHOK, K., RYAN, P. \& FLORIAN 'FLOYD, M. 2015b. EdiPulse: Turning Physical Activity Into Chocolates. Proceedings of the 33rd Annual ACM Conference Extended Abstracts on Human Factors in Computing Systems. Seoul, Republic of Korea: ACM.

ROMANZINI, M., PETROSKI, E. L. \& REICHERT, F. F. 2012. Accelerometers thresholds to estimate physical activity intensity in children and adolescents: a systematic review. Revista Brasileira de Cineantropometria \& Desempenho Humano, 14, 101-113.

RONDA, G., VAN ASSEMA, P. \& BRUG, J. 2001. Stages of change, psychological factors and awareness of physical activity levels in The Netherlands. Health Promot Int, 16, 305-14.

ROSADO, E. L., KAIPPERT, V. C. \& DE BRITO, R. S. 2013. Energy expenditure measured by indirect calorimetry in obesity. Applications of Calorimetry in a Wide Context-Differential Scanning Calorimetry, Isothermal Titration Calorimetry and Microcalorimetry. InTech.

ROSE, T., BARKER, M., MARIA JACOB, C., MORRISON, L., LAWRENCE, W., STROMMER, S., VOGEL, C., WOODS-TOWNSEND, K., FARRELL, D., INSKIP, H. \& BAIRD, J. 2017. A Systematic Review of Digital Interventions for Improving the Diet and Physical Activity Behaviors of Adolescents. $J$ Adolesc Health, 61, 669-677.

ROSEN, L. R. 2012. Rewired: Understanding the iGeneration and the Way they Learn. Teaching Theology \& Religion, 15, 288-289.

ROTH, M. \& STAMATAKIS, E. 2010. Linking young people's knowledge of public health guidelines to physical activity levels in England. Pediatr Exerc Sci, 22, 467-76.

ROTHON, C., EDWARDS, P., BHUI, K., VINER, R. M., TAYLOR, S. \& STANSFELD, S. A. 2010. Physical activity and depressive symptoms in adolescents: a prospective study. Bmc Medicine, 8, 32 .

ROULSTONE, S., LOADER, S., NORTHSTONE, K. \& BEVERIDGE, M. 2002. The speech and language of children aged 25 months: Descriptive data from the Avon Longitudinal Study of Parents and Children. Early Child Development and Care, 172, 259-268.

ROUTEN, A. C., UPTON, D., EDWARDS, M. G. \& PETERS, D. M. 2014. The effect of pedometer step goal, feedback and self-monitoring interventions on accelerometer-measured physical activity in children. Graduate journal of sport, exercise \& physical education research., 2, 37-53.

ROWICKA, G., DYLĄG, H., AMBROSZKIEWICZ, J., RIAHI, A., WEKER, H. \& CHEŁCHOWSKA, M. 2017. Total Oxidant and Antioxidant Status in 
Prepubertal Children with Obesity. Oxidative Medicine and Cellular Longevity, 2017.

ROWLAND, T., WEHNERT, M. \& MILLER, K. 2000. Cardiac responses to exercise in competitive child cyclists. Medicine and science in sports and exercise, 32, 747-752.

ROWLAND, T. W. \& GREEN, G. M. 1988. Physiological responses to treadmill exercise in females: adult-child differences. Medicine and science in sports and exercise, 20, 474-478.

ROWLANDS, A. V. 2007. Accelerometer assessment of physical activity in children: an update. Pediatr Exerc Sci, 19, 252-66.

ROWLANDS, A. V., ESTON, R. G. \& INGLEDEW, D. K. 1999. Relationship between activity levels, aerobic fitness, and body fat in 8-to 10-yr-old children. Journal of Applied Physiology, 86, 1428-1435.

ROWLANDS, A. V., PILGRIM, E. L. \& ESTON, R. G. 2008. Patterns of habitual activity across weekdays and weekend days in 9-11-year-old children. Prev Med, 46, 317-24.

ROWLANDS, A. V., THOMAS, P. W., ESTON, R. G. \& TOPPING, R. 2004a. Validation of the RT3 triaxial accelerometer for the assessment of physical activity. Medicine \& Science in Sports \& Exercise, 36, 518-524.

ROWLANDS, A. V., THOMAS, P. W., ESTON, R. G. \& TOPPING, R. 2004b. Validation of the RT3 triaxial accelerometer for the assessment of physical activity. Med Sci Sports Exerc, 36, 518-24.

ROWSELL, A., MULLER, I., MURRAY, E., LITTLE, P., BYRNE, C. D., GANAHL, K., MÜLLER, G., GIBNEY, S., LYLES, C. R. \& LUCAS, A. 2015. Views of people with high and low levels of health literacy about a digital intervention to promote physical activity for diabetes: a qualitative study in five countries. Journal of medical Internet research, 17.

RUETERBORIES, J., SPAICH, E. G., LARSEN, B. \& ANDERSEN, O. K. 2010. Methods for gait event detection and analysis in ambulatory systems. Med Eng Phys, 32, 545-52.

RYAN, R. M. \& DECI, E. L. 2000. Self-determination theory and the facilitation of intrinsic motivation, social development, and well-being. American psychologist, 55, 68.

RYSCHON, T., FOWLER, M., WYSONG, R., ANTHONY, A.-R. \& BALABAN, R. 1997. Efficiency of human skeletal muscle in vivo: comparison of isometric, concentric, and eccentric muscle action. Journal of applied physiology, 83, 867-874.

SADIK, A. 2008. Digital storytelling: A meaningful technology-integrated approach for engaged student learning. Educational technology research and development, 56, 487-506.

SALARIAN, A., RUSSMANN, H., VINGERHOETS, F. J., DEHOLLAIN, C., BLANC, Y., BURKHARD, P. R. \& AMINIAN, K. 2004. Gait assessment in Parkinson's disease: toward an ambulatory system for long-term monitoring. IEEE Trans Biomed Eng, 51, 1434-43.

SALLIS, J. F. 1991. Self-report measures of children's physical activity. J Sch Health, $61,215-9$.

SALMON, J., BOOTH, M. L., PHONGSAVAN, P., MURPHY, N. \& TIMPERIO, A. 2007. Promoting physical activity participation among children and adolescents. Epidemiologic reviews, 29, 144-159. 
SALVY, S. J., DE LA HAYE, K., BOWKER, J. C. \& HERMANS, R. C. 2012. Influence of peers and friends on children's and adolescents' eating and activity behaviors. Physiol Behav, 106, 369-78.

SALVY, S. J., ROEMMICH, J. N., BOWKER, J. C., ROMERO, N. D., STADLER, P. J. \& EPSTEIN, L. H. 2009. Effect of peers and friends on youth physical activity and motivation to be physically active. J Pediatr Psychol, 34, 217-25.

SANAEINASAB, H., SAFFARI, M., NAZERI, M., KARIMI ZARCHI, A. \& CARDINAL, B. J. 2013. Descriptive analysis of I ranian adolescents' stages of change for physical activity behavior. Nursing \& health sciences, 15, 280-285.

SANDELOWSKI, M. 2001. Real qualitative researchers do not count: the use of numbers in qualitative research. Research in nursing \& health, 24, 230-240.

SANDERCOCK, G. R. \& OGUNLEYE, A. A. 2013. Independence of physical activity and screen time as predictors of cardiorespiratory fitness in youth. Pediatr Res, 73, 692-7.

SANIGORSKI, A. M., BELL, A. C., KREMER, P. J., CUTTLER, R. \& SWINBURN, B. A. 2008. Reducing unhealthy weight gain in children through community capacity-building: results of a quasi-experimental intervention program, $\mathrm{Be}$ Active Eat Well. Int J Obes (Lond), 32, 1060-7.

SANTOS, M. P., GOMES, H. \& MOTA, J. 2005. Physical activity and sedentary behaviors in adolescents. Ann Behav Med, 30, 21-4.

SARDINHA, L. B., ANDERSEN, L. B., ANDERSSEN, S. A., QUITERIO, A. L., ORNELAS, R., FROBERG, K., RIDDOCH, C. J. \& EKELUND, U. 2008. Objectively measured time spent sedentary is associated with insulin resistance independent of overall and central body fat in 9- to 10-year-old Portuguese children. Diabetes Care, 31, 569-75.

SASAKI, J. E., DA SILVA, K. S., DA COSTA, B. G. G. \& JOHN, D. 2016. Measurement of physical activity using accelerometers. Computer-assisted and web-based innovations in psychology, special education, and health. Elsevier.

SAUVÉ, K., HOUBEN, S., MARQUARDT, N., BAKKER, S., HENGEVELD, B., GALLACHER, S. \& ROGERS, Y. LOOP: A physical artifact to facilitate seamless interaction with personal data in everyday life. Proceedings of the 2017 ACM Conference Companion Publication on Designing Interactive Systems, 2017. ACM, 285-288.

SCHAEFER, S. E., CHING, C. C., BREEN, H. \& GERMAN, J. B. 2016. Wearing, thinking, and moving: testing the feasibility of fitness tracking with urban youth. American Journal of Health Education, 47, 8-16.

SCHÄRLI, A. M., KELLER, M., LORENZETTI, S., MURER, K. \& VAN DE LANGENBERG, R. 2013. Balancing on a Slackline: 8-Year-Olds vs. Adults. Frontiers in Psychology, 4, 208.

SCHEMBRE, S. M., LIAO, Y., ROBERTSON, M. C., DUNTON, G. F., KERR, J., HAFFEY, M. E., BURNETT, T., BASEN-ENGQUIST, K. \& HICKLEN, R. S. 2018. Just-in-Time Feedback in Diet and Physical Activity Interventions: Systematic Review and Practical Design Framework. Journal of medical Internet research, 20.

SCHMIDT, R. A. \& WRISBERG, C. A. 2008. Motor learning and performance: A situation-based learning approach, Human kinetics.

SCHOELLER, D. A. 1999. Recent advances from application of doubly labeled water to measurement of human energy expenditure. The Journal of nutrition, 129, 1765-1768. 
SCHOT, P., DART, J. \& SCHUH, M. 1995. Biomechanical analysis of two changeof-direction maneuvers while running. J Orthop Sports Phys Ther, 22, 254-8.

SCHUMANN, A., ESTABROOKS, P., NIGG, C. \& HILL, J. 2003. Validation of the stages of change with mild, moderate, and strenuous physical activity behavior, intentions, and self-efficacy. International Journal of Sports Medicine, 24, 363-365.

SCHUMANN, A., NIGG, C. R., ROSSI, J. S., JORDAN, P. J., NORMAN, G. J., GARBER, C. E., RIEBE, D. \& BENISOVICH, S. V. 2002. Construct validity of the stages of change of exercise adoption for different intensities of physical activity in four samples of differing age groups. American Journal of Health Promotion, 16, 280-287.

SCRUGGS, T. E. \& MASTROPIERI, M. A. 1986. Academic characteristics of behaviorally disordered and learning disabled students. Behavioral Disorders, 11, 184-190.

SERRANO-SANCHEZ, J. A., MARTÍ-TRUJILLO, S., LERA-NAVARRO, A., DORADO-GARCÍA, C., GONZÁLEZ-HENRÍQUEZ, J. J. \& SANCHÍSMOYSI, J. 2011. Associations between Screen Time and Physical Activity among Spanish Adolescents. PLoS ONE, 6, e24453.

SHARMA, S., KALLIONIEMI, P., HEIMONEN, T., HAKULINEN, J., TURUNEN, M. \& KESKINEN, T. Overcoming socio-technical challenges for crosscultural collaborative applications. Proceedings of the 17th ACM Conference on Interaction Design and Children, 2018. ACM, 325-336.

SHARPE, P. A., WILCOX, S., ROONEY, L. J., STRONG, D., HOPKINSCAMPBELL, R., BUTEL, J., AINSWORTH, B. \& PARRA-MEDINA, D. 2011. Adherence to accelerometer protocols among women from economically disadvantaged neighborhoods. Journal of physical activity \& health, 8, 699.

SHAW, J. M., MITCHELL, C. A., WELCH, A. J. \& WILLIAMSON, M. J. 2015. Social media used as a health intervention in adolescent health: A systematic review of the literature. DIGITAL HEALTH, 1, 2055207615588395.

SHEA, J. M. \& BEAUSOLEIL, N. 2012. Breaking down 'healthism': Barriers to health and fitness as identified by immigrant youth in St. John's, NL, Canada. Sport, Education and Society, 17, 97-112.

SHEPARD, E. L., WILSON, R. P., LIEBSCH, N., QUINTANA, F., LAICH, A. G. \& LUCKE, K. 2008. Flexible paddle sheds new light on speed: a novel method for the remote measurement of swim speed in aquatic animals. Endangered Species Research, 4, 157-164.

SHEPHARD, R. \& VUILLEMIN, A. 2003. Limits to the measurement of habitual physical activity by questionnaires. British Journal of Sports Medicine, 37, 197-206.

SHEPHARD, R. J. 2003. Limits to the measurement of habitual physical activity by questionnaires. British journal of sports medicine, 37, 197-206.

SHILTS, M. K., HOROWITZ, M. \& TOWNSEND, M. S. 2004. Goal setting as a strategy for dietary and physical activity behavior change: a review of the literature. Am J Health Promot, 19, 81-93.

SHIRI, R., SOLOVIEVA, S., HUSGAFVEL-PURSIAINEN, K., TELAMA, R., YANG, X., VIIKARI, J., RAITAKARI, O. T. \& VIIKARI-JUNTURA, E. The role of obesity and physical activity in non-specific and radiating low back pain: the Young Finns study. Seminars in arthritis and rheumatism, 2013. Elsevier, 640-650. 
SHIROMA, E. J., COOK, N. R., MANSON, J. E., MOORTHY, M. V., BURING, J. E., RIMM, E. B. \& LEE, I. M. 2017. Strength Training and the Risk of Type 2 Diabetes and Cardiovascular Disease. Med Sci Sports Exerc, 49, 40-46.

SILVA, A. G., QUEIRÓS, A., NETO, M. \& ROCHA, N. P. 2016. Type and quantity of physical activity and screen based activities of students from the 7 th to the 12th grades: Characterization and association. Revista Portuguesa de Saúde Pública, 34, 236-243.

SIRARD, J. R. \& SLATER, M. E. 2009. Compliance with wearing physical activity accelerometers in high school students. Journal of Physical Activity and Health, 6, S148-S155.

SIRARD, J. R., TROST, S. G., PFEIFFER, K. A., DOWDA, M. \& PATE, R. R. 2005. Calibration and evaluation of an objective measure of physical activity in preschool children. Journal of physical activity and health, 2, 345-357.

SIRRIYEH, R., LAWTON, R. \& WARD, J. 2010. Physical activity and adolescents: an exploratory randomized controlled trial investigating the influence of affective and instrumental text messages. Br J Health Psychol, 15, 825-40.

SISSON, S. B., CHURCH, T. S., MARTIN, C. K., TUDOR-LOCKE, C., SMITH, S. R., BOUCHARD, C., EARNEST, C. P., RANKINEN, T., NEWTON JR, R. L. \& KATZMARZYK, P. T. 2009. Profiles of sedentary behavior in children and adolescents: the US National Health and Nutrition Examination Survey, 2001-2006. International Journal of Pediatric Obesity, 4, 353-359.

SKALSKI, P. \& TAMBORINI, R. 2007. The role of social presence in interactive agent-based persuasion. Media psychology, 10, 385-413.

SKINNER, H., BISCOPE, S., POLAND, B. \& GOLDBERG, E. 2003. How adolescents use technology for health information: implications for health professionals from focus group studies. Journal of medical Internet research, 5.

SLEAP, M. \& WARBURTON, P. 1996. Physical activity levels of 5-11-year-old children in England: cumulative evidence from three direct observation studies. Int J Sports Med, 17, 248-53.

SLEAP, M. \& WORMALD, H. 2001. Perceptions of Physical Activity Among Young Women aged 16 and 17 Years. European Journal of Physical Education, 6, 26-37.

SLEDDENS, S. F., GERARDS, S. M., THIJS, C., DE VRIES, N. K. \& KREMERS, S. P. 2011. General parenting, childhood overweight and obesity-inducing behaviors: a review. International journal of pediatric obesity, 6, e12-27.

SLOOTMAKER, S. M., CHINAPAW, M. J., SEIDELL, J. C., VAN MECHELEN, W. \& SCHUIT, A. J. 2010. Accelerometers and Internet for physical activity promotion in youth? Feasibility and effectiveness of a minimal intervention [ISRCTN93896459]. Prev Med, 51, 31-6.

SMAHEL, D., WRIGHT, M. F. \& CERNIKOVA, M. 2015. The impact of digital media on health: children's perspectives. Int J Public Health, 60, 131-7.

SMITH, J. \& NOBLE, H. 2014. Bias in research. Evid Based Nurs, 17, 100-1.

SNETHEN, J. A. \& BROOME, M. E. 2007. Weight, Exercise, and Health:Children's Perceptions. Clinical Nursing Research, 16, 138-152.

SOTHERN, M. S., LOFTIN, M., SUSKIND, R. M., UDALL, J. N. \& BLECKER, U. 1999. The health benefits of physical activity in children and adolescents: implications for chronic disease prevention. Eur J Pediatr, 158, 271-4.

SOUTHARD, D. R. \& SOUTHARD, B. H. 2006. Promoting physical activity in children with MetaKenkoh. Clinical and Investigative Medicine, 29, 293. 
SPARROW, W. A. 2000. Energetics of human activity, Human Kinetics.

SPELLER, V., BYRNE, J., DEWHIRST, S., ALMOND, P., MOHEBATI, L., NORMAN, M., POLACK, S., MEMON, A., GRACE, M. \& MARGETTS, B. 2010. Developing trainee school teachers' expertise as health promoters. Health Education, 110, 490-507.

SPORT WALES. '5x60'. Available: http://www.webcitation.org/73Bbuf9Fy http://sport.wales/community-sport/education/5x60.aspx.

ST LEGER, L. E. 2004. What's the place of schools in promoting health? Are we too optimistic? Health promotion international.

STAIANO, A. E., ABRAHAM, A. A. \& CALVERT, S. L. 2012. The Wii Club: Gaming for Weight Loss in Overweight and Obese Youth. Games For Health Journal, 1, 377-380.

STEELE, R. M., BRAGE, S., CORDER, K., WAREHAM, N. J. \& EKELUND, U. 2008. Physical activity, cardiorespiratory fitness, and the metabolic syndrome in youth. Journal of Applied Physiology, 105, 342-351.

STEELE, R. M., VAN SLUIJS, E. M., CASSIDY, A., GRIFFIN, S. J. \& EKELUND, U. 2009a. Targeting sedentary time or moderate-and vigorous-intensity activity: independent relations with adiposity in a population-based sample of 10-y-old British children. The American journal of clinical nutrition, 90, $1185-$ 1192.

STEELE, R. M., VAN SLUIJS, E. M., CASSIDY, A., GRIFFIN, S. J. \& EKELUND, U. 2009b. Targeting sedentary time or moderate-and vigorous-intensity activity: independent relations with adiposity in a population-based sample of 10-y-old British children-. The American journal of clinical nutrition, 90, $1185-1192$.

STEGENGA, H., HAINES, A., JONES, K. \& WILDING, J. 2014. Identification, assessment, and management of overweight and obesity: summary of updated NICE guidance. $b m j, 349$, g6608.

STEINBECK, K. S. 2001. The importance of physical activity in the prevention of overweight and obesity in childhood: a review and an opinion. Obes Rev, 2, 117-30.

STELlA, S. G., VILAR, A. P., LACROIX, C., FISBERG, M., SANTOS, R. F., MELLO, M. T. D. \& TUFIK, S. 2005. Effects of type of physical exercise and leisure activities on the depression scores of obese Brazilian adolescent girls. Brazilian Journal of Medical and Biological Research, 38, 1683-1689.

STEUDEL, K. \& BEATTIE, J. 1995. Does limb length predict the relative energetic cost of locomotion in mammals? Journal of Zoology, 235, 501-514.

STEUDEL-NUMBERS, K. L., WEAVER, T. D. \& WALL-SCHEFFLER, C. M. 2007. The evolution of human running: effects of changes in lower-limb length on locomotor economy. Journal of Human Evolution, 53, 191-196.

STEWART, A., MARFELL-JONES, M., OLDS, T. \& DE RIDDER, H. International standards for anthropometric assessment. 2011: ISAK: Lower Hutt. New Zealand.

STEWART, S. \& MITCHELL, M. 2003. Chapter 4: Instructional variables and student knowledge and conceptions of fitness. Journal of Teaching in Physical Education, 22, 533-551.

STOKOLS, D., KING, A., TALEN, E., BRASSINGTON, G. \& KILLINGSWORTH, R. 2002. Theoretical approaches to the promotion of physical activity: Forging a transdisciplinary paradigm. 
STONE, E. J., MCKENZIE, T. L., WELK, G. J. \& BOOTH, M. L. 1998. Effects of physical activity interventions in youth: review and synthesis. American journal of preventive medicine, 15, 298-315.

STONE, M. R., ROWLANDS, A. V., MIDDLEBROOKE, A. R., JAWIS, M. N. \& ESTON, R. G. 2009. The pattern of physical activity in relation to health outcomes in boys. Int J Pediatr Obes, 4, 306-15.

STORY, M., KAPHINGST, K. M. \& FRENCH, S. 2006. The role of schools in obesity prevention. The future of children, 109-142.

STRATH, S. J., KAMINSKY, L. A., AINSWORTH, B. E., EKELUND, U., FREEDSON, P. S., GARY, R. A., RICHARDSON, C. R., SMITH, D. T. \& SWARTZ, A. M. 2013. Guide to the assessment of physical activity: Clinical and research applications: a scientific statement from the American Heart Association. Circulation, 128, 2259-79.

STRATTON, G. \& MULLAN, E. 2005. The effect of multicolor playground markings on children's physical activity level during recess. Preventive medicine, 41, 828-833.

STROHACKER, K., GALARRAGA, O. \& WILLIAMS, D. M. 2014. The impact of incentives on exercise behavior: a systematic review of randomized controlled trials. Ann Behav Med, 48, 92-9.

STRONG, W. B., MALINA, R. M., BLIMKIE, C. J., DANIELS, S. R., DISHMAN, R. K., GUTIN, B., HERGENROEDER, A. C., MUST, A., NIXON, P. A., PIVARNIK, J. M., ROWLAND, T., TROST, S. \& TRUDEAU, F. 2005. Evidence based physical activity for school-age youth. J Pediatr, 146, 732-7.

STUSAK, S., SCHWARZ, J. \& BUTZ, A. 2015. Evaluating the Memorability of Physical Visualizations. Proceedings of the 33rd Annual ACM Conference on Human Factors in Computing Systems. Seoul, Republic of Korea: ACM.

STUSAK, S., TABARD, A., SAUKA, F., KHOT, R. A. \& BUTZ, A. 2014. Activity Sculptures: Exploring the Impact of Physical Visualizations on Running Activity. IEEE Trans Vis Comput Graph, 20, 2201-10.

SUNNY, C., DAVID, W. M. \& JAMES, A. L. 2009. Theory-driven design strategies for technologies that support behavior change in everyday life. Proceedings of the SIGCHI Conference on Human Factors in Computing Systems. Boston, MA, USA: ACM.

SUZUKI, H. \& KATO, H. 1995. Interaction-level support for collaborative learning: $<$ italic $>$ AlgoBlock $<$ italic $>\&$ mdash; an open programming language. The first international conference on Computer support for collaborative learning. Indiana Univ., Bloomington, Indiana, USA: L. Erlbaum Associates Inc.

SVENDSEN, G. B. 1991. The influence of interface style on problem solving. International Journal of Man-Machine Studies, 35, 379-397.

SWEEN, J., WALLINGTON, S. F., SHEPPARD, V., TAYLOR, T., LLANOS, A. A. \& ADAMS-CAMPBELL, L. L. 2014. The role of exergaming in improving physical activity: a review. J Phys Act Health, 11, 864-70.

SYLVIA, L. G., BERNSTEIN, E. E., HUBBARD, J. L., KEATING, L. \& ANDERSON, E. J. 2014. Practical guide to measuring physical activity. Journal of the Academy of Nutrition and Dietetics, 114, 199-208.

SYMONDS, J. E. \& GORARD, S. The death of mixed methods: research labels and their casualties. British Educational Research Association Annual Conference, 2008. Citeseer.

TAMMELIN, R., YANG, X., LESKINEN, E., KANKAANPAA, A., HIRVENSALO, M., TAMMELIN, T. \& RAITAKARI, O. 2014. Tracking of physical activity 
from early childhood through youth into adulthood. Med. Sci. Sports Exerc, 46, 955-962.

TAMMELIN, T., EKELUND, U., REMES, J. \& NAYHA, S. 2007. Physical activity and sedentary behaviors among Finnish youth. Med Sci Sports Exerc, 39, 106774.

TANAKA, C., TANAKA, S., KAWAHARA, J. \& MIDORIKAWA, T. 2007. Triaxial accelerometry for assessment of physical activity in young children. Obesity, $15,1233-1241$.

TANG, K.-C., NUTBEAM, D., ALDINGER, C., ST LEGER, L., BUNDY, D., HOFFMANN, A. M., YANKAH, E., MCCALL, D., BUIJS, G. \& ARNAOUT, S. 2008. Schools for health, education and development: a call for action. Health Promotion International, 24, 68-77.

TANNER, J. M. 1962. Growth at adolescence, with a general consideration of the effects of hereditary and environmental factors upon growth and maturation from birth to maturity, Oxford,, Blackwell Scientific Publications.

TAYLOR, M. J., DABNICHKI, P. \& STRIKE, S. C. 2005. A three-dimensional biomechanical comparison between turning strategies during the stance phase of walking. Hum Mov Sci, 24, 558-73.

TELAMA, R. 2009. Tracking of physical activity from childhood to adulthood: a review. Obes Facts, 2, 187-95.

TELAMA, R., YANG, X., VIIKARI, J., VALIMAKI, I., WANNE, O. \& RAITAKARI, O. 2005. Physical activity from childhood to adulthood: a 21year tracking study. Am J Prev Med, 28, 267-73.

TELFORD, R. M., TELFORD, R. D., OLIVE, L. S., COCHRANE, T. \& DAVEY, R. 2016. Why Are Girls Less Physically Active than Boys? Findings from the LOOK Longitudinal Study. PLoS ONE, 11, e0150041.

THAPAR, A., COLLISHAW, S., PINE, D. S. \& THAPAR, A. K. 2012. Depression in adolescence. The Lancet, 379, 1056-1067.

THIN, A. G., BROWN, C. \& MEENAN, P. 2013. User experiences while playing dance-based exergames and the Influence of different body motion sensing technologies. International Journal of Computer Games Technology, 2013.

THOMPSON, A. M., MCHUGH, T. L., BLANCHARD, C. M., CAMPAGNA, P. D., DURANT, M. A., REHMAN, L. A., MURPHY, R. J. \& WADSWORTH, L. A. 2009. Physical activity of children and youth in Nova Scotia from 2001/02 and 2005/06. Prev Med, 49, 407-9.

THOMPSON, D. \& BATTERHAM, A. M. 2013. Towards integrated physical activity profiling. PLoS One, 8, e56427.

THOMPSON, D., CANTU, D., BHATT, R., BARANOWSKI, T., RODGERS, W., JAGO, R., ANDERSON, B., LIU, Y., MENDOZA, J. A., TAPIA, R. \& BUDAY, R. 2014. Texting to Increase Physical Activity Among Teenagers (TXT Me!): Rationale, Design, and Methods Proposal. JMIR Research Protocols, 3, e14.

THOMPSON, D., CANTU, D., RAJENDRAN, M., RAJENDRAN, M., BHARGAVA, T., ZHANG, Y., CHEN, C., LIU, Y. \& DENG, Z. 2016. Development of a teen-focused exergame. Games for health journal, 5, 342356.

THOMPSON, D., PEACOCK, O., WESTERN, M. \& BATTERHAM, A. M. 2015. Multidimensional physical activity: an opportunity not a problem. Exercise and sport sciences reviews, 43, 67. 
THOMPSON, D. I., CANTU, D., CALLENDER, C., LIU, Y., RAJENDRAN, M., RAJENDRAN, M., ZHANG, Y. \& DENG, Z. 2018. Photorealistic Avatar and Teen Physical Activity: Feasibility and Preliminary Efficacy. Games for health journal, 7, 143-150.

TIMPERIO, A., SALMON, J. \& BALL, K. 2004. Evidence-based strategies to promote physical activity among children, adolescents and young adults: review and update. J Sci Med Sport, 7, 20-9.

TONG, X., GROMALA, D., BARTRAM, L., RAJABIYAZDI, F. \& CARPENDALE, S. 2015. Evaluating the effectiveness of three physical activity visualizationshow people perform vs. perceive. IEEE VIS Electronic Proceedings.

TORSHEIM, T., ERIKSSON, L., SCHNOHR, C. W., HANSEN, F., BJARNASON, T. \& VALIMAA, R. 2010. Screen-based activities and physical complaints among adolescents from the Nordic countries. BMC Public Health, 10, 324.

TOWNSEND, N., WICKRAMASINGHE, K., WILLIAMS, J., BHATNAGAR, P. \& RAYNER, M. 2015. Physical Activity Statistics 2015. British Heart Foundation: London: Nuffield Department of Population Health, University of Oxford

TREMBLAY, M. S., COLLEY, R. C., SAUNDERS, T. J., HEALY, G. N. \& OWEN, N. 2010. Physiological and health implications of a sedentary lifestyle. Applied Physiology, Nutrition, and Metabolism, 35, 725-740.

TREMBLAY, M. S., LEBLANC, A. G., JANSSEN, I., KHO, M. E., HICKS, A., MURUMETS, K., COLlEY, R. C. \& DUGGAN, M. 2011a. Canadian sedentary behaviour guidelines for children and youth. Applied Physiology, Nutrition, and Metabolism, 36, 59-64.

TREMBLAY, M. S., LEBLANC, A. G., KHO, M. E., SAUNDERS, T. J., LAROUCHE, R., COLLEY, R. C., GOLDFIELD, G. \& CONNOR GORBER, S. 2011b. Systematic review of sedentary behaviour and health indicators in school-aged children and youth. Int J Behav Nutr Phys Act, 8, 98.

TRIONA, L. M. \& WILLIAMS, C. Point and click or build by hand: comparing the effects of physical vs. virtual materials on middle school students' ability to optimize an engineering design. In Proc. of CogSci2005, 2005. Citeseer.

TROCHIM, W. M. \& DONNELLY, J. P. 2001. Research methods knowledge base, Atomic Dog Publishing Cincinnati, $\mathrm{OH}$.

TROOSTERS, T., GOSSELINK, R. \& DECRAMER, M. 1999. Six minute walking distance in healthy elderly subjects. Eur Respir J, 14, 270-4.

TROST, S. G. 2006. Public health and physical education.

TROST, S. G. 2007. State of the art reviews: measurement of physical activity in children and adolescents. American Journal of Lifestyle Medicine, 1, 299-314.

TROST, S. G., BLAIR, S. N. \& KHAN, K. M. 2014a. Physical inactivity remains the greatest public health problem of the 21 st century: evidence, improved methods and solutions using the ' 7 investments that work' as a framework. $\mathrm{Br}$ J Sports Med, 48, 169-70.

TROST, S. G., LOPRINZI, P. D., MOORE, R. \& PFEIFFER, K. A. 2011. Comparison of accelerometer cut points for predicting activity intensity in youth. Med Sci Sports Exerc, 43, 1360-8.

TROST, S. G., MORGAN, A. M., SAUNDERS, R., FELTON, G., WARD, D. S. \& PATE, R. R. 2000a. Children's Understanding of the Concept of Physical Activity. Pediatric Exercise Science, 12, 293-299. 
TROST, S. G., OWEN, N., BAUMAN, A. E., SALLIS, J. F. \& BROWN, W. 2002a. Correlates of adults' participation in physical activity: review and update. Medicine \& science in sports \& exercise, 34, 1996-2001.

TROST, S. G., PATE, R. R., FREEDSON, P. S., SALLIS, J. F. \& TAYLOR, W. C. 2000b. Using objective physical activity measures with youth: how many days of monitoring are needed? Medicine \& Science in Sports \& Exercise, 32, 426.

TROST, S. G., SAUNDERS, R. \& WARD, D. S. 2002b. Determinants of physical activity in middle school children. Am J Health Behav, 26, 95-102.

TROST, S. G., ZHENG, Y. \& WONG, W.-K. 2014b. Machine learning for activity recognition: hip versus wrist data. Physiological measurement, 35, 2183.

TROUT, A. L., NORDNESS, P. D., PIERCE, C. D. \& EPSTEIN, M. H. 2003. Research on the academic status of children with emotional and behavioral disorders: A review of the literature from 1961 to 2000. Journal of Emotional and Behavioral Disorders, 11, 198-210.

TSAI, H. J., TSAI, A. C., NRIAGU, J., GHOSH, D., GONG, M. \& SANDRETTO, A. 2007. Associations of BMI, TV-watching time, and physical activity on respiratory symptoms and asthma in 5th grade schoolchildren in Taipei, Taiwan. J Asthma, 44, 397-401.

TUDOR-LOCKE, C., BARREIRA, T. V. \& SCHUNA, J. J. 2015. Comparison of step outputs for waist and wrist accelerometer attachment sites. Medicine and science in sports and exercise, 47, 839-842.

TUDOR-LOCKE, C. \& BASSETT, D. R. 2004. How many steps/day are enough? Sports medicine, 34, 1-8.

TUKEY, J. W. 1977. Exploratory data analysis, Addison-Wesely.

ULLMER, B. \& ISHII, H. 2000. Emerging frameworks for tangible user interfaces. IBM Syst. J., 39, 915-931.

UNDHEIM, J. O. \& NORDVIK, H. 1992. Socio-economic Factors and Sex Differences in an Egalitarian Educational System: academic achievement in 16-year-old Norwegian students. Scandinavian Journal of Educational Research, 36, 87-98.

UNICEF The State of the World's Children 2017-Children in a Digital World, December 2017, 14.

UPHILL, M. A. 2014. Behaviour change: Physical (in)activity. The British Psychological Society.

UTTAL, D. H. \& DOHERTY, K. O. 2008. Comprehending and Learning from 'Visualizations': A Developmental Perspective. In: GILBERT, J. K., REINER, M. \& NAKHLEH, M. (eds.) Visualization: Theory and Practice in Science Education. Dordrecht: Springer Netherlands.

VAN CAUWENBERGHE, V., LABARQUE, V., TROST, S. G., DE BOURDEAUDHUIJ, I. \& CARDON, G. 2011. Calibration and comparison of accelerometer cut points in preschool children. International Journal of Pediatric Obesity, 6, e582-589.

VAN DER STEEN, S., STEENBEEK, H., WIELINSKI, J. \& VAN GEERT, P. 2012. A Comparison between Young Students with and without Special Needs on Their Understanding of Scientific Concepts. Education Research International, 2012, 12.

VAN EKRIS, E., ALTENBURG, T., SINGH, A., PROPER, K., HEYMANS, M. \& CHINAPAW, M. 2016. An evidence-update on the prospective relationship between childhood sedentary behaviour and biomedical health indicators: a systematic review and meta-analysis. Obesity reviews, 17, 833-849. 
VAN GEMERT-PIJNEN, J. E., NIJLAND, N., VAN LIMBURG, M., OSSEBAARD, H. C., KELDERS, S. M., EYSENBACH, G. \& SEYDEL, E. R. 2011. A holistic framework to improve the uptake and impact of eHealth technologies. Journal of medical Internet research, 13.

VAN GILS, F. Potential applications of digital storytelling in education. 3rd twente student conference on IT, 2005. University of Twente, Faculty of Electrical Engineering, Mathematics and Computer Science Enschede.

VAN HOYE, K., BOEN, F. \& LEFEVRE, J. 2012. The effects of physical activity feedback on behavior and awareness in employees: study protocol for a randomized controlled trial. Int J Telemed Appl, 2012, 460712.

VAN REMOORTEL, H., GIAVEDONI, S., RASTE, Y., BURTIN, C., LOUVARIS, Z., GIMENO-SANTOS, E., LANGER, D., GLENDENNING, A., HOPKINSON, N. S., VOGIATZIS, I., PETERSON, B. T., WILSON, F., MANN, B., RABINOVICH, R., PUHAN, M. A. \& TROOSTERS, T. 2012. Validity of activity monitors in health and chronic disease: a systematic review. Int J Behav Nutr Phys Act, 9, 84.

VAN SLUIJS, E. M., GRIFFIN, S. J. \& VAN POPPEL, M. N. 2007. A cross-sectional study of awareness of physical activity: associations with personal, behavioral and psychosocial factors. Int J Behav Nutr Phys Act, 4, 53.

VAN SLUIJS, E. M., SKIDMORE, P. M., MWANZA, K., JONES, A. P., CALLAGHAN, A. M., EKELUND, U., HARRISON, F., HARVEY, I., PANTER, J., WAREHAM, N. J., CASSIDY, A. \& GRIFFIN, S. J. 2008. Physical activity and dietary behaviour in a population-based sample of British 10-year old children: the SPEEDY study (Sport, Physical activity and Eating behaviour: environmental Determinants in Young people). BMC Public Health, 8, 388.

VAN WIJK, J. 2005. The value of visualization.IEEE Visualisation, pp. 79-86., In Proc.

VANDONI, M., BUZZACHERA, C. F., OTTOBRINI, S., CORREALE, L., BORRELli, P., BERZOLARI, F. G. \& CODRONS, E. 2017. Perceived and objectively measured physical activity in high school students: is there any link between aerobic fitness, psychological responses and acute exercise? Sport Sciences for Health, 13, 157-164.

VANHELST, J., BÉGHIN, L., DRUMEZ, E., COOPMAN, S. \& GOTTRAND, F. 2017. Awareness of wearing an accelerometer does not affect physical activity in youth. BMC Medical Research Methodology, 17, 99.

VASSILEV, I., ROWSELL, A., POPE, C., KENNEDY, A., O'CATHAIN, A., SALISBURY, C. \& ROGERS, A. 2015. Assessing the implementability of telehealth interventions for self-management support: a realist review. Implementation Science, 10, 59.

VELOSO-GUEDES, C. A., ROSALEN, S. T., THOBIAS, C. M., ANDREOTTI, R. M., GALHARDO, F. D. M., OLIVEIRA DA SILVA, A. M., ARAUJO, O. \& BOIN, I. F. S. F. 2011. Validation of 20-Meter Corridor for the 6-Minute Walk Test in Men on Liver Transplantation Waiting List. Transplantation Proceedings, 43, 1322-1324.

VISEU, A. \& SUCHMAN, L. 2010. Wearable augmentations: Imaginaries of the informed body. Technologized images, technologized bodies, 161-184.

VOGLER, A. J., RICE, A. J. \& GORE, C. J. 2010. Validity and reliability of the Cortex MetaMax3B portable metabolic system. Journal of sports sciences, 28, 733-742. 
VYAS, A. N., LANDRY, M., SCHNIDER, M., ROJAS, A. M. \& WOOD, S. F. 2012. Public health interventions: reaching Latino adolescents via short message service and social media. J Med Internet Res, 14, e99.

WALSBERG, G. E. \& HOFFMAN, T. C. 2005. Direct calorimetry reveals large errors in respirometric estimates of energy expenditure. Journal of Experimental Biology, 208, 1035-1043.

WALSH, J. C., CORBETT, T., HOGAN, M., DUGGAN, J. \& MCNAMARA, A. 2016. An mHealth intervention using a smartphone app to increase walking behavior in young adults: a pilot study. JMIR mHealth and uHealth, 4.

WALTHER, J. B., VAN DER HEIDE, B., KIM, S.-Y., WESTERMAN, D. \& TONG, S. T. 2008. The role of friends' appearance and behavior on evaluations of individuals on Facebook: Are we known by the company we keep? Human communication research, 34, 28-49.

WALTON, J., HOERR, S., HEINE, L., FROST, S., ROISEN, D. \& BERKIMER, M. 1999. Physical activity and stages of change in fifth and sixth graders. Journal of school health, 69, 285-289.

WARBURTON, D. E., GLENDHILL, N. \& QUINNEY, A. 2001. The effects of changes in musculoskeletal fitness on health. Can J Appl Physiol, 26, 161-216.

WARBURTON, D. E. R. \& BREDIN, S. S. D. 2017. Health benefits of physical activity: a systematic review of current systematic reviews. Curr Opin Cardiol, 32, 541-556.

WARD, D. S., EVENSON, K. R., VAUGHN, A., RODGERS, A. B. \& TROIANO, R. P. 2005. Accelerometer use in physical activity: best practices and research recommendations. Med Sci Sports Exerc, 37, S582-8.

WARD, M. M., VAUGHN, T. E., UDEN-HOLMAN, T., DOEBBELING, B. N., CLARKE, W. R. \& WOOLSON, R. F. 2002. Physician knowledge, attitudes and practices regarding a widely implemented guideline. J Eval Clin Pract, 8, 155-62.

WARREN, J., HENRY, C., LIGHTOWLER, H., BRADSHAW, S. \& PERWAIZ, S. 2003. Evaluation of a pilot school programme aimed at the prevention of obesity in children. Health promotion international, 18, 287-296.

WARTELlA, E., RIDEOUT, V., MONTAGUE, H., BEAUDOIN-RYAN, L. \& LAURICELLA, A. 2016. Teens, Health and Technology: A National Survey.

WATERS, R. L. \& MULROY, S. 1999. The energy expenditure of normal and pathologic gait. Gait Posture, 9, 207-31.

WEBB, T. L., JOSEPH, J., YARDLEY, L. \& MICHIE, S. 2010. Using the internet to promote health behavior change: a systematic review and meta-analysis of the impact of theoretical basis, use of behavior change techniques, and mode of delivery on efficacy. J Med Internet Res, 12, e4.

WEINBERG, R. \& WEIGAND, D. 1993. Goal Setting in Sport and Exercise: A Reaction to Locke. Journal of Sport and Exercise Psychology, 15, 88-96.

WEINSTEIN, N. D. 1988. The precaution adoption process. Health Psychol, 7, 35586.

WEIPPERT, M., STIELOW, J., KUMAR, M., KREUZFELD, S., RIEGER, A. \& STOLL, R. 2013. Tri-axial high-resolution acceleration for oxygen uptake estimation: Validation of a multi-sensor device and a novel analysis method. Appl Physiol Nutr Metab, 38, 345-51.

WELK, G. J. 2005. Principles of design and analyses for the calibration of accelerometry-based activity monitors. Medicine and science in sports and exercise, 37, S501-11. 
WELK, G. J. \& CORBIN, C. B. 1995. The validity of the Tritrac-R3D activity monitor for the assessment of physical activity in children. Research Quarterly for Exercise and Sport, 66, 202-209.

WELK, G. J., CORBIN, C. B. \& DALE, D. 2000. Measurement issues in the assessment of physical activity in children. Res Q Exerc Sport, 71, S59-73.

WELLS, S. L., KIPPING, R. R., JAGO, R., BROWN, J., HUCKER, D., BLACKETT, A. \& LAWLOR, D. A. 2013. Characteristics associated with requested and required accelerometer wear in children. BMJ open, 3, e003402.

WELSMAN, J. R. \& ARMSTRONG, N. 2000. Statistical techniques for interpreting body size-related exercise performance during growth. Pediatric Exercise Science, 12, 112-127.

WESTERSTAHL, M., BARNEKOW-BERGKVIST, M. \& JANSSON, E. 2005. Low physical activity among adolescents in practical education. Scand J Med Sci Sports, 15, 287-97.

WESTERTERP, K. R. 2004. Diet induced thermogenesis. Nutrition \& Metabolism, 1, 5.

WESTERTERP, K. R. 2009. Assessment of physical activity: a critical appraisal. Eur J Appl Physiol, 105, 823-8.

WESTERTERP, K. R., WILSON, S. A. \& ROLLAND, V. 1999. Diet induced thermogenesis measured over $24 \mathrm{~h}$ in a respiration chamber: effect of diet composition. Int J Obes Relat Metab Disord, 23, 287-92.

WESTON, K. L., AZEVEDO, L. B., BOCK, S., WESTON, M., GEORGE, K. P. \& BATTERHAM, A. M. 2016. Effect of Novel, School-Based High-Intensity Interval Training (HIT) on Cardiometabolic Health in Adolescents: Project FFAB (Fun Fast Activity Blasts) - An Exploratory Controlled Before-AndAfter Trial. PLoS One, 11, e0159116.

WETHINGTON, H., PAN, L. \& SHERRY, B. 2013. The association of screen time, television in the bedroom, and obesity among school-aged youth: 2007 National Survey of Children's Health. J Sch Health, 83, 573-81.

WHITTEMORE, R., JASER, S. S., JEON, S., LIBERTI, L., DELAMATER, A., MURPHY, K., FAULKNER, M. S. \& GREY, M. 2012. An internet coping skills training program for youth with type 1 diabetes: six-month outcomes. Nurs Res, 61, 395-404.

WILLIAMS, H. J., HOLTON, M. D., SHEPARD, E. L. C., LARGEY, N., NORMAN, B., RYAN, P. G., DURIEZ, O., SCANTLEBURY, M., QUINTANA, F., MAGOWAN, E. A., MARKS, N. J., ALAGAILI, A. N., BENNETT, N. C. \& WILSON, R. P. 2017a. Identification of animal movement patterns using triaxial magnetometry. Movement Ecology, 5, 6.

WILLIAMS, H. J., HOLTON, M. D., SHEPARD, E. L. C., LARGEY, N., NORMAN, B., RYAN, P. G., DURIEZ, O., SCANTLEBURY, M., QUINTANA, F., MAGOWAN, E. A., MARKS, N. J., ALAGAILI, A. N., BENNETT, N. C. \& WILSON, R. P. 2017b. Identification of animal movement patterns using triaxial magnetometry. Movement Ecology, 5, 6 .

WILLIAMS, S. \& FRENCH, D. 2011. What are the most effective intervention techniques for changing physical activity self-efficacy and physical activity behaviour-and are they the same? Health education research, 26, 308-322.

WILLIS, W. T., GANLEY, K. J. \& HERMAN, R. M. 2005. Fuel oxidation during human walking. Metabolism, 54, 793-9. 
WILSON, R. P., GRIFFITHS, I. W., LEGG, P. A., FRISWELL, M. I., BIDDER, O. R., HALSEY, L. G., LAMBERTUCCI, S. A. \& SHEPARD, E. L. 2013. Turn costs change the value of animal search paths. Ecol Lett, 16, 1145-50.

WILSON, R. P., SHEPARD, E. \& LIEBSCH, N. 2008. Prying into the intimate details of animal lives: use of a daily diary on animals. Endangered Species Research, 4, 123-137.

WILSON, R. P., WHITE, C. R., QUINTANA, F., HALSEY, L. G., LIEBSCH, N., MARTIN, G. R. \& BUTLER, P. J. 2006. Moving towards acceleration for estimates of activity-specific metabolic rate in free-living animals: the case of the cormorant. J Anim Ecol, 75, 1081-90.

WINN, C. O. N., MACKINTOSH, K. A., EDDOLLS, W. T. B., STRATTON, G., WILSON, A. M., RANCE, J. Y., DOULL, I. J. M., MCNARRY, M. A. \& DAVIES, G. A. 2017. Perceptions of asthma and exercise in adolescents with and without asthma. J Asthma, 1-9.

WINTER, D. A., PATLA, A. E. \& FRANK, J. S. 1990. Assessment of balance control in humans. Med Prog Technol, 16, 31-51.

WITTMEIER, K. D., MOLLARD, R. C. \& KRIELlAARS, D. J. 2008. Physical activity intensity and risk of overweight and adiposity in children. Obesity, 16, 415-420.

WOJCICKI, T. R., GRIGSBY-TOUSSAINT, D., HILLMAN, C. H., HUHMAN, M. \& MCAULEY, E. 2014. Promoting Physical Activity in Low-Active Adolescents via Facebook: A Pilot Randomized Controlled Trial to Test Feasibility. JMIR Res Protoc, 3, e56.

WOLIN, K. Y., HEIL, D. P., ASKEW, S., MATTHEWS, C. E. \& BENNETT, G. G. 2008. Validation of the international physical activity questionnaire-short among blacks. Journal of Physical Activity and Health, 5, 746-760.

WOLIN, K. Y., YAN, Y., COLDITZ, G. A. \& LEE, I. 2009. Physical activity and colon cancer prevention: a meta-analysis. British journal of cancer, 100, 611.

WOODWARD, J., MCFADDEN, Z., SHIVER, N., BEN-HAYON, A., YIP, J. C. \& ANTHONY, L. Using Co-Design to Examine How Children Conceptualize Intelligent Interfaces. Proceedings of the 2018 CHI Conference on Human Factors in Computing Systems, 2018. ACM, 575.

WORLD HEALTH ORGANISATION. 2011. Global Recomendations on Physical Activity for Health [Online]. Available: http://www.who.int/dietphysicalactivity/leaflet-physical-activityrecommendations.pdf?ua $=1$

WORLD HEALTH ORGANISATION. 2017. Physical Activity [Online]. Available: http://www.who.int/mediacentre/factsheets/fs385/en/

WYSE, J., MERCER, T., ASHFORD, B., BUXTON, K. \& GLEESON, N. 1995. Evidence for the validity and utility of the Stages of Exercise Behaviour Change scale in young adults. Health Education Research, 10, 365-377.

WYSZYNSKA, J., PODGORSKA-BEDNARZ, J., DEREN, K. \& MAZUR, A. 2017. The Relationship between Physical Activity and Screen Time with the Risk of Hypertension in Children and Adolescents with Intellectual Disability. Biomed Res Int, 2017, 1940602.

XU, F., WANG, X., XIANG, D., WANG, Z., YE, Q. \& WARE, R. S. 2017. Awareness of knowledge and practice regarding physical activity: A population-based prospective, observational study among students in Nanjing, China. PLoS One, 12, $\mathrm{e} 0179518$. 
YEE, N. 2006. The psychology of massively multi-user online role-playing games: Motivations, emotional investment, relationships and problematic usage. Avatars at work and play. Springer.

YEE, N. \& BAILENSON, J. 2007. The Proteus effect: The effect of transformed selfrepresentation on behavior. Human communication research, 33, 271-290.

YI, J. S., AH KANG, Y. \& STASKO, J. 2007. Toward a deeper understanding of the role of interaction in information visualization. IEEE transactions on visualization and computer graphics, 13, 1224-1231.

YOU, M.-A. \& SON, Y.-J. 2012. Prevalence of Metabolic Syndrome and Associated Risk Factors Among Korean Adolescents:Analysis From the Korean National Survey. Asia Pacific Journal of Public Health, 24, 464-471.

ZADRO, I., SEPULCRI, L., LAZZER, S., FREGOLENT, R. \& ZAMPARO, P. 2011. A protocol of intermittent exercise (shuttle runs) to train young basketball players. J Strength Cond Res, 25, 1767-73.

ZARRUGH, M. Y., N TODD, F. \& J RALSTON, H. 1974. Optimization of energy expenditure during level walking.

ZHANG, G., WU, L., ZHOU, L., LU, W. \& MAO, C. 2015. Television watching and risk of childhood obesity: a meta-analysis. The European Journal of Public Health, 26, 13-18.

ZIEVE, G. G., RICHARDSON, L. P., KATZMAN, K., SPIELVOGLE, H., WHITEHOUSE, S. \& MCCARTY, C. A. 2017. Adolescents' Perspectives on Personalized E-Feedback in the Context of Health Risk Behavior Screening for Primary Care: Qualitative Study. Journal of medical Internet research, 19.

ZIMMERMAN, E. \& WOOLF, S. H. 2014. Understanding the relationship between education and health, Institute of Medicine of the National Academies.

ZUCKERMAN, O., ARIDA, S. \& RESNICK, M. 2005. Extending tangible interfaces for education: digital montessori-inspired manipulatives. Proceedings of the SIGCHI Conference on Human Factors in Computing Systems. Portland, Oregon, USA: ACM. 


\section{APPENDICES}

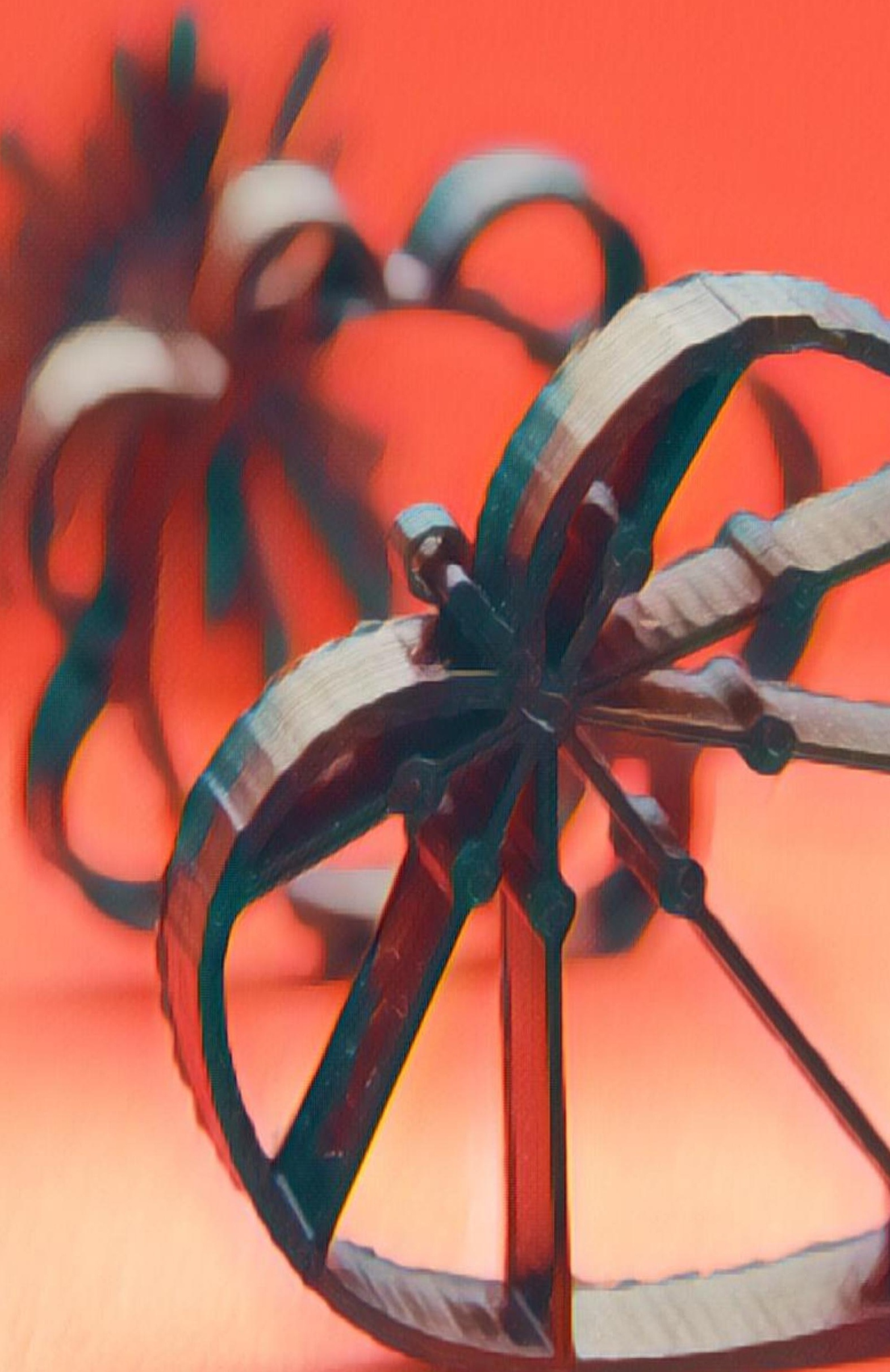




\section{Appendices}

Appendix I: Study 1 - Mixed Models

Absolute and Scaled $\dot{V} \mathrm{O}_{2}$ Models

\begin{tabular}{|c|c|c|c|c|c|c|}
\hline & \multicolumn{3}{|c|}{ Absolute $\dot{V} \mathbf{O}_{2}\left(1 \cdot \mathbf{m i n}^{-1}\right)$} & \multicolumn{3}{|c|}{ Scaled $\dot{V} \mathbf{O}_{2}\left(\mathbf{l} \cdot \mathrm{kg}^{-0.79} \cdot \mathrm{min}^{-1}\right)$} \\
\hline & Estimate ( $\beta$ ) & Std.Error & Sig. & Estimate ( $\beta)$ & Std.Error & Sig. \\
\hline Parameter & \multicolumn{6}{|c|}{ Speed } \\
\hline $5.5 \mathrm{~km} \cdot \mathrm{hr}-1$ & 0.23 & 0.03 & $0.00 * *$ & 13.87 & 1.95 & $0.00^{* *}$ \\
\hline $4.5 \mathrm{~km} \cdot \mathrm{hr}-1$ & 0.14 & 0.02 & $0.00^{* *}$ & 8.78 & 1.48 & 0.00 ** \\
\hline $3.5 \mathrm{~km} \cdot \mathrm{hr}-1$ & 0.07 & 0.02 & $0.002^{*}$ & 4.06 & 1.19 & $0.00^{* *}$ \\
\hline $2.5 \mathrm{~km} \cdot \mathrm{hr}-1$ & 1.00 & - & - & 1.00 & - & - \\
\hline Parameter & \multicolumn{6}{|c|}{ Angle } \\
\hline $180^{\circ}$ & 0.02 & 0.02 & 0.38 & 1.26 & 1.39 & 0.37 \\
\hline $90^{\circ}$ & 0.03 & 0.02 & 0.10 & 2.68 & 1.17 & 0.03 \\
\hline $45^{\circ}$ & 0.10 & 0.02 & 0.62 & 0.89 & 1.20 & 0.46 \\
\hline $0^{\circ}$ & 1.00 & - & - & 1.00 & - & - \\
\hline Parameter & \multicolumn{6}{|c|}{ Speed $x$ Angle } \\
\hline Speed $5.5 \times$ Angle $180^{\circ}$ & 0.14 & 0.05 & $0.005^{*}$ & 8.84 & 2.89 & $0.003 *$ \\
\hline Speed $5.5 \times$ Angle $90^{\circ}$ & -0.32 & 0.04 & 0.48 & -1.95 & 2.65 & 0.46 \\
\hline Speed $5.5 \times$ Angle $45^{\circ}$ & -0.02 & 0.04 & 0.67 & -1.01 & 2.60 & 0.70 \\
\hline Speed $5.5 \times$ Angle $0^{\circ}$ & 1.00 & - & - & 1.00 & - & - \\
\hline Speed $4.5 \times$ Angle $180^{\circ}$ & 0.05 & 0.04 & 0.17 & 3.59 & 2.27 & 0.12 \\
\hline Speed $4.5 \times$ Angle $90^{\circ}$ & -0.03 & 0.03 & 0.40 & -2.11 & 2.06 & 0.31 \\
\hline Speed $4.5 \times$ Angle $45^{\circ}$ & -0.02 & 0.03 & 0.50 & -1.33 & 2.07 & 0.52 \\
\hline Speed $4.5 \times$ Angle $0^{\circ}$ & 1.00 & - & - & 1.00 & - & - \\
\hline Speed $3.5 \times$ Angle $180^{\circ}$ & 0.41 & 0.03 & 0.19 & 2.77 & 1.82 & 0.13 \\
\hline Speed 3.5 x Angle $90^{\circ}$ & -0.02 & 0.03 & 0.52 & -1.15 & 1.66 & 0.49 \\
\hline Speed $3.5 \times$ Angle $45^{\circ}$ & -0.02 & 0.03 & 0.57 & -1.58 & 1.73 & 0.36 \\
\hline Speed 3.5 x Angle $0^{\circ}$ & 1.00 & - & - & 1.00 & - & - \\
\hline Parameter & \multicolumn{6}{|c|}{ Sex } \\
\hline Girl & -0.02 & 0.01 & $0.013^{*}$ & -1.48 & 0.55 & $0.008^{*}$ \\
\hline Boy & 1.00 & - & - & 1.00 & - & - \\
\hline Parameter & \multicolumn{6}{|c|}{ Covariates } \\
\hline Stature & 0.00 & 0.00 & $0.00^{* *}$ & 0.08 & 0.04 & $0.04 *$ \\
\hline Peak $\dot{V} \mathrm{O}_{2}$ & 0.08 & 0.02 & $0.00^{*}$ & - & - & - \\
\hline Scaled $\dot{V} \mathrm{O}_{2 \text { peak }}$ & - & - & - & -0.01 & 0.01 & 0.32 \\
\hline
\end{tabular}

Reference values speed $2.5 \mathrm{~km} \cdot \mathrm{hr}^{-1}$ and angle set at $0^{\circ}$. ' $\mathrm{x}$ ' indicates interaction, '*' indicates significant effect at $(p<0.05)$, '**' indicates significant effect at $(p<0.001)$ 
Straight and Turn Mean VeDBA Models

\begin{tabular}{|c|c|c|c|c|c|c|}
\hline & \multicolumn{3}{|c|}{ Straight Mean VeDBA $(g)$} & \multicolumn{3}{|c|}{ Turn Mean VeDBA $(g)$} \\
\hline & Estimate ( $\beta$ ) & Std.Error & Sig. & Estimate ( $\beta$ ) & Std.Error & Sig. \\
\hline Parameter & \multicolumn{6}{|c|}{ Speed } \\
\hline $5.5 \mathrm{~km} \cdot \mathrm{hr}-1$ & 0.35 & 0.02 & $0.00 * *$ & 0.38 & 0.02 & $0.00 * *$ \\
\hline $4.5 \mathrm{~km} \cdot \mathrm{hr}-1$ & 0.18 & 0.02 & $0.00 * *$ & 0.19 & 0.01 & $0.00 * *$ \\
\hline $3.5 \mathrm{~km} \cdot \mathrm{hr}-1$ & 0.07 & 0.02 & $0.00^{* *}$ & 0.06 & 0.01 & $0.00 * *$ \\
\hline $2.5 \mathrm{~km} \cdot \mathrm{hr}-1$ & 1.00 & - & - & 1.00 & - & - \\
\hline Parameter & \multicolumn{6}{|c|}{ Angle } \\
\hline $180^{\circ}$ & 0.001 & 0.01 & 0.94 & 0.008 & 0.01 & 0.25 \\
\hline $90^{\circ}$ & -0.002 & 0.01 & 0.86 & 0.000 & 0.01 & 0.98 \\
\hline $45^{\circ}$ & -0.001 & 0.01 & 0.93 & -0.003 & 0.01 & 0.66 \\
\hline $\mathbf{0}^{\circ}$ & 1.00 & - & - & 1.00 & - & - \\
\hline Parameter & \multicolumn{6}{|c|}{ Speed x Angle } \\
\hline Speed 5.5 x Angle $180^{\circ}$ & 0.06 & 0.03 & $0.04 *$ & 0.01 & 0.03 & 0.60 \\
\hline Speed $5.5 \times$ Angle $90^{\circ}$ & 0.02 & 0.03 & 0.50 & -0.01 & 0.03 & 0.69 \\
\hline Speed 5.5 x Angle $45^{\circ}$ & 0.04 & 0.03 & 0.19 & -0.01 & 0.03 & 0.77 \\
\hline Speed 5.5 x Angle $0^{\circ}$ & 1.00 & - & - & 1.00 & - & - \\
\hline Speed $4.5 \times$ Angle $180^{\circ}$ & 0.04 & 0.02 & 0.09 & 0.03 & 0.02 & 0.19 \\
\hline Speed $4.5 \times$ Angle $90^{\circ}$ & 0.01 & 0.02 & 0.77 & -0.008 & 0.02 & 0.69 \\
\hline Speed $4.5 \times$ Angle $45^{\circ}$ & 0.02 & 0.02 & 0.42 & -0.002 & 0.02 & 0.92 \\
\hline Speed $4.5 \times$ Angle $0^{\circ}$ & 1.00 & - & - & 1.00 & - & - \\
\hline Speed $3.5 \times$ Angle $180^{\circ}$ & 0.01 & 0.02 & 0.51 & 0.03 & 0.01 & $0.02 *$ \\
\hline Speed $3.5 \times$ Angle $90^{\circ}$ & 0.00 & 0.02 & 0.91 & 0.01 & 0.01 & 0.36 \\
\hline Speed 3.5 x Angle $45^{\circ}$ & 0.01 & 0.02 & 0.59 & 0.01 & 0.01 & 0.31 \\
\hline Speed $3.5 \times$ Angle $0^{\circ}$ & 1.00 & - & - & 1.00 & - & - \\
\hline Parameter & & & & & & \\
\hline Girl & 0.003 & 0.01 & 0.53 & 0.02 & 0.00 & $0.00 * *$ \\
\hline Boy & 1.00 & - & - & 1.00 & - & - \\
\hline Parameter & & & & iates & & \\
\hline Peak $\dot{V} \mathbf{O}_{2}$ & -0.01 & 0.01 & 0.11 & - & - & - \\
\hline Scaled $\dot{V} \mathbf{O}_{2 \text { peak }}$ & - & - & - & 0.0002 & 0.0001 & 0.10 \\
\hline
\end{tabular}

Reference values speed $2.5 \mathrm{~km} \cdot \mathrm{hr}^{-1}$ and angle set at $0^{\circ}$. ' $\mathrm{x}$ ' indicates interaction, ' ${ }^{*}$ ' indicates significant effect at $(p<0.05)$, '**' indicates significant effect at $(p<0.001)$ 


\title{
Appendix II: Information Sheets and Consent/Assent Forms
}

\author{
Study 1 - Parent Information Sheet
}

\author{
Applied Sports Technology Exercise and Medicine Research \\ Centre (A-STEM) \\ Sport and Health Portfolio, College of Engineering
}

PARENTAL INFORMATION SHEET

(Version 1.1, Date: 01/10/2014)

Project Title:

Influence of walking speed on the energetic cost of turning in children aged 10-12 years

Contact Details:

Melitta McNarry

Sam Crossley

Swansea University

Swansea University

Ph:

E-mail:

Ph: 0

E-mail:

\section{Invitation Paragraph}

Thank you for taking the time to read this information sheet, it will provide you with the details of our study and hopefully provide you with the information you require to help you decide if you want your child to participate. It is important to say at this point that the decision to take part is entirely up to you and that your child will not be at a disadvantage for future studies should you decide for them not to participate.

\section{What is the purpose of the study?}

Recent studies now suggest that there are significant impacts of turning on the overall energetic costs of movement. The purpose of the study is to find the energetic costs of turning at different speeds and angles within young children.

\section{Why has my Child been chosen??}

Your child has been asked to volunteer because they are a healthy individual of 10-12 years of age, free from injury or illness. We regret to say that those with known cardiovascular disease are not able to volunteer for this study due to the risks associated with exercise for these children. 


\section{What will happen to my child if they take part?}

Your child will have to complete a self-reported Tanner Stages form. The Tanner Stage form is an assessment for level of pubertal maturation. The reason this form is done is because different levels of maturation can alter the physical output of a child. Researchers can then understand the possible differences between children might be of influence from maturation level. Your child will complete the form in private and then seal it into an envelope. The envelope will have an assigned number that corresponds to your child's identity. Only the researchers will have access to the forms collected.

Your child will be asked to visit the lab on three occasions. On the first visit, we will measure your child's height, weight and sitting height. After this, they will practice walking and running on the treadmill (see picture below) until they are happy and relaxed with it. We will then start the actual exercise, which will start off easy and get harder and harder but will be stop when your child says they can't keep going. During this, they will be breathing into a mask to allow us to measure the air that they breathe in and out. This mask does not make breathing any harder and you can talk through it and remove it at any time they feel uncomfortable about wearing it. They will also have two small "cards" attached around their waist (see picture below). This will measure how much exercise they are doing. The exercise will last approximately 15 minutes.

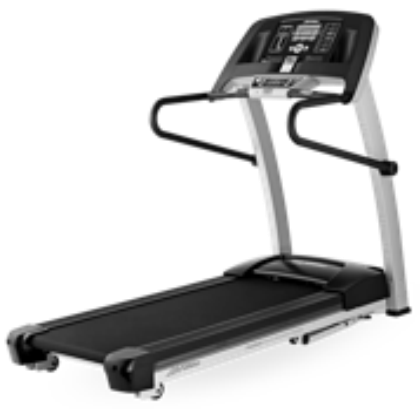

Treadmill

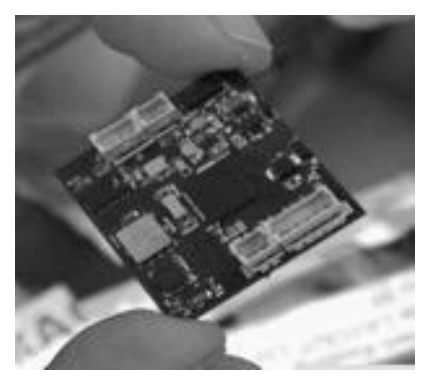

SLAM Tracker

On the second and third visit, they will repeat the turning protocol. This will involve walking in a straight line for $5 \mathrm{~m}$ then undertaking a turn at a prescribed angle and continuing to walk. The walking speeds will be $2.5,3.5,4.5,5.5 \mathrm{kph}$, in a random order. The angle of turn will be $0^{\circ}, 45^{\circ}, 90^{\circ}$ or $180^{\circ}$. Each combination of speeds and angles will be completed for 3 minutes followed by a 3-minute rest. After the 3-minute rest your child will then repeat the same protocol but with a different speed and turning angle. Total testing time should take around 1 hour 45 minutes. This test will be completed twice.

\section{What are the possible disadvantages of taking part?}

The acute risks associated with exercise are very small and will be further minimised by the health screening they will complete prior to undertaking exercise. They will not be 
disadvantaged in any way by choosing to stop participating and will not be forced to continue if you do not want to.

\section{What are the possible benefits of taking part?}

You will learn how fit your child is and about novel devices currently being developed by the College of Engineering.

\section{Will my child's taking part in the study be kept confidential?}

All information collected about your child will be kept strictly confidential. Any information that is distributed among the research team will only be identifiable by number and not name.

\section{What if I have any questions?}

If you have any questions about the study, either now or in the future, please do not hesitate to contact Melitta or Sam on the details provided at the top of this document. 


\section{CHILD INFORMATION SHEET}

(Version 1.1, Date: 01/10/2014)

\section{Project Title:}

Influence of walking speed on the energetic cost of turning in children aged 10-12 years

\section{Contact Details:}

Melitta McNarry

Swansea University

$\mathrm{Ph}$ :

E-mail:
Sam Crossley

Swansea University

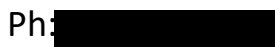

E-mail:

\section{Invitation Paragraph}

Thank you very much for agreeing to take part. This information sheet will provide you with all the information about the different tests you are going to do. If you want to stop taking part in this testing at anytime just tell one of the researchers.

\section{What is the purpose of the study?}

The purpose of this study is to find the amount of energy it takes to simply turn whilst walking at different speeds.

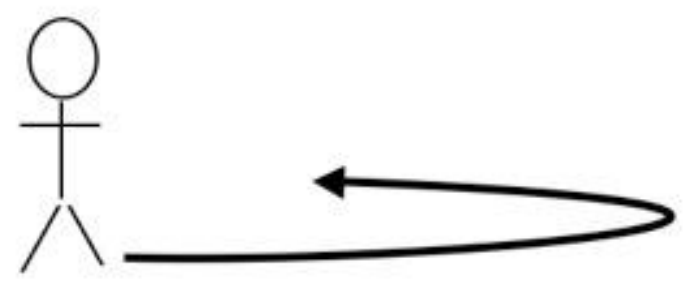

\section{Why have I been chosen??}

You have been asked to volunteer because you are a healthy individual of 10-12 years of age, free from injury or illness. Those children with illness or injury are not able to volunteer for this study.

\section{What will happen to me if I take part?}

You will first be asked to complete a special form called the Tanner stage form. This form will have pictures of male or female private parts. You must simply tick a box to which picture 
looks like you. You will complete this form away from other people. When you have completed the form, you will then put it into an envelope and seal it. Only members of the research team will be allowed to look at the forms.

You will be asked to visit the lab on three occasions. On the first visit, we will measure your height, weight and sitting height.
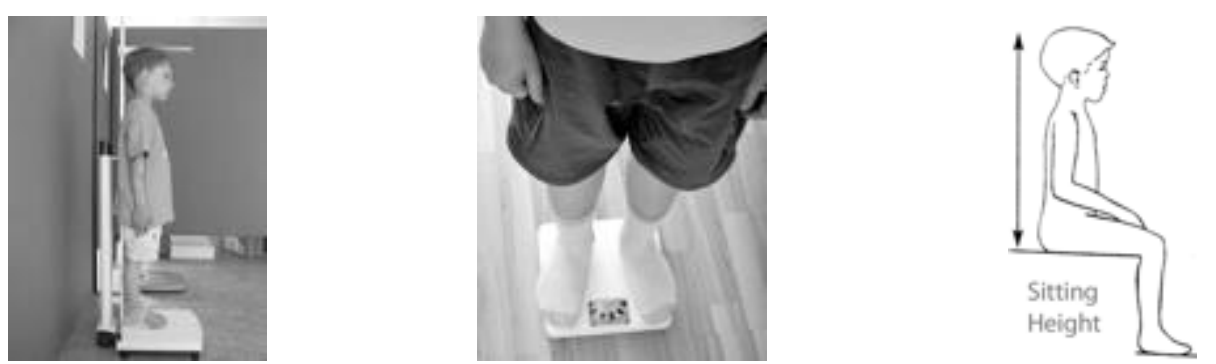

After this, you will practice walking and running on the treadmill (see picture below) until you are happy with it.

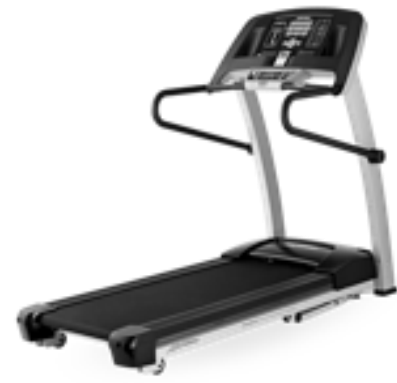

Treadmill

We will then start the exercise test, which will start off easy and get harder and harder but will stop when you decide you are done. During this exercise, you will be breathing into a mask (see picture below) to allow us to measure the air that you breathe in and out. This mask does not make breathing any harder and you can talk through it and remove it at any time you feel uncomfortable. The exercise will last approximately 15 minutes.
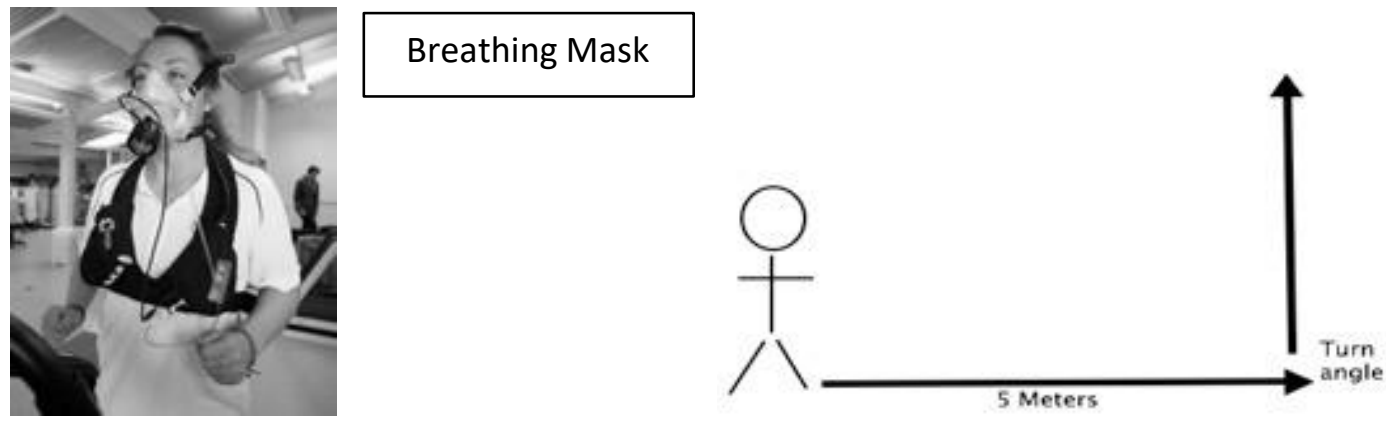

On the second and third visits, you will do a new test. This will involve walking in a straight line for 5 meters and then turning at a particular angle and continuing to walk (see picture above). 
You will be told which speed you have to walk $(2.5,3.5,4.5$ or $5.5 \mathrm{kph})$ and which angle you need to turn $\left(0^{\circ}, 45^{\circ}, 90^{\circ}\right.$ or $\left.180^{\circ}\right)$ but none of the speeds will be hard or involve running. Turn angles will be marked out on the floor. The test will take around 1 hour 45 minutes. During these tests you will have an Actisleep monitor and two small SLAM "cards" attached around your waist (see pictures below). This will measure how much exercise you are doing. This test will be completed twice.
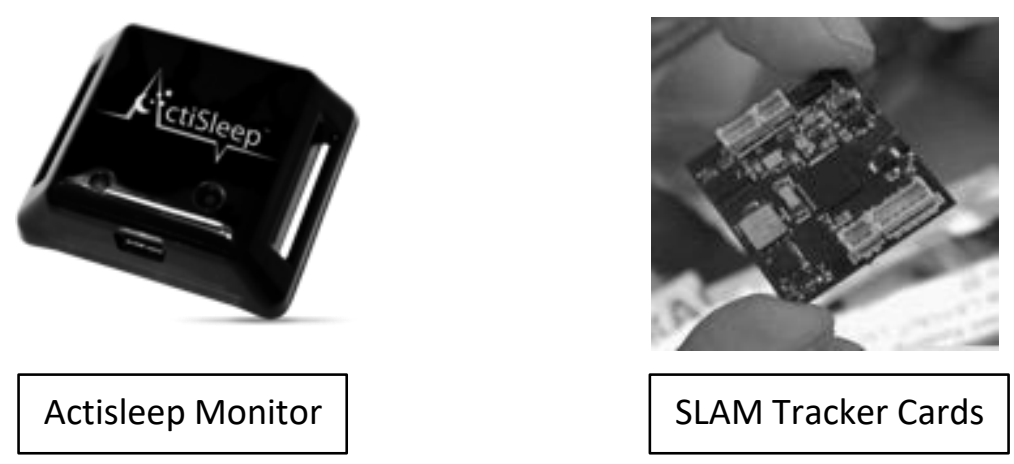

\section{What are the possible disadvantages of taking part?}

The risks associated with exercise are very small. If you want to stop at any time you can, and you will not be forced to continue if you do not want to.

\section{What are the possible benefits of taking part?}

You will learn how fit you are along with accurate measurements for height, weight and sitting height. The study will also give you an insight into sport and medical testing.

\section{Will my taking part in the study be kept confidential?}

All information collected about you will be kept strictly confidential. Any information that is distributed among the research team will identify you by number and not name.

\section{What if I have any questions?}

If you have any questions about the study, either now or in the future, please contact Melitta or Sam using the details provided at the top of this document. 
Studies 2 \& 3 - Parent Information Sheet

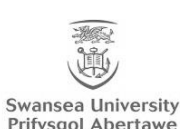

Applied Sports Technology Exercise and Medicine Research

Centre (A-STEM)

Sport and Health Portfolio, College of Engineering

\section{PARENTAL INFORMATION SHEET - PILOT STUDY}

(Version 1.1, Date: 01/10/2015)

\section{Project Title:}

Physical Activity across the Globe: The G-Sphere

\section{Contact Details:}

Sam Crossley

Phone:

Email:

Dr Melitta McNarry

Dr Kelly Mackintosh

\section{Invitation Paragraph}

Thank you for taking the time to read this information sheet, it will provide you with the details of our pilot study and help you decide if you want your child to participate. It is important to say at this point that the decision to take part is entirely up to you and that your child will not be at a disadvantage for future studies should you decide for them not to participate.

\section{What is the purpose of the study?}

This study is a 'pilot study', this means its findings and feedback will be used to design the main 'intervention study' planned for the future. The purpose of this study is to gather ideas, interpretations and feedback from the children, parents and teachers on the concept of 3D printing physical activity. In more detail, physical activity means any bodily movement from walking to running but does not include a sitting of lying position where the body is still. It is becoming increasingly important to find new ways of interpreting physical activity and especially for children. 3D-printed physical activity is a very new concept and is becoming increasingly popular in physical activity research. The intended outcome of printing physical activity for children is that it will be fun and produce more meaningful way of looking at data rather than graphs and numbers. This method will teach children about different intensities 
of physical activity and the problems associated with sedentary behaviours (being inactive e.g. watching tv/video games).

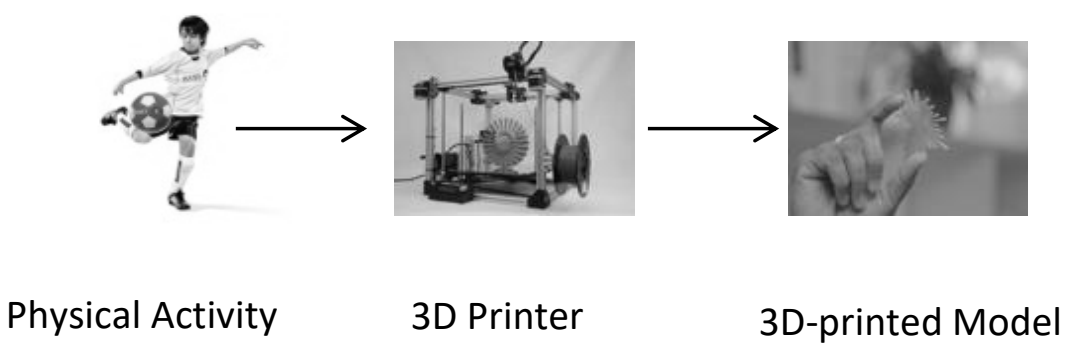

\section{Why has my Child been chosen??}

Your child has been asked to volunteer because they are of the targeted age (7-14 years) and are free from illness or injury that could affect normal movement patterns for the study design.

\section{What will happen to my child if they take part?}

As well as your child, both parents and teachers will help provide vital feedback and ideas for the design of the main intervention study. Short interviews will be conducted to find out: (i) What your child understands about keeping active and how this also relates to being less active (sedentary) (ii) to receive feedback and advice on the planned 3D-printed shapes for physical activity, (iii) provide feedback on young people's experience to the intervention plan. Finally, to enhance the methods and procedure to be used in the main intervention. Interviews will last up to 20 minutes.

\section{What are the possible disadvantages of taking part?}

It will require taking your child out of school time to complete the short interview. It will also require the participating parent to give up some time to provide feedback and thoughts on the concept.

\section{What are the possible benefits of taking part?}

Your child will be involved in designing a real science intervention. Giving vital thoughts and feedback on potential 3D-printed models that could dramatically change the way we look at physical activity.

\section{Will my child's taking part in the study be kept confidential?}

All information collected about your child will be kept strictly confidential. Any information that is distributed among the research team will only be identifiable by number and not name.

\section{What if I have any questions?}

If you have any questions about the study, either now or in the future, please do not hesitate to contact Melitta or Sam on the details provided at the top of this document. 


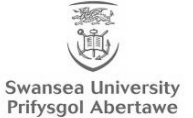
Swansea University
Prifysgol Abertawe

\author{
Applied Sports Technology Exercise and Medicine Research \\ Centre (A-STEM) \\ Sport and Health Portfolio, College of Engineering
}

\section{CHILD INFORMATION SHEET - PILOT STUDY}

(Version 1.1, Date: 01/10/2015)

\section{Project Title:}

Physical Activity across the Globe: The G-Sphere

\section{Contact Details:}

Sam Crossley

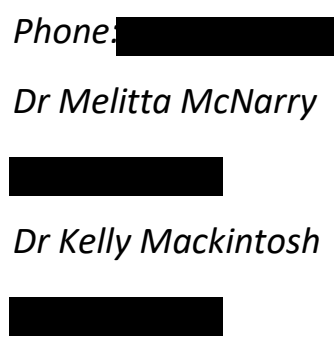

Email:

Dr Melitta McNarry

\section{Invitation Paragraph}

Thank you very much for agreeing to take part. This information sheet will provide you with all the information on what you will have to do. If you want to stop taking part in this testing at any time just tell one of the researchers.

\section{What is the purpose of the study?}

This study is called a 'pilot study', which is done to create new ideas and feedback on a certain topic. The topic in this pilot study is 3D printing your own movements. This means measuring your own movements such as walking to school or running around in the playground and then 3D printing these movements into your own model. Your movements will be measured using a special device during a day at school. Once the device has finished measuring it will be connected up to the 3D printer, which will then print your very own physical activity model (see picture below). But before we start printing your physical activity, we want to get some ideas from you about the best shapes and ways of measuring this. So, the purpose of this 'pilot' study is to help design the main study that will be completed in the future. 


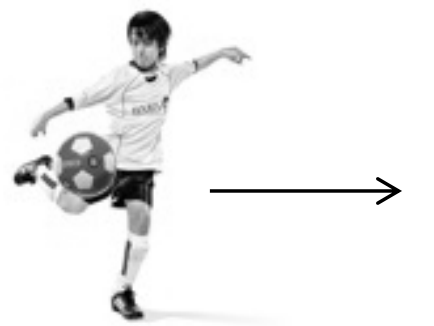

Physical Activity

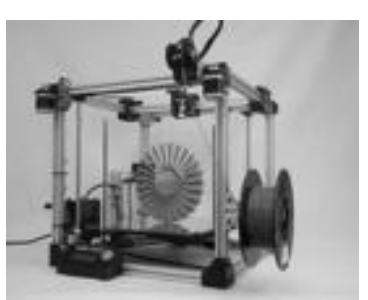

3D Printer

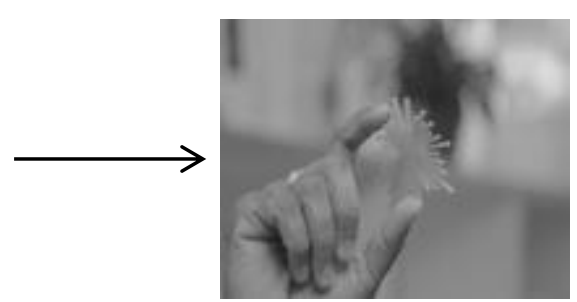

3D-printed Model

\section{Why have I been chosen??}

You have been asked to volunteer because you are of the targeted age of 7-14 years of age.

\section{What will happen to me if I take part?}

You will take part in a short talk with one of our team. In the talk we will have questions like "what 3D-printed shape do you prefer?" and "Do you understand what sedentary behavior is?". But don't worry if you don't understand the questions or know the answers, as that's all part of the study. It will also be important for you to give us ideas and even ask us questions and you will be helping to plan the study as well.

\section{What are the possible disadvantages of taking part?}

You will have to miss a small amount of school time. This may be during lesson time, break time or even after school.

\section{What are the possible benefits of taking part?}

You will be involved in designing a very new way of looking at physical activity for children. Your input will be vital for the future main study. You will also learn how 3D printing works and what a sport scientist actually does.

\section{Will my taking part in the study be kept confidential?}

All information collected about you will be kept strictly confidential. Any information that is distributed among the research team will identify you by number and not name.

\section{What if I have any questions?}

If you have any questions about the study, either now or in the future, please contact Melitta or Sam using the details provided at the top of this document. 
Study 4 - Parent Information Sheet

\author{
Applied Sports Technology Exercise and Medicine Research \\ Centre (A-STEM) \\ Sport and Health Portfolio, College of Engineering
}

\title{
PARENTAL INFORMATION SHEET - INTERVENTION
}

(Version 1.1, Date: 01/10/2015)

\section{Project Title:}

Physical Activity across the Globe: The G-Sphere

\section{Contact Details:}

Sam Crossley

Phone:

Email.

Dr Melitta McNarry
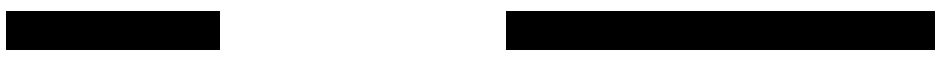

Dr Kelly Mackintosh
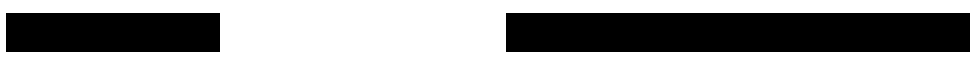

\section{Invitation Paragraph}

Thank you for taking the time to read this information sheet, it will provide you with the details of our study and hopefully provide you with the information you require to help you decide if you want your child to participate. It is important to say at this point that the decision to take part is entirely up to you and that your child will not be at a disadvantage for future studies should you decide for them not to participate.

\section{What is the purpose of the study?}

It is becoming increasingly important to find new novel ways of interpreting physical activity and especially for children. The main focus of this study is to 3D print physical activity into tangible objects that allows children to touch and interpret exactly what physical activity they have accomplished rather than looking at graphs and numbers that are not meaningful.
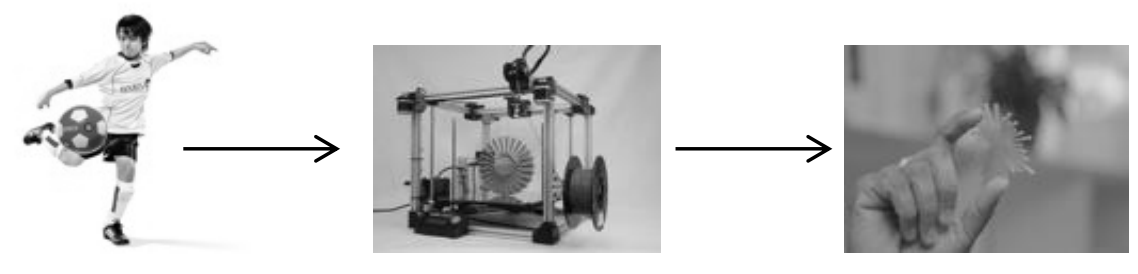

Physical Activity

3D Printer

3D-printed Model 


\section{Why has my Child been chosen??}

Your child has been asked to volunteer because they are a healthy individual between 7-14 years of age, free from injury or illness. We regret to say that those with known cardiovascular disease are not able to volunteer for this study due to the risks associated with exercise for these children.

\section{What will happen to my child if they take part?}

Your child will be invited to a class assessment during school time which will happen every 3months ( $3 \times$ assessment days) and will take approximately 1-hour to complete. The class assessment includes:

Body Measurements -Your child's weight, height, sitting height and waist circumference will be measured using accurate measures. This will take no longer than 3 minutes to complete.

Maturation Assessments - Your child will have to complete a self-reported Tanner Stages form. The Tanner Stage form is an assessment for level of pubertal maturation. The reason this form is done is because different levels of maturation can alter the physical output of a child. Researchers can then understand the possible differences between children might be of influence from maturation level. Your child will complete the form in private and then seal it into an envelope. The envelope will have an assigned number that corresponds to your child's identity. Only the researchers will have access to the forms collected. This will take approximately 5 minutes to complete.

Questionnaire - General Quality of Life Measurement (PedsQL), which assesses the healthrelated quality of life to find out how the intervention is affecting their general all-round health. This will take approximately 10-20 minutes to complete.

Multi-Stage Fitness Test - Your child will be asked to complete a bleep test which they have probably done before in PE lessons. This test is done to assess their fitness. It will require running $20 \mathrm{~m}$ shuttle runs to the sound of beeps, which increase in speed until they can no longer reach the line before the beep sounds. This will take approximately 15 minutes.

Incremental Treadmill Test - A small number of participants will be chosen to complete a more accurate measure of fitness which measures oxygen uptake. This will require visiting the Swansea University Sport Science laboratory to complete the test. This incremental test involves your children pushing themselves really hard until they cannot run anymore. Firstly, they will practice walking and running on the treadmill (see pictures below) until they are happy and relaxed with it. We will then start the actual exercise, which will start off easy and get harder and harder but will be stop when your child says they can't keep going. During this, they will be breathing into a mask to allow us to measure the air that they breathe in and out. This mask does not make breathing any harder and they can talk through it and remove it at any time they feel uncomfortable about wearing it. The exercise will last approximately 15 minutes. 


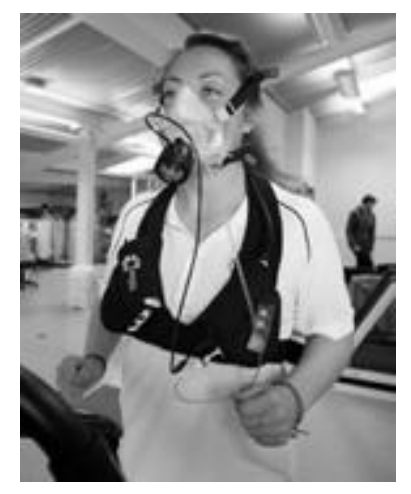

Breathing Mask

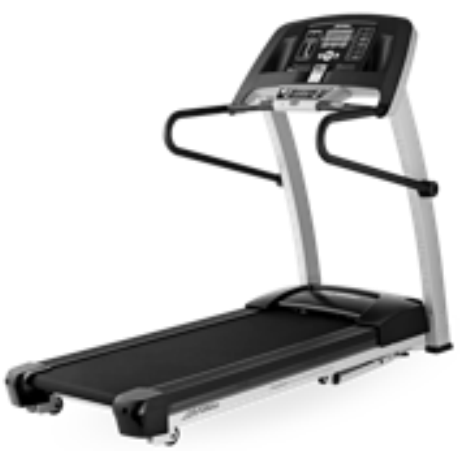

Treadmill

Your child will also complete a series of physical activity measurement and interviews at different time points. These include:

7-Day Physical Activity Measurement - Your child will wear an accelerometer device for 7days straight, so we can get an idea of how physically active your child is. At the beginning of the school week your child will be given an accelerometer to wear around the waistline. Once the 7-day period is up your child will hand in the accelerometer to the researcher, which will then be uploaded and looked at. During this period your child will also keep an activity log to record for when they remove the device (during contact sports and water activities). This measurement will be taken every 3-months and will take approximately 10 minutes to setup.

3D-Printing Models of Physical Activity - Your child will wear an accelerometer device for 1 full school day. The next day your child will hand in the accelerometer to the researcher. Your child will receive the 3D-printed model of the physical activity completed on that day of measurement. Your children will receive new 3D-printed models every 2-weeks for the first 2 months, then every 4-weeks for the next 2 months and finally 1 model for the final 2 months. All together you child will have 7 different 3D-printed models of physical activity.

Interviews - Your child will complete a short interview on their interpretations of each personal 3D-printed model. This will take approximately 20 minutes and will be completed during school time.

Focus Groups - Your child will be interviewed in groups of 5 and asked questions related to physical activity, sedentary behaviour, exercise intensities and understanding this through 3D-printed models. These sessions will be either videoed or voice recorded. This will take approximately 1-hour of their time and will be completed 3- times over the course of the 6month study.

\section{What are the possible disadvantages of taking part?}

There aren't any real risks or discomforts within the study. The incremental test and multistage fitness test require participants for push themselves maximally, which may cause slight discomfort. However, if you follow our instructions we will ensure that you are warmed up 
for this activity to reduce the risks of injury. There will be trained first aiders on hand to deal with any injuries that may occur. They will not be disadvantaged in any way by choosing to stop participating and will not be forced to continue if you do not want to.

\section{What are the possible benefits of taking part?}

You will learn how fit your child is and how physically active they are compared to current government guidelines for physical activity. They will also develop an understanding of physical activity, sedentary behaviors and different exercise intensities. They will also learn about novel devices being developed by Swansea University and new advancements in 3D printing.

\section{Will my child's taking part in the study be kept confidential?}

All information collected about your child will be kept strictly confidential. Any information that is distributed among the research team will only be identifiable by number and not name.

\section{What if I have any questions?}

If you have any questions about the study, either now or in the future, please do not hesitate to contact Melitta or Sam on the details provided at the top of this document. 


\section{Centre (A-STEM)}

Sport and Health Portfolio, College of Engineering

\section{CHILD INFORMATION SHEET - INTERVENTION}

(Version 1.1, Date: 01/10/2015)

\section{Project Title:}

Physical Activity across the Globe: The G-Sphere

\section{Contact Details:}

Sam Crossley

\section{Phone:- \\ Dr Melitta McNarry \\ Dr Kelly Mackintosh}

Email:
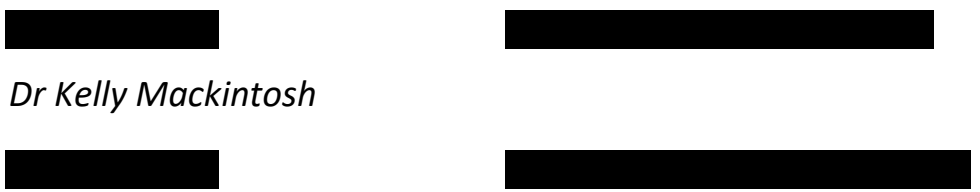

\section{Invitation Paragraph}

Thank you very much for agreeing to take part. This information sheet will provide you with all the information about the different tests you are going to do. If you want to stop taking part in this testing at any time just tell one of the researchers.

\section{What is the purpose of the study?}

The purpose of this study is to find out how physical active you are, and we will help you understand this by 3D printing your very own model of physical activity (something like the pictures below).

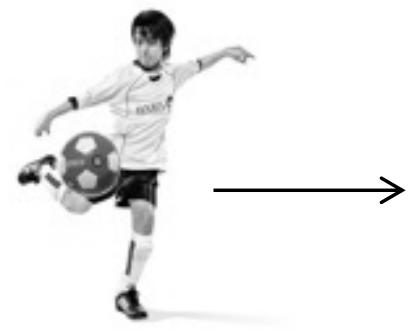

Physical Activity

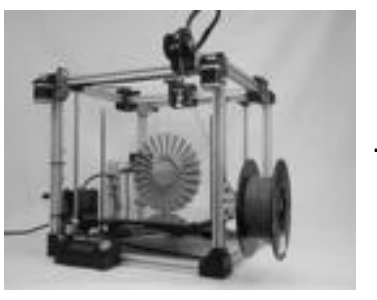

3D Printer

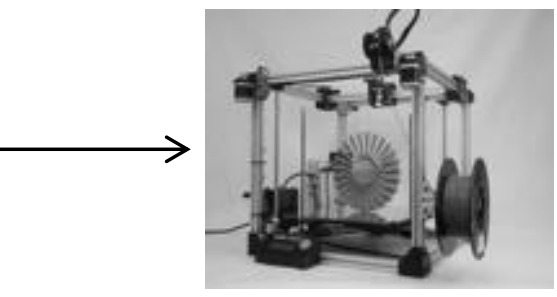

3D-printed Model 


\section{Why have I been chosen??}

You have been asked to volunteer because you are a healthy individual of 7-14 years of age, free from injury or illness. Those children with illness or injury are not able to volunteer for this study.

\section{What will happen to me if I take part?}

You will be invited to an assessment day which happens every 3-months ( $3 \mathrm{x}$ assessment days) and will take approximately 1 -hour to complete. The assessment day includes:

Maturation Assessments - You will be asked to complete a special form called the Tanner stage form. This form will have pictures of male or female private parts (which ever one corresponds to you!). You must simply tick a box to which picture looks like you. You will complete this form away from other people. When you have completed the form, you will then put it into an envelope and seal it. Only members of the research team will be allowed to look at the forms.

Body Measurements -Your weight, height, sitting height and waist circumference will be measured using accurate measures. This will take no longer than 3 minutes to complete.
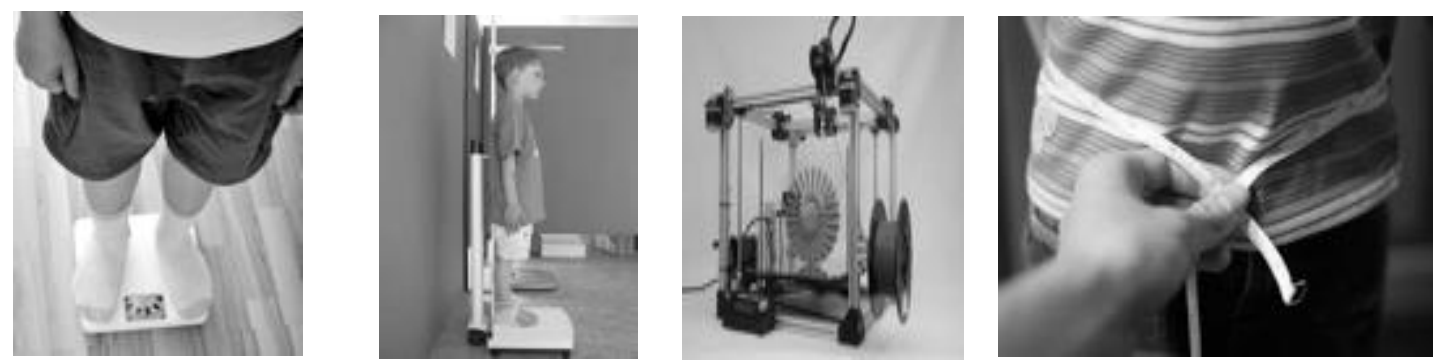

Questionnaire - General Quality of Life Measurement (PedsQL), which assesses the healthrelated quality of your life to find out how the intervention is affecting your general all round health. This will take approximately 10-20 minutes to complete.

Multi-Stage Fitness Test - You will be asked to complete a bleep test to assess fitness. It will require running $20 \mathrm{~m}$ shuttle runs to the sound of beeps, which increase in speed until you can no longer reach the line before the beep sounds. This will take approximately 15 minutes.

Incremental Treadmill Test - A small number of you will be chosen to complete a VO2 max test. This will require visiting the Swansea University Sport Science laboratory to complete the test. This incremental test involves you pushing yourselves really hard until you can't run anymore. Firstly, you will practice walking and running on the treadmill (see pictures below) until your happy and relaxed with it. We will then start the actual exercise, which will start off easy and get harder and harder but will be stopped when you have reached you limit. During test, you will be breathing into a mask to allow us to measure the air that they breathe in and out. This mask does not make breathing any harder and you can talk through it and remove it at any time you feel uncomfortable about wearing it. The exercise will last approximately 15 minutes. 


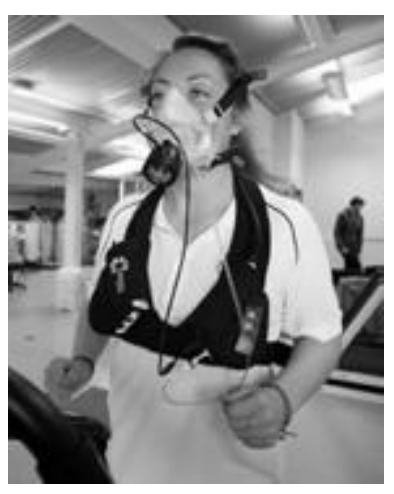

Breathing Mask

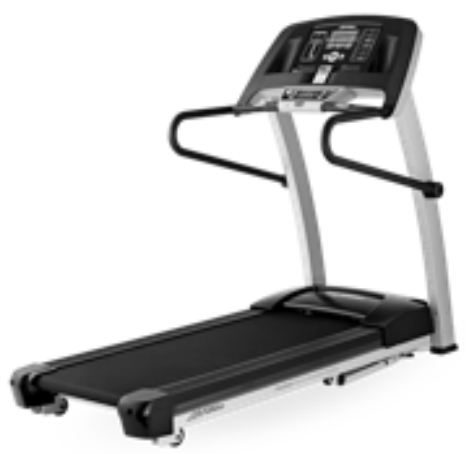

Treadmill

Your will also complete a series of physical activity measurement and interviews at different time points. These include:

7-Day Physical Activity Measurement - You will wear an accelerometer (picture below) device for 7-days straight, so we can get an idea of how physically active you are. At the beginning of the school week you will be given an accelerometer to wear around the waistline. Once the 7-day period is up you will hand in the accelerometer to the researcher, which will then be uploaded and looked at. During this period, you will also keep an activity log to record for when you remove the device during the 7-day period (to be taken off during contact sports and water activities). This measurement will be taken every 3-months and will take approximately 10 minutes to setup.

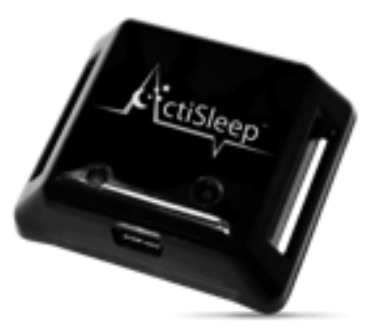

Accelerometer Device

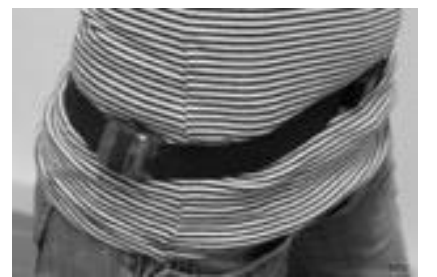

Accelerometer around waist

3D-Printing Models of Physical Activity - You will wear a similar accelerometer device to the one above for 1 full school day. The next day you will hand in the device to the researcher. You will then receive the 3D-printed model of the physical activity completed on that day of measurement the following day or end of that school week. You will complete this measurement 7 times over the 6 months intervention. You will receive a new 3D-printed model every 2-weeks for the first 2 months, then every 4-weeks for the next 2 months and finally 1 model for the final 2 months. You will be asked to safely hold onto the 3D models for later comparisons. 
Interviews - You will complete a short interview on your interpretations of your personal 3Dprinted model. This will take approximately 20 minutes and will be completed during school time.

Focus Groups - You will be interviewed in groups of 5 and asked questions related to physical activity, sedentary behaviour, exercise intensities and understanding this through 3D-printed models. These sessions will be either videoed or voice recorded. This will take approximately 1-hour of their time and will be completed 3-times over the course of the 6-month study.

\section{What are the possible disadvantages of taking part?}

The risks associated with exercise are small. You will have to complete at least 3 fitness tests, which involve full out effort. However, if you want to stop at any time you can, and you will not be forced to continue if you do not want to.

\section{What are the possible benefits of taking part?}

You will learn how fit you are and a better understanding of health and physical activity. You will learn how 3D-printing works and you will get to keep your own unique 3D-printed models of physical activity.

\section{Will my taking part in the study be kept confidential?}

All information collected about you will be kept strictly confidential. Any information that is distributed among the research team will identify you by number and not name.

\section{What if I have any questions?}

If you have any questions about the study, either now or in the future, please contact Melitta or Sam using the details provided at the top of this document. 


\section{Studies 1 to 4 - Headteacher Consent Form}

Applied Sports Technology Exercise and Medicine Research Centre (A-STEM)

Sport and Health Portfolio, College of Engineering

\section{HEADTEACHER CONSENT FORM}

(Version 1.4, Date: 25/06/2015)

Project Title:

Physical Activity across the Globe: The G-Sphere

Contact Details:

Sam Crossley

DrMelitta McNarry

DrKelly Mackintosh

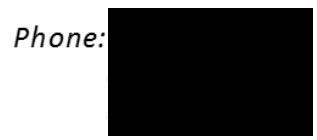

Email:

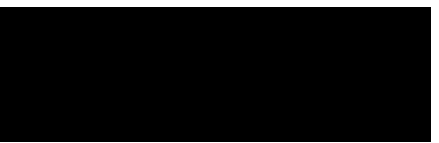

Please initial box

1. I confirm that I have read and understood the information sheet dated 01/10/2015 (version number 1.4) for the above study and have had the opportunity to ask questions.

2. I understand that my school's participation is voluntary and that I am free to withdraw at any time, without giving any reason, without my legal rights being affected.

3. I understand that sections of any of data obtained may be looked at by responsible individuals from the Swansea University or from research regulatory authorities where it is relevant to my taking part in research. I give permission for these individuals to have access to these records. Analysis will be done on anonymous data.

4. I give consent for video and voice recording and photography. (providing parent/guardian have provided consent)

5. I agree for my school to take part in the above study.

Name of School

Name of Headteacher

Date

Signature

Researcher

Date

Signature 
Applied Sports Technology Exercise and Medicine Research Centre (A-STEM) Sport and Health Portfolio, College of Engineering

PARENT CONSENT FORM

(Version 1.1, Date: 01/10/2014)

Project Title:

Influence of walking speed on the energetic cost of turning in children aged 10-12 years

Contact Details:

Dr Melitta McNarry

Sam Crossley

Swansea University

Swansea University

$\mathrm{Ph}$

E-mail

Ph:

Email:

Please initial box

1. I confirm that I have read and understood the information sheet dated $01 / 10 / 2014$ (version number 1.1) for the above study and have had the opportunity to ask questions.

2. I confirm that my child is healthy and free from illness or injury.

3. I understand that my child's participation is voluntary and that they are free to withdraw at any time, without giving any reason, without their medical care or legal rights being affected.

4. I understand that sections of any of data obtained may be looked at by responsible individuals from the University of Wales Swansea or from regulatory authorities where it is relevant to my child's taking part in research. I give permission for these individuals to have access to these records.

5. I agree for my child to take part in the above study.

Name of Subject

Name of Person taking consent

Researcher

\section{Date}

Date

Date
Signature

Signature

Signature 
Applied Sports Technology Exercise and Medicine Research Centre (A-STEM) Sport and Health Portfolio, College of Engineering

\section{CHILD ASSENT FORM}

(Version 1.1, Date: 01/10/2014)

Project Title:

Influence of walking speed on the energetic cost of turning in children aged 10-12 years

\section{Contact Details:}

Dr Melitta McNarry

Swansea University

Ph:

E-mail:

Sam Crossley

Swansea University

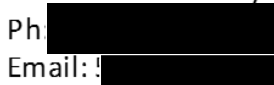

Please initial box

1. I confirm that I have read and understood the information sheet dated $01 / 10 / 2014$ (version number 1.1) for the above study and have had the opportunity to ask questions.

2. I confirm that I am healthy and free from illness and injury.

3. I understand that my participation is voluntary and that I am free to withdraw at any time, without giving any reason, without my medical care or legal rights being affected.

4. I understand that sections of any of data obtained may be looked at by responsible individuals from the University of Wales Swansea or from regulatory authorities where it is relevant to my taking part in research. I give permission for these individuals to have access to these records.

5. I agree to take part in the above study.

Name of Subject

Name of Person taking consent

Researcher
Date

Date

Date
Signature

Signature

Signature 


\section{Studies 2 to 4 - Parent Consent Form}

Applied Sports Technology Exercise and Medicine Research Centre (A-STEM)

Sport and Health Portfolio, College of Engineering

PARENT CONSENT FORM

(Version 1.1, Date: 01/10/2015)

Project Title:

Physical Activity across the Globe: The G-Sphere

Contact Details:

Sam Crossley

Dr Melitta McNarry

Dr Kelly Mackintosh
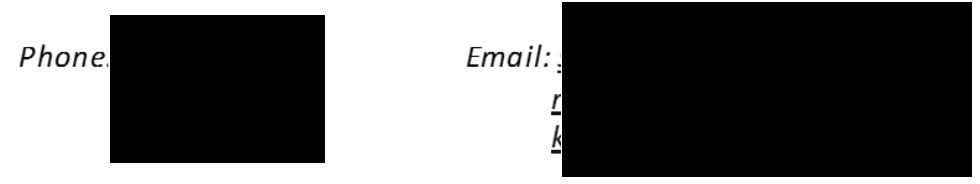

Please initial box

1. I confirm that I have read and understood the information sheet dated 01/10/2015 (version number 1.4) for the above study and have had the opportunity to ask questions.

2. I understand that my school's participation is voluntary and that I am free to withdraw at any time, without giving any reason, without my legal rights being affected.

3. I understand that sections of any of data obtained may be looked at by responsible individuals from the Swansea University or from research regulatory authorities where it is relevant to my taking part in research. I give permission for these individuals to have access to these records. Analysis will be done on anonymous data.

4. I give consent for video and voice recording and photography. (providing parent/guardian have provided consent)

5. I agree for my school to take part in the above study.

Name of Subject

Name of Person taking consent
Date

Date
Signature

Signature 


\section{Studies 2 to 4 - Child Assent Form}

Applied Sports Technology Exercise and Medicine Research Centre (A-STEM) Sport and Health Portfolio, College of Engineering

\section{CHILD ASSENT FORM}

(Version 1.1, Date: 01/10/2015)

\section{Project Title:}

Physical Activity across the Globe: The G-Sphere

Contact Details:

Sam Crossley

DrMelitta McNarry

Dr Kelly Mackintosh
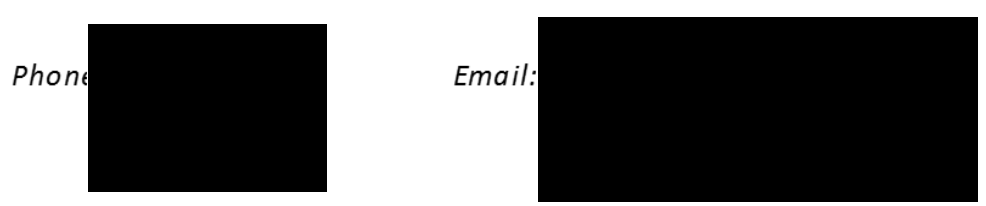

Please initial box

1. I confirm that I have read and understood the information sheet dated $01 / 10 / 2015$ (version number 1.4) for the above study and have had the opportunity to ask questions.

2. I understand that my participation is voluntary and that I am free to withdraw at any time, without giving any reason, without my medical care or legal rights being affected.

3. I understand that sections of any of data obtained may be looked at by responsible individuals from the Swansea University or from regulatory authorities where it is relevant to my taking part in research. I give permission for these individuals to have access to these records. Analysis will be done on anonymous data.

4. I give consent for video and voice recording and photography.

5. I agree to take part in the above study.

Name of Subject

Name of Person taking consent

Researcher

\section{Date}

Date

Date

\section{Signature}

Signature

Signature 


\title{
Studies 1 to 4 - Video, Audio and Photography Consent Form
}

\author{
Applied Sports Technology Exercise and Medicine Research Centre (A-STEM) \\ Sport and Health Portfolio, College of Engineering \\ CONSENT FORM FOR FILM/VIDEO, AUDIO \& PHOTOGRAPHY \\ (Version 1.1, Date: 01/10/2014)
}

Project Title:

Influence of walking speed on the energetic cost of turning in children aged 10-12 years

Contact Details:

Dr Melitta McNarry

Sam Crossley

Swansea University

Ph:

E-mail:

Swansea University

Ph:

Email:

I consent to the capture of:

$\square$ Still images (photographs)

$\square$ Moving images (film/video)

$\square$ Audio recordings

I accept that the subsequent use may be in a number of media, including but not limited to print, digital and electronic use by the University of Swansea and/or by agents authorised by the University.

The University would like to make the image(s) and/or recording(s) available to the general public via the Internet.

Please tick the box if you do not wish your child to be included in the making of image(s) and/or recordings to be made available to the public.

Name of Subject

Name of Person taking consent
Date

Date

Date
Signature

Signature

Signature 
Appendix III: Self-Report Tanner Stage Scale

$\square$ Tick the box that most closely resembles yourself.
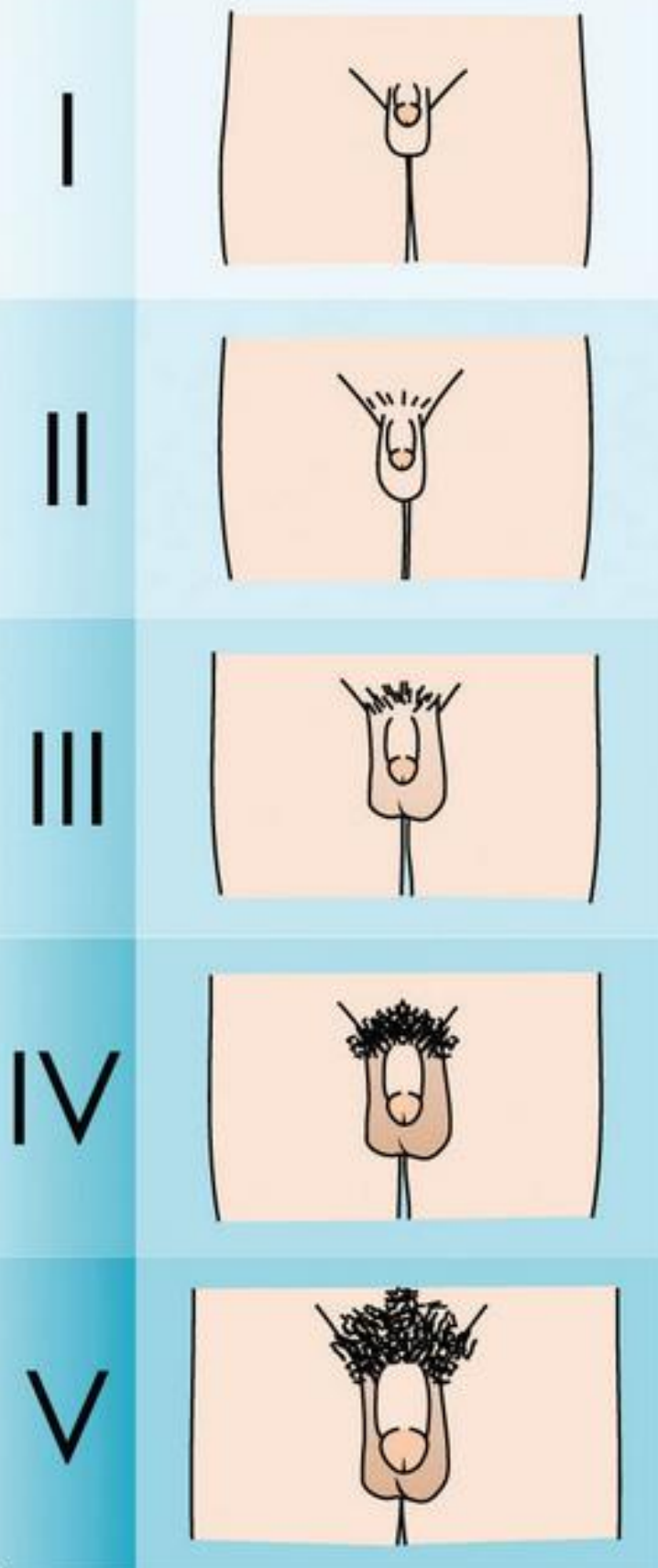
$\square$ Tick the box that most closely resembles yourself.

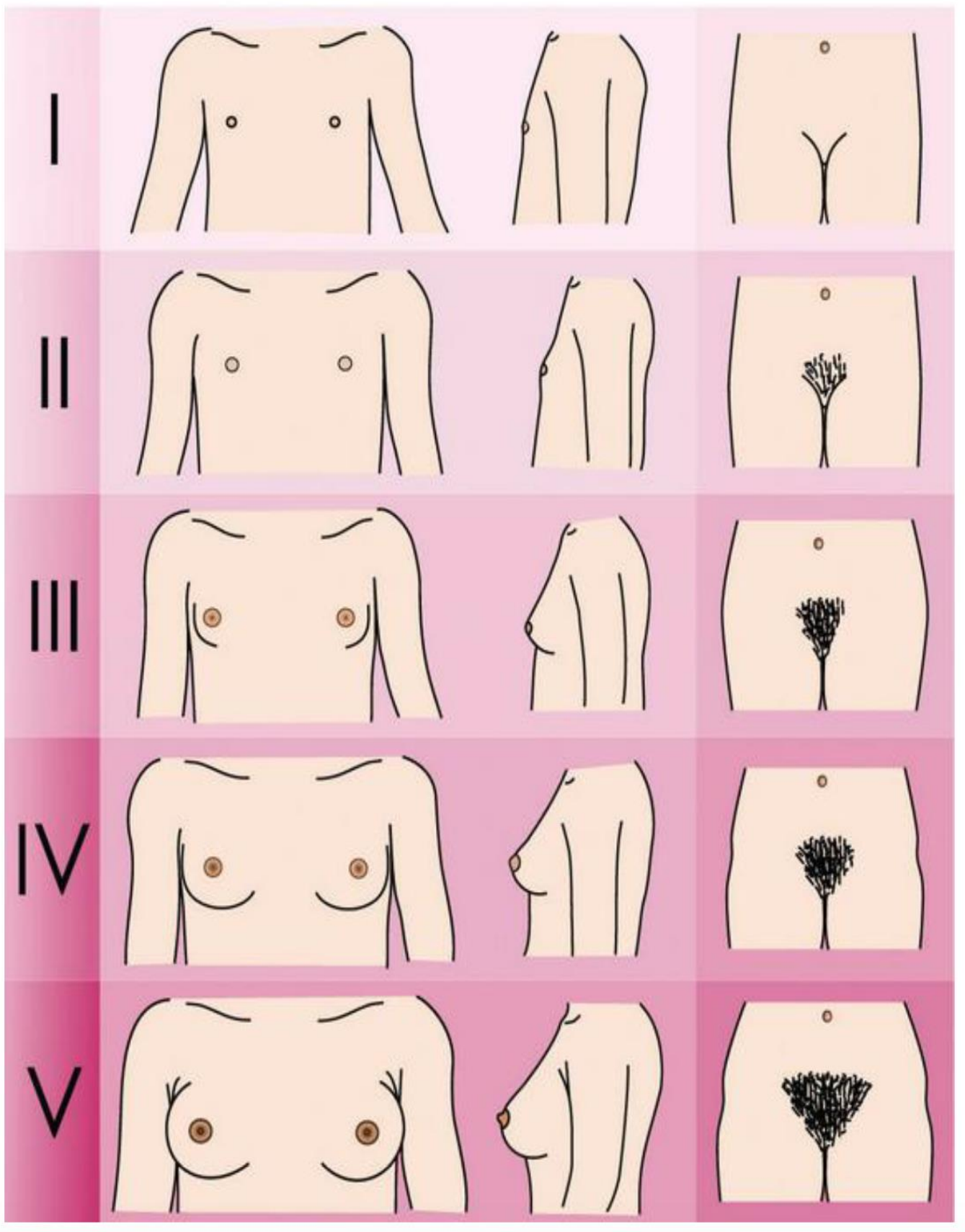


Appendix IV: Studies 2 to 4 Qualitative Questions and Sample Transcripts

Study 2 - Youths' Focus Group Questions

\section{Focus Group Questions}

\section{Structure of topics for discussion:}

- Role models

- Activities they enjoy

- Playground crazes/trends/fashion/collectables

- Fitness

- Health

- Physical Activity

- Exercise intensities/the extent (level) to which something is hard

- Sedentary Behaviour/Inactivity/inactive/still/lying

- Reinforcing factors - Family, peers and coach's/teachers influences.

- Enabling factors - Fitness, Skills, Access and Environment

- Motivation

- Goal setting

- Feedback

- $3 \mathrm{D}$ printing

- Designing 3D-printed models of physical activity

\section{Tasks/Activities for group session:}

- Video on 3D printing physical activity (approx.3mins)

- Drawing/designing your own model of physical activity

- Modeling your own model of physical activity through Play-Doh

- Picture questionnaire - picking your top 3 favourite models 


\section{Start of Procedures:}

1. Thank the group for coming/taking part

2. Handout child information sheet - explain verbally what it is and why its important to read.

3. Handout child assent form - explain verbally what it is and get them to agree/sign boxes

4. Remind and explain to them why they are being filmed.

5. Review the purpose of the group and goals of group meeting (set the stage).

6. Go over the flow of the discussion- how it will proceed, and how the members can contribute - Encouraging open participation

7. Lay out the ground rules

N.B. Given the age differences between year 3 and 9 - interaction key (different), particularly with year 3 e.g. explaining words clearly with prompts (pre-drawn pictures, written words on flip chart style). Use terms and terminology used by the children. Provide motivational comments and refrain from providing evaluation of drawings.

\section{Ground Rules for Participants:}

\section{$\underline{\text { Year } 3 \text { Script: }}$}

"So, I'm just quickly going to go over the rules of how this group discussion works. The first and most helpful rule is, that only one person speaks at a time, but don't worry, there's no right or wrong answer to these questions and its quite likely you'll all have different answers anyway! But, if you have something you would like to add to another persons answer, please wait until they have finished and then speak.

When speaking, please make sure you don't have your fingers up your nose, otherwise I won't be able to understand what you've said! If you don't have an answer, it's ok, just tell me that you're not sure or you don't know. When one of you is speaking make 
sure to listen and not to interrupt them, as you will all get a turn to give your answer. About halfway through the questions we will be doing some small fun tasks involving drawing and modeling with Play-Doh! Which I cannot wait for!

...Most importantly, remember to relax and enjoy yourself, it's all about telling me what you think and how you see things, just like having a normal conversation. The reason I'm filming this group discussion is so that I can go back over what you guys said! Only myself and other members of the will be allowed to watch the footage!

\section{Year 9 Script:}

"So, now I'm just going to go over some of the rules of the group interview. It is very important that you speak one at a time and that you listen carefully, but remember there's no right or wrong answers to these questions, it's all about what you think! Don't worry though if you don't have an answer to one of the questions, just tell me your unsure or you don't know. However, if you have something you would like to add to someone else's answer please wait until they have finished and then speak.

Around about halfway through the session we will also be doing some small tasks involving drawing and modeling using Play-Doh. So get ready to be creative!

...Most importantly, I want you guys to feel relaxed and confident to speak out, just imagine its a normal conversation, but with a camera filming us (said in a jokey way). Only myself and members of the research team will be allowed to see the footage"

\section{Start of Questions}

\section{Icebreaker:}

"Before I get started with the questions, I would like to get to know a little about yourselves. So, if I get each one of you to introduce yourself by giving your name and what you like to do for fun year $3 /$ most enjoy doing year 9 ? 
...I'll go first, my name is Sam and what I most enjoy doing/for fun is going surfing in the Gower! Ok, next (hand gesture to pupil)"

\section{Questions set 1: Role models, Activities, Playground crazes/collectables}

1. Who is your role model year ${ }^{9}$, the person you look up to ${ }^{\text {year }}{ }^{3}$ ? Why is that? (Prompts; parents, brother/sister, superhero, sportsperson, TV character?)

2. What do you like to do before or after school or even during school playtime?

\footnotetext{
"So, when I was at school, way back...almost 100 years ago, I used to collect Pokémon/Pogs/Tazos (show one of these items)"
}

3. So from this, what sort of things are you collecting at the moment, if anything?

"So do any of you like being active?...The next few questions we are going to talk about is all about this and why it is important!'

\section{Questions set 2: Fitness, Health, Physical Activity, Sedentary Behaviour and Enabling/Reinforcing factors:}

4. Can you tell me what you think 'health' means?

5. Can you tell me what you think 'fitness' means?

6. Who can tell me what physical activity means?

7. Can you tell me how active are you? (A lot? Every day? Only once a week? All day?) 
8. When and where are you most and least active? (prompts: home, school, weekend) Why is this? What kinds of activities do you do when you are active?

9. So, if you're being inactive (Prompts; being still, no movement), what sort of activities might you be doing (prompts: lying, playing video games, watching TV)? What do you think will happen to you if you carry on (go on from their examples) being inactive?

10. What sorts of things tend to stop you from being more physically active? Or Can you think of anything that stops you from being physically active? (prompts: fitness level, skills required, access to facilities, environment?)

11. Does anyone support/help you to be more physically active? (prompts: parents, carers, brothers/sisters, friends, teachers, coaches)...ok, so what do they do that specially helps you to be more physically active?

12. How do you feel about taking part in new activities? (prompts: feelings, confident, happy, scared)

\section{Questions set 3: Intensities, Motivation, Goal Setting and Feedback}

13. Some kinds of exercise make you more out of breath or more tired than others... which ones are these? Do you know what we call that? (prompt: the extent/level to which something is hard) - Intensity

14. How do you feel when you're doing low intensity activity such as...or high intensity....(prompts: my legs ache and I find it hard to breathe...)

15. Which intensities of activity are you more likely to do? (prompt: high/moderate/low?)

16. Which intensities of activity do you want to do more of? (prompt: more high intense activities - running, fast cycling, football, netball; more low activities - sitting, dancing slowly, playing catch) why is that? 
17. So, What do you think the difference is between somebody who's physically active because they want to be and someone who doesn't want to be physically active?

18. What do you think motivation means?

19. What makes you want to be physically active? (prompt: I want to be physically active to improve my health)

20. What do you think I mean by goal-setting means? (prompts: targets for the future, goals to achieve, things to better yourself)

21. When you're active are there any goals you set yourself? (prompt: like completing 12 jumps a day?)

22. Why do you think it is important for people or yourselves to set goals? (prompt: to help them be more active)

23. Do you know how much exercise you should do to be healthy?

24. Have any of you heard of the physical activity guidelines set by the government for youselves? - Yes that's right or If they don't know, mention the current government guidelines of ' 60 -minutes of moderate to vigorous physical activity a day' and then lead onto -> Do you think you meet these guidelines?

So, from all of this....

25. If I told you I could create a physical object to show you how active you had been throughout the day, would that motivate you to be more physically active? Why is this?

\section{Questions set 4: 3D printing and Designing 3D models of Physical Activity}

26. Who can tell me what a $3 \mathrm{D}$ printer is? What do you think they do? 
27. What would you think if I said we could 3D print your own personal model which shows how physically active you are?

28. Video: 3D printing physical activity (approx.3mins): "Right I'm now going to show you a short movie that I have put together myself, that should help you understand exactly what I'm on about when I say 3D printing your own physical activity. So, if you'd please sit quietly and enjoy the show...sorry no popcorn (year 9 - popcorn prohibited) allowed here!"

29. What do you think of the idea of 3D printing your own physical activity?

\section{Tasks: Drawing and creating 3D models of physical activity (3-potential tasks)}

"We would like you to help us create/design these 3D models of physical activity. So, I have setup a few tasks ( 3 tasks to be precise) that will allow you to create your own - which will involve drawing, modeling and picking models"

\section{Task 1 - Drawing: (i) What sort of model would you like to develop or represent} your own physical activity as in the video, how would it look? " $O k$, so the first task I am going to give you is a drawing one. I want you to draw your own picture of what you think physical activity would look like as a physical object. You can use labels and arrows to describe and show what your model means. So each of you grab an A4 piece of paper and a colouring pen and I'm going to give you all two minutes to complete your own drawing. No peeping at anyone else's drawing, I want them to be your personal drawing of what you think it should look like! ... When you have all completed your drawings I would also like you to talk me through what exactly you have drawn!'

(ii) Could you explain/tell me what you have drawn here? 
N.B. Get them to sit apart so not to influence each other, ask them to not look at others drawings/models Place a post-it note with participants ID on each drawing.

\section{Task 2 - Play-Doh! : (i) What sort of model would you like to develop or represent} your own physical activity as in the video, how would it look? "Ok, so for this next task, I now want you to model your own physical activity object with Play-Doh! So this is very similar to the last task we completed, but I want you to now mold a physical activity object. I am going to give you each a tub of Play-Doh and again, I'm going to give you two minutes to complete your model and then I will get you each one-by-one to explain your model to me" (Prompts; How would you show different activities and intensities in your model?)

(ii) I want you to now describe your individual model to the group and why you've made it like that...why is it big? Spiky? How you interpret it/look at it? (Prompt; shape, size- big or small, what colour would you like it to be)

N.B. Take a picture of each model created with the participant number visible in picture on a post-it note before resetting Play-Doh (label of number in black ink) Handout tubs of Play-Doh to avoid confrontation with kids picking out favourite colours.

Task 3 - Picture Questionnaire: "The final task I would like you to complete is a simple picture choosing task. What I would like you to do here is to number your top 3 favourite models, with 1 being your most favourite, 2 being your second favourite and 3 being your third favourite. Put the number 1,2 and 3 in the boxes next to the picture of the models you like. I would then like you to put a zero in the box next to the model picture that is your least favourite. Once you have all completed the questionnaire can you please hand them back to me. I would then like you to each explain to me which was your favourite model and why.' 


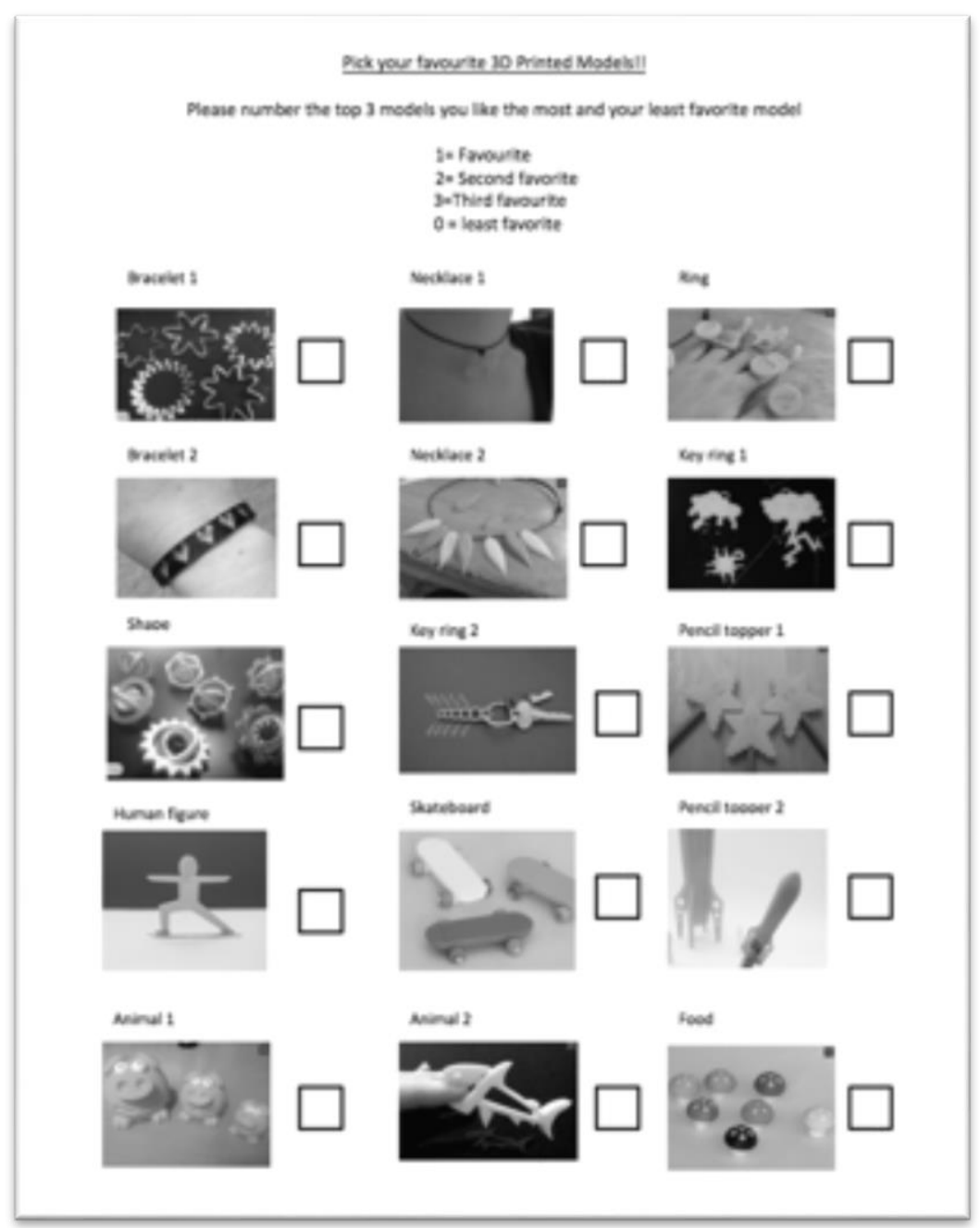

\section{Questions set 5: 3D model build, time and view}

"I'm now going to show you some cool things that I have made using a 3D printer!"

30. So here, I have some models of physical activity I have already created (show them the individual models of physical activity). (i) Now, how would you describe (interpret) this model of physical activity - What kind of activity do you think the person did to produce this large spike/blob? (One at a time show models and discuss them) - (Prompt; this one shows a day/week of physical activity) 
(ii) What do you think the bigger spikes/blobs/lines represent or show the person was doing? (Prompt; think back to intensities of physical activity or amount of physical activity - which we discussed earlier!)

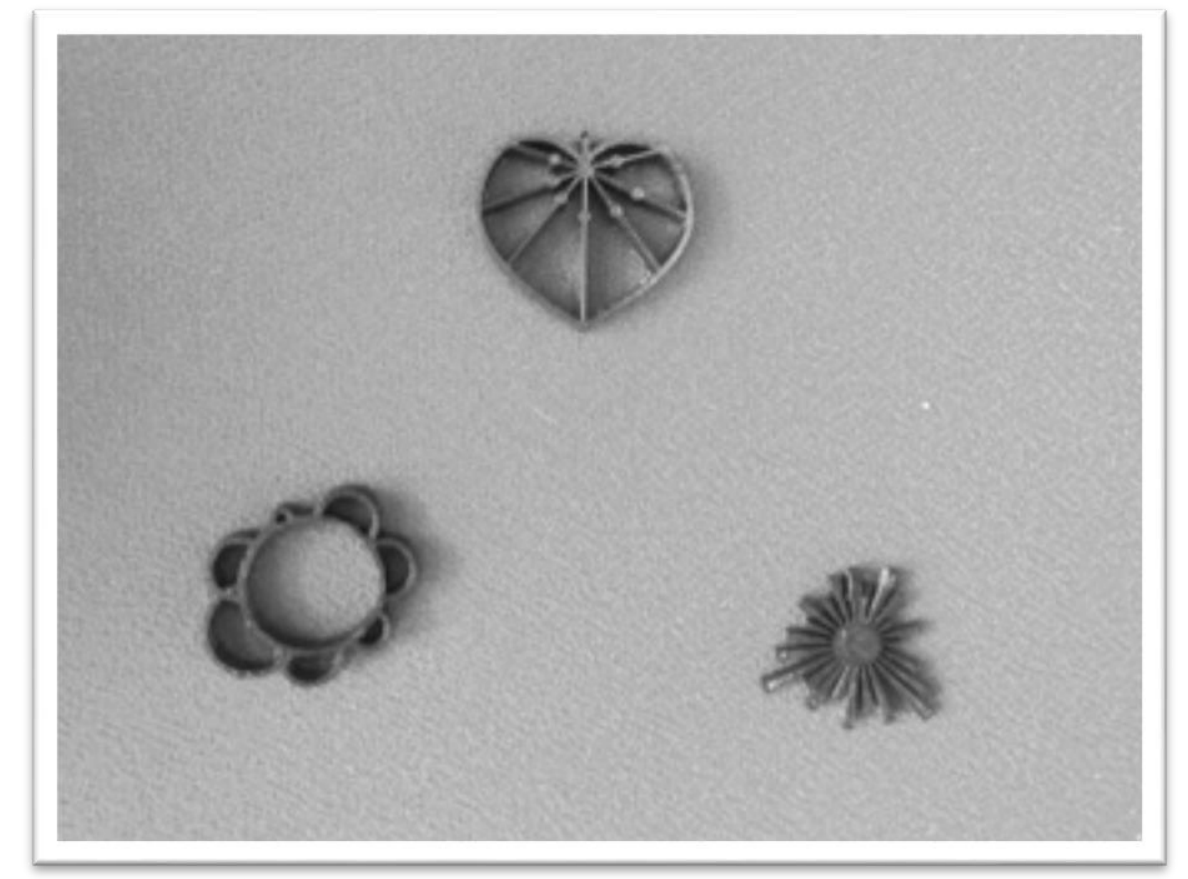

31. If you did get to make these models from your physical activity would it make you want to do more? Why is that?

32. Would you like your models of physical activity to show a full week of activity or just one whole day of activity?

33. Would you like a model that shows your whole class's physical activity?

\section{End of Questions}




\section{Focus Group Questions}

\section{Structure of topics for discussion:}

- Children's Health and Fitness

- Children and Obesity

- Children and Physical Activity

- $\quad$ Gender differences

- Motivation

- Intensities

- 3D Printing

- 3D Printing Physical Activity

- Integration into school curriculum

- Designing of intervention

*Additional activities - 3D Printing Physical Activity Video

\section{$\underline{\text { Start }}$}

- Thank the teacher/parent for taking part

- Review the purpose and goals of the interview

- State that the questions will be referenced towards children

- $\quad$ Go over the flow of the meeting

\section{Questions set 1: Children's Health and Fitness, Obesity, Physical activity, Gender}

1. How would you describe a healthy child?

2. How would you describe a fit child? 
3. What sorts of things help children to achieve a healthy well-balanced lifestyle? Which of these do you do at home?

4. What do you do at home to help your child stay healthy/get healthier?

5. What do you do at home to help your child stay fit/get fitter?

6. What changes have you noticed in school children since you went to school in regards to health and fitness?

7. Numbers of children with obesity are increasing across the developed world. What is your opinion of obesity in the school?

8. Can you tell me about anything the school does to help children manage their weight? How do you feel about the school playing a role in weight management?

9. How would you describe a child who is physically active?

10. What do you think about children's physical activity levels these days?

11. How do you encourage physical activity within your family? (prompts: travel week day vs weekend, indoor/outdoor motivation)

12. Do you think there is enough time dedicated to learning about health and fitness?

13. Where do you think children are most and least active? What is it about these environments that you think makes children more/least active? (prompt: anything within the school structure/enviroment specifically?

14. What do you think motivates children to be physically active? Do you think boys and girls are motivated differently to be physically active? 
15. Who do you think are the most influential people in encouraging activity in your child and others? And who are the role models that make children less active?

16. Do you think there is a difference between boys and girls physical activity? Why do you think this?

17. How do children feel about taking part in something new, like a new sport or physical activity?

\section{Question set 2: Intensities and Motivation}

18. What kind of intensity of physical activity do you think children enjoy the most? Do you think there is a difference between boys and girls?

19. What understanding do you think children have about the benefits of different intensities of physical activity?

20. If your child was physically active, how would you suggest reinforcing this? Does this vary between children?

21. Do you set up any physical activity goals/targets for your children? (prompt: Why not if they don't? how and when if they do?)

22. Can you recall any recent conversations you have had with your children about being physically active?

23. Do you know what the current government guidelines are for children's physical activity? (60-minutes MVPA a day)

24. Can you tell me about any ways that you try and help your children to meet these guidelines? 


\section{Questions set 3: 3D Printing, 3D-printed models of physical activity}

25. Technology has advanced a lot recently and we now have $3 \mathrm{D}$ printers. Have you heard anything about these? If so, what?

26. What do you think children will think about 3D printing or have your children had any experience of $3 \mathrm{D}$ printing before? If so can you give examples of this?

3D Printing Physical Activity video: "I have a video here showing the process of $3 D$ printing physical activity "

27. What do you think about the idea of using 3D printing to represent children's physical activity? Advantages and disadvantages to prompt if needed...

28. How do you think the children will engage with the 3D-printed models of physical activity?

29. How do you think the 3D models of physical activity could motivate children to be more physically active?

30. Do you think boys and girls would react differently to the 3D models? Why is that?

31. What do you think about using $3 \mathrm{D}$ printing physical activity as something that could be taught within the school curriculum to help educate children about the importance of keeping physically active?

32. Are there any models that you think would be good to help children to visualize physical activity? Please draw it on this A4 paper if you do!

" Here I have some models of physical activity that I have already created. The heart shape shows physical activity along lines within the heart with the blobs denoting just how much physical activity was completed that day. The round-hooped shaped model also represents a week of activity with each half circle denoting a day of activity. The 
larger the half-circle the activity completed that day. The final model shows a day of activity with each spike representing an hour of activity "

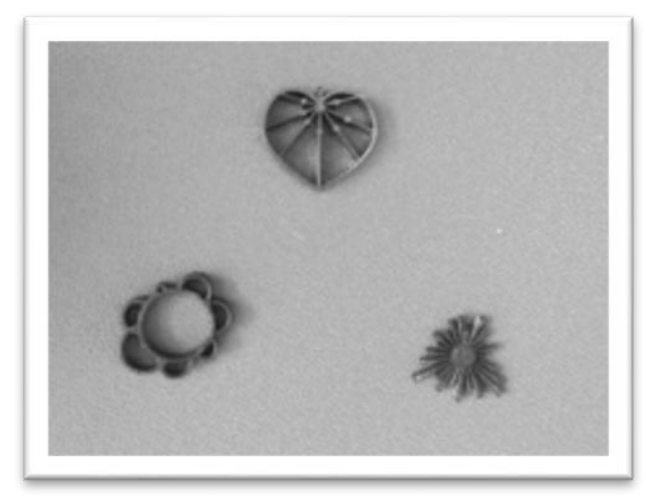

33. What do you think of these models as a motivational tool to increase children's physical activity? What do you think their understanding of them would be?

34. What do you think are the benefits and drawbacks of showing a day or a week of activity? Should be given the 3D models if they are to promote their motivation for physical activity? Every week, month or other?

35. It would be possible to produce a model that represents a group of children's physical activity such as a class. What are you thoughts on this?

\section{Questions set 4: Design of the Intervention}

The plan for the study is to design an intervention that can be done within the school to help children understand and become more physically active through 3D-printed models of physical activity.

36. What do you think about introducing this in schools? Any problems or advantages?

- Any other important comments or questions that I might have missed out? 


\section{D-printed Models of Physical Activity: Child Interview Questions}

\section{Equipment:}

1. 3D models of PA: Sun and Bar Chart (x3 large, medium and small)

2. Video camera + Tripod - setup behind the participant to capture hand movements/task displays

3. Voice recorder

4. Consent forms: Parental (to collect) and Child (to be completed)

5. Flipchart + pens

6. Laminated pictures of activity + PA intensity boxes + labels for participant numbers

7. Diagrammatic pictures of 3D models with labels

8. Accelerometer (to show the kids the type of measurement devices)

\section{Introduction:}

Hello my name is Sam and I am from Swansea University, I'm here today because I want to speak with you about some of the work I am doing at the university. The area of work I am particularly interested in is how do we get children to be more active!

I hope that all sounds ok? ...So if we start off with you just giving me your name and tell me something you enjoy doing in your free time?

Great, ok, so I am now going to go through what I am doing at the University. I am looking at 3D printing physical activity. So...

1. Can you tell me what a 3D printer is? (Prompt: what do you think they do?)

Ok yeah, so a 3D printer uses these reels of plastic to print physical objects that you can hold in your hands! Exactly what it does is to melt the plastic really really hot and 
then it squeezes it out of this tiny little nozzle, which then prints the object layer by layer until you have your physical object. Like this 3D-printed frog (show 3D-printed frog) I did earlier!
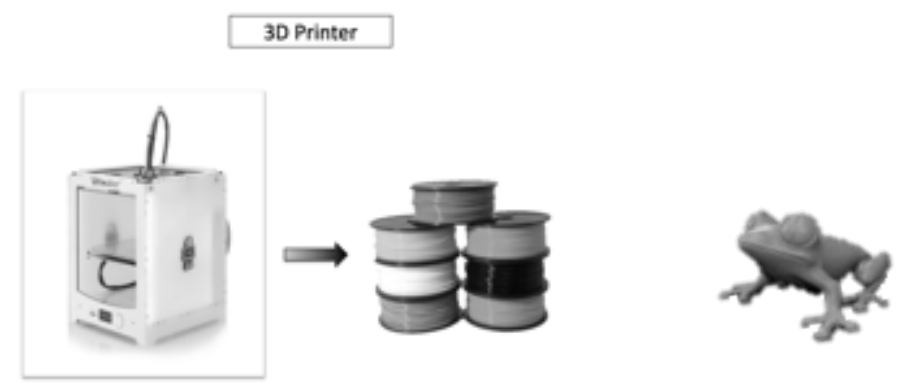

Alright so...

2. Can you tell me what you think physical activity means?

Ok, yeah so...Physical activity is any body movement, so these are like all the things you take part in everyday, like walking to school, playing in the playground or even grabbing something from the fridge!

3. So what do you think 3D printing physical activity means? How do you think those things (3D printing and PA) could work together?

Well, the combining both of those thing means we could have real physical objects that you could hold in your hands that could show you you're physical activity!

So does that all make sense now? I will get into a bit more detail later on but right now we will move onto my next question.

4. Can you think of any activity that might not be physical activity?

5. Can you think of the word that describes that activity?

Yes that's right, researchers like me call this being inactive or sedentary, which means spending time being very still 
No problem, it's being inactive/not moving or as we call it sedentary, which usually involves sitting down for long periods being very still.

Now moving back to physical activity. There are different levels of physical activity

6. Can you tell me what you think these different intensities/levels of physical activity might be?

So yeah there are different levels or intensity, you have light (easy), moderate (medium) and vigorous (hard). I now have a quick task for you to complete.

Task 1: Match the activity to the PA intensity box

Now what I want you to do is match the activity to the different physical activity level box. Once you have completed that I will then ask to describe why you have placed each activity in that physical activity box!

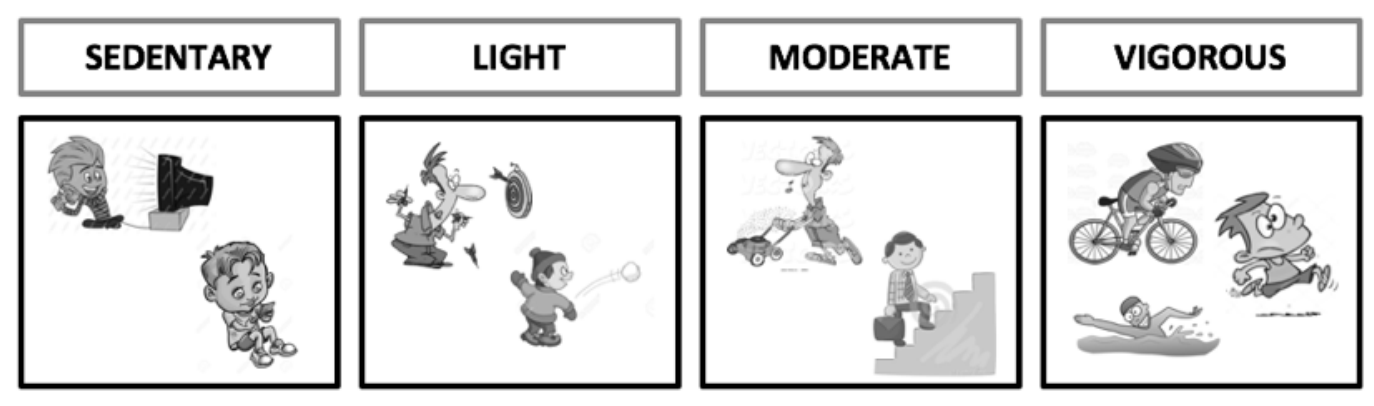

[NOTE - Take picture of each individual participants final display + place participant number in photo taken]

7. Can you talk me through why you have placed each activity within the physical activity box chosen?

So from this, what we are able to do is measure your movements using a tracking device like this one (show accelerometer), it goes around your waist and measures all the movements you have done for a whole day or even a whole week. 
This accelerometer can tell us exactly what type of physical activity level (pointing to task 1 for reference) you have been doing and for exactly how long, usually in minutes. This is information we collect is called physical activity data. The data is what tells you or myself how much activity you have been doing.

What my project is looking at, is how $3 D$ printing can show physical activity (movement) data and how it can be displayed as a 3D-printed physical object.

Does that all make sense now? Brilliant, ok, so I just have a few more questions left!

8. Can you tell me how much physical activity children your age should do to stay healthy?

Ok so the government (Prompt: Government overlooks and decide what's important for the country) has setup guidelines for you to follow, to keep you healthy and fit. The government says you should do 60 minutes of moderate that medium level of physical activity and vigorous the hard level of physical activity everyday. This level of physical activity is known to help strengthen your heart, lungs, muscles and bones!

So here we have a 3D-printed model of physical activity. Now this bar here is the target or guideline that I just told you about. So this bar represents/shows 60 minutes of moderate to vigorous physical activity. Does that make sense? Ok...

9. Can you now tell me what you think the rest of the physical activity model shows? (Prompt: how do you think this model (sun or bar chart) shows physical activity?)

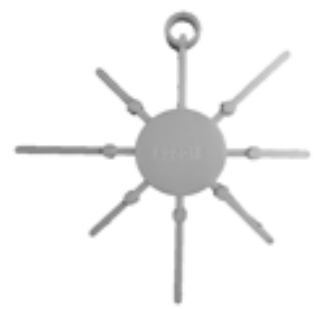


Yes, that's right, it shows how much activity a person has completed (Revisit key features that they got right by re-confirming what it is - if nothing is quite right go to questions below)

10. What do you think the lines show/bars? (Prompt: different lengths?)

Yes, what if I told you they show the different days of activity, so Monday is here and it goes around like that/across. Remember that this bar is the guideline $(60$ mins MVPA), this is your target!

11. What do you think the blobs/within bars on the suns rays/bars represent/show?

Yep, they show the different type of level of physical activity, but, they only show moderate and vigorous. This is because they are the most important levels of physical activity that make you healthy.

So these rays of the sun/bars are your moderate and vigorous physical activity complete for one day in minutes. So from here to here is vigorous and then from here to the top is your moderate physical activity...does that all make sense? (Let them make sense of it...)

So what you can do is estimate how much moderate and vigorous physical activity you've completed in that day by comparing it with the target bar that we keep on talking about.

12. So how much physical activity do you think this person has done for this day? (Prompt: how much moderate? how much vigorous?)

So imagine I gave you this physical activity-tracking device (show accelerometer) to wear around your waist for a whole week and then you go off wearing while it collects data about all of the movements you are doing. And then at the end of the week, I come back and pick up the activity-tracking device and upload the physical activity data to a computer. From the computer, I can then put the physical activity data onto an object 
like this sun shape/bar chart that's been 3D-printed. Finally, I can then give you you're personalized 3D-printed model of physical activity...to keep!

13. What would you think if you got your own personal model of physical activity?

14. What would you do with this model if you were given it at the end of the week? (Prompt: how would you use it?)

15. Then imagine we measured your physical activity again the next week and you got another model? (Hand over another of the same model - correct size) (Prompt: what would you do with it?)

16. Do you think this might make a difference to the amount or type? physical activity? (Prompt: In what way would it change you?)

Ok, so I've just got a few more questions then we are finished. These questions are more about the design.

17. What colour would you like your model to be?

Keep a tally chart of preferred colours

\begin{tabular}{|l|l|l|}
\hline Colour & Primary (tally) & Secondary (tally) \\
\hline Blue & & \\
\hline Red & & \\
\hline Green & & \\
\hline Orange & & \\
\hline Pink & & \\
\hline Purple & & \\
\hline Yellow & & \\
\hline Black & & \\
\hline White & & \\
\hline
\end{tabular}

18. How big would you like your model? From these different sizes of model? (small, medium and large models displayed)

19. What do you find the easiest part to understand about the $3 \mathrm{D}$ models of physical activity? 
20. What do you find the hardest part to understand about the $3 \mathrm{D}$ models of physical activity?

Task 2: Label the physical activity

Right, just one more thing to do and then we're done!

So what I want you to do here is to describe and label the diagram of the Sun/Bar chart to show what you think each part represents.
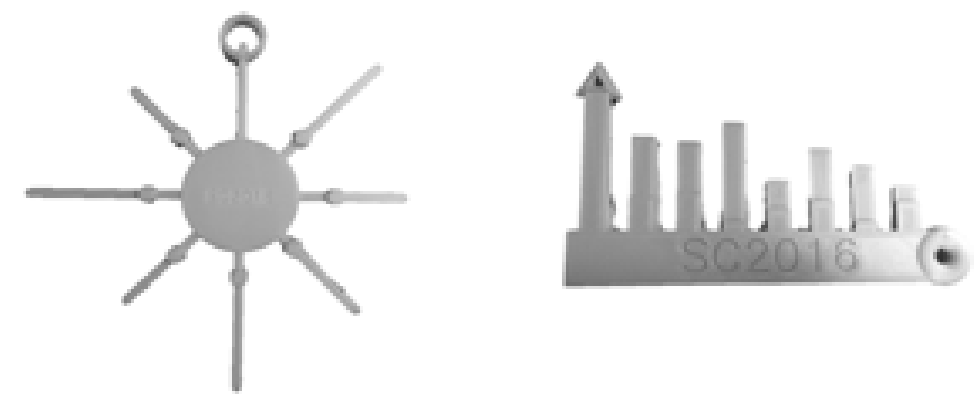

That is the end of the questions that I have, do you have anything you would like to say?

Thanks for taking part! 


\section{One to one Interview Questions: Model 1}

1. What do you think of your personal 3D-printed model of physical activity? (Prompts: Do you like your first model? Why do you like it?)

2. Can you tell me what you think physical activity means? (Prompt: Its everything movement that you do with your body)

3. Can you talk me through how your personal 3D-printed model shows your physical activity?

\section{Prompts Sun blob: Can you talk me through what the different parts of the model show?}

(i) Target bar: Is it important? What is the target? How much PA should you be doing?

(ii) Individual lines/rays: What do they represent? What happens to the lines with PA?

(iii) Blobs along the line: What are they? What do they do?

(iv) Two different lines within the lines: What do they show?

Prompts bar chart: Can you talk me through what the different parts of the model show?

(i) Target bar: Is it important? What is the target? How much PA should you be doing?

(ii) Individual bars: What do they represent? What happens to the bars with PA?

(iii) Two different bars within the bars: What do they show?

4. Can you tell me what kind of activities might be vigorous/hard and moderate/medium physical activities? (Prompts: Can you name some activities for me? What activities do you do that are hard/medium physical activities?)

5. Do you think it's important to stay physically active? (Prompts: What is the importance of the information displayed on the model?)

6. What will you do with your 3D-printed model now? (Prompts: When you go home later today/tonight?) 
Study 2 - Sample Transcript: Secondary School Adolescents Focus Group

$I=$ Interviewer

$G=\operatorname{Girl}$

$B=$ Boy

[sample of transcript taken at start of Play-Doh modelling task]

I- so for this next task, I now want you to model your own physical activity object with Play-Doh. So, this is very similar to the last task we completed, but I want you to now mold a physical activity object. I am going to give you each a tub of Play-Doh and again, I'm going to give you two minutes to complete your model and then I will get you each one-by-one to explain your model to me.

B4 - oh that's why you asked if we collect something, is it?

I- yeah that was part of my interest to see if you collect something.... because you know, if it's something you collect maybe you can base it off something that's you know

B4 - yeah, I think I...

I- something that you do at the moment or something that you might collect in the future or...

B4 - I think if I had something like this... I think I would collect them just to see my progress and what happened there, I've done one wave

I- but yeah if you can think of an idea that's something that you won't get bored off that you'll keep wanting to have these models come back to you and keep on improving on them, making them change in the way they look, cause, as you yeah, as you know a $3 \mathrm{D}$ printer you can almost doing anything with it...and it just...it can just change the look or something by just changing numbers...make them bigger or smaller or

G1 - make a bar...bar chart

B4 - I think so if you had the wave scheme....if you had different colours to show, erm, which like your, which was a really appalling day...in contrast to like a good day like that shows that you met all your needs and the five times sixty

G1 - like that's what I was thinking like with those bars like...if you have like ...like say it was a day now....like I don't know if you could like with the tracker someway like say...we'll this is...going to be a less active hour because I'm in a lesson like...or would you be able to see that I don't know with different colours or something like that, I don't know...something like that, I don't know

I- $\mathrm{mm}$.

G1 - something like that

I- yeah no it could be colour schemed

B4 - yeah no that's.

G1 - yeah for like when you should be doing like well with like being active and when you should be....you know well what to expect if you're gonna be like working in a class your going to expect to be less active than if you're playing a game of netball or whatever...

I - yeah

G1 - just having fun with play-doh, really, I can't pull it up 
G3 - know it stinks though

I- keep modeling because we don't have a lot of time. So, what are you making here?

G2 - well, each ring represents a day but they don't necessarily have to be in order, it's just what if you rings are bigger...so it means you've done the most amount of exercise and its getting smaller and smaller like the least exercise, so it's like this could be like Thursday and this could be like Saturday...

I- ok, so the most active day will be the biggest ring always

G2 - yeah

1 - and the smallest ring will be the least active day...

G2 - yeah

I- then how do you, are they colour coded or, for each day

G3 - just go for it

G2 - yeah, yeah choice, different colour

I- so you'd know what the colours are for a day

G2 - yeah or whichever, it's like

G3 - what are you doing G1?

G1 - I don't know, I'm going to do bars | think

G3 - err...it broke

G1 - different colours

G3 - I was going to that but it would take me too much time so...
G1 - Can I adapt the idea I did on the paper, yeah

I- go for it! That's the idea that like you've drawn something you've kind of got an idea and now maybe...

I - $\quad$ so what have we got here then?

B2 - so it's a surfboard and then it has that idea of how much exercise you've done on it..

G3 - that's cool

I- ah ok

B2 - and then if your collecting it could be like different colours or different shapes...

I - so what... what the different shape of the surf board or...

B2 - yeah so, if you did like lots of exercise in one day you could have a bigger surfboard

I- ok

B2 - or something like that

I- so the surfboard would change in size and then you got the bar, zig zag which shows..

B2 - yeah

I - was that showing the hours?

B2 - yeah hours

I- ok, in the day and then the surfboard would show the total amount..

B2 - yeah 
I - for that day? And then maybe you get another surfboard that's smaller that day than the hour would be smaller as well

B2 - yeah

I- ok cool, alright and then for you you've got?

G3 - that one's just like, it's a line and it show this size, this side would be like when you're the most active and this side would be like when you're the least active

I- ok, so it's similar to your picture, is that?

G3 - yeah

I- so, you modeled your picture is that right ok..

G3 - yeah...just because I didn't have much time, what are those dots for (to B4's model)

B4 - this shows the highest peak and so you can look at it and you can think what you were doing at that time of the day and then like you can put it on the other day which I haven't and then this would show like the lowest peak but like it doesn't include sleeping..

1- does it go along, so is that, are they days

B4 - yeah

I- they are days aren't they, ok
B4 - and its just think about like...

I- so that would be time like so starting off on the curb you'd say you woke up at seven...and then the black dot would show? Your highest point of activity

B4 - yeah and like so you can look and reflect on what you were doing then, and see what and how you could, maybe do something like that in the days where you haven't done as much...

I - yeah, so yeah

G1 - so this is really similar to the bars but instead cause I didn't know how to, model it like that...l just did like if it's a bigger circle then...you...your most active if its red then your sleeping, well it hasn't necessarily got to be coloured for something like that so you know you're not going to be very active then and then like yeah this would be like not as active but you should be active...if that makes sense like...

I- oh ok, and that was for, is that a whole day?

G1 - yeah well...it's a bit of a day but yes, a day potentially

I- ok, cool and then we kind of talked about yours didn't we with the...you haven't adapted it in any way or...

G2 - not really...

I - ok, so if you can leave all your models there, we'll finish there. 


\section{Study 2 - Sample Transcript: Primary School Teacher Interview}

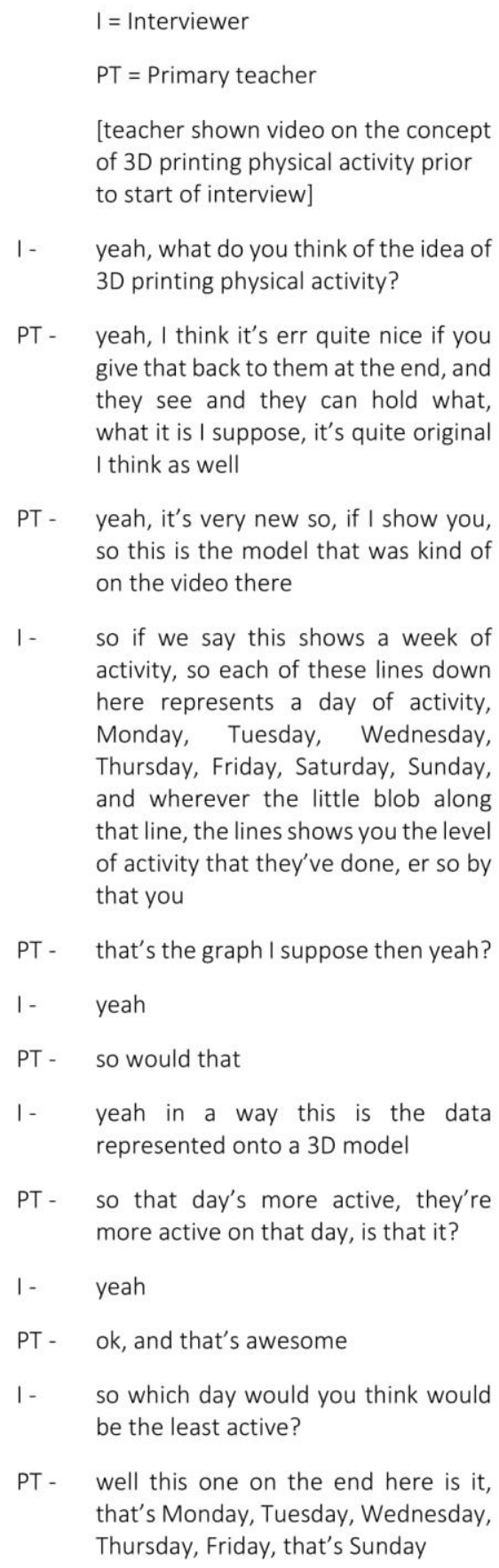

PT - yeah, I think it's err quite nice if you give that back to them at the end, and they see and they can hold what, what it is I suppose, it's quite original I think as well

PT - yeah, it's very new so, if I show you, so this is the model that was kind of on the video there

1- so if we say this shows a week of activity, so each of these lines down here represents a day of activity, Monday, Tuesday, Wednesday, Thursday, Friday, Saturday, Sunday, and wherever the little blob along that line, the lines shows you the level of activity that they've done, er so by that you

PT - that's the graph I suppose then yeah?

1- yeah

PT - so would that

1- yeah in a way this is the data represented onto a 3D model

PT - so that day's more active, they're more active on that day, is that it?

I- yeah

PT - ok, and that's awesome

1- so which day would you think would be the least active?

PT - well this one on the end here is it, that's Monday, Tuesday, Wednesday, Thursday, Friday, that's Sunday

1- yeah, yeah, so for someone they could look at that if it was a kid or whatever, and they, they

PT - that's brilliant

I- they could see

PT - you know for data handling and things like that, which they do, is something completely different then to be able to feel it

I- yeah

PT - and hold it, I think that's brilliant

I- and yeah, so you think the kids would kind of engage with this kind of idea?

PT - oh absolutely, you know they love um, they love technology and they, we're using it a lot more in schools now whereas this is, this is another step up you know it's fantastic

I- yeah, I've got another two I'll quickly just show you, so again we've got a week, a week of activity, so each of these bubbles is a day of activity, so it

PT - obviously that's the most active then yeah

I- yeah

PT - yeah that's cool as well, I like that design

1 - and then this one shows a day of activity

PT - oh ok

I- so say each of these spikes represents an hour of activity, so you know you could say that the kid woke up at seven, er and then hopefully they could count around from the hours and pin point exactly when they took part in you know playing football or

PT - yeah, like all three of those models, I think they're brilliant 
I- um, so yeah do you think this would motivate the children to be more physically active if they received these models, of personalised models of their own activity

PT - yeah I think so, I think it would make them um far more aware of how active they actually are and you know they could compare themselves with other children in the classroom, yeah I think it could motivate them definitely, they definitely, they would really enjoy the process of getting to that you know and then having a look at that and just to be able to hold it

1 - yep

PT - and see, I think it could motivated them

1- ok, and do you think um the boys and girls would react differently to them or?

PT - I feel like I'm very biased with the boys, but maybe, maybe th boys might be a little bit more interested, but I think, I think across the board they'd enjoy that, they'd be intrigued by that

I- $\quad$ yeah, um and then do you think you could see this being something being taught in the school curriculum that they could take part in, something the school could um

PT - what, with those monitors, that show in the video, like that is it? The whole process?

I- yeah, something like that or

PT - yeah

I- or you could have this in a PE lesson as a, as a lesson to, yeah get the kids to understand what physical activity is
PT - oh yeah, I think, I think that would be fantastic, you've got so much in that, yes it's a PE lesson but you've got maths, science, it's brilliant

I - yeah

PT - yeah definitely

1- and then, just out of this, have you got any kind of ideas of models yourself that you think would help the children to be active, have you got any thoughts of what models you think would work best for the kids? Not even out of these three, if you've got one of your own idea of a model that might work for them um, so the idea is that it could be anything that we could put the data onto, so for instance, over here l've got a frog, so say you know we put the data activity onto a frog and something happened to the frog, you know it increased in size in some kind of way, to show the physical activity, is there some other ideas that you can imagine that might

PT - oh wow, that's a cool idea

I- (laughs)

PT - I can't think of any really, I mean

I- is there anything that the kids, you can see are collecting at the moment that you know we could kind of follow or copy

PT - oh a certain model?

I - yeah

PT - I mean I like all three of these, and I didn't know you could do it like that, so you could take a, a model of something like that and distort it with the data

I- yeah, we can manipulate pretty much anything and have it so the data, you know the data that you collect with how active they are 
PT - yeah

I - you can then put it onto any kind of shape and maybe something happens to it, so the more vigorous activity they do

PT - maybe, maybe I don't know perhaps a 3D model of the people, like a, you could have a, you know if you can do that with a frog and distort the frog with the data, then could you use I don't know a child's, sort of, you know like a model of a child perhaps

I- ok, like a human figure

PT - yeah, figure, that's the word, yeah

I- ok

PT - maybe, I didn't realise you could do that

I- $\quad \mathrm{mm}, \mathrm{umm}$, and then do you, do you think there are any, so we've got a week here and a day here, do you think there are any benefits in showing a day or a week of activity, or any, or do you think there's another way that we can show the data in terms of time?

PT - well I think obviously you could do a long terms study couldn't you for perhaps a year but then that's, I think the children, especially the younger children would probably, maybe lose, lose their focus with it, I think a week, a week is great, and you could do that over a couple of weeks then perhaps

I- $\quad$ so as in they receive the models every week?

PT - yeah

I- and they get a weekly, you know image of their activity?

PT - yeah, and then obviously they're trying to be, I suppose you're giving them the motivation by showing them this at the end of the first week and they're being motivated then aren't they to perhaps be more active and to see the results in the following week and so on then, constantly trying to become more active really aren't they, and you know at the end you could, they could see at the end of five or seeks weeks, over half term they could place their models and compare them then couldn't then, then you're definitely motivating them, or you could do the same with the day perhaps, you know, but then that, I think a week works best isn't it, you've have that, you can print it out end of the week, hand it to the children and then they can try and improve it the following week

I- um, I mean in the future could you see the school investing in a 3D printer, with this kind of?

PT - how much are they?

I- (laughs) yeah, they're actually, commercially they are coming down in price, so for you know a standard one you'd be looking at about two grand

PT - yeah, I could see that happening, um perhaps not this year or

I- yeah, yeah

PT - but yeah in the future I think so, in the near future

I- ok um

PT - because it's all about trying to excite the children isn't it, and getting them interested

1- yeah

PT - whatever you're trying to teach them really, and you know technology is one way that definitely excites them 
and this is, and this is amazing, this is fantastic so, yeah

I- and then what do you think, we're just coming to the last few questions here, but what do you think of maybe a class model of activity

PT - oh right

I - so we combine you know the whole class's activity data together to produce a bigger, one model

PT - and compare classes then?

I- yeah you could, yeah

PT - yeah that would work, that would definitely work

I - ok, do you think there's a, one way is better than another, or do you think both would be?

PT - obviously it's difficult because in, how would you be recording this data, they're time in, it's jus their time in school I suppose isn't in?

I- $\quad$ um so if we were to get a week, we'd have to get $a$, they'd have to keep this band on

PT - for a whole week?

1- or day

PT - so when they go home as well then?

I- so when they go home, because there's times when they, you know they can take them off, but yeah, be measuring for that whole week

PT - I think it would be great, could use it as a, you know physical activity, PE awareness week or however you wanted to phrase it, but yeah I didn't realise yeah if they took it home then you could compare classes, from, like we do with healthy schools we compare what they eat, and for a week or whatever you could do that with the class as well, compare classes then

I- yeah

PT - um

I - so that would be something that you think you could see the school being interested to take part in?

PT - yeah I think so

I- yeah

PT - in terms of sort of next year is it?

I - yeah, the next academic year

PT - yeah, if not a whole school thing then definitely a class

1 - yep

PT - one class could do it, maybe mine

I - ok (laughs) I mean my last question here is, yeah what do you think about introducing this into a school, so you know into the school, is there any problems or advantages that you could see?

PT - any problems with this?

I- yeah

PT - well do we need to purchase a 3D printer for it? Or would

I - no, I think, because we have a 3D printer, we're still in, deciding how the intervention's going to work

PT - ok

I- but yeah I can tell you more about that

PT - yeah I can't see any problems with it, I think lots of teachers would be enthusiastic about it, I think parents would be interested as well, um parents are quite supportive here 
1- yeah

PT - um in terms of new things like this, I think it could happen, I don't see any problems with it, you know they take, they wear the belt for the day, they take it home then and they just bring it into school I suppose is it?

1- yeah

PT - yeah that's fine

I- ok, is there anything that I might have missed out, any other questions that you can think of?

PT - no, I think there's a lot of scope here for what you could do with it, like you mention um, it's hard for me to think of ideas because I don't know what the limitations are with it, if there are any really, but you could do a class one, you could do just pupils in a class, you could compare the whole school

I- yeah
PT - you could do staff in school

I- yeah

PT - and all models I mean I, I suppose onto those three models, and I didn't realise you could distort the figure like that

1- mm, actually that's interesting because you said staff, is it something that you think would be beneficial if the staff and the children took part?

PT - yeah I think so, you know lots of staff have the Fitbits on you know and um and this would be interesting you know if the staff got involved, the children like that as well, you know oh compare sir's what do you call that, model I suppose to our own, that would be nice to compare as well

I - wicked, that's all, brilliant

PT - cool, thank you

1- alright 
Study 3 - Sample Transcript: Primary School Child Interview

I = Interviewer

B2 $=$ Boy participant

[sample of transcript taken from start of matching activities to intensities task]

I- now what I want you to do is match the activity to the different physical activity level box.

B2 - what's he doing here [mobile phone, sitting]? Ah he's playing a game, inactive, reading a book a bit harder, stretching, walking to school playing on video games throwing, snowballs, washing, throwing darts, walking, climbing a tree. Again, playing on a computer game, swimming...it's a bit hard to learn how to swim. Why am I leaving all the hard ones to last?

I - well you can just...put them all on.

B2 - I'm done, fishing is the last one.

I- cool, right, so if you could briefly just give me a quick tour of why you put these activities in the boxes

B2 - these [points at light-intensity box] are quite easy cause all you're doing is basically moving your fingers

I- yeah

B2 - this is light [intensity] because eating food is easy, throwing darts is pretty easy but not to hit the middle.

I- hmm...true

B2 - throwing snowballs is quite easy because you can just throw them any way you like. Mowing the lawn...my dad makes me do that [all placed in light-intensity box]
I- really? Yeah, ok, my dad used to make me do it as well

B2 - ...and sweeping because you can relax while you're doing it. Throwing darts, you can just go like that, snowballs you can just go like that [all placed into light-intensity box].

I- yeah

B2 - eating, you can just go like that and sweeping all you've got to do is this so it's quite easy [placed into lightintensity box].

I- yeah

B2 - moderate [intensity], fishing, quite hard to catch a fish. Reading you've got to be able to read. Stretching, it's pretty easy [all placed in moderate-intensity box]. This one I think I need to put that one there [re-placed into light-intensity box]

I- Ok...so putting stretching into...?

B2 - yeah, stretching into there [lightintensity box], walking up steps, it's not very far to walk so I'm putting that as light [intensity]. Climbing a tree, you're not going to climb a really high tree, really, trees can be small [placed in moderate-intensity box]. And walking to school, you can't, you can live right next to a school, so sometimes, yeah [placed in light-intensity box]

1- yeah, and then over here in the vigorous-intensity box?

B2 - vigorous, stretching, playing football, swimming, hockey, running, tennis and biking, they're all, you've got to move quite a lot during. 


\section{Study 3 - Sample Transcript: Secondary School Adolescent Interview}

I = Interviewer

B1 = Boy participant

[sample of transcript taken from start of matching activities to intensities task]

I- now what I want you to do is match the activity to the different physical activity level box. Once you have completed that I will then ask to describe why you have placed each activity in that physical activity box.

B1 - yeah

I - brilliant, cool, now I'm just wondering if you could talk me through why each activity is in the specific boxes?

B1 - yeah well obviously computer games you're not, you're not doing much except moving your fingers maybe. And reading a book all you're doing is just flipping a page with almost nothing movement [all placed in sedentary box], just like that [acts out turning a page]

I- yeah

B1 - eating, maybe just a little bit of movement when you 're like bringing it up to your mouth and then when you're chewing, and then other than that it's not much [placed in lightintensity box]. But brushing [the floor] I'd say maybe a bit less because all you're doing is just one movement over and over again, and it's not a wide range of different movements and activity [placed in light-intensity box].

I- ok, yeah

B1 - walking to school you do need to walk obviously but it's not very hard [vigorous] it's just very, probably a small amount of distance.

1 - yeah

B1 - throwing a snowball not much at all, all you have to do is just craft this little ball of precipitation and throw it at someone else. Darts, all you're doing is just throwing a small dart at a small target. Stretching, even though it is vital I'd say it's not as hard as other sports [all placed in light-intensity box]

I - yeah

B1 - now mowing the lawn, you're just walking around, just almost doing little movement. Fishing you're just waiting in a boat and when a fish comes you have to reel it in, and stuff and it might get a bit harder than just sitting in a boat. That's why I didn't put it in sedentary [all placed in lightintensity box].

I - yeah

B1 - walking upstairs you have to use different, a variety of muscles to get up the stairs and different movements [placed in moderateintensity box]. Climbing up a tree you need different types of, what's it called? You need your movements to be more balanced and stuff like that [placed in moderate-intensity box]. A girl playing hockey you need to run around the pitch many times and it might get a bit tiring. Football's the same and running for long distances can be vigorous but short distances it's just a short sprint and you get tired but not as tired as you would be for a longer run [all placed in vigorous-intensity box].

I- yeah, and tennis? 
B1 - tennis I'd say is vigorous because you need to get more balance while you're in it [playing] and you need to run across the pitch to be able to actually, reach the ball.

1- yeah

B1 - you use your other arm muscles to hit it over as well [playing tennis]. Cycling, especially if you're on a road bike, you would have to use most of your leg muscles and try to stay streamlined and it would, especially if it's a longer distance than you're sprinting, it would be very vigorous cause it would hurt a lot, from experience, using most of your leg muscles. Swimming, you have to be able to do the right streamlined technique to be able to glide through the water and then you'd like, you need to be able to breathe but you need to make many movements in like one length, just to be able to do that. You need to be, if you're in a race especially you need to time your breathing. 


\section{Study 4 - Sample Transcript: Primary School Child Short Interview}

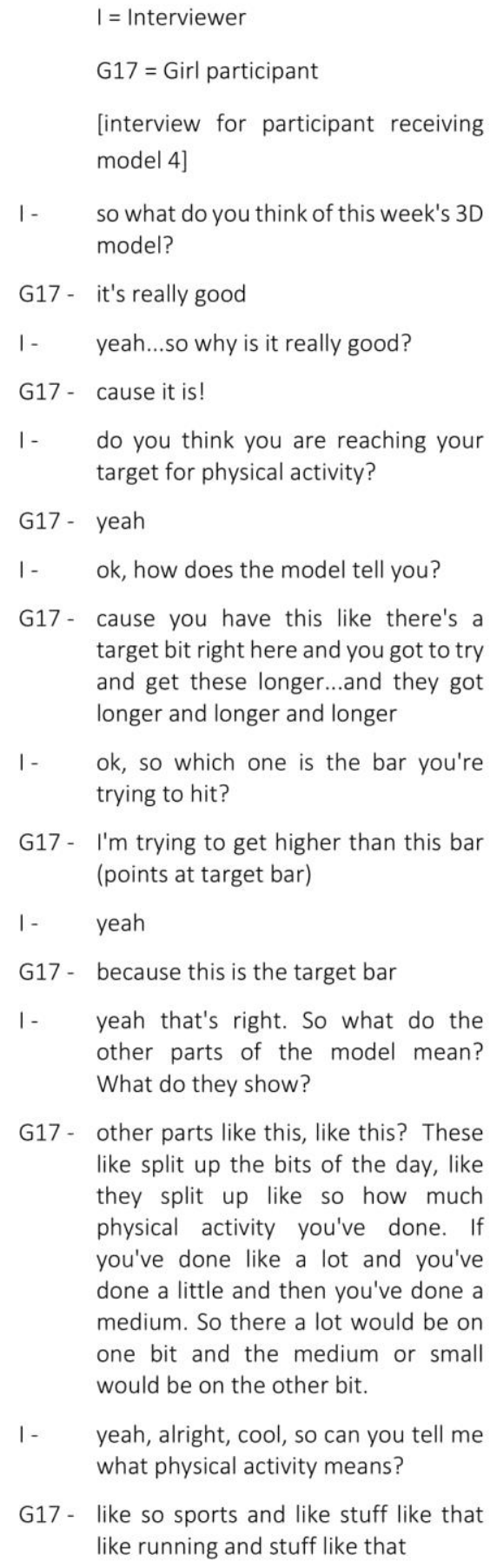

G17 - cause you have this like there's a target bit right here and you got to try and get these longer...and they got longer and longer and longer

I- ok, so which one is the bar you're trying to hit?

G17 - I'm trying to get higher than this bar (points at target bar)

1- yeah

G17 - because this is the target bar

I- yeah that's right. So what do the other parts of the model mean? What do they show?

G17 - other parts like this, like this? These like split up the bits of the day, like they split up like so how much physical activity you've done. If you've done like a lot and you've done a little and then you've done a medium. So there a lot would be on one bit and the medium or small would be on the other bit.

I- yeah, alright, cool, so can you tell me what physical activity means?

G17 - like so sports and like stuff like that like running and stuff like that

1- yeah, so what are those vigorous levels of physical activity?

G17 - like running a really long way or like running a race where they race really hard

I- then what about those moderate levels of physical activity?

G17 - running a race and coming second or in like running a little bit of a long way

I- So since receiving these models have your thoughts changed on how important it is to stay active?

G17 - erm...

I- do you think it's important to stay active? Physically active?

G17 - yeah

I- so why do you think it's important?

G17 - because it's important to stay active to be healthy

I- ok, and are there any more reasons why it is important to stay fit and healthy? What does it do to you?

G17 - because it's good for you

I - yeah, ok

G17 - and makes you feel really strong

I- yeah, ok, so what will you do with this new $3 \mathrm{D}$ model?

G17 - I'll keep it with the others so I can compare it and I might put them on my window sill

I- yeah, cool. And why would it be helpful to compare them?

G17 - I'll put my first, like my first one at the bottom [of the other 3D models] and like put them all in a pile and see which one's the biggest.

I- alright, we'll finish there 
Study 4 - Sample Transcript: Secondary School Adolescent Short Interview

\author{
I = Interviewer \\ B17 = Boy participant \\ [interview for participant receiving \\ model 4] \\ I- so, what do you think of your final \\ model for physical activity? \\ B17 - I say I could've done better by \\ wearing it every day. But like, \\ because I do a lot of contact sport, \\ I've got to take it off. \\ 1- ok, yeah. And so, do you think you \\ reached your target for physical \\ activity though? \\ B17 - on about two days I think, I reckon I \\ reached my target. \\ I- and so how do you know if you \\ reached your target? \\ B17 - by the target bar which is the arrow \\ I- ok and how much does the target bar \\ represent \\ B17 - about an hour of physical activity \\ every day \\ 1- ok, so which one is the bar you're \\ trying to hit? \\ B17 - I'm trying to get higher than this bar \\ (points at target bar) \\ I- what do the other bars tell you? \\ B17 - well theirs a bar in front of that, that \\ bar is harder physical activity, and the \\ other ones not \\ I- So, what is physical activity? You tell \\ me \\ B17 - exercise, moving around and sort of \\ 1- so what are vigorous levels of \\ physical activity? \\ B17 - sprinting, triathlons and stuff like that \\ I- and what about the moderate levels \\ of physical activity? \\ B17 - everyday walking around \\ I- since you've seen these models, have \\ your views and thoughts changed on \\ the importance of being physically \\ active? \\ B17 - I think you still have to be physical \\ and active to stay fit and healthy \\ I- so what will you do with your final \\ model now? \\ B17 - compare it to the others on the self \\ I- ok, and how will that help you? \\ B17 - to see what, how l've improved with \\ my physical activity and what I need \\ to keep on doing, what l've done \\ I- Yeah, ok, cool, we will finish there.
}


Appendix V: 3D Model Designs

Study 1 - Prototype 3D Models

\section{Bubbles}

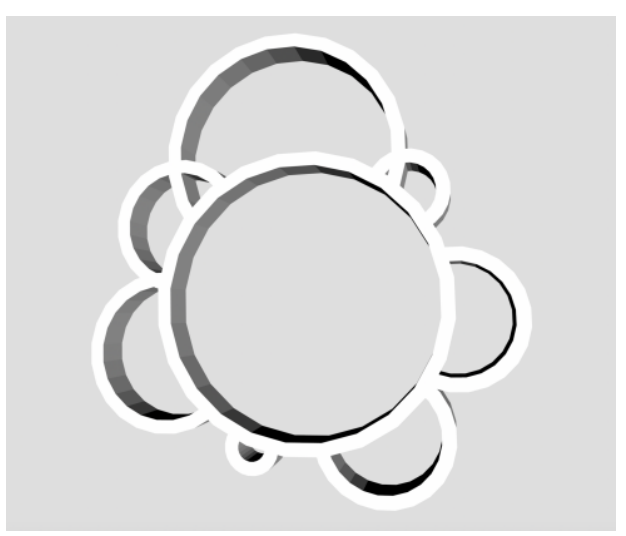

Heart

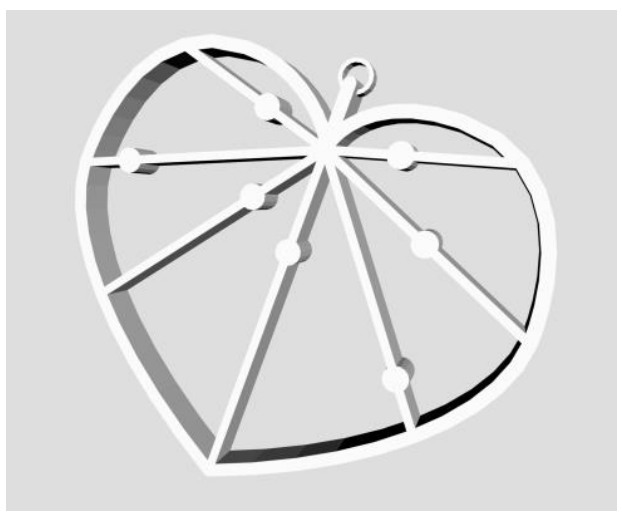

Flower

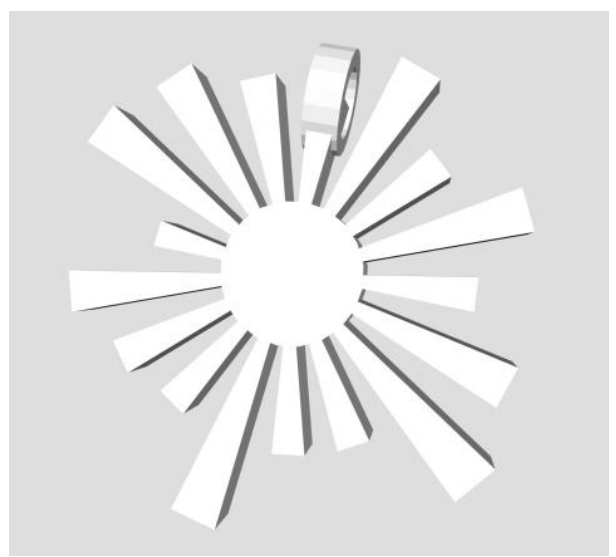




\section{Adolescents Bar Chart}

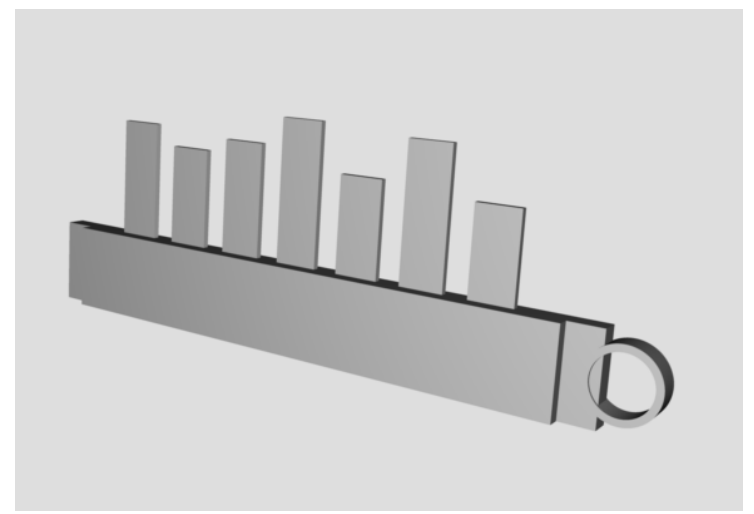

\section{Children's Bubbles 2}

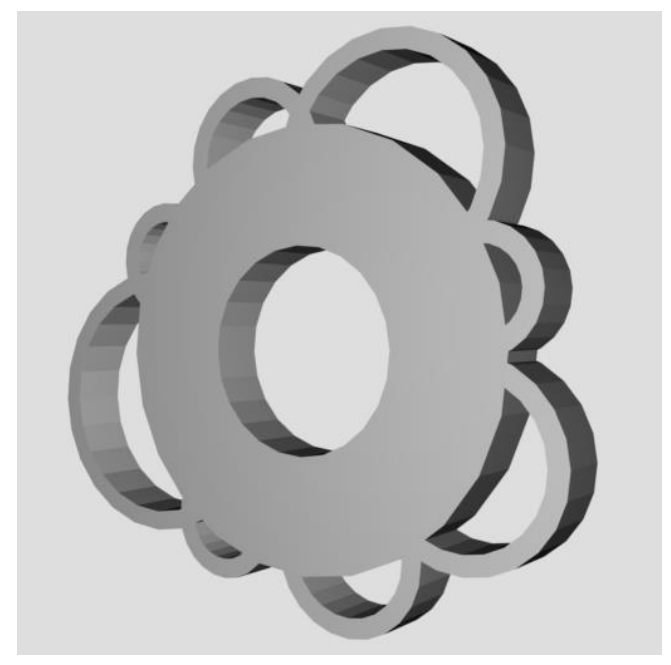

\section{Children's Star}

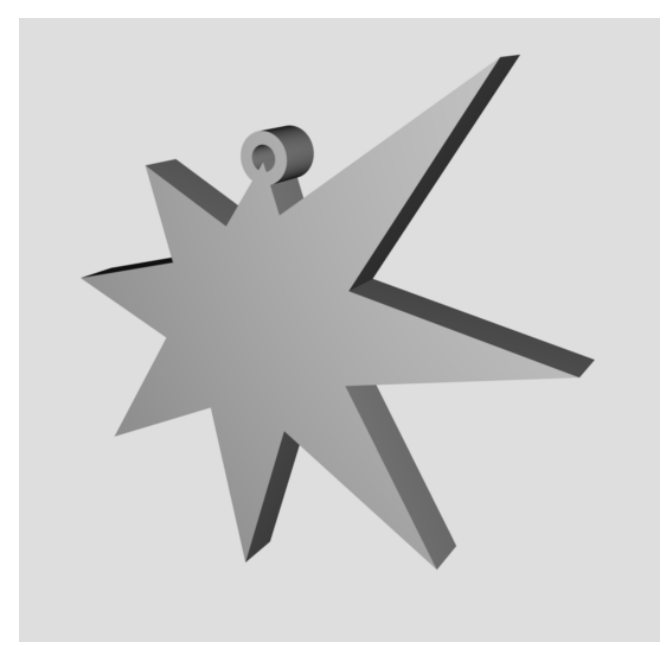


Children's Sun 1

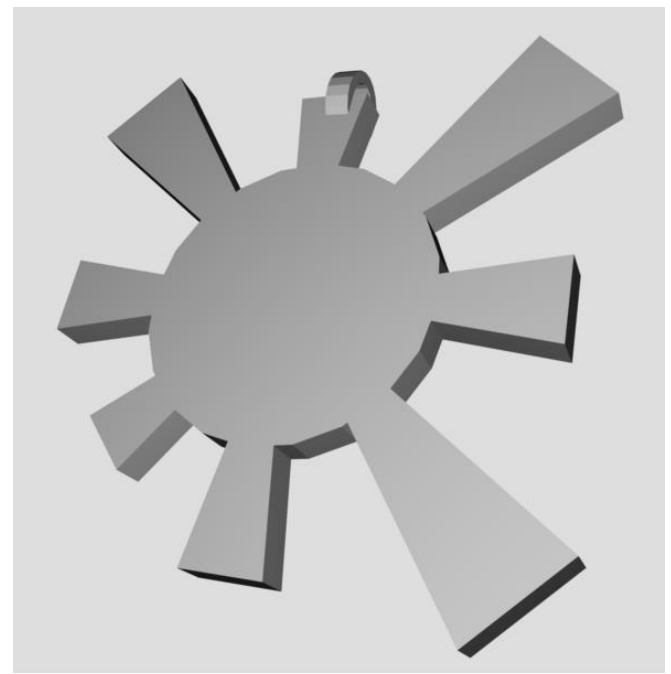

Children's Sun 2

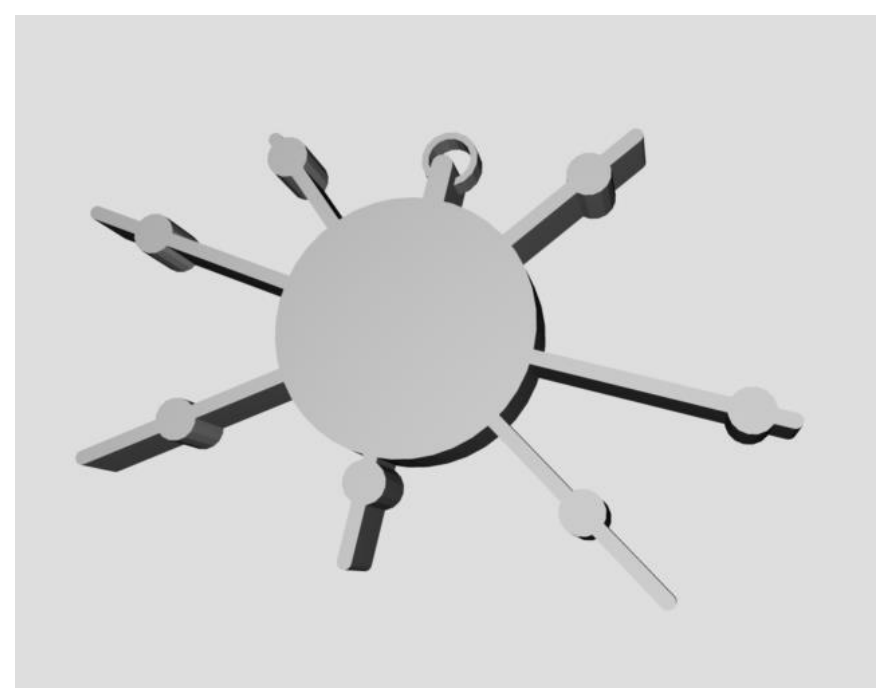




\section{Bar Chart Design}

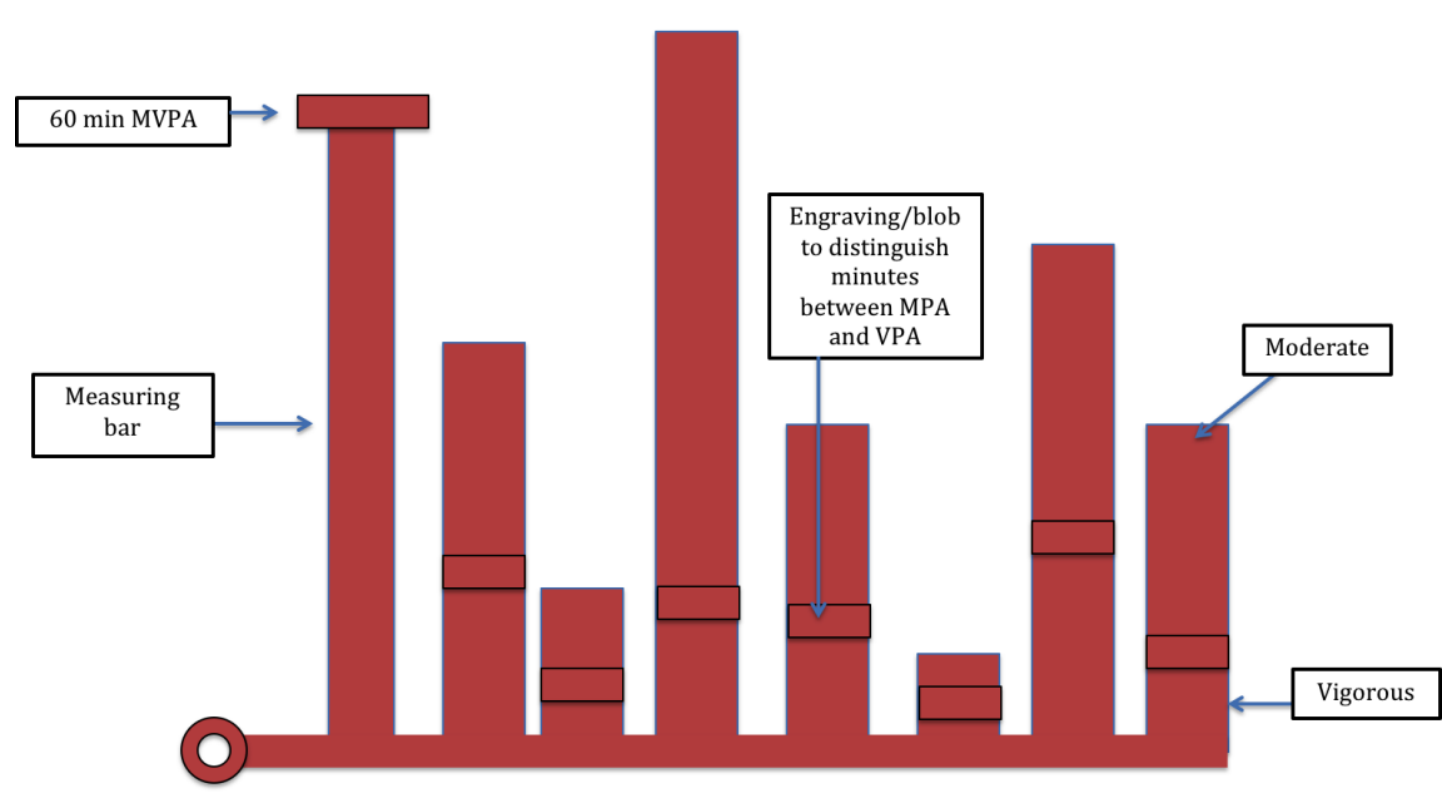

Human Figure Chart Design

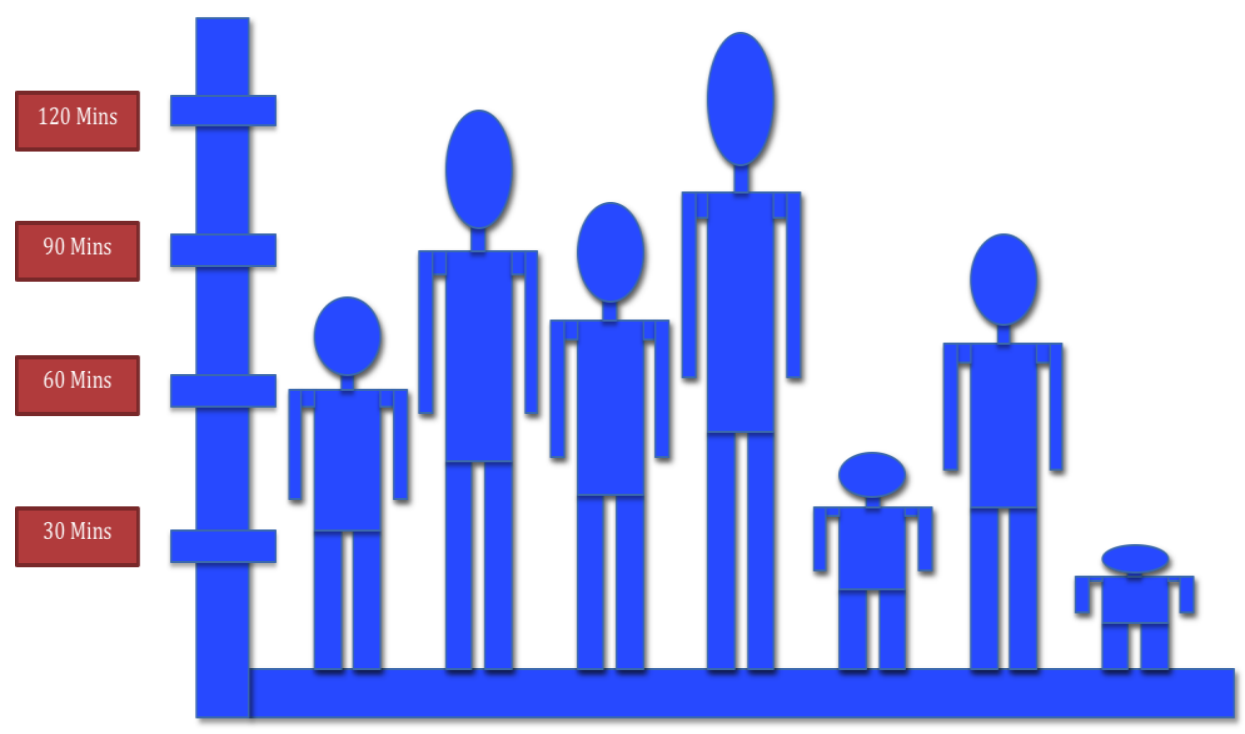




\section{Sun Design}

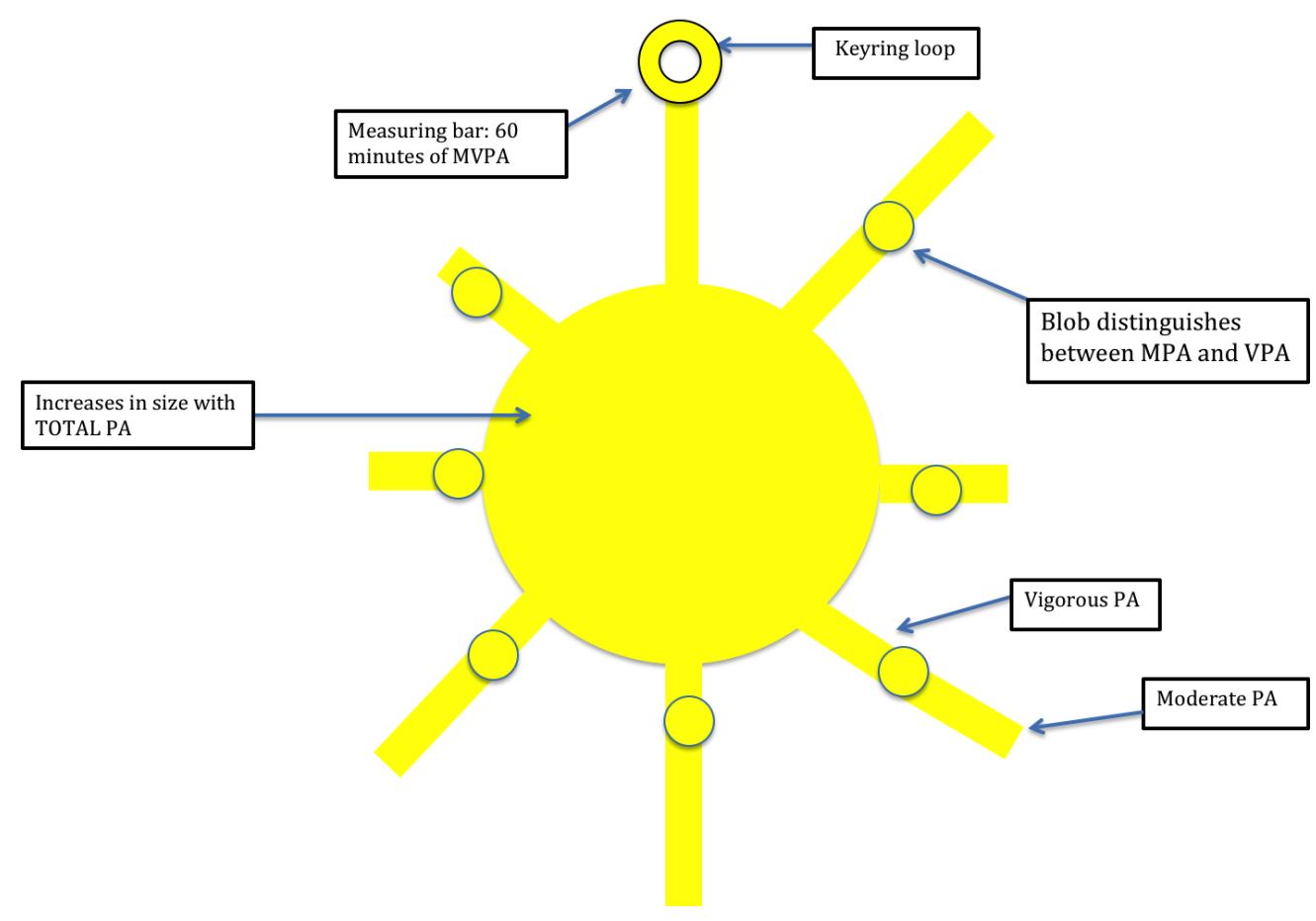

Star Design

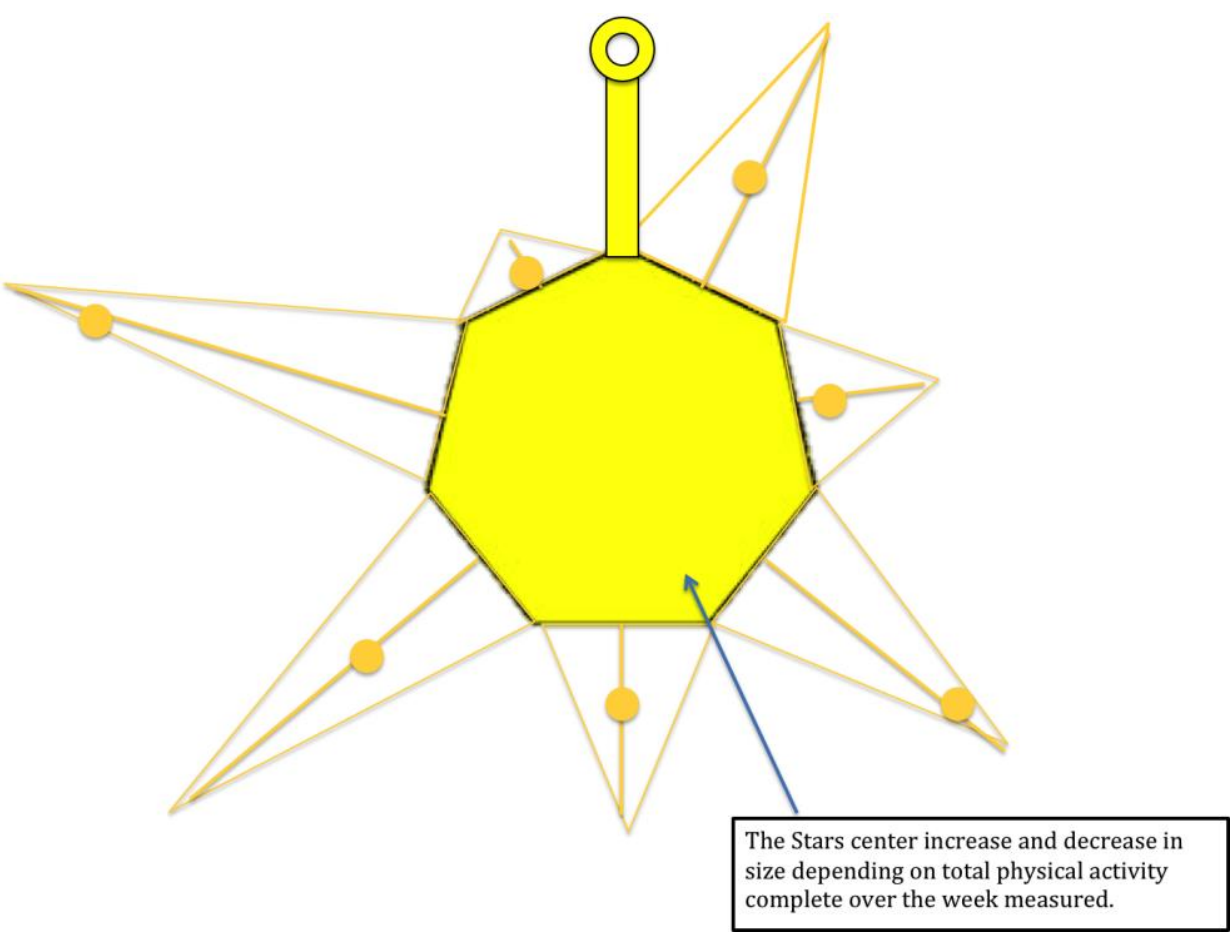


Study 1 - Children's Play-Doh Model Designs

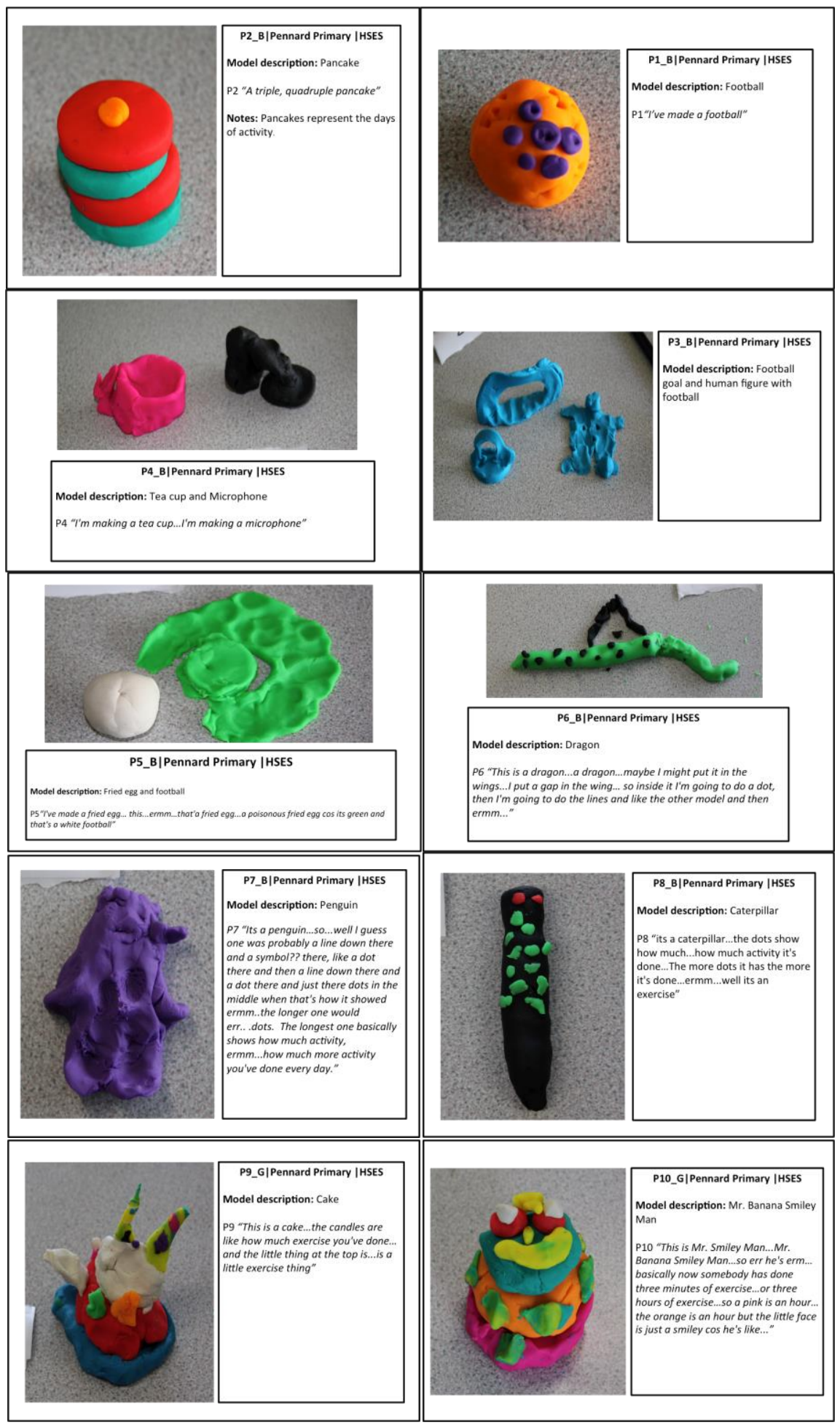




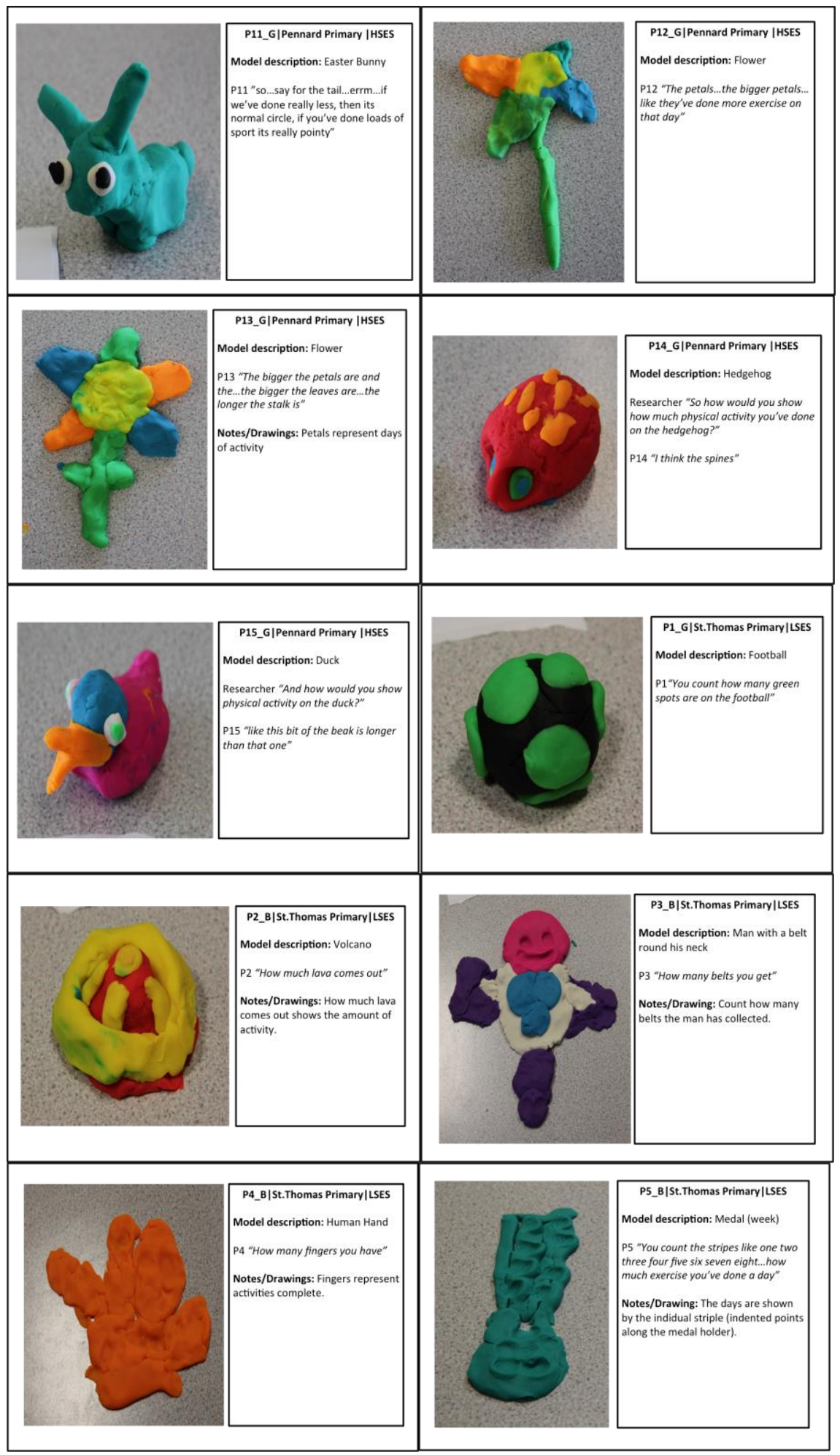




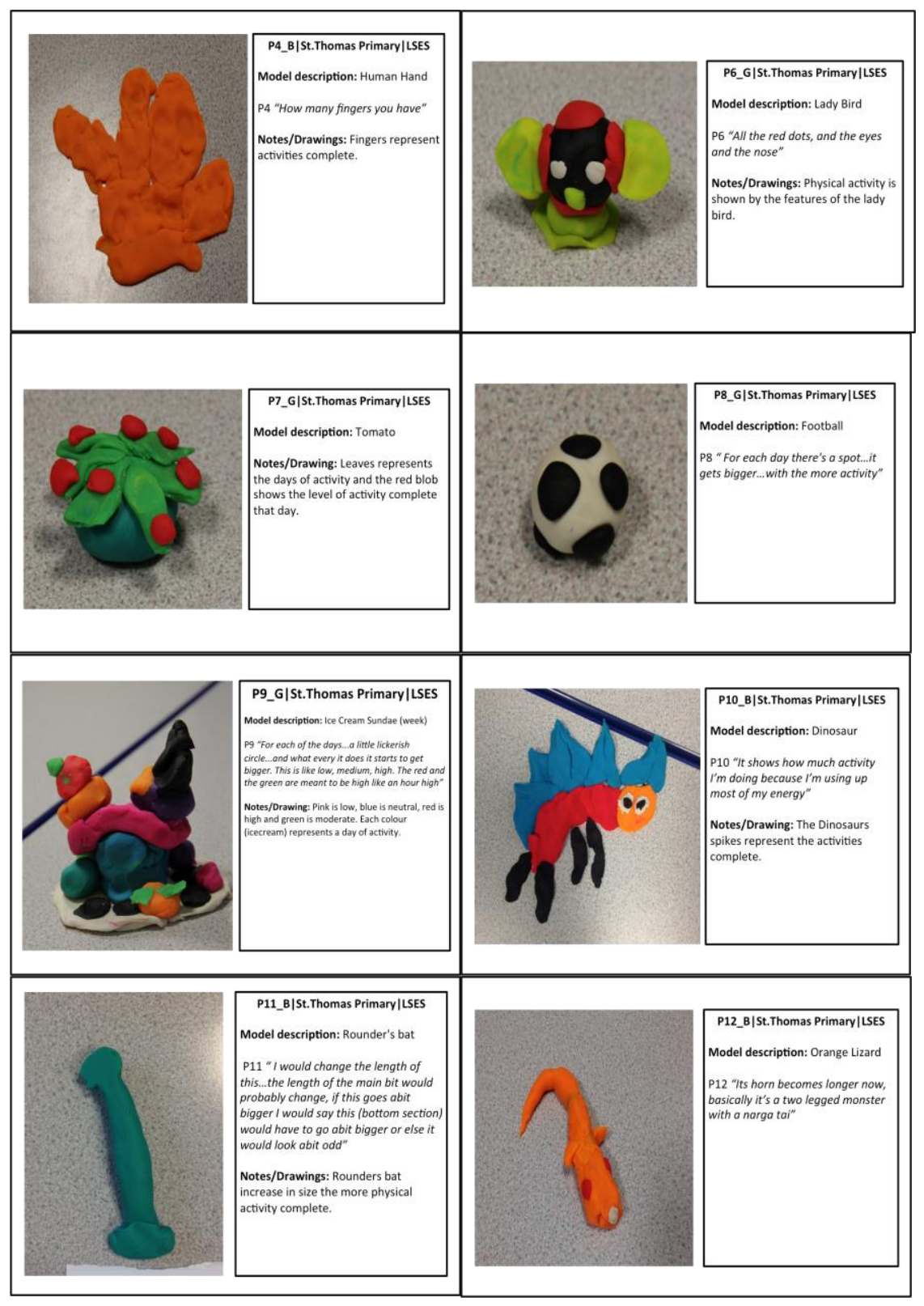




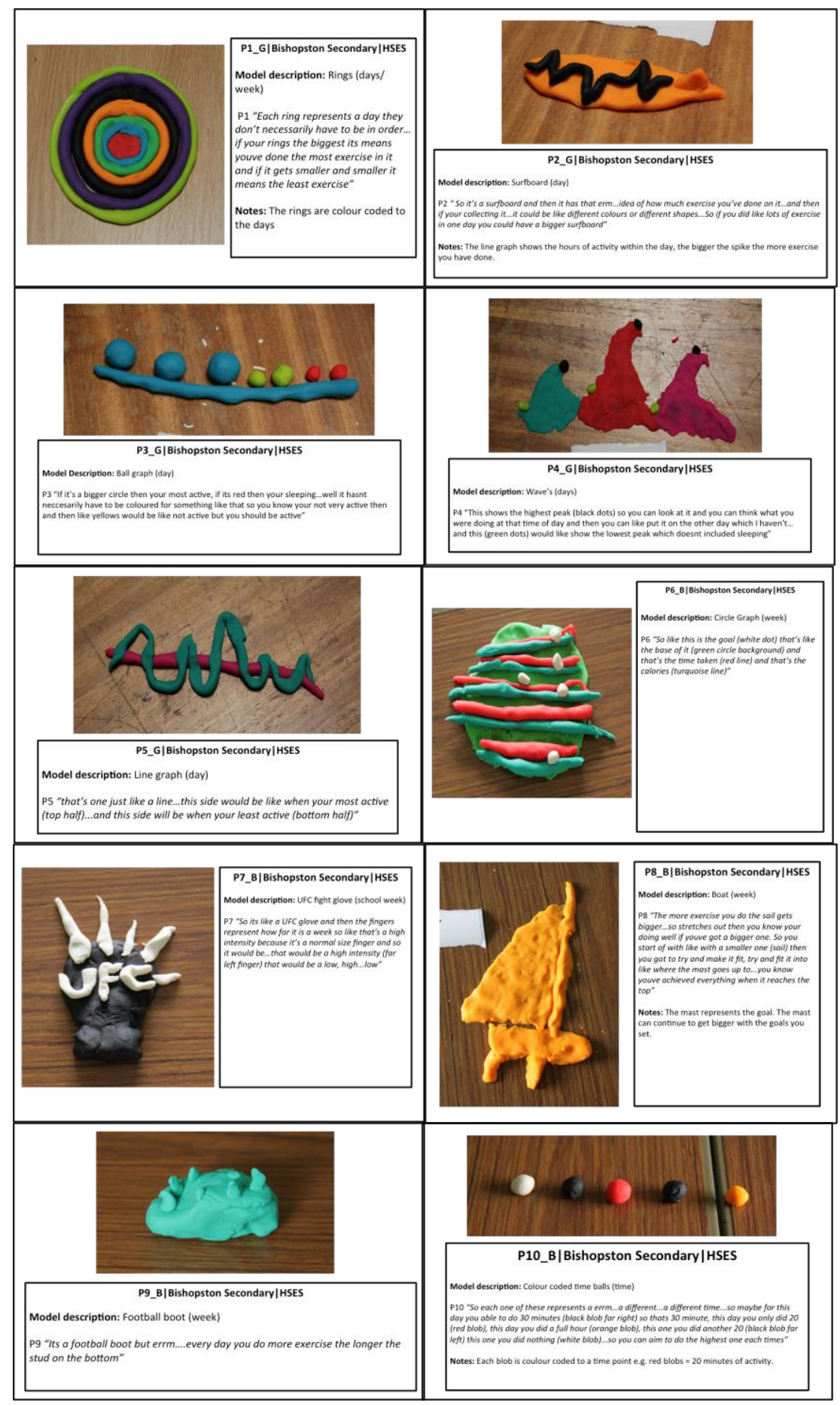




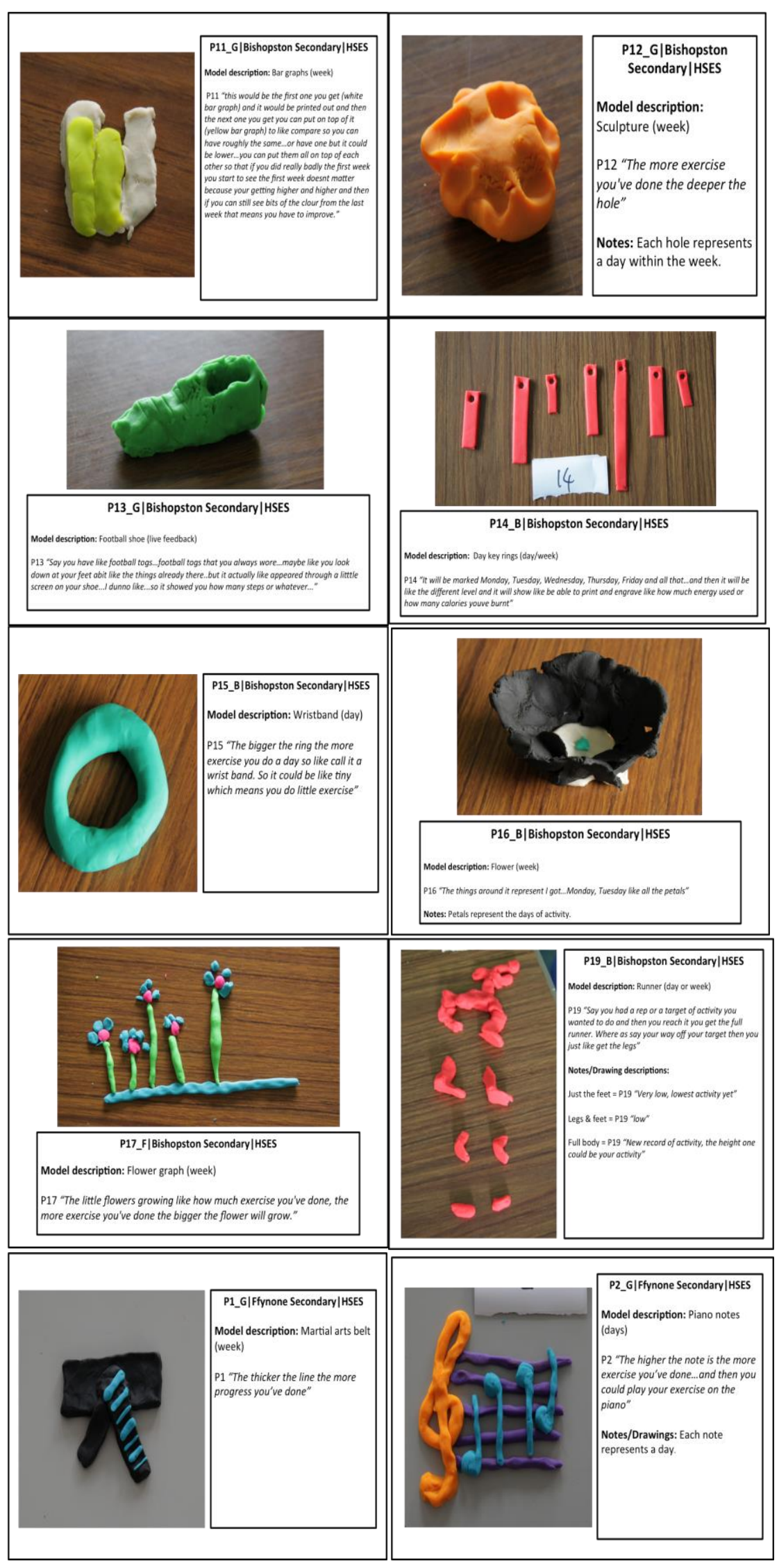




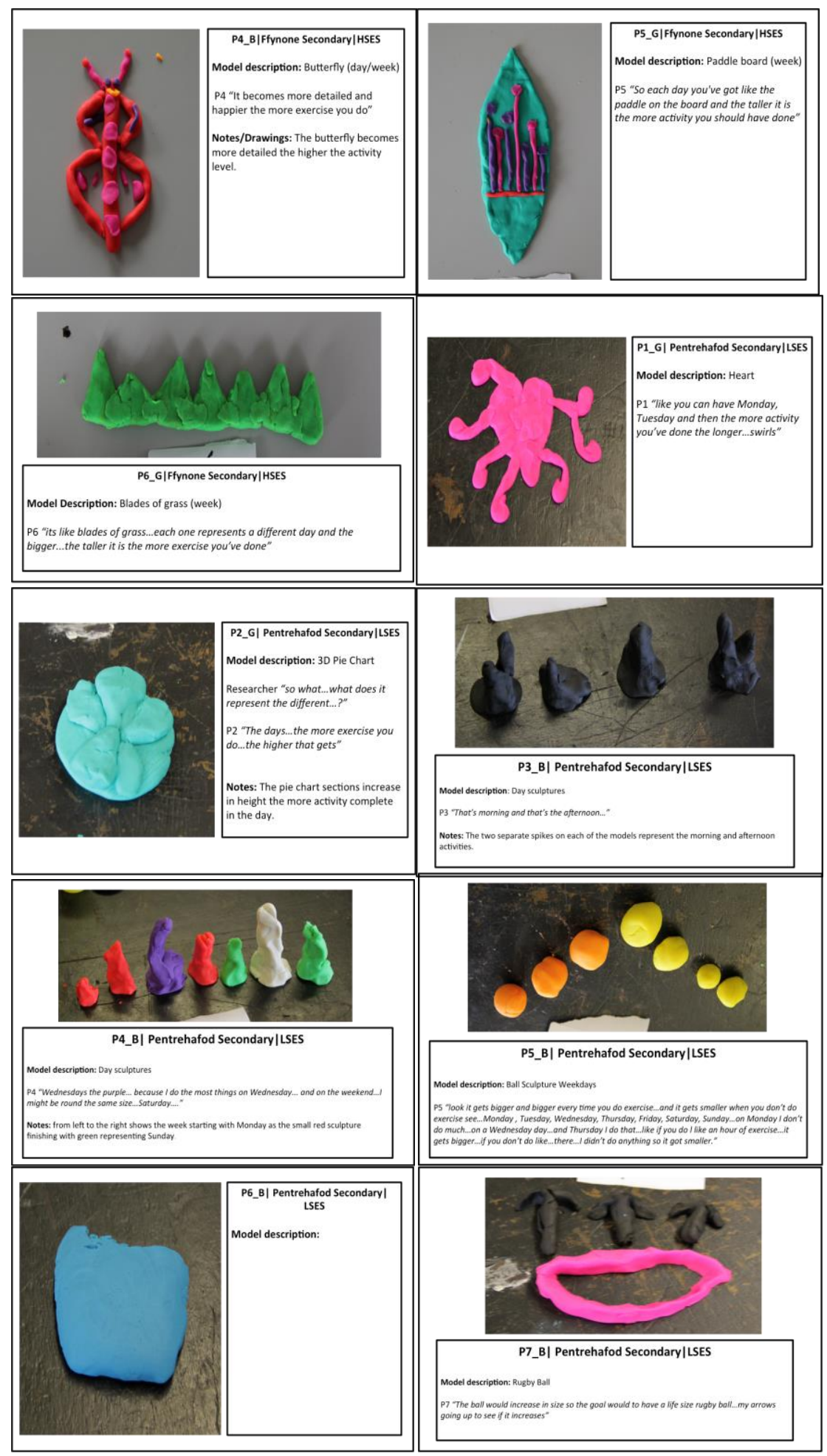




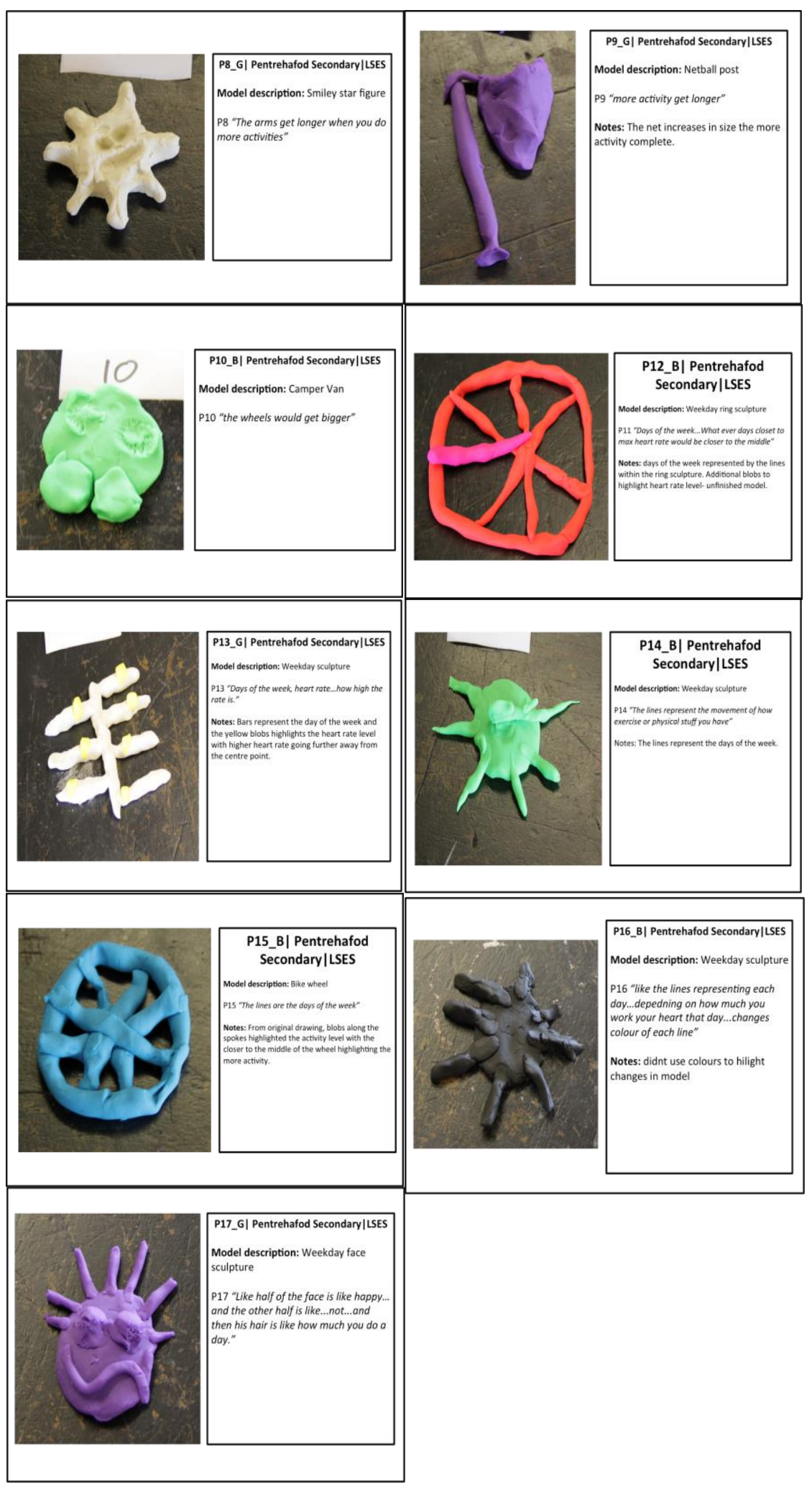




\section{Appendix VI: Scientific Outputs}

\section{ISBNPA 2017 Poster Presentation}

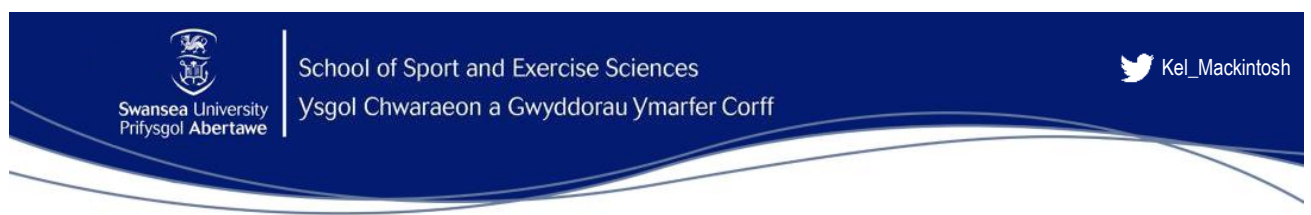

\section{Perceptions of Visualising Children's Physical Activity as a 3D Object}

Mackintosh, K.A., ${ }^{1}$ Crossley, S.G ${ }^{1}$. , Eslambolchilar, P., ${ }^{2}$ Knowles, Z.R. ${ }^{3}$, Hudson, ${ }^{\prime},{ }^{1}$ \& McNarry, M.A. ${ }^{1}$

${ }^{1}$ Applied Sports, Technology, Exercise and Medicine Research Centre, Swansea University, UK; ${ }^{2}$ School of Computer Science and Informatics, Cardiff University, UK; ${ }^{3}$ Physical Activity Exchange, Liverpool John Moores University, UK

\section{Introduction}

- The majority of children fail to meet current physical activity (PA) guidelines of 60 minutes moderate-to-vigorous PA every day [1]

- A frequently cited barrier to meeting these guidelines is that they are difficult to measure, interpret and apply.

- 3D printing enables the creation of a tangible output, providing a novel and exciting way to conceptualise children's physical activity levels (PAL).

- Therefore, the purpose of the study was to elicit children's subjective views regarding the interpretation of 3D PA models and to develop a user-informed 3D model of PA.

\section{Methods}

- Twenty-eight primary school (15 boys, $8.4 \pm 0.3$ years) and forty-two secondary school-children ( 22 boys, $14.4 \pm 0.3$ years) took part.

- Semi-structured focus groups were conducted to investigate children's perceptions regarding PAL, intensities, motivation and the potential use of 3D printing to represent PA.

- Subsequently, children created a 3D Play-Doh ${ }^{\oplus}$ model of their PAL.

- Data were transcribed verbatim and subsequently analysed inductively.

Pen profiles were constructed representing analysis outcomes via a diagram of key emergent themes.

Results
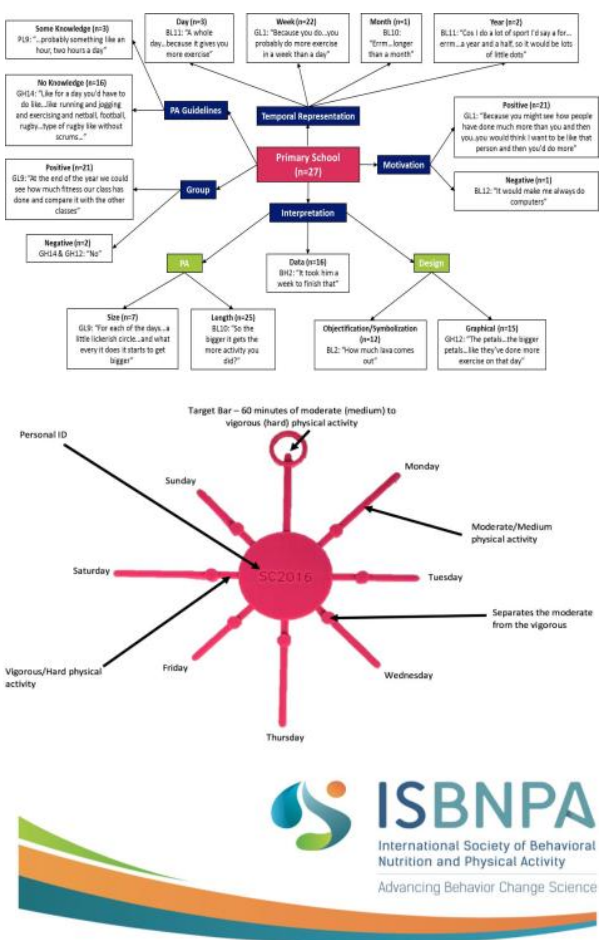

VICTORIA CANADA 7-10 JUNE
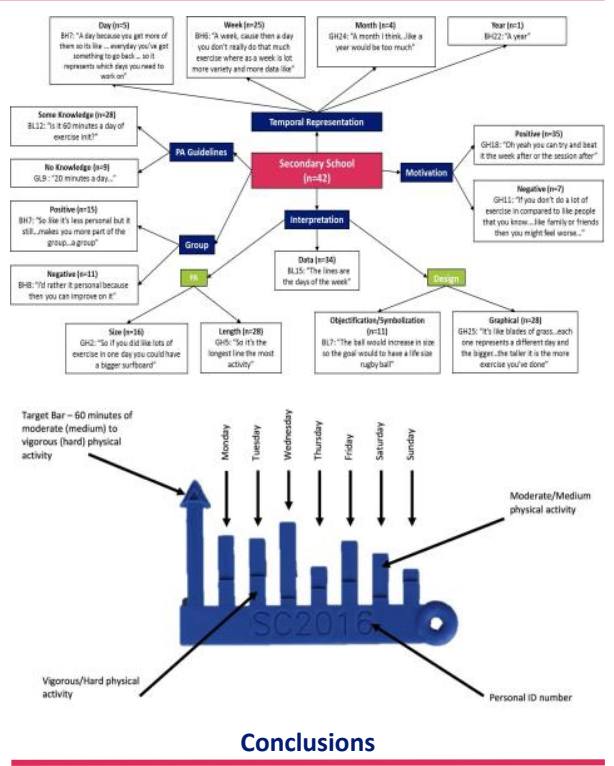

- Both primary and secondary school children engaged in the concept of personalised 3D models displaying their PAL.

- Children felt it could not only enhance their understanding, but motivate them to increase the amount and intensity they engage in.

- This study therefore suggests that 3D models may offer unique strategies for the promotion of PA in children.

References

1) Department of Health. (2011). Start Active, Stay Active: a report on physical activity for health from the four home countries' Chief Medical Officers. London, UK. 


\section{Published Paper - Energy Expenditure Associated with Walking Speed and Angle of \\ Turn in Children. European Journal of Applied Physiology.}

European Journal of Applied Physiology

https://doi.org/10.1007/500421-018-3981-1

ORIGINAL ARTICLE

\section{Energy expenditure associated with walking speed and angle of turn in children}

Sam G. M. Crossley ${ }^{1} \odot \cdot$ Kelly A. Mackintosh ${ }^{1} \odot \cdot$ Rory P. Wilson $^{2} \odot \cdot$ Leanne J. Lester $^{3} \odot \cdot$ Iwan W. Griffiths ${ }^{1} \odot$. Melitta A. McNarry ${ }^{1} \odot$

Received: 26 February 2018/ Accepted: 18 August 2018

OThe Author(s) 2018

\section{Abstract}

Purpose Recent studies have suggested that turning is power intensive. Given the sporadic and irregular movement patterns of children, such findings have important implications for the assessment of true energy expenditure associated with habitual physical activity. The purpose of this study was to investigate the influence of walking speed and angle, and their interaction, on the energy expenditure of healthy children.

Methods 20 children ( $10.1 \pm 0.5$ years; 10 boys) participated in the study. On two separate days, participants completed a turning protocol involving 3-min bouts of walking at one of the 16 speed $\left(2.5,3.5,4.5\right.$, and $\left.5.5 \mathrm{~km} \mathrm{~h}^{-1}\right)$ and angle $\left(0^{\circ}, 45^{\circ}\right.$, $90^{\circ}$, and $180^{\circ}$ ) combinations, interspersed by 3 min seated rest. The movement involved $5 \mathrm{~m}$ straight walking interspaced with prescribed turns with speed dictated by a digital, auditory metronome. Breath-by-breath gas exchange was measured, in addition to tri-axial acceleration and magnetic field intensity recorded at $100 \mathrm{~Hz}$.

Results Mixed models revealed a significant main effect for speed $(p<0.006)$ and angle $(p<0.006)$, with no significant interaction between speed and angle $(p>0.006)$. Significant differences to straight-line walking energy expenditure within speed were established for 3.5 and $5.5 \mathrm{~km} \mathrm{~h}^{-1}$ for $180^{\circ}$ turns ( 13\% and $\sim 30 \%$ increase, respectively).

Conclusion These findings highlight the importance of accounting for the magnitude and frequency of turns completed when estimating children's habitual physical activity and have significant implications for the assessment of daily energy expenditure.

Keywords Youth · Energy requirements · Velocity · Change of direction

$\begin{array}{llll}\text { Abbreviations } & \dot{\mathrm{VO}} & \text { Oxygen uptake } \\ \text { BMI } & \text { Body mass index } & \dot{\mathrm{VO}} & \text { Peak oxygen uptake } \\ \text { DBA } & \text { Dynamic body acceleration } & 6 \mathrm{MWT} & \text { Six-minute walking test } \\ \text { GET } & \text { Gas exchange threshold } & \mathrm{TCH} & \text { Tukey-Ciminera-Heyse }\end{array}$

PHV - Peak height velocity

VeDBA Vectorial dynamic body acceleration

$C_{\mathrm{r}} \quad$ The net energy cost of walking

\section{Introduction}

Communicated by Jean-René Lacour.

Sam G. M. Crossley

S.G.M.Crossley@swansea.ac.uk

Applied Sport Technology Exercise and Medicine Research Centre, Swansea University, Swansea, Wales, UK

2 Swansea Lab for Animal Movement, Biosciences, College of Science, Swansea University, Singleton Park, Swansea, Wales, UK

3 School of Human Science, The University of Western Australia, Crawley, Australia
Insufficient physical activity is one of the leading risk factors for global mortality, at least in part due to its association with obesity and non-communicable diseases (NCDs), such as cardiovascular disease, cancer, and diabetes (WHO 2017). However, despite the numerous physiological (Janssen and Leblanc 2010; Sothern et al. 1999) and psychosocial benefits (Eime et al. 2013; Nieman 2002) associated with physical activity, it is suggested that as little as $19 \%$ of boys and $16 \%$ of girls meet the current government guidelines of $60 \mathrm{~min}$ of moderate-to-vigorous physical activity every day (Townsend 
Published Paper - Perceptions of Visualizing Physical Activity as a 3D-Printed

Object: Formative Study. Journal of Medical Internet Research.

\section{JOURNAL OF MEDICAL INTERNET RESEARCH \\ Crossley et al \\ Original Paper \\ Perceptions of Visualizing Physical Activity as a 3D-Printed Object: Formative Study}

Sam Graeme Morgan Crossley ${ }^{1 *}$, BSc, PhD; Melitta Anne McNarry ${ }^{1 *}, \mathrm{BSc}, \mathrm{PhD}$; Joanne Hudson ${ }^{1 *}, \mathrm{PhD}, \mathrm{HCPC}$; Parisa Eslambolchilar ${ }^{2^{*}}$, BEng, MEng, PhD; Zoe Knowles ${ }^{3 *}, \mathrm{PhD}, \mathrm{HCPC} ;$ Kelly Alexandra Mackintosh ${ }^{1{ }^{*}}, \mathrm{BSc}, \mathrm{MSc}$, $\mathrm{PhD}$

${ }^{1}$ Applied Sport Technology Exercise and Medicine Research Centre, School of Sport and Exercise Sciences, Swansea University, Swansea, United Kingdom

${ }^{2}$ Human Factors Technology Research Priority Area, School of Computer Science and Informatics, Cardiff University, Cardiff, United Kingdom

${ }^{3}$ Physical Activity Exchange, School of Sport and Exercise Sciences, Liverpool John Moores University, Liverpool, United Kingdom

"all authors contributed equally

Corresponding Author:

Parisa Eslambolchilar, BEng, MEng, $\mathrm{PhD}$

Human Factors Technology Research Priority Area

School of Computer Science and Informatics

Cardiff University

Queen's Buildings, North Building

5 The Parade, Newport Road

Cardiff, CF24 3AA

United Kingdom

Phone: 4402920879346

Email: EslambolchilarP@cardiff.ac.uk

\section{Abstract}

Background: The UK government recommends that children engage in moderate-to-vigorous physical activity for at least 60 min every day. Despite associated physiological and psychosocial benefits of physical activity, many youth fail to meet these guidelines partly due to sedentary screen-based pursuits displacing active behaviors. However, technological advances such as 3D printing have enabled innovative methods of visualizing and conceptualizing physical activity as a tangible output.

Objective: The aim of this study was to elicit children's, adolescents', parents', and teachers' perceptions and understanding of 3 D physical activity objects to inform the design of future 3D models of physical activity

Methods: A total of 28 primary school children (aged 8.4 [SD 0.3 ] years; 15 boys) and 42 secondary school adolescents (aged 14.4 [SD 0.3 ] years; 22 boys) participated in semistructured focus groups, with individual interviews conducted with 8 teachers ( 2 male) and 7 parents ( 2 male). Questions addressed understanding of the physical activity guidelines, 3D model design, and both motivation for and potential engagement with a 3D physical activity model intervention. Pupils were asked to use Play-Doh to create and describe a model that could represent their physical activity levels (PAL). Data were transcribed verbatim and thematically analyzed, and key emergent themes were represented using pen profiles.

Results: Pupils understood the concept of visualizing physical activity as a 3D object, although adolescents were able to better analyze and critique differences between low and high PAL. Both youths and adults preferred a 3D model representing a week of physical activity data when compared with other temporal representations. Furthermore, all participants highlighted that 3D models could act as a motivational tool to enhance youths' physical activity. From the Play-Doh designs, 2 key themes were identified by pupils, with preferences indicated for models of abstract representations of physical activity or bar charts depicting physical activity, respectively,

Conclusions: These novel findings highlight the potential utility of 3D objects of physical activity as a mechanism to enhance children's and adolescents' understanding of, and motivation to increase, their PAL. This study suggests that 3D printing may offer a unique strategy for promoting physical activity in these groups.

(J Med Internet Res 2019;21(1):e12064) doi:10.2196/12064 


\title{
Published Paper - Understanding Youths'Ability to Interpret 3D-Printed Physical Activity Data and Identify Associated Intensity Levels: Mixed-Methods Study. Journal of Medical Internet Research.
}

\section{Understanding Youths' Ability to Interpret 3D-Printed Physical Activity Data and Identify Associated Intensity Levels: Mixed-Methods Study}

Sam Graeme Morgan Crossley $^{1 *}$, BSc, PhD; Melitta Anne McNarry ${ }^{1 *}$, BSc, PhD; Michael Rosenberg ${ }^{2}$, BAppSc, DipEd, MPH, PhD; Zoe R Knowles ${ }^{3 *}$, HCPC, PhD; Parisa Eslambolchilar ${ }^{4}$, BEng, MEng, PhD; Kelly Alexandra Mackintosh $^{1 *}$, BSc, MSc, PhD

\footnotetext{
${ }^{1}$ Applied Sports Science Technology and Medicine Research Centre (A-STEM), Swansea University, Swansea, United Kingdom ${ }^{2}$ School of Human Sciences, The University of Western Australia, Crawley, Australia

${ }^{3}$ Physical Activity Exchange, School of Sport and Exercise Sciences, Liverpool John Moores University, Liverpool, United Kingdom ${ }^{4}$ School of Computer Science and Informatics, Cardiff University, Cardiff, United Kingdom

"these authors contributed equally
}

\section{Corresponding Author:}

Sam Graeme Morgan Crossley, BSc, PhD

Applied Sports Science Technology and Medicine Research Centre (A-STEM)

Swansea University

Bay Campus, Engineering East Building

Swansea, SA1 8EN

United Kingdom

Phone: 4407707470931

Email: 557947@swansea.ac.uk

\begin{abstract}
Background: A significant proportion of youth in the United Kingdom fail to meet the recommended 60 minutes of moderate-to-vigorous physical activity every day. One of the major barriers encountered in achieving these physical activity recommendations is the perceived difficulty for youths to interpret physical activity intensity levels and apply them to everyday activities. Personalized physical activity feedback is an important method to educate youths about behaviors and associated outcomes. Recent advances in 3D printing have enabled novel ways of representing physical activity levels through personalized tangible feedback to enhance youths' understanding of concepts and make data more available in the everyday physical environment rather than on screen.

Objective: The purpose of this research was to elicit youths' (children and adolescents) interpretations of two age-specific 3D models displaying physical activity and to assess their ability to appropriately align activities to the respective intensity.

Methods: Twelve primary school children ( 9 boys; mean age 7.8 years; SD 0.4 years) and 12 secondary school adolescents (6 boys; mean age 14.1 years; SD 0.3 years) participated in individual semistructured interviews. Interview questions, in combination with two interactive tasks, focused on youths' ability to correctly identify physical activity intensities and interpret an age-specific $3 \mathrm{D}$ model. Interviews were transcribed verbatim, content was analyzed, and outcomes were represented via tables and diagrammatic pen profiles.

Results: Youths, irrespective of age, demonstrated a poor ability to define moderate-intensity activities. Moreover, children and adolescents demonstrated difficulty in correctly identifying light- and vigorous-intensity activities, respectively. Although youths were able to correctly interpret different components of the age-specific 3D models, children struggled to differentiate physical activity intensities represented in the models.

Conclusions: These findings support the potential use of age-specific 3D models of physical activity to enhance youths' understanding of the recommended guidelines and associated intensities.
\end{abstract}

(J Med Internet Res 2019;21(2):e11253) doi:10.2196/11253

KEYWORDS

3D printing; education; adolescent; child; comprehension; understanding; mental recall 


\section{Appendix VII: Online Articles}

The Conversation: Online Article

\section{THE CONVERSATION}

\section{How 3D printing may help cut childhood obesity by getting children active}

September 2, 2016 4.14pm BST

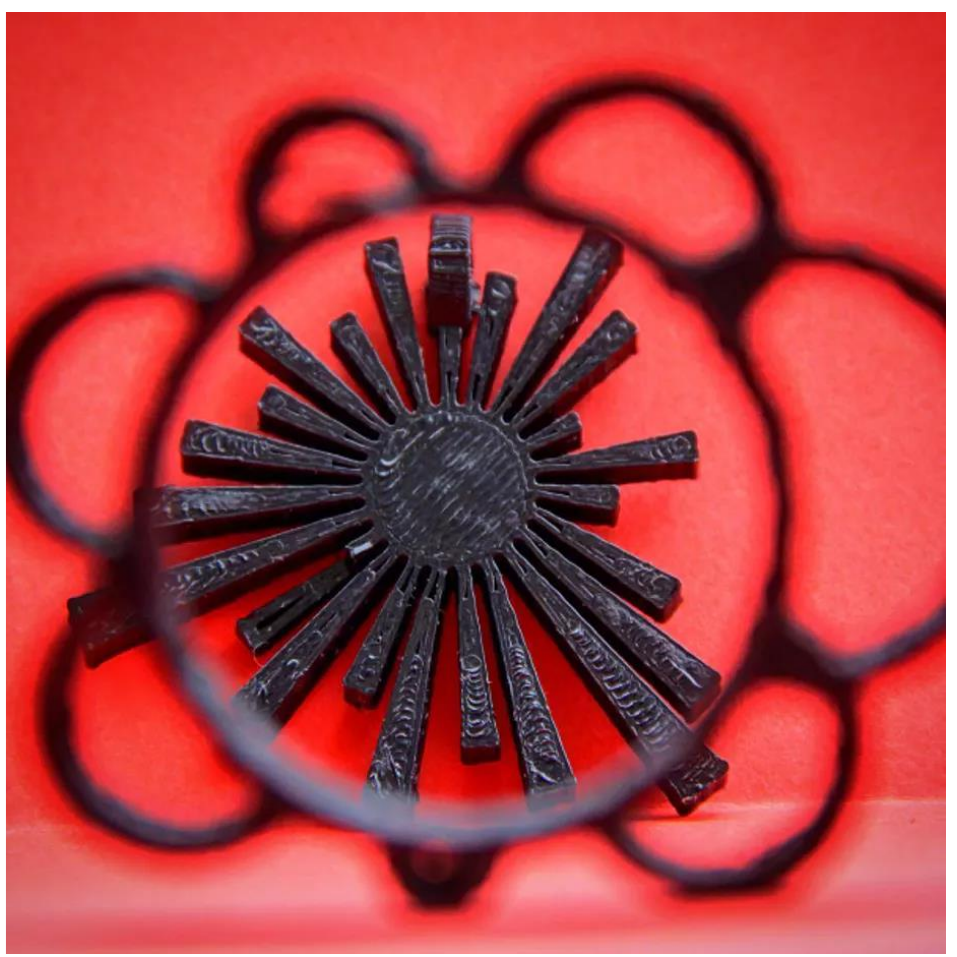

Authors

Selly Mackintosh Lecturer in Sports Science,

Swansea University

Instagram/3dphysicalactivity/Sam Crossley, Author provided

This past summer has seen a mini revolution: worried parents rejoiced as their children tore themselves away from computer and television screens to get outside and enjoy the school break albeit with the help of Pokemon Go.

Childhood obesity has reached epidemic proportions - the World Health Organisation recognises it as a major public health challenge of the 21st century. But in a world of engaging technology, mobile technology and video games of epic lengths, it can be very difficult to encourage children to move around more. 
Recent advances in accessible technologies such as wearable activity monitors, pedometers and an array of online apps have provided lots of new opportunities to gain an insight into our daily physical activity levels. Reward systems such as goal setting, physical activity profiles, real-time feedback and social support networking are some of the top strategies that are helping keep people motivated and get more active. More specifically for children, visual stimulation through phone apps - such as Pokémon Go, Motion Maze, Zombies, Run! and ibitz for kids - are proving to have fantastic results in helping children stay more physically active and interested.

This maintained interest is of paramount importance in light of recent statistics which suggest that approximately $30 \%$ of children are overweight or obese in the UK. Furthermore, childhood obesity is known to track strongly into adolescence and adulthood - evidence suggests that $80 \%$ of obese adolescents will become obese adults. In fact, studies show that if current trends continue, global obesity rates will reach $18 \%$ in men and more than $21 \%$ in women by 2025 .

\section{Pioneering solutions}

So how can we get our kids moving? At present, more than one-third of children don't achieve the recommended levels of 60 minutes of moderate-to-vigorous physical activity every day. To make matters worse, physical activity has been displaced by sedentary behaviours, such as watching television and playing video games. Children are reported to spend an average of eight hours per week playing sedentary video games and this has been shown to increase the risk of childhood obesity.

One of the major reasons that children don't meet the recommended requirements for physical activity is thought to be because it is difficult for them to understand, interpret and apply recommendations to everyday activities. Ask your average six-year-old what they think an "intense" or "vigorous" activity is or they will struggle to accurately answer. At present, official guidelines refer to these kinds of exercise intensities which are hard to align with daily activities - and it is hard to recognise the differences between them. For example, walking to school, playing in the playground and walking up stairs to the classroom are all very different exercise intensities, but how much of each do you need to do to meet the government guidelines?

Modern technology is evidently a fantastic way of getting children motivated to exercise - but it can help them understand their own physical activity and health as well. Apps targeted at an adult market focus on activities such as mapping cycling or run routes, counting steps or recording daily gym sessions. But for children the information collected needs to be a bit more tangible than just facts and figures. 
It is with this in mind that our team has been looking into helping children get more active - with the help of $3 \mathrm{D}$ printers. The Exertion Games Lab in Melbourne was the first to use $3 \mathrm{D}$ printing to visualise heart rate during physical activities. The print-outs were used to provide feedback on intensity: the larger the spike, the higher the heart rate and therefore intensity of activity.

Following on from this, our research team is now looking at different ways that children can see, feel and interact with a personal 3D model of their weekly physical activity. Early discussions with children, parents and teachers have provided many interesting, and creative models, in different shapes and styles. A selection of these models will be combined in the coming months to produce a final design that will be trialled in schools across Swansea, Wales, in 2017. We hope to explore if these tangible objects can promote healthy activity and cause long term changes in behaviour.

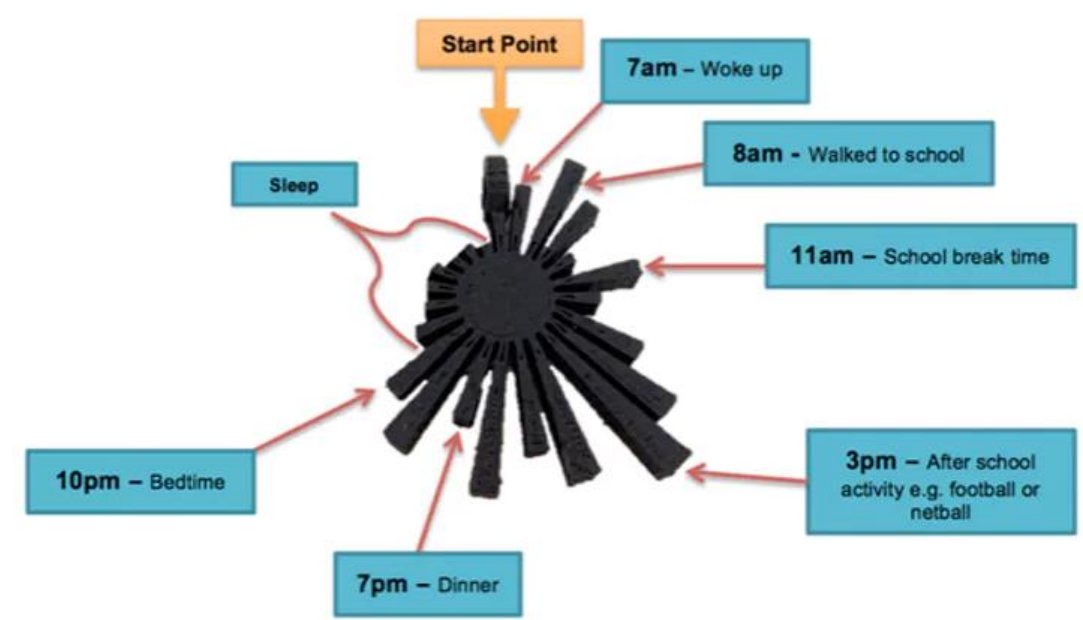

A model with activity information for one day. Sam Crossley, Author provided

We believe these models have great potential to educate and enthuse children about developing and maintaining appropriate levels of physical activity and sedentary behaviours. They could also have have a positive impact upon the future health problems of the UK, with improved social outcomes, reduced costs to the country and enhanced quality of life across the age span. And with continuous advances in the technology, who knows where this could go?

Children Childhood obesity Fitness Physical activity Child obesity Fitness trackers 3D models 3-D printing 


\section{Visualizing physical activity through 3D printing to get children moving more and sitting less}

Resource Type: Idea or inspiration

Subject Area: Physical Education \& Health

Applications/Fields: Medical/Health

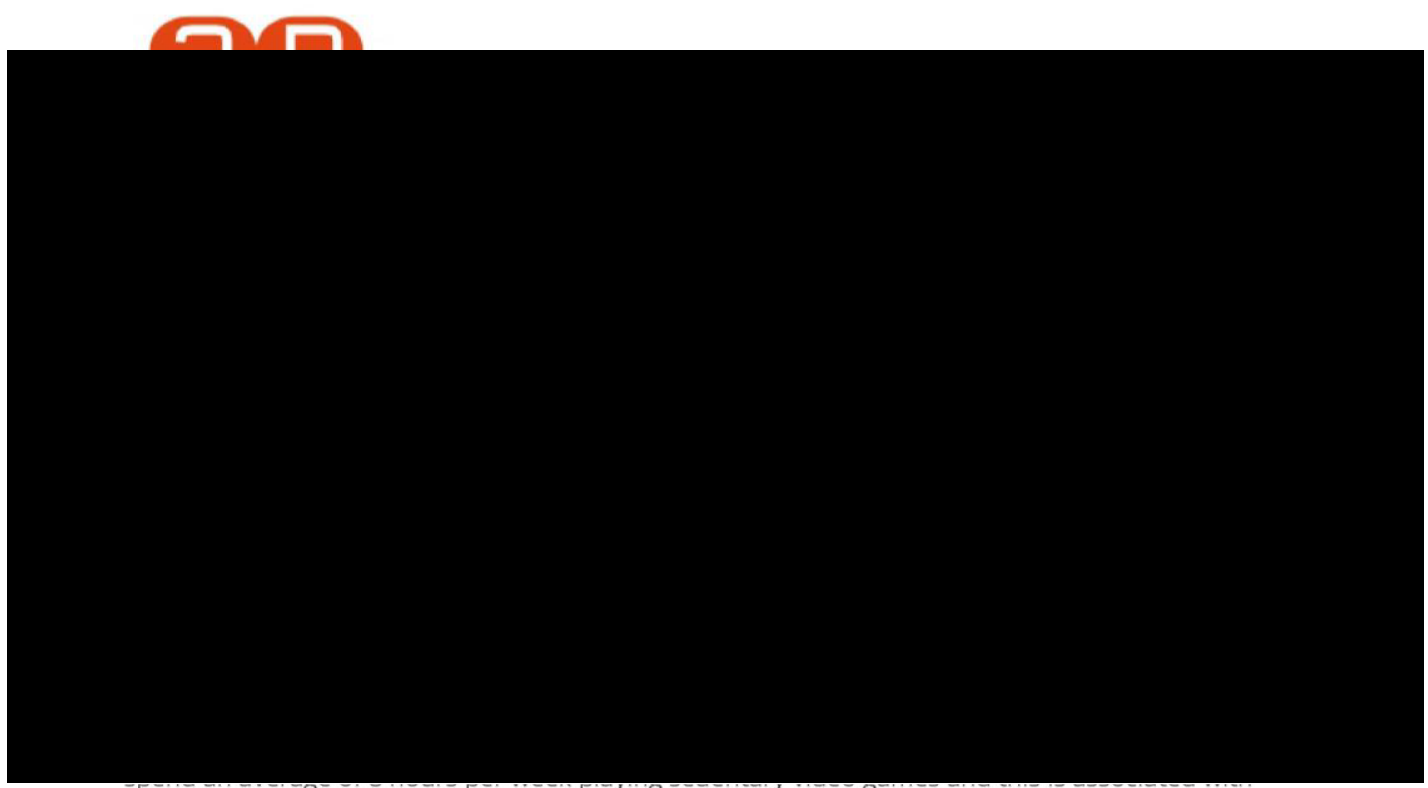

increased risk of childhood obesity. More than one third of children don't achieve the recommended levels of 60 minutes of moderate-to-vigorous physical activity (MVPA) everyday, with approximately $30 \%$ of children being overweight or obese. The really scary thing is that childhood obesity tracks into adolescence and adulthood, with figures suggesting that $80 \%$ of obese adolescents become obese adults. These figures are alarming and highlight the importance of getting children moving more and sitting less. 


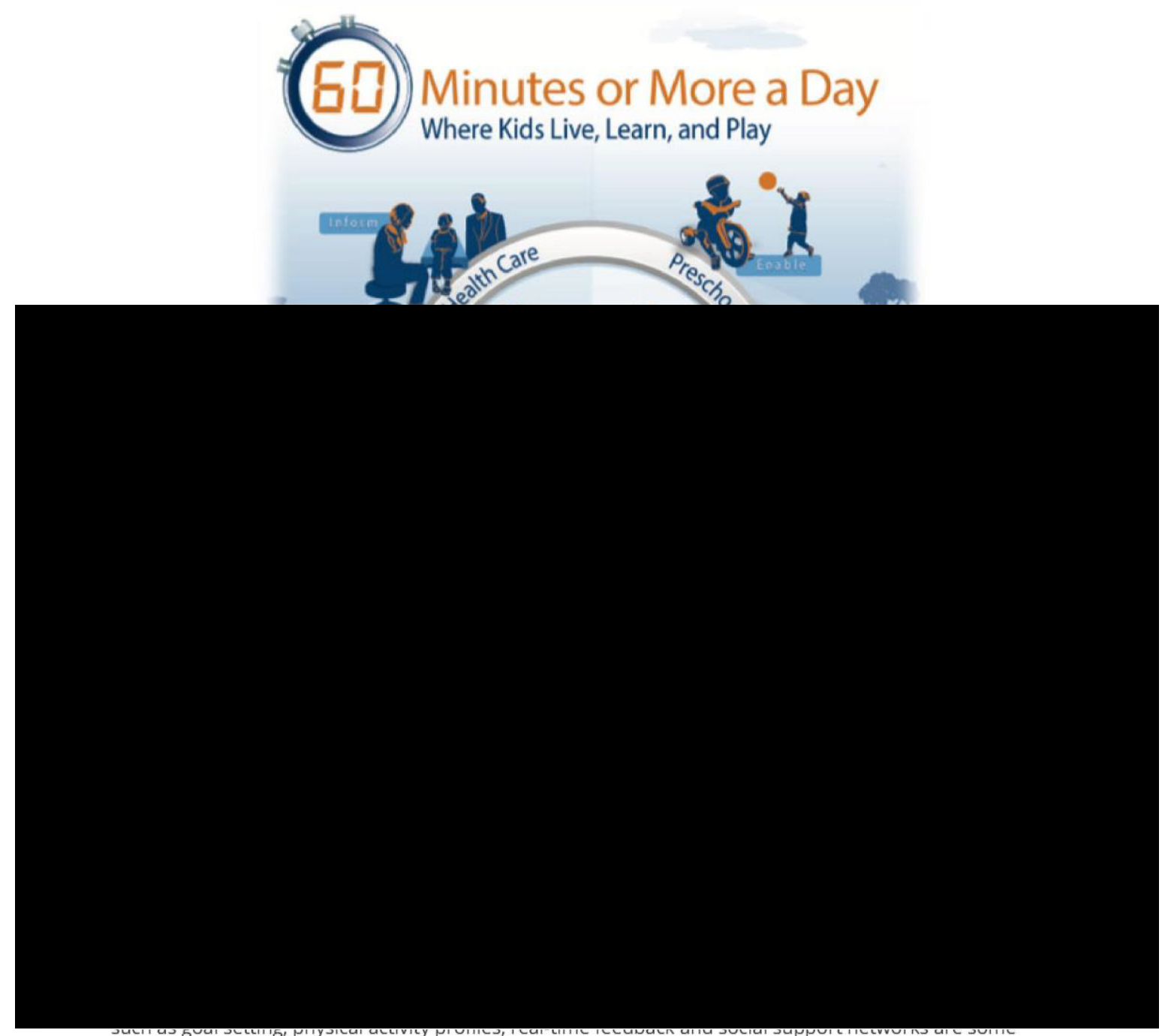

of the best ways to help people maintain their motivation to be more physically active. For children, this visual stimulation may take the form of phone apps such as Pokémon Go, Motion Maze, Zombies, Run! and ibtiz kids, which are proving to provide better results in helping children to stay more physically active and interested. But what if we could create something that's meaningful, visual and tangible for children all in one? 


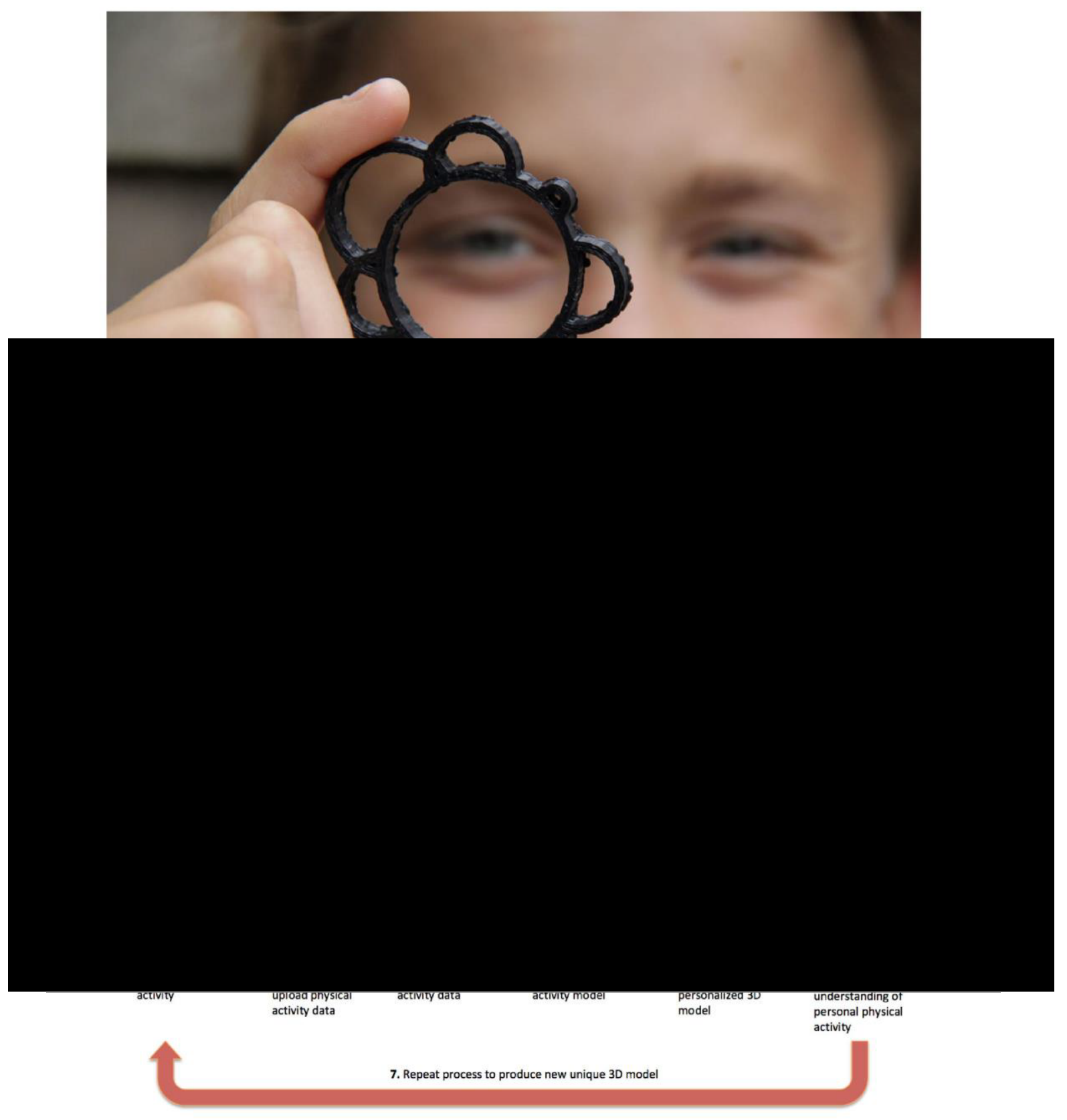

343 
more the child moves, the more the shape or size of the personalized model changes in appearance. Bringing physical activity data back to reality, something that the children can touch, collect and compare over time we think is important. We chose to use the Ultimaker because we think it goes hand in hand with the concept of making something complicated like physical activity data or 3D printing simple and relatable for everyone. The Ultimaker demonstrates this with its luminous LEDs and userfriendly menu system that the children can navigate through, additionally adding to the learning process.

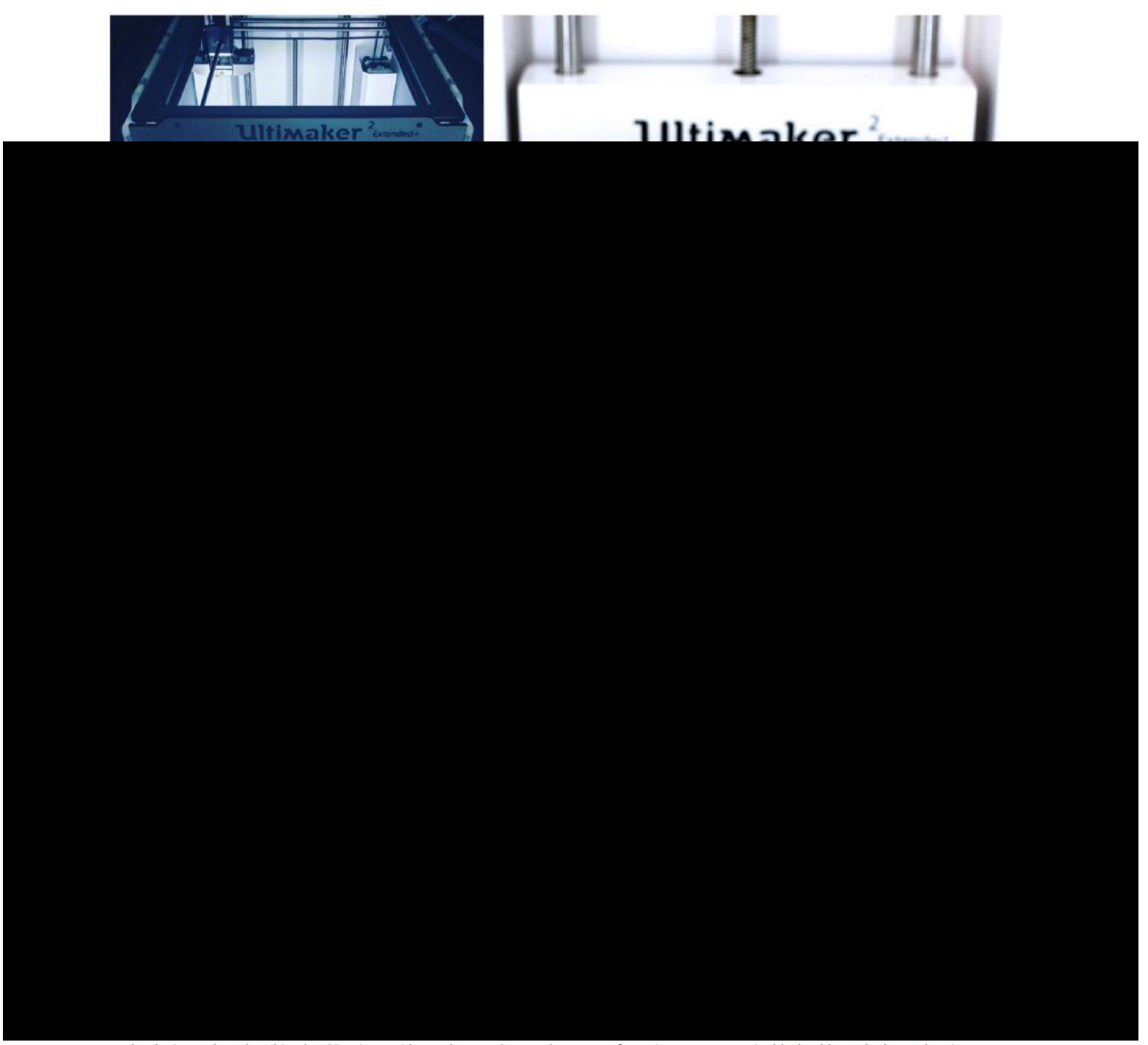

technologies such as the Ultimaker 3D printer with novel research to explore ways of creating a more sustainable, healthy and educated society. 


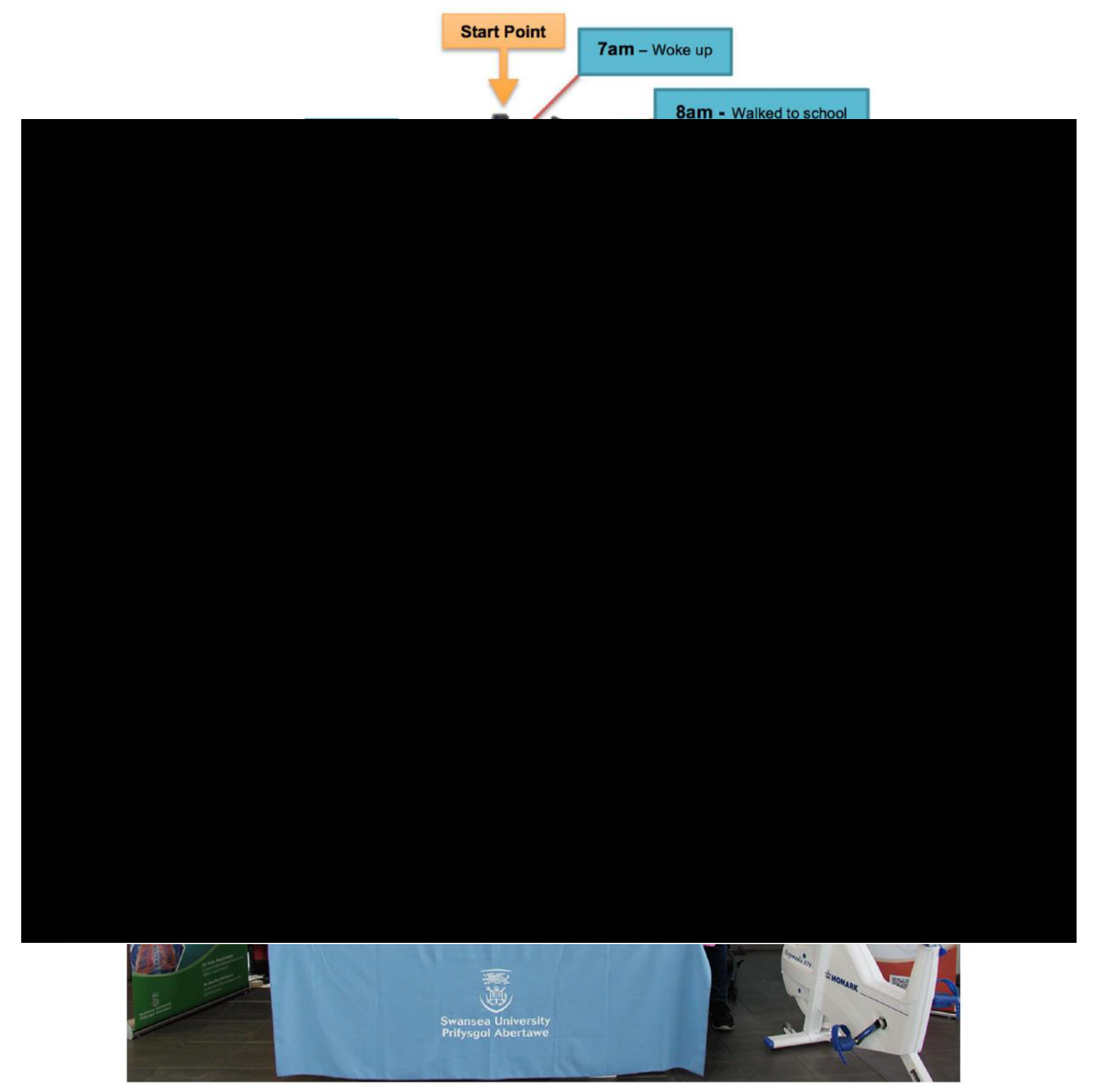

We would like to thank Sam Crossley and supervisors Dr. Melitta McNarry, Dr. Kelly Mackintosh and Dr. Parisa Eslambolchilar for sharing this great project with us, to find out more on 3D Physical Activity follow them on the social media tags below and check out their website at https://3dprintingphysicalactivity.wordpress.com/ 
AICI

\section{Using 3D Printing to Enhance Children's Understanding of Physical Activity}

16 July 2018

By Sam Crossley (1), Dr. Kelly Mackintosh (1), Dr. Melitta McNarry (1) and Dr. Parisa Eslambolchilar (2)

(1) Applied Sports, Technology, Exercise and Medicine Research Centre, Swansea University, Wales, UK

(2) School of Computer Science and Informatics, Cardiff University, Wales, UK

In the UK, more than one-third of children are not achieving the minimum recommended levels of 60 minutes of moderate-to-vigorous physical activity (MVPA) every day. While there are several reasons that children do not achieve this recommended amount, including socioeconomic status, urbanisation, social and environmental differences, screen-based technology is perhaps one of the most criticised. A recent Ofcom report shows that children spend an average of 10 hours per week playing video games, which is associated with an increased risk of childhood obesity.

Schools are recognised as key settings to promote MVPA as a large number of children can be reached through break times, in class activity breaks and physical education classes. However, it has been recently 
suggested that children have a lack of understanding of what type of activities count towards their daily MVPA target. Understanding of physical activity behaviours has been identified as an important correlate for behaviour change, through motivating an individual to get ready to make healthy changes to their daily lifestyle. The use of technology could be a great way to enhance children's understanding of physical activity levels, especially given that they are unlikely to relinquish such highly-valued, technology-based behaviours.

The release of wearable activity trackers that are more meaningful for children, such as superhero or Disney-themed trackers, represent potential tools to change their attitudes and physical activity behaviours. However, the on-screen visualisations of data interfaced on these wearable devices and adjoining screens (e.g., monitors, tablets and smartphones) are expensive and limited to stimulating a child's visual and auditory senses, which tend to ignore the abundance of other senses, such as touch, which is especially pertinent to children.

It is with this in mind that we are exploring novel ways to encourage children to be more physically active with the aid of 3D printers. The Exertion Games Lab in Melbourne was the first to utilise 3D printing to visualise adults' heart rate data during physical activities. Building on this, our research team undertook a qualitative study to explore children's perceptions of 3D printing physical activity data and invited children as codesigners using Play-Doh to inform the development of prototype 3D models. The Play-Doh model designs took the form of both abstract and graphical designs, such as a flower or paddleboard (Figure 1), which provided feedback using the petals or paddles on the board to represent different days of activity, with larger petals or paddles corresponding to higher levels of physical activity achieved for that day. 


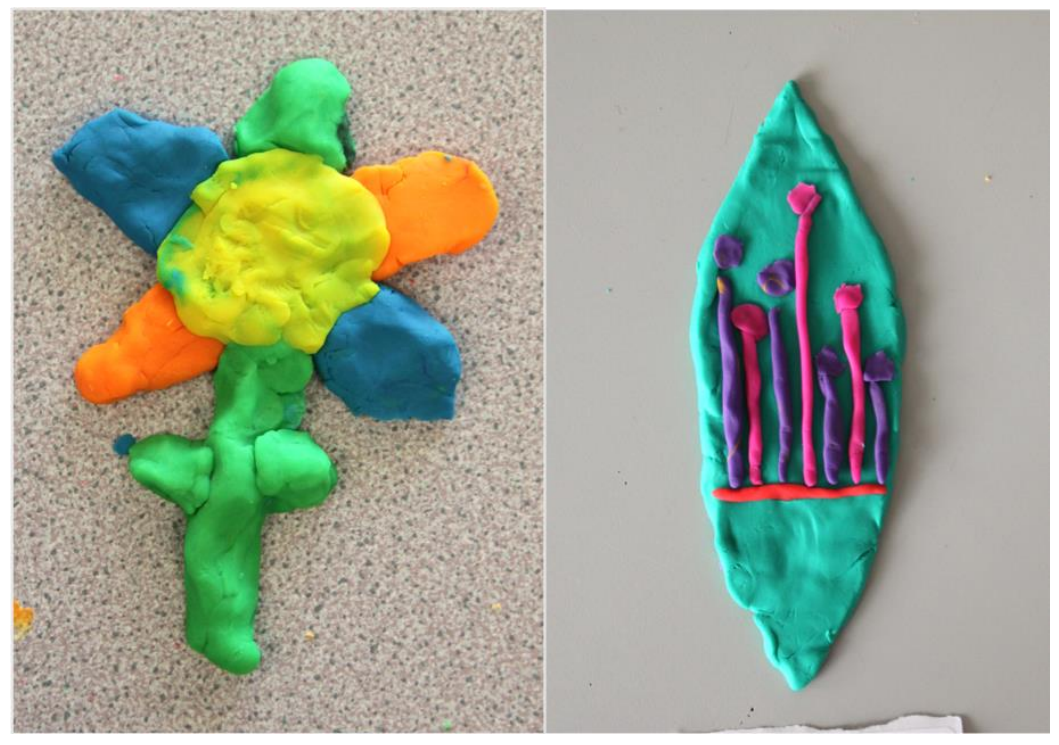

Figure 1. Children's Play-Doh Model Designs

Through an iterative design process, two age-specific 3D models of physical activity were developed, one taking the form of a 'sun' design (aged 7-8 years) and the other resembling 'bar chart' (aged 13-14 years old). The 3D models were designed to represent children's moderate and vigorous physical activity levels achieved for each day, across a week, as well as displaying the physical activity guideline of 60 minutes of MVPA (Figure 2).

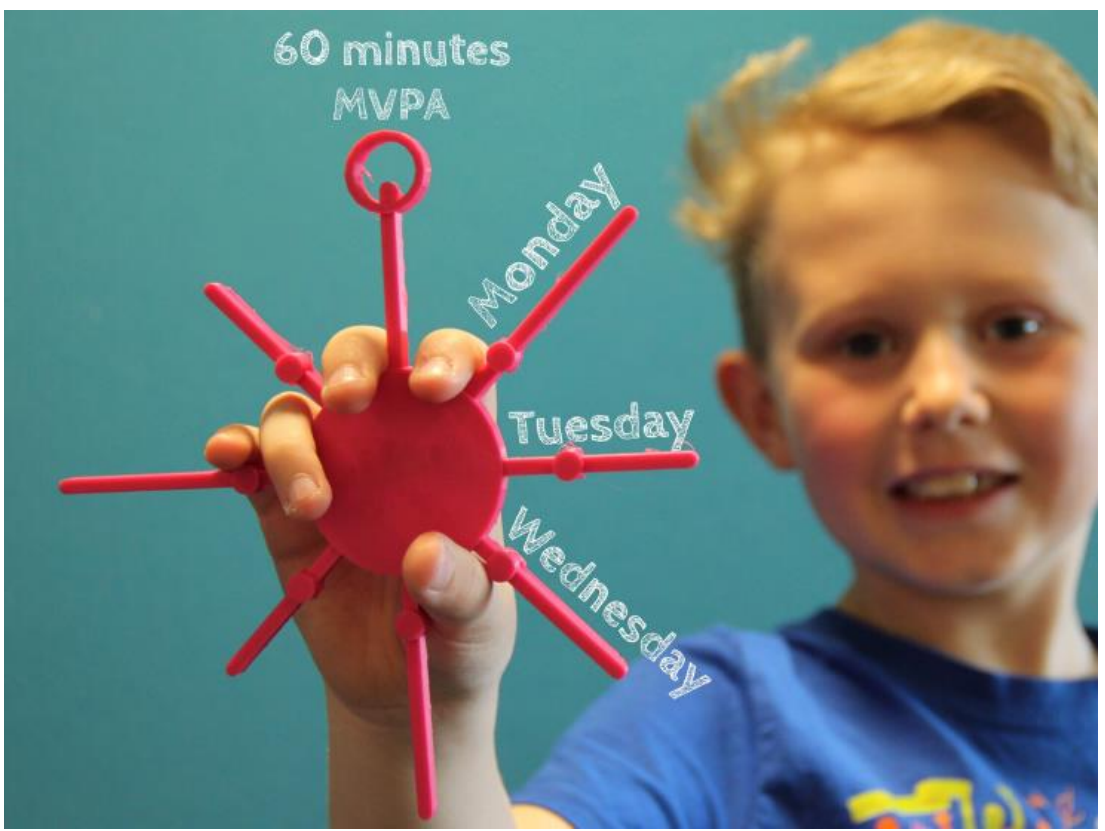

Figure 2. Children's 3D Model of Physical Activity (ages 7-8 years). 
We have examined the effectiveness of a three-month school-based intervention in South Wales, whereby ninety-six children (aged 7-14 years) were given 3D-printed models of their previous week's physical activity levels, objectively measured using an accelerometer (publications under review). Following receipt of their 3D models, each child completed a short video interview to assess their understanding of physical activity levels. Preliminary findings show that the age-specific 3D models may enhance children's understanding of physical activity levels, with $73 \%$ of children demonstrating an awareness that their behaviours were not optimal. In this light, the novel approach of 3D printing physical activity may offer a unique strategy to promote children's understanding of how much physical activity is important to gain health benefits.

\section{Questions:}

How can 3D printing be adapted to account for the more complex behaviours of physical activity?

What other populations do you think could benefit from 3D-printed feedback?

What limitations should researchers keep in mind when designing 3Dprinted feedback?

How can tangible data provide a more meaningful and rewarding experience than digital data alone? 


\section{Appendix VIII: Public Engagement Outputs}

\section{Swansea University Research as Art Competition Submission}

Visualising Children's Physical Activity through 3D-printed Artefacts

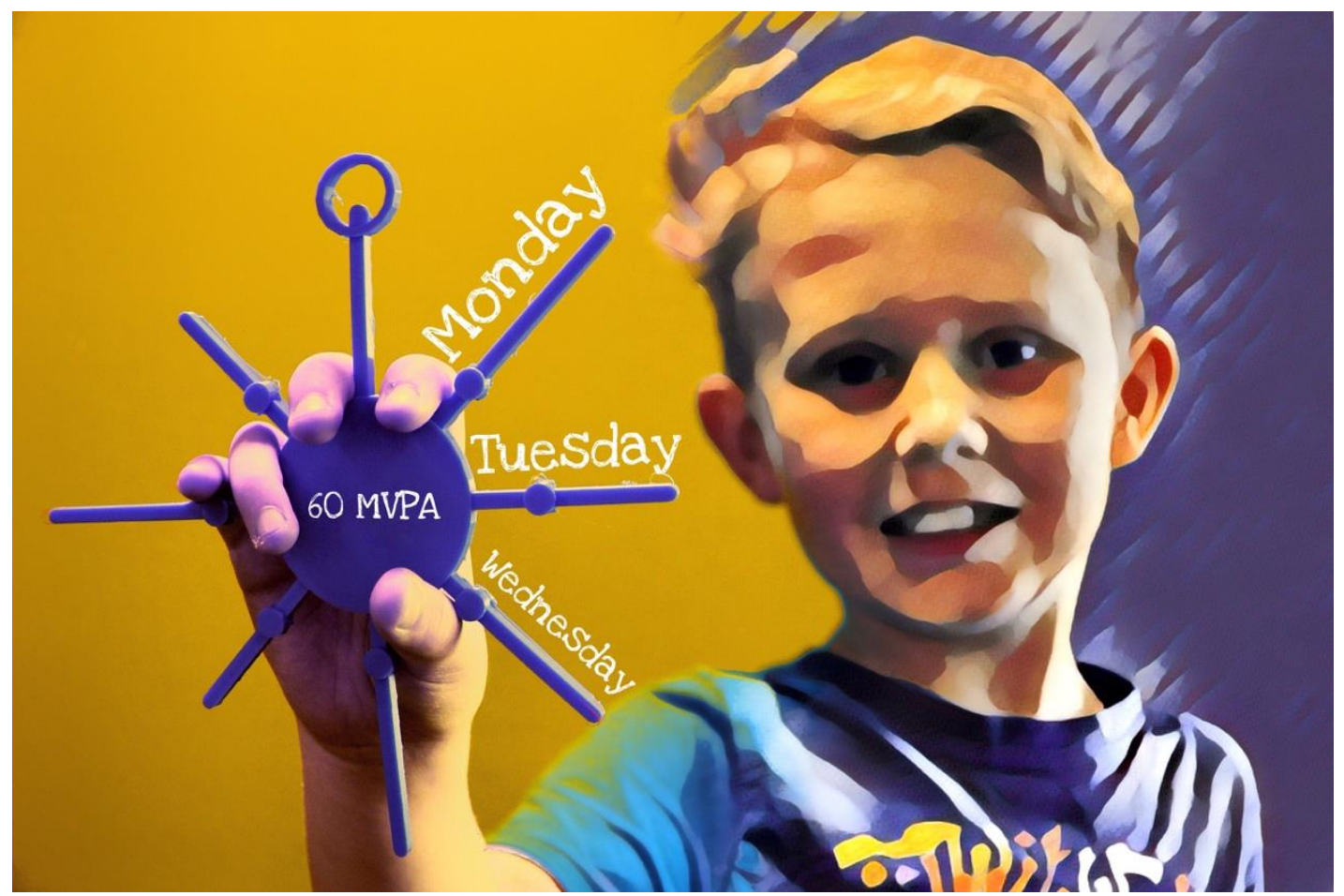

Authors: Sam Crossley, Dr Kelly Macintosh and Dr Melitta McNarry

Department: School of Sport and Exercise Sciences

Text: To lead a healthy lifestyle and maintain a healthy weight, children need to engage in at least 60 minutes of physical activity every day. However, as little as one third of children meet these guidelines and are overweight or obese. This is a contemporary issue that everyone has an opinion on. 3D modelling and printing weekly physical activity data can provide children with personalised feedback, which is not only attractive, but easy to understand. From children to grandparents, the innovative approach will capture their imagination as they try to generate unique personalised 3D shapes that can be kept as a souvenir; the more you move, the bigger the shape! Bringing physical activity data back to reality, something that the children can touch, collect and compare over time, can get children to move more and sit less. Perhaps every child needs a ray of sunshine a day for health and happiness?! 


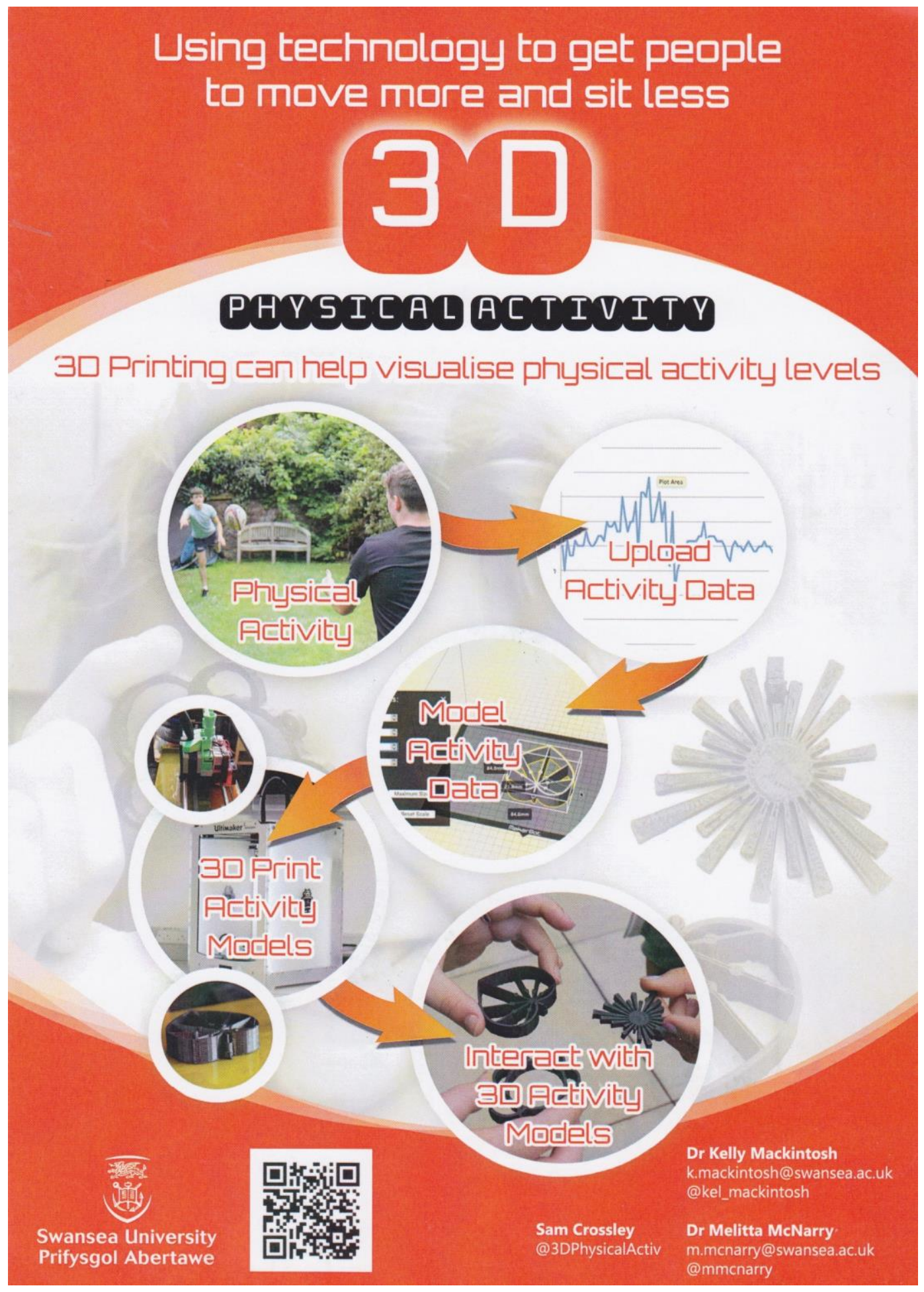




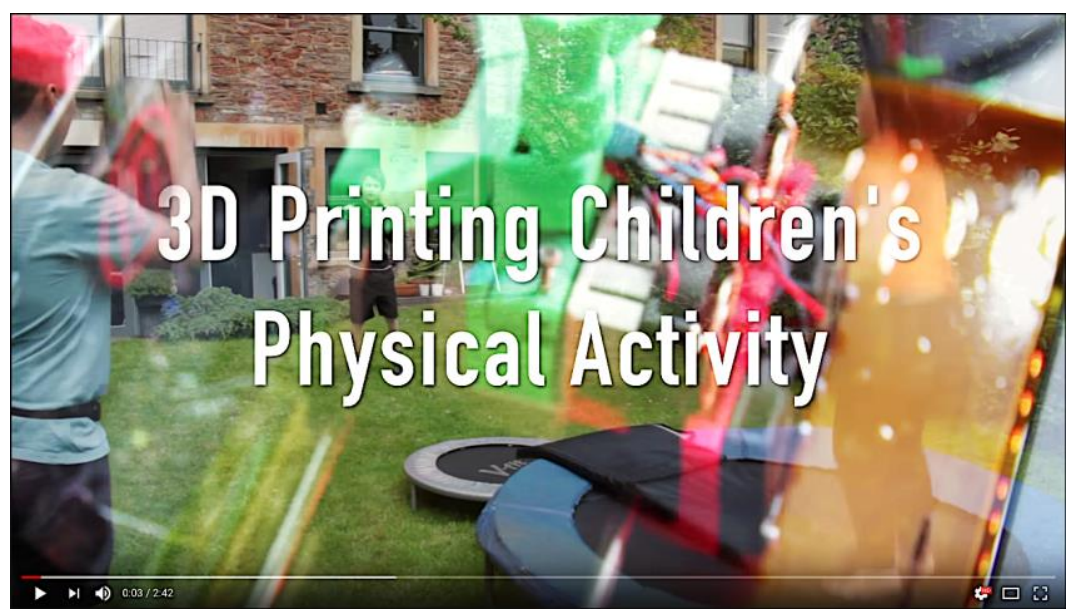

https://www.youtube.com/watch?v=9IOgg20tpPg\&t=3s

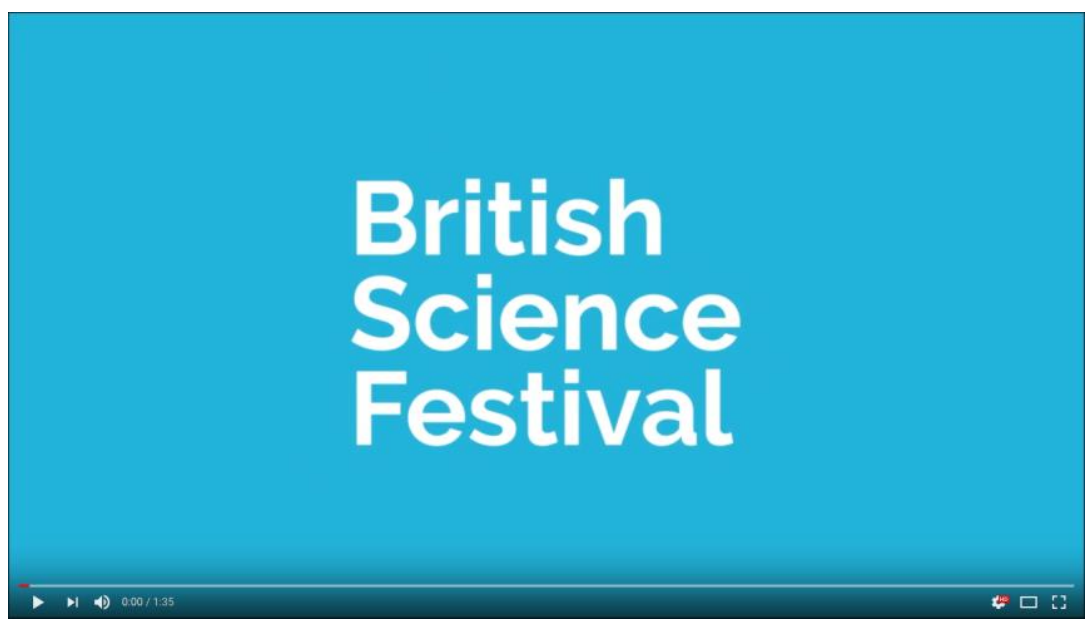

https://www.youtube.com/watch?v=F37DBtUw_Bk

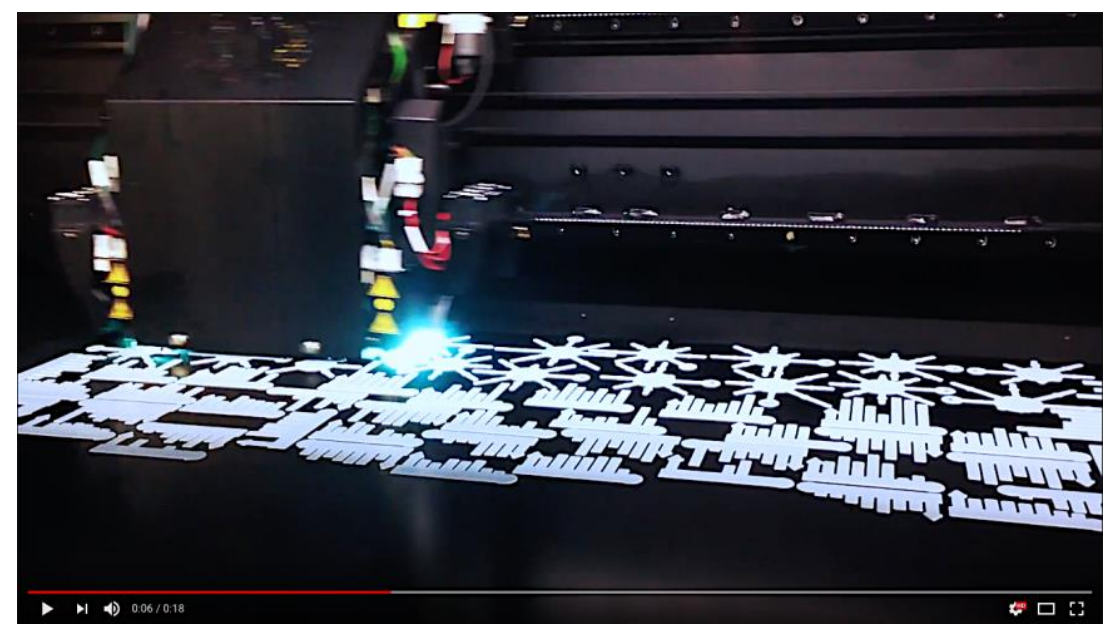

https://www.youtube.com/watch?v=BzVXSv43y4w 


\section{School Fortnightly Friday Flyer}

\section{January 2017}

\section{'We have the right to be fit \& healthy'}

Blwyddyn 3 is currently involved in a very exciting project working with Sam Crossley from Swansea University. The project involves a study of physical activity over the next term. The children have been fitted with accelerometers to track their physical activity each day. Every 2 weeks the data will be collected, a 3d printer will be used to produce a model of the activity which will also be shared with the children. It will be interesting to see what the impact of study has on the children's physical activity. The project also supports our school aim to 'Ensure the safety of every child and encourage a healthy and active lifestyle for all' I'd like to thank parents for their support with the project.

Blwyddyn 6 will taking part in the Ospreys

1- Community Project this term, Mrs Dardecker is

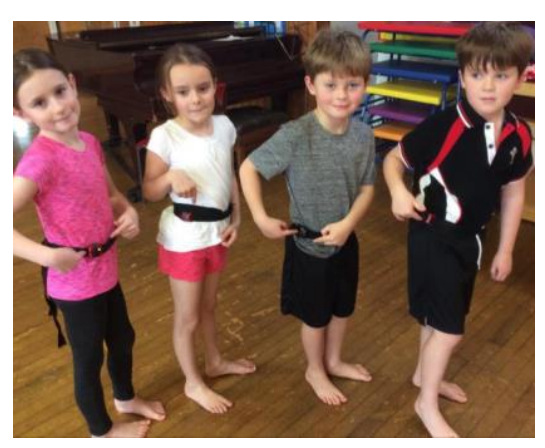
Stadium and Sony in Bridgend. The project culminates in a rugby tournament.

Dream, Believe, Achieve Together - Breuddwydio, Credu, Cylfawni Gyda'n gilydd

\section{February 2017}

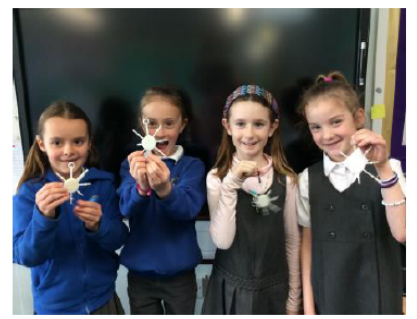

wyddyn 3 continues to be involved in a very exciting project working with Sam Crossley from Swansea University. The project involves a study of physical activity over the term. The children have been fitted with accelerometers to track their physical activity each day. Every 2 weeks the data has been collected and a 3D model has been produced to show their activity each day, I'm sure today will have added a lot! It will be interesting to see what the impact of study has on the children's physical activity. The project also supports our school aim to 'Ensure the safety of every child and encourage $a$ healthy and active lifestyle for all' Thank you to parents for reminding the children to wear their accelerometers every day.

\section{April 2017}

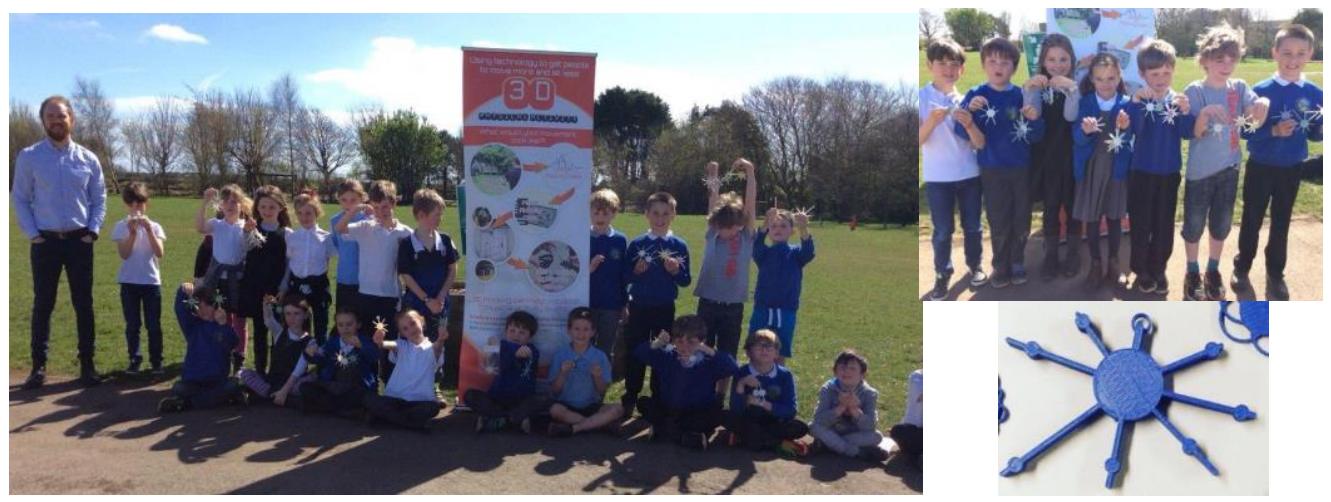

Year 3 completed their project with Sam Crossley, Phd research student from Swansea University who has been looking at physical activity and finding ways to encourage children to be more active through the use of 3D models. It's certainly been interesting to see if the 3D models have encouraged the children to be more active. We'll update you with more once the research results are in. The children explain the project here.

In Y3 we've been doing a physical activity project. We had to wear belts that measure how much you move each day. Every week we got a model shaped like a sun. The sticks on the sun are the days of the week and show how active we were on each day. In the middle of each stick is a blob that separates the medium activity from the hard activity. In the middle of the circle in the sun are numbers $1,2,3$ or 4 , so you know which week is which, we did this for 4 weeks. There is a target for 60 minutes of activity each day. I enjoyed the project and it helped me because I now want to do more activity.

Amber Y3
Y3 have been doing a physical activity project. We had to use belts that measured our physical activity and at the end of each week we would get a model that shows how much physical activity we did. The model looks like a sun and it had a target bar. The bar shows how long you should try to do physical activity for each day which was 60 minutes. There were sticks coming out of the sun that show each day of the week, on the stick were blobs which separated the hard and medium levels of activity. In total we did 4 weeks of the project. The project was fun and it has made me want to do more swimming. William Y3 
MEMS

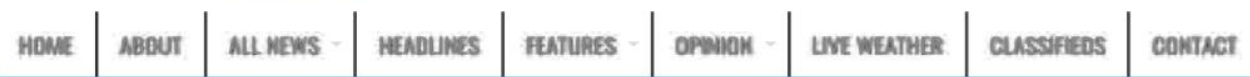

\section{PRESS RELEASE: SALCC hosts important physical activity}

\section{seminar}

\section{By Sir Arthur Lewis Community College
June 2, 2017}

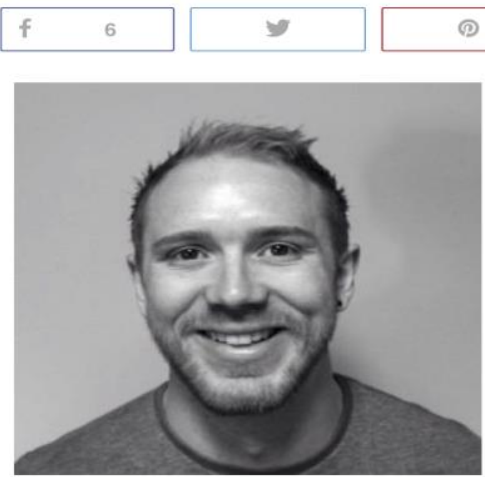

Crossley fields of study as possible, the Sir Arthur Lewis Community College in association with the Office of the Governor General, will host Sam G M and Medicine (A-STEM), Swansea University, South Wales, UK.

He will be the main facilitator at a seminar on Tuesday 6th June 2017 from 9:00 a.m. at the College, which will provide participants with insight
In keeping with its mandate to facilitate education at all levels and in as many Crossley, PhD Student Research Centre in Applied Sports, Technology, Exercise into the use of technology to promote physical activity.

The topic for discussion is "Visualizing physical activity through 3D printing to get children moving more and sitting less."

Mr. Crossley is a member of the Swansea University multidisciplinary research team involving Sport, Exercise and Health Scientists, Computer Scientists and Arts and Humanities academics.

The research is focused on a novel concept involving 3D printing, to help aid children's understanding and interpretations of how important physical activity is for staying healthy.

With a natural attraction to technology the model allows children to monitor their daily and weekly physical activity levels through a process of 3D modelling activity data, which provide children with personalized physical activity feedback, aesthetically attractive and easy to understand. The more the child moves, the more the shape or size of the personalized model changes in appearance.

This brings physical activity data back to reality, by providing something that the children can touch, collect and compare over time. The hope is that such an intervention will stimulate interest and become the catalyst for a more active lifestyle.

Attendees include various age categories from secondary schools, Physical Education and Sports Professionals, Officials from the Ministry of Education and Department of Youth and Sports as well as student athletes from SALCC.

$3(0) \&(0)$

Share This On:

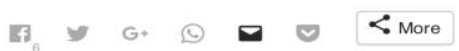

This article was posted in its entirety as received by stlucianewsonline.com. This media house does not correct any spelling or grammatical error within press releases and commentaries. The views expressed therein are not necessarily those of stlucianewsonline.com, its sponsors or advertisers.

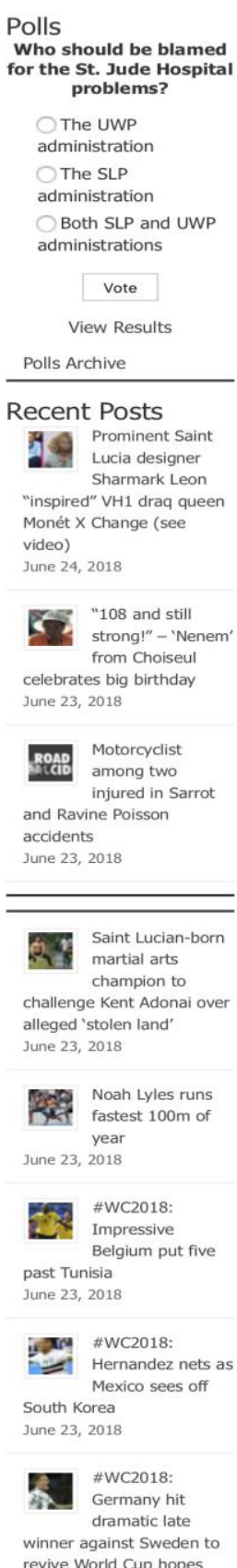
strong'" - 'Nenem' strong!" - 'Nenem" celebrates big birthday (23, 2018 une 23, 2018 injured in Sarrot and Ravine Poisson accidents June 23, 2018 martial arts champion to challenge Kent Adonai over alleged 'stolen land' June 23, 2018

20. Noah Lyles runs year
June 23, 2018

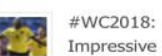
Impressive
Belgium put five past Tunisia June 23, 2018

\#WC2018: Hernandez nets as Mexico sees off South Korea une 23, 201

\#WC2018: Germany hit Germany hit dramatic late winner aganst sweden 


\section{NEMS}

\begin{tabular}{|c|c|c|c|c|c|c|c|}
\hline HOME & ABDUT & ALI NEWS & MEADUNES & FEATURES & ОРЕиок - & UVE WERTHER & CLASSIFIEDS \\
\hline
\end{tabular}

\section{PRESS RELEASE: Physical Activity Seminar hailed a success}

\section{By Sir Arthur Lewis Community College}

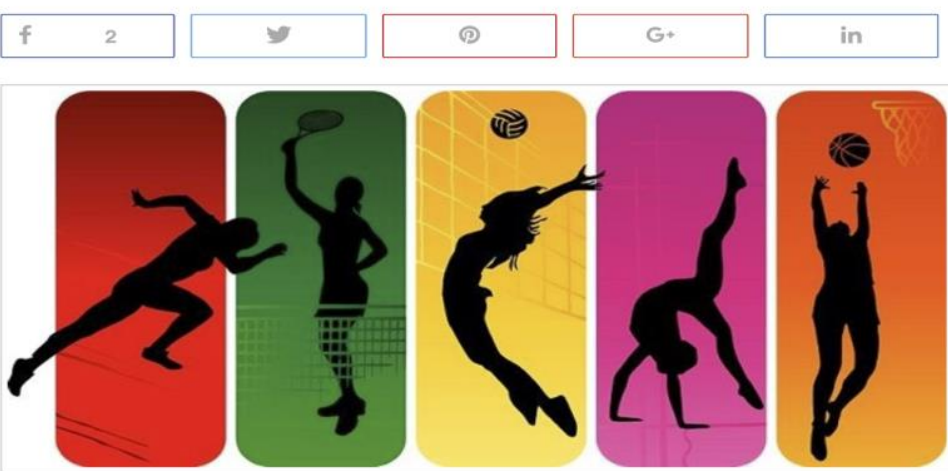

The Seminar on "Visualizing Physical Activity through 3D printing to get children moving more and sitting less." facilitated by Sam Crossley of Swansea University South Wales, UK on Tuesday 6 th June 2017 has been deemed a major success according to all attendees including Curriculum Officer for Physical Education and Sports at CAMDU, Dr. Veneta Lesporis and School Sports Coordinator, Mrs. Isabel Alexander-Marquis.

They were among students of St. Mary's College, staff of the Sir Arthur Lewis Community College and other sporting officials who listened attentively to the presentation on the scope of the research, its findings and implications for further analysis and intervention.

The research procedure utilized activity belts to record moderate and vigorous physical activity in subjects on a daily basis for a period of three months.

The data from the belts was then downloaded into a 3D Printer which produced a tangible 3D model. The models included bar graphs, animal shapes and other subject approved symbols which were adorned by the students to display their activity levels over a specific period.

Mr. Crossley explained that this initiative has great potential in motivating students to get active as well as increase their awareness of the importance of physical activity.

Questions following the presentation included gender inquiry, cultural significance of the research and feasibility for conducting similar exercises in the Caribbean Region. Vice Principal of SALCC, Dr. Merle St Clair-Auguste, highlighted the needfor the College to collaborate with more agencies to assist with the developmental needs of the country and praised the Office of the Governor General for initiating the seminar.

$3(1) \&(0)$

\section{Share This On:}

$$
\text { If } y \text { G. (1) } \bullet<<\text { More }
$$
This article was posted in its entirety as received by stlucianewsonline.com. This media house does not therein are not necessarily those of stlucianewsonline.com, its sponsors or advertisers.
Polls

Who should be blamed for the St. Jude Hospital problems?

The UWP

administration

The SLP

administration

Both SLP and UWP

administrations

vote

View Results

Polls Archive

\section{Recent Posts}

Brazilian court: Dog has visiting rights too

une 24, 2018

2. Ganja slipping out of Jamaica via courier services June 24, 2018

T\&T rocked by T2T rocked by quake

\begin{tabular}{l}
\hline Zimbabwe \\
President \\
Mnangagwa says \\
he was 'inches' from \\
Bulawayo explosion \\
June 24, 2018
\end{tabular}

[e. President Trump's press secretary kicked out of restaurant because she works for him 


\section{South Wales Evening Post: PRESS RELEASE}

\section{Bae Abertawe \\ Swansea Bay \\ You are here: Home " News » All News (General) » 3D printing can help curb childhood obesity according to Swansea experts \\ $15_{2016}^{\mathrm{sep}}$ \\ 3D printing can help curb childhood obesity according to Swansea experts}

\section{Swansea schoolchildren will be helping to shape the future as researchers believe 3D printing can help curb} childhood obesity.

In a bid to encourage children to be more active experts have used 3D printing to visualise youngsters heart rate during physical activities.

This could now be expanded and research teams will be going into schools in Swansea next year to see if they can build on the work done elsewhere.

Childhood obesity has reached epidemic proportions and the World Health Organisation has dubbed the issue as a major public health challenge of the 21 st century.

Kelly Mackintosh, senior lecturer in sports science, from Swansea University, and Melitta McNarry, who is also a senior lecturer in sports science, writing in a joint article for The Conservation, said that although parents had been glad with the way Pokemon Go had helped encourage their children to be active they said there was scope for a greater use of technology to encourage children to be active.

They said: "Recent advances in accessible technologies such as wearable activity monitors, pedometers and an array of online apps have provided lots of new opportunities to gain an insight into our daily physical activity levels.

"Reward systems such as goal setting, physical activity profiles, real-time feedback and social support networking are some of the top strategies that are helping keep people motivated and get more active.

"More specifically for children, visual stimulation through phone apps - such as Pokémon Go, Motion Maze, Zombies, Run! and ibitz for kids - are proving to have fantastic results in helping children stay more physically active and interested.

"This maintained interest is of paramount importance in light of recent statistics which suggest that approximately 30 per cent of children are overweight or obese in the UK.

"Furthermore, childhood obesity is known to track strongly into adolescence and adulthood - evidence suggests that 80 per cent of obese adolescents will become obese adults.

"In fact, studies show that if current trends continue, global obesity rates will reach 18 per cent in men and more than 21 per cent in women by 2025."

She added: "Our team has been looking into helping children get more active - with the help of 3D printers. The Exertion Games Lab in Melbourne was the first to use 3D printing to visualise heart rate during physical activities.

"The print-outs were used to provide feedback on intensity: the larger the spike, the higher the heart rate and therefore intensity of activity.

"Following on from this, our research team is now looking at different ways that children can see, feel and interact with a personal 3D model of their weekly physical activity.

"Early discussions with children, parents and teachers have provided many interesting, and creative models, in different shapes and styles.

"A selection of these models will be combined in the coming months to produce a final design that will be trialled in schools across Swansea, Wales, in 2017."

The article by Dr Kelly Mackintosh and Dr Melitta McNarry was originally published on http://theconversation.com">The Conversation. Read the https://theconversation.com/how-3d-printing-may-help-cut-childhood-obesity-by-getting-children-active-63533">original article. South Wales Evening Post

\section{Related Pages}
$\rightarrow$ News
Business Events
Bay of Life Newsletter
- Local Media
Sports Events 